\title{
Final Technical Report on STTR Project DE-FG02-06ER86281

\author{
Particle Tracking in Matter-Dominated Beam Lines \\ (G4beamline)
}

\section{Executive Summary}

This project has been for software development of the G4beamline [1] program, which is a particle-tracking simulation program based on the Geant4 toolkit [2], optimized for beam lines. This program can perform more realistic simulations than most alternatives, while being significantly easier to use by physicists. This project has fostered the general acceptance of G4beamline within the muon community, and has assisted in expanding its role outside that community. During this project, the G4beamline user community has grown from about a halfdozen users to more than 200 users around the world. This project also validated our business decision to keep G4beamline an open-source program, judging that an STTR project would provide more development resources than would marketing and selling the program. G4beamline is freely available to the physics community, and has been well validated against experiments and other codes within its domain. Muons, Inc. continues to support and develop the program, and a major part of the company's continued success and growth is directly related to our expertise in applying this program to interesting applications.

The basic software architecture of G4beamline was established before this project began. It is highly modular, well documented, and is an excellent software platform for further developments in the area of particle simulations. In particular, its modularity permits multiple developers to work completely independently on separate features. Highlights of developments during this project include:

- Tremendous expansion of the user base

- Support for installations on Windows and Mac OS X (initially it was Linux only)

- Greatly expanded User's Guide, including "tips and techniques”

- A graphical user interface

- Automatic tuning for many parameters of beamline elements

- Greatly expanded suite of tests (there are currently 90 regression tests)

- Developed the HistoRoot program to assist users in generating histograms

- Simplified the build procedure so users can easily build the program from source

- Implemented a direct interface to the NIST material database for common materials

- Visualization using Open Inventor, by far the most user-friendly driver available

- Extended the repertoire of solids

- Implemented a simple track-fitting algorithm for evaluating resolutions of detectors

- Added the ability to create movies of particle motions

- Implemented collective tracking by modifying the Geant4 kernel to track many particles in parallel, using steps in time (not space)

- Implemented two space charge computations (Lienard-Wiechert and a much more efficient Green's function convolution in the beam frame using FFTs)

- Implemented MPI for parallel computations

- Numerous minor new features and bug fixes 


\section{Final Technical Report on STTR Project DE-FG02-06ER86281 \\ Particle Tracking in Matter-Dominated Beam Lines (G4beamline)}

\section{Table of Contents}

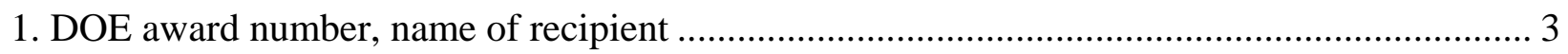

2. Project Title, Name of PI, Subgrant................................................................................. 3

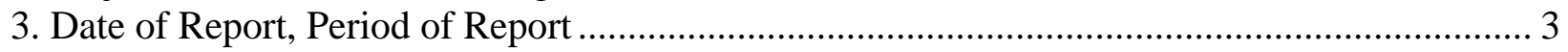

4. Comparison of Accomplishments and Goals.......................................................................... 3

5. Summary of Accomplishments and Project Activities .............................................................. 3

6. Products or Technology Transfer........................................................................................ 4

a. Publications and Conference Papers ..................................................................................... 4

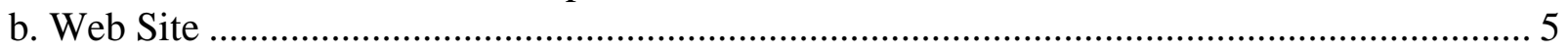

c. Networks or Collaborations Fostered ………………......................................................... 5

d. Technologies/Techniques ................................................................................................... 6

e. Inventions/Patent Applications ......................................................................................... 6

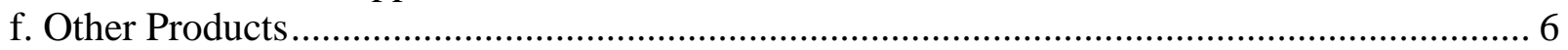

7. Computer Modeling Information ........................................................................................... 7

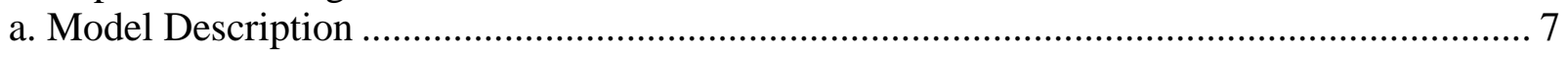

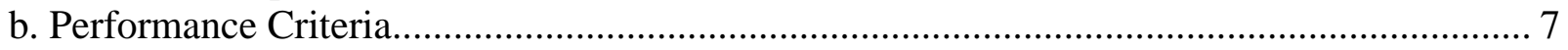

c. Test Results and Validation ........................................................................................... 7

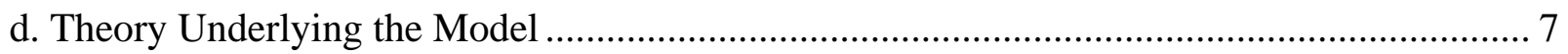

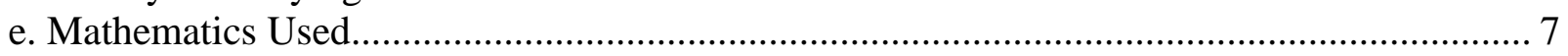

f. Summary of Theoretical Strengths and Weaknesses ................................................................ 7

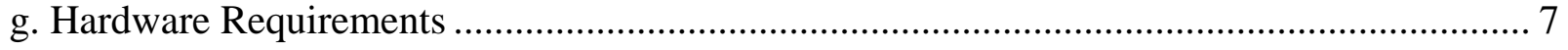

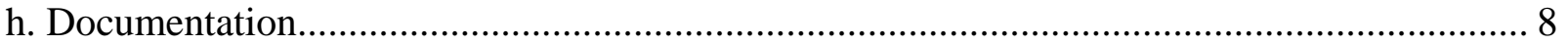

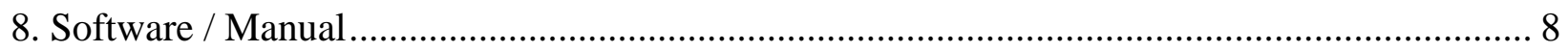

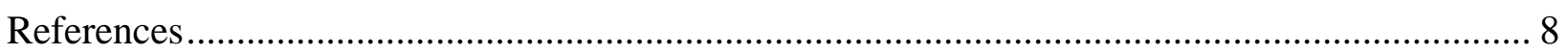

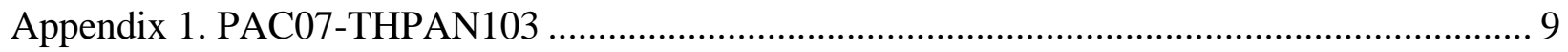

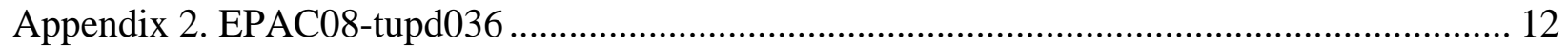

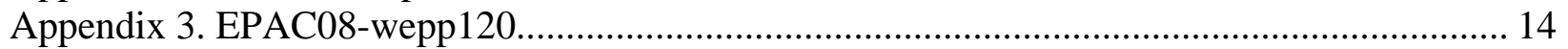

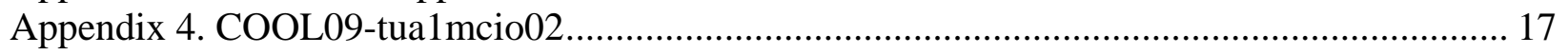

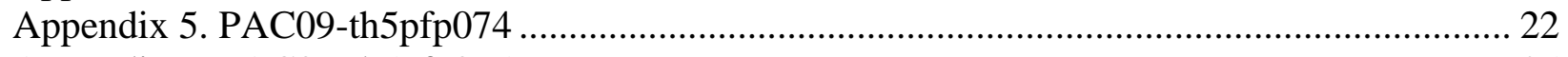

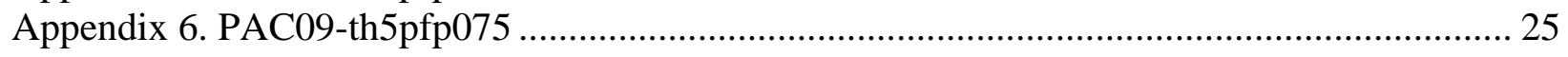

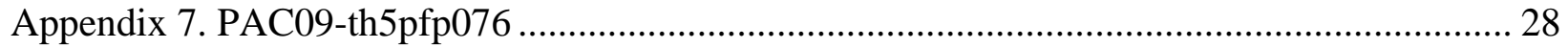

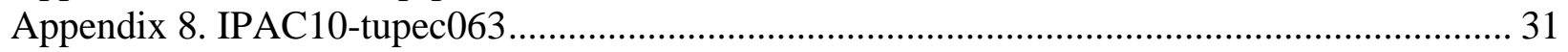

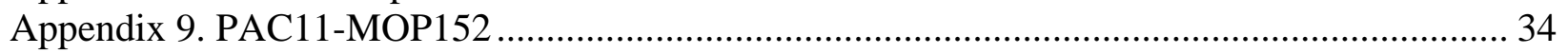

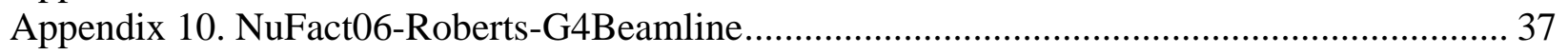

Appendix 11. NuFact09-wg3_roberts-missingprocesses............................................................... 52

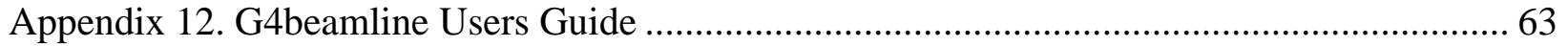

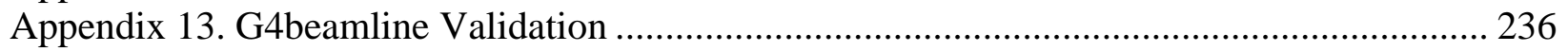




\section{Particle Tracking in Matter-Dominated Beam Lines (G4beamline)}

1. DOE award number: STTR Project DE-FG02-06ER86281, Name of Recipient: Muons, Inc.

2. Project Title: Particle Tracking in Matter-Dominated Beam Lines

Name of PI: Dr. Thomas J. Roberts

Research Partner: Illinois Institute of Technology

Name of JLab subgrant PI: Dr. Daniel M. Kaplan

3. Date of Report: May 16, 2011

Period of Report: July, 2006 to December, 2009

\section{Comparison of Accomplishments and Goals:}

All of the primary goals of this project have been met; some secondary ones have not.

The primary goal for Phase II of this project was the implementation of space charge computations. This turned out to be considerably more complicated than was initially estimated. As a result, there was no time left to implement the polarized muon physics processes. The very low energy physics processes (below $\sim 100 \mathrm{eV}$ ), proved to be far too complex, as they require a detailed enumeration of the properties and energy levels of every atom and molecule in the materials to be considered.

\begin{tabular}{|l|l|l|}
\hline Proposal & Technical Objective (Major) & Accomplishment \\
\hline \multirow{4}{*}{ Phase I } & Graphical User Interface & Complete \\
\cline { 2 - 3 } & $\begin{array}{l}\text { User-friendly package for creating Root } \\
\text { histograms }\end{array}$ & Complete, HistoRoot is available \\
\hline \multirow{3}{*}{ Phase II } & Space charge effects & $\begin{array}{l}\text { Complete, two different } \\
\text { implementations }\end{array}$ \\
\cline { 2 - 3 } & Parallelization of tracking & Complete, using MPI \\
\cline { 2 - 3 } & Low energy processes & $\begin{array}{l}\text { Partial implementation, valid down } \\
\text { to a few hundred eV }\end{array}$ \\
\cline { 2 - 3 } & Muon polarized physics & Not begun \\
\hline Both & Support and enhance G4beamline & $\begin{array}{l}\text { Complete during project, ongoing } \\
\text { afterward }\end{array}$ \\
\hline
\end{tabular}

\section{Summary of Accomplishments and Project Activities:}

- During the course of this project, the G4beamline user base expanded greatly, from about a half-dozen users to more than 200.

- G4beamline is now supported for installation on:

o Linux (Red-Hat derived systems such as Scientific Linux 4.0 and later)

o Windows (Xp, Vista, and Windows 7)

o Mac OS X (10.5 (Leopard) and 10.6 (Snow Leopard)), Intel processors

0 Other systems by building from source

- The user documentation has been expanded, including an extensive "tips and techniques" section in the User's Guide. 


\section{Particle Tracking in Matter-Dominated Beam Lines (G4beamline)}

- The Graphical User Interface has been implemented and extended, and the HistoRoot program was developed to assist users in creating Root histograms. Screenshots of both appear on the first page of Appendix 2.

- This project supported two postdocs at IIT, Shahid Ahmed and Dazhang Huang.

- During this project we submitted seven bug reports to the Geant4 collaboration, and attended every Geant4 user forum. We considered joining the Geant4 collaboration, but they have no mechanism to include a corporation (only universities and national labs).

- G4beamline has been enhanced and supported, including many new features:

0 Automatic tuning for many parameters of beamline elements

o Extended suite of regression tests

o Simplified build procedure, making building from source easy for users

o Direct interface to the NIST materials database for common materials

o Visualization using Open Inventor, the most user-friendly driver available

o Expanded repertoire of solids

o Simple track-fitting algorithm for evaluating the resolutions of detectors

o Ability to create movies of particle motions

o Numerous minor new features and bug fixes

- Collective tracking was implemented by replacing much of the Geant4 kernel, so an arbitrary number of tracks are tracked in parallel for individual time steps. This permits any number of collective computations to be implemented at each time step, such as space charge, wake fields, beam-beam interactions, etc.

- Four independent implementations of space charge computations were built, based on the collective computation infrastructure. The two best implementations were selected for release:

o "spacechargelw" is a command that implements the Lienard-Wichert E and B fields, which are added in to the Geant4 global field routine, and are thus used in the tracking of particles. This computation scales as (number of particles) ${ }^{2}$, and becomes computationally infeasible for more than $\sim 1,000$ particles.

o "spacecharge" is a command that implements solving Poisson's equation on a grid in the beam frame, using a convolution of the Green's function via FFTs. This is far more efficient computationally, and can be used for up to about a million particles.

The advantage of having two independent implementations is that the L-W computation is rigorously correct, and can be used to verify the validity of the far more efficient Green's function computation.

- Parallelization of tracking was implemented using MPI, the message-passing interface that has become the standard for parallel computations on clusters and supercomputers.

- The low-energy processes implemented by the Geant4 collaboration have been incorporated into G4beamline, providing electromagnetic interaction models valid down to $\sim 100 \mathrm{eV}$ (and valid to $>100 \mathrm{TeV}$ ).

\section{Products or Technology Transfer:}

The primary product of this project is the G4beamline program, which is freely available for download from our web site http://g4beamline.muonsinc.com/. In addition, we have presented 11 conference papers on G4beamline, which has served as primary advertising of its capabilities.

\section{a. Publications and Conference Papers:}




\section{Particle Tracking in Matter-Dominated Beam Lines (G4beamline)}

Copies of these papers are provided in the listed appendix.

\begin{tabular}{|c|c|c|}
\hline Appendix & Conference & Title \\
\hline 1 & $\begin{array}{l}\text { PAC07, Albuquerque, NM, } \\
\text { June 2007, IEEE, APS, LANL }\end{array}$ & $\begin{array}{l}\text { "G4BEAMLINE SIMULATION PROGRAM } \\
\text { FOR MATTER-DOMINATED BEAMLINES” }\end{array}$ \\
\hline 2 & \multirow[t]{2}{*}{$\begin{array}{l}\text { EPAC08, Genoa, Italy, June } \\
\text { 2008, EPS-AG, IEEE, APS }\end{array}$} & $\begin{array}{l}\text { “G4BEAMLINE SIMULATIONS FOR } \\
\text { DETECTOR DEVELOPMENT” }\end{array}$ \\
\hline 3 & & $\begin{array}{l}\text { "G4BEAMLINE PARTICLE TRACKING IN } \\
\text { MATTER-DOMINATED BEAM LINES" }\end{array}$ \\
\hline 4 & $\begin{array}{l}\text { COOL09, Lanzhou, China, } \\
\text { Sept. 2009, IMP and CAS }\end{array}$ & $\begin{array}{l}\text { "STUDY OF COLLECTIVE EFFECT IN } \\
\text { IONIZATION COOLING" }\end{array}$ \\
\hline 5 & \multirow{3}{*}{$\begin{array}{l}\text { PAC09, Vancouver, Canada, } \\
\text { May 2009, IEEE, APS, } \\
\text { TRIUMF }\end{array}$} & $\begin{array}{l}\text { "PUTTING SPACE CHARGE INTO } \\
\text { G4BEAMLINE” }\end{array}$ \\
\hline 6 & & $\begin{array}{l}\text { "SIMULATION TOOLS FOR THE MUON } \\
\text { COLLIDER DESIGN FEASIBILITY STUDY” }\end{array}$ \\
\hline 7 & & $\begin{array}{l}\text { "PARTICLE TRACKING IN MATTER } \\
\text { DOMINATED BEAM LINES” }\end{array}$ \\
\hline 8 & $\begin{array}{l}\text { IPAC10, Kyoto, Japan, Aug. } \\
\text { 2010, IEEE, APS, Univ. Kyoto } \\
\end{array}$ & $\begin{array}{l}\text { "PARTICLE TRACKING IN MATTER } \\
\text { DOMINATED BEAM LINES” }\end{array}$ \\
\hline 9 & $\begin{array}{l}\text { PAC11, New York, NY, April } \\
\text { 2011, IEE, APS, BNL } \\
\end{array}$ & $\begin{array}{l}\text { “G4BEAMLINE PARTICLE TRACKING IN } \\
\text { MATTER DOMINATED BEAM LINES” }\end{array}$ \\
\hline 10 & $\begin{array}{l}\text { NuFact06, Irvine, CA, Aug. } \\
\text { 2006, UCI }\end{array}$ & $\begin{array}{l}\text { "G4Beamline - A 'Swiss Army Knife' for } \\
\text { Geant4” }\end{array}$ \\
\hline 11 & $\begin{array}{l}\text { NuFact09, Chicago, IL, July } \\
\text { 2009, IIT }\end{array}$ & $\begin{array}{l}\text { "Physics Processes Missing from our Current } \\
\text { Simulation Tools" }\end{array}$ \\
\hline
\end{tabular}

b. Web Site: http://g4beamline.muonsinc.com/

G4beamline is an open-source project supported by Muons, Inc. The website includes:

- A general description of the program

- A videotape of a G4beamline tutorial given at Fermilab

- A screen-cast of using G4beamline for a simple example

- A movie of particle transport, created by G4beamline

- Downloadable distributions for:

o Windows (Windows Xp, Windows Vista, and Windows 7)

o Linux (Scientific Linux 4, and later RedHat-derived distributions)

o Mac OS X (10.5 [Leopard] and later), Intel CPUs

o Complete program source (including non-system libraries)

All distributions include a set of examples, the suite of $>80$ tests, the "G4beamline User's Guide”, and the "G4beamline Validation” document; the source distribution includes Doxygen [3] documentation of the G4beamline source code.

\section{c. Networks or Collaborations Fostered:}




\section{Particle Tracking in Matter-Dominated Beam Lines (G4beamline)}

All of the above conference papers were presented as posters at the conference. This has proven to be an excellent way to advertise the capabilities of G4beamline, to interact with current users, and to attract new users. Hundreds of people have expressed interest during these conferences, and many have become actual users of G4beamline.

\section{d. Technologies/Techniques:}

The technologies used in G4beamline are the usual techniques of software development, plus well-known algorithms for particle tracking and the simulation of physics processes. G4beamline improves and enhances these in the following ways:

- Excellent user interface: Programs written by physicists often have difficult or arcane user interfaces; G4beamline stands out in that its user interface was designed by an expert, providing on-line help, self-documenting input, commonality of design, and an input file describing the system that is readable without reference to detailed documentation (everything is named, not represented by arbitrary small integers).

- Excellent user documentation: The G4beamline User's Guide is comprehensive and written from the perspective of a user (not that of a code developer).

- Complete internal documentation: Doxygen [3] is used to provide developersearchable hyperlinked documentation for every class and function in the code. All code adheres to our coding standards, and algorithms that are not obvious are fully commented.

- Highly modular code: by separating features into individual commands that implement them, multiple developers can work in parallel on independent features; this works so well that no code management system is needed to manage parallel developments.

- Modular physics processes: As in Geant4, multiple physics processes can be attached to any particle to be applied while tracking that particle; similarly, multiple collective computations can be registered to apply to the entire ensemble of tracks in collective mode.

\section{e. Inventions/Patent Applications:}

Most of Muons, Inc. inventions are particularly useful for large projects built by the US Government, which will have rights to our inventions as part of SBIR-STTR agreements. It is difficult to imagine commercially important applications for muon colliders in the time frame of a patent. We are pleased, however, to contribute to the progress toward the energy frontier, which has tremendous importance to humanity as a source of fundamental knowledge of our universe.

G4beamline has many applications to other facilities. However, the community it serves is rather small and generally unwilling to spend large amounts on such software. Our decision to keep G4beamline as an open-source project was based on our judgment that this STTR program would provide considerably more development resources than would charging users for its use.

\section{f. Other Products:}

The source code, executable objects, and documentation for G4beamline are described in sections 7 and 8 below. 
Particle Tracking in Matter-Dominated Beam Lines (G4beamline)

\section{Computer Modeling Information}

G4beamline is a particle-tracking simulation program based on the Geant4 toolkit, optimized for beam lines.

\section{a. Model Description}

G4beamline uses the Geant4 toolkit [2] for most modeling, and therefore implements most of what is known about the interactions of elementary particles with matter. Details are given in the Geant4 website [2] and in the G4beamline User’s Guide (Appendix 12).

\section{b. Performance Criteria}

The performance of G4beamline is highly dependent on the complexity of the system being simulated. On a modern system, it can range from 5,000 events per second for a small and simple system, to several seconds per event for a large and complex one. As G4beamline implements a realistic model of the system, and tracks particles individually, it runs considerably slower than programs that use approximations such as envelope calculations, matrix-based tracking, and simple elements (e.g. no fringe fields, or only impulse approximations to them).

\section{c. Test Results and Validation}

G4beamline has been extensively tested and validated against experiments and other codes. The results are given in the "G4beamline Validation” document (Appendix 13).

\section{d. Theory Underlying the Model}

See the Geant4 website [2], especially the Physics Manual.

\section{e. Mathematics Used}

See the Geant4 website [2], especially the Physics Manual.

\section{f. Summary of Theoretical Strengths and Weaknesses}

See the Geant4 website [2], especially the Physics Manual.

\section{g. Hardware Requirements}

G4beamline is distributed to run on the following systems:

- Windows (Windows Xp, Windows Vista, and Windows 7)

- Linux (Scientific Linux 4, and later RedHat-derived distributions)

- Mac OS X (10.5 [Leopard] and later), Intel CPUs

Most modern computers can run one of the above distributions; the restrictions are primarily based on the software and operating system, not the hardware. For computer systems other than 
Particle Tracking in Matter-Dominated Beam Lines (G4beamline)

the above, the source distribution can be built, given appropriate compilers and software components (the details are given in the README files of the distributions).

\section{h. Documentation}

The primary documentation of G4beamline is its User’s Guide (Appendix 12). Internal documentation of the code is contained in the Doxygen documentation, which is included in the source distribution.

\section{Software / manual}

The G4beamline User’s Guide is appended to this report as Appendix 12. It is a 173-page manual on the use of G4beamline, from a user perspective.

It is infeasible to attach the source code and executable files to this report; they total well over 200 Megabytes of compressed information. Older releases more than triple this number. A CDROM containing the latest release (version 2.08) will be sent separately. Note, however, that the source code, executable object code, data files, and documentation are all available for download from our web site, http://g4beamline.muonsinc.com, and will remain so for the foreseeable future.

\section{References}

[1] G4beamline - http://g4beamline.muonsinc.com

[2] The Geant4 toolkit - http://geant4.cern.ch

[3] Doxygen - http://doxygen.org 


\title{
G4BEAMLINE SIMULATION PROGRAM FOR MATTER-DOMINATED BEAMLINES*
}

\author{
Thomas J. Roberts ${ }^{\#}$, Muons, Inc., Batavia, IL, U.S.A. \\ Daniel M. Kaplan, Illinois Institute of Technology, Chicago, IL, U.S.A.
}

\section{Abstract}

G4beamline is a single-particle simulation program optimized for the design and evaluation of beam lines. It is based on the Geant4 toolkit [1], and can implement accurate and realistic simulations of particle transport in both EM fields and matter. This makes it particularly well suited for studies of muon collider and neutrino factory design concepts. G4beamline includes a rich repertoire of beamline elements and is intended to be used directly, without $\mathrm{C}^{++}$programming, by accelerator physicists. The program has been enhanced to handle a large class of beamline and detector systems, and is available on Linux, Windows, and Macintosh platforms.

\section{INTRODUCTION}

Potential new facilities such as neutrino factories and muon colliders present new and interesting problems in accelerator physics involving both the decay of unstable particles and the traversal of matter. Realistic modeling of such systems requires the simulation of individual particle behavior in both matter and electromagnetic fields. As most accelerator physics programs handle neither decays nor materials, G4beamline has been developed to provide a flexible, user-friendly program to perform such simulations without user programming. By using the Geant4 toolkit [1], it incorporates most of what is known about the interactions of particles in matter, as well as their decays and behavior in EM fields.

\section{DESCRIPTION}

A key advantage of using G4beamline is that its description of the simulation is commensurate with the complexity of the system being simulated, instead of being a significantly more complicated custom $\mathrm{C}^{++}$ program. Other important aspects are:

- A physicist-readable ASCII file to specify the simulation, with auxiliary files for field maps, etc.

- A rich repertoire of beamline elements that can be combined to define new and customized elements

- A general set of initial beam specifications (including a cosmic-ray muon "beam" and external files)

- Beam tracks can be input and output using several formats including ASCII and Root [2] files

- Many parameters can be automatically tuned (RF cavity timing and gradients, bending magnet fields, etc.)

\footnotetext{
*Supported by DOE STTR grant DE-FG02-6ER86281

\#tjrob@muonsinc.com
}

- Visualization of the system is included, using many viewers (OpenGL, VRML, Open Inventor, etc.)

- Support for parallel jobs on multiple CPUs

- The historoot program, which makes it easy for nonexperts to generate Root histograms and plots

The basic structure of a simulation is to first define the beamline elements to be used (magnets, RF cavities, etc.), including their geometry, materials, and local fields. Then these elements are placed into the world, usually along the nominal beam centerline; each placement can have a position, rotation, and its own field value. Parameters for the elements can be defined in the input file or on the command line, so scripting is straightforward. Individual particles can be traced, beam profiles can be generated and displayed, and virtual detectors can be used to sample the beam at any point.

The tracking of particles through the simulated system is as accurate and realistic as the Geant4 toolkit implements. The input file selects from any of the Geant4 physics use cases, and can set values for the various Geant 4 tracking-accuracy parameters. This permits users to make trade-offs between CPU time and simulation accuracy. Similarly, G4Beamline permits the specification of magnetic map parameters, permitting a trade-off between memory usage (and the CPU time to generate the map) and simulation accuracy.

While G4Beamline can make it rather simple to specify a simulation, it cannot substitute for knowledge and experience about the problem domain or about particletracking simulations in general. Like all computer programs, G4Beamline is prone to "garbage in, garbage out", especially when used by unskilled users. It is strongly suggested that visualization be used to verify the geometry of the simulation and that a handful of particles be tracked properly through it. Whenever possible one should arrange to track through a simple geometry that can be compared to independent results, to make sure that what one thinks is happening actually does occur in the simulation.

\section{EXAMPLES}

Figures 1-7 show the G4beamline graphical user interface and some of the many systems that have been simulated. 


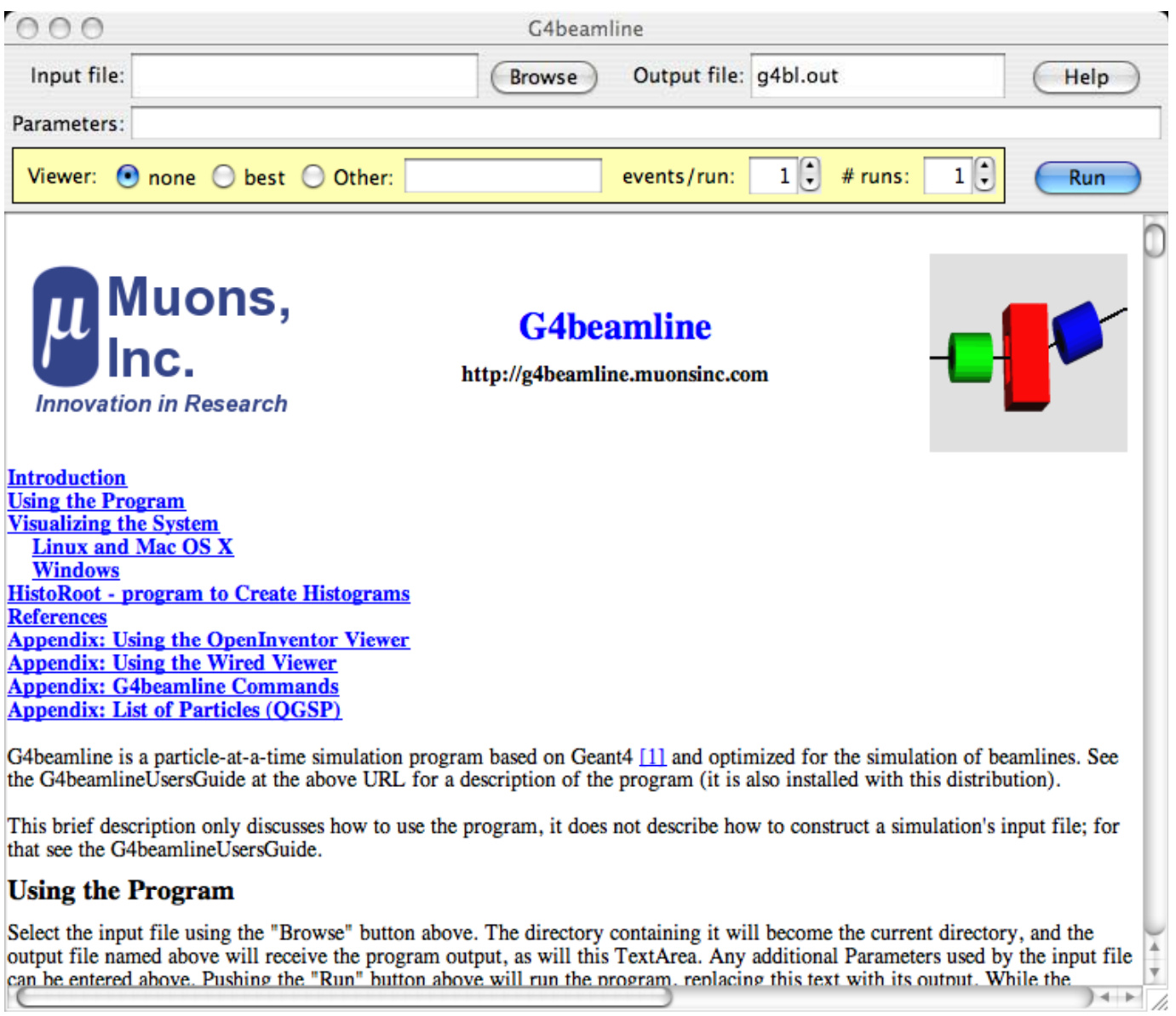

Figure 1: The G4beamline graphical user interface.

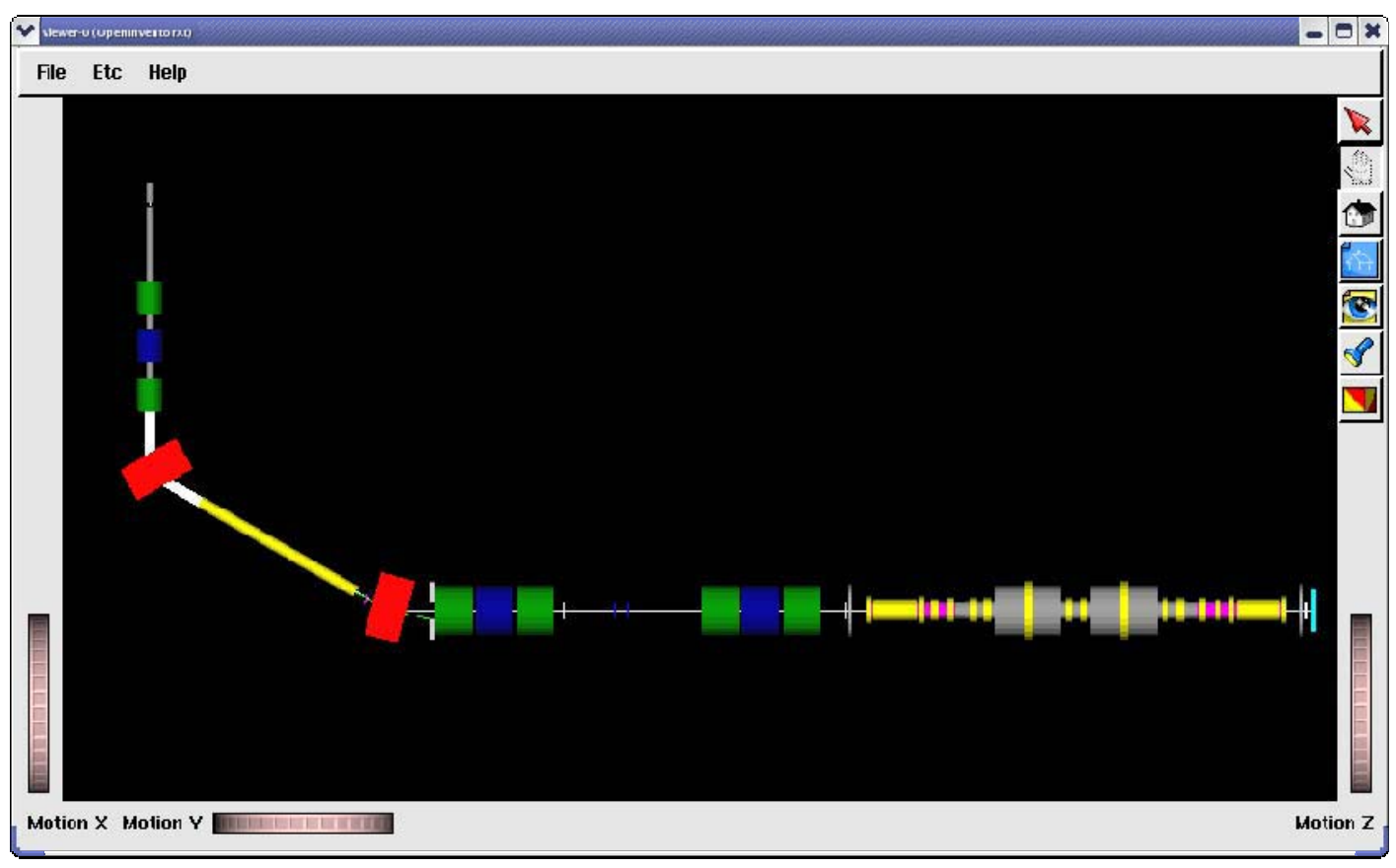

Figure 2: The MICE muon beamline and cooling channel [3] - a detailed and realistic simulation using G4beamline. Quadrupoles are green (HF) and blue (HD), dipoles are red, solenoids are yellow, beam pipes are gray, vacuum chambers are white, the two RF cavities are gray, and the calorimeter at the end is light blue. The pion production target is at the top left inside the ISIS synchrotron (not shown). 


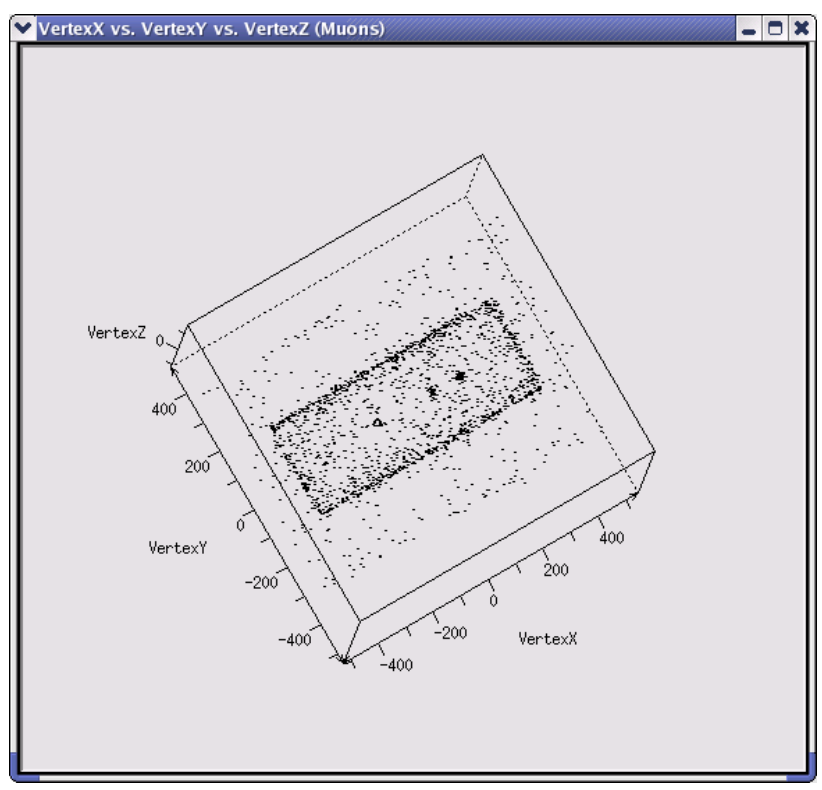

Figure 3: Cosmic Ray muons scanning a shipping container.
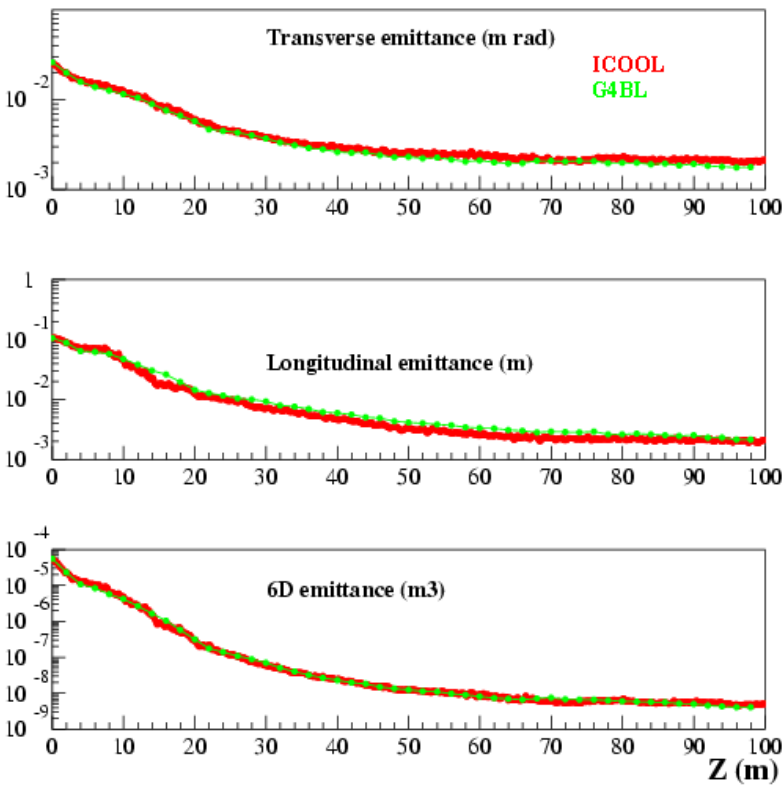

Figure 4: Emittance evolution in a Helical Cooling Channel.

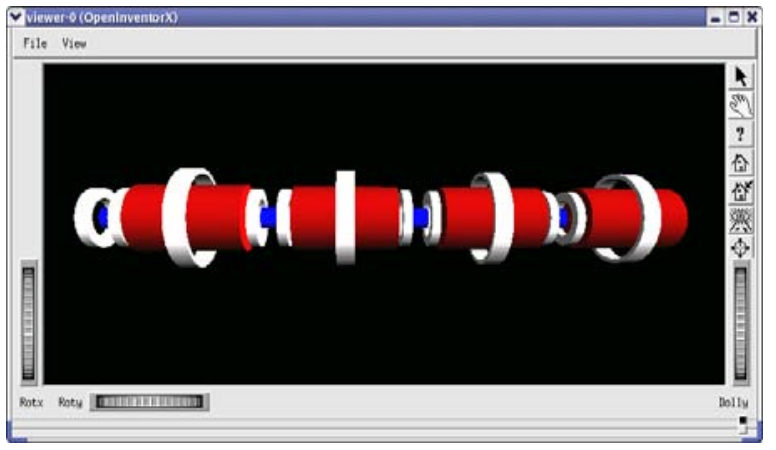

Figure 5: A section of the Study 2 SFOFO cooling channel. Solenoids are white, RF cavities are red, and the liquid hydrogen absorbers are blue.

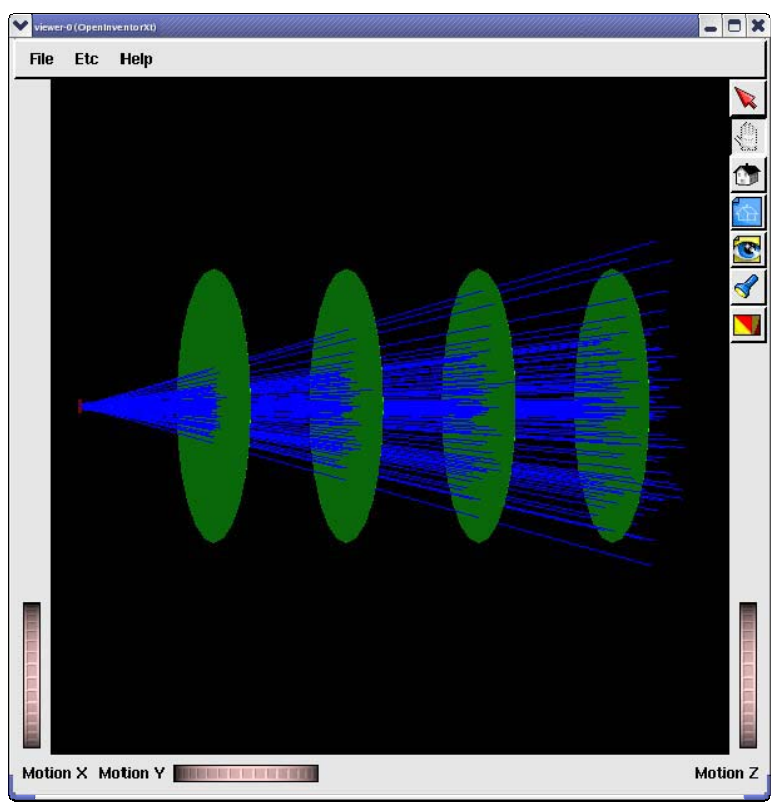

Figure 6: example1.in - a simple Gaussian beam into four circular detectors.

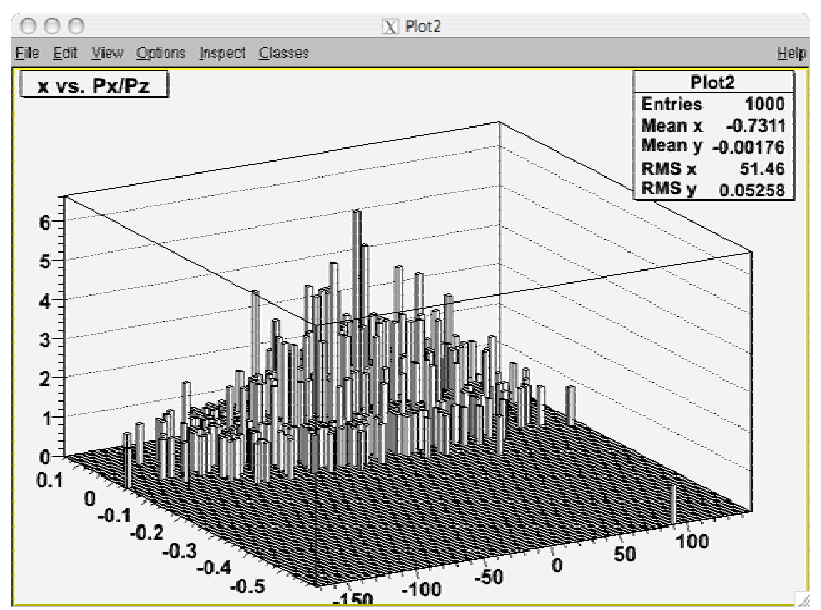

Figure 7: A 2-D histogram showing the outlier event that greatly affects the emittance computation.

\section{SUMMARY}

G4beamline has been in use for several years by a halfdozen research groups to simulate several dozen different systems. It has achieved its goal of being easy to use, and the learning curve for experienced accelerator physicists is rather short for a program of this scope. With sufficient attention to detail, it is possible to make the simulation quite realistic. The development of G4beamline is ongoing, and its features and facilities are expanding. Comments and suggestions from potential or actual users are welcome (see http://g4beamline.muonsinc.com).

\section{REFERENCES}

[1] http://geant4.cern.ch.

[2] http://root.cern.ch.

[3] http://mice.iit.edu 


\title{
G4BEAMLINE SIMULATIONS FOR DETECTOR DEVELOPMENT*
}

\author{
Thomas J. Roberts, Kevin Beard (Muons, Inc., Batavia, Illinois, USA 60510)
}

\section{Abstract}

The G4beamline program [1] is a useful and steadily improving tool to quickly and easily model beamlines and experimental equipment without user programming. As it is based on the Geant4 toolkit [2], G4beamline includes most of what is known about the interactions of particles and matter, including time-varying electromagnetic fields. We are continuing the development of G4beamline to extend its domain to include detector systems. G4beamline is open source and is freely available at http://g4beamline.muonsinc.com.

\section{INTRODUCTION}

G4beamline [1] is a general and flexible tool for the simulation and evaluation of beamlines and related systems, using single particle tracking. The Geant 4 toolkit [2] has proven to be an excellent choice for the basis of the program, as it is comprehensive, accurate, and actively supported by a strong and vibrant collaboration. Unlike many physics programs, particular attention has been paid to G4beamline's user interface, with the result that it has a considerably shorter learning curve than most comparable programs. Most accelerator physicists can read and understand its description file without reference to the documentation, and can learn how to develop their own simulations with minimal effort. Extensive online help is available within the program to assist users in developing their simulations. Figure 1 shows the G4beamline graphical user interface (GUI) screen, including an index and the beginning of its Help text.

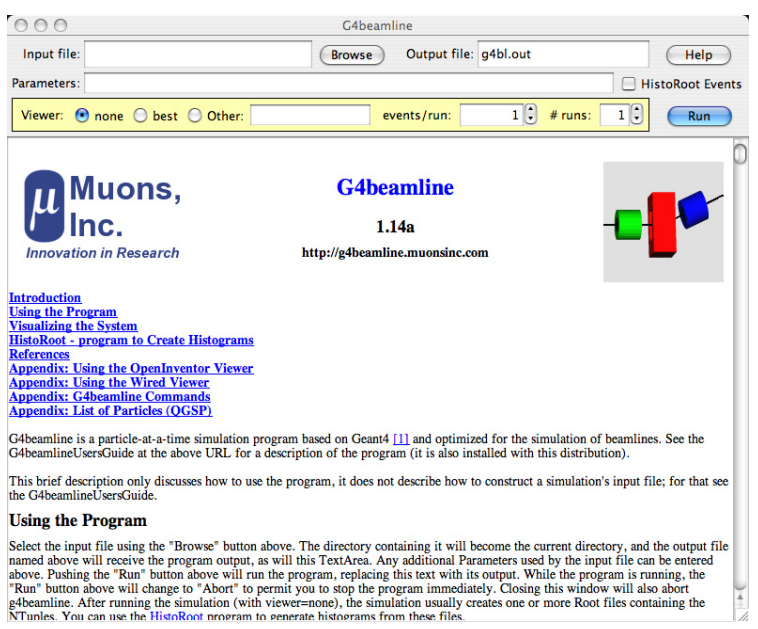

Figure 1 : The G4beamline GUI screen.

To facilitate the generation of histograms and plots, the G4beamline distribution includes the historoot program that provides a user-friendly graphical interface to Root [3]. While general Root programming can be used to

* Supported in part by DOE STTR grant DE-FG02-06ER86281. create plots, most users find the interface shown in Figure 2 to be more usable and efficient.

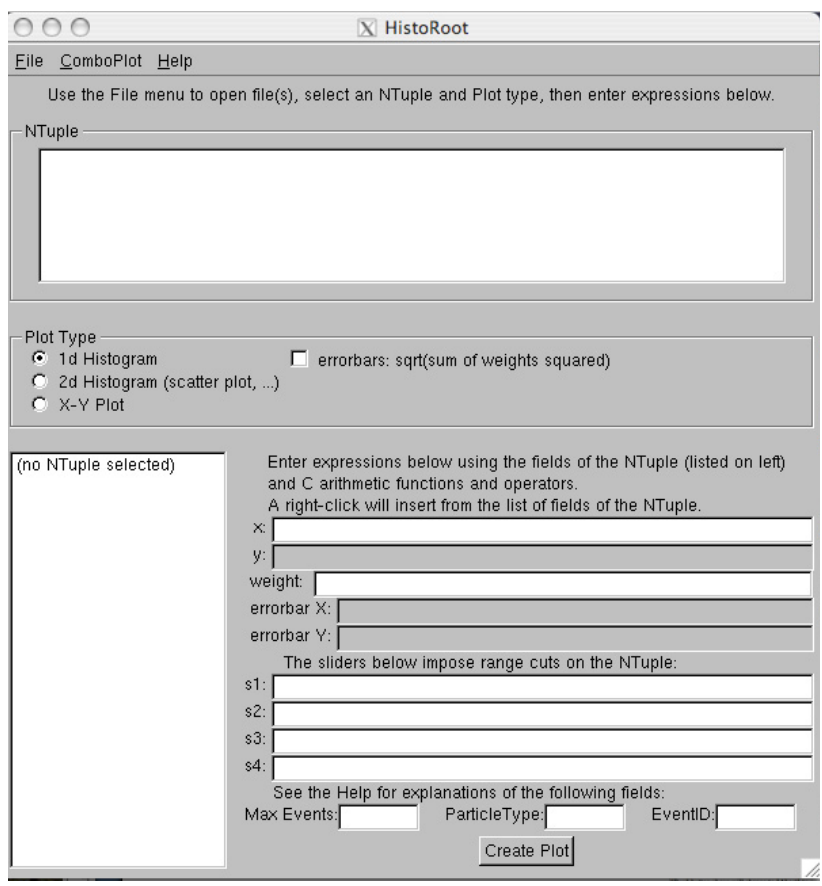

Figure 2 : The historoot GUI screen.

At present, G4beamline has extensive features for specifying and simulating beamlines, but cannot simulate detectors or include their data into its output file. Our plans include extending the program to interface to the Geant4 facilities for detectors: active volumes, hits, and digitization. These capabilities will enable the user to generate a Root output file that is similar in many ways to the data files from actual experiments. In addition, we will interface to the existing Geant4 processes for optical photons and very low energy physics, extending them where necessary to be able to simulate the inner workings of a large class of detectors.

\section{DESCRIPTION}

As the G4beamline code was designed from its inception to be easily extensible, we can add these new features to it without disturbing its current capabilities, or forcing existing users to change their input decks. This is easily accomplished by simply implementing the new features in new commands; adding new commands merely requires deriving a new class for each of them from the appropriate base class, and putting their source code into the proper directory. In particular, no changes to existing code are required to add new commands (the list of commands is dynamically built from the files located in the source directory). There will be a new infrastructure 
required to implement the expanded Root output file capabilities.

Another common feature of detectors is that they can be segmented, with a separate readout per segment. To make it easy to simulate a large number of identical detectors (a multi-wire proportional chamber, a scintillating fiber hodoscope, etc.), an interface to the Geant4 "parameterized placement" will be implemented. The user will define a single wire or fiber, and then use a single command to place many copies into a single plane or unit. The output file will include identification of which detector was hit, as well as position information, energy deposited, timing information, etc.

Initially we will focus on three detector types during the implementation of the new capabilities:

1. A bulk detector that responds to the energy deposited in its volume (e.g. a Sodium Iodide crystal).

2. A plane of identical detectors that respond with individual hits (e.g. a proportional wire chamber).

3. A new type of fast timing detector with better than 5 picoseconds resolution (see below).

In the style of G4beamline, these will all be extensively configurable by the user, but with sensible defaults to simplify learning how to use them.

\section{BULK DETECTOR}

This will be a generic detector that responds with a pulse-height output related to the energy deposited in the detector volume. Initially it will have a simple, userconfigurable mapping from energy deposition to pulse height to permit the code to be used for various physical situations. Relaxation time of the detector will also be user configurable.

\section{PLANE OF IDENTICAL DETECTORS}

This will be a generic detector consisting of a plane of identical segments that responds with a list of hit segments. It will have a user-configurable probability of reporting a hit based on the number of charged particles that traverse each individual segment's volume. That will permit the code to be used for different detector technologies (e.g. multi-wire proportional chambers, scintillating fiber hodoscopes, etc.).

\section{FAST TIMING DETECTOR}

Initial prototype work by an informal collaboration [4] has shown that it is feasible to develop detectors for charged particles that have considerably better timing resolution than current detectors: resolutions less than 5 picoseconds appear likely. This technology is based on a micro-channel plate coupled to a collection anode; when combined with integrated readout electronics it offers the possibility of segmentation in two dimensions with pixels on the order of half a centimeter square (and perhaps smaller). To optimize the design, detailed simulations of the detector are required; this includes particle simulations as well as electronics simulations that will be performed separately. One of the key enablers of this technology is the ability to design a custom ASIC with the bandwidth to implement multiple TDCs with picosecond resolution. The basic concept is shown in figure 3 .

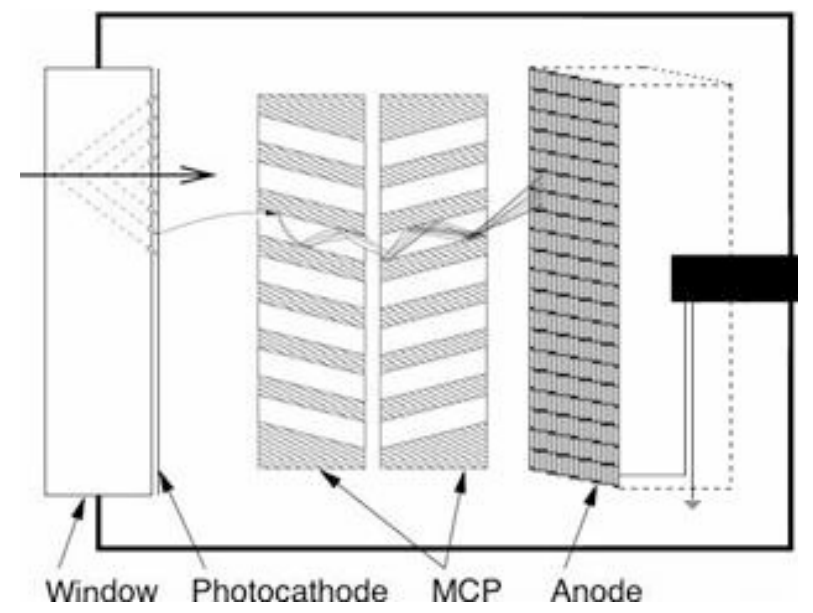

Figure 3 : Schematic of the fast timing detector. A relativistic charged particle produces Cherenkov light in the window. This radiation is converted into electrons by a photocathode. The electrons produce a shower in the micro-channel plates (MCP), and the electrons are deposited on the segmented anode to be detected. Not shown: the anode has equal-time connections from each segment to the output. The drawing is not to scale.

\section{SUMMARY}

As the Geant4 toolkit already has most of the capabilities and physics processes required, the actual software development required for these enhancements will be rather modest. The new capabilities will permit G4beamline to simulate a modest particle experiment (simple trigger, tracking, particle ID, basic calorimetery), as well as the beamlines and systems that it already handles. In addition, it will become possible to simulate the inner workings of a large class of interesting detectors.

\section{REFERENCES}

[1] G4beamline - http://g4beamline.muonsinc.com

[2] Geant4 Toolkit - http://geant4.cern.ch

[3] Root - http://root.cern.ch

[4] Fast Timing Workshop http://www.hep.anl.gov/ertley/tof/talks.html 


\section{G4BEAMLINE PARTICLE TRACKING IN MATTER-DOMINATED BEAM LINES}

Thomas J. Roberts, Kevin Beard (Muons, Inc., Batavia, Illinois, USA 60510), Dazhang Huang, Shahid Ahmed, Daniel M. Kaplan, Linda Spentzouris, (Illinois Institute of Technology, Chicago, Illinois, USA 60616)

\section{Abstract}

The G4beamline program [1] is a useful and steadily improving tool to quickly and easily model beamlines and experimental equipment without user programming. Unlike most accelerator physics codes, it easily handles a wide range of materials and fields, being particularly well suited for the study of muon and neutrino facilities. As it is based on the Geant4 toolkit [2], G4beamline includes most of what is known about the interactions of particles and matter. We are continuing the development of G4beamline to facilitate its use by a larger set of beam line and accelerator developers. It is open source and freely available at http://g4beamline.muonsinc.com.

\section{INTRODUCTION}

As accelerator facilities get more complex and more expensive, accurate and comprehensive simulations of their performance are required long before construction begins. There are many choices and optimizations to be made, and new concepts to be explored, so flexible and user-friendly simulation programs become essential to streamline the design process. For future facilities such as muon colliders and neutrino factories, the muon cooling sections demand simulations that accurately compute the interactions of particles in matter, along with associated magnetic and RF fields. The Geant4 toolkit [2] is an excellent choice as the basis of such a program, as it is comprehensive, accurate, and actively supported by a vibrant collaboration. G4beamline [1] was conceived as a user-friendly interface between accelerator physicists and the $\mathrm{C}++$ code of Geant 4 - to facilitate the frequent evaluation of new concepts and design changes by physicists, without the complexities of $\mathrm{C}++$ programming. An important aspect of G4beamline is that its description of the simulated system is far more understandable by physicists than the corresponding Geant $4 / \mathrm{C}++$ code would be.

\section{DESCRIPTION}

An obvious aspect of G4beamline is that its user interface has been designed with physicist-users in mind. The system to be simulated is described in a single ASCII file using an object-oriented language specifically designed for this application. Most accelerator physicists can read and understand such a description file without reference to the G4beamline documentation, and can learn how to develop their own simulations with minimal effort. Extensive online help is available within the program to assist users in developing their simulations. Figure 1 shows the G4beamline graphical user interface (GUI) screen, including an index and the beginning of its Help text.

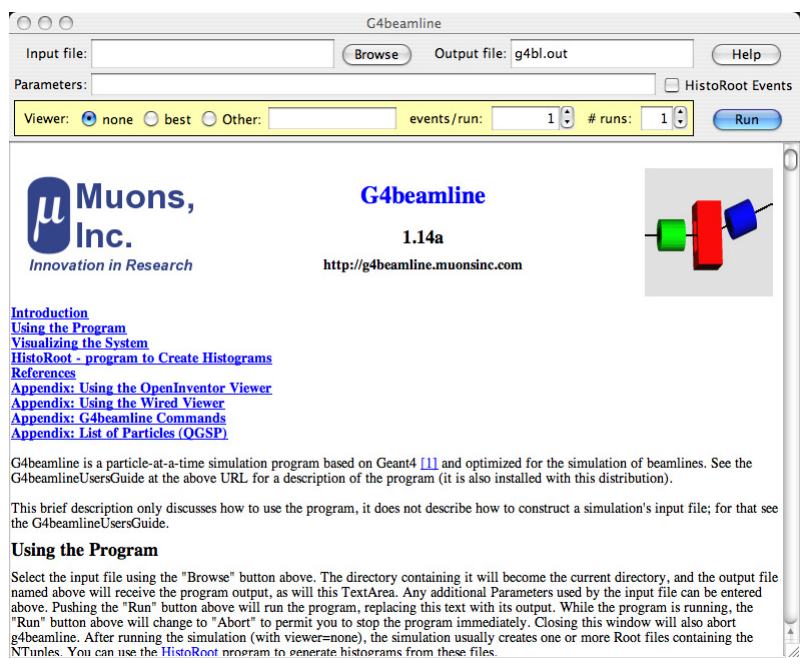

Figure 1. The G4beamline GUI screen.

To facilitate the generation of histograms and plots, the G4beamline distribution includes the historoot program that provides a user-friendly graphical interface to Root [3]. While general Root programming can be used to create plots, most users find the interface shown in Figure 2 to be more usable and efficient.

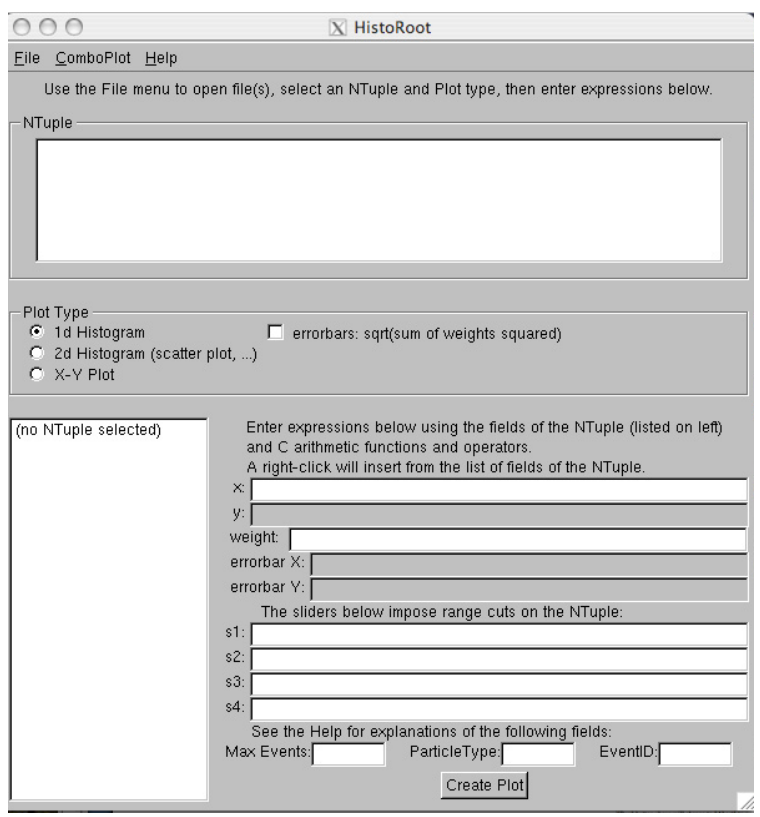

Figure 2. The historoot GUI screen.

A09 Muon Accelerators and Neutrino Factories 
The major aspects of G4beamline are:

- Accurate and realistic simulations using the Geant4 toolkit

- A physicist-readable ASCII file to specify the simulation, with auxiliary files for field maps, etc.

- A rich repertoire of beamline elements that can be combined to define new and customized elements

- A general set of initial beam specifications (including a cosmic-ray muon "beam" and external files)

- Beam tracks can be input and output using several formats including ASCII and Root [3] files - this permits easy interfacing to other programs (e.g. partitioning complex systems, verification of results)

- Many parameters can be automatically tuned (RF cavity timing and gradients, bending magnet fields, etc.)

- Visualization of the system is included, using many viewers (OpenGL, VRML, Open Inventor, etc.)

- Support for parallel jobs on multiple CPUs

- The historoot program, which makes it easy for nonexperts to generate Root [3] histograms and plots

The basic structure of a simulation is to first define the beamline elements to be used (magnets, RF cavities, etc.), including their geometry, materials, and local fields. Then these elements are placed into the world, usually along the nominal beam centerline; each placement can have a position, rotation, and its own field value. Parameters for the elements can be defined in the input file or on the command line, so scripting is straightforward. Individual particles can be traced, beam profiles can be generated and displayed, and virtual detectors can be used to sample the beam at any point.

The tracking of particles through the simulated system is as accurate and realistic as the Geant4 toolkit implements. The input file selects from any of the Geant 4 physics use cases, and can set values for the various Geant 4 tracking-accuracy parameters. This permits users to make trade-offs between CPU time and simulation accuracy. Similarly, G4beamline permits the specification of magnetic map parameters, permitting a trade-off between memory usage (and the CPU time to generate the map) and simulation accuracy.

While G4beamline can make it rather simple to specify a simulation, it cannot substitute for knowledge and experience about the problem domain or about particletracking simulations in general. Like all computer programs, G4beamline is prone to "garbage in, garbage out", especially when used by unskilled users. It is strongly suggested that visualization be used to verify the geometry of the simulation and that a handful of particles are tracked properly through it. Whenever possible, one should arrange to track through a simple geometry that can be compared to independent results, to make sure that what one thinks is happening actually does occur in the simulation.

\section{SOFTWARE DEVELOPMENT}

G4beamline is being developed using modern software development techniques. In particular, our methodology requires that feature documentation be written before the code, and the documentation is contained within the code, so there is always comprehensive and up-to-date documentation available to users. There are two levels of documentation:

- User documentation describing how to use the code

- Internal documentation describing what the code does and how it works

The first is intended for users and is contained in Help text within the code to implement always-available online help. The latter is intended only for developers, and is contained in structured comments that the doxygen [4] system converts into hyper-linked HTML. By keeping all documentation in the code it is easier for developers to keep them in sync; automated tools format it for presentation to users (e.g. the User's Guide).

\section{RECENT FEATURES}

Several recently added features make G4beamline more usable by physicists:

1. An event-list interface between historoot and G4beamline. The user can select an outlier event in a histogram or plot and easily re-run G4beamline with visualization to see what that particular event did.

2. More Geant4 objects have been implemented, expanding the internal repertoire of G4beamline.

3. A direct interface to the NIST material database gives users the ability to use many common materials without manually looking up their properties.

4. Rudimentary track fitting is implemented, intended for estimating required resolutions in proposals. This is not robust enough to serve as an analysis of real experiments, but combined with the inherent flexibility of G4beamline this becomes a powerful tool for the early design of experiments.

5. Do loops and if-then-else have been implemented to simplify repetitive tasks and input file options.

\section{NEW FEATURES}

We are currently developing the following new features:

1. Space Charge. Current expectations for muon colliders indicate that space charge may be a problem in the final stages of cooling. We are implementing computations of space charge based on several existing programs (Orbit [5], TREDI [6], Parmela [7]). We intend to extend this to computations inside absorbers. This includes radical revisions to the Geant4 G4RunManager and physics processes to track particles in parallel.

2. Polarization physics. Many new muon facilities, up to and including a muon collider, can benefit from the ability to use polarized muons. This requires modeling the polarization, especially in the interactions with matter in a cooling channel. 
3. Very low energy physics. We are interested in simulating the inner workings of advanced detector systems, atomic traps, and the details of surface effects. This requires accurate modeling at the eV level and perhaps below.

4. Automated parallelization. Computer farms and multiCPU systems are now common, and we intend to enhance G4beamline to automatically take advantage of such parallelism. At present the user must do this manually.

\section{APPLICATIONS}

At present, more than a dozen research groups are using G4beamline. In addition to the primary use of investigating many aspects of muon cooling for a muon collider or neutrino factory, applications include: analyzing test beamlines and target halls, feasibility studies for several new experiments and facilities, cosmic-ray muon tomography, and detector design. Out of these, we have selected two examples to display: the Mu2E experiment's beamline and detector [8], and the trapping of antiprotons in an atomic trap [9].

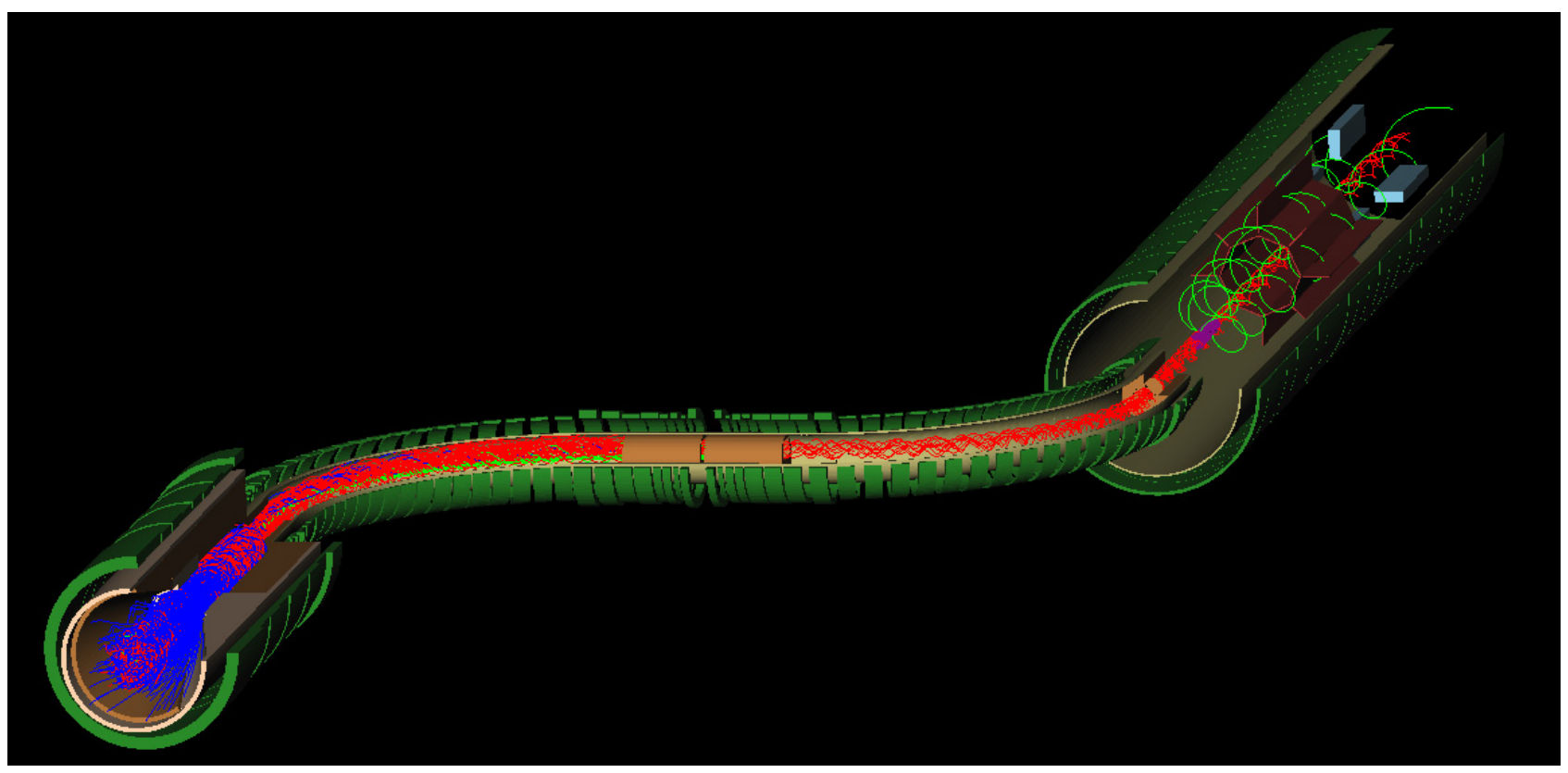

Figure 3. The Mu2E primary target, muon transport, stopping target, spectrometer, and calorimeter.

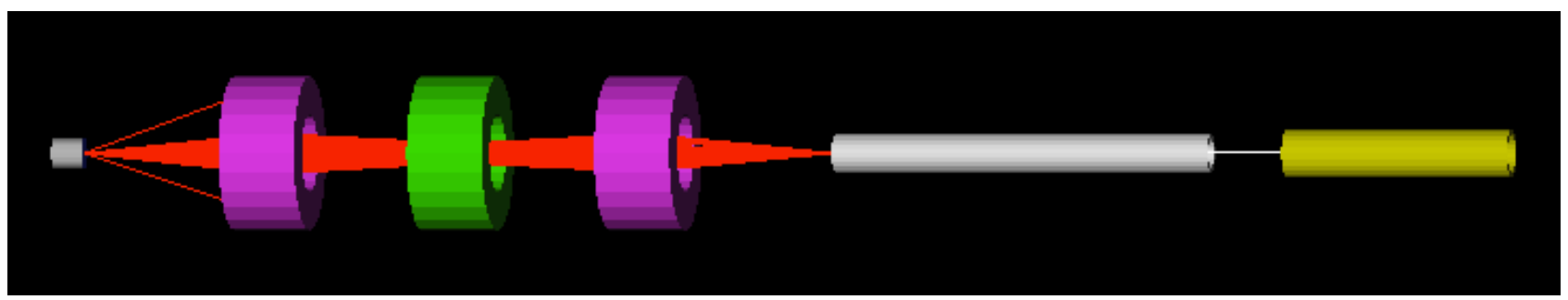

Figure 4. Antiprotons $(1 \mathrm{GeV} / \mathrm{c})$ incident on a lead degrader, a quad triplet (FDF in this plane), a pulsed linac antiproton decelerator, and the HiPat atomic trap (idealized).

\section{SUMMARY AND FUTURE}

G4beamline is a highly flexible and user-friendly program for simulating beamlines both with and without matter. Our commitment to supporting it, our current funding for new features, and our growing user base ensure that it will remain useful for the foreseeable future.

\section{REFERENCES}

[1] G4beamline - http://g4beamline.muonsinc.com

[2] Geant4 Toolkit - http://geant4.cern.ch
[3] Root - http://root.cern.ch

[4] Doxygen - http://www.doxygen.org

[5] http://neutrons.ornl.gov/APGroup/Codes/orbit.htm

[6] TREDI - http://www.tredi.enea.it

[7] Parmela

[8] Mu2E - http://mu2e.fnal.gov

[9] Anti-hydrogen gravity experiment http://capp.iit.edu/hep/pbar 


\title{
STUDY OF COLLECTIVE EFFECT IN IONIZATION COOLING *
}

\author{
D. Huang, Illinois Institute of Technology, Chicago, IL 60616, USA \\ K.Y. Ng, Fermilab Batavia, IL 60510, USA \\ T.J. Roberts, Muons Inc., Batavia, IL 60510, USA
}

\section{Abstract}

As a charged particle passes through a non-gaseous medium, it polarizes the medium and induces wake fields behind it. The interaction with wake fields perturbs the stopping power for the beam particles that follow. The perturbation strongly depends on the densities of both the incident beam and the medium. To understand this collective effect, detailed studies have been carried out. Both analytic and simulation results are obtained and compared.

\section{INTRODUCTION}

The study of the physics of a charged particle passing through a non-gaseous medium is of long history $[1,2,3]$. For a single particle, if its momentum is high enough, it will lose energy through both ionization process and density effect. The latter has been systematically studied. For a beam consisting of a large number of particles, the interaction among the beam particles should also be taken into account in order to describe the process correctly.

Essentially, the density effect is introduced by the polarization of the medium. The electric fields from the polarized medium molecules generate wake fields behind the incident particle, which perturb the motion of the beam particles following. If the particle density of the beam is high enough, the wake will enhance the stopping power for beam particles, and may possibly increase the rate of ionization cooling.

In this article, we derive the expressions for the wake electric field introduced by a single incident charged particle and its perturbation on the stopping power. This is extended to a two-particle system and a multi-particle system with various distributions. The comparison with simulations is next demonstrated. Finally, the damping mechanism on the wake is discussed, and its effect on the stopping-power enhancement is found to be important.

\section{WAKE ELECTRIC FIELD}

First, let us focus on a single particle of charge $e$ moving with velocity $v$ in the $z$ direction, where $-e$ is the electron charge. The particle is at longitudinal position $z=z_{1}$ at time $t=0$. Cylindrical coordinates are used with $\vec{\rho}$ denoting the transverse directions. The scalar potential generated by both the incident particle and polarized medium in the Coulomb gauge is given by

$$
\phi(\vec{r}, t)=\frac{e}{\pi v} \int d \omega \int \frac{\kappa d \kappa J_{0}(\kappa \rho)}{\kappa^{2}+\omega^{2} / v^{2}} \frac{e^{i \frac{\omega}{v}\left(z-z_{1}-v t\right)}}{\varepsilon\left(k^{2}, \omega\right)},
$$

* Work supported by USDOE Grant DE FG0292ER40747 and USDOE STTR Grant DE FG02 08ER86281. where $J_{0}$ is the Bessel function. The wave number vector is denoted by $\vec{k}=\left(\vec{\kappa}, k_{z}\right)$ and the frequency by $\omega$. In the above, the integration over $k_{z}$ and $\vec{\kappa} \cdot \vec{\rho}$ have already been carried out. The polarization of the medium is described by the dielectric constant, which in a dispersive medium takes the form

$$
\varepsilon\left(k^{2}, \omega\right)=1-\omega_{p}^{2} \sum_{j} \frac{f_{j}}{\omega^{2}-\omega_{j}^{2}+i \omega \Gamma_{j}},
$$

where $f_{j}$ is the fraction of bound electrons that oscillate with the bound frequency $\omega_{j}$ and damping rate $\frac{1}{2} \Gamma_{j}$, with $\sum_{j} f_{j}=1$. In the above, $\omega_{p}=\sqrt{4 \pi n_{e} e^{2} / m_{e}}$ is the plasma frequency, where $m_{e}$ is the electron mass and $n_{e}$ the electron density. We make the assertion that $\omega_{p}$ is much larger than the bound frequencies and damping rates. ${ }^{1}$ Then $\frac{1}{2} \Gamma_{j}$ can be replaced by the infinitesimal positive number $\epsilon$, leading to

$$
\frac{1}{\varepsilon}=\frac{\omega^{2}}{(\omega+i \epsilon)^{2}-\omega_{p}^{2}}=\frac{\omega^{2}}{\left(\omega-\omega_{p}+i \epsilon\right)\left(\omega+\omega_{p}+i \epsilon\right)} .
$$

Contour integration over $\omega$ can now be performed giving

$$
\begin{aligned}
& \phi(\vec{r}, t)=e \int d \kappa \frac{\kappa^{2} J_{0}(\kappa \rho)}{\kappa^{2}+\omega_{p}^{2} / v^{2}} e^{-\kappa\left|z-z_{1}-v t\right|} \\
& +\frac{2 e \omega_{p}}{v} \int d \kappa \frac{\kappa J_{0}(\kappa \rho)}{\kappa^{2}+\omega_{p}^{2} / v^{2}} \sin \frac{\omega_{p}}{v}\left(z-z_{1}-v t\right) \theta\left(z_{1}+v t-z\right) .
\end{aligned}
$$

The limits of integration are from $\kappa=0$ to

$$
\kappa=\frac{\omega_{p}}{v} \sqrt{x_{m}^{2}-1} \quad \text { with } \quad x_{m}=\frac{2 \gamma m_{e} v^{2}}{\hbar \omega_{p}}
$$

which corresponds to the maximal momentum transfer in a collision. In the above, $\gamma=1 / \sqrt{1-v^{2} / c^{2}}, c$ is the velocity of light, and $\hbar$ is the reduced Planck constant. The second term in Eq. (4) is the potential coming from the polarization of the medium, and the first term is the mediummodified self-field of the incident charged particle. The longitudinal and transverse electric fields derived from the second term vanish in front of the particle and are therefore the wake fields. Behind the particle, they take the form:

$$
\begin{aligned}
& E_{z}^{w}(\vec{r}, t)=-\frac{2 e \omega_{p}^{2}}{v^{2}} \int d \kappa \frac{\kappa J_{0}(\kappa \rho)}{\kappa^{2}+\omega_{p}^{2} / v^{2}} \cos \frac{\omega_{p}\left(z-z_{1}-v t\right)}{v}, \\
& E_{\rho}^{w}(\vec{r}, t)=+\frac{2 e \omega_{p}^{2}}{v^{2}} \int d \kappa \frac{\kappa^{2} J_{1}(\kappa \rho)}{\kappa^{2}+\omega_{p}^{2} / v^{2}} \sin \frac{\omega_{p}\left(z-z_{1}-v t\right)}{v} .
\end{aligned}
$$

Evaluating at the particle location $\left(z=z_{1}+v t, \rho=0\right)$, we obtain the longitudinal field on axis,

$$
E_{z}^{w}=-\frac{2 e \omega_{p}^{2}}{v^{2}} \ln x_{m}
$$

${ }^{1}$ We believe the bound frequencies are one order of magnitude smaller than $\omega_{p}$ in liquid hydrogen. 
The corresponding energy loss per unit time or stopping power is

$$
\frac{d W}{d t}=e v E_{z}^{w}=-\frac{2 e^{2} \omega_{p}^{2}}{v} \ln x_{m}
$$

Behind the particle, the electric wake can be very well approximated by extending the upper limit of the $\kappa$ integrations to infinity, resulting in

$$
\begin{aligned}
& E_{z}^{w}=\frac{2 e \omega_{p}^{2}}{v^{2}} K_{0}\left(\frac{\omega_{p} \rho}{v}\right) \cos \left[\left(\frac{z-z_{1}}{v}-t\right) \omega_{p}\right], \\
& E_{\rho}^{w}=\frac{2 e \omega_{p}^{2}}{v^{2}} K_{1}\left(\frac{\omega_{p} \rho}{v}\right) \sin \left[\left(\frac{z-z_{1}}{v}-t\right) \omega_{p}\right],
\end{aligned}
$$

with $K_{0,1}$ the modified Bessel functions of the second kind.

The vector potential contributes only to the mediummodified self-field when bound frequencies are neglected. The electric field derived from it consists of two parts: one part cancels the medium-modified stationary self-field from the scalar potential in Eq. (4), while the other represents the medium-modified self-field of a moving charge. The total self-field can be written as

$$
\begin{gathered}
E_{z}^{s}=e \int d \kappa \frac{\kappa^{3} J_{0}(\kappa \rho)}{\kappa^{2}+\frac{\omega^{2}}{v^{2}}} e^{-\gamma \sqrt{\kappa^{2}+\frac{\omega_{p}^{2}}{c^{2}}}\left|z-z_{1}-v t\right|}, \\
E_{\rho}^{s}=e \int d \kappa \frac{\gamma \kappa^{2} J_{1}(\kappa \rho)}{\kappa^{2}+\frac{\omega_{p}^{2}}{v^{2}}} \sqrt{\kappa^{2}+\frac{\omega_{p}^{2}}{c^{2}}} e^{-\gamma \sqrt{\kappa^{2}+\frac{\omega_{p}^{2}}{c^{2}}}\left|z-z_{1}-v t\right|} .
\end{gathered}
$$

In the absence of the medium $\left(\omega_{p}=0\right)$, it reduces to the familiar pancake self-field,

$$
E_{z}^{s}=\frac{e \gamma Z}{\left(\rho^{2}+\gamma^{2} Z^{2}\right)^{3 / 2}}, \quad E_{\rho}^{s}=\frac{e \gamma \rho}{\left(\rho^{2}+\gamma^{2} Z^{2}\right)^{3 / 2}},
$$

where $Z=z-z_{1}-v t$. In the presence of the medium, the self-field decays very much faster with respect to $Z$. For a bunch with longitudinal and transverse radii $\gg v / \omega_{p}$, the self-field has almost no influence compared with the wake fields. Therefore we ignore it in the rest of our discussions.

\section{TWO-PARTICLE SYSTEM}

Now let us discuss the stopping power for a two-particle system. The particles are denoted by 1 and 2 , respectively, with the charge density

$$
\rho(\vec{r}, t)=e\left[\delta\left(\vec{r}-\vec{r}_{1}-\vec{v}_{1} t\right)+\delta\left(\vec{r}-\vec{r}_{2}-\vec{v}_{2} t\right)\right] .
$$

The electric field at $(\vec{r}, t)$ is

$$
\begin{aligned}
& \vec{E}(\vec{r}, t)=-\frac{i 2 e}{4 \pi^{2}} \int d^{3} k \int d \omega \frac{\vec{k} e^{-i \omega t}}{\varepsilon k^{2}} \times \\
& \times\left[e^{i \vec{k} \cdot\left(\vec{r}-\vec{r}_{1}\right)} \delta\left(\vec{k} \cdot \vec{v}_{1}-\omega\right)+e^{i \vec{k} \cdot\left(\vec{r}-\vec{r}_{2}\right)} \delta\left(\vec{k} \cdot \vec{v}_{2}-\omega\right)\right] .
\end{aligned}
$$

The energy gained per unit time by the two particles are

$$
\begin{aligned}
\frac{d W_{1,2}}{d t} & =-\frac{i 2 e^{2}}{4 \pi^{2}} \int d^{3} k \frac{\vec{k} \cdot \vec{v}_{j}}{k^{2} \varepsilon\left(k^{2}, \vec{k} \cdot \vec{v}_{1,2}\right)} \times \\
& \times\left[1+e^{ \pm i \vec{k} \cdot\left(\vec{r}_{1}-\vec{r}_{2}\right) \pm i\left(\vec{k} \cdot\left(\vec{v}_{1}-\vec{v}_{2}\right) t\right)}\right] .
\end{aligned}
$$

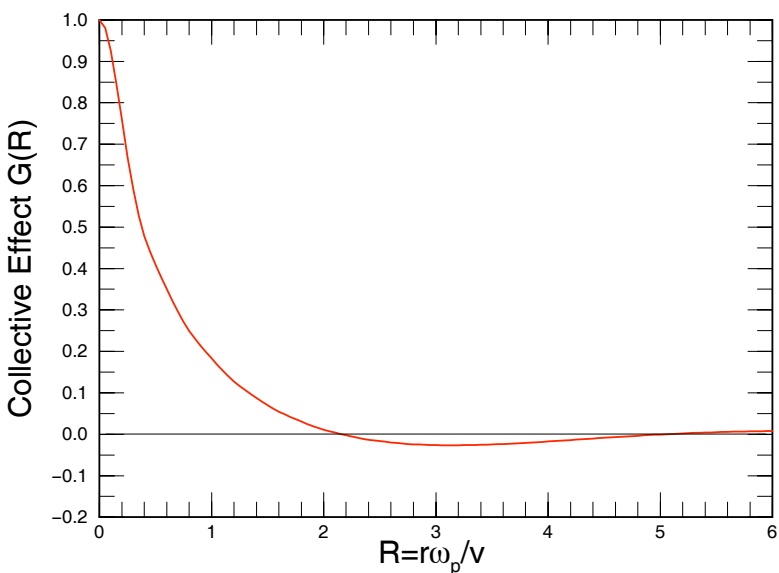

Figure 1: Stopping power enhancement due to collective wake effect on a two-particle system as a function of $R=r \omega_{p} / v$, where $r$ is the separation between the two particles.

For the special case where $\vec{v}_{1}=\vec{v}_{2}=\vec{v}$, we have the average energy loss per particle per unit time or stopping power

$$
\frac{d W}{d t}=-\frac{i e^{2}}{2 \pi^{2}} \int d^{3} k \frac{\vec{k} \cdot \vec{v}}{k^{2} \varepsilon\left(k^{2}, \vec{k} \cdot \vec{v}\right)}[1+\cos (\vec{k} \cdot \vec{r})],
$$

where $\vec{r}=\vec{r}_{1}-\vec{r}_{2}$. Here, only the imaginary part of $1 / \varepsilon$ contributes. After averaging over all orientations of this two-particle system, we arrive at

$$
\left\langle\frac{d W}{d t}\right\rangle_{\text {angles }}=-\frac{e^{2} \omega_{p}^{2}}{v} \ln x_{m}[1+G(R)],
$$

where the correlation function or stopping power enhancement is defined as

$$
G(R)=\frac{-\frac{\sin R x_{m}}{R x_{m}}+\frac{\sin R}{R}-\mathrm{Ci}\left(R x_{m}\right)+\mathrm{Ci}(R)}{\ln x_{m}},
$$

and $\operatorname{Ci}(x)=-\int_{x}^{\infty} d y \cos y / y$ is the cosine integral. Here, the distance of separation of the two particles, $R=r / a_{I}$, has been normalized to $a_{I}=v / \omega_{p}$ or the interaction length. The correlation function [4] as depicted in Fig. 1 shows that $G(R)=1$ at $R=1$ and decreases rapidly when $R \gg$ 1 with oscillation period equal to the plasma wavelength $\lambda_{p}=2 \pi a_{I}$. It is interesting to point out that the averaging over all orientations cancels out all self-field contribution.

\section{VARIOUS BEAM DISTRIBUTIONS}

In this section we will discuss the stopping power enhancement for a particle in the center of a bunch as a result of the polarization wake. Various bunch distributions are used and their importance analyzed.

\section{Uniformly Distributed Sphere}

Let us start with a uniformly distributed spherical $N_{b^{-}}$ particle bunch of radius $r_{0}$. The extra collective stopping power $G_{t}$ received by the particle at bunch center is obtained by integrating the two-particle correlation function $G(R)$ of Eq. (15) over all the particles in the bunch. We get 


$$
G_{t}=\frac{3 N_{b}}{R_{0}^{3}} \int_{0}^{R_{0}} R^{2} G(R) d R=N_{b} \frac{f\left(R_{0}\right)-f\left(R_{0} x_{m}\right)}{\ln x_{m}},
$$

with $R_{0}=r_{0} \omega_{p} / v$, the reduced bunch radius and

$$
f(u)=\left(\frac{1}{u^{3}}+\frac{1}{u}\right) \sin u-\frac{\cos u}{u^{2}}-\operatorname{Ci}(u) .
$$

Since $x_{m}$ is usually a very big number, the above can be readily approximated as

$$
G_{t} \approx \frac{3 N_{b} \sin R_{0}}{R_{0}^{3} \ln x_{m}} .
$$

As an example, consider a $\gamma=2.2$ bunch containing $N_{b}=1 \times 10^{12}$ muons going through liquid hydrogen of density $\rho_{H_{2}}=0.07099 \mathrm{~g} / \mathrm{cm}^{-3}$. The electron density is $n_{e}=\rho_{H_{2}} N_{A}=4.275 \times 10^{28} \mathrm{~m}^{-3}$, where $N_{A}$ is Avogadro's number. The plasma frequency is therefore $\omega_{p}=$ $1.166 \times 10^{16} \mathrm{~s}^{-1}$ leading to $x_{m}=2.323 \times 10^{5}$. If bunch is a uniformly distributed sphere of radius $r_{0}=1 \mathrm{~mm}$, $R_{0}=4.369 \times 10^{4}$ and the envelope of $G_{t}$ is \pm 0.0029 . However, since the bunch edge can never be made sharper than the interaction length $a_{I}=2.289 \times 10^{-8} \mathrm{~m}$, the rapid oscillation of $G_{t}$ with $R_{0}$ with period $\lambda_{p}$ implies the enhancement of stopping power from correlation is essentially zero.

\section{Cylindrical Bunch}

Let us consider next distributions having the separable form $f(z, \rho)=f_{z}(z) f_{\rho}(\rho)$. One example is a bunch with uniform distribution in the transverse direction, but that is tapered at both end longitudinally, or

$$
f_{z}(z)=\frac{A_{n}}{\hat{z}}\left(1-\frac{z^{2}}{\hat{z}^{2}}\right)^{n}, \quad f_{\rho}(\rho)=\frac{2 \pi \rho}{\pi \hat{\rho}^{2}},
$$

where $A_{n}=\Gamma\left(n+\frac{3}{2}\right) /[\sqrt{\pi} \Gamma(n+1)]$ for any $n>-1, \hat{\rho}$ is the transverse radius of the bunch, and $\pm \hat{z}$ are the longitudinal extents of the bunch. We can no longer apply the expression of the all-orientation-averaged correlation function $G(R)$ of Eq. (15), because the distribution is now different in different directions. Instead, we start from Eq. (9) to compute $G_{t}$, the collective stopping power enhancement for a particle at the center of the bunch, by the integration,

$G_{t} \ln x_{m}=N_{b} \int_{0}^{\hat{z}} d z f(z, \rho) \cos \left(\frac{z}{a_{I}}\right) \int_{0}^{\hat{\rho}} d \rho f_{\rho}(\rho) K_{0}\left(\frac{\rho}{a_{I}}\right)$,

and obtain easily

$$
G_{t} \ln x_{m}=\frac{\sqrt{\pi} A_{n}}{\left(\omega_{p} \hat{\rho} / v\right)^{2}}\left(\frac{2 v}{\omega_{p} \hat{z}}\right)^{n+\frac{1}{2}} J_{n+\frac{1}{2}}\left(\frac{\omega_{p} \hat{z}}{v}\right) .
$$

We see clearly that there is an oscillation in the Bessel function $J_{n+\frac{1}{2}}$ which gives positive or negative enhancement depending very sensitively on the half bunch length $\hat{z}$. In fact, when $n$ is an integer, the Bessel function reduces to sine and cosine with period $\lambda_{p}$, and the result will be similar to that of the uniform distribution in a sphere discussed earlier. We learn from Eq. (21) that the longitudinal and transverse beam sizes behave very differently, and it is the longitudinal that introduces the oscillations. To avoid oscillations, we need to go to a distribution without finite longitudinal boundaries.

\section{Lorentzian Distribution}

Let us keep the transverse distribution to be finite and uniform, but let the longitudinal distribution be

$$
f_{z}(z)=\frac{z_{1}}{\pi} \frac{1}{z^{2}+z_{1}^{2}},
$$

where $z_{1}$ is the half longitudinal length at half maximum. The correlation stopping power enhancement $G_{t}$ for a particle at the bunch center is found to be

$$
G_{t} \ln x_{m}=\frac{N_{b} e^{-z_{1} / a_{I}}}{\left(\hat{\rho} / a_{I}\right)^{2}} .
$$

If $\hat{\rho}=z_{1}=1 \mathrm{~mm}, \hat{\rho} / a_{I}=z_{1} / a_{I}=4.37 \times 10^{4}$. Then the stopping-power enhancement is $G_{t}=45.3 \times 10^{-19000}$, which is essentially zero. In order to have a more reasonable effect at the bunch intensity of $1 \times 10^{12}$, we require $\hat{\rho} / a_{I}=z_{1} / a_{I}=20$. Then $G_{t}=0.417$. If $\hat{\rho} / a_{I}=z_{1} / a_{I}=$ $25, G_{t}=0.0018$ instead. However, one must remember that at $a_{I}=v / \omega_{p}=2.289 \times 10^{-8} \mathrm{~m}$, these examples correspond to $z_{1}=4.6 \times 10^{-7}$ and $5.7 \times 10^{-7} \mathrm{~m}$, or bunches of sub-micron lengths. At this moment, the most aggressive cooling scheme proposed by Neuffer [5] is to have an eventual bunch length of $30 \mathrm{~cm}$ and transverse radii $r_{0}=50 \mu \mathrm{m}$.

\section{Tri-Gaussian Distribution}

Let

$$
f_{z}(z)=\frac{e^{-z^{2} /\left(2 \sigma_{z}^{2}\right)}}{\sqrt{2 \pi} \sigma_{z}} \quad \text { and } \quad f_{\rho}(\rho)=\rho \frac{e^{-\rho^{2} /\left(2 \sigma_{\rho}^{2}\right)}}{\sigma_{\rho}^{2}} .
$$

The stopping power enhancement can be integrated readily to the exponential function. However, when $\sigma_{\rho} / a_{I} \gg 1$, it can be neatly reduced to

$$
G_{t} \ln x_{m}=\frac{N_{b} e^{-\left(\sigma_{z} / a_{I}\right)^{2} / 2}}{\left(\sigma_{\rho} / a_{I}\right)^{2}},
$$

which is much smaller than the case of the Lorentzian distribution. Here, we again see that the transverse and longitudinal behave every differently. Although both are Gaussian distributed, the longitudinal decay of the collective stopping power enhancement is Gaussian, while the transverse decay is $\left(a_{I} / \sigma_{\rho}\right)^{2}$, which is very much milder.

\section{Exponential Distribution}

In order to achieve a larger stopping power enhancement, the distribution must roll off very rapidly from the center of the bunch. A possible distribution is the exponential

$$
f_{z}(z)=\frac{e^{-|z| / z_{1}}}{2 z_{1}} \quad \text { and } \quad f_{\rho}(\rho)=\frac{e^{-\rho / \rho_{1}}}{\rho_{1}} .
$$

When $z_{1} / a_{I} \gg 1$ and $\rho_{1} / a_{I} \gg 1, G_{t}$ is given by

$$
G_{t} \ln x_{m} \approx \frac{\pi N_{b}}{8\left(\rho_{1} / a_{I}\right)\left(z_{1} / a_{I}\right)^{2}} .
$$

Or $G_{t}=3.81 \times 10^{-4}$ at $\rho_{1}=z_{1}=1 \mathrm{~mm}$. If either the bunch sizes are each reduced 5 times or the bunch intensity is increased by a factor of 125 , the collective effect enhancement will become $4.8 \%$, and thus more significant. 
In Neuffer's scheme [5] with transverse bunch radii $\rho_{1}=$ $50 \mu \mathrm{m}$ and bunch length $z_{1}=30 \mathrm{~cm}$, we get a stopping power enhancement of $G_{t}=8.47 \times 10^{-8}$, which increases rapidly to $7.62 \times 10^{-3}$ when the bunch length is reduced to $z_{1}=1 \mathrm{~mm}$. Here, again we notice that the longitudinal bunch length is the most important factor that determines the enhancement. For example, if we divide a bunch up longitudinally into bunchlets of shorter lengths but with the particle density unchanged, the stopping power enhancement for the bunch particles will be increased.

\section{COMPARISON WITH SIMULATION}

OOPICPro [6] developed by Tech-X Corporation is able to simulate a charged particle beam passing through matter. We simulate a $\gamma=2.2$ tri-Gaussian muon bunch with rms radii $1 \mathrm{~mm}$ traveling through a plasma medium of electron density $n_{e}=4.28 \times 10^{18} \mathrm{~m}^{-3}$ (which is very much less than that in liquid hydrogen). The peak beam density is $5 \times 10^{19} \mathrm{~m}^{-3}$, corresponding to total bunch intensity $N_{b}=0.787 \times 10^{12}$. The longitudinal wake is shown in Fig. 2. The same wake can be computed by integrating our derived wake in Eq. (9) over all the particles in the bunch in a fake liquid hydrogen medium of the same low density. The field patterns in both the longitudinal and transverse directions agree with the simulation results. For example, the oscillation wavelength is $\lambda_{p}=1.43 \mathrm{~cm}$ corresponding to the plasma frequency of $\omega_{p}=1.17 \times 10^{11} \mathrm{~s}^{-1}$. However, OOPICPro gives the peak longitudinal wake electric field $1.02 \times 10^{8} \mathrm{~V} / \mathrm{m}$, while the computed one in Fig. 3 gives $4 \times 10^{8} \mathrm{~V} / \mathrm{m}$. The discrepancy may come from the fact that OOPICPro treats the medium as a fully ionized plasma while we treat the medium as a liquid with polarized molecules. This difference will be examined next.

\section{RELAXATION AND DAMPING}

We need to address the question of relaxation or damping of the plasma oscillation to determine whether the oscillatory wake can be established and sustained.

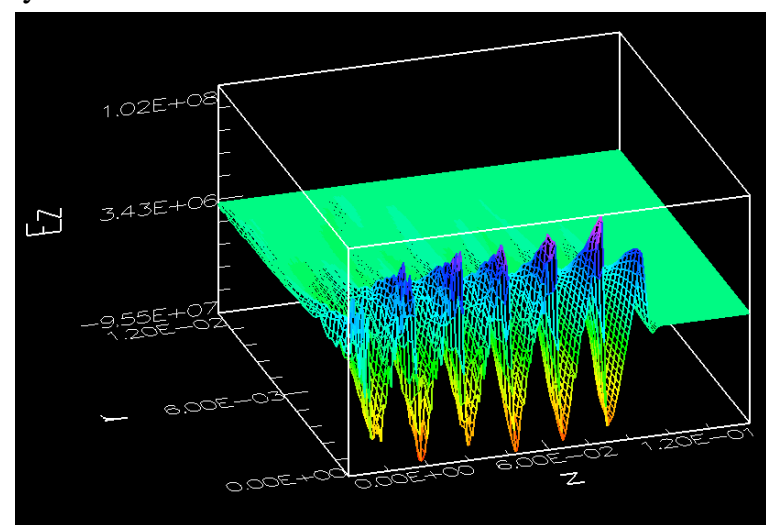

Figure 2: (Color) The longitudinal wake electric field behind an incident muon bunch simulated by OOPICPro with electron density $4.28 \times 10^{18} \mathrm{~m}^{-3}$ and peak particle density $5 \times 10^{19} \mathrm{~m}^{-3}$. The bunch is tri-Gaussian distributed of rms radii $1 \mathrm{~mm}$ in all directions with $\gamma=2.2$ muons. Both the longitudinal and transverse axes $\left(z\right.$ and $r$ ) are in $\mathrm{m}$ while $E_{z}$ is in $\mathrm{V} / \mathrm{m}$.

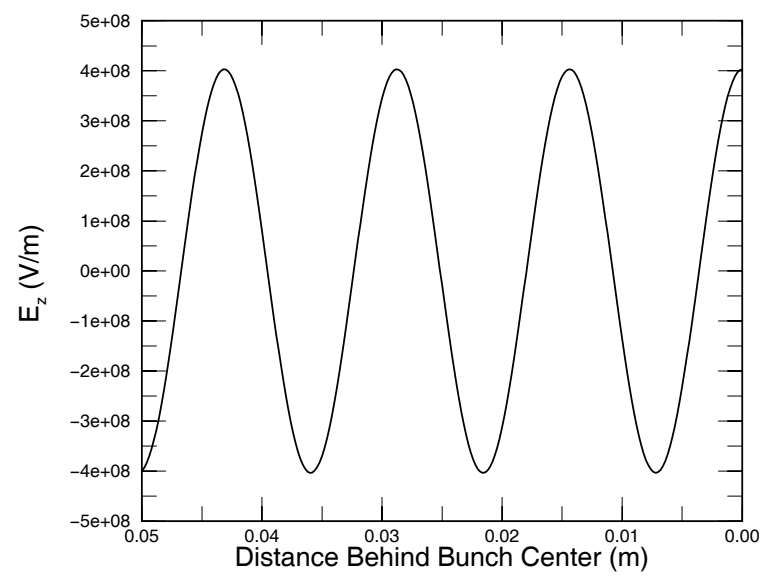

Figure 3: Computed longitudinal wake behind a tri-Gaussian bunch. Bunch sizes and medium density are the same as in the OOPICPro simulation in Fig. 3 . The bunch center is at the origin and $2 \times 10^{5}$ macro-particles have been used.

\section{Cold Plasma}

In a fully ionized plasma, electrons are free to move around as a thermal gas. In the presence of the incident muon beam, the electrons are driven into oscillations about the background ions at plasma frequency. At the same time these electrons collide with the ions. If a collision takes place within one period of plasma oscillation, the plasma oscillation will be disturbed. Thus collision with ions serves as a damping mechanism. The collision frequency of an electron with the ionic background is given by [7] $\nu_{e}=2.9 \times 10^{-9} n_{i} T_{e}^{-3 / 2} \ln \Lambda \mathrm{s}^{-1}$, where $n_{i}$ is the ionic density in $\mathrm{m}^{-3}, \ln \Lambda \approx 10$ is the cutoff logarithm, and $T_{e}$ is the thermal temperature of the electrons in $\mathrm{eV}$. For the above OOPICPro simulation, we substitute $n_{i}=4.28 \times 10^{18} \mathrm{~m}^{-3}$ and $T_{e}=1.72 \times 10^{-3} \mathrm{eV}$ (corresponding to $20 \mathrm{~K}$ ) to obtain $\nu_{e}=1.74 \times 10^{12} \mathrm{~s}^{-1}$, which is comparable to the plasma frequency of $\omega_{p}=1.17 \times 10^{11} \mathrm{~s}^{-1}$. So the wake will be heavily perturbed. We do see some damping of the simulated wake in Fig. 2, but not as heavy as estimated. The discrepancy may come from the relatively higher temperature of the ionized electrons, which may not even be in thermal equilibrium. Since the collision frequency increases as $n_{i}$ while the plasma frequency increases with $\sqrt{n_{i}}$, the damping of plasma oscillation will become more severe as the plasma density increases. Such a simulation has been carried out using OOPICPro with the plasma density increased $10^{4}$-fold to $n_{i}=4.28 \times 10^{22} \mathrm{~m}^{-3}$ while all other parameters remain unchanged. The result in Fig. 4 shows that the on-axis longitudinal wake is damped almost immediately as soon as it is generated. The maximum $\left|E_{z}\right|$ is less than $8.5 \times 10^{5} \mathrm{~V} / \mathrm{m}$ as compared with $1 \times 10^{8} \mathrm{~V} / \mathrm{m}$ in Fig. 2 .

\section{Liquid Hydrogen}

In producing Fig. 3, we employed the wake expression without consideration of damping and the electron density lowered to $n_{e}=4.28 \times 10^{18} \mathrm{~m}^{-3}$ so as to compare with simulation. Now let us come back to the case of real liquid hydrogen where the bound-electron density is 


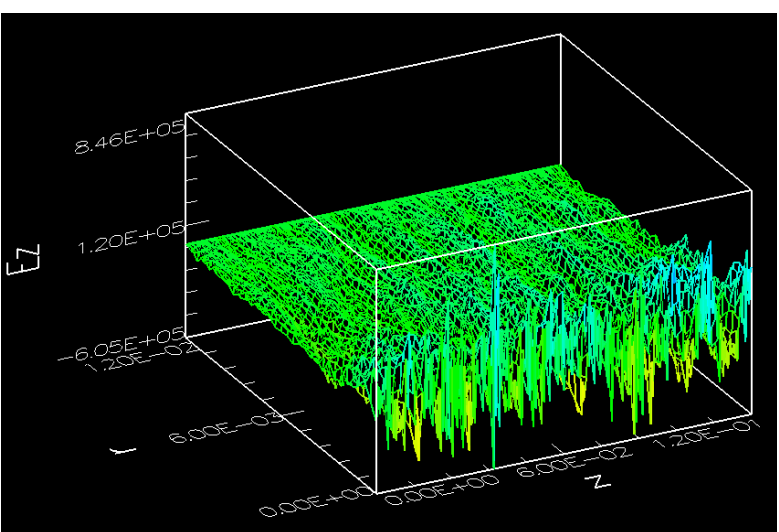

Figure 4: (Color) OOPICPro simulation for a muon beam in a plasma. All parameters are the same as in Fig. 2, except that the plasma density has been increased to $4.28 \times 10^{22} \mathrm{~m}^{-3}$. The $z$ and $r$-axes are in $\mathrm{m}$, while $E_{z}$ is in $\mathrm{V} / \mathrm{m}$.

$n_{e}=4.270 \times 10^{28} \mathrm{~m}^{-3}$ and study possible damping of the wake. At $\gamma=2.2$, the energy loss in liquid hydrogen is $d W / d x=-4.5 \mathrm{MeV}-\mathrm{cm}^{2}-\mathrm{g}^{-1}$. For a bunch with crosssectional radii $r=1 \mathrm{~mm}$, consisting of $1 \times 10^{12}$ muons, the density of ionized electrons is

$$
n_{e i}=-\frac{\rho_{H_{2}} N_{b} d W / d x}{\pi r^{2} I},
$$

where the medium density is $\rho_{\mathrm{H}_{2}}=0.07099 \mathrm{~g} / \mathrm{cm}^{3}$ and the ionization energy is $I=35 \mathrm{eV}$. We obtain $n_{e i}=$ $2.9 \times 10^{23} \mathrm{~m}^{-3}$, which is five orders of magnitude less than the density of the bound electrons and can therefore be neglected. The damping of the wake can come from collisions between the neutral polarized hydrogen molecules, since directional changes of the polarized molecules will disturb the plasma oscillations. The mean thermal velocity of the $\mathrm{H}_{2}$ molecules at $20 \mathrm{~K}$ is $v_{\mathrm{H}_{2}}=235 \mathrm{~m} / \mathrm{s}(3$ degrees of freedom considered). The typical cross section for the hydrogen molecule in the hard-ball model is $\sigma_{H_{2}} \approx 3 \times 10^{-20} \mathrm{~m}^{2}$. Thus the collision frequency is $\nu_{\mathrm{H}_{2}} \approx 4 n_{\mathrm{H}_{2}} \sigma_{\mathrm{H}_{2}} v_{\mathrm{H}_{2}} \approx 45 \times 10^{11} \mathrm{~s}^{-1}$, with $n_{\mathrm{H}_{2}}$ the density of the $\mathrm{H}_{2}$ molecules. This is still many order smaller than the plasma frequency of $\omega_{p}=1.17 \times 10^{16} \mathrm{~s}^{-1}$.

Another possibility of damping comes from the damping rates of the bound frequencies of the $\mathrm{H}_{2}$ molecules. We asserted earlier that the bound frequencies $\omega_{j}$ are an order of magnitude smaller than $\omega_{p}$. It is reasonable to assume that the damping rates $\frac{1}{2} \Gamma_{j}$ of the bound frequencies are the same order of magnitude as $\omega_{j}$. Let us simplify the problem by including only one damping rate $\frac{1}{2} \Gamma$. Then $\epsilon$ in Eq. (3) will be replaced by $\frac{1}{2} \Gamma$, and there will be the extra factor of $e^{-\Gamma Z / 2 v}$ in the wake expressions of Eqs. (6) and (9), where $Z=z-z_{1}-v t$. Since $2 v / \Gamma \ll \sigma_{z}$, the bunch length, in the computation of stopping power enhancement in Eq. (20), the longitudinal beam distribution can be replaced by the peak beam density. Taking the tri-Gaussian distribution as an example, instead of Eq. (25), we obtain

$$
G_{t} \ln x_{m} \approx \frac{N_{b}}{\sqrt{2 \pi}\left(\sigma_{\rho} / a_{I}\right)^{2}\left(\sigma_{z} / a_{I}\right)} \frac{\Gamma}{2 \omega_{p}} .
$$

For a beam with $\sigma_{\rho}=\sigma_{z}=1 \mathrm{~mm}$ and $N_{b}=1 \times 10^{12}$, $\Gamma / 2 \omega_{p} \sim 0.1$ implies $G_{t} \sim 3.9 \times 10^{-5}$. In addition, if the transverse distribution is exponential, $\left(\sigma_{\rho} / a_{I}\right)^{2}$ in the denominator is replaced by $4\left(\sigma_{\rho} / a_{I}\right) / \sqrt{2} \pi$ and we have $G_{t} \sim 190 \%$ instead. In short, the enhancement becomes much larger in the presence of some amount of damping.

\section{CONCLUSIONS}

The perturbation of stopping power due to collective effect as a charged particle beam traverses a medium is studied in detail. This effect is introduced by the polarization of the medium and depends on a variety of factors such as beam distribution, beam density, and medium density.

The magnitude of the collective perturbation is fundamentally determined by the ratio of the separation of beam particles and the interaction length in the polarized medium, which is also a function of the velocity of incident particles. As this ratio decreases, the collective effect becomes more significant.

The damping of the wake also plays an important role in the wake field. Without any damping consideration, the wake oscillates sinusoidally with period $\lambda_{p}=2 \pi a_{I}$. Since the average separation of the incident beam particles is usually much larger than the interaction length, the wake field perturbation on stopping power is negligibly small. Damping comes from two sources: one is the collision rate between absorber molecules, which is slow and insignificant, the other is the damping rates of the bound frequencies of absorber electrons. Under certain circumstances, a shorter damped wake enhances collective perturbation.

The model used in the analysis employs the dielectric constant $\varepsilon$ in the form of Eq. (3) where bound frequencies are considered small and neglected. Further analysis should take account of the contribution of bound frequencies to the wake and their effects on the stopping-power enhancement should be fully investigated.

Whether an enhancement of stopping power will increase the ionization-cooling rate has not been proven. In our future analysis, we will study the perturbation on the stopping power for particles traveling at various angles relative to the traveling direction of the bunch center. This will allow us to determine precisely the effect on the cooling rate of the beam due to interaction with the wake generated in the medium.

\section{REFERENCES}

[1] E. Fermi, Phys. Rev. 57, (1940) 485.

[2] J. Neufeld and R.H. Ritchie, Phys. Rev. 98, (1955) 1632.

[3] W. Brandt, A. Ratkowski and R.H. Ritchie, Phys. Rev. Lett. 33, (1974) 1325.

[4] N.R. Arista and V.H. Ponce, J. Phys. C 8 (1975) L188. Results depicted in Fig. 3 of Ref. [3] are incorrect.

[5] D. Neuffer, LEMC09 presentation.

[6] OOPICPro, Tech-X Corp., http://www.txcorp.com.

[7] J.D. Huba, NRL Plasma Formulary, 2009, p.33. 


\title{
PUTTING SPACE CHARGE INTO G4BEAMLINE*
}

\author{
K.B.Beard ${ }^{\#}$, T.J.Roberts, Muons, Inc. Batavia, IL, 60510, U.S.A.
}

\section{Abstract}

The G4beamline program is based on the wellestablished Geant4 toolkit used to simulate the interactions of particles and photons with matter. Until now, only a single particle at a time could be tracked and there are no interactions between particles. Recent designs for high pressure RF cavities and other novel devices achieving extreme muon cooling require that the effect of space charge be included in the simulations. A new tracking manager in G4beamline propagates a number of particles (typically 1,000$10,000)$ in parallel, stepping all particles in time. This allows all of the usual Geant 4 physics interactions to be applied, plus collective computations. A simple macroparticle-based model is used to represent $\sim 10^{8}$ charges with an ellipsoidal charge density. At intervals the appropriate macroparticle size and shape are recalculated, the electric and magnetic fields are determined, and an impulse is applied to the simulated particles. Comparisons to a space charge experiment are presented.

\section{INTRODUCTION}

The Geant4[1] toolkit is the leading tool in the world for simulating the interaction of particles and photons with matter, but mastering Geant4 requires an extensive knowledge of $\mathrm{C}++$ as well as the physics. The G4beamline[2] program provides a simple interface to the power of Geant4, allowing one to create and run simulations in hours rather than months.

To date, Geant 4 considers each particle in turn; a particle may stop, decay, scatter, but essentially only one pariticle is considered at a time. G4beamline has provided a means of considering many particles moreor-less simulatenously, but that still leaves the question of how to best describe the interactions among the particles. The most important of these is space charge.

This particle-particle interaction was encapsulated into a single collective routine, allowing developers and users to easily insert their own models without modifying G4beamline itself. This first attempt only considers space charge in vacuum, but for our applications of interest this method must be extended to cover space charge in matter, too.

\section{CONCEPT}

The electromagnetic interaction of two charge particles is well understood; the whole problem of space charge in beams is that of using a very small number of "particles $\left(\sim 10^{3}\right)$ to predict the behavior of many $\left(10^{9-20}\right)$ particles. Such "macroparticles", essentially large overlapping blobs of charge, came into wide use; some more recent codes use particle-in-cell

* Supported in part by USDOW Contract DE-FG02-6ER86281. \# beard@muonsinc.com
(PIC) models with many more point-like particles and fields calculated on a grid.

The macroparticle model is very attractive for use with G4beamline - the number of particles used is very small $\left(10^{4}\right)$ and the calculation intuitive. A package called kbb_sc5 was linked into G4beamline; this package was developed solely to test various macroparticle models and to compare the results with various versions of the well established Parmela [3] [4] space charge program. While kbb_sc5 is very similar in concept to TJNAF Parmela, it contains no Parmela code.

\section{PHYSICAL MODEL}

The simplest possible model is to just naively apply Coulomb's law to point particles. While some kind of screening is required to avoid singularities when two particles get very close, the method is trivial and ensured all the units were correctly chosen.

The first improvement was to put in the relativistically correct interaction where the moving particle creates both an electric and a magnetic field. As in TJNAF Parmela, the retarded time was not used by default, but may be optionally enabled.

The next was to replace the point-like particles with macroparticles. For the purpose of their propagation, macroparticles are point-like; for the purpose of calculation the electric field from their charge distribution, they are extended globular objects that may be either uniformly or non-uniformly charged inside with either a hard or soft edge. Each of the N macroparticles is assigned 1/Nth the charge of the whole bunch.

Table 1. kbb_sc5 bit-packed options.

\begin{tabular}{|c|c|c|c|c|c|}
\hline bit & option & 101510 & 1318 & 3206 & 99590 \\
\hline 1 & KBB_SC_OPT_DISABLE & & & & \\
\hline 2 & KBB_SC_OPT_ENABLE & $*$ & * & * & * \\
\hline 4 & KBB_SC_OPT_MACROPARTICLE & $*$ & * & * & * \\
\hline 8 & KBB_SC_OPT_FUZZYEDGE & & & & \\
\hline 16 & KBB_SC_OPT_DISCARDOUTLIERS & & & & \\
\hline 32 & KBB_SC_OPT_XYZMACROPARTICLE & & * & & \\
\hline 64 & KBB_SC_OPT_PARMELALIKE & & & & \\
\hline 128 & KBB_SC_OPT_JACKSON & $*$ & & $*$ & \\
\hline 256 & KBB_SC_OPT_COULOMB & T & * & & * \\
\hline 512 & KBB_SC_OPT_CUBICMP & r & & & \\
\hline 1024 & KBB_SC_OPT_CENTEROFMASS & * & * & * & * \\
\hline 2048 & KBB_SC_OPT_RETARDED & * & & * & \\
\hline 4096 & KBB_SC_OPT_BOOST2CM & & & & \\
\hline 8192 & KBB_SC_OPT_REITZMIILFORD & 1 & & & \\
\hline 32768 & KBB_SC_OPT_KLUDGEDRIFT & $*$ & & & * \\
\hline 65536 & KBB_SC_OPT_TIMEZONE & $*$ & & & * \\
\hline 1073741824 & KBB_SC_OPT_RUNSELFTEST & & & & \\
\hline
\end{tabular}

The dimensions of the macroparticles are derived from the bunch and usually updated from time-to-time 
based on the distribution of the entire bunch. The best formula for this is open to debate [6].

Since the physics model in kbb sc5 is controlled by a bit packed integer, it is easy to change the model and compare results (Table 1).

\section{COMPARISON WITH OTHER CODES}

A simple field free drift space was used for the first tests. A single bunch of macroparticles was injected at one end of a field free region and allowed to drift; the paths of the particles under various models were compared.
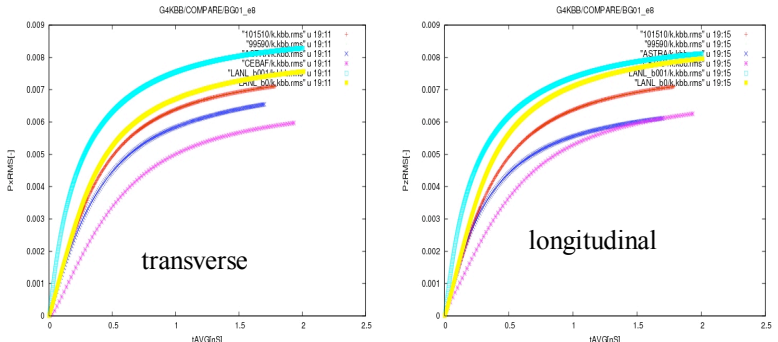

Figure 1: Simulated results for PxRMS and PzRMS $(\mathrm{MeV} / \mathrm{c})$ for a spherical bunch in various models. The $\mathrm{kbb}$ sc5 results are in shown in red.

The first test just used a $16 \mathrm{pC}$ spherical Gaussian bunch with a $1 \mathrm{~mm}$ radius and $\gamma=0.1$. The $\mathrm{kbb} \_\mathrm{sc} 5$ results for the simplest and better models lie atop one another and between the ASTRA, LANL Parmela $(b=0$ $\& b=0.001$ ), and CEBAF Parmela results.

The next test used a uniform "slug" of electrons with radius $=1 \mathrm{~cm}$, length $=0.909 \mathrm{~cm}, \mathrm{Q}=6 \mathrm{nC}$, and $\gamma=1$ that had been used for earlier comparisons.[6]
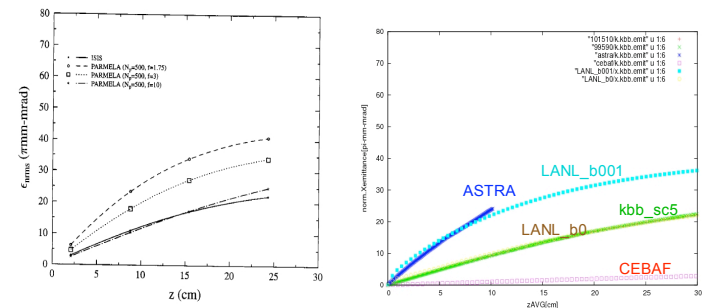

Figure 2: Emittance results for ISIS and CEBAF Parmela [6] and results from ASTRA, LANL Parmela with parameter $b=0$ and 0.001 , CEBAF Parmela (with default parameters), and kbb_sc5 for a uniform slug of charge.

Even in this fairly simple case, the results strongly depended on the program and the parameters chosen. The CEBAF Parmela run used the default parameters for the CEBAF injector, a poor match for the slug.

The agreement of various quantities between the various simulations varied widely depending on the circumstances and the quantity of interest with no obvious pattern to suggest that one code was consistently far superior to the others.

\section{COMPARISON WITH REAL DATA}

While new space charge codes are often tested by comparing with existing space charge codes [5] [6], it is much better to compare to real data.

Fortunately, a very careful experiment to measure space charge and compare with existing models has recently been published [7] [8] [9]. This electron photoinjector beamline consists of a photocathode, electrostatic gun, emittance compensation solenoid, slits, scanners, and imaging systems.

This beamline is unusual in that it is primary purpose is to study space charge. The slits allow sampling of the distribution transversely, but rather than moving the slits the beam is displaced to the side by a matched pair of air core dipoles.

The important components of the beamline were modeled in G4beamline (Figures 3 and 4) using only 75 non-comment lines in the input file, 2 field maps, and an initial distribution based on the paper.[9]

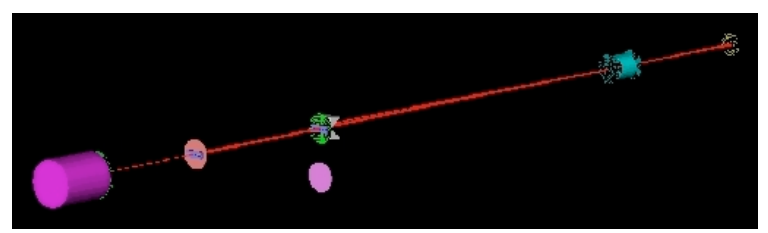

Figure 3: The Cornell beamline line in g4beamline; cathode is on the right; dump on the left; the viewer is retracted.

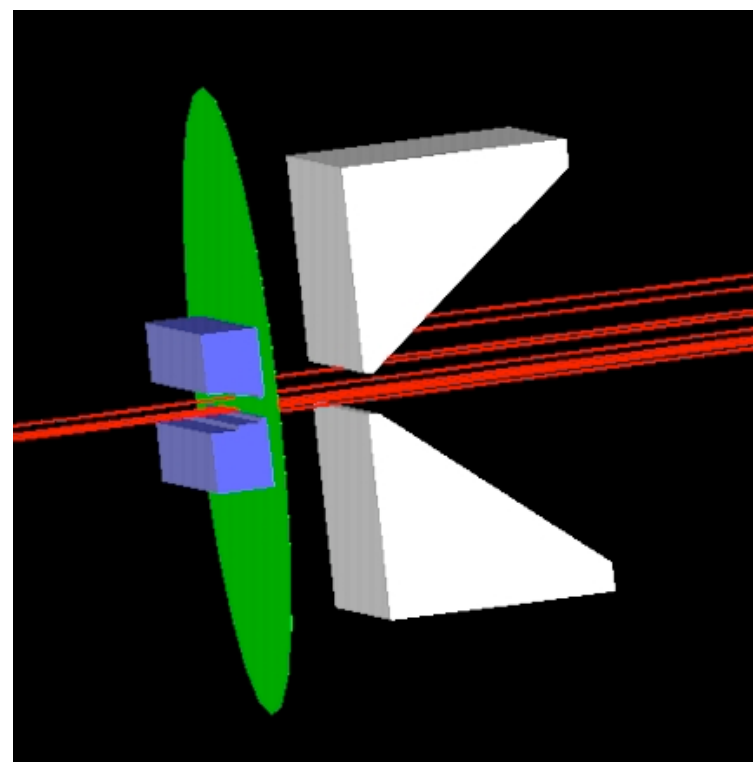

Figure 4: Close up of armor (white) and fine (blue) copper slits; the virtual detector (green) records the electrons (red) but has no physical existence.

Initial testing revealed some discrepancies between this model and data from the beamline, so a direct comparison between the data and our simulation isn't possible at this time. These deficiencies will be corrected and the simulations rerun later. 
The first measurement was to retract the slits and vary the current of the emittance compensation solenoid, then compare low and high current behavior at the image screen, $124.4 \mathrm{~cm}$ from the photocathode.

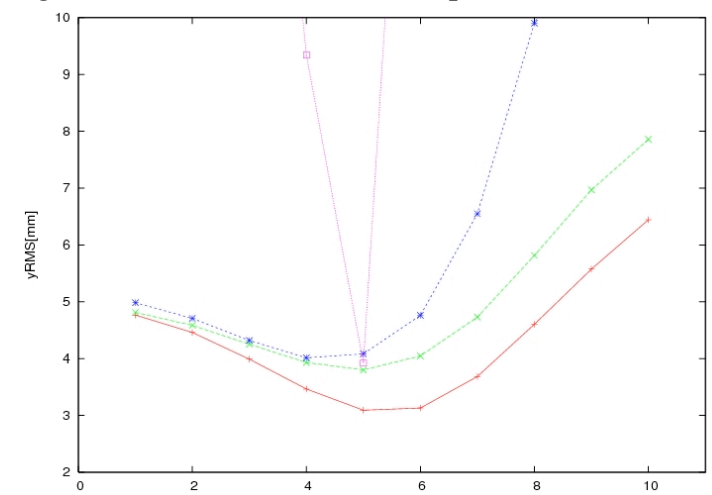

Figure 5: YRMS as a function of solenoid current for 0.5 (red), 20 (green), and 80 (blue) pC/bunch using a realistic interaction mode 1 (option $=3206$ ) and 80 (magenta) $\mathrm{pC} /$ bunch using the simplest model (option=1318).

A comparison of the simplest macroparticle model and a much more sophisticated model shows that the simplest model greatly overpredicts the space charge, as would be expected. The qualitative behavior agrees with the data; the differences are likely due to our misunderstanding about the solenoid's construction and will be addressed later.

The next step involves inserting the slits and using them to scan the beam transversely. This is done using two air-core dipoles to move the entire beam to the side.

A problem arises when the beam strikes a narrow slit, as most of the beam (and the macroparticles) are lost, resulting in very poor statistics. This is a general problem with of the macroparticle method that we are investigating.

For faster testing, the slit was opened to $+/-1 \mathrm{~mm}$.

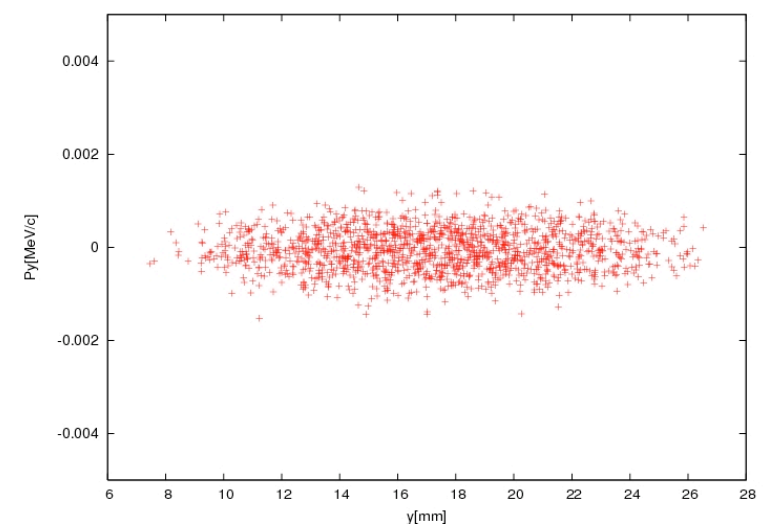

Figure 6: Py vs y just after slit A for a $20 \mathrm{pC}$ bunch (option=3246).

\section{CONCLUSION}

These very preliminary results demonstrate that the collective mode modifications to g4beamline were successful, but the evidence suggests that the physical model needs to be improved before any hard conclusions can be made about the quality of this macroparticle space charge model. It also exposed several obstacles that need to be overcome before a practical system can be constructed, the most important here being of how to deal with the loss of simulated macroparticles.

\section{REFERENCES}

[1] http://www.geant4.org/geant4

[2] http://g4beamline.muonsinc.com

[3] L.Young and J.Billen, PAC 2003 Conference Proceedings

[4] K.B.Beard and B.Yunn, Jefferson Lab technical note JLAB-TN-03-023 (2003)

[5] C.Limborg, et al, PAC 2003 Conference Proceedings

[6] H.Liu, Jefferson Lab technical note CEBAF-TN-94-040 (1994)

[7] I.V.Bazorav, et al, PRST Accel \& Beams 11, 1000703 (2008)

[8] C.Gulliford, Cornell Univ. Internal Report, 2006 (http://www.lepp.conrell.edu/ ib38/reu/06/ gulliford.pdf)

[9] I.V.Bazarov, et al, PAC 2007 Conference Proceedings 


\title{
SIMULATION TOOLS FOR THE MUON COLLIDER DESIGN FEASIBILITY STUDY*
}

\author{
Thomas J. Roberts ${ }^{\#}$, Muons, Inc. Batavia IL 60510, U.S.A. \\ Richard Fernow, Brookhaven National Laboratory, Upton, NY 11973, U.S.A.
}

\begin{abstract}
The U.S. muon collider community is mobilizing itself to produce a "Design Feasibility Study" (DFS) for a muon collider. This is happening on an aggressive schedule and must include the best possible simulations to support and validate the technical design. The DFS for a muon collider will require innovative new approaches to many aspects of accelerator design, and the simulations to support it will require tools with features and capabilities that are equally innovative and new. Two computer programs have emerged as the preferred and most commonly used simulation tools within the muon collider community: ICOOL (primary author: Dr. Fernow), and G4beamline (primary author: Dr. Roberts). We describe the ongoing development and testing of both tools for the DFS, including a common suite of tests to ensure that both tools give accurate and realistic results, as well as innovative user-friendly interfaces with emphasis on graphical user interfaces and windows.
\end{abstract}

\section{THE DESIGN FEASIBILITY STUDY}

This study will involve extensive simulations of new accelerator designs, including the interactions of protons in a target, the decay of pions to muons in a magnetic field, the capture and phase rotation of muon beams in magnetic fields and RF cavities, the passage of muon beams through matter in order to cool them, the acceleration of muon beams to very high energy, and the circulation of muon beams in storage rings and their intersection at the center of a modern particle detector. The understanding of muon decay backgrounds will be an important aspect of these simulations, including the power deposited in accelerator structures, and tracks in the intersection-region detectors. Many of those tasks are beyond the capabilities of traditional accelerator-physics codes, so programs have been developed specifically to perform such simulations. The community feels strongly that at least two such programs should be available, so they can crosscheck each other, increasing the confidence that the results are correct and that facilities designed using the programs will operate as intended.

At present this Design Feasibility Study (DFS) is just starting up. The details of its budget and scope are still being negotiated, and the design of the actual muon collider facility to be considered is essentially undetermined - the first few years of the study will be devoted to evaluating and selecting the components of the

\footnotetext{
* Work supported in part by USDOE STTR Grant DE-FG0208ER86281 and DE-AC02-98CH10886.

\# tjrob@muonsinc.com
}

final design. It is clear that extensive simulations will be required to do that, and the current plans are to use both ICOOL [1] and G4beamline [2]. The recent history of the muon collider community shows that new ideas for muon cooling are still being invented, and it is likely that during the period of the DFS innovative new ideas will be discovered, evaluated, and perhaps incorporated into the final design of the facility. This implies that the simulation programs used for such evaluations may need to be modified to accommodate the new ideas and concepts. So specific tool development will be necessary to support the DFS, as well as routine bug fixes and program enhancements. As it is important to retain two independent simulation programs (to crosscheck each other), no attempt will be made to merge them; we do intend to implement a common test suite to verify their simulation accuracy with each other and with real experiments (discussed below).

In short, this project is an enabler for the much larger DoE project that is the muon collider Design Feasibility Study (DFS). The software support that is the focus of this project will enable all of the participants in the DFS to perform their required simulations more effectively and with greater confidence in their accuracy. As the muon collider itself includes completely new types of accelerator structures (e.g. muon cooling), innovative solutions will be required throughout both projects.

\section{BACKGROUND: ICOOL}

ICOOL [1] is a particle beam simulation code that has played a major role in the design of ionization cooling channels for muon colliders and neutrino factories since 1996. It provides the flexibility to quickly examine widely different ideas for configuring muon collider front ends. For example, setting up many preliminary field configurations is accomplished in ICOOL using predefined, analytic field models. This simplifies the adjustment of parameters for the field to obtain some desired result. Comparisons of results from ICOOL and Geant4-based codes have generally shown good agreement. One program is frequently used to check results from the other.

ICOOL uses a command input file consisting of five parts: simulation control variables, beam definition, control of physics interactions, diagnostics, and region definition. The program tracks the particles to the end of a given region and generates any desired diagnostics. It then continues to track the surviving particles to the end of the next region. This program structure was adopted to make it possible to include space charge interactions. There is no practical limit on the number of regions in a problem. 
The user can choose to generate Gaussian or uniform initial particle distributions inside the program. Available particles are electrons, muons, pions, kaons and protons of either sign. It is possible to generate a number of important correlations in the initial beam distribution. The input beam information may also be read in from an existing set of particle data on an external file. There is no practical limit on the number of particles. Currently the first 300,000 particles are stored in memory and particles in excess of this are stored on a direct access disk file. The program can save the particle state after any region in the same format required for input, so problems can be run in stages

Muon and pion decays can be simulated. Energy loss, straggling and scattering in matter are simulated as continuous processes. The code currently uses a set of 17 internally defined elements and compounds that are of greatest interest for ionization cooling. The mean value of the energy loss is computed using the Bethe-Bloch formalism including the density effect. Fluctuations in the energy loss may be sampled from Gaussian, Landau or Vavilov distributions. Multiple Coulomb scattering may be sampled from Gaussian distributions, from Rutherford single scattering or from the Moliere distribution.

ICOOL has been used to model some very complicated muon beam channels. These include the $550 \mathrm{~m}$ long front end for the Feasibility Study 2 Neutrino Factory design [3] and the $295 \mathrm{~m}$ long Study 2a Neutrino Factory design [4]. Modeling the Feasibility Study 2 channel used 1466 separate regions in ICOOL, consisting of liquid hydrogen absorbers with aluminum windows and pillbox RF cavities with beryllium windows, all immersed in a tapered, alternating direction, periodic solenoid field lattice. The code was also used to design the RFOFO ring [5], which was one of the first successful ring coolers for $6 \mathrm{D}$ emittance reduction. It has also been used to do the preliminary designs of all the subsystems for the highemittance muon collider scenario currently being studied for the muon collider.

\section{BACKGROUND: G4BEAMLINE}

Geant4 [6] is an internationally supported particle tracking toolkit that was developed to simulate particle interactions in large detectors for high energy and nuclear physics experiments, and includes most of what is known about the interactions of particles and matter, including time-varying electromagnetic fields. G4beamline [2] is a program that provides a highly flexible and user-friendly interface to the Geant4 capabilities relevant to simulating beamlines, requiring no programming by users.

G4beamline was conceived and designed as a flexible interface to the Geant4 toolkit specifically intended to permit the rapid prototyping and evaluation of different accelerator and beamline designs. It has become a "Swiss Army Knife" for Geant4. Its internal programming infrastructure is fully object-oriented, highly modular, and designed to be easy to extend. No changes to its existing infrastructure are expected for this project.
G4beamline uses an ASCII file to specify all aspects of the simulation. It consists of a series of commands that control the simulation, define elements to be used in the simulation, place elements into the simulated world, and direct the generation of results. The complexity of the input file is comparable to the complexity of the system to be simulated (compared to system-specific simulation programs which are much more complex than the system itself). G4beamline has a rather large repertoire of common elements used in particle accelerators and detectors, such as bending magnets, quadrupole magnets, RF cavities, etc. In addition, G4beamline makes it easy to lay out beamlines that involve bending magnets and the resulting rotations of elements - centerline coordinates are available that automatically handle the details so the user does not need to laboriously compute the locations and rotations of beamline elements.

Once the system to be simulated is specified in the input file, particles are tracked using the full accuracy of the Geant 4 toolkit. To permit trade-offs between accuracy and computation time, several different models for hadronic interactions are available, and over 100 megabytes of experimental data are available for use by the more detailed models; much of these data are specific to neutrons and photons. The result is that G4beamline can provide a realistic assessment of how the real system will behave. To assist the user in verifying that the simulated system accurately represents the real system, extensive visualization capabilities are included in G4beamline, so the user can see what is being simulated, and where the simulated particles go.

To assist the user in obtaining answers from a simulation run, a user-friendly histogram program is included with G4beamline. Called HistoRoot, it permits the user to easily obtain histograms and plots from the G4beamline output files.

\section{SOFTWARE DEVELOPMENT METHODOLOGY}

Modern software development does not occur independent of applications. The effective implementation of new capabilities for ICOOL and G4beamline requires that we use the programs while developing them. So the software development teams will also directly participate in the simulations required as part of the DFS. This ensures that we do not spend time implementing useless features, and also helps us refine and optimize the user interface to the new features. In the software engineering literature this aspect of our methodology is known as "rapid prototyping", and we often implement one or more prototypes of a new feature before finalizing its implementation.

Experience shows that while this is not directly in line with a top-down management style, in practice the effort expended in re-work and refinement of prototypes is more than compensated by the improved quality of the result. So we cannot describe in detail the steps required to 
implement the new features. Similarly, we cannot describe the bug fixes that will be implemented.

All software developed in this project will be freely available to participants in the DFS. All G4beamline code will remain open source and freely available on the web. ICOOL is also freely downloadable from the web. New tools will be open source and available on the web unless they rely on software that prevents it.

\section{DEVELOPMENT PLANS}

At present we have identified the following items common to both programs:

- A common test suite for ICOOL and G4beamlne.

- A common repository for subsystem designs.

- Common code for assessing cooling channel performance, perhaps based on the program ecalc 9 [7].

- Converters among ICOOL and G4beamline data files, including Root [8] files.

- A common system description, perhaps an automated translation from G4beamline to ICOOL input decks.

\section{ICOOL Plans}

We plan to maintain and upgrade ICOOL as necessary, at least through the completion of the Muon Collider Design Feasibility Study. The code should have the capability of simulating any muon beam channel needed for the front end of the collider. This includes channels for muon decay, bunching, phase rotation, transverse cooling, charge separation, 6D emittance cooling, bunch merging, charge recombination, and final cooling to very small muon beam emittances. The DFS calls for a decision on the baseline design of the accelerator and its technical specifications to be made around the end of 2011. The ultimate goal is to use the code to make an end-to-end simulation of the entire collider front end. The results of the front end simulations must be carefully compared with the results of the same channels using G4Beamline, and any discrepancies must be understood.

We will prepare a list of extensions to ICOOL that are needed to perform the desired simulations of the DFS. This list will be maintained for the duration of both this project and the DFS, and they will be implemented as resources permit. Note that the DFS is likely to involve new and innovative techniques, and this list will change over time.

\section{G4beamline Plans}

We plan to maintain and upgrade G4beamline for the foreseeable future - it has become an important part of the culture of Muons, Inc., and is being used by a diverse and growing user community. We ultimately intend that the program will be able to simulate every aspect of the muon collider, from the initial gas bottle of the proton driver to the detection of particles in the collider detector. A major goal is to use the code to make an end-to-end simulation of the entire collider front end, the muon accelerator, and the intersecting storage rings; perhaps simulations of the proton driver, the pion production target, and the colliding detector will also be performed. The results of the front end simulations must be carefully compared with the results of the same channels using ICOOL, and any discrepancies must be understood.

We will prepare a list of extensions to G4beamline that are needed to perform the desired simulations of the DFS. This list will be maintained for the duration of both this project and the DFS, and they will be implemented as resources permit. Note that the DFS is likely to involve new and innovative techniques, and this list will change over time.

There is a rather large list of new features suggested by users for G4beamline. We will prioritize them according to their effort and relevance to the DFS, and will implement them as resources permit. The current list is always available at $\mathrm{http}: / / \mathrm{g} 4$ beamline.muonsinc.com (link at the top).

We intend to implement a Graphical User Interface to G4beamline that is a user-friendly editor for its input files. The user will be presented with a view of the system to be simulated, along with other windows containing libraries of elements, plus the usual toolbars and menus. The user can define new libraries of elements, and add new customized or generic elements to libraries. To construct the system, the user simply drags an element from a library into the world, rotating and placing it as necessary. Each element will have individually editable parameters, and the user will be able to customize the menus, toolbars, libraries, and other aspects of the GUI. Interestingly, the initial library of generic elements will be automatically built from the G4beamline source, eliminating any need to keep the GUI and the program in sync. This new editor will be integrated into the existing GUI that runs G4beamline, and it will be coordinated with the item mentioned above for having a common system description for ICOOL and G4beamline.

\section{CONCLUSION}

The muon collider Design Feasibility Study will place demands on the tools it uses to be complete, accurate, and up-to-date. The project described in this paper is an enabler for the study, providing support for maintaining those tools.

\section{REFERENCES}

[1] R. Fernow, Proc. 2005 Part. Acc. Conf. Knoxville, TN, p. 2651; R. Fernow, Proc. 1999 Part. Acc. Conf., New York, NY, p. 3020.

[2] G4beamline: http://g4beamline.muonsinc.com

[3] Technical Report No. BNL-52623, edited by S. Ozaki, R. Palmer, M. Zisman and J. Gallardo, 2001.

[4] J.S. Berg et al., Phys. Rev. ST-AB 9, 011001 (2006).

[5] R. Palmer et al., Phys. Rev. ST-AB 8, 061003 (2005).

[6] Geant4: http://geant4.cern.ch

[7] Ecalc9: http://nfmcc-docdb.fnal.gov/cgi-bin/ ShowDocument?docid $=280$

[8] Root: http://root.cern.ch 


\title{
PARTICLE TRACKING IN MATTER DOMINATED BEAM LINES
}

\author{
T. Roberts ${ }^{\#}$, K. Beard, Muons, Inc., Batavia IL, USA \\ S. Ahmed, D. Huang, D. M. Kaplan, L. Spentzouris, IIT, Chicago, IL, USA
}

\begin{abstract}
The G4beamline program [1] is a useful and steadily improving tool to quickly and easily model beam lines and experimental equipment without user programming. Unlike most accelerator physics codes, it easily handles a wide range of materials and fields, being particularly well suited for the study of muon and neutrino facilities. As it is based on the Geant4 toolkit [2], G4beamline includes most of what is known about the interactions of particles with matter. We are continuing the development of G4beamline to facilitate its use by a larger set of beam line and accelerator developers. It is open source and freely available at http://g4beamline.muonsinc.com.
\end{abstract}

\section{INTRODUCTION}

As accelerator facilities become more complex and more expensive, accurate and comprehensive simulations of their performance are required long before construction begins. There are many choices and optimizations to be made, as well as new concepts to be explored, so flexible and user-friendly simulation programs become essential to streamline the design process. For future facilities such as muon colliders and neutrino factories, the muon cooling sections demand simulations that accurately compute the interactions of particles in matter, along with associated magnetic and RF fields. The Geant4 toolkit [2] is an excellent choice as the basis of such a program, as it is comprehensive, accurate, and actively supported by a vibrant collaboration. G4beamline [1] was conceived as a user-friendly interface between accelerator physicists and the $\mathrm{C}++$ code of Geant 4 to facilitate the rapid evaluation of new concepts and design changes by physicists without the complexities of $\mathrm{C}++$ programming. An important aspect of G4beamline is that its description of the simulated system is far more comprehensible by unspecialized physicists than the corresponding Geant4 $\mathrm{C}++$ code would be, and no more complicated than is the system itself.

\section{DESCRIPTION}

An obvious aspect of G4beamline is that its user interface has been designed with physicist-users in mind. The system to be simulated is described in a single ASCII file using an object-oriented language specifically designed for this application. Most accelerator physicists can read and understand such a descriptive file without reference to the G4beamline documentation, and can learn how to develop their own simulations with minimal effort.

\footnotetext{
* Work supported in part by USDOE STTR Grant

DE-FG02-08ER86281.

\# tjrob@muonsinc.com
}

Extensive online help is available within the program to assist users in developing their simulations. Figure 1 shows the G4beamline graphical user interface (GUI) screen, including a hyperlinked index and the beginning of its Help text.

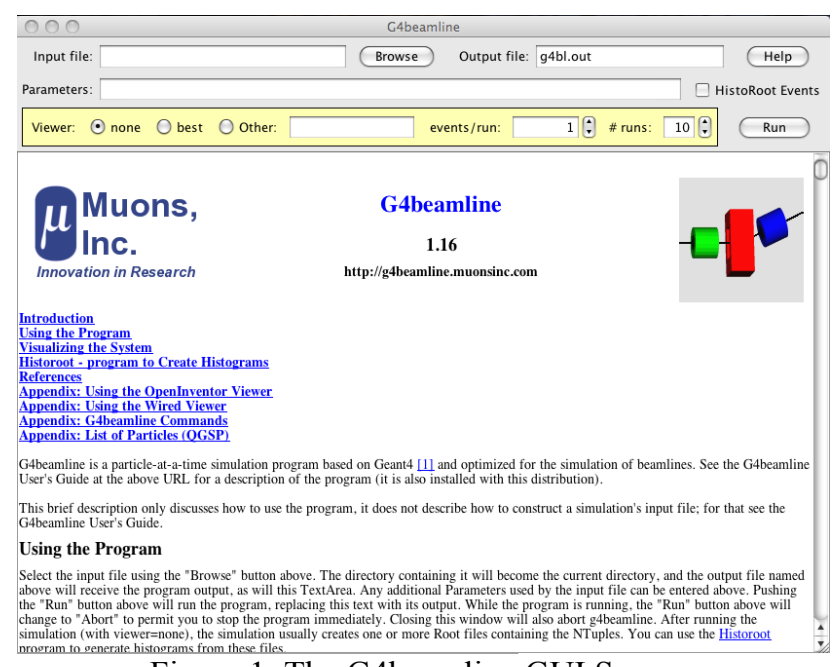

Figure 1. The G4beamline GUI Screen.

To facilitate the generation of histograms and plots, the G4beamline distribution includes the HistoRoot program [3], which provides a user-friendly graphical interface to ROOT [4]. While general ROOT programming can used to create plots, most users find the interface shown in Figure 2 to be more usable and efficient.

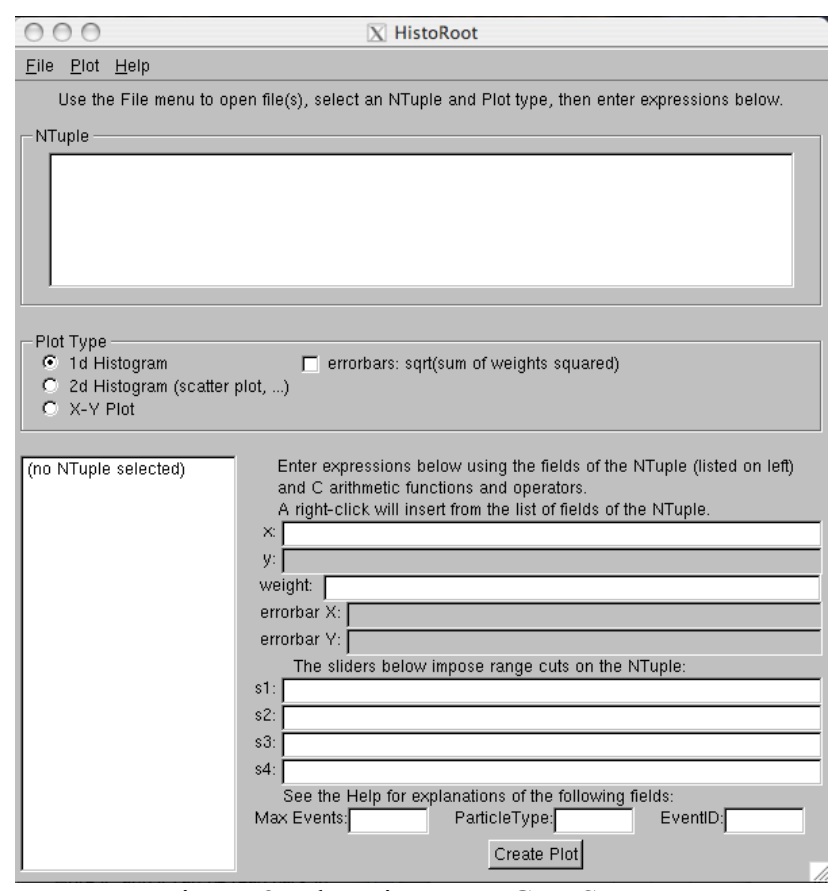

Figure 2. The HistoRoot GUI Screen.

Beam Dynamics and Electromagnetic Fields 
The major aspects of G4beamline are:

- Accurate and realistic simulations using the Geant4 toolkit

- A physicist-readable ASCII file to specify the simulation, with auxiliary files for field maps, etc.

- A rich repertoire of beamline elements that can be combined to define new and customized elements

- A general set of initial beam specifications (including a cosmic ray muon "beam" and external files)

- Input and output of beam tracks using several formats including ASCII and ROOT [4] files - this permits easy interfacing to other programs (e.g., for partitioning of complex systems and verification of results)

- Automatic tuning of many parameters (RF cavity timing and gradients, bending magnet fields, etc.)

- Included visualization of the simulated device or system using many viewers (OpenGL, VRML, Open Inventor, etc.) - provides a powerful visual check that the system implemented is the system desired, and lets you see tracks and their interactions with materials

- Support for parallel jobs on multiple CPUs

- The HistoRoot program, which makes it easy for nonexperts to generate ROOT [4] histograms and plots

The basic structure of a simulation is to first define the beamline elements to be used (magnets, RF cavities, etc.), including their geometry, materials, and local fields. Then these elements are placed into the "world", usually along the nominal beam centerline; each placement can have a position, rotation, and its own field value. Parameters for the element can be defined in the input file or on the command line, so scripting is straightforward. Individual particles can be traced, beam profiles can be generated and displayed, and "virtual detectors" can be used to sample the beam at any point.

The tracking of particles through the simulated system is as accurate and realistic as the Geant4 toolkit implementation. The input file selects any one of the Geant 4 physics lists, and can set values for the various Geant4 tracking accuracy parameters. This permits users to make trade-offs between CPU time and simulation accuracy. Similarly, G4beamline permits the specification of magnetic map parameters, permitting a trade-off between memory usage (and the CPU time needed to generate the map) and simulation accuracy.

While G4beamline can make it rather simple to specify a simulation, it cannot substitute for knowledge and experience about the problem domain or about particle tracking simulations in general. Like all computer programs, G4beamline is prone to "garbage in, garbage out," especially in the hands of unskilled users. It is strongly suggested that visualization be used to verify the geometry of the simulation and the proper tracking of at least a handful of particles. Whenever possible, one should arrange to track through a simple geometry that can be compared to independent results, to make sure that what one thinks is happening actually does occur in the simulation.

\section{EXAMPLE}

An example of using G4beamline is shown in Figure 3. This is the Mu2e experiment at Fermilab [5], searching for the lepton-violating conversion of $\mu^{-}$to $\mathrm{e}^{-}$near an atomic nucleus. Many aspects of the design of this experiment have been explored and evaluated using G4beamline and other programs.

\section{SOFTWARE DEVELOPMENT}

G4beamline is being developed using modern software development techniques. In particular, our methodology requires that feature documentation be written before the code, and that the documentation is contained within the code, so there is always comprehensive and up-to-date documentation available to users. There are two levels of documentation:

- User documentation describing how to use the code

- Internal documentation describing what the code does and how it works.

The first is intended for users and is contained in Help text within the code to implement always-available online help. The latter is intended only for developers, and is contained in structured comments that the doxygen [6] system converts into hyper-linked HTML. Keeping all documentation within the code makes it easy for developers to keep everything in sync; automated tools format it for presentation to users (e.g., the User's Guide).

\section{RECENT FEATURES}

Several recently added features make G4beamline more powerful and more usable by physicists:

1. The User's guide has been considerably enhanced and numerous tips and techniques have been discussed for the best performance of simulations and generation of results.

2. An event-list interface between HistoRoot and G4beamline. The user can select an outlier event in a histogram or plot and easily re-run G4beamline with visualization to see what that particular event did.

3. More Geant4 objects have been implemented, expanding the repertoire of G4beamline.

4. A direct interface to the NIST material database [7] gives users the ability to use many common materials without manually looking up their properties.

5. Rudimentary track fitting is implemented, sufficient to evaluate momentum and mass resolutions; combined with the inherent flexibility of G4beamline this becomes a powerful tool for the early design of experiments.

6. Visualization has been enhanced by adding the feature to generate movies of events showing the time evolution of Ntuple values. 


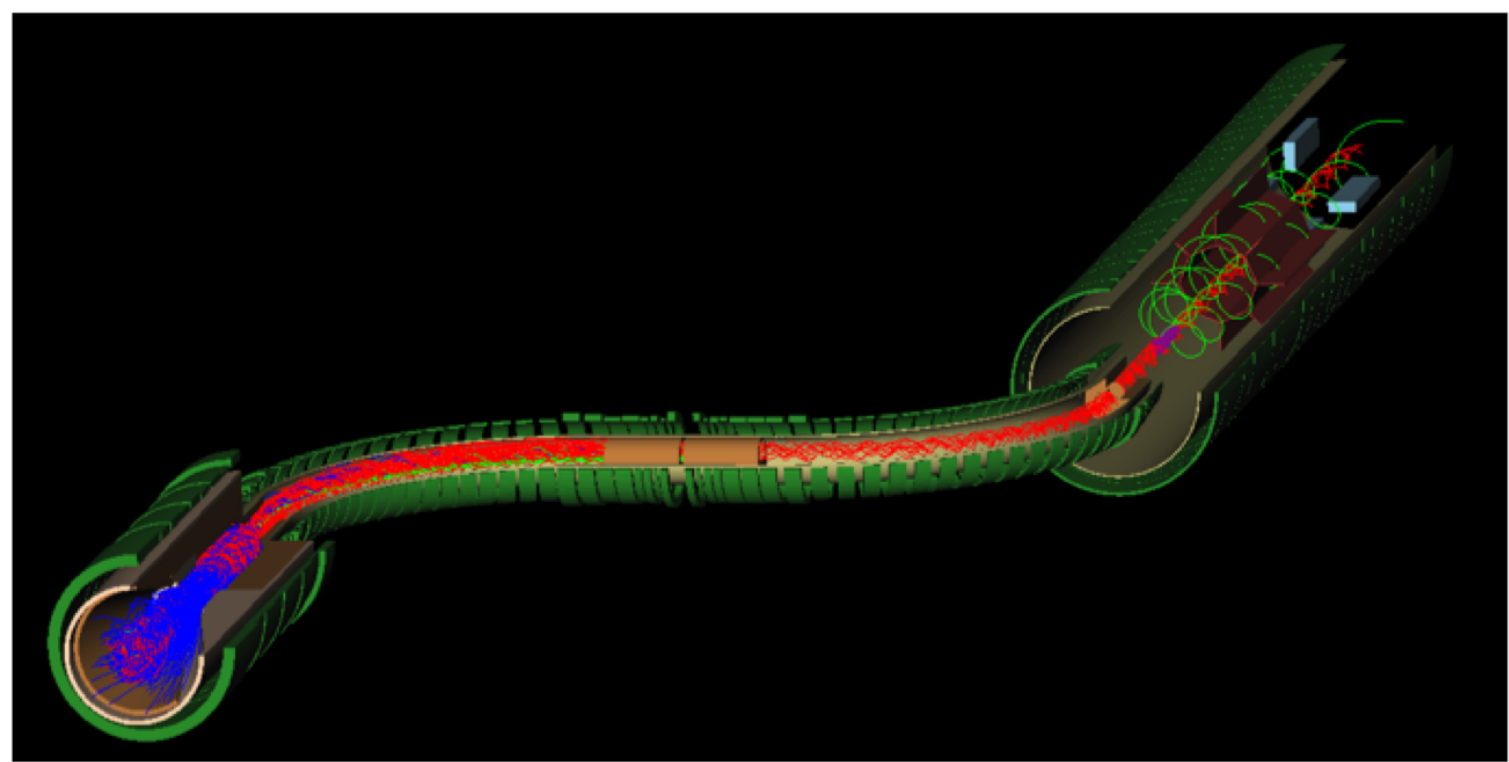

Figure 3: G4beamline visualization of the Mu2e experiment. The production target is at the left, followed by the S-shaped transport solenoid, to the stopping target, with the electron spectrometer and calorimeter behind; $\pi^{-}$are blue, $\mu^{-}$are red, and $\mathrm{e}^{-}$are green.

\section{FUTURE PHYSICS INTERESTS}

We are currently developing or planning the development of the following new features:

1. Space Charge: Current design projections for muon colliders indicate that space charge may be a problem in the final stage of cooling. The implementation of space charge physics in vacuum is almost completed and validation is progressing. The physics model is similar to the existing programs such as ORBIT [8], TREDI [9] and PARMELA [10]. This includes radical revisions to the Geant4 G4Runmanager and G4Eventmanager to track particles in parallel.

2. Collective effects in matter: at the end of a muon cooling channel, very high beam densities are expected, and the collective effects between the beam and the material in the absorbers are expected to be important. This requires an interdisciplinary approach that includes atomic, accelerator, and plasma physics.

3. Polarization physics: Many new muon facilities including muon colliders can benefit from using polarized muons. This requires modeling of polarization especially during the interaction with matter in a cooling channel.

4. Very low energy physics: We are interested in simulating the inner workings of advanced detector systems, atomic traps, and some of the details of surface effects. These require accurate modeling at energies of a few tens or hundreds of $\mathrm{eV}$.

5. Automated parallelization: Computer farms and multi-CPU systems are now common, and we will enhance G4beamline to automatically take advantage of parallelism. At present users can do this manually.

\section{SUMMARY}

G4beamline is a highly flexible and user-friendly program for simulating beamlines both with and without matter. The learning curve is rather short for a program of this scope, and with sufficient attention to detail, the simulation can be quite realistic. We are continually advancing the applicability of the code. In addition to the primary use of investigating many aspects of muon cooling for a muon collider or neutrino factory, applications have included: target hall and test beam design evaluation, feasibility studies for new facilities and experiments, cosmic-ray muon tomography, and detector design. Our commitment to supporting it, our current funding for new features, and our growing user base ensure that G4beamline will remain useful for the foreseeable future.

\section{REFERENCES}

[1] G4beamline - http://g4beamline.muonsinc.com

[2] Geant4 Toolkit - http://geant4.cern.ch

[3] Inspired by HistoScope - http://fermitools.fnal.gov/ abstracts/histoscope/abstract.html

[4] ROOT - http://root.cern.ch

[5] Mu2e - http://mu2e.fnal.gov

[7] Doxygen - http://www.doxygen.org

[7] http://www.nist.gov/srd/materials.htm

[7] Orbit - http://neutrons.ornl.gov/APGroup/ Codes/orbit.htm

[7] TREDI - http://www.tredi.enea.it

[10] PARMELA - http://laacg1.lanl.gov/laacg/services/ serv_codes.phtml\#parmela 


\title{
PARTICLE TRACKING IN MATTER DOMINATED BEAM LINES*
}

\author{
T. Roberts ${ }^{\#}$, K. Beard, Muons, Inc., Batavia IL, USA \\ S. Ahmed, D. Huang, D. M. Kaplan, L. Spentzouris, IIT, Chicago, IL, USA
}

\section{Abstract}

The G4beamline program [1] is a useful and steadily improving tool to quickly and easily model beam lines and experimental equipment without user programming. It has both graphical and command-line user interfaces. Unlike most accelerator physics codes, it easily handles a wide range of materials and fields, being particularly well suited for the study of muon and neutrino facilities. As it is based on the Geant4 toolkit [2], G4beamline includes most of what is known about the interactions of particles with matter. We are continuing the development of G4beamline to facilitate its use by a larger set of beam line and accelerator developers. A major new feature is the calculation of space-charge effects. G4beamline is open source and freely available at:

\section{http://g4beamline.muonsinc.com}

\section{INTRODUCTION}

As accelerator facilities become more complex and more expensive, accurate and comprehensive simulations of their performance are required long before construction begins. There are many choices and optimizations to be made, as well as new concepts to be explored, so flexible and user-friendly simulation programs become essential to streamline the design process. For future facilities such as muon colliders and neutrino factories, the muon cooling sections demand simulations that accurately compute the interactions of particles in matter, along with associated magnetic and RF fields. The Geant4 toolkit [2] is an excellent choice as the basis of such a program, as it is comprehensive, accurate, and actively supported by a vibrant collaboration. G4beamline [1] was conceived as a user-friendly interface between accelerator physicists and the $\mathrm{C}++$ code of Geant 4 to facilitate the rapid evaluation of new concepts and designs by physicists without the burden of $\mathrm{C}++$ programming. An important aspect of G4beamline is that its description of the simulated system is far more comprehensible by physicists than the corresponding Geant $4 \mathrm{C}++$ code would be, and no more complicated than is the system itself.

\section{DESCRIPTION}

An obvious aspect of G4beamline is that its user interface has been designed with physicist-users in mind. The system to be simulated is described in a single ASCII file using an object-oriented language specifically designed for this application. Most accelerator physicists can read and understand such a descriptive file without reference to the G4beamline documentation, and can

\footnotetext{
* Work supported in part by USDOE STTR Grant DE-FG02-08ER86281.

\# tjrob@muonsinc.com
}

learn how to develop their own simulations with minimal effort. Extensive online help is available within the program to assist users in developing their simulations. Figure 1 shows the G4beamline graphical user interface (GUI) screen, including a hyperlinked index and the beginning of its Help text.

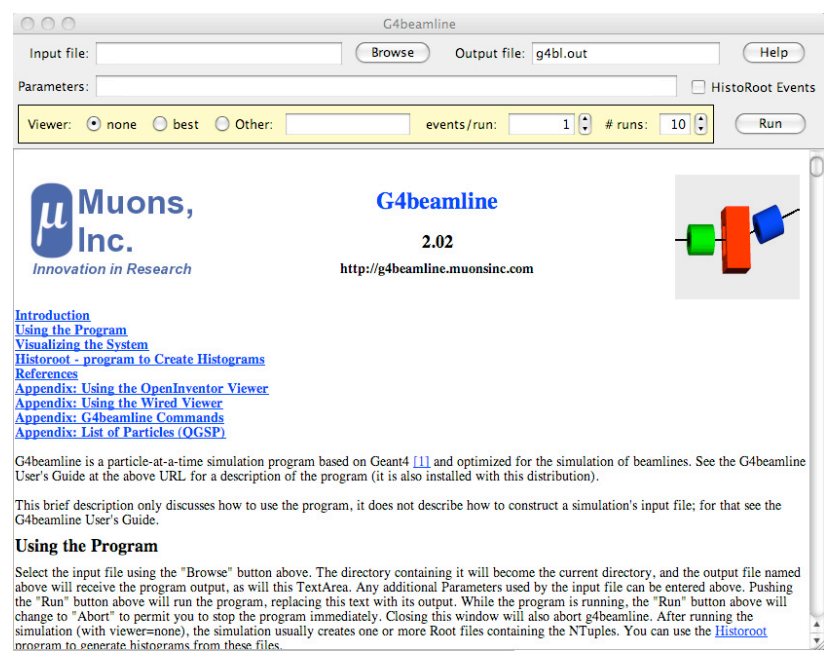

Figure 1: The G4beamline GUI Screen.

To facilitate the generation of histograms and plots, the G4beamline distribution includes the HistoRoot program, which provides a user-friendly graphical interface to ROOT [3]. While general ROOT programming can be used to create plots, most users find the interface shown in Figure 2 to be much more usable and efficient.

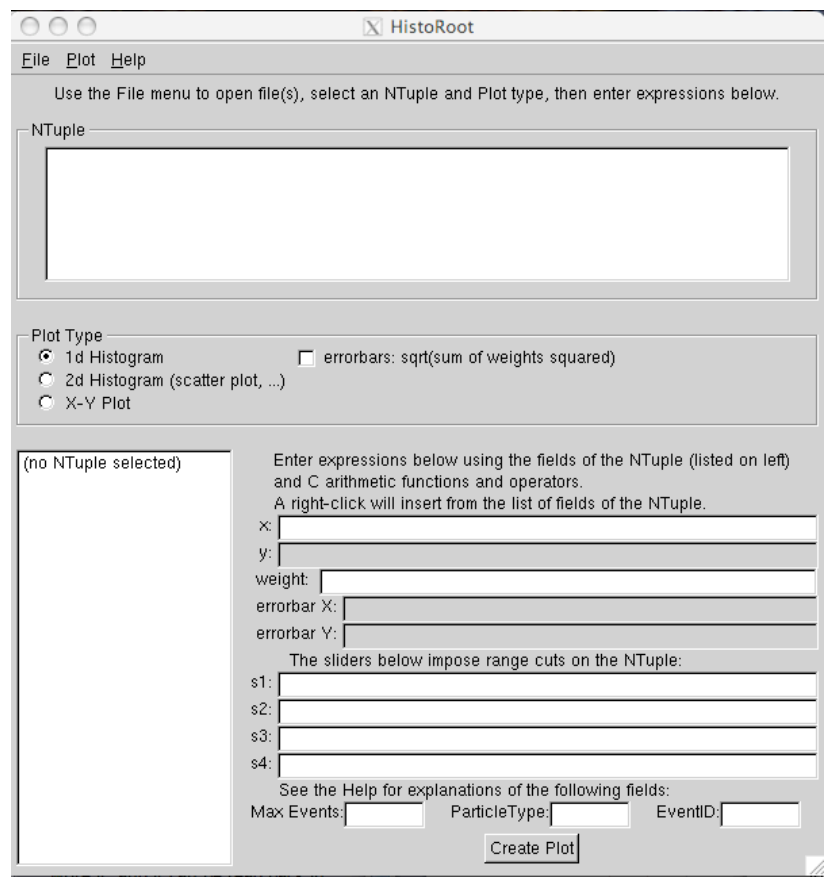

Figure 2: The HistoRoot GUI Screen. 
The major aspects of G4beamline are:

- Accurate and realistic simulations using the Geant4 toolkit

- A physicist-readable ASCII input file to specify the simulation, with auxiliary files for field maps, etc.

- A rich repertoire of beamline elements that can be combined to define new and customized elements

- A general set of initial beam specifications (including a cosmic ray muon "beam" and external files)

- Input and output of beam tracks using several formats including ASCII and ROOT [3] files, supporting easy interfacing to other programs (e.g., for partitioning of complex systems and for verification of results)

- Automatic tuning of many parameters (RF cavity timing and gradients, bending magnet fields, etc.)

- Included visualization of the simulated device or system using many viewers (OpenGL, VRML, Open Inventor, etc.) - provides a powerful visual check that the system implemented is the system desired, and lets you see tracks and their interactions with materials

- Support for parallel jobs on multiple CPUs

- The HistoRoot program, which makes it easy for non-experts to generate ROOT [3] histograms and plots

The basic structure of a simulation is to first define the beamline elements to be used (magnets, RF cavities, etc.), including their geometry, materials, and local fields. Then these elements are placed into the "world", usually along the nominal beam centerline; each placement can have a position, rotation, and its own field value. Parameters for the element can be defined in the input file or on the command line, so scripting is straightforward. Individual particles can be traced, beam profiles can be generated and displayed, and "virtual detectors" can be used to sample the beam at any point.

The tracking of particles through the simulated system is as accurate and realistic as the Geant4 toolkit implementation. The input file selects any one of the Geant4 physics lists, and can set values for the various Geant4 tracking accuracy parameters. This permits users to make trade-offs between CPU time and simulation accuracy. Similarly, G4beamline permits the specification of magnetic map parameters, permitting a trade-off between memory usage (and the CPU time needed to generate the map) and simulation accuracy.

While G4beamline can make it rather simple to specify a simulation, it cannot substitute for knowledge and experience about the problem domain or about particle tracking simulations in general. It is strongly suggested that visualization be used to verify the geometry of the simulation and the proper tracking of at least a handful of particles. Whenever possible, one should arrange to track through a simple geometry that can be compared to independent results, to make sure that what one thinks is happening actually does occur in the simulation.

\section{SOFTWARE DEVELOPMENT}

G4beamline is being developed using modern software development techniques. In particular, our methodology requires that feature documentation be written before the code, and that the documentation is contained within the code, so there is always comprehensive and up-to-date documentation available to users. There are two levels of documentation:

- User documentation describing how to use the code

- Internal documentation describing what the code does and how it works.

The first is intended for users and is contained in Help text within the code to implement always-available online help. The latter is intended only for developers, and is contained in structured comments that the doxygen [4] system converts into hyper-linked HTML. Keeping all documentation within the code makes it easy for developers to keep everything in sync; automated tools format it for presentation to users (e.g., the User's Guide).

\section{RECENT FEATURES}

Several recently added features make G4beamline more powerful and more usable by physicists:

1. The User's guide has been considerably enhanced and numerous tips and techniques have been discussed for the best performance of simulations and generation of results.

2. More Geant 4 objects have been implemented, expanding the repertoire of G4beamline.

3. The Geant4 General Particle Source can now be used to generate beams.

4. The build structure has been completely revised, improving the installers and making it much easier for users to build G4beamline and add new code.

5. Visualization has been enhanced by adding the feature to generate movies of events showing the time evolution of tracks.

6. Space Charge: Current design projections for muon colliders indicate that space charge may be a problem in the final stage of cooling. The computation uses Lienard-Wiechert potentials, and its CPU time scales as the square of the number of macroparticles used. This includes radical revisions to the code of the Geant4 RunManager and EventManager to track particles in parallel.

7. Low energy physics: new physics lists are available that include the Geant4 low-energy electromagnetic models.

8. Automated parallelization: Computer farms and multi-CPU systems are now common, and MPI [5] is used to take advantage of parallelism - a single set of output files contains the computations of multiple processes running on multiple CPUs. 


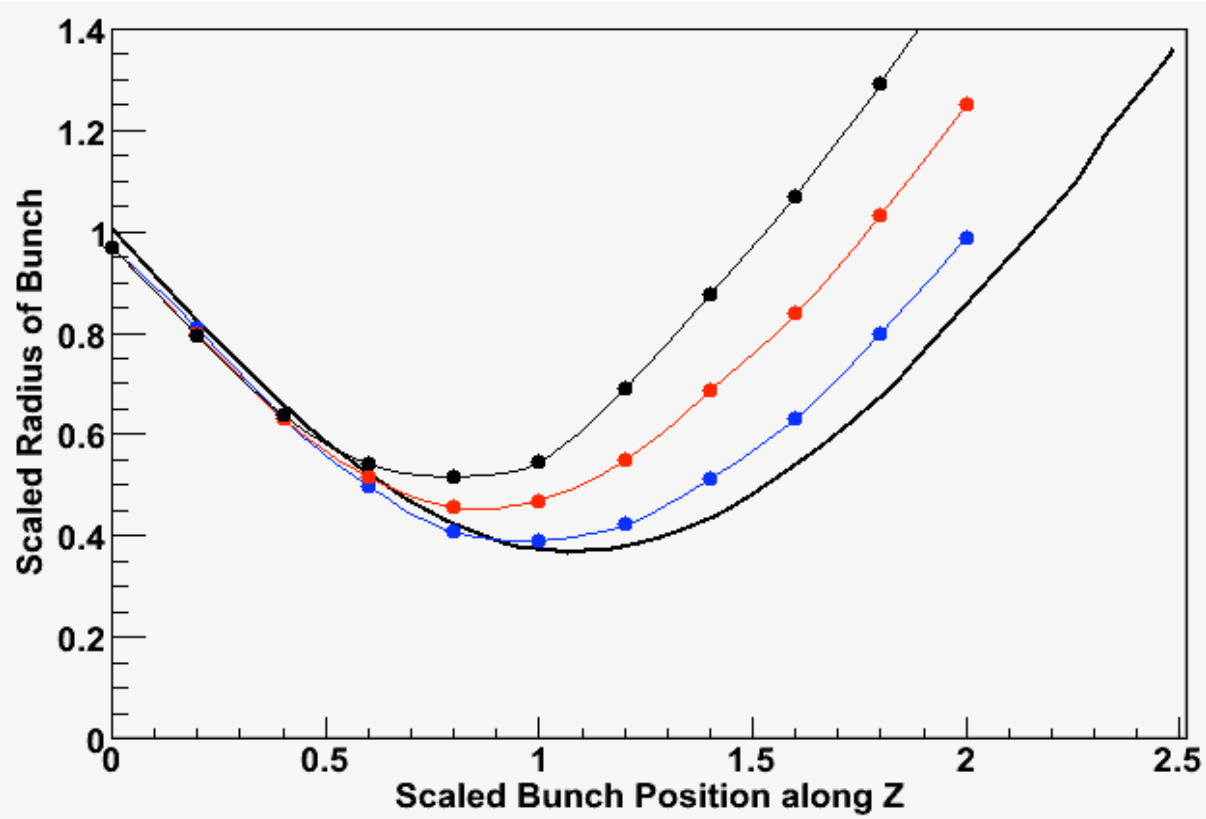

Figure 3: Comparison of G4beamline's space charge computation to a calculation in Reiser's textbook [6], showing transverse beam size as a function of propagation distance. The initial beam is a uniform cylinder, and would be focused to a point at $Z=1$ without space charge. The solid line is the calculation for a continuous charge density; the points are for computations using 100 (black), 1,000 (red), and 10,000 (blue) macro-particles.

\section{SPACE CHARGE VALIDATION}

Figure 3 shows a comparison of the G4beamline space charge computation to a calculation from Reiser's textbook [6] of how space charge forces affect the beam size as it propagates. The initial beam is a uniform cylinder with converging trajectories; the space charge defocusing is evident, as without it the beam would be focused to a point at $Z=1$. The values plotted are appropriately scaled, so the plot is unchanged for variations in beam momentum, bunch radius, total charge, and particle type. The points plotted are for the $95^{\text {th }}$ percentile of macro-particle radii, which is why they are initially below the line. At large Z, some macro-particles have experienced an abnormally large interaction with other macro-particles, so the points are above the line, and higher-charge macro-particles are affected more strongly. Overall, the agreement with the calculation is quite good, but requires a large number of macro-particles for accurate agreement with the analytic result.

\section{FUTURE PHYSICS INTERESTS}

We are currently developing or planning the development of the following new features:

1. Improving the space charge computation: the current computation scales as $\mathrm{N}^{2}$, which makes it extremely CPU intensive; we intend to implement a much faster algorithm based on Fast Fourier Transforms that will significantly reduce the CPU usage.

2. Collective effects in matter: at the end of a muon cooling channel, very high beam densities are expected, and the collective effects between the beam and the material in the absorbers are expected to be important. This includes effects due to the polarization of the material, as well as the plasma generated by the ionization of the material by the beam. This requires an interdisciplinary approach that includes atomic, accelerator, and plasma physics.

3. Polarization physics: Many new muon facilities including muon colliders can benefit from using polarized muons. This requires modeling of polarization especially during the interaction with matter in a cooling channel.

\section{SUMMARY}

G4beamline is a highly flexible and user-friendly program for simulating beamlines both with and without matter. We are continually advancing the applicability of the code. In addition to the primary use of investigating many aspects of muon cooling for a muon collider or neutrino factory, applications include: target hall and test beam design evaluation, feasibility studies for new facilities and experiments, cosmic-ray muon tomography and detector design. Our commitment is to provide support; current funding for new features and our growing user base ensure that G4beamline will remain useful for the foreseeable future.

\section{REFERENCES}

[1] G4beamline - http://g4beamline.muonsinc.com

[2] Geant4 Toolkit - http://geant4.cern.ch

[3] ROOT - http://root.cern.ch

[4] Doxygen - http://www.doxygen.org

[5] MPI - http://www.open-mpi.org

[6] Reiser, M., Theory and Design of Charged Particle Beams, Wiley, 2008. 


\title{
G4BEAMLINE PARTICLE TRACKING IN MATTER DOMINATED BEAM LINES*
}

\author{
T. J. Roberts ${ }^{\#}$, K. B. Beard, Muons, Inc., Batavia, IL, USA \\ S. Ahmed", D. Huang ${ }^{\S}$, D. M. Kaplan, IIT, Chicago, IL, USA
}

\begin{abstract}
The G4beamline program [1] is a useful and steadily improving tool to quickly and easily model beam lines and experimental equipment without user programming. It has both graphical and command-line user interfaces. Unlike most accelerator physics codes, it easily handles a wide range of materials and fields, being particularly well suited for the study of muon and neutrino facilities. As it is based on the Geant4 toolkit [2], G4beamline includes most of what is known about the interactions of particles with matter. We are continuing the development of G4beamline to facilitate its use by a larger set of beam line and accelerator developers. A major new feature is the calculation of space-charge effects. G4beamline is open source and freely available at:

http://g4beamline.muonsinc.com
\end{abstract}

\section{INTRODUCTION}

As accelerator facilities become more complex and more expensive, accurate and comprehensive simulations of their performance are required long before construction begins. There are many choices and optimizations to be made, as well as new concepts to be explored, so flexible and user-friendly simulation programs become essential to streamline the design process. For future facilities such as muon colliders and neutrino factories, the muon cooling sections demand simulations that accurately compute the interactions of particles in matter, along with associated magnetic and RF fields. The Geant4 toolkit [2] is an excellent choice as the basis of such a program, as it is comprehensive, accurate, and actively supported by a vibrant collaboration. G4beamline [1] was conceived as a user-friendly interface between accelerator physicists and the $\mathrm{C}++$ code of Geant 4 to facilitate the rapid evaluation of new concepts and designs by physicists without the burden of $\mathrm{C}++$ programming. An important aspect of G4beamline is that its description of the simulated system is far more comprehensible by physicists than the corresponding Geant $4 \mathrm{C}++$ code would be, and unlike the code, it is no more complicated than is the system itself.

\section{DESCRIPTION}

An obvious aspect of G4beamline is that its user interface has been designed with physicist-users in mind. The system to be simulated is described in a single ASCII file using an object-oriented language specifically designed for this application. Most accelerator physicists can read and understand the input file without reference to the G4beamline documentation, and can learn how to

* Work supported in part by USDOE STTR Grant DE-FG02-08ER86281. * tjrob@muonsinc.com

"Current address: Jefferson Lab, Newport News, VA, USA.

${ }^{\S}$ Current address: Shanghai Institute of Applied Physics, China. develop their own simulations with minimal effort. Extensive online help is available within the program to assist users in developing their simulations. Figure 1 shows the G4beamline graphical user interface (GUI) screen, including a hyperlinked index and the beginning of its Help text.

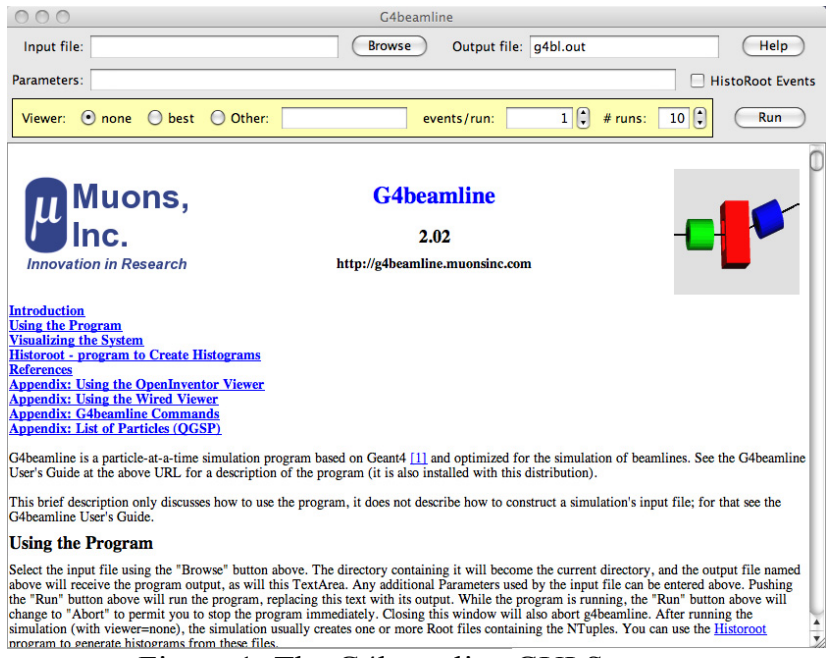

Figure 1: The G4beamline GUI Screen.

To facilitate the generation of histograms and plots, the G4beamline distribution includes the HistoRoot program, which provides a user-friendly graphical interface to ROOT [3]. While general ROOT programming can be used to create plots, most users find the interface shown in Figure 2 to be much more usable and efficient.

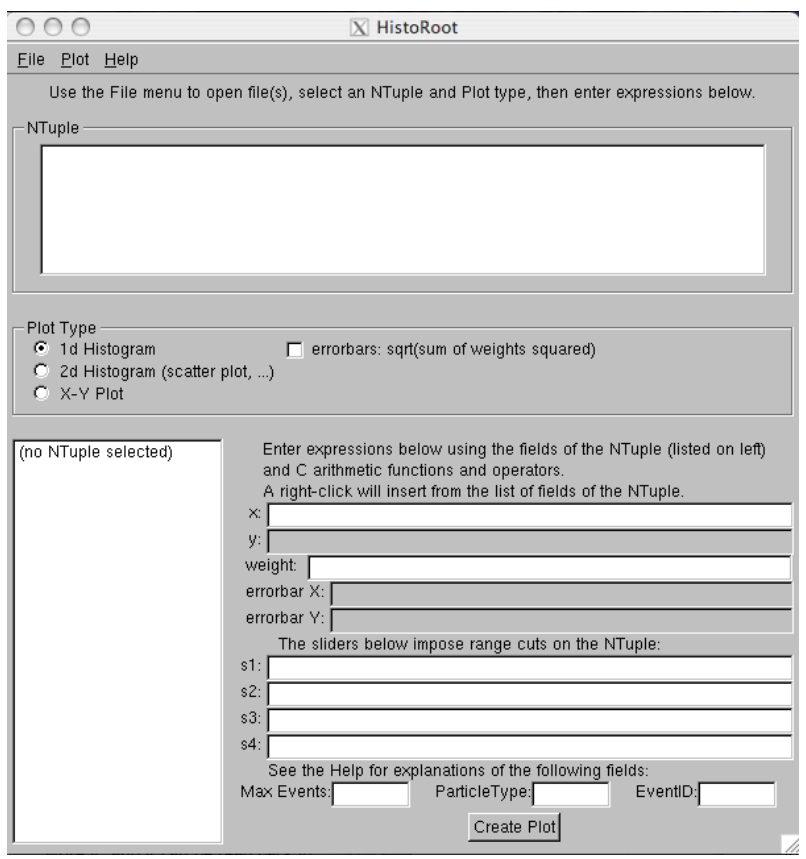

Figure 2: The HistoRoot GUI Screen. 
The major aspects of G4beamline are:

- Accurate and realistic simulations using the Geant4 toolkit

- A physicist-readable ASCII input file to specify the simulation, with auxiliary files for field maps, etc.

- Centerline coordinates make it easy to place elements along the beamline

- A rich repertoire of beamline elements suitable for a large class of accelerator, beamline, and detector systems, that can be combined to define new and customized elements

- A general set of initial beam specifications (including the General Particle Source, a cosmic ray muon "beam", and external files)

- Input and output of beam tracks using several formats including ASCII and ROOT [3] files, supporting easy interfacing to other programs (e.g., for partitioning of complex systems and for verification of results)

- Automatic tuning of many parameters (RF cavity timing and gradients, bending magnet fields, etc.)

- Included visualization of the simulated device or system using many viewers (OpenGL, VRML, Open Inventor, etc.) - provides a powerful visual check that the system implemented is the system desired, and lets you see tracks and their interactions with materials

- Support for parallel jobs on multiple CPUs

- Available for Linux, Windows, and Mac OS systems, as well as source code; it is Open Source

- The HistoRoot program, which makes it easy for nonexperts to generate ROOT [3] histograms and plots

The basic structure of a simulation is to first define the beamline elements to be used (magnets, RF cavities, etc.), including their geometry, materials, and local fields. Then these elements are placed into the "world", usually along the nominal beam centerline; each placement can have a position, rotation, and its own field value. Parameters for the element can be defined in the input file or on the command line, so scripting is straightforward. Individual particles can be traced, beam profiles can be generated and displayed, and "virtual detectors" and "zntuples" can be used to sample the beam at any location.

The tracking of particles through the simulated system is as accurate and realistic as the Geant4 toolkit implementation. The input file selects any one of the Geant 4 physics lists, and can set values for the various Geant 4 tracking accuracy parameters. This permits users to make trade-offs between CPU time and simulation accuracy. Similarly, G4beamline permits the specification of magnetic map parameters, permitting a trade-off between memory usage and simulation accuracy.

While G4beamline can make it rather simple to specify a simulation, it cannot substitute for knowledge and experience about the problem domain or about particle tracking simulations in general. It is strongly suggested that visualization be used to verify the geometry of the simulation and the proper tracking of at least a handful of particles. Whenever possible, one should arrange to track through a simple geometry that can be compared to independent results, to make sure that what one thinks is happening actually does occur in the simulation.

\section{SOFTWARE DEVELOPMENT}

G4beamline is being developed using modern software development techniques. In particular, our methodology requires that feature documentation be written before the code, and that the documentation is contained within the code, so there is always comprehensive and up-to-date documentation available to users. There are two levels of documentation:

- User documentation describing how to use the code

- Internal documentation describing what the code does and how it works

The first is intended for users and is contained in Help text within the code to implement always-available online help. The latter is intended only for developers, and is contained in structured comments that the doxygen [4] system converts into hyper-linked HTML. Keeping all documentation within the code makes it easy for developers to keep everything in sync; automated tools format it for presentation to users (e.g. the User's Guide).

\section{RECENT FEATURES}

Several recently added features make G4beamline more powerful and more usable by physicists:

1. The User's guide has been considerably enhanced including numerous tips and techniques to help users apply the program to their problems, get the best simulation performance, and generate results.

2. More Geant4 objects have been implemented, expanding the repertoire of G4beamline.

3. Visualization has been enhanced with the implementation of transparency in objects and the ability to draw E \& B field lines

4. Space Charge: current design projections for muon colliders indicate that space charge may be a problem in the final stages of cooling. This computation uses FFTs for the efficient convolution of the Green's function with the charge distribution on a grid in the beam rest frame; it can use up to about 1 million macro-particles. This includes radical revisions to the code of the Geant4 RunManager and EventManager to track particles in parallel for steps in time.

5. Synchrotron radiation is now supported for e and $\mu$.

6. $\mu$-pair production is now supported.

7. Automated parallelization: Computer farms and multi-CPU systems are now common, and MPI [5] can be used to take advantage of parallelism - a single set of output files contains the computations of multiple processes running on multiple CPUs. 


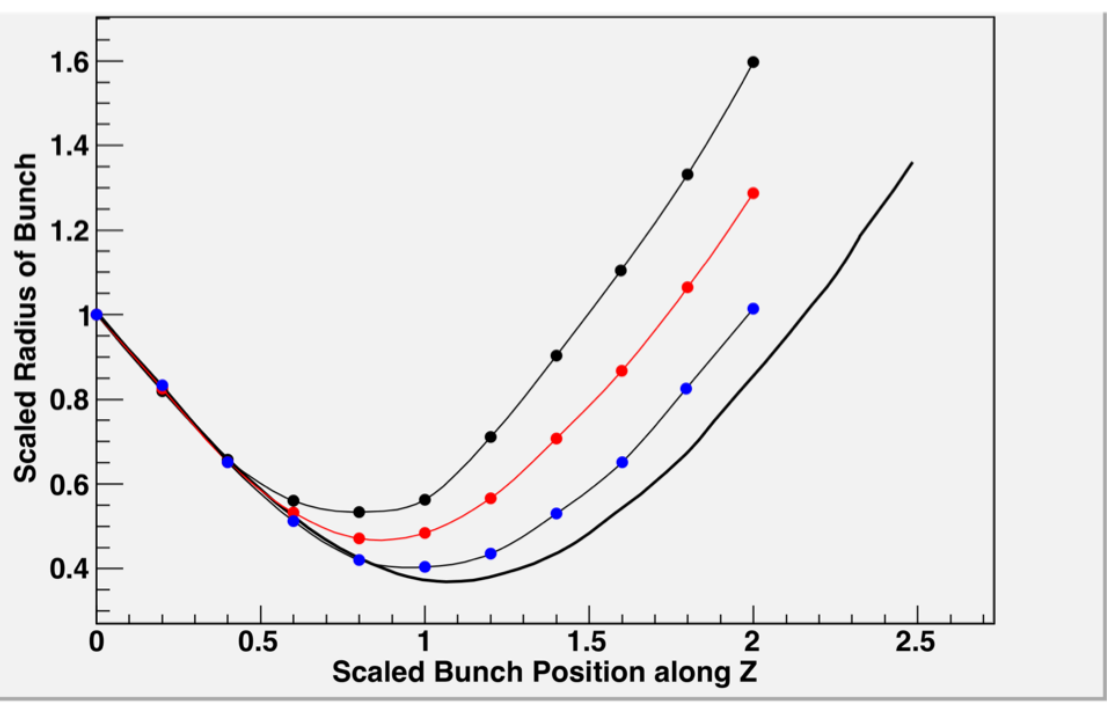

Figure 3: Comparison of G4beamline's space charge computation to a calculation in Reiser's textbook [6], showing scaled transverse beam size as a function of scaled propagation distance $Z$. The initial beam is a uniform cylinder which would be focused to a point at $Z=1$ without space charge. The solid black line is the calculation for a continuous charge density corresponding to $10^{11} \mu^{+}$; the points are for computations using 1,000 (black), 10,000 (red), and 100,000 (blue) macro-particles, each modeling a bunch also totaling $10^{11} \mu^{+}$(decays disabled).

\section{SPACE CHARGE VALIDATION}

Figure 3 shows a comparison of the G4beamline space charge computation to a calculation from Reiser's textbook [6] of how space charge forces affect the beam size as it propagates. The initial beam is a uniform cylinder with converging trajectories; the space charge defocusing is evident, as without it the beam would be focused to a point at $Z=1$. The values plotted are scaled by a function of beam momentum, bunch radius, total charge density, and particle type (see [6]), so the plot is independent of them. Because the macro-particles have huge charges, some macro-particles have experienced an abnormally large interaction with other macro-particles, so the points are above the line, and higher-charge macroparticles are affected more strongly. Overall, the agreement with the calculation is quite good, but requires a large number of macro-particles for accurate agreement with the analytic result.

\section{"MISSING" PHYSICS PROCESSES}

The MAP Collaboration [7] has identified many physics processes that are not in our current tool set; we need to understand each process, and for the important ones either acquire new tools or implement them in our current tools; G4beamline is the prime candidate for the latter.

- Some minor single-particle processes

- Collective effects in vacuum:

- Wake fields and beam loading

- Beam-beam interactions

- Electron cloud effects

- Decay and interactions of macro-particles

- Collective effects in matter:

- Space charge screening and dielectric polarization

- Plasma effects from ionized atoms and their free electrons
- Energy loss and multiple scattering from ionized and excited atoms, and effects from the presence of plasma

- Intra-beam screening affecting energy loss and multiple scattering

- Effects of matter on wake fields and electron cloud

Efforts are underway to analyze and understand these processes; tool acquisition and/or implementation of important ones will follow.

\section{SUMMARY}

G4beamline is a highly flexible and user-friendly program for simulating beamlines both with and without matter. Its learning curve is rather short for a program of this scope, and with sufficient attention to detail the simulation can be quite realistic. We are continually advancing the applicability of the code. In addition to the primary use of investigating many aspects of muon cooling for a muon collider or neutrino factory, current applications include: target hall and test beam design evaluation, feasibility studies for new facilities and experiments, cosmic-ray muon tomography, and detector design, resolution, and background studies. Our commitment to supporting it, our current funding for new features, and our growing user base ensure that G4beamline will remain useful for the foreseeable future.

\section{REFERENCES}

[1] G4beamline - http://g4beamline.muonsinc.com

[2] Geant4 Toolkit - http://geant4.cern.ch

[3] ROOT - http://root.cern.ch

[4] Doxygen - http://www.doxygen.org

[5] MPI - http://www.open-mpi.org

[6] Reiser, M., Theory and Design of Charged Particle Beams, Wiley, 2008.

[7] The Muon Accelerator Program, http://map.fnal.gov 


\title{
G4Beamline
}

\section{A "Swiss Army Knife" for Geant4}

\author{
http://g4beamline.muonsinc.com
}

\author{
Tom Roberts
}

Muons, Inc.

$\int \begin{aligned} & \text { Muons, } \\ & \text { Inc. }\end{aligned}$ 


\section{Basic Approach}

- A general, flexible, and extensible program for Geant4 simulations using single particle tracking, optimized for beamlines and other applications (e.g. there is a cosmic-ray "beam").

- Requires no programming by users, but is sophisticated enough to simulate the Study II SFoFo Cooling Channel, and flexible enough to do the MICE beamline, cosmic-ray studies, and many others.

- Provide user-friendly input generation, visualization of the system, and analysis of the simulation results. The specification of the simulation is no more complicated than the system to be simulated.

- Include realistic and accurate particle tracking and interactions (incl. EM, weak, and hadronic).

- Includes support for scripting and parallel jobs on a cluster. 


\section{Using the Program}

- The basic idea is to define each beamline element, and then place each one into the beamline at the appropriate place(s).

- All aspects of the simulation are specified in a single ASCII input file:

- Geometry

- Input Beam

- Physics process selection

- Program control parameters

- Generation of output NTuples

- The input file consists of a sequence of commands with named arguments

- Command and argument names are spelled out, so the input file becomes a record of the simulation that is readable by others 


\section{Using the Program}

- The beamline elements implemented are:

- absorber - a material absorber with shaped containment and safety windows

- box - a material in the shape of a box

- corner - rotate the centerline coordinates, for bend or secondary target

- cosmicraybeam - a "beam" of cosmic-ray muons

- fieldmap - read a field map from a file, for E and/or B

- genericbend - a generic bending magnet

- genericquad - a generic quadrupole magnet

- helicaldipole - a helical dipole magnet for 6-D muon cooling

- idealsectorbend - a sector bending magnet

- pillbox - a pillbox RF cavity, including optional windows

- polycone - a material in the shape of multiple cones

- solenoid - a single-coil magnet

- sphere - a material in the shape of a sphere

- trap - a material in the shape of a trapezoid

- tubs - a material in the shape of a cylinder or pipe

- virtualdetector - a 'perfect' detector for monitoring the beam 
Muons,

Inc.

\section{Using the Program}

- Simulation control commands:

- beam - specify the incoming beam (from a file or randomly generated)

- reference - specify a reference particle

- place - position a previously-defined object into the simulation

- material - specify the properties of a new material

- geometry - perform geometrical tests for invalid intersections of objects

- param - define parameters for program or input file

- particlecolor - specify the display colors for particle types

- particlefilter - cut particles by type or momentum, force decays, etc.

- physics - defines the physics processes and controls them

- trackcuts - impose specific cuts on tracks

- Beamline layout commands:

- start - defines the starting point and orientation of the beamline

- corner - inserts a corner into the Centerline coordinates

- cornerarc - inserts a corner into the Centerline coordinates, with path length of an arc 


\section{Using the Program}

- Complex manual procedures have been automated:

- Field maps of solenoids can be automatically determined by specifying the required accuracy

- RF Cavities can be tuned, for timing and gradient; both can be fixed or automatically tuned

- Geometry layout has been vastly simplified

- Beam elements are simply lined up along the $\mathrm{Z}$ axis

- Centerline coordinates behave naturally for bending magnets or secondary targets

- Elements may overlap (e.g. nested pipes), but not intersect

- Many elements can be the parent of other elements

- Specific offsets in X, Y, and/or Z can be specified when needed

- Rotations are specified naturally

- Y30,Z90 is a 30 degree rotation around $\mathrm{Y}$ followed by a 90 degree rotation around Z

- Axes are for the parent volume and thus do not change

- Automatic geometry testing detects invalid intersections

- All of the Geant4 8.1 physics use cases are available by name.

- Beam tracks can be generated internally, or read from a file

- Most Geant4 visualization drivers are supported by name.

- Open Inventor is included, and is by far the most user friendly 


\section{Using the Program}

- The result is a program that reduces the complexity of the user input to that of the system being simulated (a major drawback of any simulation program, including those using Geant 4 , is that $\mathrm{C}++$ simulation code is considerably more complex than the problem).

- While $\mathrm{C}++$ programming is not required to use the program, knowledge of the problem domain is absolutely required, as is enough experience to distinguish sensible results from nonsense.

- Visualization is highly recommended, to verify that the geometry is correct and makes sense. 


\section{Example Uses - MICE}

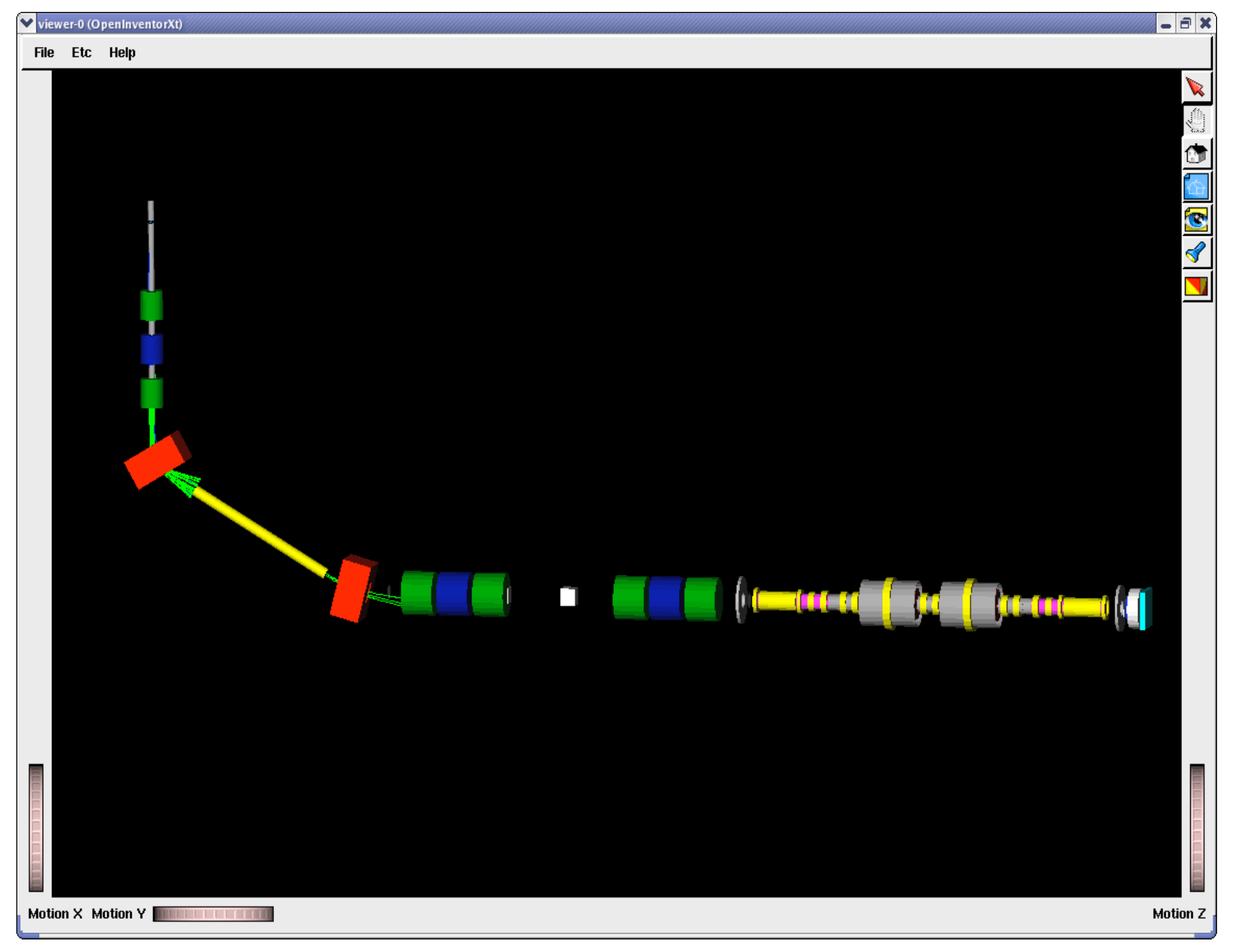




\section{Example Uses - 6D Muon Cooling}

\section{Evolution of Beam emittance}
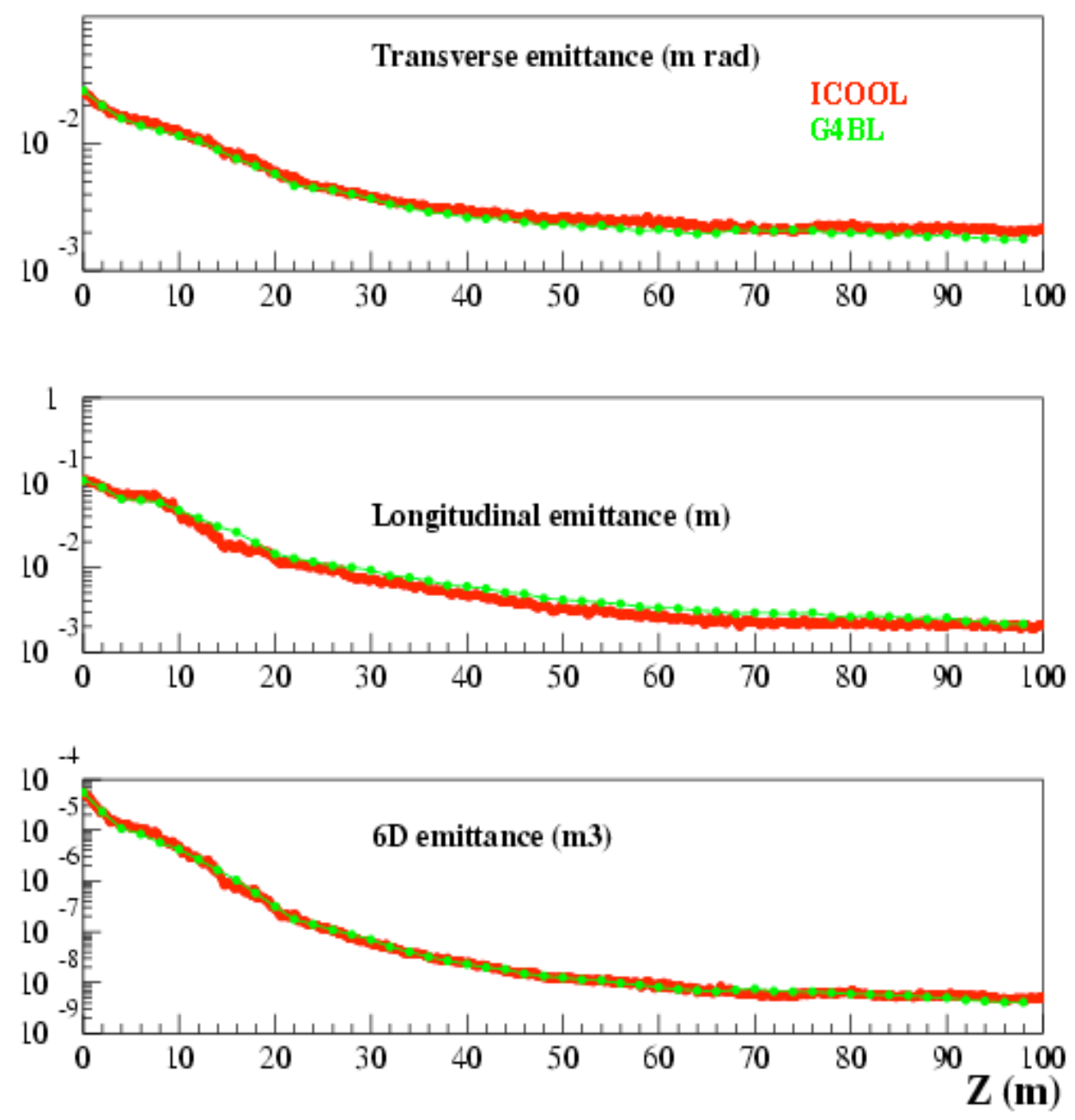


\section{$\boldsymbol{\mu}_{\text {Inc. }}^{\text {Muons, }}$ Example Uses - Cosmic Ray Tomography}

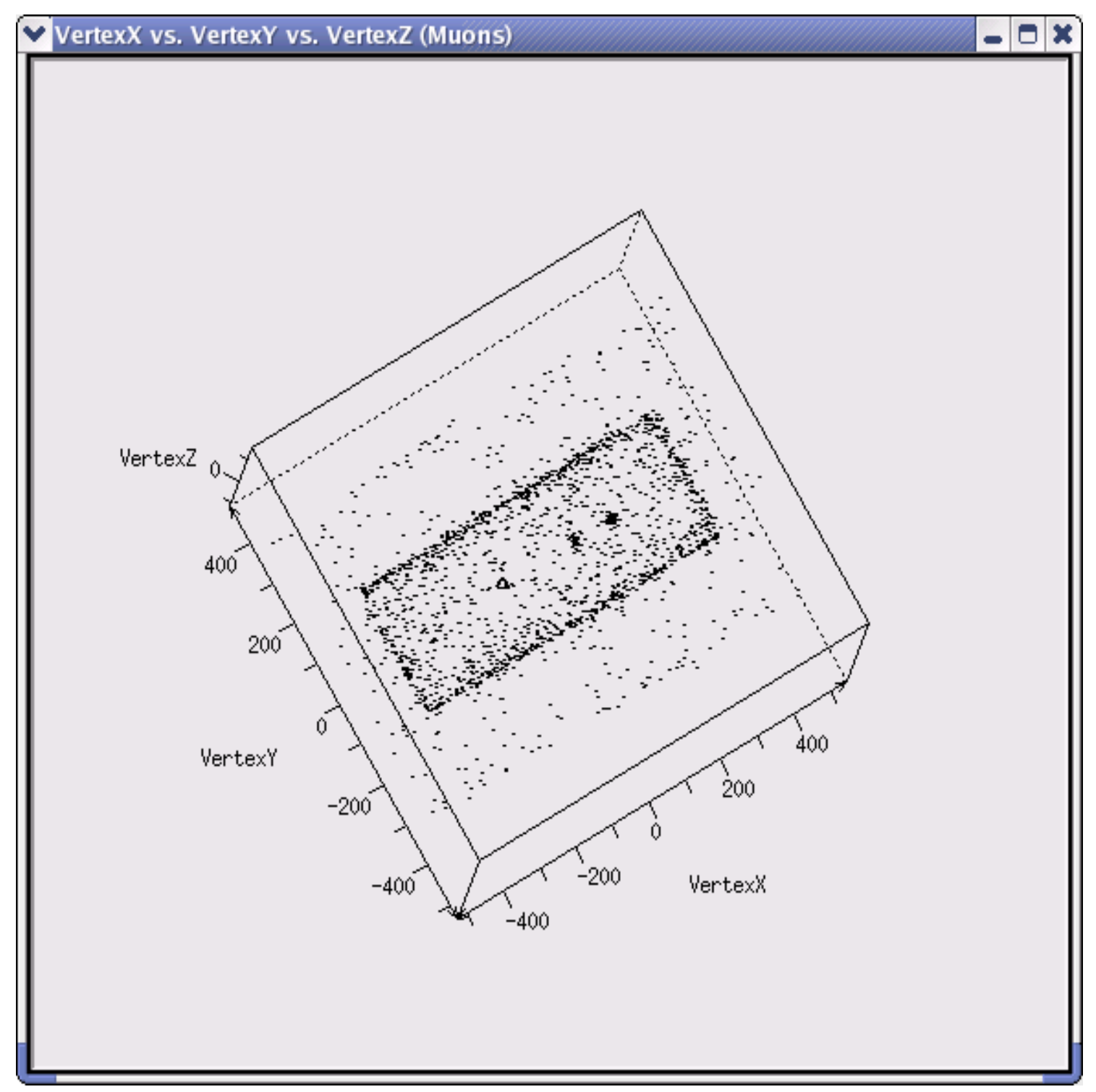




\section{example1.in}

\# example1.in - put beam into 4 detectors physics LHEP_BIC

beam gaussian particle $=$ mu $+n E v e n t s=1000$ beamZ $=0.0 \backslash$

sigmaX $=10.0$ sigma $Y=10.0$ sigmaX $p=0.100$ sigma $Y p=0.100 \backslash$

meanMomentum $=200.0$ sigmaP $=4.0$ meanT $=0.0$ sigmaT $=0.0$

\# BeamVis just shows where the beam comes from

Box BeamVis width $=100.0$ height $=100.0$ length $=0.1$ color $=1,0,0$

\# define the detector (used 4 times)

detector Det radius $=1000.0$ color $=0,1,0$

\# place BeamVis and four detectors, putting their number into their names

place BeamVis $z=0$

place Det $z=1000.0$ rename $=$ Det\#

place Det $z=2000.0$ rename $=$ Det\#

place Det $z=3000.0$ rename $=$ Det\#

place Det $z=4000.0$ rename $=$ Det\# 


\section{example1.in}

viewer-0 (OpenGLImmediateX)

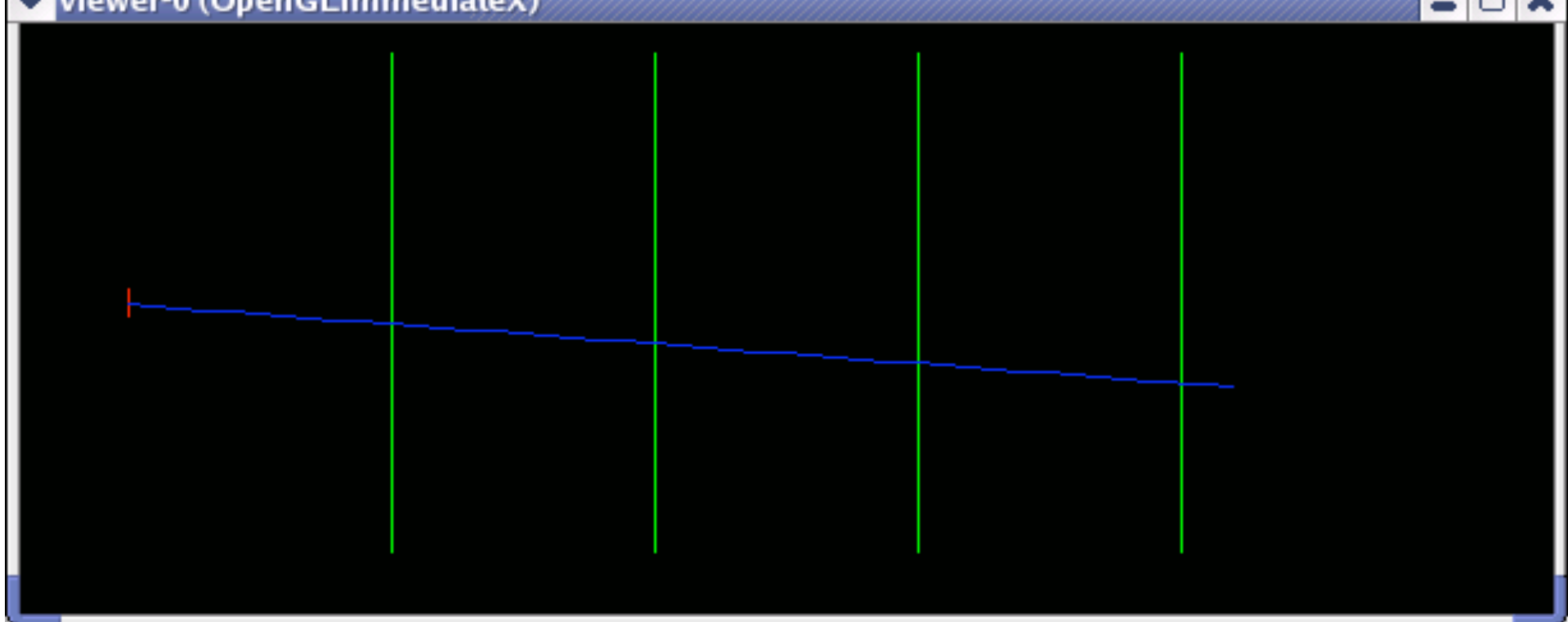

Visualizations of example1.in, using OpenGL (above), and OpenInventor (right). Above is a plan view, while at right is a 3-D view.

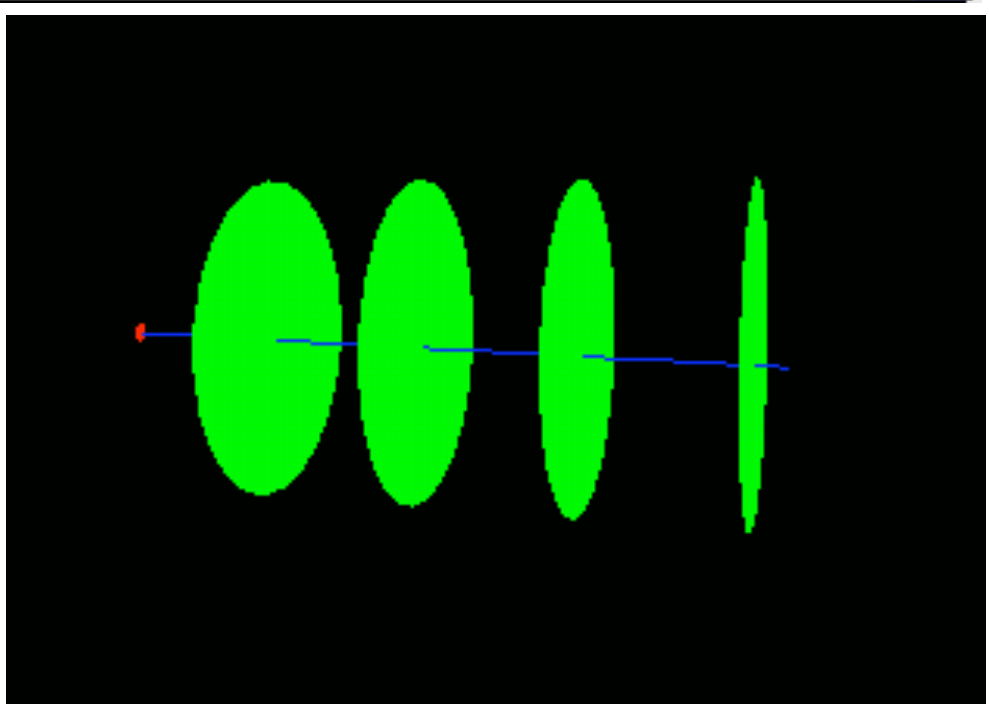




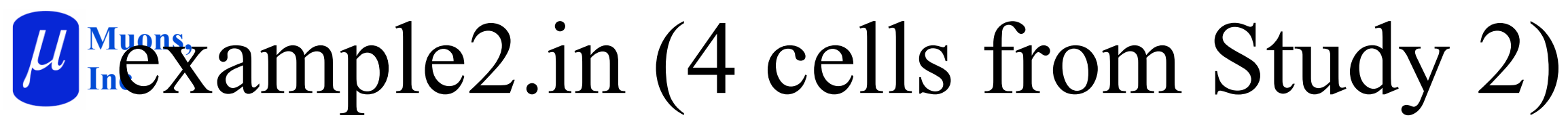

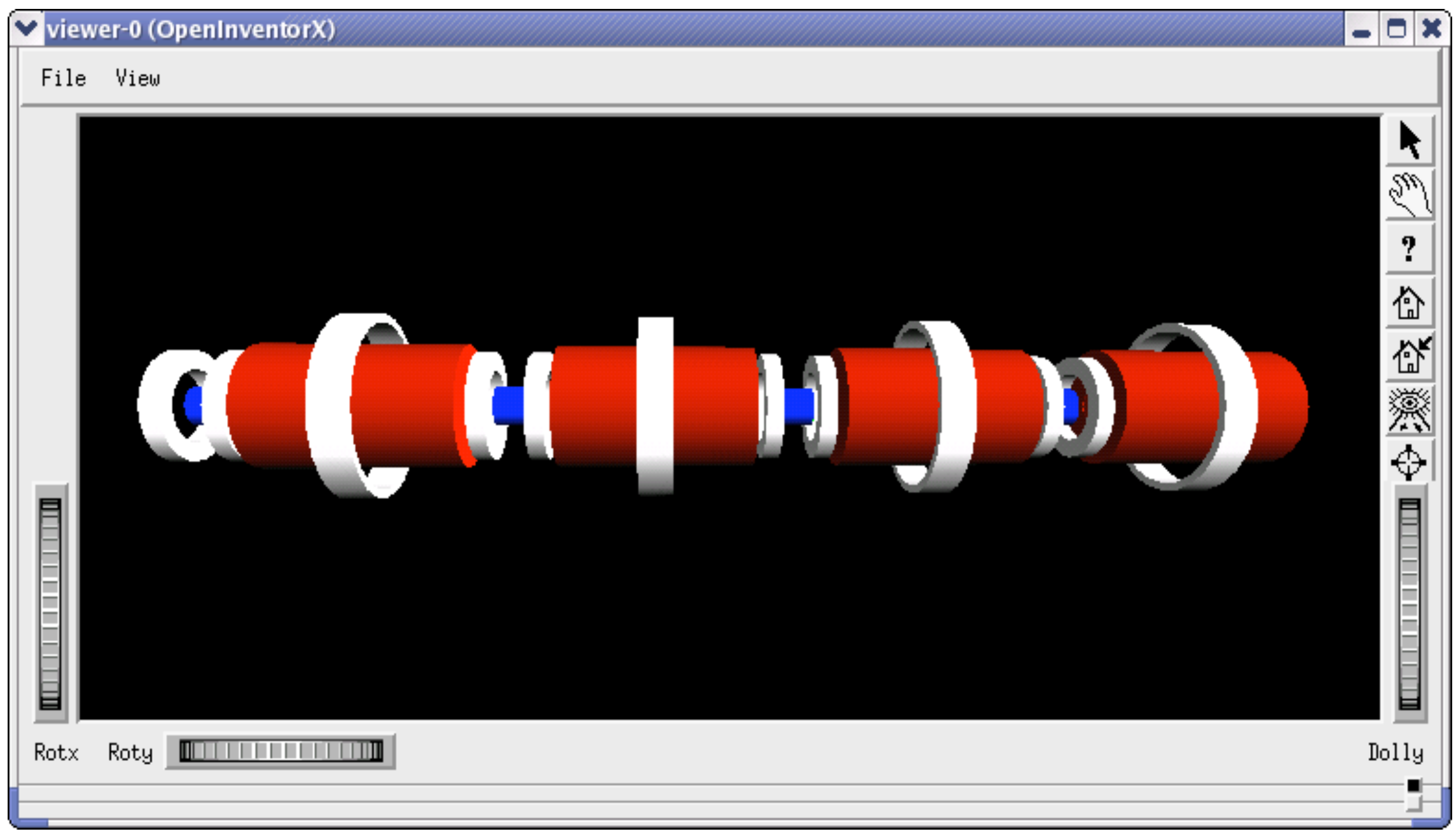


Inc.

- Visualization of the MICE beamline via Open Inventor

- Generation of histograms and manipulating them via HistoScope 


\section{Summary}

- G4Beamline is a simulation program capable of accurate and realistic simulations via singleparticle tracking.

- It has an intuitive, user-friendly interface that reflects the complexity of the problem, and is directly readable by physicists familiar with the problem domain.

- Simulations of complex accelerator systems can be performed without $\mathrm{C}++$ programming.

\section{http://g4beamline.muonsinc.com}




\title{
Physics Processes \\ Missing from our Current Simulation Tools
}

\author{
This is the current list - \\ Please help us to complete it.
}

\section{Tom Roberts}

Muons, Inc. 


\section{Classes of Processes}

- Single-particle processes

- Collective effects in vacuum

- Collective effects in matter

- Polarized muon processes

- Normalization

- RF breakdown

- Neutrino processes

- Details

Some of these are implemented in other tools; we need to consider adding to our toolbox and/or enhancing the tools we use. 
- Accurate multiple-scattering model

- Several are available in ICOOL

- Coming to G4beamline

- Energy-loss straggling model (incl. length dependence)

- Vavilov model vs. Striganov model

- Material dependence of energy loss and straggling

- ICOOL and G4beamline seem to disagree for $\mathrm{LiH}$

- Correlation of energy loss with multiple scattering angle

- Effect of magnetic field on multiple scattering

- Radiative energy loss in matter for high-energy muons $(>\sim 200 \mathrm{GeV})$

- May not be needed if no material is in the beam (vacuum windows, instrumentation, residual gas, ...) 


\section{Collective Effects in Vacuum}

- Space charge

- Basic computation in ICOOL

- Coming soon to G4beamline

- Wake fields

- Beam loading

- Beam-beam interactions

- Electron cloud effects

- Decay of macro-particles

- Interactions of macro-particles 


\section{Collective Effects in Matter}

- Space charge screening by material

- Bunch effect on the density term of the single-particle formula for energy loss (plasma density)

- Bunch-induced polarization of material causing intensitydependent increase in energy loss

- Can induce an instability

- Effect of matter on wake fields

- For all of the above:

- Consider dependency on material properties

- Consider time dependence

- Head vs. tail of a single bunch

- Effects involving successive bunches in a train 


\section{Polarized Muon Processes}

- Effect of ionization cooling on polarization.

- For applications other than a muon collider or neutrino factory

- Might it be interesting in a collider to trade a factor of $\sim 100$ reduction in luminosity for partially polarized beams? 


\section{Normalization}

- Uncertainties remain about the accuracy of the pion production models used.

- Cavitation and other distortions of the mercury jet production target 


\section{RF Breakdown}

- While not directly part of the particle simulations, modeling RF breakdown is an important aspect of the overall simulation of a muon collider.

- Vacuum

- High-pressure $\mathrm{H}_{2}$

- Surface effects and processing

- This is so important that experiments are required.

- Will affect technology used, so must be done early 


\section{Neutrino Processes}

- Surface radiation assessment will require accurate simulations of neutrino interactions

- Need to significantly increase neutrino interaction cross-sections to make simulations feasible 


\section{Details}

- 3-D effects in RF cavity field models

- Realistic, non-pillbox cavities

- Couplers

- HOM absorbers

- Engineering assessments

- Thermal loads from the beam

- Thermal loads from muon decay

- Radiation levels

- Surely others...

- Ability to model a large and complex detector for background studies

- Surely more... 


\section{Summary}

- There are a number of complicated and subtle physics processes not included in our current simulation tools.

- We must expand the list to be as complete as possible.

- There are of course engineering details that won't be needed until accurate simulations of a specific design are needed.

- We ultimately need a set of publications or MuCool notes, estimating the importance of each one.

- We need to implement those that can significantly affect our modeling of facilities.

- There are many other simulation tools available, and we need to assess whether to acquire and use additional tools, or to enhance the ones we already use. 


\title{
G4beamline User's Guide 2.08
}

\author{
Tom Roberts \\ Muons, Inc. \\ tjrob@muonsinc.com
}

February, 2011

http://g4beamline.muonsinc.com

Copyright (C) 2004 - 2011 by Tom Roberts; all rights reserved.

See license and distribution terms in section 1.2. 


\section{G4Beamline User's Guide Contents}

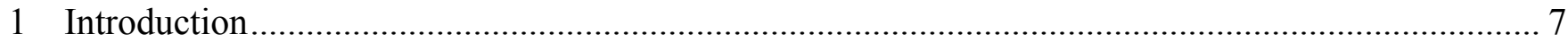

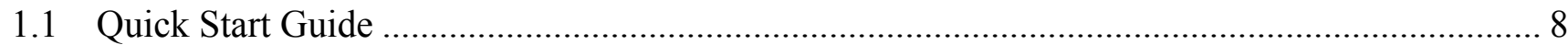

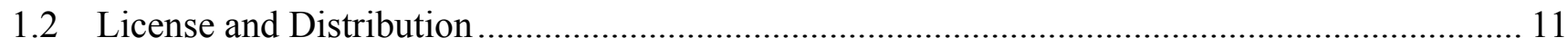

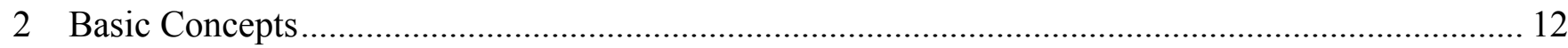

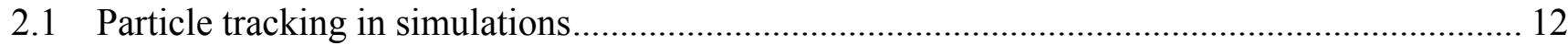

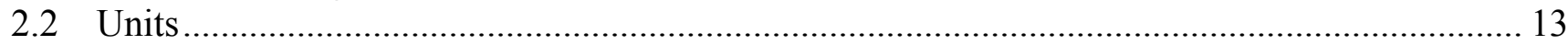

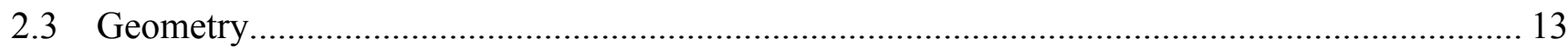

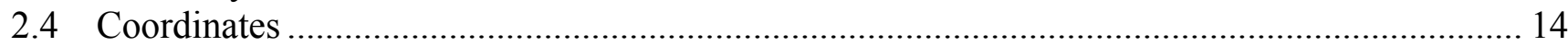

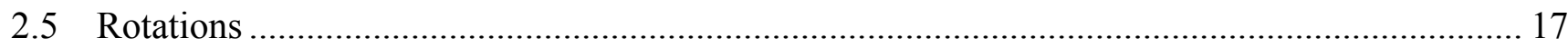

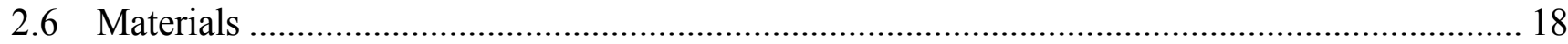

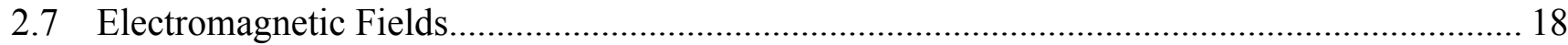

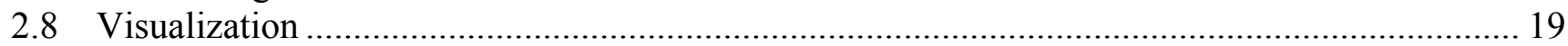

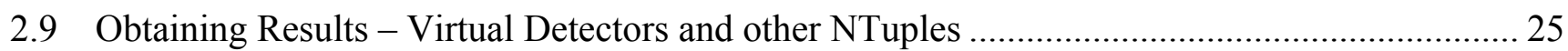

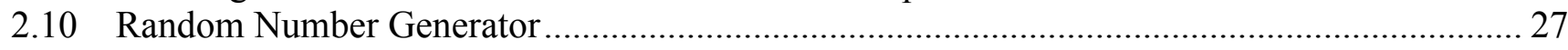

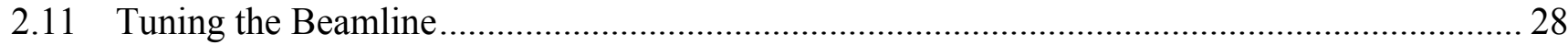

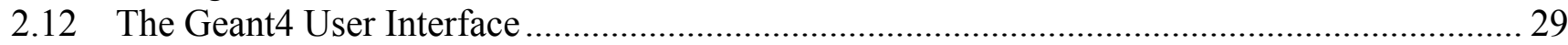

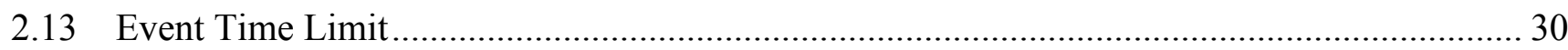

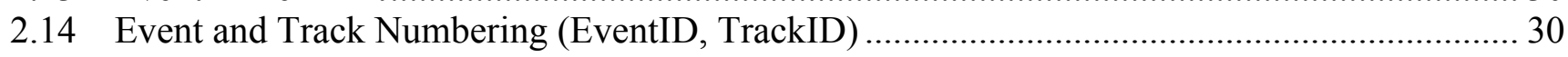

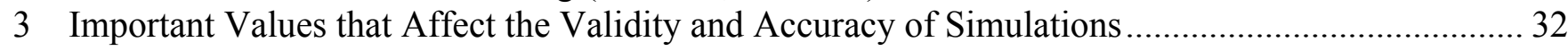

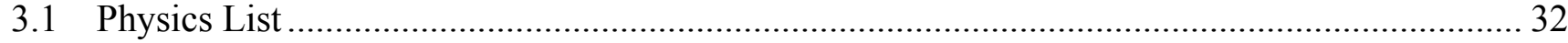

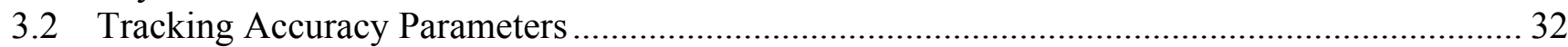

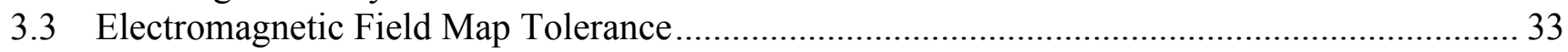

3.4 Secondary Creation Threshold in Physics Processes..................................................................... 33

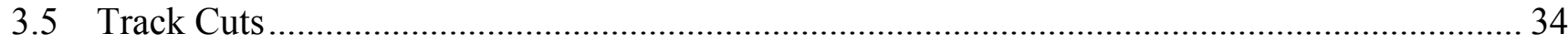

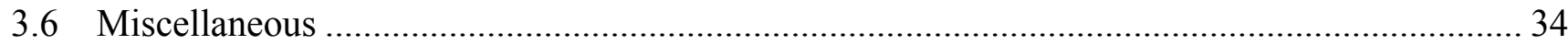

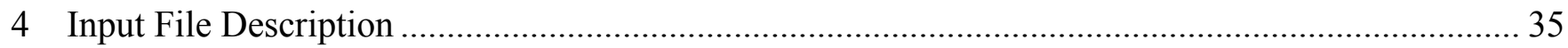

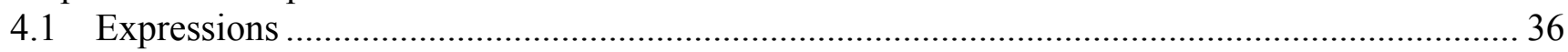

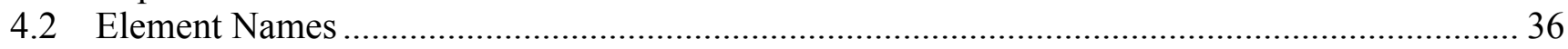

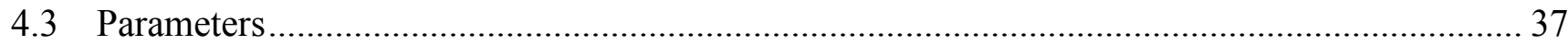

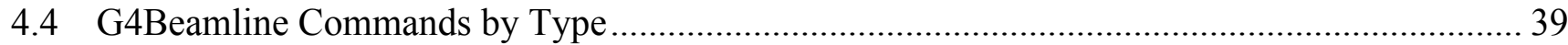

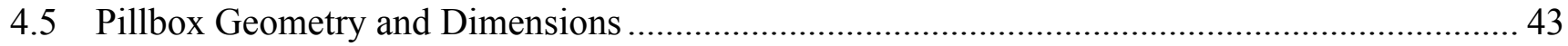

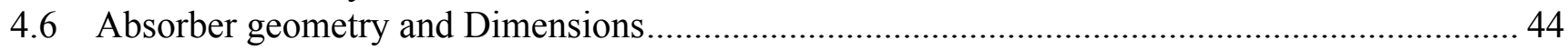

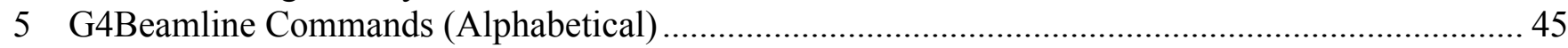

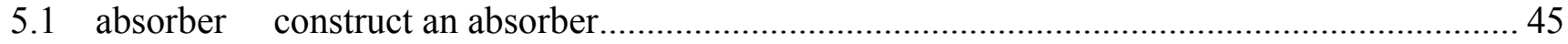

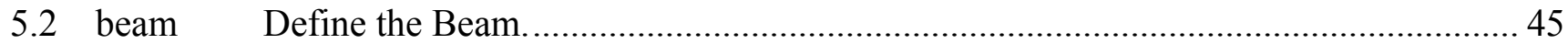

5.3 beamlossntuple NTuple containing particle tracks when lost................................................. 47

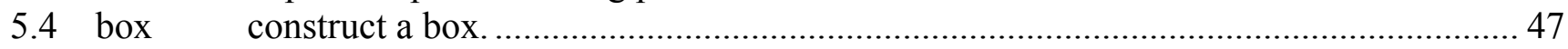

5.5 bug1021 Workaround to improve accuracy of bug1021 in E field ........................................ 48

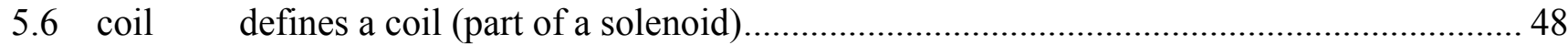

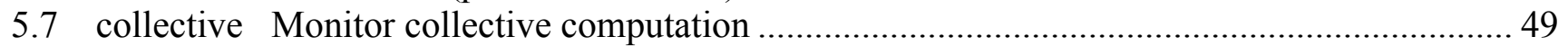




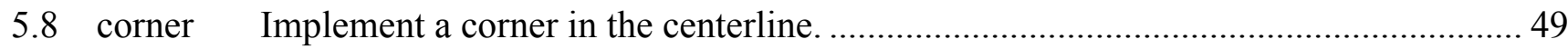

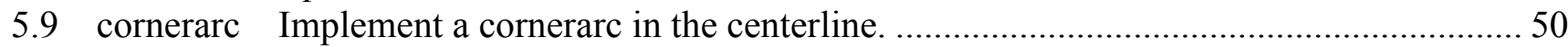

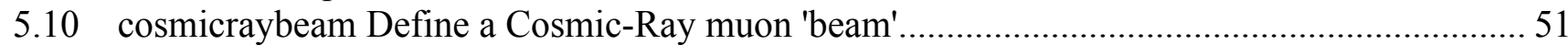

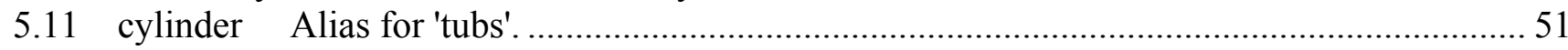

5.12 define defines a macro (argument-expanded set of commands).......................................... 51

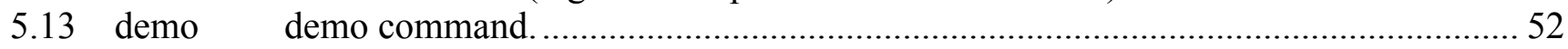

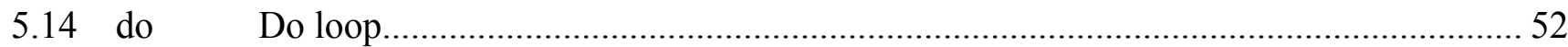

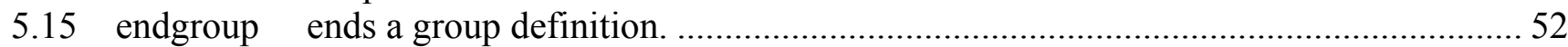

5.16 eventcuts Implements cuts on event number via lists in files.................................................5 52

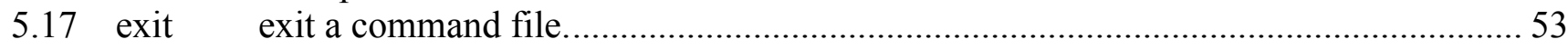

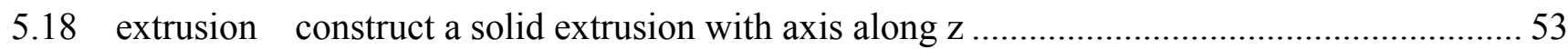

5.19 fieldexpr implements a field map, E and/or B, from expressions......................................... 54

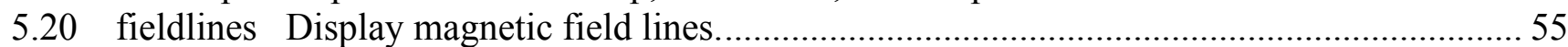

5.21 fieldmap implements a field map, $\mathrm{E}$ and/or B, from a file. ……………………...............5 56

5.22 fieldntuple Generates an NTuple from B and E fields at specified points.................................56

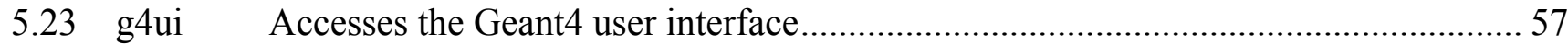

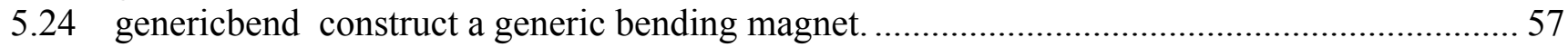

5.25 genericquad construct a generic quadrupole magnet............................................................5 57

5.26 geometry Arranges to perform a geometry test. ..................................................................5 58

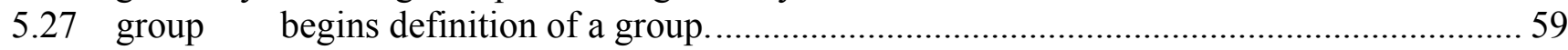

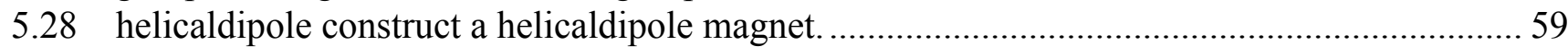

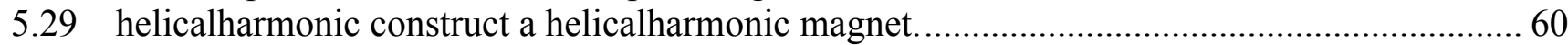

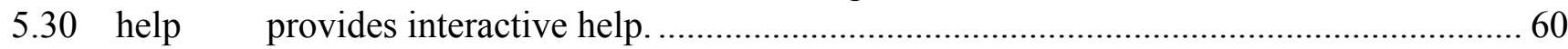

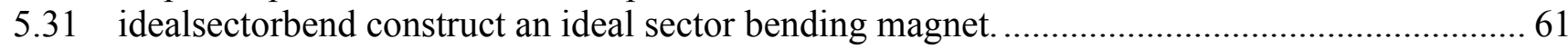

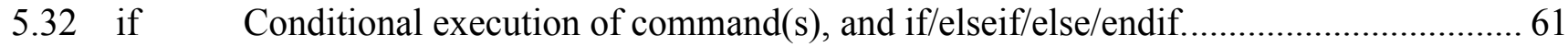

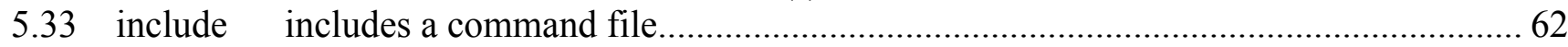

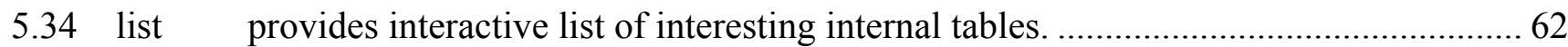

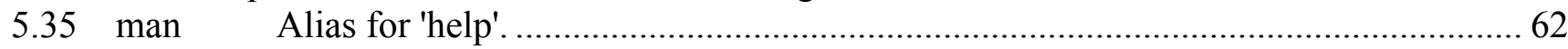

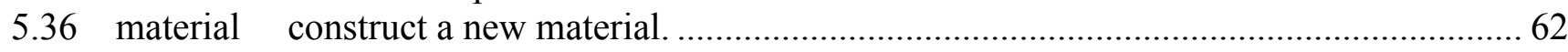

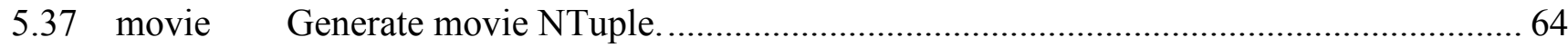

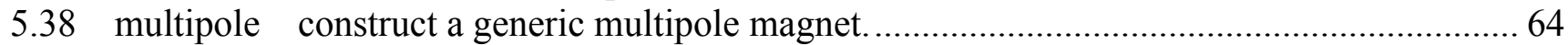

5.39 muminuscapturefix Fixes up the G4MuonMinusCaptureAtRest process. ..................................65

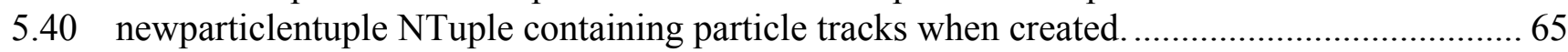

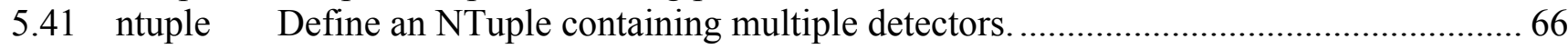

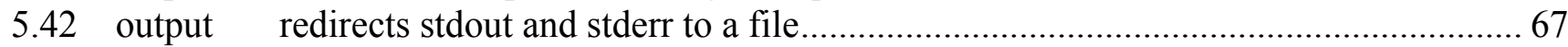

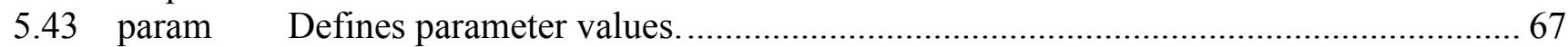

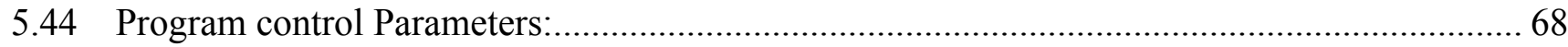

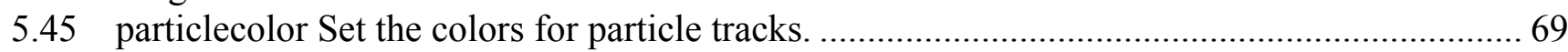

5.46 particlefilter Will kill particles from a list, or force particles to decay.......................................... 69

5.47 particlesource Interface to the Geant4 General Particle Source. .................................................. 70

5.48 physics Defines the physics processes and controls them. ................................................. 70

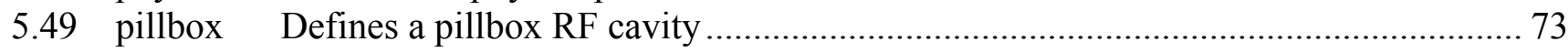

5.50 place places an element into the current group (or world).................................................. 74

5.51 polycone construct a polycone with axis along z................................................................ 75

5.52 printf prints track variables and expressions................................................................... 75

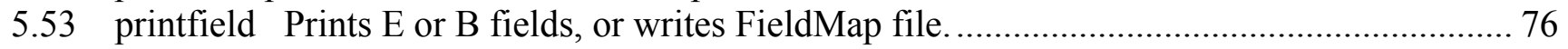


5.54 probefield Prints B and E fields at specified points............................................................. 77

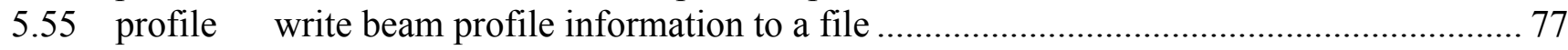

5.56 randomseed control pseudo random number generator seeds ............................................... 78

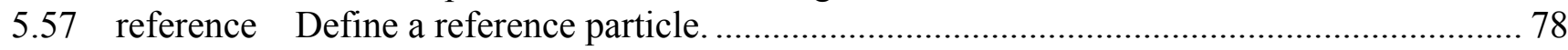

5.58 setdecay Set lifetime, decay channels, and branching ratios for a particle's decay............... 79

5.59 showmaterial Set the colors for selected materials................................................................ 79

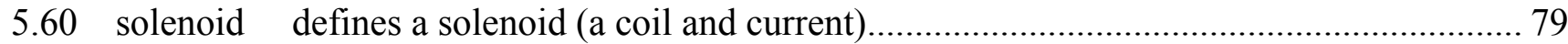

5.61 spacecharge Beam-frame Green's function space charge computation .................................... 80

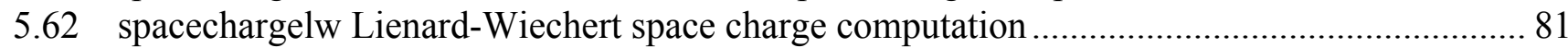

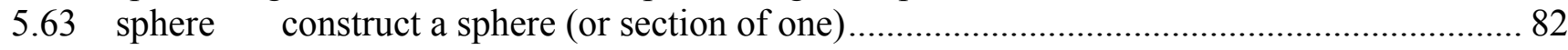

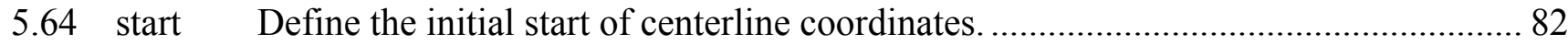

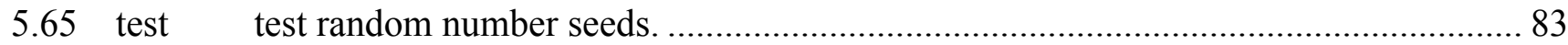

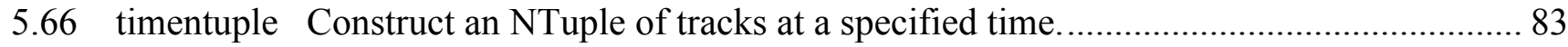

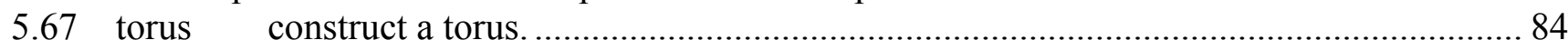

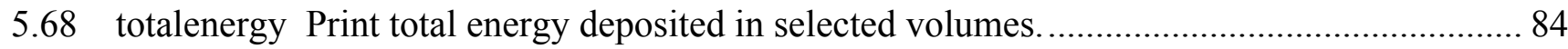

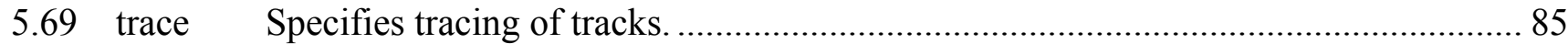

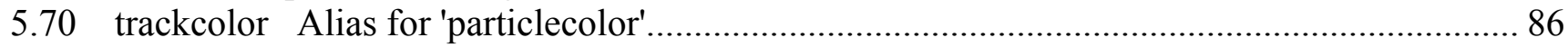

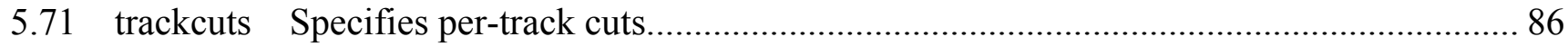

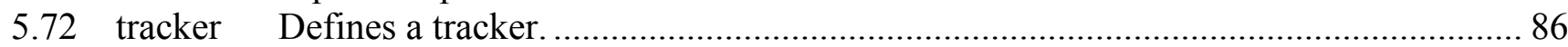

5.73 trackermode Sets mode for all trackers, manages track fitting................................................. 88

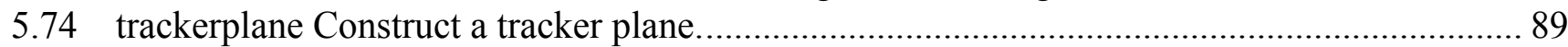

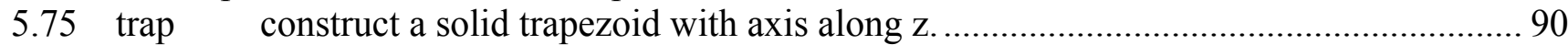

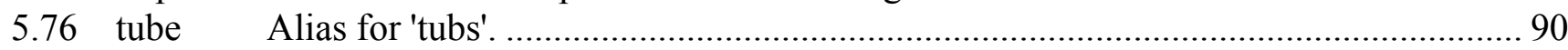

5.77 tubs construct a tube or cylinder with axis along z...................................................... 91

5.78 tune Tune a variable used as argument to other elements. ………………….................... 91

5.79 usertrackfilter Construct a usertrackfilter that filters tracks via user code. ............................... 92

5.80 virtualdetector Construct a VirtualDetector that generates an NTuple...................................92

5.81 zntuple Generate an NTuple for each of a list of $Z$ positions............................................... 93

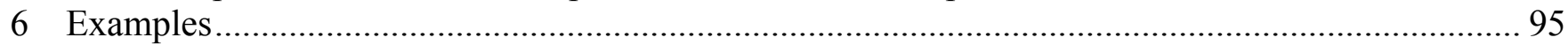

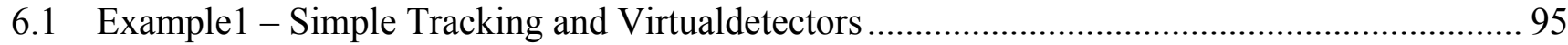

6.2 Example2 - 4 Cells of the Study 2 Cooling Channel .............................................................. 95

6.3 Example3 - Simple Computation of Multiple Scattering ........................................................... 97

6.4 Example4-8 GeV Proton beam into a Tungsten Target ........................................................ 97

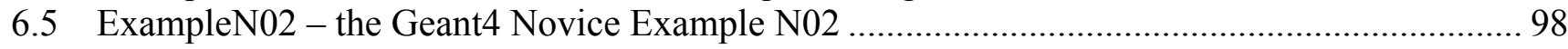

6.6 triplet.sh - tune a quad triplet for point-to-point focus ................................................................. 98

6.7 emittancematch.sh - attempt to match a quad triplet into a solenoid ........................................... 100

6.8 ExampleAUG05 - the MICE Muon Beam Line...................................................................... 100

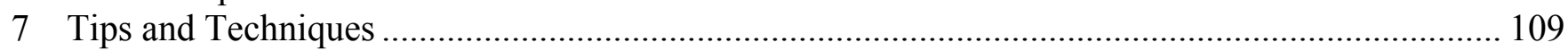

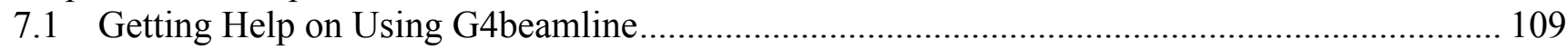

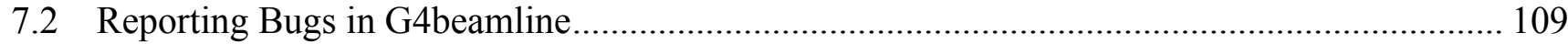

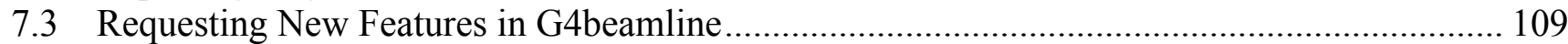

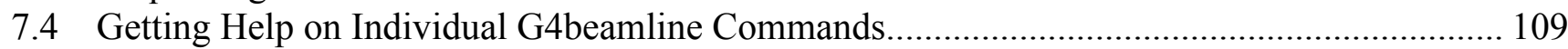

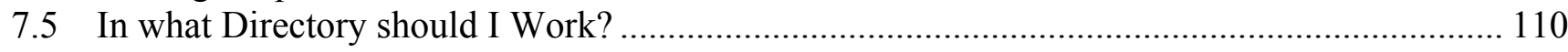

7.6 Files Common to Multiple Simulations .................................................................................. 110

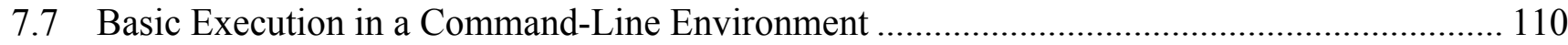

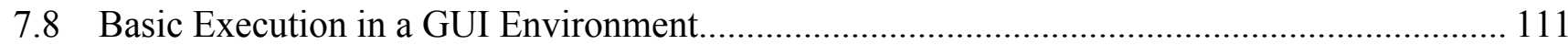




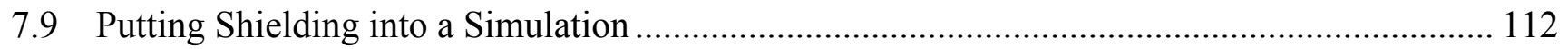

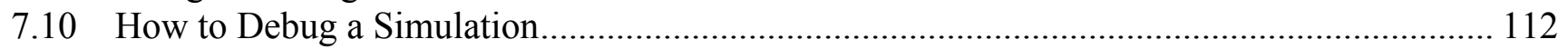

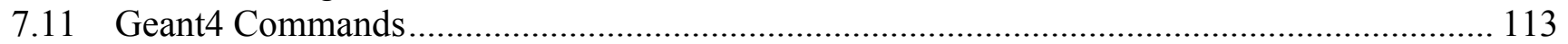

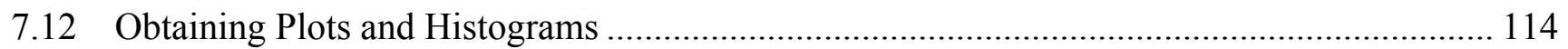

7.13 Obtaining Pictures of the System and Events .................................................................... 115

7.14 Warning and Error messages - Which Ones can be ignored................................................... 116

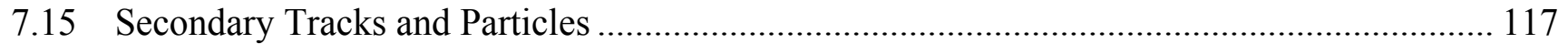

7.16 Finding Example Input Files using the XXX command..................................................... 118

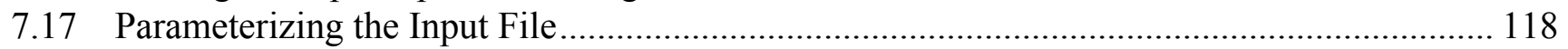

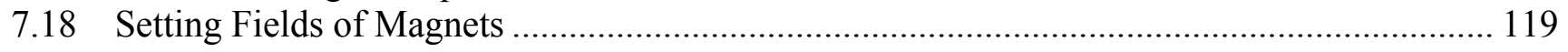

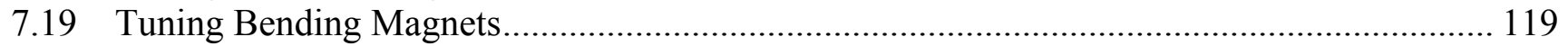

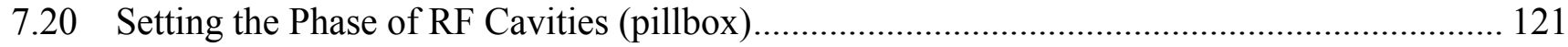

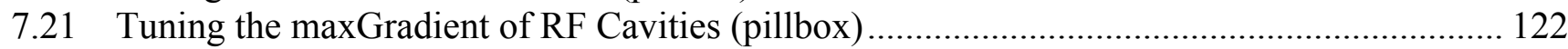

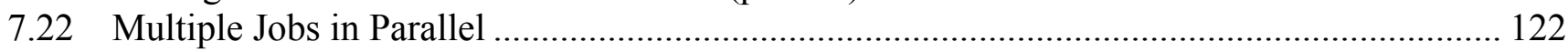

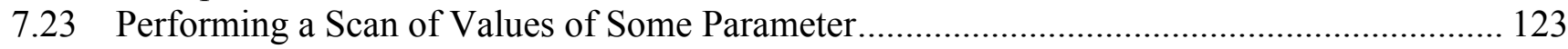

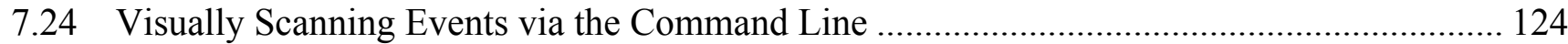

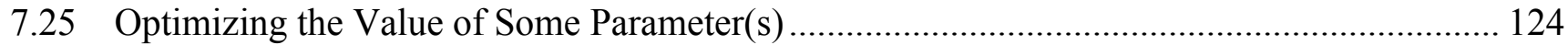

7.26 Using Two or More Reference Particles........................................................................... 125

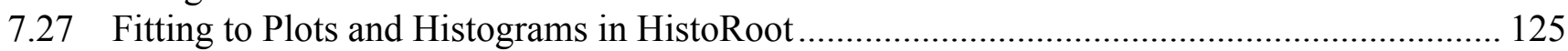

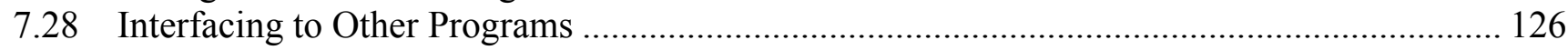

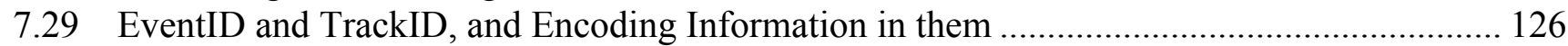

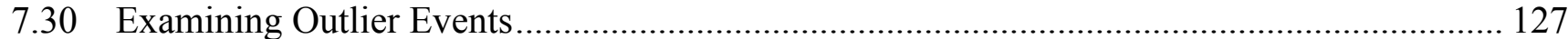

7.31 Increasing the Number of Events Displayed Visually ............................................................. 129

7.32 Building G4beamline, Adding Your Own Code................................................................... 129

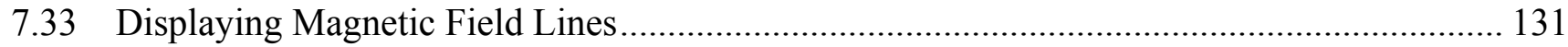

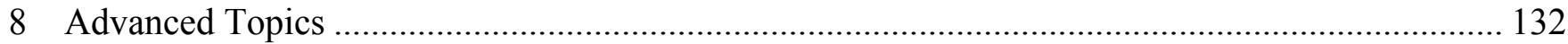

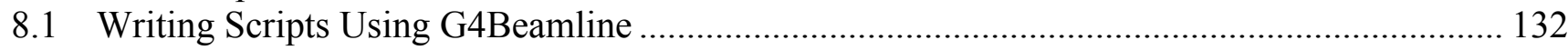

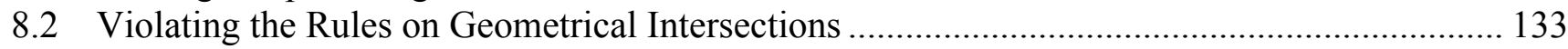

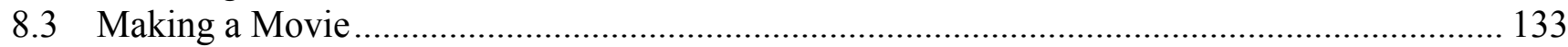

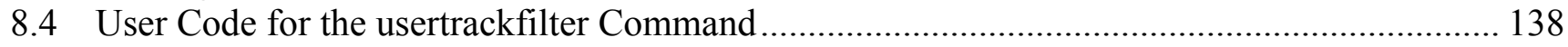

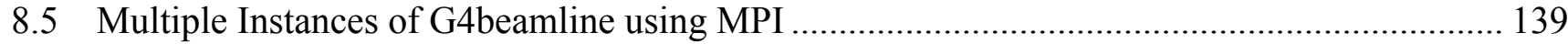

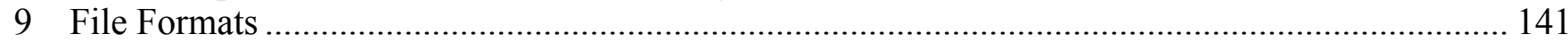

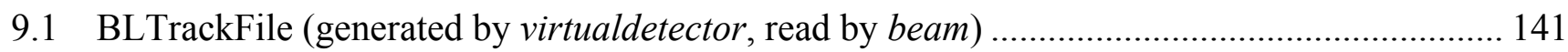

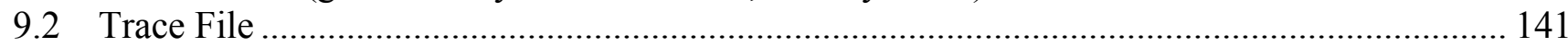

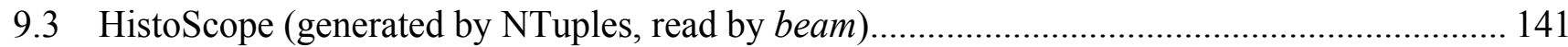

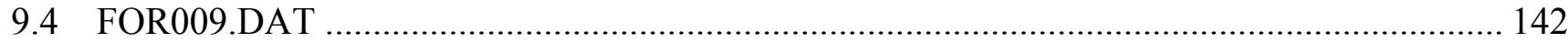

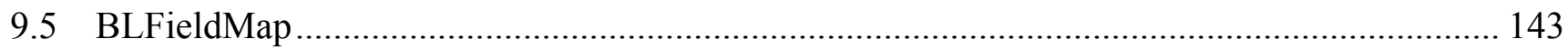

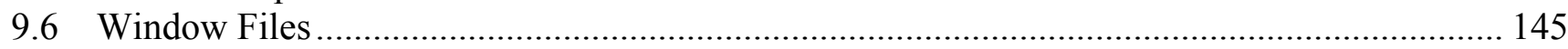

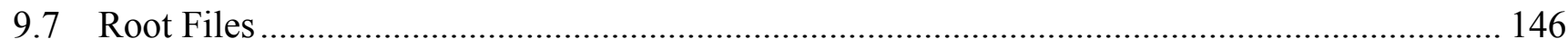

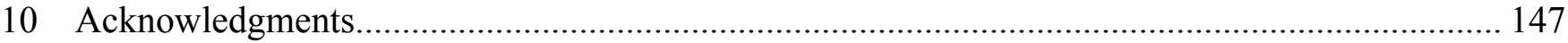

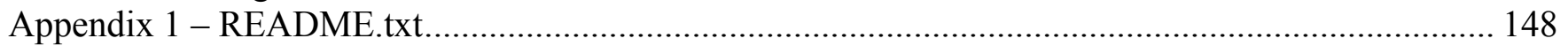

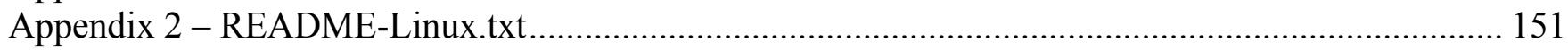

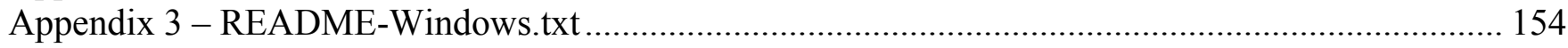

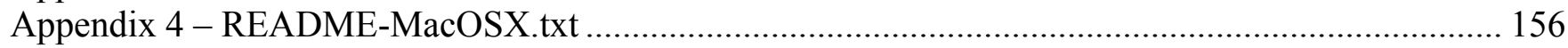

Appendix 5 - Annotated Output from Example1 ……….............................................................. 159

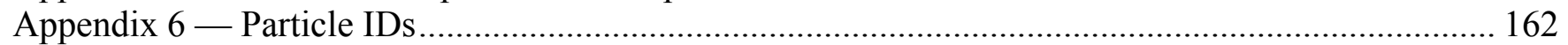




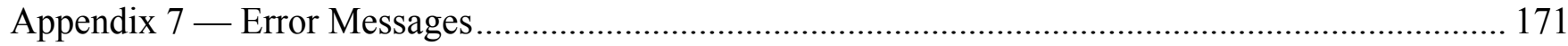

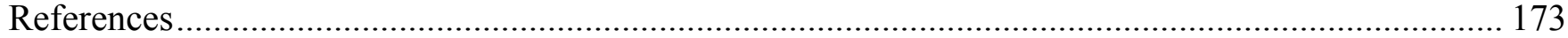




\section{Introduction}

G4Beamline is a particle tracking and simulation program based on the Geant4 toolkit [1] that is specifically designed to easily simulate beamlines and other systems using single-particle tracking. It is flexible enough to simulate complex beamlines like the MICE muon beam, and the Neutrino Factory Study 2 SFOFO muon-cooling channel. Because of its simple and straightforward method of specifying the system to be simulated, it is also well suited for quickly answering questions about particle interactions and tracking (e.g. "On average, how much energy does a $150 \mathrm{MeV}$ proton lose in a $1 \mathrm{~mm} \mathrm{Al}$ window?", "How large does the multiple-scattered beam grow 20 meters downstream of the window?"). As a Cosmic Ray "muon beam" is included, the notion of "beamline" can be rather more general than usual.

The primary advantage of using G4beamline is that its description of the simulation is commensurate with the complexity of the system being simulated, instead of being a significantly more complicated $\mathrm{C}++$ program. Most users need not face the challenges of learning $\mathrm{C}++$ programming and the details of the Geant4 toolkit - to use G4beamline there is no need to know $\mathrm{C}++$, to learn the many aspects of the Geant4 toolkit API, to face the non-trivial challenges of installing the Geant4 toolkit and all its required libraries, and to learn how to solve any problems that arise while linking a complicated and rather large program. All of that is done during the production of the G4beamline distribution. Users with special needs can download and install the source distribution, and learn how to build the program, which will permit them to add their own $\mathrm{C}++$ code and custom commands to G4beamline.

The basic structure of a G4Beamline simulation is to first define beamline elements (magnets, windows, RF cavities, etc.), including their geometry, materials, fields, etc., and then to place them into the world, usually along the beam direction. As bending magnets can be modeled, the "beam direction" can change - see "Centerline Coordinates" below; it remains simple to place elements along the nominal beam centerline. It should be noted that a G4beamline simulation is closer to specifying a real beamline that it is to the abstractions and approximations used in most accelerator-physics codes. All descriptions and configurations are contained in a single ASCII input file, which also provides values for various program parameters, the initial beam, etc.

The tracking of particles through the simulated system is as accurate and realistic as the Geant 4 toolkit implements. The input file selects from any of the Geant 4 physics use cases, and can set values for the various Geant4 tracking-accuracy parameters. This permits users to make trade-offs between CPU time and simulation accuracy. Similarly, G4Beamline permits the specification of magnetic map parameters, permitting a trade-off between memory usage (and the CPU time to generate the map) and simulation accuracy.

While G4Beamline can make it rather simple to specify a simulation, it cannot substitute for knowledge and experience about the problem domain or about particle-tracking simulations in general. Like all computer programs, G4Beamline is prone to "garbage in, garbage out", especially when used by unskilled users. It is strongly suggested that you use visualization to verify the geometry of your simulation and that a handful of particles are tracked properly through it. Whenever possible you should arrange to track through a simple geometry that you can compare to independent results, to make sure that what you think is happening actually does occur in the simulation. 
This document does not discuss compiling and building the G4Beamline program. That is an advanced topic complicated by the complexity of linking a Geant4 executable with diverse visualization drivers. Most users will not need to do that, and can just use the distribution as is. The README-*.txt and BUILD.txt files in the distribution and the appendices provide considerable detail about how to build the program.

\subsection{Quick Start Guide}

This section gives a quick overview of how to install and run G4beamline. It cannot discuss all the subtleties involved in performing simulations. New users should note in particular section 3 on important values that affect the accuracy, and section 7 on tips and techniques.

\subsubsection{Installation}

\subsubsection{Windows}

\section{Prerequisites:}

- Java SE Runtime Environment 1.5 or later (http://java.sun.com) - if you have the JDK installed that includes the runtime environment.

- Root 1.14 or later (http://root.cern.ch).

Root is not required if you will use only ASCII output files (though you will probably find the historoot program useful to generate plots of Root and ASCII files, and it requires Root).

The distribution file is G4beamline-VERSION.msi, available from http://g4beamline.muonsinc.com. Simply download it and execute it with administrator privileges. By default, it will install files into "C: Program Files $\backslash$ MuonsInc", and place icons onto your Desktop and into the Start menu. It will also place a copy of the examples into "My Documents $\backslash$ G4beamline Examples". Delete the downloaded file after installing it.

\subsubsection{Linux (Intel)}

\section{Prerequisites:}

- Java SE Runtime Environment 1.5 or later (http://java.sun.com) - if you have the JDK installed that includes the runtime environment.

- Root 1.14 or later (http://root.cern.ch).

Java is not required if you will only use G4beamline from a command shell (i.e. not use the GUI). Root is not required if you will use only ASCII output files (though you probably will find the historoot program useful to generate plots of Root and ASCII files, and it requires Root).

The distribution file is g4beamline-VERSION-Linux-g++.tgz, available from http://g4beamline.muonsinc.com. Simply download it and un-tar it in your \$HOME:

$$
\text { tar -xzf g4beamline-VERSION-Linux-g++.tgz }
$$

Delete the downloaded file after doing this. Now you need to add the G4beamline programs into your PATH: 
cd g4beamline-VERSION-Linux-g++

. / setup

The setup script will guide you through the process. It puts 2-lie shell scripts into $\$ \mathrm{HOME} / \mathrm{bin}$ (or other directory you specify). You can also add \$HOME/g4beamline-VERSION-Linux-g++/bin to your PATH manually. The setup script also creates G4beamline.desktop and Historoot.desktop in \$HOME/Desktop (if it exists); these define icons in the format used by both KDE and Gnome, and most other desktop managers.

\subsubsection{Mac OS X (Intel)}

\section{Prerequisites:}

- Java SE Runtime Environment 1.5 or later (http://java.sun.com) - if you have the JDK installed that includes the runtime environment. Java is normally installed on Mac OS X.

- Root 1.14 or later (http://root.cern.ch).

Java is not required if you will only use G4beamline from a command shell (i.e. not use the GUI). Root is not required if you will use only ASCII output files (though you probably will find the historoot program useful to generate plots of Root and ASCII files, and it requires Root).

The distribution file is g4beamline-VERSION-Darwin-g++.dmg, available from http://g4beamline.muonsinc.com. Simply download it, open it, and drag the G4beamline and Historoot icons to your /Applications folder. Drag the documentation and examples folders to your desktop, if desired. Then eject the installer disk image and move the downloaded file to the trash. As usual, you can then drag the application icons from /Applications to the Dock.

\subsubsection{Initial Test}

After installing G4beamline, the first thing to do is to try it on some of the example files. You can double-click the G4beamline icon or select it from the Start menu; on Windows/Cygwin, Linux, or Mac you can also execute the command g4blgui. This should bring up the G4beamline GUI window (if it doesn't, there is a problem with either your Java installation or your PATH). Click on the Browse button and navigate to the install-directory/examples and select example1.in (on Windows, go to "My Documents $\backslash$ G4beamline Examples"). Clicking on the Run button should execute the example1 simulation (takes just a few seconds). Try selecting the best viewer, select (say) 10 events/run, and click Run again to see the OpenInventor display of the system and a handful of events.

If you have $t c l$ and the bash shell available (Linux, Mac, Windows with cygwin), you can run the full test suite of G4beamline. Simply cd to the install-directory/test and issue the command ./all. Individual tests can be run individually, if desired. At present, it takes about 5 minutes to run all tests on a reasonably modern computer.

\subsubsection{The G4beamline Command Line}

On Linux and Mac OS X, the command line is the traditional way to run programs. On Windows this is possible, but you must install the cygwin environment first (http://www.cygwin.com) and use its bash shell rather than the Windows $\mathrm{cmd}$. You can execute the G4beamline GUI in all environments via the command g4blgui executed via an absolute path. 
The g4beamline command specifies the input file, plus whatever additional parameters are needed (see the param command). Its syntax is:

$$
\text { g4beamline input. file namel=valuel name2=value2 ... }
$$

Here input.file is the filename of the file describing the simulation ('-" means stdin), and namel and name 2 are parameters to be passed to the program or to input.file. Note that users will rarely (if ever) execute this command directly - most users will use the $g 4 b l$ script instead of G4Beamline itself; its arguments are the same:

$$
\text { g4bl input. file namel=valuel name2=value2 ... }
$$

The $g 4 b l$ script will ensure that the necessary libraries from the binary distribution will be found and used, and sets G4BL_DIR for finding viewer.def and the physics data files. Note that the $g 4 b l$ script relies on being executed via a full pathname, so the distribution bin directory should be put into your PATH (or you can manually type the full path to $g 4 b l$ - see README.txt in Appendix 1). G4Beamline has no such expectation, but to run it you need to have all the necessary libraries available in your environment (which happens when you setup to build G4Beamline).

In addition, the $g 4 b l$ command can contain commands to be interpreted before reading the input.file. These are interpreted in the order given, after all the parameters on the command-line are defined. If the command has arguments, it must be quoted because of the spaces between arguments. The usual commands this is used for are eventcuts (used by g4blgui) and movie (used by users to add the movie NTuples to an existing simulation):

$$
\text { g4bl input. file movie namel=valuel name2=value2 } \ldots
$$

For visualization, the viewer. def file is looked for in the current directory and then in the directory from G4BL_DIR in your environment. If you aren't using the $g 4 b l$ script, you probably want to set that variable to the distribution directory, so you don't need to link viewer. def into every working directory (G4BL_DIR is set when you setup to build G4Beamline, and also by the $g 4 b l$ script).

G4beamline has two modes of operation: visualization and tracking. If the parameter viewer has been set to anything other than none, it will run in visualization mode, which displays an image of the simulated system (as specified in the viewer. def file for the viewer used). For viewer=none (the default), G4beamline will run in tracking mode. In visualization mode any NTuples defined in the input.file will not be written to files. The simplest and most common way to invoke visualization mode is to put an argument viewer=best onto the command line, or select a viewer in the GUI. See section 2.8 for a list of the supported visualization drivers. 
A very useful trick is to run G4Beamline interactively, just to issue help and list commands:

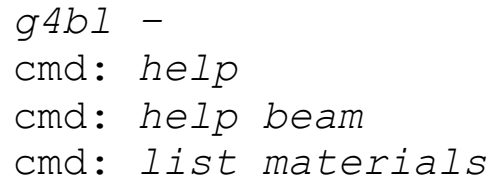

You may find it useful to keep this open in one window while you edit your input.file in another window.

Another useful trick is to add "steppingVerbose $=1$ " to the command line. This will print one line per physics step, so you want to limit the number of events to some small number. The prints are about 120 characters wide by default, so it's best to do this in a wide window. You can change the information printed, see section 3.2.1.

A large number of useful tips and techniques are described in section 7.

\subsubsection{The historoot Program}

The G4beamline distribution includes the historoot program, which makes generating histograms and plots easy. While everything historoot does can be done via Root commands and code, the historoot program makes it unnecessary to know Root and $\mathrm{C}++$, and guides you through most common tasks. The historoot program can read multiple Root files (TFile), and can generate plots from the TNtuple-s in them. It can also read ASCII files (in either .csv or column format) and convert them to TNtuple-s for plotting (see the Help for details on the ASCII file format).

To run historoot, simply double-click its icon or enter its command:

$$
\text { historoot [file1] [file2] [...] }
$$

The file arguments are optional, and it will auto-detect whether they are Root files or ASCII files.

historoot was inspired by the HistoScope program [3], though its user interface is completely different. Having sliders that interactively impose cuts on the histogram is a great convenience.

\subsection{License and Distribution}

This program is free software; you can redistribute it and/or modify it under the terms of the GNU General Public License as published by the Free Software Foundation; either version 2 of the License, or (at your option) any later version.

This program is distributed in the hope that it will be useful, but WITHOUT ANY WARRANTY; without even the implied warranty of MERCHANTABILITY or FITNESS FOR A PARTICULAR PURPOSE. See the GNU General Public License for more details.

http://www.gnu.org/copyleft/gpl.html

This product includes software developed by Members of the Geant4 Collaboration (http://cern.ch/geant4). 


\section{Basic Concepts}

Any physical component of the simulation is called a beamline element, or just an element. Every element inherently has a geometrical shape and a material; some elements also have electromagnetic fields associated with them. Some elements are quite simple, merely defining a box or tube made of some material; other are quite complex, defining in detail the construction and electromagnetic fields of a complicated object such as an RF cavity.

Elements can often be nested inside each other; any element can be placed inside another element, but not all elements can act as the parent of others (in general, simple elements can be parents, complex ones cannot; most elements that generate an electromagnetic field cannot be parents). The notes and help for each element describe whether or not it can be the parent to other elements. This is the natural way Geant4 builds the geometry hierarchically: a "daughter" volume is said to be placed into its "mother" or "parent" volume (every element is also a volume). The "World" volume is the ancestor of all volumes, and in G4beamline the World volume automatically grows to hold everything that is placed into it. In G4beamline, individual elements have their own material(s), and when a daughter is placed inside a parent, the daughter's material applies inside the daughter, and the parent's material applies inside the parent outside any daughters; many elements use their parent's material by default.

G4Beamline attempts to model the simulated world in as simple and straightforward manner as possible, so in most cases a single object you might place into a real beamline will be represented as a single beamline element in the program. So, for example, a genericbend element represents a bending magnet and its field; the genericbend command defines an individual magnet type, including all geometrical details that the element can represent, and all details of the field that it can model; each placement of the magnet puts the geometrical object into the simulation and also generates the magnetic field appropriate to the magnet. Other elements behave similarly.

In the Geant4 toolkit, only simple geometrical shapes are available, and complicated objects must be built up from cylinders, boxes, etc. In G4Beamline there is a rather large list of pre-defined elements that are much more complicated, such as an entire RF cavity (including time-dependent E- and B-fields, optional windows, etc.), or a complete absorber assembly (modeled after the absorbers of the MICE experiment, suitable for neutrino factory cooling channel simulations). G4Beamline includes simple pipes, cylinders, spheres, boxes, extruded polygons, and polycones, so you can build up complicated objects from them; in general, it is both easier and better to use the pre-defined elements whenever they are suitable for your needs. In cases where the pre-defined elements are not sufficient, it is possible to add additional elements to the program (this requires $\mathrm{C}++$ programming and the installation of the complete G4Beamline build environment - see BUILD.txt in the distribution and the doxygen documentation of the classes).

\subsection{Particle tracking in simulations}

The accuracy of a simulation of tracking particles through a system depends primarily on how realistic the simulation is. This has many aspects:

- How closely the geometry of the simulation matches the real geometry

- How accurately the real electromagnetic fields are modeled in the simulation

- How accurately the material properties of the real objects are modeled in the simulation

- How well the modeled beam distributions match the real beam 
- How accurately the program tracks particles

- How accurately the implementations of physical processes match the real world

- Etc.

While G4Beamline provides facilities for the user to specify most of these, and gives them reasonable default values, it is really the user's responsibility to ensure that the result of a simulation is accurate enough to be useful.

In G4Beamline, the Geant4 particle tracking is used directly with the tracking-accuracy parameters of Geant4 settable by the user (see section 3.2.2).

\subsection{Units}

In G4Beamline, all physical sizes and dimensions are specified in millimeters, just as in Geant4. Most other units are as in Geant4, except rotation angles are in degrees and EM fields are in Tesla and MegaVolts/meter. The units of all arguments to all commands are given in section 5 of this document and in the output from "help commandname".

\subsection{Geometry}

As in Geant4, geometry in G4Beamline is specified at the element level and in the placement of elements. Each element has an inherent shape and size, which are usually parameterized, that determine the basic geometry of the element in isolation. Elements can be placed into the simulated World, or into other elements placed into the world, with specified geometrical relationships consisting of both rotations and offsets relative to the parent's coordinates. This will become obvious when you look at the various element definition commands (e.g. the box and $t u b s^{I}$ commands), and the place command.

Because of the way Geant 4 does tracking, there are restrictions on the geometrical intersections of elements:

- Every daughter element must be wholly contained in its parent

- No sibling elements can intersect each other in any way

This results in a strict hierarchical arrangement of element volumes, with the World volume as the root and outermost volume from which all other volumes are descended. The World volume, and the group element, will automatically expand to the size required to hold all daughter elements placed into them.

G4Beamline has a geometry test that will verify these requirements for points distributed on the surfaces of every element. Due to round off in tracking, the geometrical intersections have a default accuracy of 10 microns; the geometry test has a default tolerance of 2 microns; in practice these are usually reasonable values, but they can be changed when required by simulations at a different scale.

Note that the Geant4 toolkit has "logical volumes", "physical volumes" and "placements". In G4Beamline, these are not visible to the user; elements are modeled as single objects, and you simply place the objects where they belong, keeping in mind the above requirements on intersections. The $\mathrm{C}++$ code implementing each element deals with the Geant4 details, of course. Unlike Geant4, in

1 "Tubs" is the name Geant 4 uses for a tube or a cylinder (a tube with innerRadius $=0$ ).

2/11/11 TJR G4Beamline User's Guide 
G4Beamline an element that generates an electromagnetic field is a single object that includes both its geometrical volume(s) and its field.

An additional limitation of the geometry in G4beamline is the requirement that any element that is to be the parent of other elements be placed after the children are placed into it. Once a given element has been placed (anywhere), it can no longer be used as the parent of other elements.

\subsection{Coordinates}

There are four basic types of coordinates used in G4Beamline:

1. Global coordinates

2. Local coordinates

3. Centerline coordinates

4. Reference coordinates

All coordinate systems are Cartesian, with axes labeled X,Y,Z,T. For geometrical placement, $T$ is irrelevant (but it is needed during tracking). $\mathrm{X}, \mathrm{Y}, \mathrm{Z}$ are always right handed, with the $+\mathrm{Z}$ direction in the nominal direction of the beam, $\mathrm{X}$ is normally beam left, and $\mathrm{Y}$ is normally up.

\subsubsection{Global Coordinates}

Global coordinates are merely the local coordinates of the World volume - the outermost physical volume of the simulation, which defines the entire world known to the program. All beamline elements and physical objects of the simulation are contained within the world volume. Note that the World volume automatically expands to hold any elements placed into it, so the user need not be concerned with how large it is. Any particle that reaches an edge of the World volume is killed right there.

Tracking is performed using global coordinates, and electromagnetic fields are always determined using global coordinates (but they are specified using the local coordinates of their element).

\subsubsection{Local Coordinates}

There is a set of local coordinates for each and every element. If the element has an associated field, the field of the element is specified in local coordinates, and the program automatically keeps track of the global $\Leftrightarrow$ local coordinate transforms required for each placement of the element (including rotations for vector fields).

Whenever an element is placed inside a parent element (e.g. a group or a box), the parent's local coordinates are used in the place command, except for the world volume. When placing an element into the World volume you can specify either Centerline coordinates (the default) or global coordinates (the local coordinates of the parent, here the World volume).

\subsubsection{Centerline Coordinates}

Centerline coordinates are intended to represent the nominal centerline of a beamline, with the $\mathrm{Z}$ axis running down the center of the beamline, the $\mathrm{X}$ axis is beam left, and the $\mathrm{Y}$ axis is up. This includes corners generated by bending magnets. The centerline coordinates are initially identical to the global coordinates, but the start, corner, and cornerarc commands will change that (each bending magnet is normally immediately followed by a matching corner or cornerarc command). By default, elements 
placed into the World volume use centerline coordinates to specify the placement, but global coordinates can be used if desired. Centerline coordinates apply only to the World volume. They are piecewiselinear, and inside bending magnets they will differ from the theoretical reference-orbit coordinates commonly used in accelerator physics; the cornerarc command will approximate reference-orbit coordinates by using three corners chosen to have the same length along $\mathrm{Z}$ as the reference-orbit coordinates have along the arc. Note that the corner and cornerarc commands cannot have an angle greater than $90^{\circ}$; if necessary, this limit can be worked around by using two cornerarc-s in a row with half the desired angle.

In $\mathrm{X}$ and $\mathrm{Z}$, centerline coordinates correspond to what a surveyor might draw on the floor - a piecewiselinear centerline along which the various beamline elements will be placed. Note that just like a real beamline, in G4beamline the fields of bending magnets must be adjusted ("tuned") so that the desired reference particle will travel along the centerline of the beamline. In general, the fringe fields of bending magnets will require them to be offset slightly from their nominal position relative to a corner of the centerline. See section 7.18 for a description of how to place and tune bending magnets.

If your input.file has no start, corner, or cornerarc commands, then the centerline coordinates will be the same as the global coordinates, everywhere.

Note that in the input file, each start, corner, or cornerarc command affects the centerline coordinates immediately. This means that all elements to be placed between a given pair of corners must be put into the input file between those corner commands. If you attempt to place an object using a $\mathrm{Z}$ position in front of a previous corner or cornerarc, or behind a following one, it will almost surely not do what you intend. Because of this, it is quite difficult to place any element inside a bending magnet using centerline or global coordinates; the best way to do that is to use the genericbend object as the parent for the placement.

Because centerline coordinates can only apply when placing elements into the World volume, bending magnets must normally be placed into the World volume, not into a group or other parent volume.

NOTE: the transform from global to centerline coordinates must be performed at the start of tracking each particle. This transform can be ambiguous in certain regions near corners - see the figure at left (in

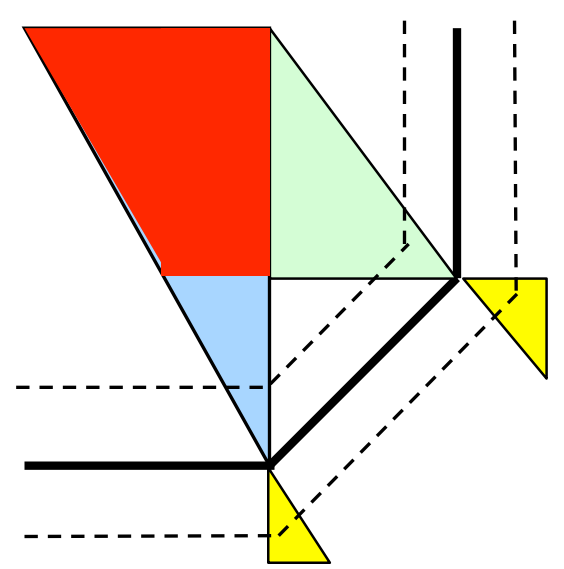
which the heavy black line is the centerline with 2 corners and 3 segments): the blue and green ambiguities are minor, because they involve adjacent regions, but the red one is serious because it involves non-adjacent segments; a ring is much worse. The yellow regions appear to belong to no segment, and are simply assigned to the next downstream segment. The tracking of particles handles the change of centerline segments naturally, moving from segment to segment in order, but any jump or start must deal with the ambiguities (beam particles or secondaries). The solution is to specify a radiusCut for every start, corner, and cornerarc command (this is a limit on a particle's radial distance from the centerline, indicated by the dashed lines in the figure). This removes the serious ambiguities, and the remaining minor ones are resolved by using the earliest region of the centerline for which the global coordinate point is inside the radiusCut. Most beamlines have apertures that can be used to determine an 
appropriate radius $C u t$ in each segment of the centerline. If no radiusCut is specified the ambiguities can become so bad that they prevent the use of centerline coordinates, especially if there are many corners, large angles, or if the tune command is used (which "jumps" the tune particle); simulating a ring may not work at all without a radiusCut (and needs the ring argument to the start command).

\subsubsection{Reference Coordinates}

Reference coordinates are the usual coordinates used by accelerator physicists, with the $\mathrm{Z}$ axis along the reference orbit, $X$ to the beam left, and Y up. In G4beamline, the actual track of the reference particle is used for the $\mathrm{Z}$ axis. As the reference track is stepwise linear between the physics steps of the tracking, it is usually necessary to specify a rather small maxStep (often $10 \mathrm{~mm}$ is about right). Note these coordinates can be used only for output; objects cannot be placed using them, and tracking always happens in global coordinates.

As an example, consider a system having a uniform By $=8 \mathrm{~T}$ with vacuum everywhere, and a Gaussian beam injected with $\mathrm{Py}=0$ centered on the $+\mathrm{Z}$ axis; there is a particlefilter to limit the number of circles each particle can take. Each particle (including the reference particle) traces $61 / 2$ circles that lay right on top of each other. When using global coordinates for the trace, the circles are quite evident; using reference coordinates, the tracks are sinusoids (see figures below).

\section{Global Coordinates view $-\mathbf{Z}$ vs. $X$}

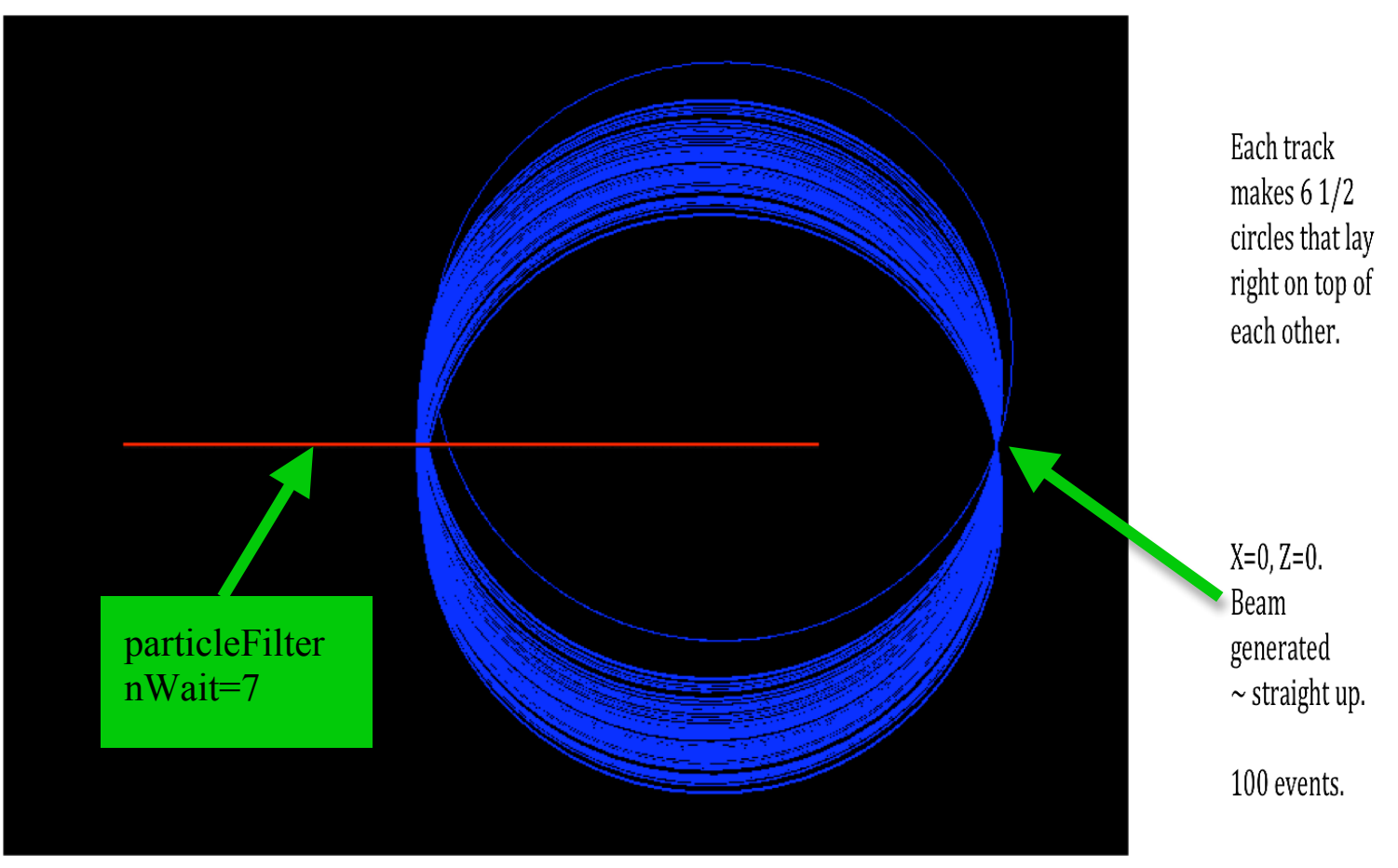




\section{Reference Coordinates view - Z vs. X}

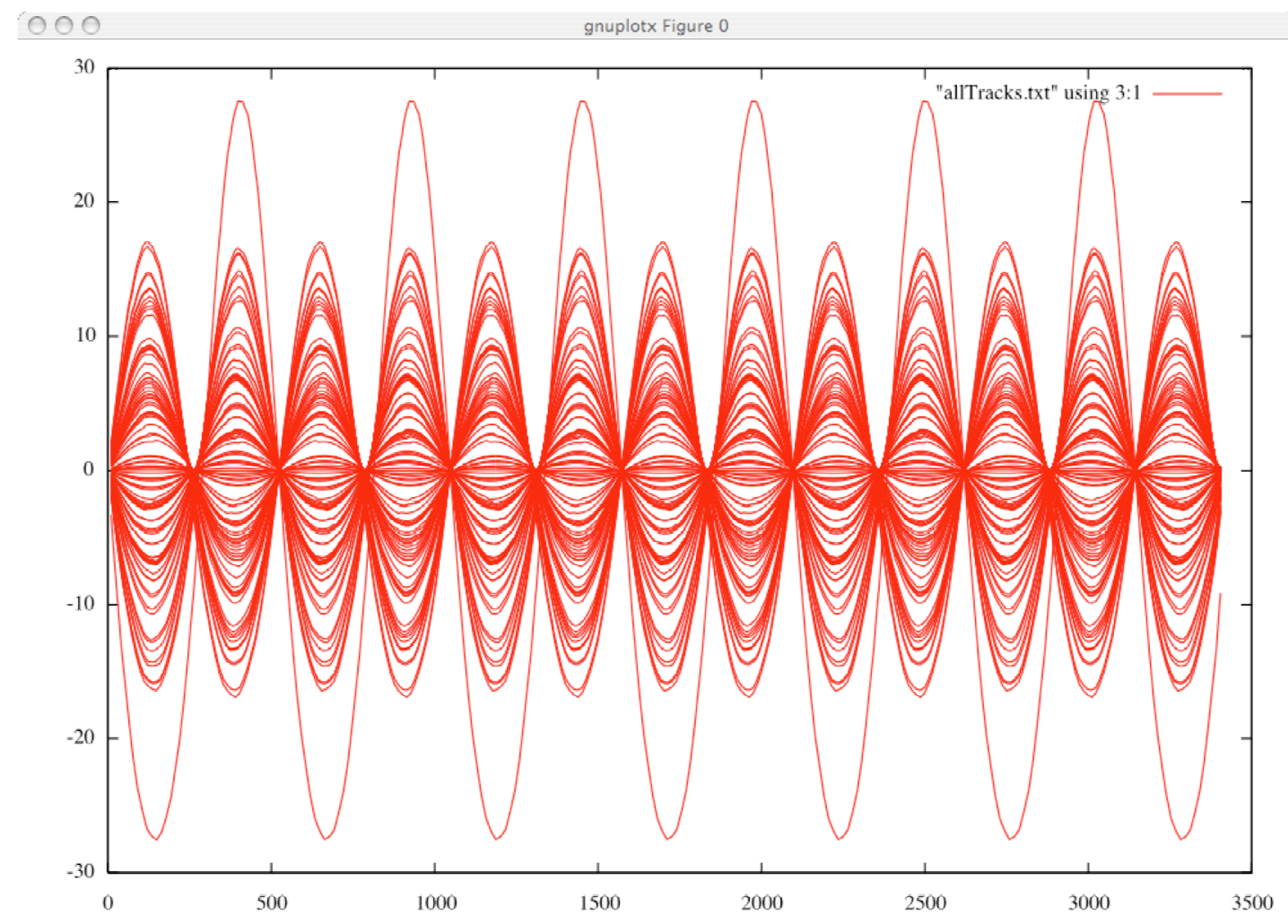

\subsection{Rotations}

In G4Beamline, all rotations are specified relative to the fixed coordinate axes of the parent volume into which a given element is being placed. This is the natural way to think of placing an object into a room, where the object is the element being placed, and the room is the parent volume into which it is placed.

Rotations can be specified in the place command, and in a few other commands. They are specified by a rotation parameter, the value of which is a comma-separated list of rotations around the parent's coordinate axes, which are applied in order (left-to-right) to the object; values are in degrees. So "rotation=Y90,X30" means:

1. Start with the object's local coordinate axes aligned with the parent's coordinate axes

2. Rotate the object (and its local coordinate axes) by 90 degrees around the parent's Y axis

3. Then rotate the object (and its local coordinate axes) by 30 degrees around the parent's X axis. Negative and decimal values of the angle are permitted. The direction of a positive rotation is given by the right-hand rule: use your right hand to grasp the rotation axis with your thumb in its positive direction; a positive rotation goes in the direction of your fingers.

If both a rotation and an offset are specified when placing an element, the rotation occurs first, then the offset. This makes sense, as the location of the center of the object is specified when placing objects. 
When the parent is placed with a rotation and/or an offset, the relationship between daughter and parent remains unchanged, so the effect on the daughter is cumulative, all the way back to the World volume.

When used in a corner or cornerarc command, the rotation is relative to the previous centerline coordinates. When placing into the World volume using centerline coordinates, the rotation is relative to the current centerline coordinates (as is natural). So, for example, one can simply place genericquad elements using centerline coordinates without rotation or offset and the nominal beam centerline will go right down their center.

Note: this is quite different from the way the Geant4 toolkit itself specifies rotations and placements.

\subsection{Materials}

Every physical object inherently is constructed out of some material. G4Beamline uses the Geant 4 methods of specifying materials. The NIST database is available, and is used to define any material that is not already defined (as long as it is known to the database). This database includes all elements with $\mathrm{Z}<=98$ plus a rather large list of other materials, including most of the ones commonly used in simulations. See "help material" for details (or the material command in section 4 below). Additional materials can be defined as needed, via the material command, but that is needed only for unusual materials or gases with nonstandard density. The Geant4 NIST database prepends "G4_" to all material names, but in G4beamline that prefix is optional (G4_Al and Al are the same material, aluminum).

Every element command has a material argument (or similar) to set the material for the physical object (some elements have more than one type of material parameter). In many cases, the default is that of the parent volume, but refer to the individual command descriptions for the actual default values. The material of the World volume is determined by the parameter worldMaterial, which defaults to Vacuum.

\subsection{Electromagnetic Fields}

In the real world, electromagnetic fields are generated by real structures. This is also true in G4Beamline, where various elements include their electromagnetic fields. So a genericbend object includes not only the geometric shape of its iron and coils, it also includes its magnetic field. Placing a genericbend element into the world places both its iron and its field; any rotations or offsets used in the place command apply to both the iron and the field.

As G4Beamline is intended to model beamlines, in many cases the field of an element is only valid inside its aperture (plus perhaps a short distance away for a fringe field). This is so for genericbend and genericquad, but solenoid and fieldmap have fields throughout space (which are truncated according to the tolerance specified or the limits of the map).

In Geant4 programs, computing the field is usually the dominant CPU time used during tracking. In order to minimize this CPU usage, G4Beamline optimizes the computation of the field by using a global bounding box around the field of each element. To compute the field at a given point, the program loops over all elements with fields, but only evaluates those fields for which the point is inside the bounding box. The bounding box is determined in global coordinates, as is the point, so checking the bounding box is fast (often the conversion from global to local coordinates is more expensive than evaluating the 
field). For rotated elements the bounding box can be much larger than the field region itself; in practice this is not a problem as the particle beam is usually localized and the "excess regions" of the bounding boxes are usually well outside the beam region.

Beware of placing two elements that generate fields adjacent to each other so their bounding boxes share a side, unless the fields for at least one are very small on the shared side. If both elements have nonzero fields, there can be a small region surrounding their shared surface in which both are evaluated, or in which neither are evaluated. This region is typically picometers or less in length, but while integrating the equations of motion it could be hit. This normally only applies to artificial elements such as fieldexpr and fieldmap, because realistic elements have fields that decay with distance and bounding boxes out where they have gone to zero (within tolerance).

Note: this is quite different from Geant4, where geometry and fields are strictly separate.

\subsection{Visualization}

The ability to easily visualize the system being simulated is an important part of G4Beamline. Without looking at the system in detail, it is easy to make simple mistakes that completely invalidate the results of the simulation. Users are strongly urged to use visualization frequently.

In order to be visible, a given beamline element must be given a color. Every element accepts an argument "color=1,1,1", where the 3 floating-point values are for Red,Green,Blue respectively, and must be between 0.0 and 1.0. "color=invisible" or "color $=$ "" is invisible, "color $=1,1,1$ " is white, "color $=0,0,0 "$ is black, "color $=0,1,1$ " is yellow, "color $=0.6,0.6,0.6$ " is gray, etc. Most elements have a default color of 1,1,1 (white).

By default, particle tracks are visible: red for negatives, blue for positives, and green for neutrals. See the particlecolor command to change this. If you run pions or muons you may be surprised to see all the neutrinos from their decays; see the trackcuts command to avoid tracking them. You may also be surprised by delta-rays, which are really low-energy electrons $\left(\mathrm{e}^{-}\right)$; trackcuts can eliminate them, too. For example, when studying $\pi^{+} \rightarrow \mu^{+} \rightarrow \mathrm{e}^{+}$decays, the following commands are useful to color them differently and to eliminate the neutrinos and other extraneous particles (e.g. delta rays):

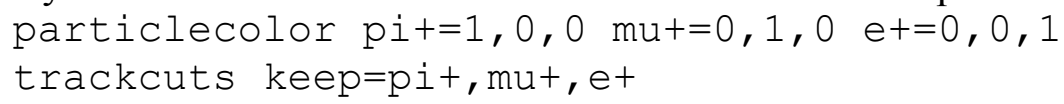

Once your beamline elements have been given colors (in your input file), and so have your particles, to actually visualize the simulation you need to select a visualization driver. G4Beamline supports the following visualization drivers from Geant4:

\begin{tabular}{|l|l|l|l|}
\hline Descriptive Name & Name & Type & Description \\
\hline- & best & Interactive & $\begin{array}{l}\text { Alias for the best viewer available, } \\
\text { currently OIX/OIWin32. }\end{array}$ \\
\hline OpenInventor & OIX & Interactive & $\begin{array}{l}\text { Renders in 3d using either solid } \\
\text { shading or wireframe mode }(+ \\
\text { others), and permits the easy } \\
\text { rotation, scaling, and movement of } \\
\text { the image using the mouse. }\end{array}$ \\
\hline
\end{tabular}




\begin{tabular}{|l|l|l|l|}
\hline OpenInventor & OIWin32 & Interactive & $\begin{array}{l}\text { The name of OpenInventor on } \\
\text { Windows. }\end{array}$ \\
\hline OpenGLImmediateX & OGLIX & Interactive & Basic OpenGL 3-D viewer. \\
\hline OpenGLStoredX & OGLSX & Interactive & Basic OpenGL 3-D viewer. \\
\hline OpenGLImmediateXm & OGLIXm & Interactive & $\begin{array}{l}\text { Like OGLIX, except has Motif } \\
\text { widgets to permit the easy rotation, } \\
\text { scaling, and movement of the image. } \\
\text { If you cannot get OpenInventor to } \\
\text { work, try this one next. }\end{array}$ \\
\hline OpenGLStoredXm & OGLSXm & Interactive & $\begin{array}{l}\text { Like OGLSX, except has Motif } \\
\text { widgets to permit the easy rotation, } \\
\text { scaling, and movement of the image }\end{array}$ \\
\hline ASCIITree & ATree & Offline & $\begin{array}{l}\text { A simple hierarchical description } \\
\text { written to stdout. }\end{array}$ \\
\hline DawnFile & DAWNFILE & Offline & Use the DAWN viewer. \\
\hline GAGTree & GAGtree & Offline & $\begin{array}{l}\text { Another geometry description } \\
\text { written to stdout. }\end{array}$ \\
\hline HepRepFile & HepRepFile & Offline & Use the WIRED viewer (Java). \\
\hline HepRepFile & HepRepXML & Offline & Use the WIRED viewer (Java). \\
\hline Wired & Wired & Offline & Synonym for HepRepFile. \\
\hline Wired3 & Wired3 & Offline & Synonym for HepRepFile. \\
\hline RayTracer & RayTracer & Offline & $\begin{array}{l}\text { Generates JPEG files; use any image } \\
\text { viewer for them. }\end{array}$ \\
\hline VRML1FILE & VRML1FILE & Offline & Use a VRML viewer. \\
\hline VRML2FILE & VRML2FILE & Offline & Use a VRML viewer. \\
\hline
\end{tabular}

Interactive viewers display the simulated system and events while G4Beamline is running; Offline viewers write the image to a file for viewing using another program.

The parameter that controls visualization is called viewer, and to use a given visualization driver, simply add viewer=best to your command line (or name whichever driver you want from the list above). This puts G4Beamline into a mode to perform visualization rather than tracking beam through the simulated system. The viewer parameter can be set via param commands in the input.file, and what matters is its value at the end; it is usually most convenient to only set it on the command line.

Visualization requires the file viewer.def, which contains help text and the internal Geant 4 commands required to invoke the selected viewer (it is a simple ASCII file with format documented in its comments). viewer.def is searched for in the current directory and then in the directory named in \$G4BL_DIR (which normally points to the G4Beamline distribution directory; it is set by the $g 4 b l$ script, or you can put it into your environment).

The G4beamline visualizations display all events of a run in a single image; some viewers consider each image to be an "event", but for beamlines multiple events in an image are often useful. Once the viewer has been selected, if you are running g4beamline manually you need to start a run by waiting for the "idle>" prompt and then typing "/run/beamOn 23" (or however many events you want to display). When G4Beamline presents you with an "idle>" prompt, this is the Geant4 internal command processor.

Viewer.def uses these commands to setup and invoke the selected visualization driver, and to arrange for 
tracks to appear in the images. See the Geant4 User's Guide [1] for details about these commands. Typing either "exit" or ${ }^{\wedge} \mathrm{C}(\mathrm{Ctrl}-\mathrm{C})$ will terminate the program. The "idle $>$ " prompt appears after the commands in viewer. def have been executed and the visualization is set up. If you are finished, simply exit the program $\left({ }^{\wedge} \mathrm{C}\right)$, or issue "/run/beamOn 23" to run the next 23 events and visualize them (i.e. generate another image containing those events). The Offline drivers are smart enough to generate a new image file for every beamOn command (filenames vary for each driver). The g4blgui graphical user interface program automatically handles all this.

\subsubsection{Additional Visualization Techniques}

These techniques use the Geant4 user interface, via the $g 4 u i$ command. You may want to look at the

Geant4 help for these commands; to do so, go to the examples directory and type (any input file will do):

$$
\text { g4bl example1. in viewer=best }
$$

Once the viewer opens its window, you will get an Idle $>$ prompt to which you can reply help $<\mathrm{CR}>$ and obtain help on any Geant4 UI command.

Note there are lots of options for the Geant4 viewers, and this is only a short list of those that users have requested in the past. If there is some aspect of drawing that you want, chances are fairly good that it is available.

\subsubsection{Different Drawing Scales}

Many beamlines are much longer than they are wide or tall. This makes the transverse dimensions difficult to see in a viewer, and when magnification is increased to see them, only a very small portion of the beamline is visible at a time. This can be addressed by using different drawing scales for $\mathrm{x}, \mathrm{y}$, and $\mathrm{z}$. The command to scale $\mathrm{x}$ by 2 , $\mathrm{y}$ by 3 , and $\mathrm{z}$ by 4 is:

$$
\text { g4ui when=4 "/vis/viewer/scaleTo } 23 \text { 4" }
$$

\subsubsection{Changing the Background Color}

To set the background color to white, use this command:

$$
\text { g4ui when=4 "/vis/viewer/set/background } 111 "
$$

The 3 values are for red, green, and blue, and should each be a value between 0 and 1; black is " 000 ". A fourth value for opacity can be given.

\subsubsection{Drawing Axes}

Drawing the coordinate axes can make it easier to find your way around the image of the system in a viewer. You can draw a set of axes at any desired location with any desired length. To draw coordinate axes of $100 \mathrm{~mm}$ length at $\mathrm{x}=1 \mathrm{y}=2 \mathrm{z}=3(\mathrm{~mm})$ :

$$
\text { g4ui when=4 "/vis/scene/add/axes } 123100 \text { mm" }
$$




\title{
2.8.1.4 Cut-Away and Section Planes
}

The Geant 4 drawing interface includes cut-away and section planes. The interface is quirky and you'll have to search the Geant4 documentation and just play with it. Not all viewers support this. The commands are: /vis/viewer/set/sectionPlane and/vis/viewer/addCutawayPlane.

\subsubsection{Starting up in Wireframe Mode}

\author{
g4ui when=4 "/vis/viewer/set/style wireframe"
}

\subsubsection{Filters}

The Geant 4 drawing interface includes filters that permit you to only draw certain particle types. The interface is quirky, and you'll have to search the Geant4 documentation and just play with it.

\subsubsection{The OpenInventor Visualization Driver}

Because the OpenInventor driver (OIX/OIWin32) is so much more useful than the other drivers, it is the driver of choice (linked to the name "best"). This driver will permit you do to almost everything that any other driver can do, the exception being the Wired driver lets you modify the graphical attributes of individual objects by picking and editing (that is in the works for OpenInventor).

Like other X-windows programs, the OpenInventor driver of G4Beamline can operate in either local or remote (networked) mode. The X display server must have the 3-D display capabilities of OpenGL installed. When you startup the OpenInventor viewer, the window looks like this (exampleAUG05.in):

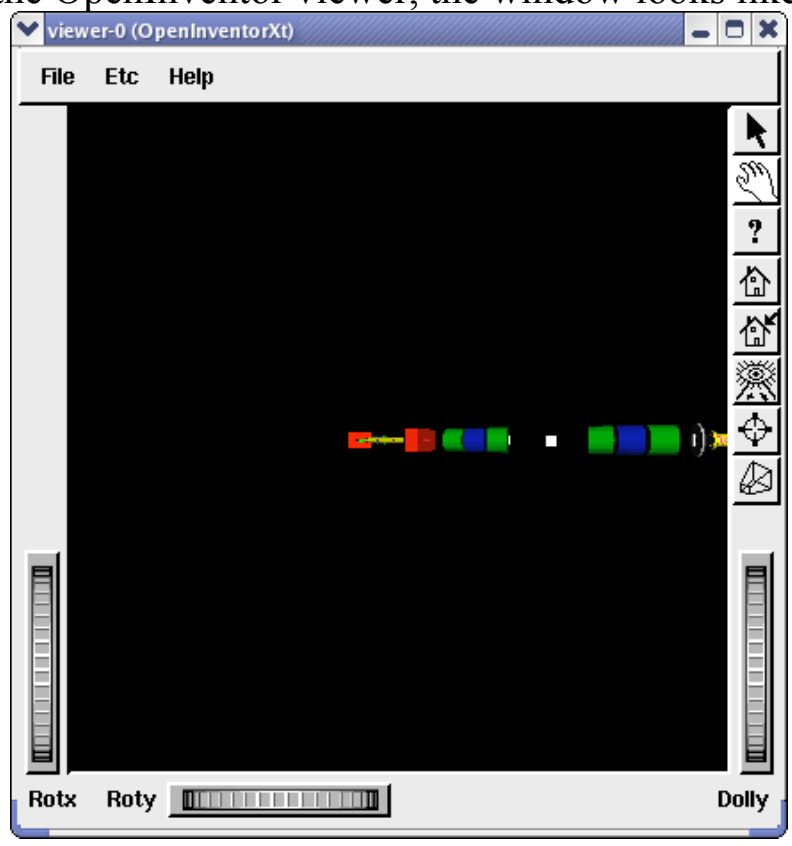

The Rotx and Roty wheels rotate the image relative to the viewer, and the Dolly wheel moves in and out with a corresponding change of scale. The buttons along the right edge do the following:

\begin{tabular}{|c|l|}
\hline Arrow & Select pick mode and cursor \\
\hline Hand & Select move mode and cursor (default) \\
\hline
\end{tabular}




\begin{tabular}{|c|l|}
\hline$?$ & Help (not implemented) \\
\hline House & Restore display to Home position \\
\hline House with arrow & Set Home position to be the current display \\
\hline Eye & Zoom so everything is visible \\
\hline Gunsight & Not implemented \\
\hline Perspective box outline & Select isometric or projected display \\
\hline
\end{tabular}

When the Hand cursor is active the mouse behaves like this:

Left Button Click and drag to rotate the display. It acts as if there were an invisible sphere surrounding the image, and the mouse movements rotate that sphere in any direction. This is quite different from the Rotx and Roty wheels, and takes some getting used to.

Middle Button Click and drag to pan the display - this moves the visible objects around without rotation.

Right Button Brings up a menu with various functions, Draw Styles, and other items. The most useful is DrawStyles/StillDrawStyle/Wireframe, which will draw in the wireframe mode rather than as solid surfaces. This permits you to look inside objects, and is considerably faster drawing.

The Etc menu has several useful items:

\begin{tabular}{|c|l|}
\hline Set Solid & Set the G4 mode to Solid (default) \\
\hline Set (G4) reduced wire frame & $\begin{array}{l}\text { Set the G4 mode to wireframe, } \\
\text { real edges only }\end{array}$ \\
\hline Set (G4) full wire frame & $\begin{array}{l}\text { Set the G4 mode to wireframe, } \\
\text { including triangles to fill surfaces }\end{array}$ \\
\hline Visible mothers + invisible daughters & $\begin{array}{l}\text { Daughter volumes are invisible } \\
\text { (default) }\end{array}$ \\
\hline Visible mothers + visible daughters & Make all objects visible \\
\hline
\end{tabular}

The key to understanding how objects are displayed is to know that Geant4 has a solid/wireframe choice, and OpenInventor has a solid/wireframe choice, and they are different:

\begin{tabular}{|c|c|l|}
\hline $\begin{array}{c}\text { G4 mode } \\
\text { (Etc menu) }\end{array}$ & $\begin{array}{c}\text { OI mode } \\
\text { (Right click menu) }\end{array}$ & Description \\
\hline Solid & Solid (“as is") & Draw solid surfaces \\
\hline Solid & Wireframe & Draw wireframes with triangles filling visible surfaces \\
\hline $\begin{array}{c}\text { Reduced } \\
\text { Wireframe }\end{array}$ & Either & Draw reduced wireframes, showing only real edges \\
\hline $\begin{array}{c}\text { Full } \\
\text { Wireframe }\end{array}$ & Either & Draw wireframes with triangles filling visible surfaces \\
\hline
\end{tabular}

For instance, in G4 reduced wireframe mode a sphere is invisible, as it has no real edges; in G4 full wireframe mode it has triangles filling its visible surface (in such a way you can tell it is a sphere). In reduced wireframe mode, a cylinder is drawn as two circles; unfortunately there is a bug and some objects are not drawn at all (use other modes to see them).

Note that by default all daughter volumes are invisible. In wireframe mode you would expect to see them, but to do so you must invoke Etc/VisibleMothers+VisibleDaughters. 
The OpenInventor driver has many more features, but this should get you started. It will also do animation: with the hand cursor left click and drag the image, letting go of the button while moving the image will continue to rotate until you left click on it (works best locally).

\subsubsection{A Trick for Visually Scanning Events}

The simplest way to scan events visually is to use the g4blgui program, but it can be done manually via the command line. The OpenInventor driver (OIX) has the ability to exit the viewer and return to the Geant 4 command input. This can be used to quickly scan events visually. To do this, first create a text file called beamon.txt containing a large number of identical lines:

/run/beamOn 1

/run/beamOn 1

/run/beamOn 1

... many more identical lines

Then invoke g4beamline like this:

G4bl input.file viewer=best $<$ beamon.txt

Once the viewer appears, it will display the first event. To see the next event, select the File/Escape menu item. This will suspend the OpenInventor viewer, and the Geant4 input routine will read the next line of beamon.txt - that causes it to track one event and refresh the viewer with it. Just keep selecting File/Escape to sequence through events one at a time. This is essentially what the g4blgui program does when a viewer is selected.

\subsubsection{Troubleshooting}

Visualization is usually the only part of G4beamline that causes problems for users getting it to work on their systems. In general, on Linux and Mac OS X a working X-windows display is required; this means on Linux you usually will run one of the common desktops (KDE or Gnome), and use a terminal window to execute the G4beamline program. On Mac OS X you need to run the X11 application, and either run $g 4 b l$ from an $x t e r m$ or specify the DISPLAY manually.

G4beamline visualization requires that both the Motif and the OpenGL extensions be configured on the display. This means that both the X-windows client (the G4beamline program) and the X-windows display server (the display+keyboard+mouse) need them installed. If you run G4beamline on your desktop Linux system, this usually happens naturally, but if you connect from your desktop to a Linux server and run G4beamline there, then you must make sure these extensions are installed on both systems. In particular, if you use the Exceed program on a Windows desktop to connect to a Linux server, be sure to install Exceed's OpenGL extension. This also applies to Mac users (G4beamline is an $\mathrm{X}$-windows application, not a native Mac application).

On Windows, all necessary graphics extensions should be installed by default. Remote displays (e.g. via Citrix) should work as usual.

If you run g4bl with a viewer parameter, and it crashes with the error message "Xlib: extension GLX missing on display :0.0." (or similar), this means you must install the OpenGL extension ( $g l x$ ) on the 
system you are using to display. That message may well be followed by a lengthy trace of function calls. If, instead, it crashes with the error message "error while loading shared libraries: libGL.so.1: cannot open shared object file: No such file or directory" (or similar), this means you must install some necessary system library on the system you are using to run g4beamline - see README-*.txt for details.

G4beamline X-windows visualization will operate over a network, but its performance will be slower than running locally, even over a $100 \mathrm{Mbit} / \mathrm{s}$ LAN. The easiest way to do this is to use ssh with Xwindows forwarding enabled (the $-\mathrm{X}$ switch, which is often supplied by default when the ssh client is run from a terminal window). In some cases (particularly on a Macintosh) the $-Y$ switch may be needed instead.

Note for NVidia display users on older versions of Linux: There are two common X-windows drivers for NVidia displays on Linux, named $n v$ and $n v i d i a$. The $n v$ driver is open source and comes with the Linux distribution, but may not have the OpenGL extension (called glx); the nvidia driver can be downloaded from http:/www.nvidia.com and does have the OpenGL extension (it also uses a kernel module called nvidia). A comparison of these drivers on my laptop:

\begin{tabular}{|c|c|c|c|c|c|c|}
\hline Driver & $\begin{array}{c}\text { OpenGL } \\
(g l x)\end{array}$ & $\begin{array}{l}\text { 1400x1050 } \\
\text { Resolution }\end{array}$ & $\begin{array}{c}\text { Other } \\
\text { Resolutions }\end{array}$ & $\begin{array}{c}\text { Suspend to } \\
\text { RAM }\end{array}$ & $\begin{array}{l}\text { External } \\
\text { Monitor }\end{array}$ & $\begin{array}{c}\text { Twinview } \\
\text { Mode }\end{array}$ \\
\hline nv & No & Yes & Yes & Yes & No & No \\
\hline nvidia & Yes & Yes & Yes & No & Yes & Yes \\
\hline
\end{tabular}

In Twinview mode the laptop's LCD and the external monitor are two physical displays in a single logical screen, and windows can be dragged from one to the other. Because of the usefulness of suspend to RAM on a laptop, I have scripts that permit me to easily select which driver is used when I startup Xwindows. More recent Linux distributions don't have this confusion.

\subsection{Obtaining Results - Virtual Detectors and other NTuples}

While Geant 4 can be used to make quite detailed models of detectors and their readouts, at present G4Beamline does not attempt to do so. The primary purpose of G4Beamline is to track particles and see where they go (or don't go). The most common way to obtain output is by using a command that generates an NTuple.

\subsubsection{Track NTuples}

The following commands generate an NTuple for tracks:

- A virtualdetector is an idealized perfect detector that "detects" every track that hits it, and measures all of the track properties with the resolution of a 32-bit float (including position, 3momentum, particle type, event \#, etc.). It is "virtual" because any material (including Vacuum) can be used and by default it uses the material of its parent so it doesn't affect the tracking of particles. Multiple tracks from a given event that hit the virtualdetector will be stored as separate rows in the NTuple (with the same EventID), as will multiple hits from a single track (with the same EventID and TrackID). The measurements for all such tracks are output to a file as an NTuple.

- A zntuple is similar to a virtualdetector, except that no physical volume is used; whenever a track reaches the specified $\mathrm{Z}$ position (centerline coordinates), it is sampled in the same manner 
as a virtualdetector. All zntuple-s are named "Z100" with the number being its Z position rounded to the nearest millimeter. Note that the zntuple can span multiple physical volumes, while the virtualdetector cannot.

- A timentuple samples the tracks at a specified time.

- A newparticlentuple samples tracks when they are created.

- A beamlossntuple samples tracks as they are killed.

- An ntuple is a collection of the data for one or more other NTuple-s, with each row of the NTuple being a single event or track, and the columns being the union of all included NTuple-s, in order. Any NTuple generated by any of the above commands, including ntuple itself, can be included in this NTuple, as long as the other commands precede this one in the input.file.

- A trace is an NTuple generated for a single track. The rows of the NTuple are the series of steps taken by tracking, and the columns are the same as for a virtualdetector plus the electromagnetic fields (B and E). Each track is a single NTuple (a separate file in ASCII formats).

Normally each virtualdetector generates an NTuple for its hits (singles), as does zntuple. The ntuple command can be used to combine multiple virtualdetector and zntuple data into a single NTuple, with each row of the NTuple containing all data for a single event (if multiple tracks of the event hit a given virtualdetector or zntuple, only the first is kept in the NTuple).

By default, all NTuples are written to a single Root [5] output file. A Root-based histogramming program, historoot, is included in the G4Beamline distribution, and it can generate histograms in a very flexible and intuitive manner from Root files and ASCII files, via a graphical user interface. In the future, G4Beamline may be extended to use AIDA for NTuples as well.

The format $=$ ascii argument to these commands writes an ASCII file for each NTuple containing one track per line, with comments at the start giving the variable names and their units. The historoot program can read these ASCII files, including the variable names from the initial comment.

The standard fields of an NTuple generated for tracks contains the following variables (in order):

\begin{tabular}{|l|l|}
\hline \multicolumn{1}{|c|}{ Name } & \multicolumn{1}{c|}{ Description } \\
\hline $\mathrm{x}, \mathrm{y}, \mathrm{z}$ & The position of the track, in the selected coordinates. Millimeters. \\
\hline $\mathrm{Px}, \mathrm{Py}, \mathrm{Pz}$ & The momentum of the track, in the selected coordinates. MeV/c. \\
\hline $\mathrm{t}$ & The global time of the track. Nanoseconds. \\
\hline PDGid & $\begin{array}{l}\text { The ID of the particle, using the assigned value from the Particle } \\
\text { Data Group. }\end{array}$ \\
\hline EventID & The event number. \\
\hline TrackID & The track identifier. \\
\hline ParentID & The track identifier for this track's parent; 0 if this is a beam track. \\
\hline Weight & The weight of the track (defaults to 1.0). \\
\hline
\end{tabular}

TrackIDs are supposed to be unique within each event; they normally start at 1 for the beam particle(s). Secondary particles' TrackIDs normally start at 1000 and increment sequentially for additional secondaries.

The trace command adds the following fields to the NTuple, after the standard fields:

\begin{tabular}{|c|l|}
\hline Name & \multicolumn{1}{|c|}{ Description } \\
\hline $\mathrm{Bx}, \mathrm{By}, \mathrm{Bz}$ & The magnetic field at the track's position, in the selected \\
\hline
\end{tabular}




\begin{tabular}{|l|l|}
\hline & coordinates. Tesla. \\
\hline Ex, Ey, Ez & $\begin{array}{l}\text { The electric field at the track's position, in the selected coordinates. } \\
\text { Megavolts/meter. }\end{array}$ \\
\hline
\end{tabular}

The format $=$ Extended, format $=$ asciiExtended, or format $=$ rootExtended argument to these commands adds the following fields to the NTuple, after the standard fields and the B and E fields from the trace format:

\begin{tabular}{|l|l|}
\hline \multicolumn{1}{|c|}{ Name } & \multicolumn{1}{c|}{ Description } \\
\hline ProperTime & The proper time of the track since it was created. Nanoseconds. \\
\hline PathLength & The total path length of the track since it was created. Millimeters. \\
\hline PolX, PolY, PolZ & The polarization of the track in the selected coordinates. \\
\hline InitialKE & The Kinetic Energy of the track when it was created. MeV. \\
\hline
\end{tabular}

\subsection{2 printf}

The printf command generates a printout in a user-specified format for every track that reaches its specified $\mathrm{Z}$ position; the standard fields above can be used in expressions to be printed via conversion specifications in the printf format string. Output can be to stdout or to a file, and multiple printf-s to the same file will be output to the file in the order tracks reach their Z positions, and in the order the printf-s appear in the input file for the same $Z$ position. This permits ASCII output of track information in any desired ASCII format, perhaps to feed directly into some other program.

\subsection{3 profile}

The profile command prints a computation of the means, sigmas, and emittances for all tracks at a given $\mathrm{Z}$ position.

\subsection{4 totalenergy}

The totalenergy command totals up the energy deposited by all tracks of a run in selected physical volumes, and prints the results at the end of the run. Output defaults to stdout, but can be put into a file. With appropriate post-processing this can be used to estimate heat loads in specified volumes.

\subsection{Random Number Generator}

G4Beamline uses the default Geant4 random number generator (which comes from CLHEP [2] - see the CLHEP documentation for details). It is an excellent pseudo-random number generator that essentially guarantees no repeat sequences when seeded by any integer between 0 and 900 million. At the start of each event, G4Beamline seeds the random number generator with the event number. This permits the user to submit multiple jobs in parallel with confidence, as long as the beam command arguments are arranged so no two jobs run events with the same event numbers - quite effective when using a Linux cluster. This also permits the user to re-run the same events (as long as the input file remains unchanged - the slightest change can cause tracking to use a different number of random numbers, and thus the runs of the same event number won't be the same). A good use of this last capability is to find an "outlier" event in some histogram, determine its event number (via narrow cuts in historoot) and re-run it with visualization to see what actually happened. 
Note that due to their internal designs, Root NTuples cannot precisely represent event numbers larger than 16 million (a float with 24 bits of mantissa). This does not affect the ability to handle larger datasets, it just means that the event numbers will be rounded to a 24-bit mantissa.

\subsection{Tuning the Beamline}

Just like a real beamline, a simulated beamline must be tuned to maximize transmission and achieve various desired characteristics. G4beamline is not intended for the design of beamlines, but rather is best used for the evaluation of them. Conventional beam optics design programs can be used to determine the beamline elements and their parameters. These programs, however, usually make approximations and ignore many subtle effects that G4beamline implements (e.g. fringe fields, non-Gaussian multiple scattering tails, energy loss straggling, hadronic interactions in collimators and other apertures, nonGaussian tails in beam profiles, etc.). The result is that just as in the real world the design programs give only approximate values.

So two methods of tweaking the tune are provided:

1. The reference command can tune its referenceMomentum in order to make the reference particle have a specified tuneMomentum at a specified tuneZ position (centerline coordinates). This is most useful for a beamline with material that induces a significant energy loss in the reference particle, such as muon cooling channels.

2. The tune command can be used to tune any tunable argument to any set of elements. This includes the $B y$ field argument of genericbend and idealsectorbend, and the maxGradient argument of pillbox.

See the online help, or section 5, for details about these commands. Tests 29-32 verify the operation of these tunes, and provide working examples.

To aid the user in fine tuning the beamline, g4beamline implements the ability to track a "Tune" particle and a "Reference" particle before tracking the beam (both are controlled by the reference command). These particles are tracked with all stochastic processes disabled, and nominally go right down the centerline of the beam. The Tune particle is used to tune all desired beamline elements, and the tuning process often forces it to be tracked multiple time through sections of the beamline; after tuning is completed then the Reference particle is tracked to give a clean readout of the reference trajectory, and computation of the reference coordinates (if used).

The Tune particle can be used to:

1. Automatically set the timing of RF cavities so the Reference particle arrives at the desired RF phase.

2. Automatically set the gradient of RF cavities so the desired acceleration of the reference particle is achieved.

3. Automatically set the field of bending magnets so the Reference particle is parallel to the centerline downstream of the magnet.

4. Automatically determine the initial momentum of the Reference particle so that it has a specified momentum at some later point in the beamline.

Unfortunately, this method cannot be used to tune the position of bending magnets (because of their fringe fields, the center of a bending magnet must normally be offset by a small distance downstream of the corner in order to make the Reference particle go right down the centerline after the magnet). 
As an example, consider tuning the $B y$ value of a genericbend:

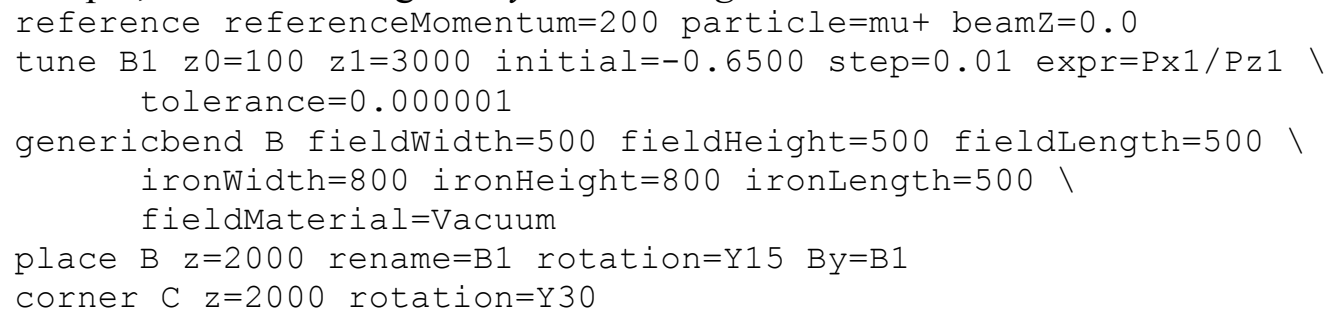

The tune command specifies to tune the value "B1" so that at $\mathrm{Z}=3000$ the expression "Px1/Pzl" will be zero to within the tolerance of 0.000001 . That is, that the Reference particle be parallel to the centerline within a microradian. The genericbend named "B1" uses the tune variable "B1" as its By argument value. What happens is that when the Tune particle reaches $\mathrm{z} 0=100$, it is saved and tracked to $\mathrm{z} 1=3000$ (which goes through the genericbend B1). When the Tune particle reaches $\mathrm{z} 1=3000$, the expr "Px1/Pzl" is evaluated, and if it is within tolerance of zero the tuning is complete. If it is out of tolerance, a simple linear solver is used to update the value of the variable $B 1$, and the saved track from $\mathrm{z} 0=100$ is retracked to $\mathrm{zl}=3000$ and the process is repeated. If the process does not converge with 10 attempts, the tuning fails and the program exits; information about each step is written to stdout.

The other tune procedures are similar, in that the Tune particle track is saved upstream of the region to be tuned, and downstream of the region its properties are used to modify a variable that affects the tuning, and the saved track is re-tracked until the result is within tolerance.

In practice, it is often necessary to use multiple tune commands. They must be properly nested so each tuning region is either disjoint from the others, or is wholly contained in one or more other regions (separation of $\sim 0.1 \mathrm{~mm}$ is usually adequate). For instance, in a muon-cooling channel with bending magnets and RF cavities it is probably necessary to tune the reference momentum, the bending magnets, and the RF gradient(s).

Note that g4beamline can only tune based on the Tune particle, and can only tune tunable arguments to certain commands. These limitations are due to technical details of the implementation, and cannot easily be relaxed. No aspect of the geometry can be tuned via the Tune particle. Note this also does not include tuning quadrupoles to achieve a specified focus, because that requires tracking many beam particles and tuning to achieve a specified distribution. Because g4beamline can be easily scripted, however, it is possible to do this manually via scripts or an external program. Such an external method can tune any parameter or argument to any element, including geometry. See sections 7.18 and 7.21 for details.

Examples: The program triplet.sh in the examples directory focuses a quad triplet for a point-to-point focus. In the test directory, test 29 , test 30 , test 31 , test 32 , and test 35 verify the tuning of each of the above quantities.

\subsection{The Geant4 User Interface}

Geant 4 has its own command line processing and user interface. For the most part this is not used in g4beamline. Some Geant4 commands can be simply placed in the input.file because all input lines 
beginning with "/" are interpreted as Geant4 commands; they are executed immediately upon being encountered in the input.file. In most cases, that is not what is needed, because the commands must be deferred until the geometry has been set up - see the g4ui command for that.

The viewer.def file contains Geant4 commands used to initialize and use the various visualization drivers. The selected section is automatically invoked when visualization is specified via the viewer $=\ldots$ argument to the command line.

\subsection{Event Time Limit}

Every event is subject to a per-event time limit. This is controlled by the parameter eventTimeLimit, which defaults to 30 (seconds), but can be set by the param command. As long as the program is taking physics steps, this time limit will cause the current event to be killed and the next event to be simulated (restarting the time limit). In case the code gets into an infinite loop, an alarm is set at the start of each event for eventTimeLimit +10 , and if this fires, the entire program will be aborted (recovery is not possible); this prevents it from exhausting your CPU allocation on a cluster.

Note that systems with stable particles and electromagnetic traps can run literally forever - see the maxTime argument to the trackcuts command to limit this (defaults to $1 \mathrm{~ms}$ in the lab). You may not think your system has this property, but low energy delta rays $\left(\mathrm{e}^{-}\right)$can surprise you. Note also that putting a medium- or high-energy electron or photon into a block of material can generate many thousands of secondaries, which can take a very long time to track.

\subsection{Event and Track Numbering (EventID, TrackID)}

EventID is intended to identify a particular event (which may have multiple tracks). In G4beamline, each beam track is a separate event. For internally generated beams, EventID is incremented for each beam track and is thus unique within a given run. For beams read from external files, the assignment of EventID-s to tracks is contained in the file - this means that multiple events can have the same EventID, and that EventID-s might not be monotonically increasing.

Moreover, NTuple-s use float-s to hold each data item, and a float has only 24 bits of mantissa. This implies that any event number $\geq 16,777,216$ will be truncated. This applies to both reading and writing Root and ASCII files. Two workarounds avoid this truncation for many cases:

- BLTrackFile format avoids the truncation internally, both reading and writing.

- Commands that generate a track NTuple will map format=ascii to format=bltrackfile. This includes: beamlossntuple, newparticlentuple, timentuple, virtualdetector, zntuple.

The result is that this truncation of EventID occurs only for Root NTuples and in the ntuple command; neither of these can be avoided without massive changes that adversely affect users.

TrackID is intended to identify a track within an event. For internally generated beams the primary track has TrackID $=1$, but for beams read from external files the assignment of TrackID to tracks is contained in the file - this means that multiple tracks with identical EventID-s can have the same TrackID-s.

Moreover, secondary tracks can be generated during the simulation of an event, and the TrackID-s for them can potentially conflict with TrackID-s read from a file. By default, secondary TrackID-s are 
assigned starting at 1001 (see secondaryTrackID of the beam command). If a beam track from an external file has a TrackID $\geq$ secondaryTrackID, a warning will be printed. For beam files containing TrackID-s $\geq 1001$, setting secondaryTrackID in the beam command can avoid both the potential confusion and the many warnings. 


\section{Important Values that Affect the Validity and Accuracy of Simulations}

Particle simulations are inherently complicated and CPU intensive. The Geant 4 toolkit necessarily provides facilities to permit users to make trade-offs between accuracy and CPU time. G4beamline likewise considers this a user issue, and provides interfaces to Geant4 mechanisms as well as some new capabilities. To perform simulations that yield results you can trust, you must make some choices that affect the accuracy and validity of the results. It is often equally important to avoid becoming overwhelmed by useless information. Features related to these are described in this section.

\subsection{Physics List}

The Geant4 toolkit contains a large number of physics processes that implement the transportation, decays, and interactions of particles. In addition, Geant4 is extensible, and users can construct their own physics processes. These processes are numerous and complex, and often have complex relationships between different processes, so it is a challenge for users to become sufficiently knowledgeable to select the necessary processes and implement them in code. As a result, the Geant 4 collaboration has constructed a number of "physics lists" that are crafted from the library of physics processes to be suitable for a specific user domain.

G4beamline shields the user from much of this complexity, and only offers to the user the choice among the Geant4 physics lists, plus a few additional lists constructed for special purposes. In particular, it is not possible for users to implement a new physics process in G4beamline without building the program yourself (that is rather complicated, but you can request a new feature to have us do it - see section 7.3). The physics list is selected by the physics command, and the set of available lists is given in the help text for that command (see section 7.4).

As a rule of thumb, for incident particles with kinetic energies above $12 \mathrm{GeV}$ or so, the "standard" physics list is QGSP. For incident particles with kinetic energies above about $100 \mathrm{MeV}$, the "standard" physics list is QGSP_BIC (it is noticeably slower than QGSP below $\sim 12 \mathrm{GeV}$ ). The electromagnetic processes of these physics lists are advertised to be valid above a few hundred electron Volts; Geant 4 has a set of low energy electromagnetic processes, indicated by a suffix "_EMX" in the physics list name. The suffix "_EMV” indicates electromagnetic processes tuned for better CPU performance with only slightly less precision.

Unfortunately, the documentation of the different Geant4 physics list is incomplete, and does not really give a complete picture of each list's strengths and weaknesses or region of applicability and validity. We have a request in to the Geant 4 collaboration to improve this documentation.

\subsection{Tracking Accuracy Parameters}

There are a number of values in Geant 4 that affect the accuracy of its tracking. The important ones are implemented in G4beamline as parameters, and they can be set anywhere in your input.file with the param command (the final value is what matters). These control such things as how close to a geometric 
boundary a track must come before it is considered to have hit the volume, how far a track can deviate from a straight line during a single step (due to a magnetic field), etc. They are:

\begin{tabular}{|l|c|l|}
\hline Parameter Name & Default & Description \\
\hline deltaChord & $3 \mathrm{~mm}$ & \\
\hline deltaIntersection & $0.1 \mathrm{~mm}$ & \\
\hline deltaOneStep & $0.01 \mathrm{~mm}$ & \\
\hline epsMax & 0.05 & \\
\hline epsMin & $2.5 \mathrm{E}-7$ & \\
\hline maxStep & $100 \mathrm{~mm}$ & $\begin{array}{l}\text { The maximum step permitted to be taken. Note this is a limit } \\
\text { on the physics step; integrating the equations of motion will } \\
\text { take multiple "steps" within this. }\end{array}$ \\
\hline minStep & $0.01 \mathrm{~mm}$ & $\begin{array}{l}\text { How close to a specified Z position a step must occur (the two } \\
\text { steps surrounding the Z position are then linearly interpolated). }\end{array}$ \\
\hline zTolerance & $2 \mathrm{~mm}$ &
\end{tabular}

These parameters specify the trade-off between tracking accuracy and CPU time. These are reasonable values for beamlines or detectors of ordinary sizes (with scales from roughly a few millimeters to a few tens of meters; the length of a narrow beamline can reasonably extend to a few kilometers). If you have objects smaller than a millimeter or so, or are working on scales larger than a kilometer or so, you should consider changing these values appropriately. If you have small objects spread over a large volume, in general you should select parameter values appropriate for the small objects and realize that the simulation will most likely require lots of CPU time.

\subsection{Electromagnetic Field Map Tolerance}

Several elements that implement electromagnetic fields internally construct a field map, because evaluating the field during tracking is too CPU intensive. These are: coil and fieldexpr. They each have a tolerance argument that is used to determine the required grid spacing of the map. Its value obviously affects the accuracy of the simulation. This is of course also true for externally supplied EM field maps.

\subsection{Secondary Creation Threshold in Physics Processes}

An important trade-off between physics accuracy and CPU time in Geant 4 is the production of lowenergy delta rays $\left(\mathrm{e}^{-}\right)$and other particles, which diverge at low energies. The approach is to only generate a secondary when the range of the particle in the current material exceeds a user-specified minimum range cut (which is converted to a kinetic-energy threshold for each material). Particles with shorter ranges are not produced as secondaries, but are lumped into the continuous ionization energy loss in the material. In G4beamline, this is set by the minRangeCut argument to the physics command; its default value is $1 \mathrm{~mm}$. If your simulation has objects smaller than that, or you are working at scales much larger than that, you should consider changing its value, unless you just don't care about these low-energy particles. 


\subsection{Track Cuts}

The trackcuts command applies cuts to every track both before starting to track it, and at every step while tracking. These can obviously affect the realism and accuracy of the simulation; they also can be quite useful for weeding out extraneous information (e.g. the neutrinos generated by pion and muon decay). The cuts it can apply are:

\begin{tabular}{|l|c|l|}
\hline Argument Name & Default & Description \\
\hline kill & (empty) & Comma-separated list of particles to kill. \\
\hline keep & (empty) & $\begin{array}{l}\text { Comma-separated list of particles to keep (kill all others); } \\
\text { ignored if empty. }\end{array}$ \\
\hline killSecondaries & 0 & Set nonzero to kill all secondaries. \\
\hline kineticEnergyCut & $0 \mathrm{MeV}$ & Minimum kinetic energy to track. \\
\hline kineticEnergyMax & Infinite MeV & Maximum kinetic energy to track. \\
\hline maxTime & $1000000 \mathrm{~ns}$ & Maximum lab time to track. \\
\hline
\end{tabular}

In addition, there is a processing time limit implemented as a parameter, eventTimeLimit (defaults to 30 seconds). This applies to the real time used during the processing of all tracks in the event. For simulations in which showers are important (and are not suppressed), this may need to be increased. Unfortunately, it is implemented as a real time limit, not a CPU time limit, so on excessively loaded systems it may also need to be increased (not usually a problem).

\subsection{Miscellaneous}

- There is a bug in Geant4 tracking that makes gross errors when tracking a particle that stops and turns around (e.g. it is traveling exactly opposite to an E field sufficient to stop it). This has been reported as Geant4 bug \#1021, and the command bug1021 implements a work-around that resolves the issue with an accuracy of a few microns.

- A number of Geant4 processes have auxiliary commands that can vary their operation. See the Geant 4 documentation for details; in most cases, these are not important, but when they are, the g4ui command can be used to execute the necessary Geant 4 commands.

- Geant4 tracking requires that the simulated world's geometry be a rigorous hierarchical structure; violations of this requirement can cause gross tracking errors. By default, G4beamline performs a geometry test to check for invalid volume overlaps and conformance to this requirement; failures generate a warning but permit the simulation to proceed. The geometry command controls its parameters. Section 8.2 discusses when these rules can be violated without affecting the simulation results.

- The pseudo random number generator is normally seeded with the event number immediately before starting to process an event. The generator used has excellent properties when seeded with integers from 0 thru 900,000,000. This behavior can be changed via the randomseed command.

- As in real beamlines, shielding is required in realistic simulations. See section 7.9.

- Individual physics processes can be disabled via the disable and doStochastics arguments to the physics command. A list of the physics processes used in the current physics list is available from the list command. 


\section{Input File Description}

A G4Beamline simulation is completely specified in a single ASCII input file, plus whatever auxiliary files it references (e.g. magnetic field maps, window profiles, etc.).

Each line in the input file is either a comment or a command. Comments are blank lines, lines beginning with '\#', and lines beginning with '*' (which are printed to stdout, giving a convenient method to document your output files). Lines beginning with '/, are commands passed directly to the Geant4 user interface command interpreter (this happens before the geometry is initialized; see the $g 4 u i$ command to defer execution of Geant4 UI commands). Lines beginning with "!" are passed directly to the shell (with the '!' stripped).

A command is a single line beginning with the command name (optionally preceded by white space), followed by any number of arguments. Lines can be continued by preceding the ending newline with a backslash (\). Commands can have any combination of positional and named arguments, which are separated by white space (spaces or tabs). A named argument is of the form "name=value", where the name is like a $\mathrm{C}$ identifier except it can also contain the characters ' + ' and '-' (which are needed for particle names). If spaces or tabs are present in the value, the entire value must be enclosed in single- or double-quotes; this delimiter cannot appear in the value. Any argument that is not named is positional, and its value can also be enclosed in single- or double-quotes; positional arguments are conventionally given first, but can be intermixed with named arguments. The order of positional arguments is important, but the order of named arguments is not.

This command has 2 named arguments:

param histoFile=histoscope.hst histoInterval=100000

This command has 1 positional and 4 named arguments:

tubs Target outerRadius $=10$ length $=100$ material=W color $=1,1,1$

This command has 3 positional arguments (the last 2 are each themselves a command that will be invoked when the defined command MyMacro is used):

define MyMacro ।

"tubs $\$ 1$ outerRadius=10 length=100 material=\$2 color=\$3" । "place $\$ 1 "$

That macro is used like this:

MyMacro TubsName Fe 1,0,1

Command files can be nested; see the include command.

Simple macros can be defined, with arguments; see the define command.

The value of a named or positional argument can come from a parameter by using a ' $\$$ ':

param $\mathrm{H}=10.0 \mathrm{~W}=20.0 \mathrm{~L}=30.0$

box BoxName width $=\$ W$ height $=\$ H$ length $=\$ \mathrm{~L}$

Note that \$parameter substitution occurs only within the value of a parameter; this can be combined with arithmetic expressions for numeric arguments:

box Box.\$H.\$W. \$L width=\$W+10 height $=\$ H * 2.0$ length=sqrt (\$L)

The param command is unique in that when the value of any argument is a valid numerical expression containing at least one operator, the expression will be evaluated and the parameter will be defined as 
the number (rather than the string of the expression). This permits the computation of rotations (the value of the rotation argument is a string containing multiple numerical values):

param angle=atan2 $(\$ a, \$ b) * 180 / 3.14159$

place Element rotation=X\$angle, Y90

\subsection{Expressions}

Expressions can be used for the value of any double argument to any command. They are evaluated during command processing, and generally must involve only double values and the operators and functions listed below. Parameter expansion (\$paramname) can be used as long as the value of paramname is valid where it appears in the expression. Some commands expand the expressions to include certain variables (e.g. the $B x$ argument to fieldexpr can be an expression involving $\mathrm{x}, \mathrm{y}, \mathrm{z}$ ).

In the param command, if the value of an argument is a valid numerical expression, the parameter is set to the numerical value rather than to the expression string.

The following arithmetic operators can be used in expressions: $+-* / \wedge()$

The following comparison operators can be used in expressions; they evaluate to a value of 1.0 (true) or 0.0 (false): $<<=>>===$ !=

The ?: operator is not implemented; $\wedge$ means exponentiation to an integer power.

The following functions can be used in expressions: $\operatorname{abs}(), \min (), \max (), \operatorname{sqrt}(), \operatorname{pow}(), \sin (), \cos (), \tan ()$, $\operatorname{asin}(), \operatorname{acos}(), \operatorname{atan}(), \operatorname{atan} 2(), \sinh (), \cosh (), \tanh (), \exp (), \log (), \log 10()$, floor(), ceil( $)$. All take 1 argument except $\min (), \max ()$, pow(), and atan2() take 2 arguments.

The following constants are pre-defined: pi, e, gamma, radian $(=1.0)$, rad $(=1.0)$, degree (=pi/180.), deg (=pi/180.).

\subsection{Element Names}

When you create an element (e.g. using the tubs command), you must specify its name as the first positional argument. When you place it into the simulated world, its name must be the first positional argument to the place command (it can be renamed when placed). Element names can be any string, but conventionally obey the rules of a $\mathrm{C}$ identifier.

Element names are necessary to correlate the definition and placement of each element. They are also used to report errors in the geometry test, and are listed in the traces when stepping. The name of a virtualdetector becomes the name of its NTuple.

When elements are placed inside another element, the name of the parent is prepended to the name of the placed daughter, so the name shows its element hierarchy. This makes it desirable to capitalize the first letter of each name. The "World" volume-name is not prepended (otherwise it would apply to every element name). The rename $=$ argument to the place command can be used to override the name of the element (the default is without the parent's name, but rename $=+X y z$ will prepend the parent's name to 
Xyz); '\#' can be used to automatically number multiple placements (see the place command in section 4 for details).

There are several independent name spaces used by various commands:

\begin{tabular}{|c|c|c|}
\hline Type & Defined by & Used by \\
\hline Element names & Element definition commands & place command \\
\hline Virtualdetector names & Name of the virtualdetector, as placed & ntuple command \\
\hline Material names & $\begin{array}{c}\text { Material command } \\
\text { (many materials are predefined). }\end{array}$ & $\begin{array}{c}\text { Element definition commands, } \\
\text { material=name argument }\end{array}$ \\
\hline Coil names & coil command & solenoid command \\
\hline
\end{tabular}

\subsection{Parameters}

Parameters are named ASCII strings that can be used in the input.file to set command arguments, and some are used to control the program. They can be set either on the command line or by the param command. They are stored internally as ASCII strings, and when they represent an integer or floatingpoint value they are converted when used (invalid strings are errors). When a parameter that has not been defined is used, it will be automatically defined from the environment if possible; otherwise using an undefined parameter generates an error (this is especially useful in Grid jobs, which generally need \$OSG_WN_TMP in every filename).

A parameter can be used in the value of any argument to any command:

tubs Name length $=\$$ length outerRadius $=\$$ radius

In this example, it is necessary to define the parameters length and radius before this command is issued; that can be either earlier in the input.file or on the command line. Here the string definitions of both parameters length and radius must be valid floating-point numbers:

$$
\begin{aligned}
& \text { \# (earlier in the input.file) } \\
& \text { param length }=100.0 \text { radius }=10.0
\end{aligned}
$$

Or on the command line:

$$
\text { g4bl input.file length }=100.0 \text { radius }=10.0 \quad[\ldots]
$$

Note that once the parameter is defined, it can be used any number of times to give the values of command arguments. That can be used to avoid having to change many identical numerical values throughout the input.file. It can also be used to collect all of the variable values of input.file in one place near the top, or even in a separate definition file (see the include command).

This is a limited macro facility, and can only be used to substitute the string value of a parameter into a command argument (named or positional). For integer and double (real) arguments, arithmetic expressions involving constants can be used (including common functions like $\sin ()$ and $\operatorname{sqrt}())$ ):

$$
\begin{aligned}
& \text { param } a=3.0 \quad b=2.0 \text { name=Name } \\
& \text { tubs } \$ \text { name lengt } h=\$ a+\$ b \text { outerRadius=sqrt }(\$ b) * 10
\end{aligned}
$$

In the param command only, argument values that are a valid numeric expression with at least one operator are converted to the value of the expression. This permits such parameters to be used to perform computations for rotations (and colors, if desired):

param angle=atan2 $(\$ a, \$ b) * 180 / 3.14159$ 
NOTE: \$name used in a macro body (the define command) is substituted when the macro is defined, not when the macro is expanded. $\$$ \$name in a macro body will be expanded when the macro is invoked.

It is possible to re-define the value of a given parameter name within input.file; the value substituted for \$name is the most recent definition preceding its use. Because this can be confusing to humans, it is discouraged (except for the do command). The -unset argument to param will set parameters only if they are not already defined; this is useful to put default values of parameters into input.file while permitting them to be overridden on the command line; it does not work for parameters used to control the program (they are pre-defined with default values).

\subsubsection{Program Control Parameters}

Several parameters are used internally by G4Beamline to control its operation:

\begin{tabular}{|l|l|l|}
\hline Name & Default & Description \\
\hline viewer & "none" & $\begin{array}{l}\text { Determines the mode of the program, visualization or } \\
\text { tracking. Also determines the visualization driver to use. } \\
\text { See section 2.8 above. }\end{array}$ \\
\hline steppingVerbose & 0 & When nonzero causes each step to be printed to stdout. \\
\hline steppingFormat & (see below) & The format of lines printed by steppingVerbose. \\
\hline histoFile & g4beamline.root & The histogram output filename. \\
\hline histoUpdate & 0 & $\begin{array}{l}\text { When nonzero causes G4Beamline to write the histoFile } \\
\text { after each block of events have been tracked. }\end{array}$ \\
\hline worldMaterial & Vacuum & The material of the World volume. \\
\hline Zcl & - & $\begin{array}{l}\text { Initially } 0, \text { the place command sets } Z c l \text { to the downstream Z } \\
\text { position of the placed element, when using Centerline } \\
\text { coordinates (not for Global coordinates or placements into } \\
\text { elements other than the World). The user can set Zcl, but } \\
\text { that has no effect on the place command. }\end{array}$ \\
\hline
\end{tabular}

The steppingFormat parameter controls how the steppingVerbose printing is performed. It is a sequence of names of track attributes (separated by commas or spaces):

$\begin{array}{ll}\text { TAG } & \text { Prints a single ">" (useful at the start of a line for grep-ing the output) } \\ \text { N } & \text { Step number } \\ \text { GLOBAL } & \text { The global coordinates }(\mathrm{X}, \mathrm{Y}, \mathrm{Z}, \mathrm{T}) \\ \mathrm{CL} & \text { The centerline coordinates }(\mathrm{X}, \mathrm{Y}, \mathrm{Z}, \mathrm{dxdz}, \mathrm{dydz}) \\ \text { CLX } & \text { The centerline coordinates, extended precision for tuning } \\ \text { KE } & \text { Kinetic Energy } \\ \text { STEP } & \text { The length of the step } \\ \text { VOL } & \text { The name of the current physical volume } \\ \text { PROCESS } & \text { The process name that terminated the step } \\ \text { B } & \text { The magnetic field (Bx,By,Bz) } \\ \text { E } & \text { The electric field (Ex,Ey,Ez) } \\ \text { P } & \text { The momentum (Px,Py,Pz) } \\ \text { MAT } & \text { The material } \\ \text { ID } & \text { Event\#, TrackID, parented }\end{array}$


SEG The centerline coordinate segment number

NEWLINE Prints a ' $\mathrm{n}$ ' (always added to end)

The default format is "N GLOBAL CL KE STEP VOL PROCESS".

See the printf command for another way to display track information, and the trace command for a third.

\subsubsection{Tracking Parameters}

Several parameters are used internally by G4Beamline to control the Geant4 tracking of particles:

\begin{tabular}{|l|l|l|}
\hline Name & Default & Description \\
\hline deltaChord & $3.0 \mathrm{~mm}$ & See the Geant4 User's Guide [1] for a description. \\
\hline deltaIntersection & $0.1 \mathrm{~mm}$ & See the Geant4 User's Guide [1] for a description. \\
\hline deltaOneStep & $0.01 \mathrm{~mm}$ & See the Geant4 User's Guide [1] for a description. \\
\hline epsMax & 0.05 & See the Geant4 User's Guide [1] for a description. \\
\hline epsMin & $2.5 \mathrm{e}-7$ & See the Geant4 User's Guide [1] for a description. \\
\hline maxStep & $100.0 \mathrm{~mm}$ & $\begin{array}{l}\text { The default maximum physics step size (can be overridden } \\
\text { in each element). }\end{array}$ \\
\hline minStep & $0.01 \mathrm{~mm}$ & The minimum physics step size. \\
\hline
\end{tabular}

In most cases, the default values for all the tracking parameters are appropriate. Note that Geant 4 tracking uses two different step sizes:

Physics step Defaults to $100 \mathrm{~mm}$, and determines the maximum step for applying physics processes. At each step it is set to the smallest value of:

$\circ$ the current value of maxStep

0 the distance to the next geometrical boundary

○ the smallest value determined by any active physics process

Integration step Determined automatically from the other tracking parameters (primarily deltaChord), and determines the accuracy of the integration of the equations of motion. In a magnetic field this is of course a helix. There can be many integration steps within a single physics step, and usually are in a magnetic field.

In particular, the accuracy of the tracking is usually not significantly improved for small physics step sizes. For instance, tracking $250 \mathrm{MeV} / \mathrm{c}$ muons through 1 meter of liquid hydrogen in a 2 Tesla solenoid (including multiple scattering and energy loss) shows no significant difference between maxStep $=1$ and maxStep $=1000$ in position or momentum histograms ${ }^{2}-$ the execution time differs by a factor of about 50 , so don't set maxStep to a small value unless you have a specific reason to do so.

\subsection{G4Beamline Commands by Type}

This section is just the output of the "help" command, with a little re-formatting.

\footnotetext{
${ }^{2}$ This comparison can only be done statistically, not on an individual track basis, because different values of maxStep inherently use different numbers of random numbers, causing variations in statistical processes like multiple scattering and ionization energy loss.
} 


\subsubsection{The help command}

$\begin{array}{ll}\text { help } & \text { provides interactive help. } \\ \text { man } & \text { Alias for 'help'. }\end{array}$

\subsubsection{Program control commands:}

bug1021 Workaround to improve accuracy of bug1021 in E field

define defines a macro (argument-expanded set of commands).

do Do loop.

endgroup ends a group definition.

exit exit a command file.

g4ui Accesses the Geant4 user interface

geometry Arranges to perform a geometry test.

group begins definition of a group.

if Conditional execution of command(s), and if/elseif/else/endif.

include includes a command file.

list provides interactive list of interesting internal tables.

muminuscapturefix Fixes up the G4MuonMinusCaptureAtRest process.

output redirects stdout and stderr to a file

param Defines parameter values.

randomseed control pseudo random number generator seeds

showmaterial set the colors for selected materials.

trackermode sets mode for all trackers, manages track fitting.

tune Tune a variable used as argument to other elements.

\subsubsection{Centerline layout commands:}

corner Implement a corner in the centerline.

cornerarc Implement a cornerarc in the centerline.

start Define the initial start of centerline coordinates.

\subsubsection{Beam definition commands:}

beam Define the Beam.

cosmicraybeam Define a Cosmic-Ray muon 'beam'.

particlesource Interface to the Geant4 General Particle Source.

reference Define a reference particle.

\subsubsection{Auxiliary definition commands:}

material construct a new material.

particlecolor set the colors for particle tracks.

physics Defines the physics processes and controls them.

trackcolor Alias for 'particlecolor'.

\subsubsection{Beamline element definition commands:}

absorber

box

coil

cylinder

extrusion construct an absorber

construct a box.

defines a coil (part of a solenoid)

Alias for 'tubs'.

construct a solid extrusion with axis along $z$ 
fieldexpr implements a field map, E and/or B, from expressions.

fieldmap implements a field map, $E$ and/or B, from a file.

genericbend construct a generic bending magnet.

genericquad construct a generic quadrupole magnet.

helicaldipole construct a helicaldipole magnet.

helicalharmonic construct a helicalharmonic magnet.

idealsectorbend construct an ideal sector bending magnet.

multipole construct a generic multipole magnet. decay.

particlefilter will kill particles from a list, or force particles to

pillbox Defines a pillbox RF cavity

polycone construct a polycone with axis along $z$

solenoid defines a solenoid (a coil and current)

sphere construct a sphere (or section of one)

torus construct a torus.

trap construct a solid trapezoid with axis along $z$.

tube Alias for 'tubs'.

tubs construct a tube or cylinder with axis along $z$.

\subsubsection{The place command:}

place

places an element into the current group (or world).

\subsubsection{Track and Event cuts:}

eventcuts Implements cuts on event number via lists in files.

trackcuts Specifies per-track cuts.

\subsubsection{Data output commands:}

beamlossntuple NTuple containing particle tracks when lost. fieldntuple Generates an NTuple from B and E fields at specified points. newparticlentuple NTuple containing particle tracks when created. ntuple printf Define an NTuple containing multiple detectors.

printfield prints track variables and expressions

probefield profile timentuple totalenergy

trace tracker Prints E or B fields, or writes FieldMap file. Prints $B$ and $E$ fields at specified points. write beam profile information to a file Construct an NTuple of tracks at a specified time. Print total energy deposited in selected volumes. trackerplane Construct a tracker plane. usertrackfilter Construct a usertrackfilter that filters tracks via user code.

virtualdetector Construct a VirtualDetector that generates an NTuple. zntuple Generate an NTuple for each of a list of $z$ positions.

Other commands: collective demo fieldlines movie

Monitor collective computation demo command. Display magnetic field lines. Generate movie NTuple. 
setdecay Set lifetime, decay channels, and branching ratios for a particle's decay.

spacecharge Beam-frame Green's function space charge computation

spacechargelw Lienard-Wiechert space charge computation

test test random number seeds.

\subsubsection{Program control Parameters:}

$\mathrm{ZCl}$

deltachord

deltaIntersection

deltaonestep

epsMax

epsMin

eventTimelimit

histoFile

histoupdate

maxstep

minstep

steppingFormat

steppingVerbose

viewer

worldMaterial

zTolerance

steppingFormat is

EXT

TAG

$\mathrm{N}$

NSTEP

GLOBAL

XYZT

CL

CLX

$\mathrm{KE}$

STEP

STEPLEN

VOL

VOLNAME

PROCESS

B

E

$P$

MAT

ID

PART

SEG

WT

NL

NEWLINE

$\backslash \mathrm{n}$
Last centerline $\mathrm{Z}$ position used (updated continuously)

Geant4 tracking parameter

Geant4 tracking parameter

Geant4 tracking parameter

Geant4 tracking parameter

Geant4 tracking parameter

CPU Time Limit (sec)

Default (Root) NTuple output filename

Output update interval (events)

Maximum physics step size (mm)

Minimum step size (mm)

Format for printing steps

Set nonzero to print each step

Visualization driver selected (default=none)

Material of the World volume

Tolerance for $Z$ steps (mm)

a space- or comma-separated list of items:

toggle extended precision ( 3 more digits)

print a '>' (useful to grep output)

step number

Synonym of $\mathrm{N}$

$\mathrm{X}, \mathrm{Y}, \mathrm{Z}, \mathrm{T}$ in global coords

Synonym of GLOBAL

$\mathrm{X}, \mathrm{Y}, \mathrm{Z}, \mathrm{dxdz}, \mathrm{dydz}$ in $\mathrm{CL}$ coords

extended precision CL

kinetic energy

step length

Synonym of STEP

volume name

Synonym of VOL

process name

magnetic field

electric field

3-momentum

material name

event ID, track ID, parent ID

particle name

centerline coord segment number

weight

<newline>

Synonym of NL

Synonym of NL 


\subsection{Pillbox Geometry and Dimensions}

The geometrical dimensions of the pillbox are given in the figure below showing a radial cross-section. The pipe, walls, and collars are always made of copper; the stepped windows (win1 and win2) are made of winMat, and the interior of the cavity is cavityMaterial. The entire pillbox is cylindrically symmetric around the beam axis. The win1-s, the win2-s, the collars, the walls, and the pipe can each be omitted by setting the corresponding thickness to zero.

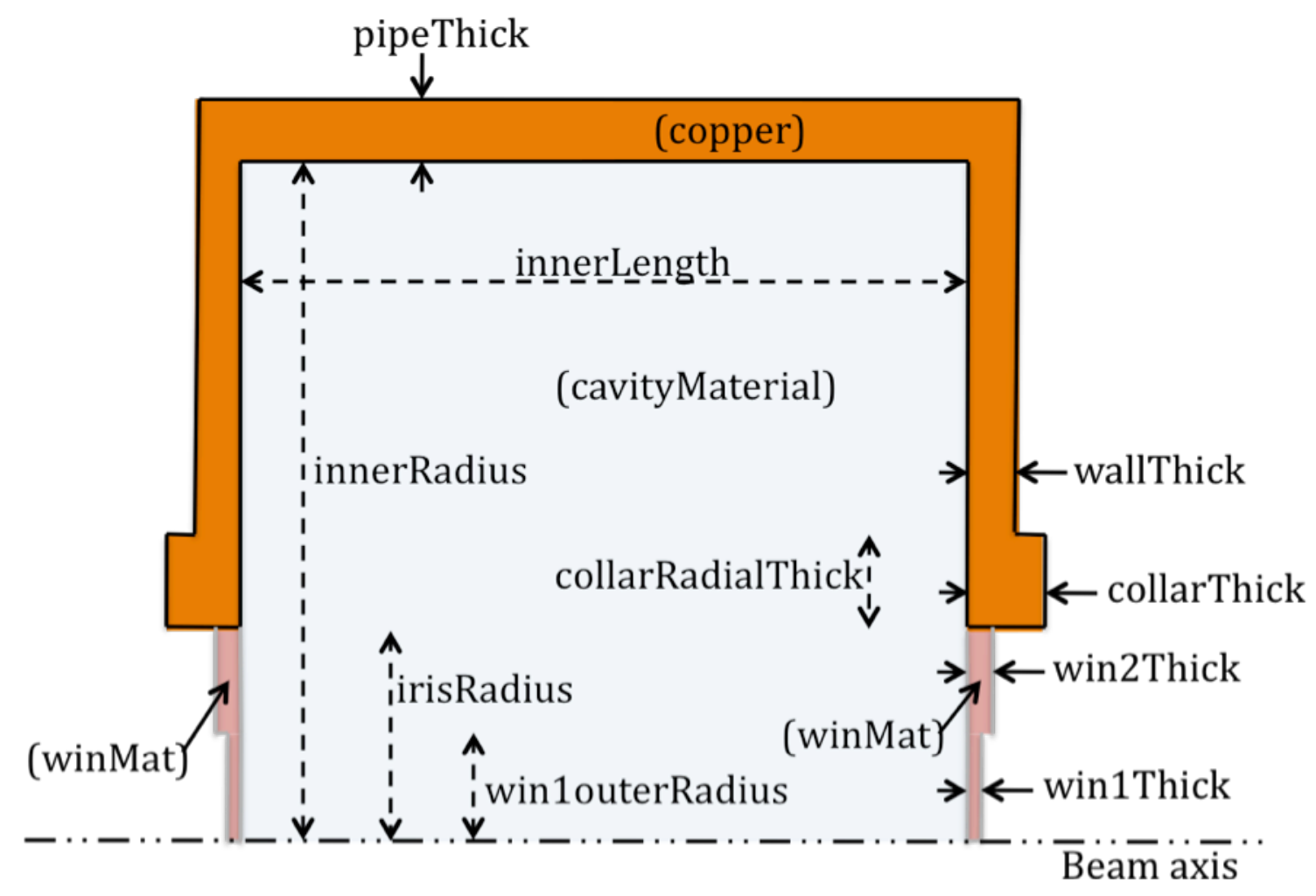




\subsection{Absorber geometry and Dimensions}

The absorber is cylindrically symmetric around the beam axis. The beampipe's radii come from the flange specifications in the files that define the profiles of the absWindow and the safetyWindow; the beampipe's length is extended to the same length as the safetyWindow-s on the beam axis. The safetyDistance is between the inner faces of the two windows on the beam axis. If safetyWindow is omitted, all references to it are changed to absWindow, and there are no volumes of safetyMaterial.

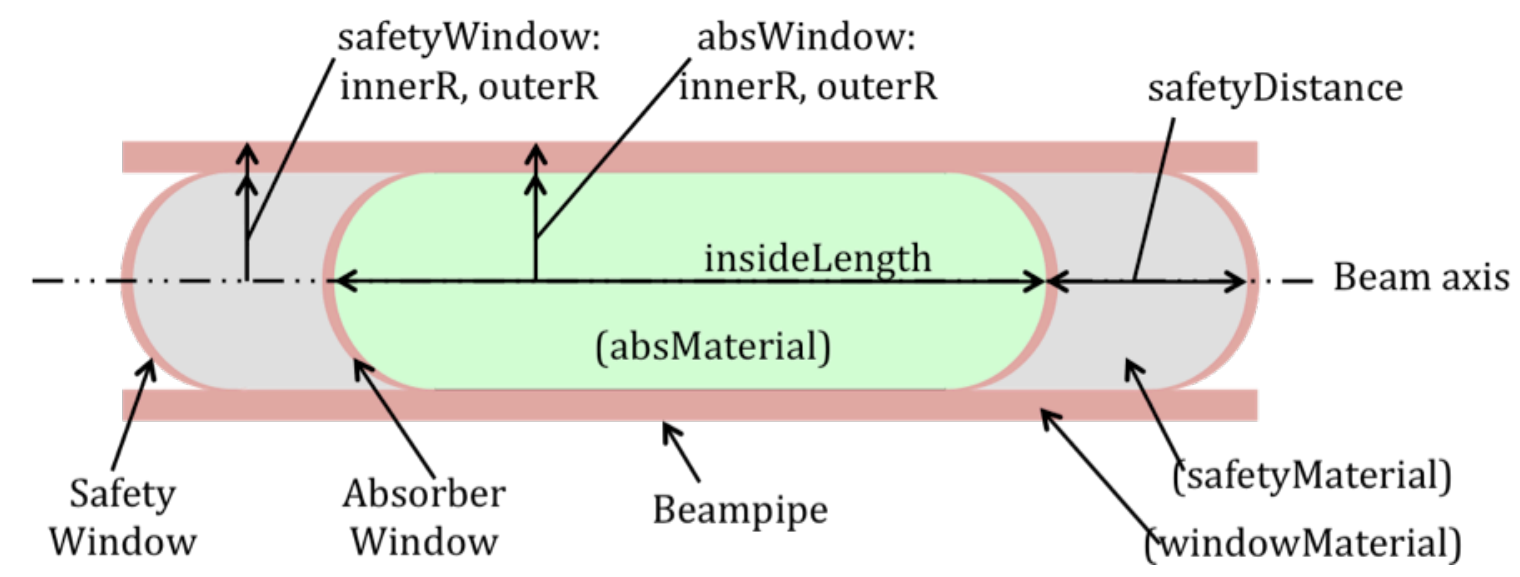




\section{G4Beamline Commands (Alphabetical)}

This section is just the output of the "help *" command, with a little re-formatting.

\section{1 absorber construct an absorber}

The absorber has two windows with beampipe and an absorber material. Optionally it has an additional two safety windows with beampipe. The WindowShape(s) are read from a file, and they determine the thickness and length of the beampipe(s). For geometry testing, acts like a cylinder enclosing the windows. For placing children, acts like a cylinder inside the central absorber.

Note that section 4.5 of the User's Guide has a dimensioned drawing of an absorber.

Named Arguments (\#=cannot be changed in place cmd): absWindow The name of the absorber window. \# safetyWindow The name of the safety window. \# insidelength Absorber length inside windows (mm) \# absMaterial The material of the absorber \# windowMaterial The material of the window(s) \# safetyMaterial The material inside the safety windows. \# safetyDistance Distance between absorber and safety windows(mm) \# color The color of the absorber (''=invisible) maxstep The maximum stepsize in the element (mm)

\section{2 beam Define the Beam.}

The beam command is: beam type $\arg 1=\mathrm{v} 1 \ldots$

Types are: gaussian, rectangular, ellipse, ascii, and root.

Gaussian beams are randomly generated to emanate from beamX, beamY, beamz with the given sigmas; negative sigma means flat with |sigma| as halfwidth.

Rectangular beams are randomly generated to emanate from the rectangle beamHeight by beamWidth centered at beamX, beamY, beamz .

Ellipse beams are randomly generated on the ellipses in $(X, X p)$, $(Y, Y p),(T, E)$, with meanE determined from meanP and the sigmas used as half-widths; tracks are generated on the ellipse with uniform density when plotted with scales such that the ellipse is a circle.

ASCII beams are read from a file using the format specified; the formats supported are: BLTrackFile 
Root beams are read from a .root file using the TNtuple named directory/name in the file. It must have the same fields as used in BLTrackFile format. Note that EventIDs greater than $16,777,216$ will be trncated.

When reading a file (ascii or root), beamX and beamY are added to input tracks; if beamz is set it will overwrite the $z$ of the track, but if it is not set the $z$ of the track in the file is kept.

\section{All coordinates are centerline coordinates.}

Multiple beam commands can be given, and they will generate events in the order they appear in input.file.

Events are generated starting at firstEvent, until either nEvents have been generated or lastEvent would be exceeded.

For gaussian, rectangular, and ellipse beams, the beam particle can be given as either a particle name or its integer PDGid. Some common beam particle names are: proton, anti_proton, pit, pi-, mu+, mu-, e+, e-, kaon+, kaon-, kaon0, nu_e, anti_nu_e. See the User's Guide for a complete list of particle names.

Named Arguments:

\begin{tabular}{|c|c|}
\hline cle & Beam particle name \\
\hline nEvents & $\begin{array}{l}\text { Number of events to process (default=1 for } \\
\text { generating events, default=ALL for reading files) } \\
\text { set to lastEvent-firstEvent+ } 1 \text { if both are set. }\end{array}$ \\
\hline firstEvent & $\begin{array}{l}\text { First event \# to process (default is the next } \\
\text { sequential eventID, } 1 \text { if none) }\end{array}$ \\
\hline lastEvent & Last (highest) event \# to process \\
\hline beamx & Beam location in $\mathrm{x}(\mathrm{mm})$ \\
\hline beamY & Beam location in $\mathrm{Y}(\mathrm{mm})$ \\
\hline beamz & Beam location in $\mathrm{Z}(\mathrm{mm})$ \\
\hline $\max R$ & Beam maximum radius ( $\mathrm{mm}$ ) \\
\hline rotation & Rotation of the beam \\
\hline $\begin{array}{l}\text { renumber } \\
\text { weight }\end{array}$ & $\begin{array}{l}\text { If nonzero, renumber events sequentially. } \\
\text { Weight for events, overwritten by value from input } \\
\text { file (1.0). }\end{array}$ \\
\hline seconda & KID The next TrackID for secondaries (1001). \\
\hline meanMom & Gaussian Beam mean momentum ( $\mathrm{MeV} / \mathrm{C})$ \\
\hline meanP & Synonym for meanMomentum. \\
\hline sigmax & Gaussian Beam sigma in $\mathrm{X}(\mathrm{mm})$ \\
\hline sigmaY & Gaussian Beam sigma in $\mathrm{Y}(\mathrm{mm})$ \\
\hline sigmaz & Gaussian Beam sigma in $\mathrm{z}(\mathrm{mm})$ \\
\hline sigmaxp & Gaussian Beam sigma in $\mathrm{dxdz}$ (slope) \\
\hline sigmaYp & Gaussian Beam sigma in dydz (slope) \\
\hline sigmaP & Gaussian Beam sigma in $\mathrm{P}(\mathrm{MeV} / \mathrm{C})$ \\
\hline sigmat & Gaussian Beam sigma in $T$ ( $\mathrm{ns}$ ) \\
\hline sigmaE & Elliptical Beam sigma in $\mathrm{E}$ (MeV) \\
\hline meanXp & Gaussian Beam mean in Xp (slope) \\
\hline meanYp & Gaussian Beam mean in Yp (slope) \\
\hline $\operatorname{meanT}$ & Gaussian Beam mean in $\mathrm{T}$ (ns) \\
\hline beamHeight & Rectangular Beam height (mm) \\
\hline
\end{tabular}




$\begin{array}{ll}\text { beamWidth } & \text { Rectangular Beam width (mm) } \\ \text { filename } & \text { input file name } \\ \text { file } & \text { synonym for filename. } \\ \text { directory } & \text { Root-file directory of NTuple } \\ \text { category } & \text { Deprecated synonym for directory. } \\ \text { uid } & \text { HistoScope uid of NTuple } \\ \text { name } & \text { Root name of NTuple. } \\ \text { format } & \text { ASCII file format (Default=BLTrackFile) } \\ \text { beamXp } & \text { Synonym for meanXp. } \\ \text { beamYp } & \text { Synonym for meanYp. }\end{array}$

\section{3 beamlossntuple NTuple containing particle tracks when lost.}

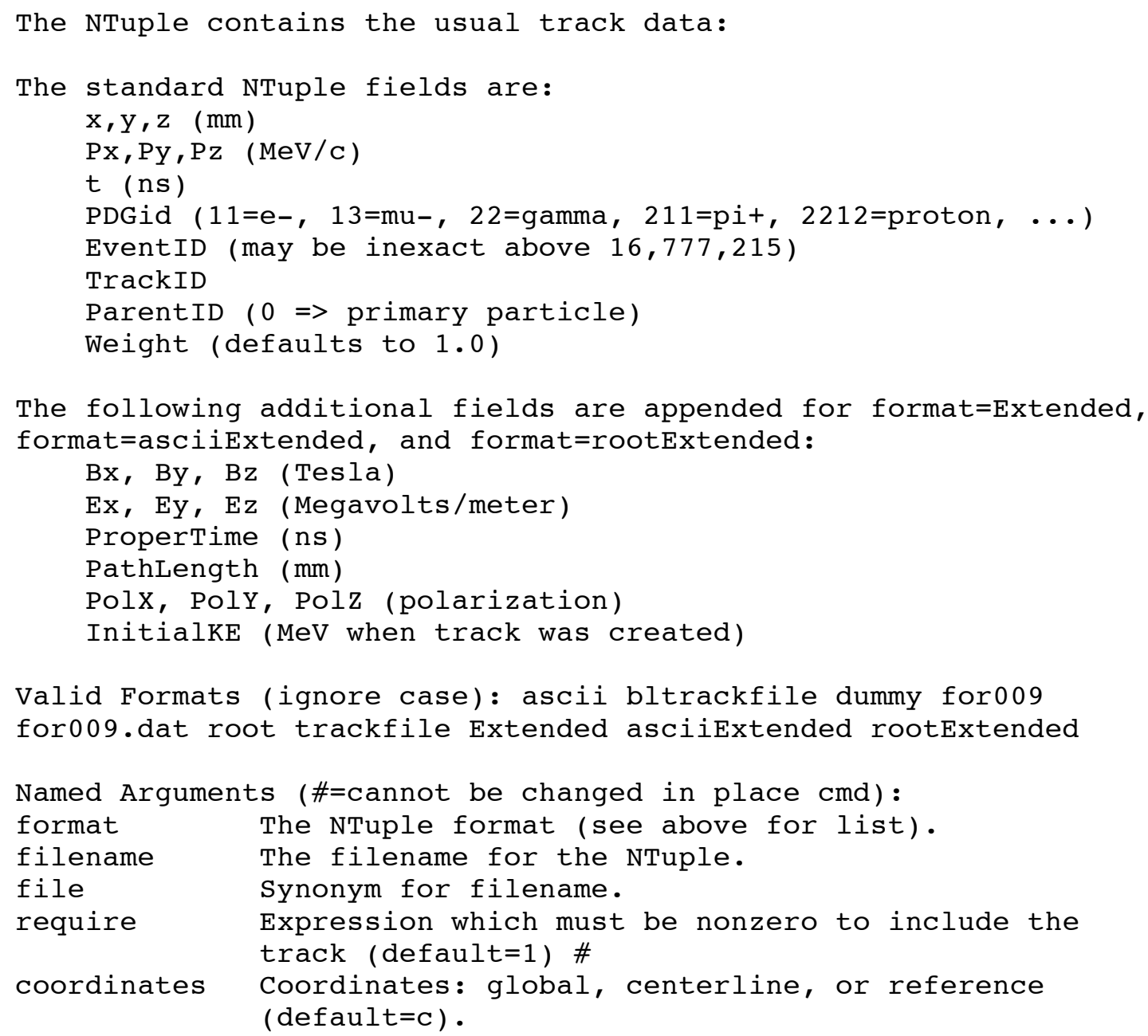

\section{4 box construct a box.}

This is a direct interface to G4Box. 
Named Arguments:

height The height of the box (mm)

width The width of the box ( $\mathrm{mm}$ )

length The length of the box (mm)

maxstep The maximum stepsize in the element (mm)

material The material of the box

color The color of the box (''=invisible)

kill Set nonzero to kill every track that enters.

\section{5 bug1021 Workaround to improve accuracy of bug1021 in E field}

When a charged particle turns around in an E field, a bug in the Geant4 transportation process can sometimes give it a wildly-incorrect kinetic energy. This workaround computes the distance to turn-around, and limits the step to half that vlue until minstep is reached; at that point the track is reflected.

Simulations in which there are no E fields, or no charged particle ever gets below $\sim 0.001 \mathrm{MeV}$ in an $\mathrm{E}$ field, have no need to apply this workaround.

Named Arguments:

minstep Minimum step in space $(\mathrm{mm}$, default=0.002)

\section{6 coil defines a coil (part of a solenoid)}

A coil is a geometrical tube that can carry current when part of a solenoid. The field is computed for a set of nsheets infinitely-thin current sheets evenly spread radially. The solenoid specifies the actual current. For tracking the computation is too slow, so a field map on a grid in $r$ and $z$ is computed and written to filename (defaults to coilname.dat). While there are lots of parameters specifying the field map it is recommended to simply accept the defaults for all but innerRadius, outerRadius, length, material, and possibly tolerance. The other parameters will be determined so that the largest error is less than tolerance times the value of $\mathrm{Bz}$ at the center of the coil. If mapFile is given, the file is read in BLFieldMap format. The cache file contains the parameters, and is a field map in a binary format; it is automatically regenerated if any parameter changes.

Note that maxR and maxz are by default determined to be large enough so that the field falls below tolerance; this can be quote large.

Named Arguments (\#=cannot be changed in place cmd): innerRadius Inside radius of the coil $(\mathrm{mm})$ \# outerRadius Outside radius of the coil (mm) \# length Length of the coil along $\mathrm{z}(\mathrm{mm})$ \# 


\begin{tabular}{|c|c|}
\hline $\begin{array}{l}\text { material } \\
\text { tolerance }\end{array}$ & $\begin{array}{l}\text { The material of the conductor (default=Cu) \# } \\
\text { The acceptable tolerance of the map. \# }\end{array}$ \\
\hline nSheets & Number of sheets used in the computation \# \\
\hline $\operatorname{maxR}$ & $\begin{array}{l}\text { Maxmum } r \text { value for the map (automatically } \\
\text { determined by default). \# }\end{array}$ \\
\hline $\max Z$ & $\begin{array}{l}\text { Maxmum z value for the map (automatically } \\
\text { determined by default). \# }\end{array}$ \\
\hline $\mathrm{dR}$ & $\begin{array}{l}\text { R interval between points of the map (automatically } \\
\text { determined by default). \# }\end{array}$ \\
\hline $\mathrm{dz}$ & $\begin{array}{l}\mathrm{z} \text { interval between points of the map (automatically } \\
\text { determined by default). \# }\end{array}$ \\
\hline filename & Filename for cache; deaults to name.dat. \# \\
\hline mapFile & Filename for map (e.g. from TOSCA). \# \\
\hline
\end{tabular}

\section{7 collective Monitor collective computation}

This command computes the means and sigmas related to the time stepping in BLRunManager (global coordinates), generating a Timestep NTuple. If the simulation has multiple bunches, this NTuple combines them all (and is thus almost useless).

This command can also generate field NTuple-s at specified points in $\mathrm{x}, \mathrm{y}, \mathrm{z}$-- unnamed parameters should be ' $\mathrm{x}, \mathrm{y}, \mathrm{z}$ ' values for monitoring $\mathrm{E}$ and $\mathrm{B}$ fields (global coordinates).

NOTE: This command must come AFTER other commands that compute collective fields; otherwise stale field values will be used from the previous time step. If deltat is set $>0.0$, this command will put the RunManager into collective mode and set its deltaT; otherwise the previous commands should do that, and this command won't modify deltat.

Named Arguments:

deltat Time step (-1 ns).

format Format of NTuples.

filenamePrefix Prefix of NTuple filenames.

\section{8 corner Implement a corner in the centerline.}

The centerline is bent by a rotation. Every track that enters the volume also gets rotated. The $\mathrm{z}$ value is for the corner and the front face of the volume (if any). If the corner is paired with a bending magnet or other mechanism to bend the beam, no volume should be used.

NOTE: This command is self-placing, do not use the place command; it also affects all following placements, and it cannot be issued inside a group. If radius=height=width=0 then no volume is 
associated with the corner, and a bending magnet should be placed nearby to bend the particles around the corner. Normally the bending magnet is placed before the corner, and is rotated by half the bend angle. For a sector bend, it's usually best to use the cornerarc command rather than this one.

NOTE: all placements before this command must have $z$ values before the corner, and all placements after this command must have $\mathrm{z}$ values after the corner. Note also that the angle is limited to 90 degrees.

Note that the radiuscut is important to reduce or eliminate ambiguities in the global to centerline coordinate transform. It can also be used to 'shield' the beamline to prevent particles from taking unusual paths around the outside of beamline elements.

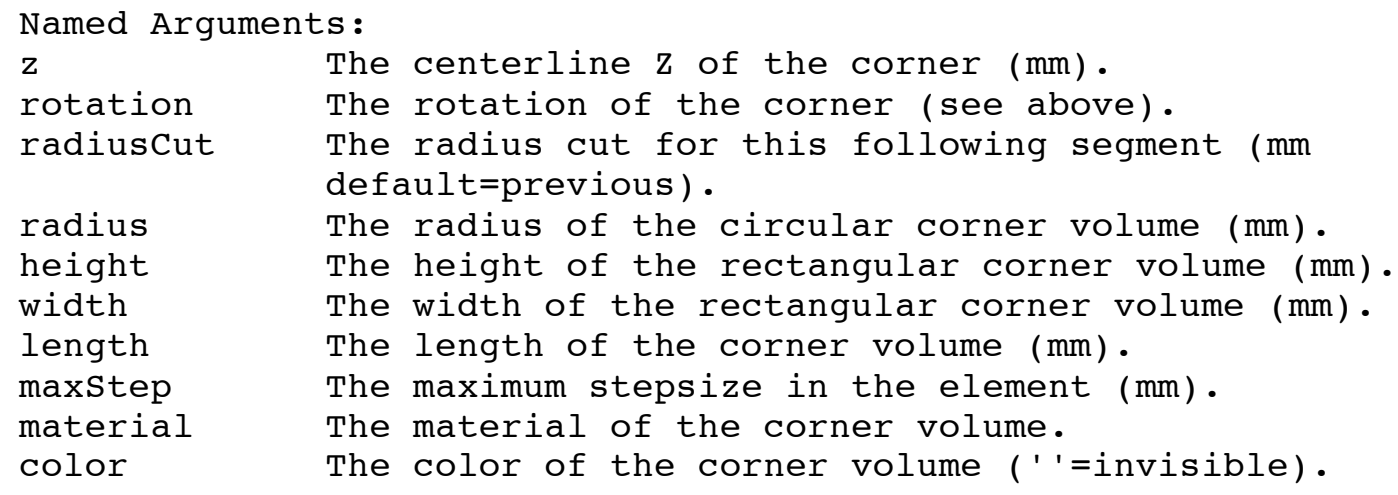

\section{9 cornerarc Implement a cornerarc in the centerline.}

The centerline is bent by a rotation. Three corners are used to approximate an arc; the total angle and path-length are equal to those for the arc. Should be used immediately after an idealsectorbend or a genericbend. The $\mathrm{z}$ value is for the front face of the arc.

NOTE: This command is self-placing, do not use the place command; it also affects all following placements, and it cannot be issued inside a group.

This command is well matched to a sector bend, but can also be used with a normal bending magnet -- normally the magnet is placed before the cornerarc and is rotated by half the bend angle.

The only useful rotations are those around the centerline $\mathrm{z}$.

NOTE: all placements before this command must have $z$ values before the corner, and all placements after this command must have $\mathrm{z}$ values after the corner. Note also that the angle is limited to 90 degrees. 
Note that the radiuscut is important to reduce or eliminate ambiguities in the global to centerline coordinate transform. It can also be used to 'shield' the beamline to prevent particles from taking unusual paths around the outside of beamline elements.

Named Arguments:

z The centerline $\mathrm{z}$ of the cornerarc (mm).

centerRadius The radius of the centerline arc ( $\mathrm{mm})$.

angle The angle of bend, $>0=$ left, <0=right (degrees).

rotation The rotation of the cornerarc (see above).

radiuscut The radius cut for this following segment (mm).

\title{
5.10 cosmicraybeam Define a Cosmic-Ray muon 'beam'.
}

\author{
The muon beam is nominally headed in the $+z$ direction, implying \\ that $+z$ is physically DOWN. The beam intersects a box defined by \\ beamWidth, beamHeight, and beamLength, centered at $\mathrm{X}=\mathrm{Y}=0$ and \\ beamz. For each event a point is selected randomly within this \\ box, angles theta and phi and the muon momentum are generated \\ according to a fit to their sea-level distributions, the track is \\ extended backwards to the 'celestial sphere', and that is the \\ initial beam position for the event. The muon flux through the \\ rectangle at $Z=$ beam $Z$ is used to display an estimate of the \\ sea-level exposure time for the run. \\ Named Arguments : \\ nEvents Number of events to process \\ beamz Beam location in $\mathrm{Z}(\mathrm{mm})$ \\ radius Radius of celestial sphere (mm) \\ beamHeight Rectangular Beam height (mm) \\ beamWidth Rectangular Beam width ( $\mathrm{mm})$ \\ beamLength Rectangular Beam length (mm)
}

\subsection{1 cylinder Alias for 'tubs'.}

\subsection{2 define defines a macro (argument-expanded set of commands).}

The first argument is the macro name, additional arguments become lines in the body of the expanded macro. The macro name becomes a command with up to 9 positional arguments. When the command is issued, the body is expanded and executed, with these substitutions:

$\$ 0 \quad$ Macroname

\$1-\$9 Positional arguments of the command 
\$\# \# macro expansions (for generating unique names)

NOTE: \$paramname is expanded in the define command, but

$\$ \$$ paramname is expanded when the macro is invoked.

\subsection{3 demo demo command.}

This demo command takes both positional and named args, and is the prototype class for all commands. All argument values are merely displayed. 'demo default name=value...' sets default values.

Named Arguments:

s1 a demo string argument.

s2 a demo string argument.

d1 a demo double argument.

d2 a demo double argument.

\subsection{4 do Do loop.}

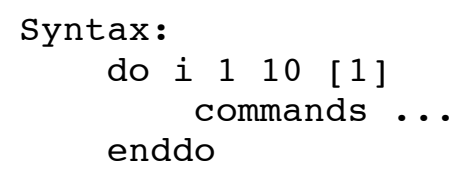

Sets the parameter $i$ to values $1,2,3, \ldots 10$, and executes the commands. The increment is 1 by default, and negative increments are allowed (with limits reversed). 'do $i$ 1 $0^{\prime}$ and 'do $i \quad 0 \quad 1-1$ ' will execute no commands. After completion, i retains its last value. Do-s and multi-line if-s can be nested in any manner.

Note: the do command will not work from standard-input or as a command in a single-line if.

\subsection{5endgroup ends a group definition.}

The group may then be placed as any other element. If the group was not given a length via an argument, endgroup computes the length and adjusts the offsets of all elements in the group so they refer to the center of the group.

\subsection{6 eventcuts Implements cuts on event number via lists in files.}

The files are ASCII, with one event number per line. If the keep file is not empty, only event numbers listed in it will be analyzed (except those listed in the skip file). The skip file 
lists event numbers that will be skipped, and event numbers listed in both files will be skipped. When reading the files, lines beginning with '\#' are ignored; blank lines are interpreted as event 0 .

Named Arguments :

keep The file containing a list of event numbers to analyze (default is all).

skip The file containing a list of event numbers to skip.

filename Synonym for keep.

file Synonym for keep.

\subsection{7 exit exit a command file.}

The exit command ceases reading the input file, and starts the simulation immediately (ignoring the remainder of the input file).

\subsection{8 extrusion construct a solid extrusion with axis along $z$}

This is a basic interface to G4ExtrudedSolid. A simple polygon in the $\mathrm{X}-\mathrm{Y}$ plane is extruded along $\mathrm{z}$, with optional scales in $\mathrm{XY}$ at the two ends (which generate a linear scaling along $\mathrm{z}$ ).

The polygon must be simple (no two sides intersect, no two vertices are equal). The vertices are listed starting from any vertex and traversing the polygon in either direction without lifting the pencil from the paper (Geant4 requires the traversal to be clockwise but this element internally reverses it if required). For an N-sided polygon give $\mathrm{N}$ vertices -- a side will be added from last to first to close the polygon; $\mathrm{N}$ is determined by counting the entries in the vertices argument.

Note that while you cannot make an extrusion with a hole, you can make such an object in two parts or by placing a daughter volume in this one.

Note the position placed is $x=0, y=0, z=0$, which is centered along $z$, but need not be near the center of the polygon in $X Y$.

With scalel!=scale2 this is not really an extrusion; by making one of them 0.001 or so, you can construct a sharp apex.

Any $\mathrm{x}$ or $\mathrm{y}$ value in vertices can be an expression using double constants and the usual $\mathrm{C}$ operators and functions.

Named Arguments (\#=cannot be changed in place cmd): length Length of the extrusion (mm). \#

vertices List of vertices of the XY polygon (mm): 
scale1

scale2

maxstep

material

color

$\mathrm{kill}$

vertexes
' $\mathrm{x} 0, \mathrm{y} 0 ; \mathrm{x} 1, \mathrm{y} 1 ; \ldots$ '; a line from last to first is added. A $2 \mathrm{~mm}$ square is: ' $-1,-1 ;-1,1 ; 1,1 ; 1,-1$ ' \# The XY scale at the upstream $(-z)$ end $(1.0)$. \# The XY scale at the downstream $(+z)$ end $(1.0)$. \# The maximum stepsize in the element ( $\mathrm{mm}$ )

The material of the extrusion The color of the extrusion (''=invisible) Set nonzero to kill every track that enters. synonym for vertices. \#

\subsection{9 fieldexpr implements a field map, $E$ and/or B, from expressions.}

A fieldexpr element can be either a box or a cylinder; set length and radius for cylinder, set length and width and height for a box. Units are Tesla, MegaVolts/meter, mm, and ns. Expressions for the field components can use $\{x, y, z\}$ for a box or $\{z, r\}$ for a cylinder; the time expression can use $\{t\}$. If present, the time expression multiples all components. The number of points in the map is increased until the largest map error divided by the maximum field is smaller than tolerance, or $1 \mathrm{M}$ points is exceeded. Similarly for the time dependence.

For time dependence: if t-timeoffset<tmin, the value at tmin is used; if t-timeoffset>tmax, the value at tmax is used.

\begin{tabular}{|c|c|}
\hline factorB & Factor for the B-field $(1.0)$. a \\
\hline factorE & Factor for the E-field $(1.0)$. a \\
\hline timeoffset & Time offset (ns). \\
\hline $\mathrm{Bx}$ & Expression for $B x$ (Tesla), use $\{x, y, z\} . \#$ \\
\hline By & Expression for By (Tesla), use $\{x, y, z\} . \#$ \\
\hline $\mathrm{Bz}$ & Expression for $B z$ (Tesla), use $\{x, y, z\}$ or $\{r, z\} . \#$ \\
\hline $\mathrm{Br}$ & Expression for $\operatorname{Br}$ (Tesla), use $\{r, z\} . \#$ \\
\hline Ex & Expression for $\operatorname{Ex}(M V / m)$, use $\{x, y, z\} . \#$ \\
\hline Ey & Expression for Ey $(M V / m)$, use $\{x, y, z\}$. \# \\
\hline $\mathrm{Ez}$ & Expression for $\operatorname{Ez}(M V / m)$, use $\{x, y, z\}$ or $\{r, z\}$. \# \\
\hline $\operatorname{Er}$ & Expression for $\operatorname{Er}(M V / m)$, use $\{r, z\} . \#$ \\
\hline time & Expression for time-dependence factor, use $\{t\}$. \# \\
\hline $\mathrm{nX}$ & Number of grid points in $\mathrm{x}$. \# \\
\hline$n Y$ & Number of grid points in $\mathrm{y} \cdot \#$ \\
\hline $\mathrm{nZ}$ & Number of grid points in $\mathrm{z}$. \# \\
\hline $\mathrm{nR}$ & Number of grid points in r. \# \\
\hline $\mathrm{nT}$ & Number of grid points in $t$. \# \\
\hline tolerance & Required relative accuracy $(0.001)$. \# \\
\hline length & Length of field map $(\mathrm{mm})$. \# \\
\hline width & Width of rectangular field map (mm). \# \\
\hline height & Height of rectangular field map $(\mathrm{mm})$. \# \\
\hline radius & Radius of cylindrical field map $(\mathrm{mm})$. \# \\
\hline tmin & Minimum value of $t$ (ns). \# \\
\hline tmax & Maximum value of $t(n s)$. \# \\
\hline
\end{tabular}




\subsection{0 fieldlines Display magnetic field lines.}

Field lines are drawn starting within a circle specified as center and radius (global coordinates); the plane of the circle is normal to the $B$ field at its center. Field lines are distributed within the circle with a density inversely proportional to $|\mathrm{B}|$. While it is attempted to keep their spacing as uniform as possible, there are both ambiguity and randomness involved in placing the lines within the circle. Lines are placed within the circle from its center outward, and if $|B|<$ minfield then no more lines are placed.

nLines is only approximate, and the actual number of lines drawn will be within a factor of 2 of the value. Asking for fewer than 10 or more than 1000 lines is likely to be ineffective. Unnamed parameters can contain specific $x, y, z$ values of points to start a field line.

Lines are drawn in both directions starting from the plane of the circle, and each half-line stops when it again reaches that plane. Line drawing also stops whenever $|B|$ is less than minfield or when it leaves the world. If you are interested in field lines far outside the magnets, you may need to add some object with a large value of $\mathrm{x}, \mathrm{y}$, and/or $\mathrm{z}$, in order to expand the world volume. With $d l=1$ and subdivide=10, the accuracy of their meeting is usually better than $0.1 \mathrm{~mm}$ after several tens of meters.

This command does nothing if not in visualization mode. For best results, use the Open Inventor viewer, and give your magnets a transparency of about 0.3 (e.g. color $=1,1,1,0.3$ ); if necessary, use the right-button menu to set the DrawStyle/TransparencyType to 'sorted object add'. With field lines in 3-d, you will want the ability to zoom, rotate, and move the image interactively.

Setting exit=1 will display the system and the field lines, without any event tracks; exiting the viewer will then exit the program. With exit=0, field lines are drawn only at the end of the first run.

Named Arguments:

\begin{tabular}{|c|c|}
\hline venter & $\begin{array}{l}\text { Time at which field lines are plotted }(0 \mathrm{~ns}) \text {. } \\
\text { Center of circle }(\mathrm{x}, \mathrm{y}, \mathrm{z}) \text { to start lines }(\mathrm{mm} \text {, } \\
\text { global). }\end{array}$ \\
\hline dius & Radius of circle to start lines (mm). \\
\hline in & Approximate number of field lines to plot (100). \\
\hline & Interval between points plotted (10 mm) \\
\hline lor & Color of field lines (white $=" 1,1,1 "$ ). \\
\hline nField & Minimum B field $(0.001$ tesla) \\
\hline xPoints & Max \# points plotted in a line $(10000)$. \\
\hline hdivide & $\begin{array}{l}\text { \# field integration points between plotted points } \\
(10) \text {. }\end{array}$ \\
\hline & oints in $\mathrm{x}$ and $\mathrm{y}(128)$. \\
\hline & Set nonzero to display field lines and exit (0). \\
\hline & $\begin{array}{l}\text { Set nonzero to start from square rather than circle } \\
(0) \text {. }\end{array}$ \\
\hline
\end{tabular}


Efield

forever
Set nonzero to draw E field (0); minfield is in MegaVolts/meter.

Set nonzero to draw lines until maxpoints is reached or $|B|<$ minfield, not stopping at the initial plane.

\title{
5.21 fieldmap implements a field map, $E$ and/or $B$, from a file.
}

\author{
Reads an input file in BLFieldMap format to define $\mathrm{E}$ and/or $\mathrm{B}$ \\ fields, optionally with time dependence. See the Users Guide for \\ a description of the BLFieldMap format. \\ Named Arguments (\#=cannot be changed in place cmd) ( $@=$ Tunable): \\ filename Filename for the Field Map. \# \\ file Synonym for filename. \# \\ current Current of the B-field. a \\ gradient Gradient of the E-field. A \\ timeoffset Time offset (ns).
}

\subsection{2 fieldntuple Generates an NTuple from B and E fields at specified points.}

Intended primarily for debugging. This command makes it easy to plot fields as a function of position and time, using existing NTuple plotting tools. Outputs $\mathrm{x}, \mathrm{y}, \mathrm{z}, \mathrm{t}, \mathrm{Bx}, \mathrm{By}, \mathrm{Bz}, \mathrm{Ex}, \mathrm{Ey}, \mathrm{Ez}$ into an NTuple. Units are mm, ns, Tesla, and MegaVolts/meter. Runs after the reference particle is tracked. Only global coordinates are used.

The single positional argument is the name of the NTuple. Named arguments $\{x, y, z, t\}$ are of two forms specifying coordinate values: $\mathrm{x}=\mathrm{xmin}, \mathrm{xmax}, \mathrm{dx}$ or $\mathrm{x}=\mathrm{x} 1: \mathrm{x} 2: \mathrm{x} 3: \ldots$ which generate the obvious loops (single value is OK). Expressions can be used. Omitted coordinates are held fixed at 0.0 .

Named Arguments:

\begin{tabular}{|c|c|}
\hline $\begin{array}{l}\text { category } \\
\text { format } \\
\text { filename }\end{array}$ & $\begin{array}{l}\text { The category of the NTuple. } \\
\text { NTuple format (see above for list). } \\
\text { Name of file. }\end{array}$ \\
\hline $\mathrm{x}$ & Loop for $\mathrm{x}$ values: Xmin, $\mathrm{xmaz}, \mathrm{dX}$ or $\mathrm{x} 1: \mathrm{X} 2: \ldots(\mathrm{mm})$ \\
\hline y & Loop for $y$ values: Ymin, Ymaz, $\mathrm{dY}$ or $\mathrm{Y} 1: \mathrm{Y} 2: \ldots$ (mm) \\
\hline $\mathrm{z}$ & Loop for $z$ values: $\mathrm{Zmin}, \mathrm{Zmaz}, \mathrm{dz}$ or $\mathrm{z} 1: \mathrm{z} 2: \ldots$ (mm) \\
\hline$t$ & Loop for $t$ values: Tmin, Tmaz, $\mathrm{dT}$ or $\mathrm{T} 1: \mathrm{T} 2:$. \\
\hline exit & after generating NTuple (0). \\
\hline file & 10 \\
\hline
\end{tabular}




\subsection{3g4ui Accesses the Geant4 user interface}

Each positional argument is executed as a Geant4 UI command, according to the when parameter. No positional arguments means open a UI session on stdout/stdin. For a given value of when, the UI commands from all g4ui commands are executed in order.

Named Arguments:

when $\quad 0$ =before reference, 1 =before beam, 2=after beam, $3=$ cannot be used, $4=v i s u a l i z a t i o n$.

\subsection{4 genericbend construct a generic bending magnet.}

The field region is a box with By specified. A fringe field computation based on the method of COSY INFINITY is included by default, extending the field in a rectangle extending the straight aperture along the local $z$. This is first order only, and assumes the magnet is infinitely wide; the fringe field extends outside of the magnet aperture only in a region extending the aperture in $x$ and $y$. As the fringe field is first order only, it is slightly non-Maxwellian.

By default, the aperture is filled with a box volume of the fieldMaterial; this prevents placing any object inside the aperture. With openAperture=1 no aperture volume is used, and objects can be placed into the parent volume that are inside the aperture.

Named Arguments (\#=cannot be changed in place cmd) ( $@=$ Tunable): fieldWidth The width of the field region (mm)

fieldHeight fieldLength ironWidth The height of the field region ( $\mathrm{mm}$ ) The length of the field region ( $\mathrm{mm}$ ) ironHeight ironLength

By The width of the iron region ( $\mathrm{mm}$ ) The height of the iron region $(\mathrm{mm})$ The length of the iron region ( $\mathrm{mm}$ ) The magnetic field (Tesla) a

maxstep The maximum stepsize in the element (mm)

fieldMaterial the material of the field region.

fieldColor The color of the field region.

ironMaterial The material of the iron region.

ironcolor The color of the iron region.

kill

fringe

Set nonzero to kill particles hitting the iron. Fringe field computation, set to 0 to disable, or a fringefactor comma-separated list of 6 Enge function parameters. openAperture set nonzero to omit the aperture volume. \#

\subsection{5 genericquad construct a generic quadrupole magnet.}


The field region is a tubs with gradient specified. A positive gradient yields a horizontally-focusing quad for positive particles. If apertureRadius $>0$ the quad has a circular aperture. For a 'rounded +' aperture using circles for the poles, set poleTipRadius, coilRadius, coilHalfwidth. Due to visualization bugs, in the latter case you cannot see through the aperture; it is solid black. A fringe field computation based on the method of COSY INFINITY is included by default, extending the field region. This is first order only, and the fringe field extends outside of the magnet aperture only in a cylinder extending the aperture straight along local z. As the fringe field is first order only, it is slightly non-Maxwellian.

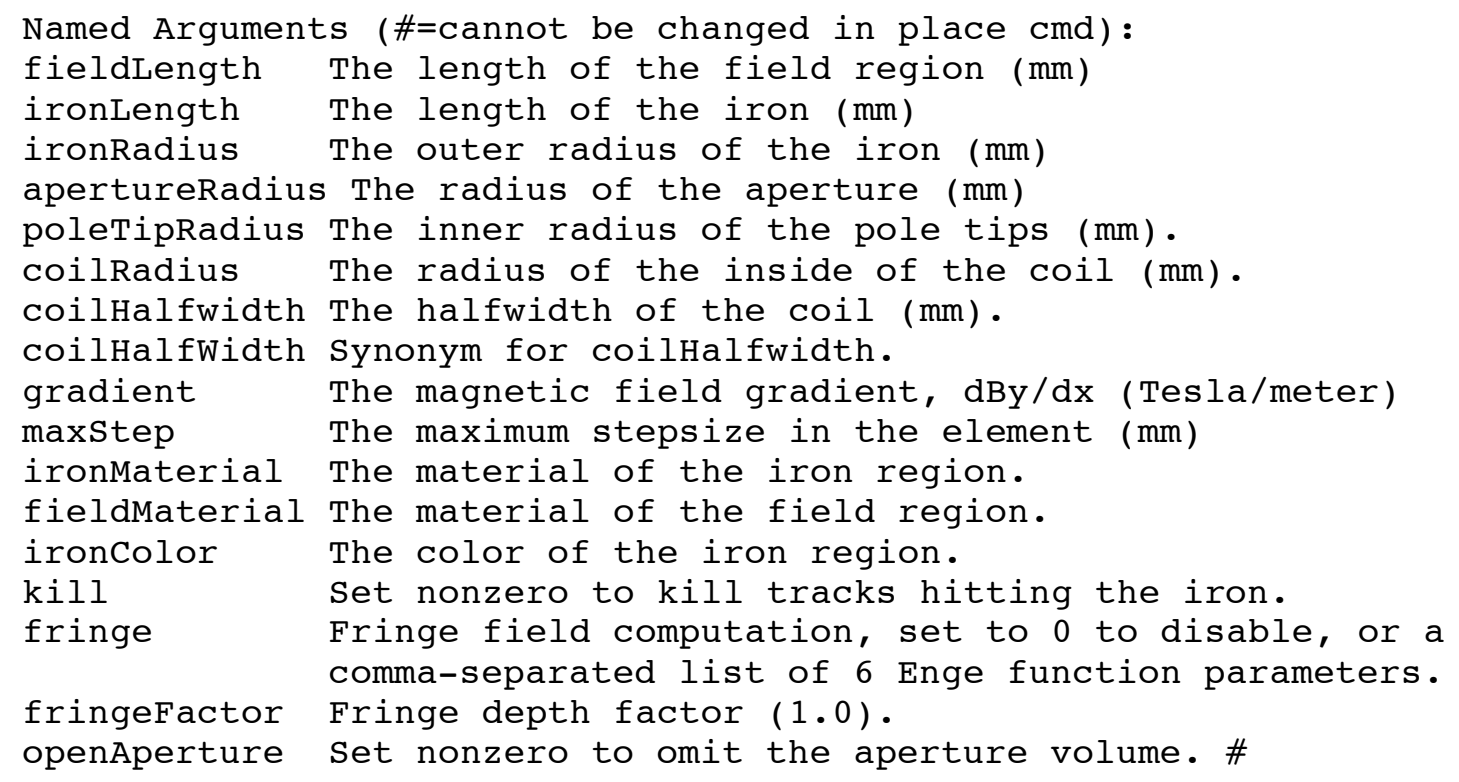

\subsection{6 geometry Arranges to perform a geometry test.}

The geometry test checks nPoints points on the surface of each element, verifying that they are inside the parent element and that they are not inside any sibling element. The default of 100 points is usually sufficient; 0 means omit the geometry test. The first 20-40 points (depending on element) test 'corners', the rest are randomly distributed on the surface. The default tolerance is $0.002 \mathrm{~mm}$.

Named Arguments :

nPoints The number of surface points to test per element printGeometry set nonzero to print the entire geometry. visual Set nonzero to display the geometry test points. tolerance Tolerance for inside/outside tests (mm). 


\subsection{7 group begins definition of a group.}

A group is a collection of elements that can be placed together, preserving their relative positions. The group is a Logicalvolume in the geant4 geometry -- this means that a group cannot overlap any other group or object, even if the overlapping portion of the group is empty. If you need to permit overlaps, consider using a macro instead (the define command).

If the group is given a length, then children can be placed at specific $z$ offsets relative to the center of the group. If the group is not given a length, then children can only be placed sequentially along $\mathrm{z}$, and the length will be computed by the endgroup command. Width and height are computed from the largest child or the argument. If radius is set to 0 , the group will be a cylinder with radius determined by the largest width or height placed into it; if $>0$ then that is the fixed radius. If radius is not set then a box is used.

Note: when placing objects into a group, if the rename argument is used, it should begin with $a$ ' + ' to include the group's name in the object's name; otherwise there may be multiple objects with the same name -- this is only a major problem for virtualdetector-s and other output objects. The group's name is included by default if no rename is used on the place command.

Named Arguments (\#=cannot be changed in place cmd):

length Overall group length along $\mathrm{z}(\mathrm{mm})$ \#

width Overall group width $(\mathrm{mm})$ \#

height Overall group height (mm) \#

radius Radius for a cylindrical group ( $\mathrm{mm}$ ) \#

material Material of the volume outside children

color Color of the volume of the group

maxstep The maximum stepsize in the volume (mm)

\subsection{8 helicaldipole construct a helicaldipole magnet.}

The field region is a cylinder with a helical dipole field plus a solenoidial field. The simple model=1 provides just a sine and cosine transverse dependence, while the maxwellian model=2 has both dipole and quadrupole terms. Both the dipole scale bD [T] and quadrupole scale bQ $[\mathrm{T} / \mathrm{m}]$ are now at rho=0; the user must determine the correct values externally.

Note that this Element generates a magnetic field only, and only within the cylinder defined by length and radius. So it has no solid associated with it, and is invisible.

Named Arguments:

radius The radius of the field region ( $\mathrm{mm}$ )

model The model of field calculated(simple=1, rpj and ysd model=2, ttominaka et al model=3), modulations in 


$\begin{array}{ll} & \text { bd, bq, bz= } 4 \\ \text { length } & \text { The length of the field region (mm) } \\ \text { bD } & \text { The dipole magnitude at rho=0 (Tesla). } \\ \text { lambda } & \text { Helix period along the } \mathrm{z} \text { axis (mm). } \\ \text { phi0 } & \text { The phase of the XY field at the entrance (deg). } \\ \text { Bsolenoid } & \text { The value of Bsolenoid (Tesla). } \\ \text { bQ } & \text { The quadrupole magnitude at rho=0 (Tesla). } \\ \text { bs } & \text { The sextupole magnitude at rho=0 (Tesla). } \\ \text { rr } & \text { Reference radius (mm) } \\ \text { psio } & \text { The offset between the dipole term and the } \\ \text { ez } & \text { quadrupole term (Degrees). } \\ & \text { The base electric field inside the helix channel } \\ & (\mathrm{GV} / \mathrm{m}) .\end{array}$

\subsection{9 helicalharmonic construct a helicalharmonic magnet.}

Creates a cylindrical region containing the field of a magnetic helical harmonic of given order [n]. The field is defined by the value of the $(n-1)$ order derivative [b] of the vertical field component (when the initial phase is 0 ) with respect to the horizontal coordinate at the center of the helix:

$\mathrm{b}=\mathrm{d}^{\wedge}(\mathrm{n}-1)$ B_phi/dr^(n-1) @ $[\mathrm{r}=0 \& \mathrm{phi}-\mathrm{k} * \mathrm{z}+\mathrm{phi} 0=0]$, where $\mathrm{k}=2 * \mathrm{pi} / \mathrm{lambda}$ is the helix's wave number, [lambda] is the length of the helix's period, and phio is the initial phase. The field components in the cylindrical frame are given by:

B_phi $=(2 /(n * k)) \wedge(n-1) * b *(I[n-1](n * k * r)-I[n+1](n * k * r)) *$ $\cos (n *(p h i-k * z+p h i 0))$,

B_r $=(2 /(n * k)) \wedge(n-1) * b *(I[n-1](n * k * r)+I[n+1](n * k * r)) *$ $\sin (\mathrm{n} *(\mathrm{phi}-\mathrm{k} * \mathrm{z}+\mathrm{phi}))$,

B_z $=-2 *(2 /(n * k)) \wedge(n-1) * b * I[n](n * k * r) * \cos (n *(p h i-k * z+p h i 0))$, where $I[n](x)$ is the modified Bessel function of the first kind of order $[n]$.

Note that this Element generates magnetic field only, and only within the cylinder defined by length and radius. So it has no solid associated with it, and is invisible.

Named Arguments:

radius The radius of the field region ( $\mathrm{mm}$ )

length The length of the field region ( $\mathrm{mm})$

$\mathrm{n} \quad$ Order of helical harmonic (i.e. $\mathrm{n}=1$ for dipole)

b (n-1)-order derivative of the field at the center $\left(\mathrm{T} / \mathrm{m}^{\wedge}(\mathrm{n}-1)\right)$

lambda Helix period along the $z$ axis (mm).

phio The phase of the XY field at the entrance (rad).

\subsection{0 help provides interactive help.}

help with no arguments lists all commands. 'help command' gives more detailed help on that command. 'help *' gives detailed help for all commands. If the first argument is -exit the program will 
exit after printing the help.

\title{
5.31 idealsectorbend construct an ideal sector bending magnet.
}

\author{
The field region is a sector with By specified. Unlike most \\ Elements, the position of the idealsectorbend is the center of \\ the front face of its field (aperture). angle>0 bends to the left \\ around $\mathrm{Y}$; angle<0 bends right. The only useful rotations are \\ around the centerline $\mathrm{Z}$. Should be followed immediately by a \\ cornerarc. Note that $-90<$ angle< 90 degrees. \\ Named Arguments ( $@=$ Tunable): \\ angle Angle of bend (degrees).

\subsection{2 if Conditional execution of command(s), and if/elseif/else/endif.}

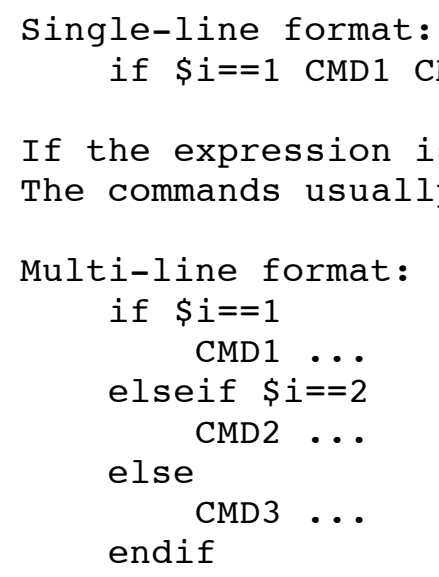

The commands are executed in the usual way; elseif and else are optional, but endif is mandatory. Any number of elseif-s and commannds can be used. Do-s and multi-line if-s can be nested in any manner. 


\title{
5.33 include includes a command file.
}

include requires one argument, the file to include.

\subsection{4 list provides interactive list of interesting internal tables.}

\author{
list with no arguments lists all lists except processes. 'list \\ name' lists that specific one. 'list -exit name(s)' will exit \\ after listing. List names are: \\ commands all commands \\ materials currently known materials \\ physics all physics lists \\ particles currently known particles \\ processes currently known physics processes $* * *$ \\ NOTE: the particles and processes lists are not populated until \\ the physics list is selected (via the physics command). Different \\ physics lists use different processes and particles. \\ ***NOTE: listing processes will prevent any simulating, as will a \\ non-empty particle list. \\ Named Arguments : \\ particle Comma-separated list of particles for which details \\ will be printed
}

\subsection{5man Alias for 'help'.}

\subsection{6 material construct a new material.}

This is an interface to G4Material. This command is rarely required, because elements and most common materials are available via the NIST database. Any material available from the NIST database can simply be used; if it is unknown then it will be automatically defined from the database. Uncommon materials or nonstandard densities must be defined with this command.

The first argument to this command is the material name, which is always required; density is also required. The command to define an element (e.g. with non-standard density) is:

material $\mathrm{H} 2 \mathrm{Z}=1 \mathrm{~A}=1.01$ density $=0.000090$

A mixture or compound is a combination of known materials and/or elements; the command is: material water $\mathrm{H}, 0.11190,0.8881$ density $=1.0$

The numbers following the element names are their mass fractions (note that WATER is available from the NIST $d b$ ). Either type of command can optionally have: pressure, temperature, state. 
With no arguments, this command prints the current material table. Note that 'G4_' is prepended to the names of most materials that are obtained from the NIST database; 'G4_Al' and 'Al' refer to the same material (unless one was previously defined using this command).

The following materials will be automatically created on first use:

$\mathrm{H}$ He Li Be B C N O F Ne Na Mg Al Si P S Cl Ar K Ca Sc Ti $\mathrm{V}$ Cr Mn $\mathrm{Fe} C \mathrm{Co} \mathrm{Ni} \mathrm{Cu} \mathrm{Zn}$ Ga Ge As Se Br Kr Rb Sr Y $\mathrm{Zr}$ Nb Mo TC Ru Rh Pd Ag $\mathrm{Cd}$ In $\mathrm{Sn} \mathrm{Sb}$ Te I Xe Cs Ba La Ce Pr Nd Pm Sm Eu Gd Tb Dy Ho Er Tm $\mathrm{Yb}$ Lu Hf $\mathrm{Ta} W$ Re Os Ir Pt Au Hg Tl Pb Bi Po At Rn Fr Ra AC Th Pa U Np Pu Am Cm Bk Cf A-150_TISSUE ACETONE ACETYLENE ADENINE ADIPOSE_TISSUE_ICRP AIR ALANINE ALUMINUM OXIDE AMBER AMMONIA ANILINE ANTHRA $\bar{C} E N E$ B-100_BONE BAKELITE BARIUM_FLUORIDE BARIUM_SULFATE BENZENE BERYLLIUM_OXIDE BGO BLOOD_ICRP BONE_COMPACT_ICRU BONE_CORTICAL_İCRP BORON_CARBIDE BORON_OXIDE BRAIN__ICRP BUTTANE N-BUTYL_ALCOHOL C-552 CADMIUM_TELLURIDE CADMIUTM_TUNGSTATE CALCIUM_CARBONATE CALCIUM_FLUORIDE CALCIUM_OXIDE CALCIUM_SULFATE CALCIUM_TUNGSTATE CARBON_DIOXIDE CARBON TETRACHLORIDE CELLULOSE CELLOPHANE CELLULOSE BUTYRATE CELLULŌSE_NITRATE CERIC_SULFATE CESIUM_FLUORIDE CESİUM_IODIDE

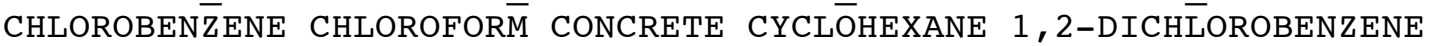
DICHLORODIETHYL ETHER 1,2-DICHLOROETHANE DIETHYL ETHER N,N-DIMETHYL_FORMAMIDE DIMETHYL_SULFOXIDE ETHANE ETHYL_ALCOHOL ETHYL_CELLULOSE ETHYLENE EYE_LENTS_ICRP FERRIC_OXIDE FERROBORIDE FERROŪS_OXIDE FERROUS_SULFATE $\bar{E}$ FREON-12 FREON-12B2 FREON-13 FREON-13B1 FREON-13I1 GADOLINIUM_OXYSULFIDE GALLIUM_ARSENIDE GEL_PHOTO_EMULSION Pyrex_Glass GLASS_LEAD GLASS_PLATE GLUCOSE GLUTAMINE GLYCEROL GUANIN̄E GYPSUM N-ḦEPTANE N-HEXANE KAPTON LANTHANUM_OXYBROMIDE LANTHANUM_OXYSULFIDE LEAD_OXIDE LITHIUM_AM IDE LITHIUM_CARBONATE LITHIUM_FLUORIDE LITHIUM_HYDRIDE LITHIUM_IODIDE LITHIUM_OXIDE LITHIUM_TETRABORATE LUNG_ICRP M3_WAX MAGNESIŪM_CARBONATE MAḠNESIUM_FLUORIDE MAGNESIUM_OXIDE MAGNESIUM_TETRABORATE MERCURIC_IODIDE METHANE METHANOL MIX_D_WAX MS20_TISSŪE MUSCLE_SKELETAL_ICRP MUSCLE_STRIATED_ICRU

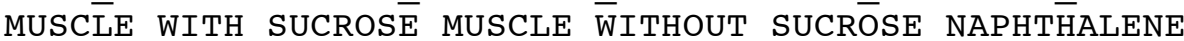
NITROBENZENE NITROUS_OXIDE NYLON-8062 NYLON-6-6 NYLON-6-10 NYLON-11_RILSAN OCTAN̄E PARAFFIN N-PENTANE PHOTO_EMULSION PLASTIC_SC_VINYLTOLUENE PLUTONIUM_DIOXIDE POLYAC̄RYLONITRILE POLYCARBONATE POLYCHLOROSTYRENE POLYETHYLENE MYLAR PLEXIGLASS POLYOXYMETHYLENE POLYPROPYLENE POLYSTYRENE TEFLON POLYTRIFLUOROCHLOROETHYLENE POLYVINYL_ACETATE POLYVINYL_ALCOHOL POLYVINYL BUTYRAL POLYVINYL CHLORIDE POLYVINYLIDENE CHLORIDE POLYVINYLIDENE_FLUORIDE POLYYVINYL_PYRROLIDONE POTASSTIUM_IODIDE POTASSIUM_OXIDE PROPANE IPROPANE $\bar{N}-$ PROPYL_ALCOHOL PYRIDINE RUBBER_BUTYYL RUBBER_NATURAL RUBBER_NEOPREN̄E SILICON_DIOXIDE SILVER_BROMIDE SILVER_CHLORIDE SILVEER_HALIDES SILVER_IODIDE SKIN_ICRP SODIUM_CARBONATE SODIUM_IODIDE SODIUM_MONOX̄IDE SODIŪM_NITRATE STTILBENE SUCROSE TËRPHENYL TESTES'ICRP TETRACHLOROETHYLENE THALLIUM_CHLORIDE TISSUE_SOFT_ICRP TISSUE_SOFT_ICRU-4 TISSUE-METHANE TISSUE-PROPANE TITANIUM_DIOXIDE TOLUENE TRICHLOROETHYLENE TRIETHYL_PHOSPHATE 


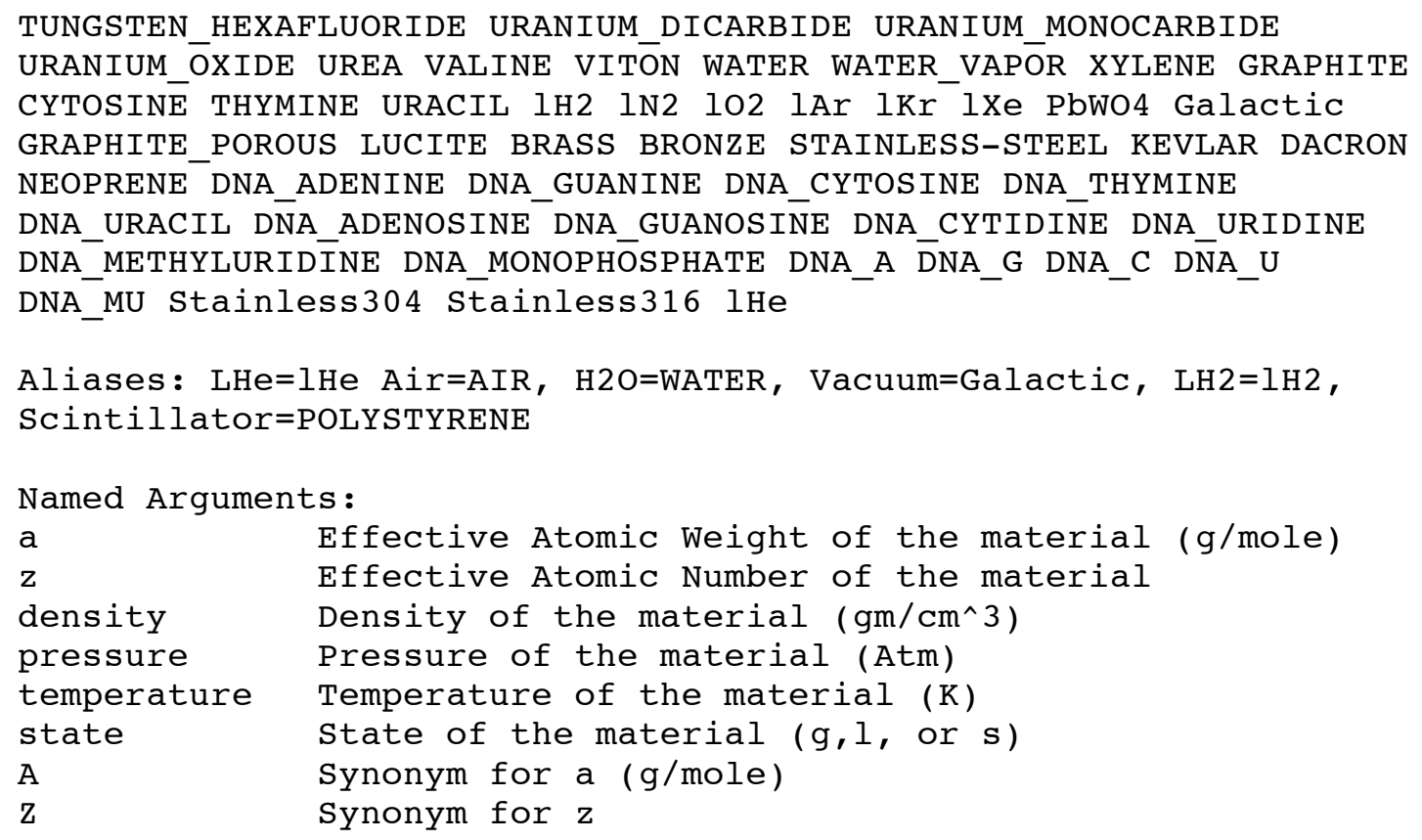

\subsection{7 movie Generate movie NTuple.}

This command outputs a set of NTuples suitable for generating a movie.

Named Arguments:

coordinates Coordinates: global, centerline, or reference $($ default $=r)$.

\subsection{8 multipole construct a generic multipole magnet.}

Multipole magnetic fields from dipole through dodecapole are implemented with a cylindrical field region and optional surrounding iron (ironLength $=0$ or ironRadius $=0$ omits it). All fields with positive strengths are oriented so in the $\mathrm{X}-\mathrm{Z}$ plane for $x>0$ (beam left) the field is purely By. Negative strengths are allowed and reverse the field. The fringe field computation is not implemented.

Named Arguments (\#=cannot be changed in place cmd): fieldLength The length of the field region (mm)

ironLength The length of the iron (mm) ironRadius The outer radius of the iron (mm) apertureRadius The radius of the aperture (mm) ironMaterial The material of the iron region. fieldMaterial the material of the field region. dipole Strength of dipole (Tesla) quadrupole Strength of quadrupole $(\mathrm{T} / \mathrm{m})$ 


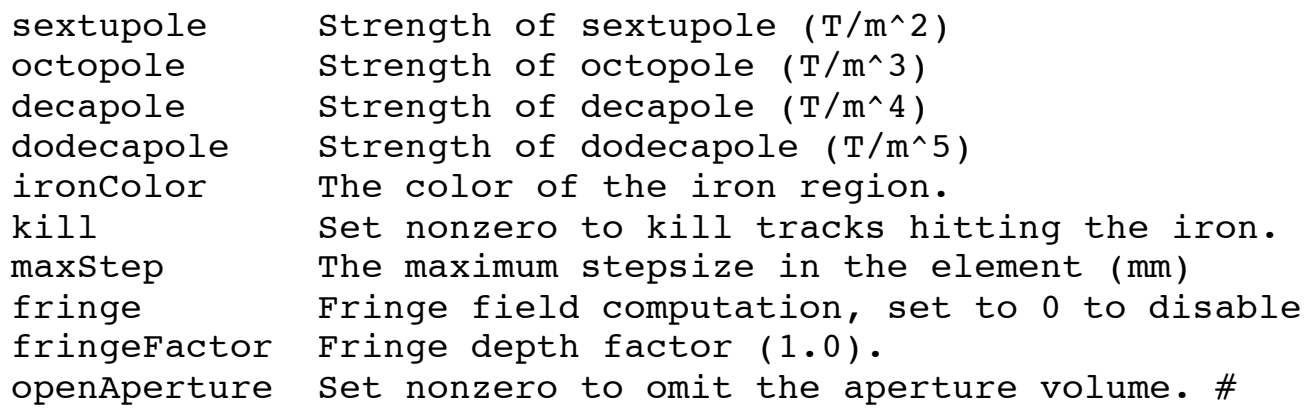

\title{
5.39 muminuscapturefix Fixes up the G4MuonMinusCaptureAtRest process.
}

\author{
This class adds extra neutrons to mu- capture. The neutrons are \\ added with a Poisson distribution having a mean of \\ neutronMeanNumber, and with an exponential distribution in \\ kinetic energy: \\ ( 1 /neutronMeanEnergy) * exp ( $-\mathrm{KE} /$ neutronMeanEnergy) \\ As the muonic atom cascades to its ground state it forgets the \\ incident mu-direction, so the extra neutrons are generated \\ isotropically in the lab. \\ The extra neutrons are added only to those captures that are \\ hadronic (i.e. not decay in orbit). The value of \\ neutronMeanNumber should reflect this. \\ The default values correspond to Aluminum. \\ Named Arguments: \\ neutronMeanNumber Mean mumber of extra neutrons per nuclear \\ capture (2.5). \\ neutronMeanEnergy Mean energy of neutron spectrum (MeV)
}

\subsection{0 newparticlentuple NTuple containing particle tracks when created.}

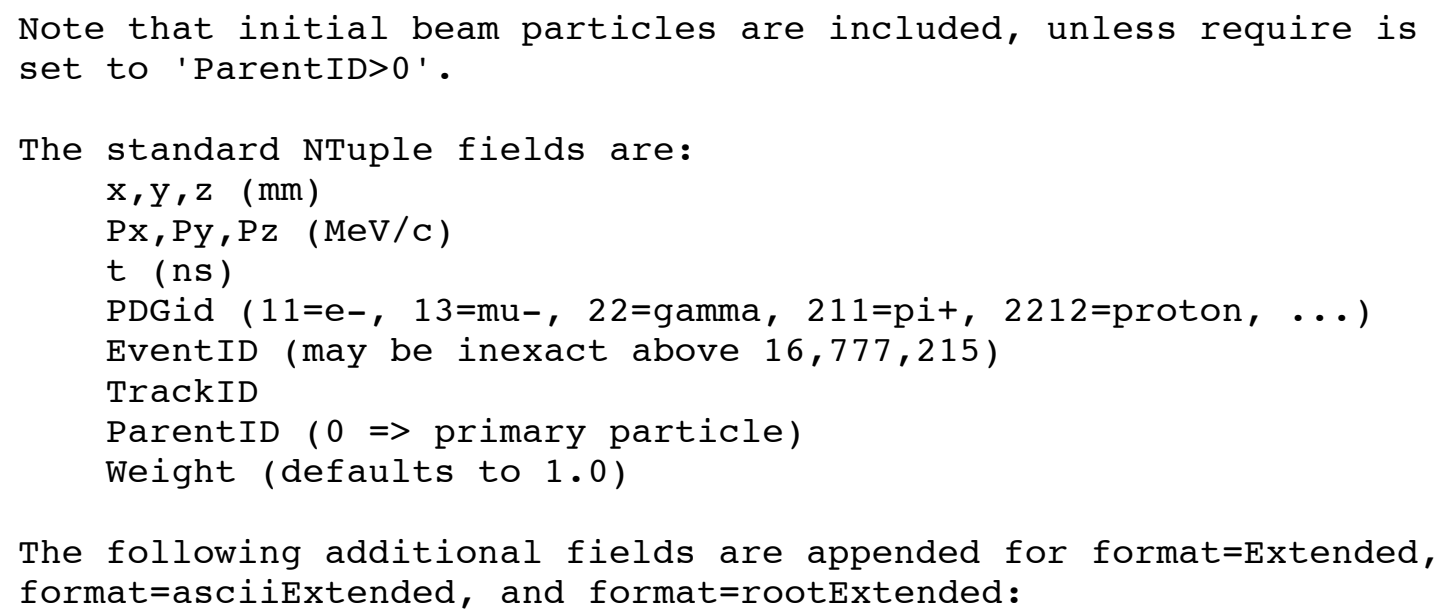




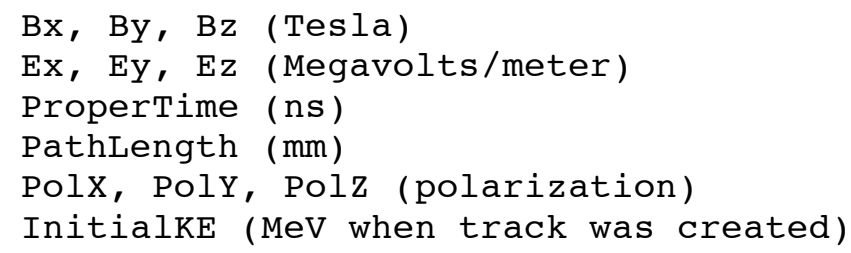

Valid Formats (ignore case): ascii bltrackfile dummy for009 for 009 .dat root trackfile Extended asciiExtended rootExtended

Named Arguments (\#=cannot be changed in place cmd):

format The NTuple format (see above for list).

filename The filename for the NTuple.

file

require

coordinates Synonym for filename. Expression which must be nonzero to include the track (default=1) \# Coordinates: global, centerline, or reference $($ default $=c)$.

\subsection{1 ntuple Define an NTuple containing multiple detectors.}

An ntuple holds the data from multiple detectors in a single NTuple, with one row (entry) per event or per track. This permits the generation of plots that compare different detectors. Up to 64 detectors can be used. While 'detector' is used in this description, any existing NTuple can be used, generated by any command, such as: virtualdetector, zntuple, beamlossntuple, timentuple, and newparticlentuple.

By default, an entry in this ntuple is made for each event satisfying the require, veto, and minHit conditions; if perTrack=1 then an entry is made for each track that satisfies the require, veto, and minHit conditions. Any hit in any detector matching the patterns in veto will prevent the event/track from being entered into the ntuple.

If multiple hits occur in a given detector during the event or track, only the first one is kept in this ntuple. Detectors are specified by patterns identical to UNIX file-matching, so '*Det*' matches any detector with 'Det' anywhere in its name, etc. The 'required' argument permits events to be omitted unless all of the matching detectors were hit at least once in the event or track. The patterns in 'required' are applied only to detectors in the ntuple, so a simple '*' only matches detectors named in the 'detectors' argument.

NOTE: the name of a detector is by default the concatenation of its ancestors' names before its own (except World), unless rename $=$ NAME was used in its place command. The patterns are applied to the names of the virtualdetectors as they were placed (including rename), not the bare name of the virtualdetector command. If 'rename=det\#' was used when placing the virtualdetector-s, you probably want a * to match the \#, or list 


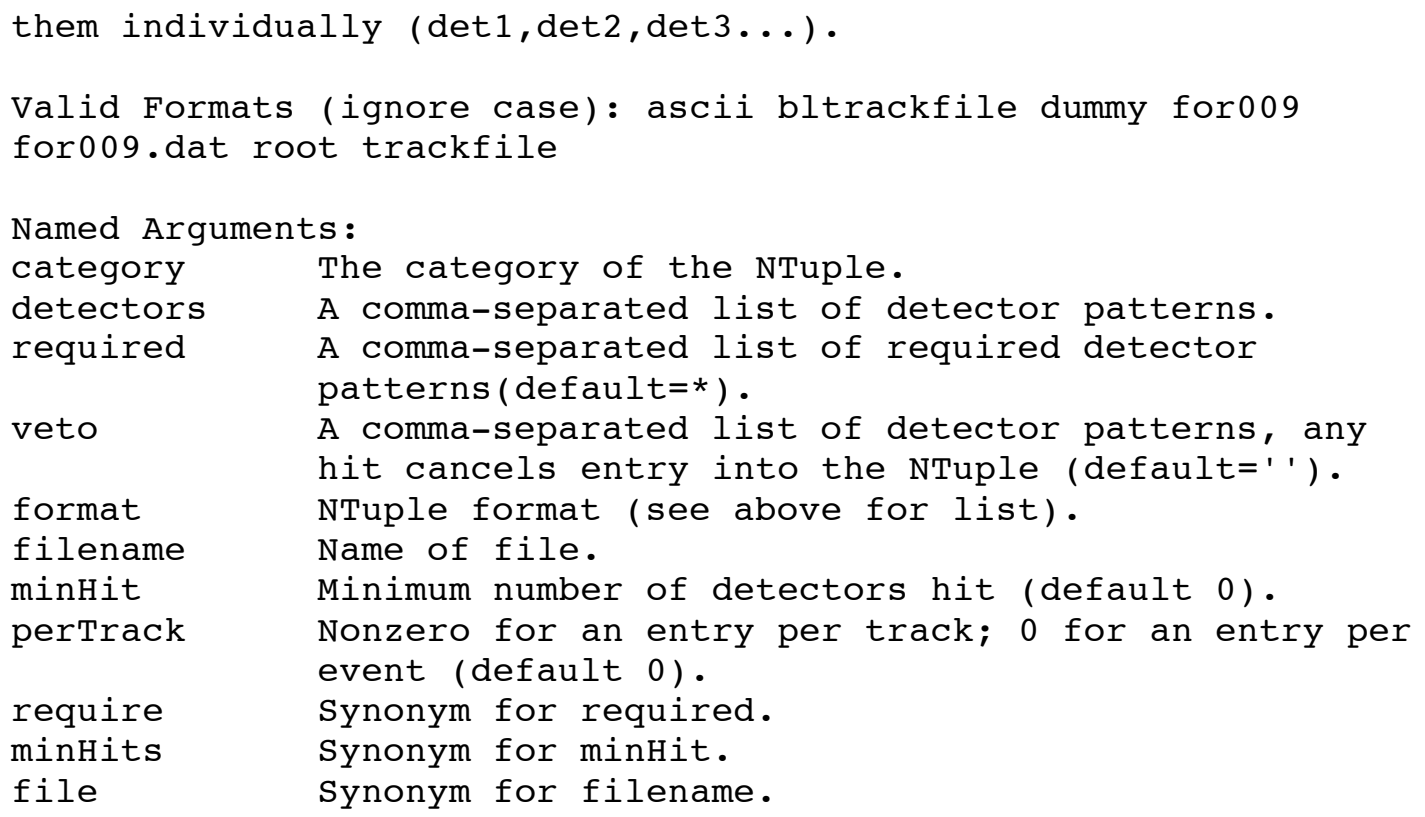

\subsection{2 output redirects stdout and stderr to a file}

output requires one argument, the new output file. Any output generated before this command will not appear in the file, so this command should come at the start of the input.file, preceded only by setting parameters. After the redirection, it will re-print the G4beamline version and the current parameter values to the file. The most common usage is to redirect output to a file named like the .root file, when that is determined by parameter values:

param -unset param $1=1.0$ param $2=3.0$

param histofile=\$param1, \$param2

output \$histofile.out

\subsection{3 param Defines parameter values.}

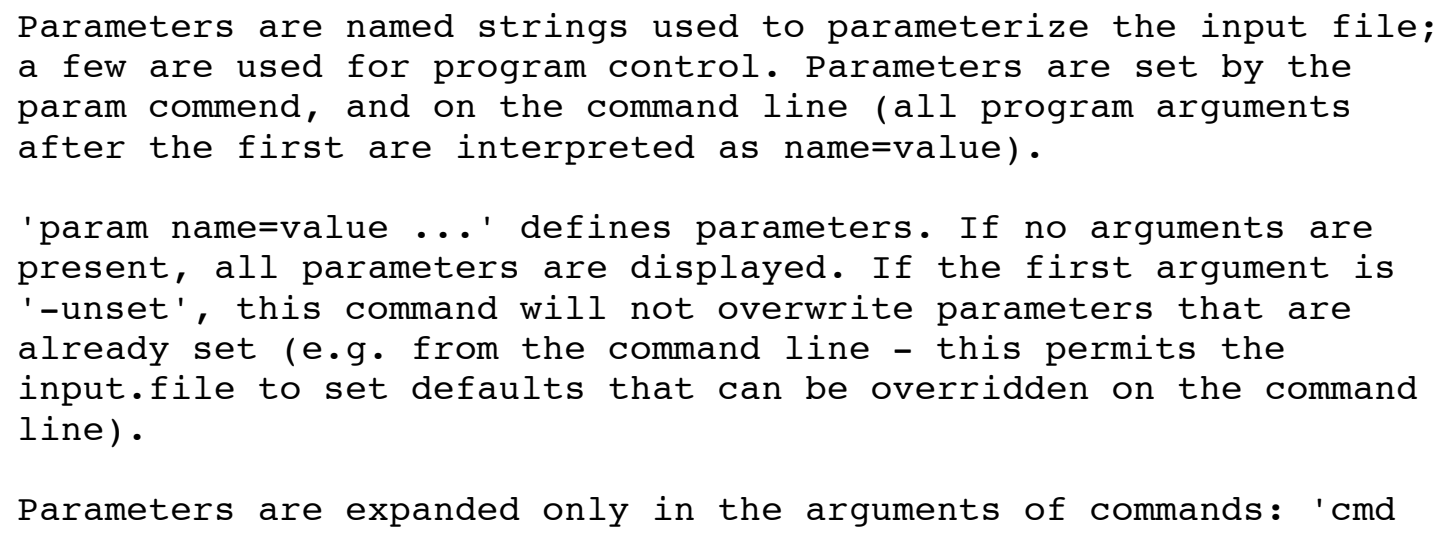




\begin{abstract}
argname $=\$$ paramname [...]'; real-valued expressions for arguments can use \$paramname as a value, as long as paramname contains a valid real expression.

Parameters are most useful for setting global things like viewer and histofile, or as parameters used in the input.file.

When a parameter is used, if it has not been defined, it will be defined from the environment if possible; if it is not defined in the environment then this generates an error message.

The values of parameters are strings, but if the value of a parameter is set to a valid real expression including at least one operator $\left\{+-* /^{\wedge}\langle>=() ! \sim \& \%| ?\right\}$, the parameter value will be set to the numerical value of the expression to 8 significant digits.

NOTE: pre-defined Program control parameters (listed below) are defined before the command-line and are not affected by -unset.
\end{abstract}

\title{
5.44 Program control Parameters:
}

$\mathrm{Zcl}$

deltachord

deltaIntersection

deltaOnestep

epsMax

epsMin

eventTimelimit

histoFile

histoupdate

maxstep

minstep

steppingFormat

steppingVerbose

viewer

worldMaterial

zTolerance

steppingFormat is

EXT

TAG

$\mathrm{N}$

NSTEP

GLOBAL

XYZT

CL

CLX

$\mathrm{KE}$

STEP

STEPLEN

VOL

VOLNAME

PROCESS

B

E

Last centerline $\mathrm{z}$ position used (updated continuously)

Geant4 tracking parameter

Geant4 tracking parameter

Geant4 tracking parameter

Geant4 tracking parameter

Geant4 tracking parameter

CPU Time Limit (sec)

Default (Root) NTuple output filename

Output update interval (events)

Maximum physics step size (mm)

Minimum step size (mm)

Format for printing steps

Set nonzero to print each step

Visualization driver selected (default=none)

Material of the World volume

Tolerance for $\mathrm{Z}$ steps (mm)

a space- or comma-separated list of items:

toggle extended precision ( 3 more digits)

print a ' $>$ ' (useful to grep output)

step number

Synonym of $\mathrm{N}$

$\mathrm{X}, \mathrm{Y}, \mathrm{Z}, \mathrm{T}$ in global coords

Synonym of GLOBAL

$\mathrm{X}, \mathrm{Y}, \mathrm{Z}, \mathrm{dxdz}, \mathrm{dydz}$ in $\mathrm{CL}$ coords

extended precision $\mathrm{CL}$

kinetic energy

step length

Synonym of STEP

volume name

Synonym of VOL

process name

magnetic field

electric field 


$\begin{array}{ll}\text { P } & \text { 3-momentum } \\ \text { MAT } & \text { material name } \\ \text { ID } & \text { event ID, track ID, parent ID } \\ \text { PART } & \text { particle name } \\ \text { SEG } & \text { centerline coord segment number } \\ \text { WT } & \text { weight } \\ \text { NL } & \text { <newline> } \\ \text { NEWLINE } & \text { Synonym of NL } \\ \text { In } & \text { Synonym of NL }\end{array}$

\subsection{5 particlecolor Set the colors for particle tracks.}

Arguments are of the form 'name $=1,1,0$ ', where name is the standard name of a particle, and $1,1,0$ is the $R, G, B$ value desired for its color ('' for invisible) The special names plus, minus, and neutral will set colors for unnamed particles of each charge. The name reference will apply to the reference track (defaults to invisible).

\subsection{6 particlefilter Will kill particles from a list, or force particles to decay.}

A particlefilter will force the decay of certain particles when they enter the physical volume of the element. The list of affected particles is in the 'decay' argument, and the normal Decay process is disabled for them. In addition, the 'kill' argument is a list of particles to kill when they enter the element, and the 'keep' argument will kill all particles not named, if it is not empty.

require is an expression invloving track parameters that will kill the track if it evaluates to zero (use a comparison operator). The variables available are:

$\mathrm{x}, \mathrm{y}, \mathrm{z}, \mathrm{t}, \mathrm{Px}, \mathrm{Py}, \mathrm{Pz}, \mathrm{Ptot}, \mathrm{PDGid}$, EventID, TrackID, ParentID Units are $\mathrm{mm}, \mathrm{ns}, \mathrm{MeV} / \mathrm{c}$.

If nWait $>1$, particles will not be killed until they hit this element nwait times; this can be used to limit the number of revolutions around a ring. Decays are unaffected by nWait. referenceWait does the same for the reference particle the element can be either a cylinder or a box: set length and radius, or set length and width and height.

Named Arguments (\#=cannot be changed in place cmd): radius The radius of the cylindrical particlefilter ( $\mathrm{mm})$. innerRadius The inner radius of the cylindrical particlefilter ( $0 \mathrm{~mm}$, solid).

height The height of the rectangular particlefilter (mm). width The width of the rectangular particlefilter (mm). 


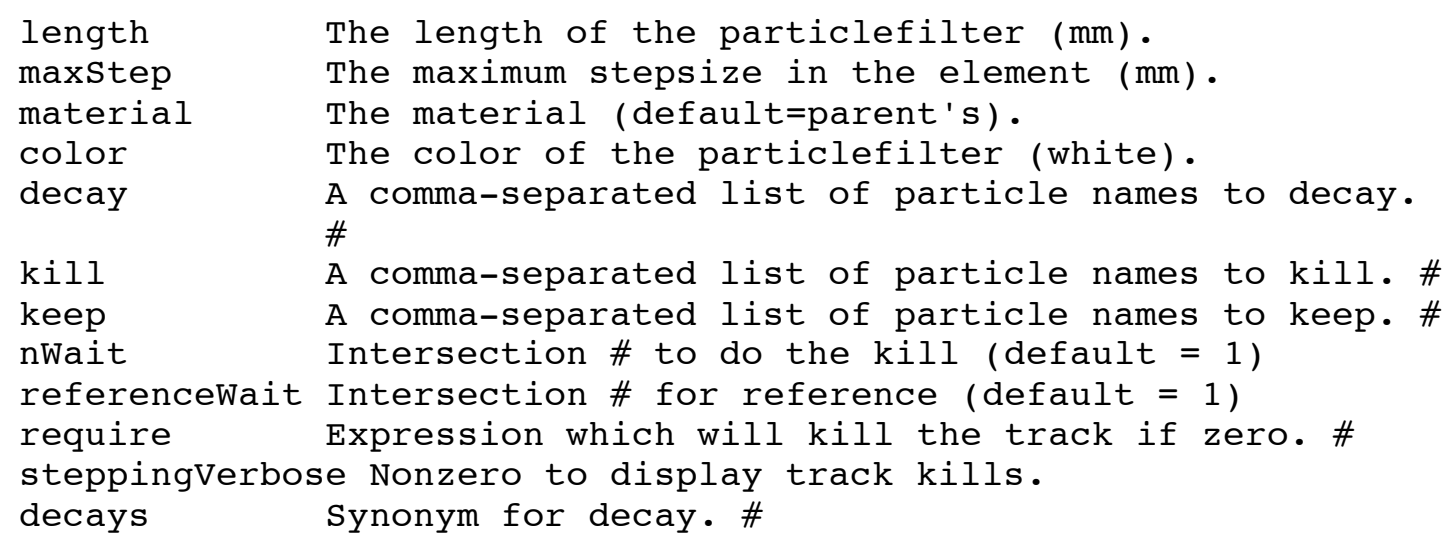

\subsection{7 particlesource Interface to the Geant4 General Particle Source.}

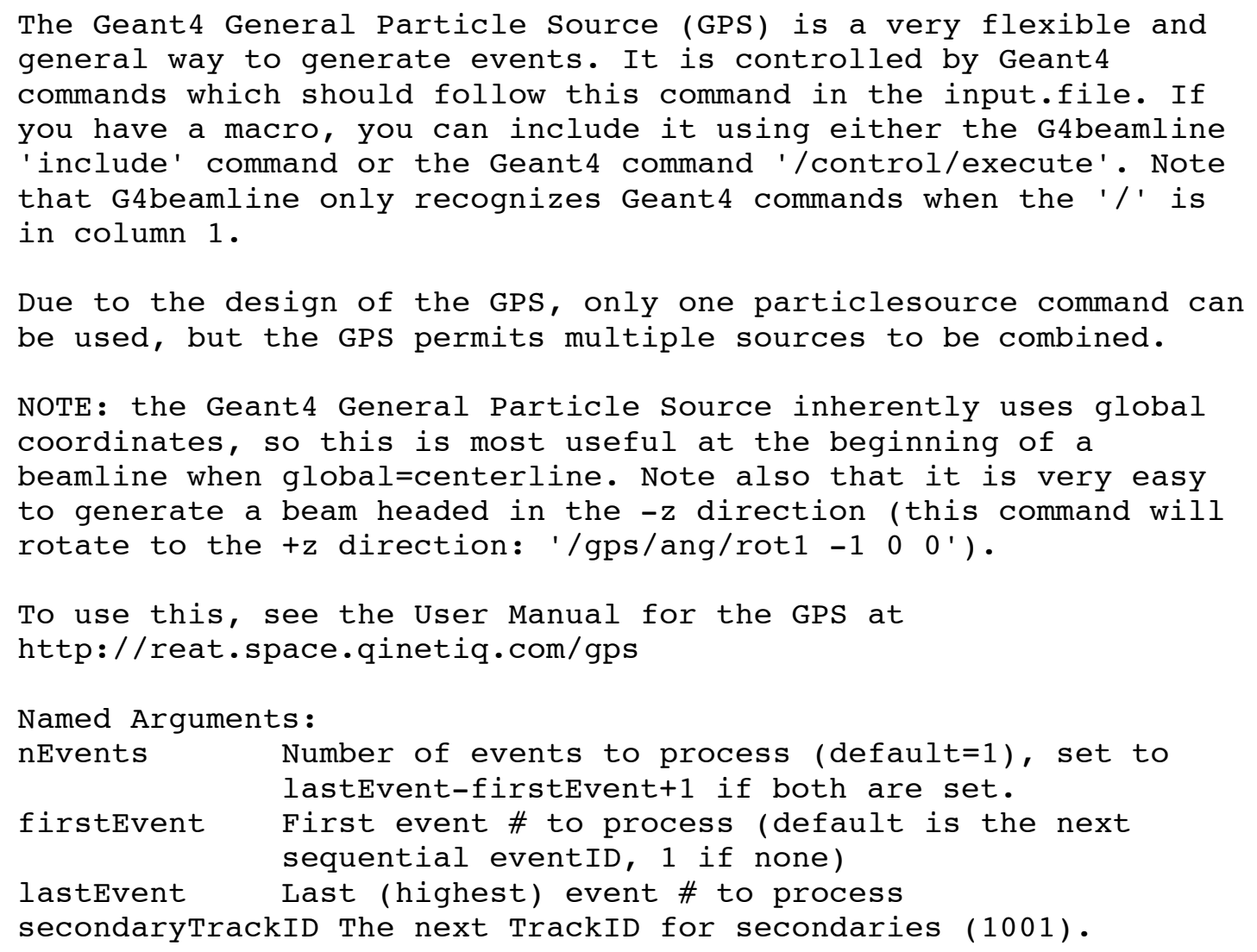

\subsection{8 physics Defines the physics processes and controls them.}

Exactly one physics command must be present. This command implements the geant4 physics lists of physics processes. The command is 'physics QGSP' for the QGSP set, and similarly for the other sets. With no argument it prints the available physics 
lists. Note that stochastic processes are always disabled while tracking the tune and reference particles. The only non-stochastic processes are Transportation and ionization energy loss (with fluctuations disabled). For muon beam studies you may want to disable the Decay process.

NOTE: this command defines the particles used throughout the simulation, so this command must come before others that use particle names.

NOTE: the rare decy mode for $\mathrm{pi}+/ \mathrm{pi}-$ to e nu is added.

The default all-around physics list for HEP is called 'QGSP_BERT' .

Named Arguments :

disable Comma-separated list of processes to disable (e.g. 'Decay, msc' ) .

inactivate Synonym of disable.

deactivate Synonym of disable.

dostochastics set to zero to disable all stochastic processes.

minRangecut Minimum range cut for particle production (1 $\mathrm{mm}$ )

list Nonzero to list the processes (later in output). gammaToMuPair Nonzero to add gamma->mu+mu- (0).

synchrotronRadiation Nonzero to add synchrotron radiation to the physics list for $e-$ and $e+$.

synchrotronRadiationMuon Nonzero to add synchrotron radiation to the physics list for mu- and mu+ NOTE: This is experimental, and may not work correctly.

----- PHYSICS LISTS -----

Further guidance in selecting physics lists is available at: http://geant4.web.cern.ch/geant4/support/physicsLists/referencePL/index.shtml

The default all-around physics list for HEP is called 'QGSP_BERT'.

LHEP uses exclusively parameterized modeling.

FTF lists use the FRITIOF description of string excitation and fragmentation.

QGSP lists use the quark gluon string model

QGSC are as QGSP except applying CHIPS modeling for the nuclear de-excitation.

BERT uses Geant4 Bertini cascade below $10 \mathrm{GeV}$.

-BIC uses Geant4 Binary cascade below $10 \mathrm{GeV}$.

EMV suffix indicates a faster but less accurate EM modeling.

EMX suffix indicates the low-energy EM processes.

-HP suffix uses the data driven high precision neutron package (thermal to $20 \mathrm{MeV}$ ).

-NQE suffix indicates a list for comparison with earlier release.

List of available physics lists:

CHIPS No synopsis available.

FTFP_BERT For calorimetry. The FTF model is based on the FRITIOF description of string excitation and fragmentation. Uses 
Geant4 Bertini cascade for primary protons, neutrons, pions and Kaons below $\sim 10 \mathrm{GeV}$.

FTFP BERT EMV Like FTFP BERT but with faster EM modeling. FTFP_BERT_EMX Like FTFP_BERT but with low-energy EM modeling. FTFP_BERT TRV A variant of QGSP BERT where the Geant4 Bertini cascade is only used for particles below $\sim 5.5 \mathrm{GeV}$. FTF_BIC No synopsis available.

$\mathrm{LBE}^{-} \quad$ For low background experiments (e.g. underground)

LHEP For calorimetry -- is the fastest, when it comes to CPU. It uses the LEP and HEP parametrized models for inelastic scattering. The modeling parametrizes the final states individual inelastic reactions, so you will not see resonances, and the detailed secondary angular distributions for $\mathrm{O}(10 \mathrm{MMeV})$ reactions may not be described perfectly. The average quantities will be well described.

LHEP_EMV Like LHEP but with faster EM modeling.

QBBC $^{-}$No synopsis available.

QGSC_BERT For calorimetry and high energy physics trackers -- is as QGSP for the initial reaction, but uses chiral invariant phase-space decay (multi-quasmon fragmentation) to model the behavior of the system's fragmentation. Uses Geant4 Bertini cascade for nucleon and pion induced reactions.

QGSP For calorimetry and high energy physics trackers and high-energy and medium-energy production targets -- uses theory driven modeling for the reactions of energetic pions, kaons, and nucleons. It employs quark gluon string model for the 'punch-through' interactions of the projectile with a nucleus, the string excitation cross-sections being calculated in quasi-eikonal approximation. A pre-equilibrium decay model with an extensive evaporation phase to model the behavior of the nucleus 'after the punch'. It uses current best pion cross-section.

QGSP_BERT Like QGSP, but using Geant4 Bertini cascade for primary protons, neutrons, pions and kaons below $\sim 10 \mathrm{GeV}$. In comparison to experimental data we find improved agreement to data compared to QGSP which uses the low energy parameterised (LEP) model for all particles at these energies. The Bertini model produces more secondary neutrons and protons than the LEP model, yielding a better agreement to experimental data.

QGSP_BERT_EMV Like QGSP_BERT but with faster EM modeling. QGSP_BERT_EMX Like QGSP_BERT but with low-energy EM modeling. QGSP_BERT_HP Like QGSP_BERT but with_HP modeling for neutrons. QGSP_BERT_NOLEP No synopsis available.

QGSP_BERT_TRV No synopsis available.

QGSP_BERT_CHIPS No synopsis available.

QGSP_BIC Like QGSP, but using Geant4 Binary cascade for primary protons and neutrons with energies below $\sim 10 \mathrm{GeV}$, thus replacing the use of the LEP model for protons and neutrons In comparison to teh LEP model, Binary cascade better describes production of secondary particles produced in interactions of protons and neutrons with nuclei. 
QGSP_BIC_EMY No synopsis available.

QGSP_BIC_HP Like QGSP_BIC but with_HP modeling for neutrons.

QGSP_FTFP_BERT No synopsis available.

QGS BIC No synopsis available.

QGSP_INCL_ABLA No synopsis available.

Shielding No synopsis available.

\subsection{9 pillbox Defines a pillbox RF cavity}

A Pillbox RF cavity is the basic RF element used to construct a linac. The phaseAcc parameter sets the phase of the tune particle at the center of the cavity, and the timing offset of the cavity is determined from that the first time that the Tune particle is tracked through the cavity. Zero degrees is the rising zero-crossing of the Ez field. If timeoffset is specified, it is used rather than setting it from the Tune particle.

The Pipe, walls, and collars are always made of copper. Pipe, wall, collar, win1, and win2 can be omitted by setting their thickness to 0 . Common usage is to set the collar values so by placing multiple pillboxes sequentially the collars form a beam pipe between them.

Note that section 4.4 of the User's Guide has a dimensioned drawing of a pillbox.

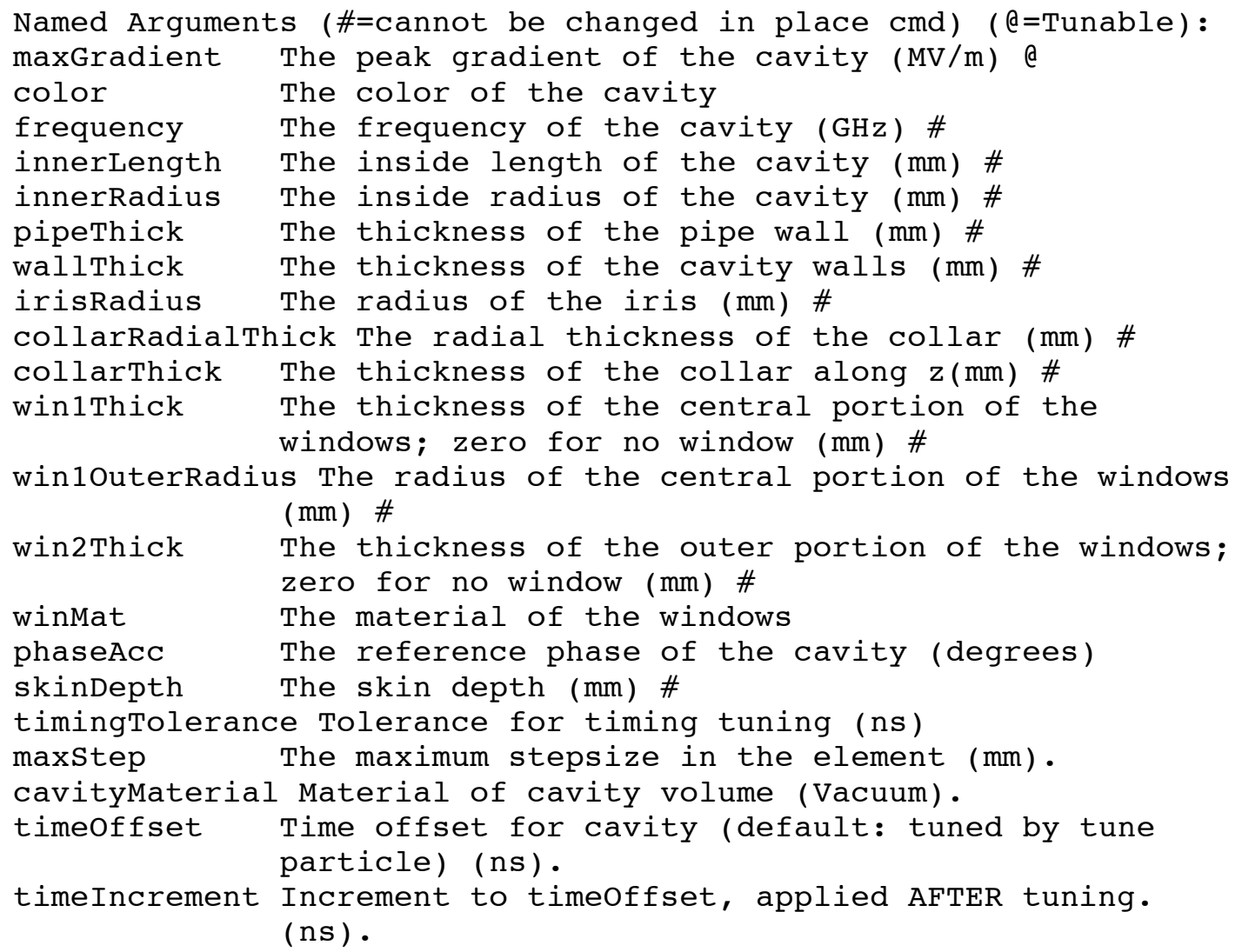


fieldMapFile Filename for BLFieldMap (pillbox if null). \# kill

Set nonzero to kill tracks that hit the pipe, walls, or collars (0).

\subsection{0 place places an element into the current group (or world).}

Every element can be placed multiple times into the beamline. For most elements the geometrical centerpoint is placed; for polycone the local $\mathrm{x}=\mathrm{y}=\mathrm{z}=0$ point is placed. If front is nonzero then the front of the element is placed. If $z$ is specified, then the element is placed at that $z$ position relative to the center of the enclosing group. If $z$ is not specified, then the element is placed immediately downstream (higher $z$ ) of the previous element in the group, or at the upstream edge of the group if this is the first element in the group.

The 'rename' argument can be used to change the name of the element (applies to traces and other uses of object names, such as the NTuple name of a virtualdetector). When placing into a group or other object, the rename argument should normally begin with '+' to include the parent's name; otherwise multiple placements of the parent will generate multiple objects with identical names -- that should be avoided for output objects like virtualdetector. Without a rename argument, the parent's name is included automatically.

When multiple copies are placed, $z$ refers to the first, and the rest are placed sequentially along $z$. When placing an element into the World group, Centerline coordinates are used unless coordinates=global is present. When centerline coordinates are used, the parameter ' $\mathrm{Zcl}$ ' is set to the highest $\mathrm{Z}$ value used; this is normally the $\mathrm{z}$ value for the front of the next element when placed sequentially (i.e. with no $z$ value given).

Rotations: The rotation parameter can be used to rotate this element relative to the enclosing group. The object is rotated, not the axes. Rotations are specified as a comma-separated list of axes and angles (in degrees): rotate $=\mathrm{Z} 90, \mathrm{x} 45$ rotates first by 90 degrees around $Z$ and then by 45 degrees around $X$. The axes are the local $\mathrm{X}, \mathrm{Y}, \mathrm{Z}$ coordinate axes of the enclosing group (centerline or global coordinate axes for the World group); groups can be rotated when placed, and are rotated as a rigid unit (including children).

If parent=name is present, then the name must be an element that accepts children, and it is used as the enclosing group; in this case the size of the group is implied by the size of the parent element, and $z$ must be given (defaults to 0 ). Note that a given element cannot be the parent of any other element once it has been placed, so you must place children into their parent before placing their parent.

If the special element 'OFFSET' is given, $x, y$, and $z$ specify 
offsets for every following place command into the current group (incl. World), that gives a $z$ position.

Named Arguments:

$\begin{array}{ll}z & \begin{array}{l}\mathrm{z} \text { position of element's center relative to the } \\ \text { center of the enclosing group (mm) }\end{array} \\ \mathrm{x} & \mathrm{X} \text { position of element's center [default=0] (mm) } \\ \mathrm{y} & \text { Y position of element's center [default=0] (mm) } \\ \text { parent } & \text { Parent element name (must accept children). } \\ \text { rename } & \text { Name to use for this placement; '\#' will be } \\ & \text { substituted by the number of placements. If the } \\ & \text { value begins with '+', it is replaced with the } \\ \text { popies } & \text { Number of copies (placed sequentially along } z) . \\ \text { front } & \text { Nonzero to specify zor the front, not the center. } \\ \text { rotation } & \text { Rotation of this object. } \\ \text { coordinates } & \text { Coordinates: global or centerline (default=c). }\end{array}$

\subsection{1 polycone construct a polycone with axis along $z$}

This is a direct interface to G4Polycone. For a solid polycone, omit innerRadius and it will be filled with zeroes. The number of entries in $z$, innerRadius, and outerRadius must be the same. Note that a polycone is placed at its $z=0, r=0$ point, which need not be its geometric center.

Named Arguments (\#=cannot be changed in place cmd): innerRadius Comma-separated list of inner radii (mm) \# outerRadius Comma-separated list of outer radii (mm) \# z

initialPhi The initial Phi value (deg; 0 for all)

finalphi The final Phi value (deg; 360 for all)

maxstep The maximum stepsize in the element (mm)

material The material of the polycone

color The color of the polycone (''=invisible)

kill Set nonzero to kill every track that enters.

\subsection{2 printf prints track variables and expressions}

This is an interface to the $\mathrm{C}$ printf() function. The first positional argument is the format, and the following positional arguments are double expressions printed with the $\%$ fields in the format. Up to 16 expressions can be printed. The print is performed only if the 'required' expression is nonzero, as each track reaches one of the $z$ positions in the ' $z$ ' argument (centerline coordinates). Multiple printf commands with the same 'file' will be combined into the file as tracks reach any of their $\mathrm{z}$ positions. More than 16 expressions can be broken into multiple printf-s with noNewline=1 for all but the last. 
The following variables can be used in expressions:

$\mathrm{x}, \mathrm{y}, \mathrm{z}, t, \mathrm{Px}, \mathrm{Py}, \mathrm{Pz}$

PDGid, EventID, TrackID, ParentId, Weight

$\mathrm{Bx}, \mathrm{By}, \mathrm{Bz}, \mathrm{Ex}, \mathrm{Ey}, \mathrm{Ez}$

tune (nonzero only for the Tune particle)

reference (nonzero only for the Reference particle)

beam (nonzero for any Beam particle)

Each value in $z$ and zloop can be an expression using double constants and the usual $\mathrm{C}$ operators and functions.

Example:

printf $z=0$ 'Momentum is $\% .3 f \mathrm{GeV} / \mathrm{C}$ ' $\operatorname{sqrt}(\mathrm{Px} * \mathrm{Px}+\mathrm{Py} * \mathrm{Py}+\mathrm{Pz} * \mathrm{Pz}) / 1000$

NOTE: if format begins 'Ptot=...' the parsing will think it is a named argument; put a space before the '=' to avoid that error.

Named Arguments:

Z

Comma-separated list of $\mathrm{Z}$ positions for printing $(\mathrm{mm})$

zloop Loop in $z$, first:last:incr $(\mathrm{mm})$

require logical expression for cutting (default=true)

file Output filename (default=stdout)

filename Synonym for file

noNewline set nonzero to omit final newline.

coordinates Coordinates: global, centerline, or reference $($ default $=c)$.

\subsection{3 printfield Prints E or B fields, or writes FieldMap file.}

Prints the value of the electromagnetic field components. For type=print, prints one component of the field in a 2-d table. Any coordinate plane can be printed (XY ... ZT). For type=grid or type=cylinder, writes a file in fieldmap format. Global coordinates are used. Units are Tesla for B and MV/meter for $\mathrm{E}$.

NOTE: This command cannot handle time dependency in the output BLFieldMap file, but can in the printout.

Note: if you want to plot field vs position or time, the 'fieldntuple' command is probably better, as it is not limited to the 2-d paper, is easier to use, and lets you use existing NTuple plotting tools. If you just want to test a few points, the 'probefield' command lets you do that interactively.

Named Arguments:

$\begin{array}{ll}\text { type } & \text { print, grid, or cylinder. } \\ \text { exit } & \text { Set nonzero to exit after printing field } \\ \text { Arguments for type=print: } & \\ \text { field } & \text { The field to print (Bx,By, Bz, Ex, Ey, Ez, Btot, Etot). } \\ \text { layout } & \text { Layout (RowCol) - } 2 \text { chars 'AB' each of }\{x y z t\} . \\ x & \text { The starting value of } x(m) . \\ y & \text { The starting value of } y(m) .\end{array}$




\begin{tabular}{|c|c|}
\hline $\mathbf{z}$ & The starting value of $\mathrm{z}(\mathrm{mm})$. \\
\hline$t$ & The starting value of time (ns). \\
\hline drow & The incr between points in each row (mm|ns). \\
\hline dcol & The incr between points in each column (mm $\mid \mathrm{ns}$ ). \\
\hline nrow & The number of rows. \\
\hline ncol & The number of columns. \\
\hline Arguments for & type=grid: \\
\hline file & Filename to write fieldmap to. \\
\hline comment & Comment for fieldmap. \\
\hline $\mathrm{x} 0$ & Initial value of $\mathrm{x}(\mathrm{mm}$, default $=0)$. \\
\hline Y0 & Initial value of $\mathrm{Y}(\mathrm{mm}$, default $=0)$. \\
\hline $\mathrm{ZO}$ & Initial value of $\mathrm{Z}(\mathrm{mm}$, default $=0)$. \\
\hline $\mathrm{nX}$ & Number of points in $\mathrm{x}$. \\
\hline $\mathrm{nY}$ & Number of points in $\mathrm{Y}$. \\
\hline $\mathrm{nz}$ & Number of points in $\mathrm{Z}$. \\
\hline$d X$ & Interval in $\mathrm{X}$ between points $(\mathrm{mm})$. \\
\hline$d Y$ & Interval in $\mathrm{Y}$ between points $(\mathrm{mm})$. \\
\hline $\mathrm{dz}$ & Interval in $\mathrm{Z}$ between points $(\mathrm{mm})$. \\
\hline Arguments for & type=cylinder: \\
\hline file & Filename to write fieldmap to. \\
\hline comment & Comment for fieldmap. \\
\hline $\mathrm{ZO}$ & Initial value of $\mathrm{z}(\mathrm{mm}$, default $=0)$. \\
\hline $\mathrm{nR}$ & Number of points in $R$. \\
\hline$d R$ & Interval in $\mathrm{R}$ between points ( $\mathrm{mm}$ ). \\
\hline $\mathrm{nZ}$ & Number of points in $\mathrm{Z}$. \\
\hline $\mathrm{Az}$ & cerval in $\mathrm{Z}$ between points $(\mathrm{mm})$. \\
\hline
\end{tabular}

\subsection{4 probefield Prints B and E fields at specified points.}

Intended primarily for debugging. Prints $\mathrm{Bx}, \mathrm{By}, \mathrm{Bz}$ in Tesla, and $\mathrm{Ex}, \mathrm{Ey}, \mathrm{Ez}$ in MegaVolts/meter. Each input line is $\mathrm{x}, \mathrm{y}, \mathrm{z}, \mathrm{t}$ separated by spaces or commas; omitted values are set to 0.0 . Runs after the reference particle is tracked. Only global coordinates are used.

Named Arguments:

file Filename for reading list of points (- = stdin)

\subsection{5 profile write beam profile information to a file}

This command accumulates the moments of the track distributions during the run, and at the end of run prints the mean, sigma, emittance, alpha, and beta (Twiss parameters) for the tracks. Each $\mathrm{z}$ position generates a line in the output file.

Each value in $\mathrm{z}$ and zloop can be an expression using double constants and the usual $\mathrm{C}$ operators and functions.

Named Arguments: 


$\begin{array}{ll} & (\mathrm{mm}) \\ \text { zloop } & \text { Loop in } \mathbf{z} \text { first:last:incr (mm) } \\ \text { require } & \text { logical expression for cutting (default=true) } \\ \text { particle } & \text { Name of particle to profile (default=mu+) } \\ \text { file } & \text { Output filename (default=stdout) } \\ \text { filename } & \text { Synonym for file } \\ \text { coordinates } & \text { Coordinates: centerline or reference (default=c). }\end{array}$

\title{
5.56 randomseed control pseudo random number generator seeds
}

\author{
This randomseed command controls the pseudo random number \\ generator seed at the start of each event. The unnamed argument \\ can be any of (case insensitive): \\ EventNumber \\ None \\ Time \\ Set 12345 \\ Now 12345 \\ EventNumber is the default and permits events to be re-run; None \\ does not re-seed the PRNG at each event, and Time is like None \\ after seeding with the time of day in microseconds; Set (Now) \\ seeds the generator immediately with the value of the second \\ argument (a long), and then acts like None.
}

\subsection{7 reference Define a reference particle.}

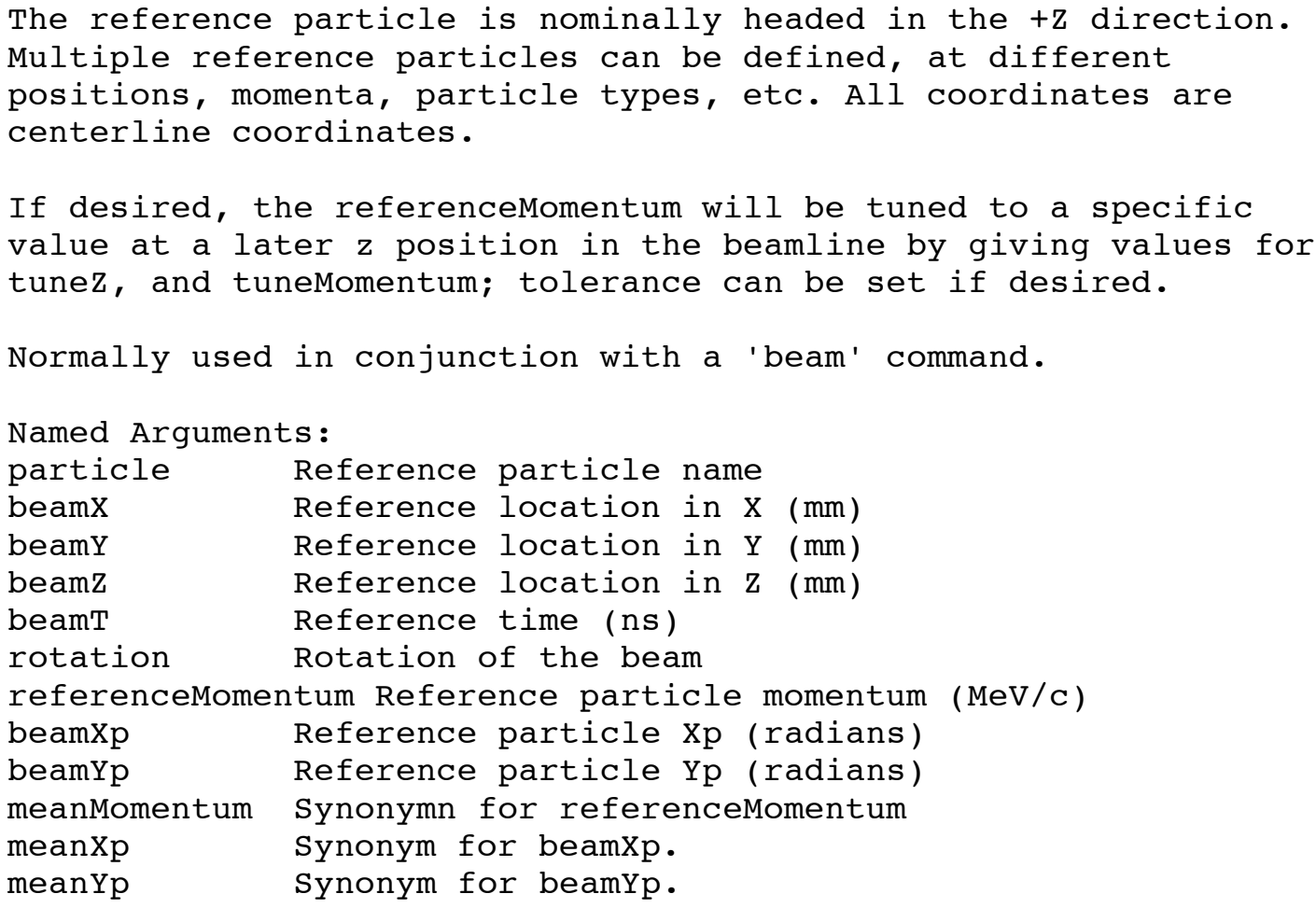


tunez

tuneMomentum

tolerance

notfield

noEloss $z$ position for momentum tuning.

Desired momentum for momentum tuning. tolerance for momentum tuning $(0.001 \mathrm{MeV} / \mathrm{c})$. Set nonzero to make this Tune and Reference particle not respond to $\mathrm{E}$ fields (ICOOL style) Set nonzero to make this Tune and Reference particle not respond to ionization energy loss (ICOOL style)

\title{
5.58 setdecay Set lifetime, decay channels, and branching ratios for a particle's decay.
}

The particle is specified by name as the first positional argument.

The lifetime of the particle can be set, unless it is a short-lived particle (for which lifetime is fixed at 0 -- these are particles like quarks, Zs, and Ws). Units are ns.

Decay channels are specified 'daughter1, daughter2=BR', where the daughter names are separated by commas, and the branching ratio is a value between 0 and 1 (inclusive); the order of daughters does not matter. The sum of all BRs must be 1.0 . It is best to use existing channels for the particle, because the code for the decay distribution is retained; new decay channels are given a default phase-space distribution, which is probably valid only for a 2-body decay of a spin 0 particle. New channels are limited to 4 daughters. Note that all desired decay channels must be listed.

Example to force fast decay $(0.1 \mathrm{~ns})$ of pit to a positron: setdecay pi+ lifetime $=0.1$ e+, nu_e $=1.0$

\subsection{9 showmaterial Set the colors for selected materials.}

\begin{abstract}
Arguments are of the form 'name $=1,1,0$ ', where name is the name of a material, and $1,1,0$ is the $R, G, B$ value desired for its color ('' for invisible) set hideothers=1 to make all other materials invisible. BEWARE: 'Vacuum' and 'vacuum' are different materials, as are 'Iron' and 'Fe'.
\end{abstract}

\subsection{0 solenoid defines a solenoid (a coil and current)}

A solenoid is a coil and a current. If alternate is nonzero, then each placement of the solenoid (or an enclosing group) will flip the sign of current. 
Named Arguments (\#=cannot be changed in place cmd): coilName The name of the coil (must exist) \#

current The current density in the conductor (Amp/mm^2)

color The color of the solenoid (' '=invisible).

alternate Set nonzero to alternate sign each placement.

kill Set nonzero to kill all tracks that hit the coil.

coil Synonym for coilname. \#

\subsection{1 spacecharge Beam-frame Green's function space charge computation}

This is a space charge computation for bunched beams. It uses a grid in the beam frame to solve Poisson's equation via a Green's function with infinite boundary conditions; the E field is boosted back to the lab frame $\mathrm{E}$ and $\mathrm{B}$ for tracking.

Macro-particles are used to enable the simulation of larger bunches than can be feasibly simulated as individual particles; the macro-particles have zero radius, but are pro-rated into the nearest eight grid points when placed into the grid. This computation can handle up to about a million macro-particles, but 100,000 is more sensible for all but the simplest physical situations.

The bunch is created from the beam tracks before tracking begins. There is one bunch for each reference particle. Particles in the bunch must be the same particle as the reference, must initially be within $\{d x, d y, d z\}$ of the reference particle, and when boosted to the reference particle's rest frame must initially have beta < maxBeta.

After boosting the particles to the beam frame, they are placed into the grid, pro-rating to the eight nearest grid points. The grid is dynamically re-sized to keep the 99th percentile of the particles between 0.5 and 0.67 of the grid size. This maintains a reasonable balance between resolution of grid points within the bunch and covering all of the particles. Particles located at $>85 \%$ of the grid size do not contribute to the field computation, but are tracked using the field of the rest of the bunch (and other bunches).

The grid has $\{\mathrm{nx}, \mathrm{ny}, \mathrm{nz}\}$ points; there is a small computational advantage to using powers of 2 , but any values $>1$ can be used. For efficiency, the convolution of the Green's function with the charge grid is performed using FFTs; the grid is doubled in each dimension with the proper symmetry applied to the Green's function, so the cyclical convolution of the FFTs gives the proper potential with infinite boundary conditions.

Outside the grid an approximation is used. An approximation grid is constructed, with the same size of the Poisson grid, but using \{nxApprox, nyApprox, nzApprox\} points. Particles are placed into this approximation grid, and the mean position is kept as well as the charge. Approximation grid points with less than $1 \%$ of the 
total charge are consolidated with their inner neighbors. The non-zero approximation grid points are treated as point charges when computing the potential outside the grid. For reasonably Gaussian bunches, $\{7,7,7\}$ are reasonable values for the approximation grid sizes.

The $\mathrm{E}$ field in the beam frame is computed via the derivatives of the linear interpolating function using the eight nearest grid points, and is boosted back to the lab frame $\mathrm{E}$ and $\mathrm{B}$ for tracking by the usual Geant4 routines.

Bunch particles that get destroyed cease contributing to the bunch. As the bunch particles are selected during start-up, no additional particles are ever added to a bunch. This algorithm handles multiple bunches of any particle types.

NOTE: For now, the reference MUST be parallel to the $\mathrm{z}$ axis.

Named Arguments :

deltat Time step (ns).

charge Charge of macro particles (times particle charge).

$\mathrm{nx} \quad$ Number of grid points in $\mathrm{x}(65)$.

ny Number of grid points in $y(65)$.

$\mathrm{nz} \quad$ Number of grid points in $\mathrm{z}(65)$.

$\mathrm{dx} \quad$ Max distance of particle to reference in $\mathrm{x}(\mathrm{mm})$

dy Max distance of particle to reference in $y(\mathrm{~mm})$

$\mathrm{dz} \quad$ Max distance of particle to reference in $\mathrm{z}(\mathrm{mm})$

nxApprox \# bins in $x$ in approximation (7).

nyApprox \# bins in $y$ in approximation (7).

nzApprox \# bins in $\mathrm{z}$ in approximation (7).

maxBeta Max beta ( $\mathrm{v} / \mathrm{c}$ ) of particle in beam frame (0.1). verbose Non-zero for verbose prints (0).

ignorefieldWhenTracking For testing only (0).

useApproximationonly For testing only (0).

fixedGrid Nonzero prevents re-sizing the grid (0).

percentile Percentile of charge distribution used for grid sizing (99).

minActive Minimum \# active tracks in bunch; if $<0$ is of initial bunch size (-95).

\subsection{2 spacechargelw Lienard-Wiechert space charge computation}

This is a space charge computation that uses macro-particles to simulate more particles than is feasible to track individually. Each macro-particle is tracked as a single particle, but its charge is multiplied by the macro-particle charge when computing the field. The radius of the macro-particle is used to avoid the singularity from a point charge; outside the radius the macro-particle is treated as a point charge; inside the radius the point-charge field is multiplied by ( $\mathrm{r} / \mathrm{radius})^{\wedge} \mathrm{K}$ (radius and $\mathrm{K}$ are parameters).

The trajectory of every particle is kept, and when computing the 
field at a point, the intersection of the point's past lightcone with the trajectory is used to determine the field from the macro-particle; there is a loop over all particles except the one currently being tracked. This computation scales as $\mathrm{N}^{\wedge} 2$, where $\mathrm{N}$ is the number of macro-particles; that makes it computationally infeasible for more than a few hundred macro-particles. But for the particles used, it is correct to within the following approximations: a) using macro-particles, b) linearly interpolating between steps, c) omitting the radiation term in the L-W potential.

The fields are computed using eq. 63.8-9 (p 162) of Landau and Lifshitz, _Classical_Theory_of_Fields_, ignoring the radiation term. The computed fields are used in the usual Geant4 tracking. Particle creation and destruction are handled properly.

This algorithm is primarily intended to test other space charge algorithms.

Named Arguments :

deltat

Time step (ns).

radius

Radius of macro-particles (mm).

charge Charge of macro-particles (times particle charge).

trackTwice $0=$ linear extrapolation, $1=$ track (0)

verbose Non-zero for verbose prints (0).

$\mathrm{K} \quad$ Exponent for macro-particle density (1).

ignorefieldWhenTracking For testing only (0).

\subsection{3 sphere construct a sphere (or section of one)}

This is a direct interface to G4Sphere.

Named Arguments:

innerRadius The inside radius of the sphere (mm)

outerRadius The outer radius of the sphere (mm)

initialPhi The initial Phi value (deg; 0 for all)

finalphi The final Phi value (deg; 360 for all)

initialtheta The initialtheta of the sphere (deg, 0 for all)

finalTheta The finalTheta of the sphere (deg, 180 for all)

maxstep The maximum stepsize in the element (mm)

material The material of the sphere

color The color of the sphere (''=invisible)

kill Set nonzero to kill every track that enters.

\subsection{4 start Define the initial start of centerline coordinates.}

If used, this command must come before any other command that puts an element into the world or affects the centerline coordinates (place, beam, corner, cornerarc, and reference commands). This command may not always be needed, but it is 
needed to eliminate the ambiguities in the global to centerline coordinate transform, and when simulating a ring to ensure that sensible values of the centerline coordinates are used.

Note that the radiusCut is important to reduce or eliminate ambiguities in the global to centerline coordinate transform. It can also be used to 'shield' the beamline to prevent particles from taking unusual paths around the outside of beamline elements.

Named Arguments:

$\begin{array}{ll}\mathrm{x} & \text { The global } \mathrm{x} \text { position of the start. } \\ \mathrm{y} & \text { The global } \mathrm{y} \text { position of the start. } \\ \mathrm{z} & \text { The global } \mathrm{z} \text { position of the start. } \\ \text { initialz } & \text { The initial centerline } \mathrm{z} \text { value. } \\ \text { rotation } & \text { The initial rotation of the centerline. } \\ \text { radiuscut } & \text { The radius cut for the initial segment (mm). } \\ \text { ring } & \text { Set nonzero to indicate a ring is present. }\end{array}$

\subsection{5 test test random number seeds.}

Test

\subsection{6 timentuple Construct an NTuple of tracks at a specified time.}

A time NTuple generates an NTuple of every track at a specified global time. It uses a linear interpolation in the step that straddles the required time, so accuracy will suffer for large steps. The NTuple uses centerline coordinates, if available.

The standard NTuple fields are:

$\mathrm{X}, \mathrm{y}, \mathrm{z}(\mathrm{mm})$

$\mathrm{Px}, \mathrm{Py}, \mathrm{Pz} \quad(\mathrm{MeV} / \mathrm{C})$

$t$ (ns)

PDGid $(11=e-, \quad 13=m u-, 22=$ gamma, $211=$ pit , 2212=proton, ...) EventID (may be inexact above $16,777,215$ )

TrackID

ParentID ( 0 => primary particle)

Weight (defaults to 1.0 )

The following additional fields are appended for format=Extended, format=asciiExtended, and format=rootExtended:

$\mathrm{Bx}, \mathrm{By}, \mathrm{Bz}$ (Tesla)

$\mathrm{Ex}, \mathrm{Ey}, \mathrm{Ez}$ (Megavolts/meter)

ProperTime (ns)

PathLength ( $\mathrm{mm})$

PolX, Poly, Polz (polarization)

InitialKE (MeV when track was created)

Valid Formats (ignore case): ascii bltrackfile dummy for 009 
for009.dat root trackfile Extended asciiExtended rootExtended

Named Arguments (\#=cannot be changed in place cmd):

time The global time of the sampling (ns).

format The NTuple format (see above for list).

filename The filename of the NTuple.

file

Synonym for filename.

require Expression which must be nonzero to include the track (default=1) \#

coordinates Coordinates: global, centerline, or reference $($ default $=c)$.

\title{
5.67 torus construct a torus.
}

This is a direct interface to G4Torus. The major radius is in the $\mathrm{X}-\mathrm{Y}$ plane, with phi=0 along $\mathrm{X}$.

Named Arguments:

innerRadius The inner radius of the torus (mm)

outerRadius The outer radius of the torus ( $\mathrm{mm}$ )

majorRadius The major radius of the torus ( $\mathrm{mm}$ )

initialphi The initial phi around major radius (0 degrees).

finalphi The final phi around major radius (360 degrees).

maxstep The maximum stepsize in the element (mm)

material The material of the torus

color The color of the torus ('"=invisible)

kill Set nonzero to kill every track that enters.

\subsection{8 totalenergy Print total energy deposited in selected volumes.}

\author{
At end of run, prints the total energy deposited in the selected \\ volumes. \\ Volume-name patterns are like UNIX filename patterns: e.g. \\ '*[AB]*' matches any name containing an $A$ or a $B$. \\ Tracks that are killed have their kinetic energy summed into the \\ volume where they were killed, unless they are killed because \\ they leave the World. \\ With ancestors=1, energy deposited in matching volumes is added \\ into their ancestors; energy deposited directly into those \\ ancestors is not summed into them unless their names also match. \\ That is, if $A$ is a child of $B$, but only $A$ matches the list of \\ volume-names, energy deposited into $A$ will be reported in both $A$ \\ and B, but energy deposited directly into B is ignored. \\ Named Arguments : \\ volumes \\ ancestors \\ Comma-separated list of Volume-Name patterns (*) \\ set nonzero to sum energy into all ancestor
}


enclosing

filename

file (enclosing) volumess (0).

Synonym for ancestors.

Filename to write summary (stdout).

Synonym for filename.

\subsection{9 trace Specifies tracing of tracks.}

Generates a separate NTuple for each track, with 1 row per step, unless oneNTuple is nonzero (in which case all tracks are put into a single NTuple). So format=ascii generates one file per track with names generated by the pattern in filename (first od is replaced by event \#, second od is replaced by trackId); for oneNTuple, the default filename is AllTracks.txt.

Note that without a trace command no traces are generated, so to trace just the tune and reference particles include a trace command with no arguments.

In collective tracking mode, oneNTuple must be nonzero, and the entries will be generated only at collective steps (usually at a specified deltat).

Unlike other NTuple commands, the require expression applies to entire tracks, not individual entries.

The standard NTuple fields are:

$\mathrm{x}, \mathrm{y}, \mathrm{z}(\mathrm{mm})$

$\mathrm{Px}, \mathrm{Py}, \mathrm{Pz} \quad(\mathrm{MeV} / \mathrm{c})$

$t$ (ns)

PDGid $(11=e-, \quad 13=$ mu,$- 22=$ gamma, $211=$ pit , 2212=proton, ...)

EventID (may be inexact above 16,777,215)

TrackID

ParentID ( 0 => primary particle)

Weight (defaults to 1.0 )

The trace includes the following fields:

$\mathrm{Bx}, \mathrm{By}, \mathrm{Bz}$ (Tesla)

Ex, Ey, Ez (Megavolts/meter)

The following additional fields are appended for format=Extended, format=asciiExtended, and format=rootExtended:

ProperTime (ns)

PathLength (mm)

PolX, Poly, Polz (polarization)

InitialKE (MeV when track was created)

Valid Formats (ignore case): ascii bltrackfile dummy for 009

for 009 .dat root trackfile Extended asciiExtended rootExtended

Named Arguments (\#=cannot be changed in place cmd):

nTrace Number of tracks to trace.

format Format of the NTuple (see above for list).

oneNTuple Nonzero to put all traces into a single NTuple.

primaryonly Nonzero to trace only primary tracks. 
filename

file

require

coordinates
Filename (EvodTrkod.txt or AllTracks.txt) • synonym for filename.

Expression which must be nonzero to trace the track (default=1) \#

Coordinates: global, centerline, or reference

$($ default $=c)$.

\subsection{0 trackcolor Alias for 'particlecolor'.}

\subsection{1 trackcuts Specifies per-track cuts.}

Applied to each track before tracking, and at each step.

Named Arguments:

kill List of particles to kill (comma separated).

keep List of particles to keep (kill all others).

killsecondaries set nonzero to kill all secondaries.

kineticEnergyCut Minimum K.E. to track ( $0 \mathrm{MeV})$.

kineticEnergyMax Maximum K.E. to track (infinite MeV).

maxTime Maximum lab time to track (1000000 ns).

keepPrimaries set nonzero to keep tracks with ParentID==0

regardless of other tests.

steppingVerbose set nonzero to print kills (defaults to parameter value).

\subsection{2 tracker Defines a tracker.}

A tracker consists of several trackerplane-s and can fit a track to wire hits and times in the trackerplanes. This is a simple algorithm that does not handle backgrounds or multiple hits. It assumes that every track hits each trackerplane at most once. It is intended to be used to explore resolutions and the effects of survey errors. A tracker is a logical combination of its trackerplanes -- the tracker cannot be placed, but its trackerplanes must be placed into the system.

The fitting algorithm used requires that all of its parameters have comparable scales, so the 'scalex', 'scalexp', 'scaleptot', and 'scaleT' arguments should be set to the approximate sigmas of the tracker. They should be within a factor of 10 of the actual values, but closer is better. At the end of fitting tracks a summary is printed that flags each parameter with 'RESCALE' if its scale is too different from its sigma (factor of 5 or more).

NOTE: if the tracker cannot measure Ptot, then 'scalePtot' MUST be set to zero. If the tracker cannot measure $T$, then scaleT MUST 
be set to zero. Parameters with zero scales are held fixed at their true-track values.

A tracker has 3 modes of operation:

true Each track of each event is written to a TrackerHits NTuple if it hits all trackerplanes; the NTuple includes both the true track values

fit and the individual wire hits for each trackerpla run, and the fit track is written to a TrackerFit NTuple (includes true values).

ignore Any track is ignored.

See the 'trackermode' command to control the mode of trackers.

The TrackerHits NTuple written in 'true' mode contains: true_x, true_y, true_z, true_Px, true_Py, true_Pz, true_t, true_PDGid, true_EventID, true_TrackID, true_ParentID, true_Weight, ... plus 1 hit and 1 time per trackerplane. (the first 12 are the same as a BLTrackFile.)

The TrackerFit Ntuple written in 'fit' mode contains: $\mathrm{x}, \mathrm{y}, \mathrm{z}, \mathrm{Px}, \mathrm{Py}, \mathrm{Pz}, t, \mathrm{PDGid}$, EventID, TrackID, ParentID, true_Px, Weight, ChisqPerDF, nDF, nHit, nIter, true_x, true_y, true_z,

true_Py, true_Pz, true_t. (the first 12 are from the fit and are the same as a BLTrackFile.)

The parameters of the fit are: $x, y, d x d z, d y d z$, Ptot, time. You must ensure that there are at least as many data points as free parameters (scaleT=0 fixes time; scaleptot=0 fixes Ptot). Each trackerplane with nonzero wirespacing provides a data point; each trackerplane with nonzero sigmaT provides a data point; trackerplanes that measure both provide two data points. You must have enough trackerplanes to meet this requirement, and must set minHits large enough to meet it. The TrackerFit NTuple has a field nDF that gives the number of degrees of freedom for the fit, which is defined as (\#DataPoints)-(\#FreeParameters); it also has nHit which gives the number of trackerplane-s hit.

Note the tracker can simulate survey errors -- see the 'trackerplane' command for details (each plane can have different errors).

Both the true and the fit tracks are reported at reportz, which defaults to the $\mathrm{z}$ position of the tracker.

Note that beamlossntuple and newparticlentuple will get many entries per track when used in mode=fit -- the fit runs the track many times through the tracker (30-100, up to maxiter).

NOTE: the trackermode command must preceed all tracker commands in the input file, and each tracker command must preceed all of its trackerplane commands. The trackerz value must also preceed 
all trackerplane-s, but reportz can be equal to or anywhere after trackerz.

Note that each trackerplane must have a unique name. This means you should either have a separate trackerplane command for each one (with unique name), or use the rename= argument to the place command (again with unique name). If you use groups for trackerplane-s, use rename=+ in the group.

Named Arguments :

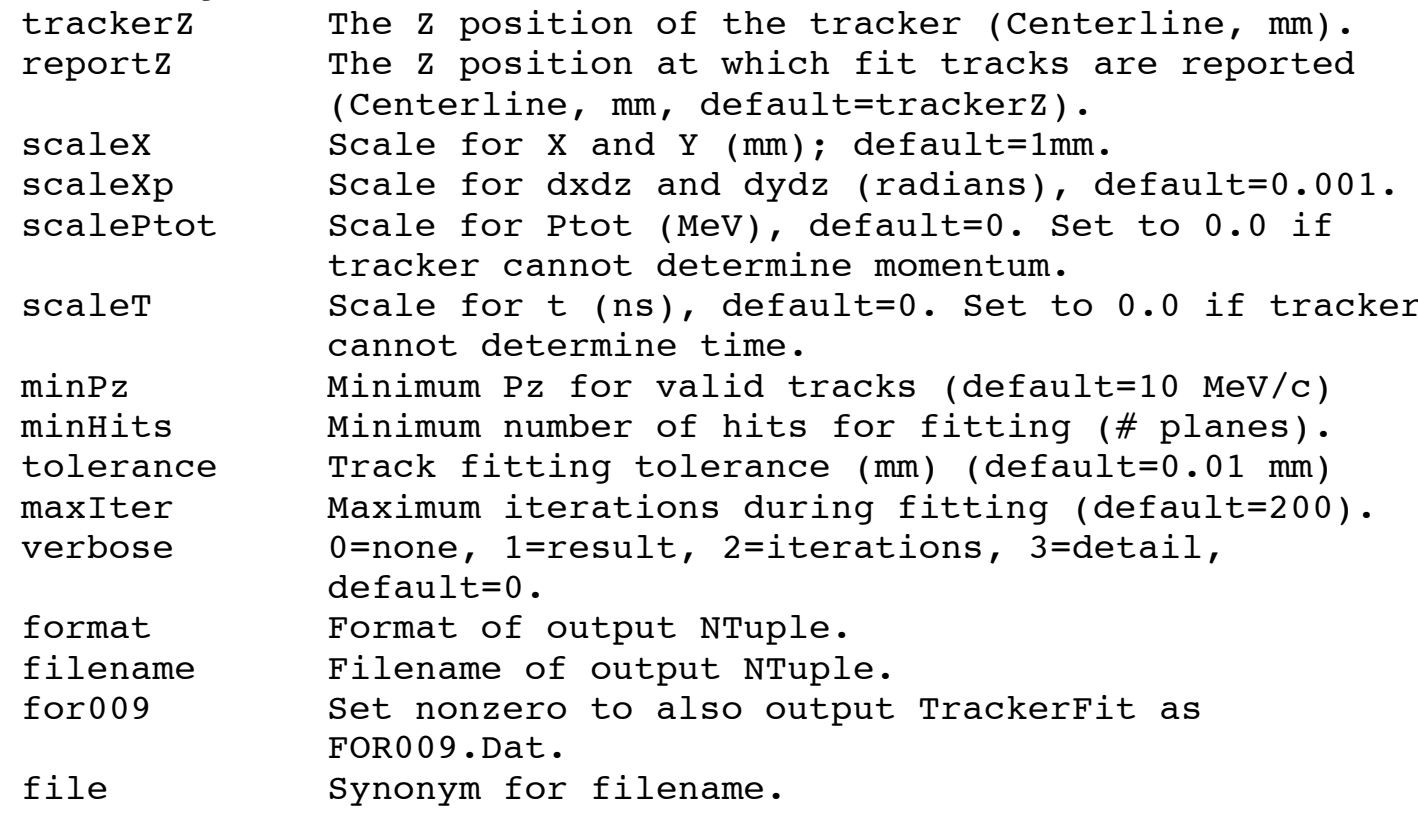

\subsection{3 trackermode Sets mode for all trackers, manages track fitting.}

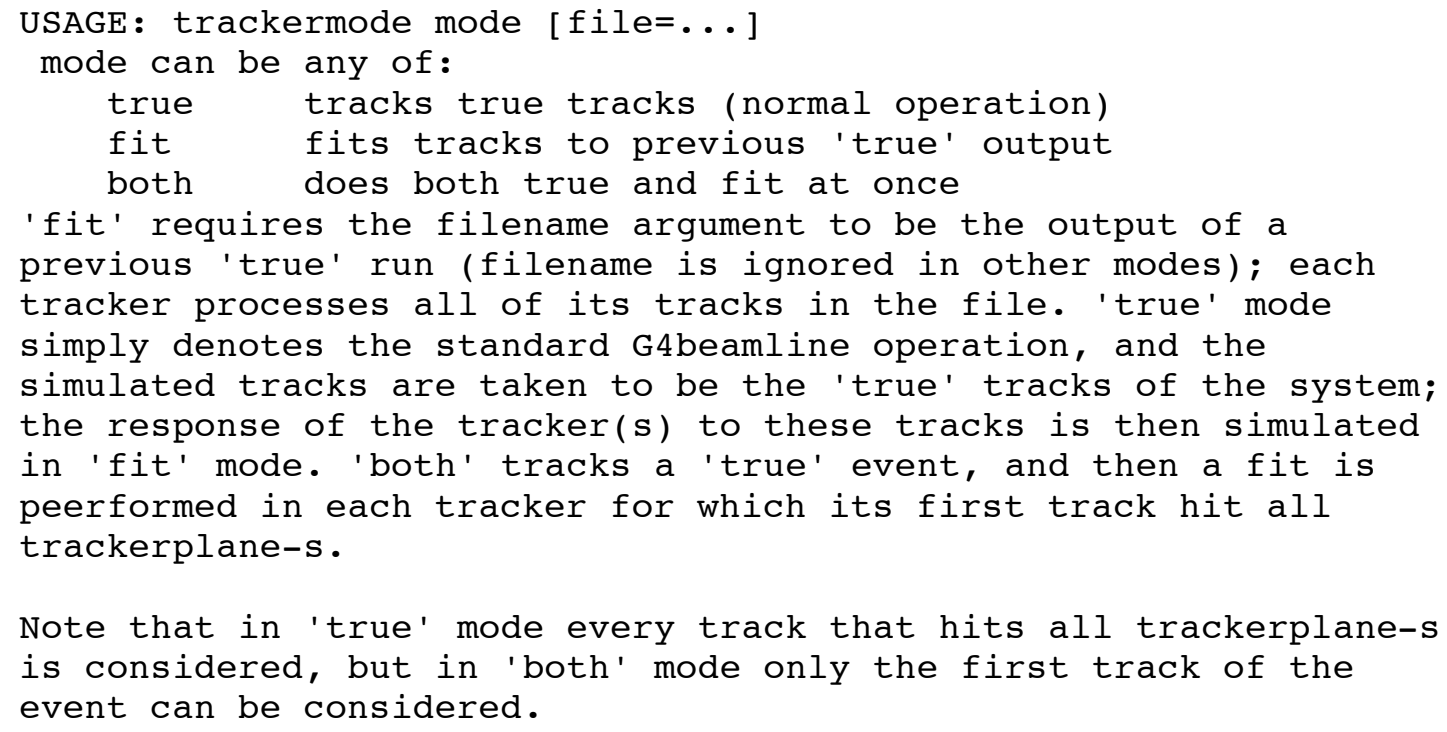

Note that in 'true' mode every track that hits all trackerplane-s is considered, but in 'both' mode only the first track of the event can be considered.

One trackermode command controls the mode of all trackers. 'true' 
mode is normal G4beamline operation, and is the same as if no trackermode command was present.

Note that the geometry of the system must not change between a 'true' run and a 'fit' run. You can, however, make small variations in fields to explore how errors in setting them will affect the fitted tracks. The trackerplane command can simulate survey errors.

NOTE: the trackermode command must preceed all tracker commands in the input file.

Named Arguments :

filename Filename to read for fitting tracks.

file Synonym for filename.

\subsection{4 trackerplane Construct a tracker plane.}

A trackerplane belongs to a specific tracker, and represents one measuring element of the tracker. While the term 'wire' is used, a trackerplane can model any planar measuring device that measures one dimension using equally spaced detectors that are either on or off (hit or not hit). A trackerplane can be circular (specify radius and possibly innerRadius) or rectangular (specify height and width).

The wires are at angle theta from the vertical, so theta=0 means vertical wires that measure $\mathrm{x}$; theta $=90$ means horizontal wires that measure $\mathrm{y} ;$ theta $=180$ also measures $\mathrm{x}$, but increasing $\mathrm{x}$ means decreasing wire \#.

Setting wirespacing=0 means there are no wires, which is useful for a trackerplane that models a scintillator used for track timing (set sigmaTime $>=0$ to indicate that).

sigmaTime>0 means this plane can measure the time of the track with that resolution. A Gaussian random number is added to the true track's time at this plane when reading the TrackHit NTuple.

Survey errors can be modeled using the err-arguments -- the values they specify are applied during trackfitting (but not for true tracks). errType: 'fixed' means error values given are the actual values, 'gaussian' means error values are the sigma of a Gaussian random number, 'rect' means error values are the half-width of a uniform random number. The random number is picked before the run begins; the random-number seed is set from the clock so every run will have different random errors.

trackerplane has 3 modes:

true The trackerplane reports the hit wire and time to the tracker;

fit The trackerplane reports the Chisq contribution of the fit track distance to the true track's hit wire 


\subsection{7 tubs}

\section{construct a tube or cylinder with axis along $z$.}

This is a direct interface to G4Tubs, which can implement a tube or cylinder; either can subtend less than 360 degrees in phi.

Named Arguments:

innerRadius The inside of the tube, 0.0 for cylinder (mm)

outerRadius The outer radius of the tube or cylinder (mm)

initialPhi The initial Phi value (deg; 0 for all)

finalphi The final Phi value (deg; 360 for all)

length The length of the tube or cylinder ( $\mathrm{mm}$ )

maxstep The maximum stepsize in the element (mm)

material The material of the tube or cylinder

color The color of the tube or cylinder (''=invisible)

kill Set nonzero to kill every track that enters.

radius Synonym for outerRadius ( $\mathrm{mm}$ )

\subsection{8 tune Tune a variable used as argument to other elements.}

This command samples the Tune particle track at $z 0$, samples it again at $z 1$, and varies its tune variable in order to bring the expression to zero. The tune variable is the first positional argument, and can be used in the argument expression(s) for tunable arguments located after z0. Due to the simple solver used, there should be an approximately linear dependence between the tune variable and the expression. This is suitable for tuning the By field of a genericbend or the maxGradient of a pillbox. At each solver step the saved Tune particle is re-started from $z 0$, and when it reaches $\mathrm{zl}$ the next step in the solver is taken.

Note that multiple tune commands can be used together, as long as their z0-z1 regions are properly nested by at least $0.5 \mathrm{~mm}$ (i.e. each pair of regions must either not overlap at all, or one must be wholly contained in the other). This command can be complicated to use; see the User's Guide for more description and examples.

Named Arguments:

$\mathrm{z} 0$

$\mathrm{z} 1$

initial initialstep step

start

expr

tolerance maxIter
The starting $\mathrm{z}$ position in $\mathrm{CL}$ coordinates. The ending $z$ position in CL coordinates. Initial value of the variable 'name' Initial step ( 0 to disable tuning) Synonym for initialstep An expression that must be nonzero to start tuning (default=1) The expression to tune to zero

The tolerance for expr to be zero The maximum number of iterations (10). 


\subsection{9 usertrackfilter Construct a usertrackfilter that filters tracks via user code.}

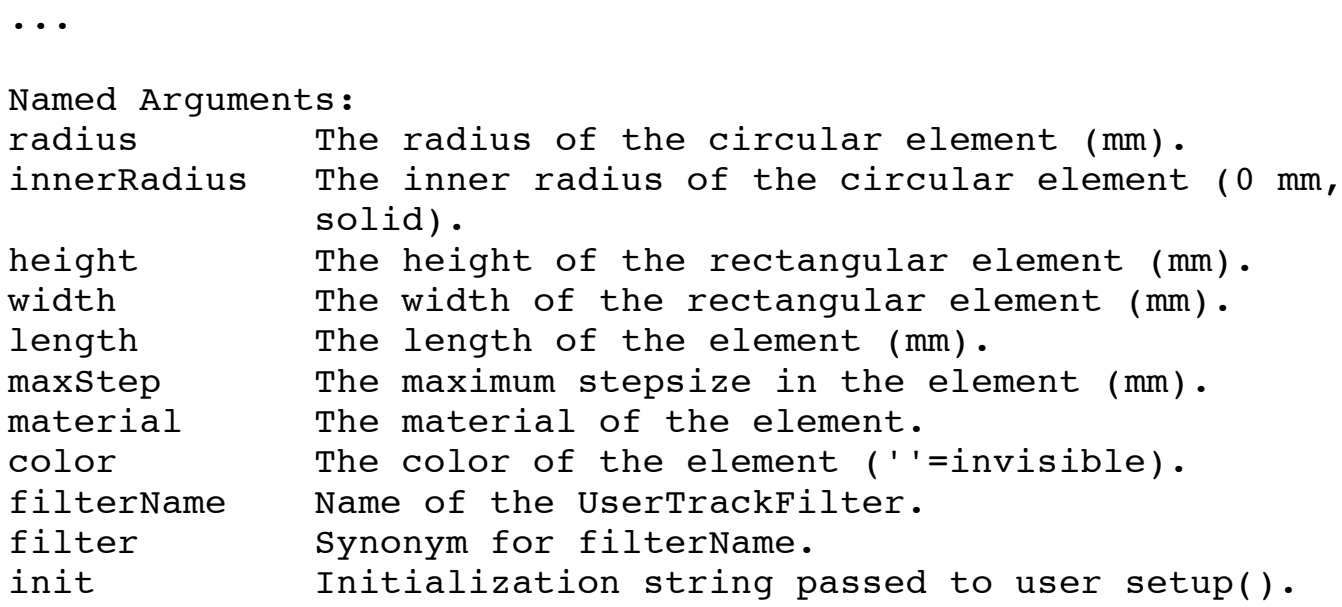

\subsection{0 virtualdetector Construct a VirtualDetector that generates an NTuple.}

A VirtualDetector generates an NTuple of any track when it enters the physical volume of the virtualdetector. It may be placed via multiple place commands (usually with a 'rename=det\#' argument to distinguish the different placements). If material is not specified, it uses the material of the enclosing element. Every placement creates an individual NTuple. For a circular VirtualDetector give radius; for a rectangular one give height and width; length is usually left at $1 \mathrm{~mm}$, but can be set to correspond to the length of a physical detector. The NTuple by default uses centerline coordinates. The NTuple of the virtualdetector can be included in an ntuple command by including a pattern that matches its name in the 'detectors' argument to the ntuple command. Note that must match the name as placed (i.e. includes rename=), not the name given to this command. The nosingles argument may be useful in this case to avoid a huge NTuple of singles (an empty NTuple may be created).

Note that secondary particles created within the virtualdetector will not get an entry until they have taken one step. They are guaranteed to do so.

The standard NTuple fields are:

$\mathrm{x}, \mathrm{y}, \mathrm{z}(\mathrm{mm})$

$\mathrm{Px}, \mathrm{Py}, \mathrm{Pz} \quad(\mathrm{MeV} / \mathrm{C})$

$t$ (ns)

PDGid $(11=e-, \quad 13=m u-, 22=$ gamma, $211=$ pit, 2212=proton, ...)

EventID (may be inexact above $16,777,215$ )

TrackID

ParentID ( $0=>$ primary particle)

Weight (defaults to 1.0 ) 
The following additional fields are appended for format=Extended, format=asciiExtended, and format=rootExtended:

$\mathrm{Bx}, \mathrm{By}, \mathrm{Bz}$ (Tesla)

Ex, Ey, Ez (Megavolts/meter)

ProperTime (ns)

Pathlength (mm)

PolX, Poly, Polz (polarization)

InitialKE ( $\mathrm{MeV}$ when track was created)

Valid Formats (ignore case): ascii bltrackfile dummy for 009

for 009 .dat root trackfile Extended asciiExtended rootExtended

Named Arguments (\#=cannot be changed in place cmd):

radius The radius of the circular VirtualDetector (mm).

innerRadius The inner radius of the circular VirtualDetector (0

$\mathrm{mm}$, solid).

height The height of the rectangular VirtualDetector ( $\mathrm{mm})$.

width The width of the rectangular VirtualDetector (mm).

length The length of the VirtualDetector (mm).

maxstep The maximum stepsize in the element (mm).

material The material of the virtualdetector.

color The color of the Virtualdetector (''=invisible).

noSingles Set to 1 to omit the NTuple for singles.

format NTuple format: (see above for list).

filename filename ('' uses name to determine filename)

file alias for filename

require Expression which must be nonzero to include the track (default=1) \#

referenceparticle set to 1 to include the Reference Particle.

coordinates Coordinates: global, centerline, or reference (default=c) .

kill

Set to 1 kill all tracks after entering them into NTuple (s) .

\subsection{1 zntuple Generate an NTuple for each of a list of $Z$ positions.}

Generates an NTuple like a virtualdetector, but without a physical volume. Tracks are forced to take steps within $2 \mathrm{~mm}$ surrounding each desired $z$ position, and they are interpolated to the desired $z$ position. Each $z$ position generates a separate NTuple named $z 123$ (etc.). z accepts a list of z positions, and zloop can generate a set of equally spaced $z$ positions; both can be used.

Each value in $\mathrm{z}$ and zloop can be an expression using double constants and the usual $\mathrm{C}$ operators and functions.

The standard NTuple fields are:

$\mathrm{x}, \mathrm{y}, \mathrm{z}(\mathrm{mm})$

$\mathrm{Px}, \mathrm{Py}, \mathrm{Pz} \quad(\mathrm{MeV} / \mathrm{c})$

$t$ (ns)

PDGid $(11=e-, \quad 13=$ mu- , 22=gamma, 211=pit, 2212=proton, ...)

EventID (may be inexact above $16,777,215$ ) 


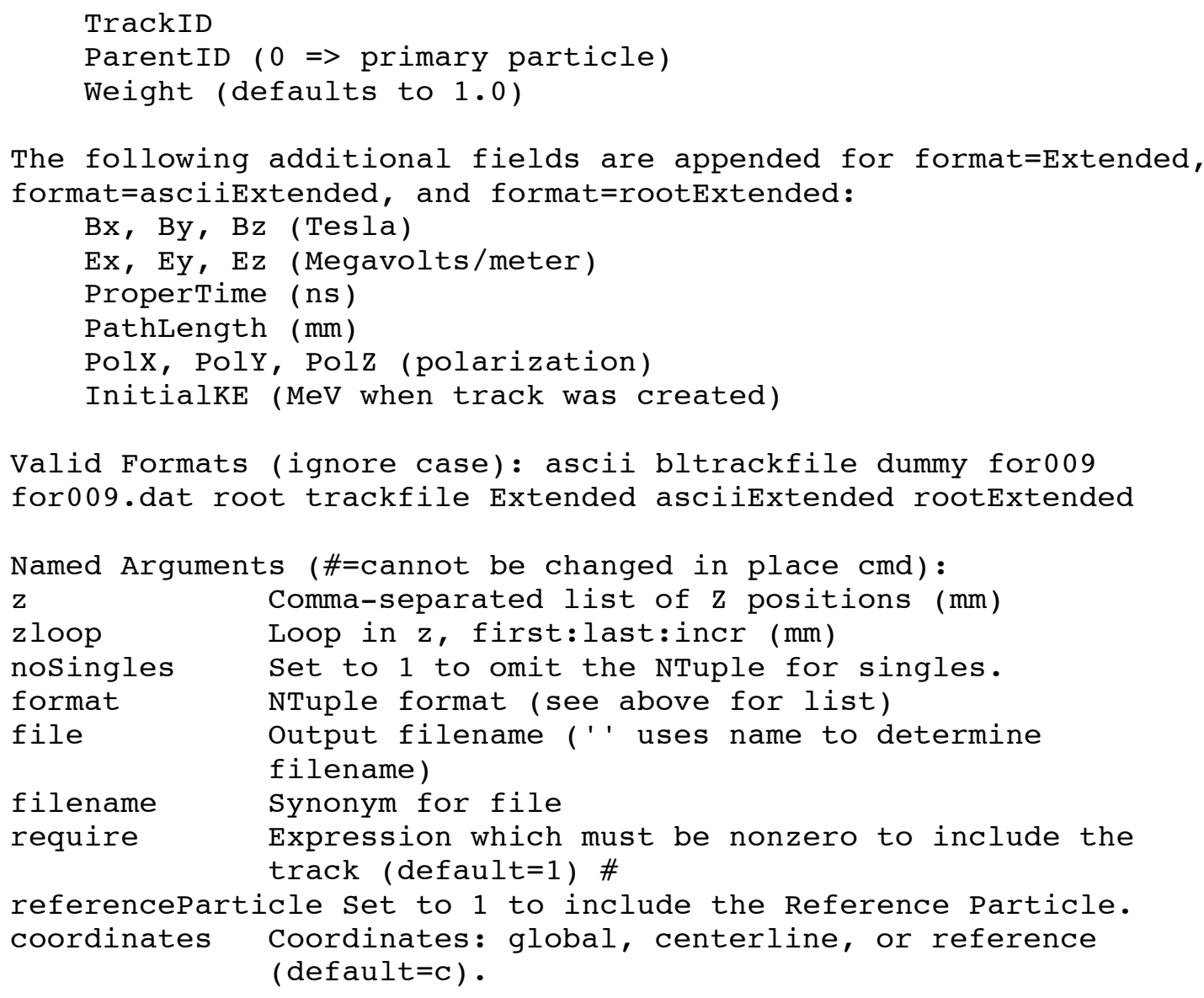




\section{Examples}

On Linux and Mac OS, the examples are found in the "examples" directory under the install directory. On Windows, they are found there also, and a copy is placed into "My Documents $\backslash$ G4beamline Examples" ("My Documents" => "Documents" on Vista).

The annotated output from example1 is given in Appendix 5.

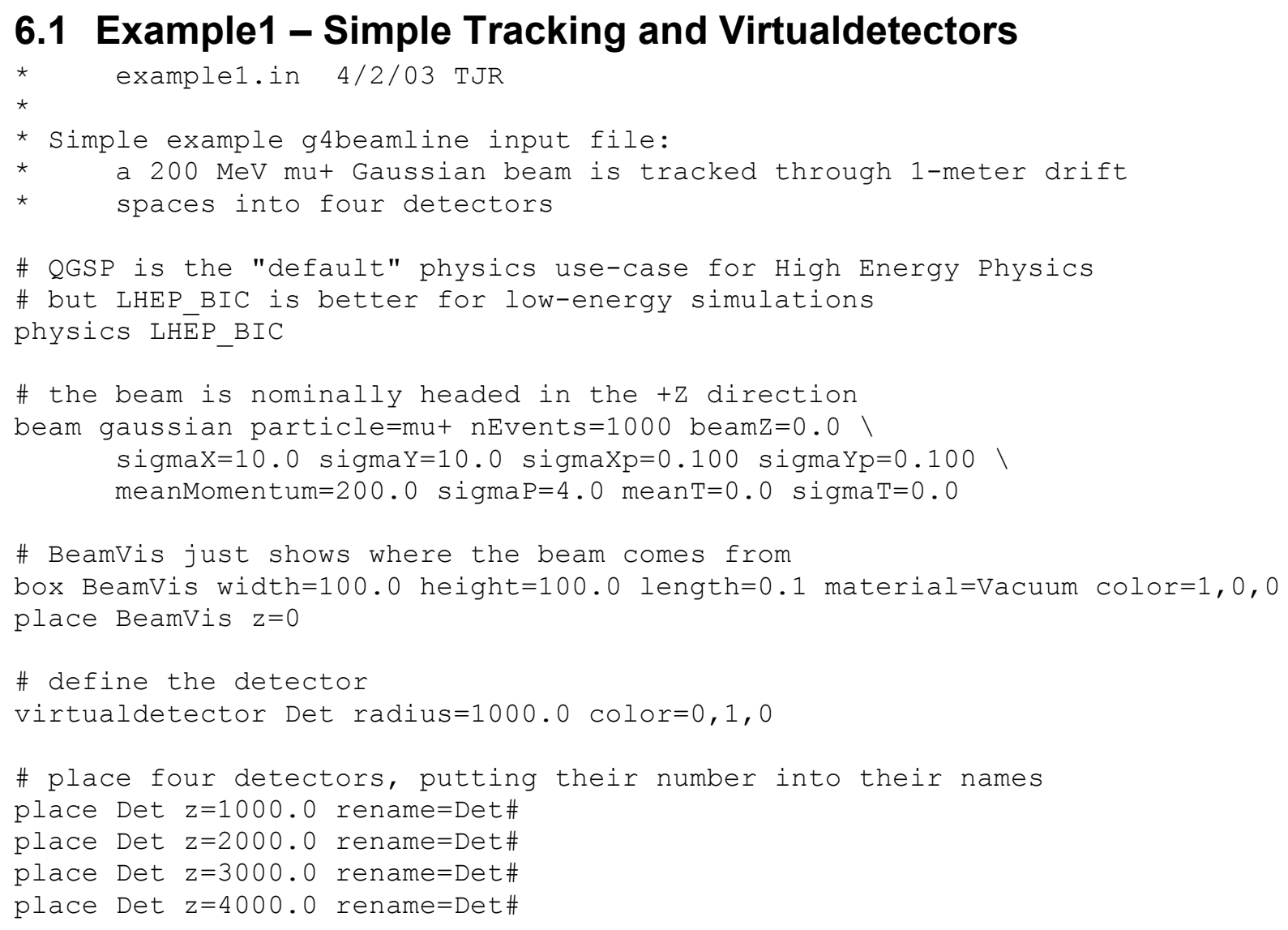

\subsection{Example2 - 4 Cells of the Study 2 Cooling Channel}

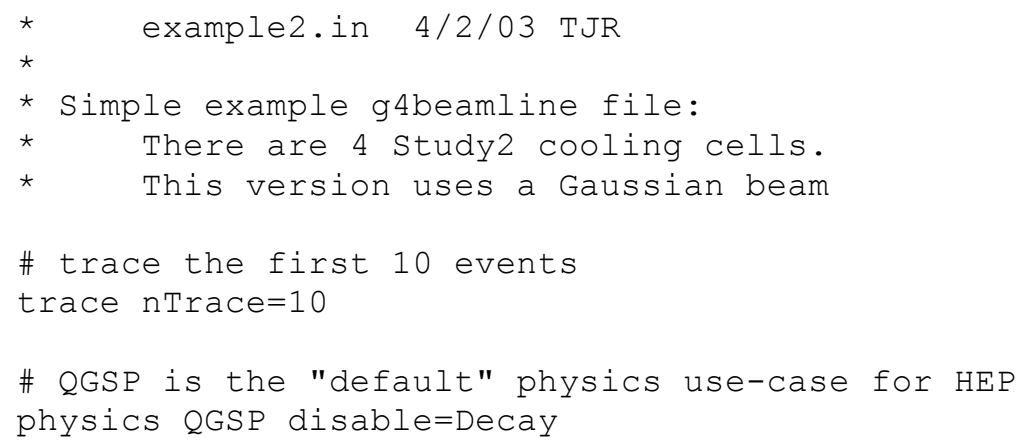




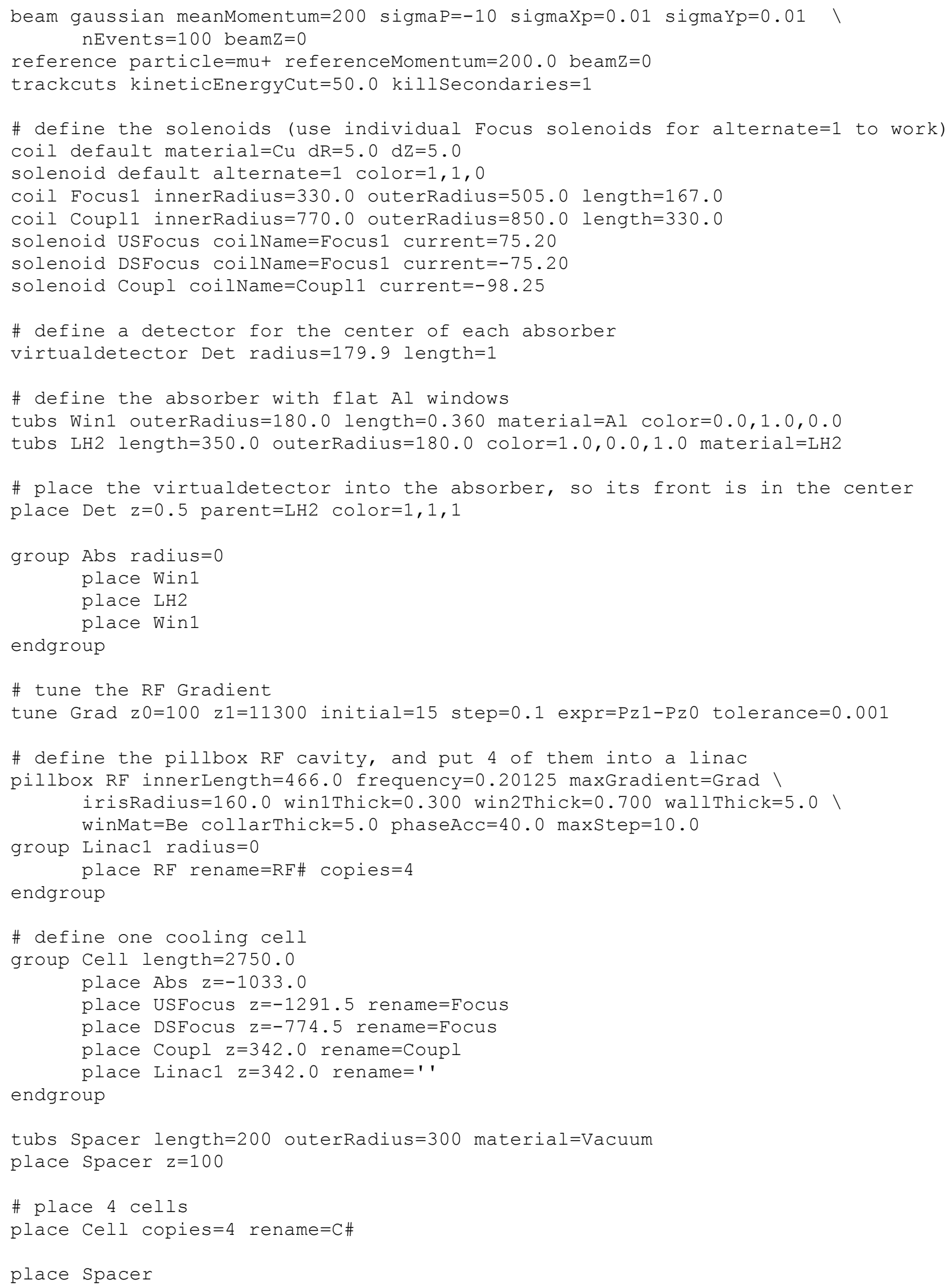




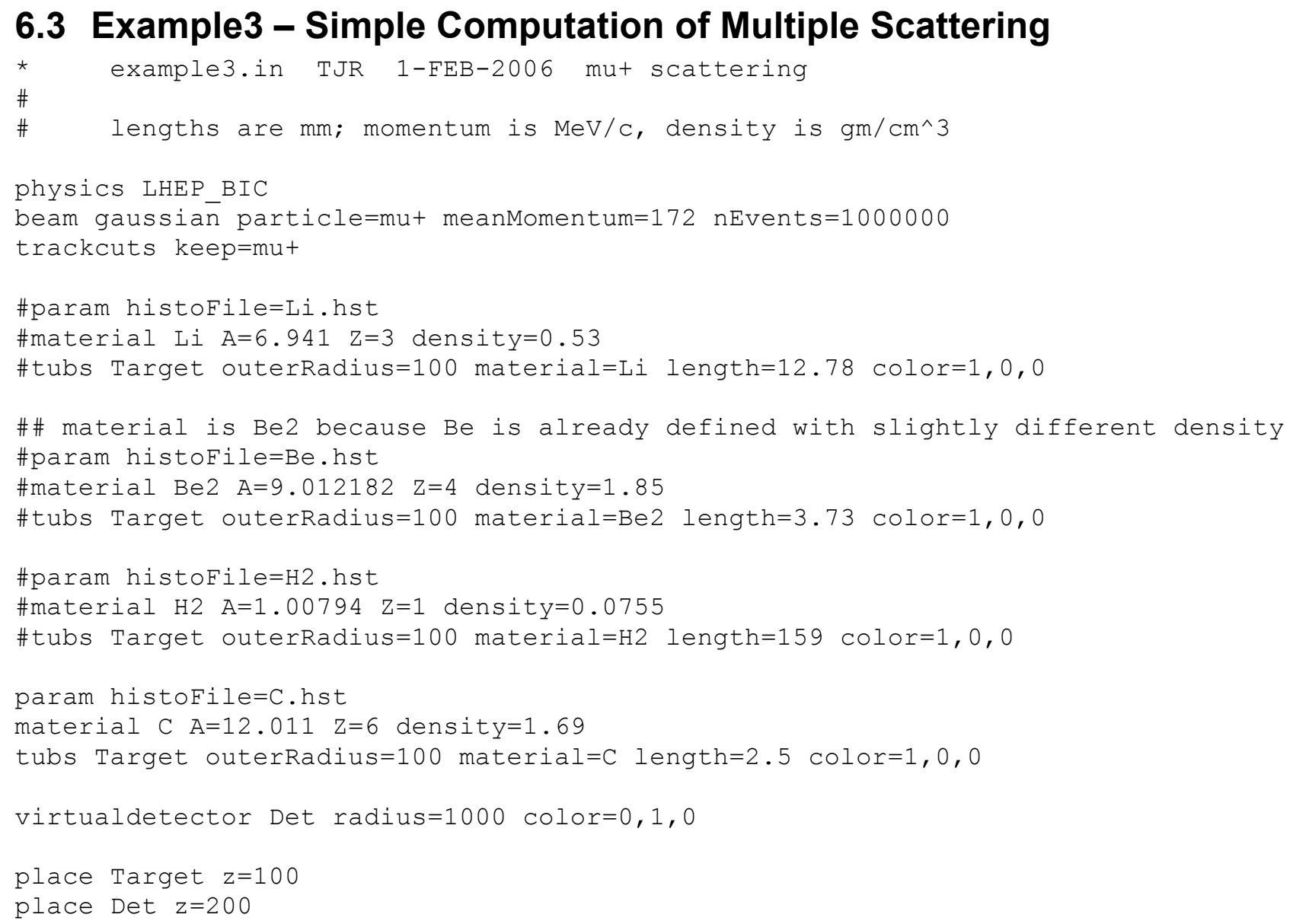

\subsection{Example4 - $8 \mathrm{GeV}$ Proton beam into a Tungsten Target}

\# example4.in - $8 \mathrm{GeV}$ proton beam into a tungsten target

\# The "default" physics list is QGSP BERT

physics QGSP_BERT

\# the beam is $8 \mathrm{GeV}$ kinetic energy, the mass of a proton is $938.272 \mathrm{MeV} / \mathrm{C}^{\wedge} 2$ param $\mathrm{M}=938.272 \mathrm{KE}=8000.0$

param $\mathrm{P}=\operatorname{sqrt}((\$ \mathrm{M}+\$ \mathrm{KE}) *(\$ \mathrm{M}+\$ \mathrm{KE})-\$ \mathrm{M} * \$ \mathrm{M})$

\# a zero-emittance beam is unrealistic, but simple; it easily fits through

\# a $1 \mathrm{~mm}$ hole in the backward detector. It emanates from $z=0$.

beam gaussian meanMomentum $=\$ P$ nEvents $=1000$ particle=proton

\# the target is a tungsten rod $20 \mathrm{~cm}$ long and $1 \mathrm{~cm}$ in diameter; make it red cylinder Target outerRadius $=5$ length $=200$ material=W color $=1,0,0$

place Target $z=200$ 


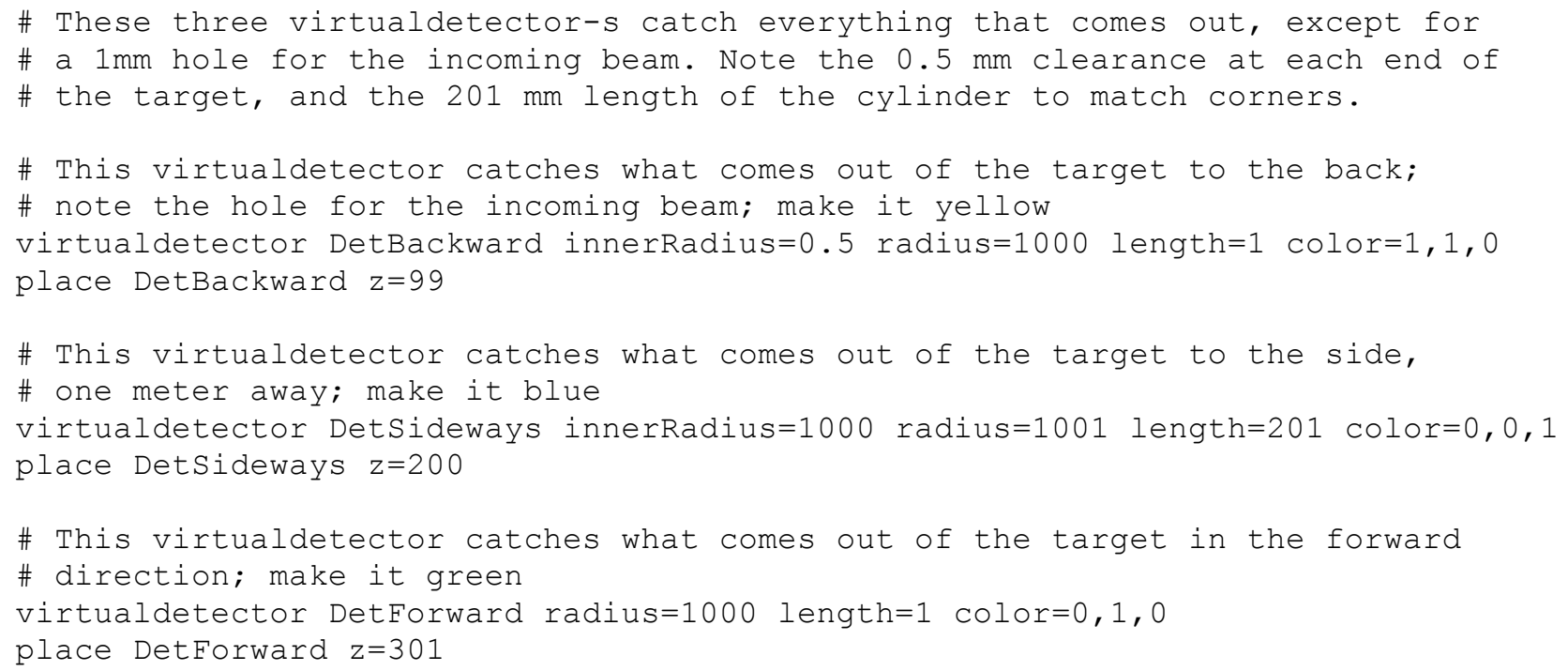

\subsection{ExampleN02 - the Geant4 Novice Example N02}

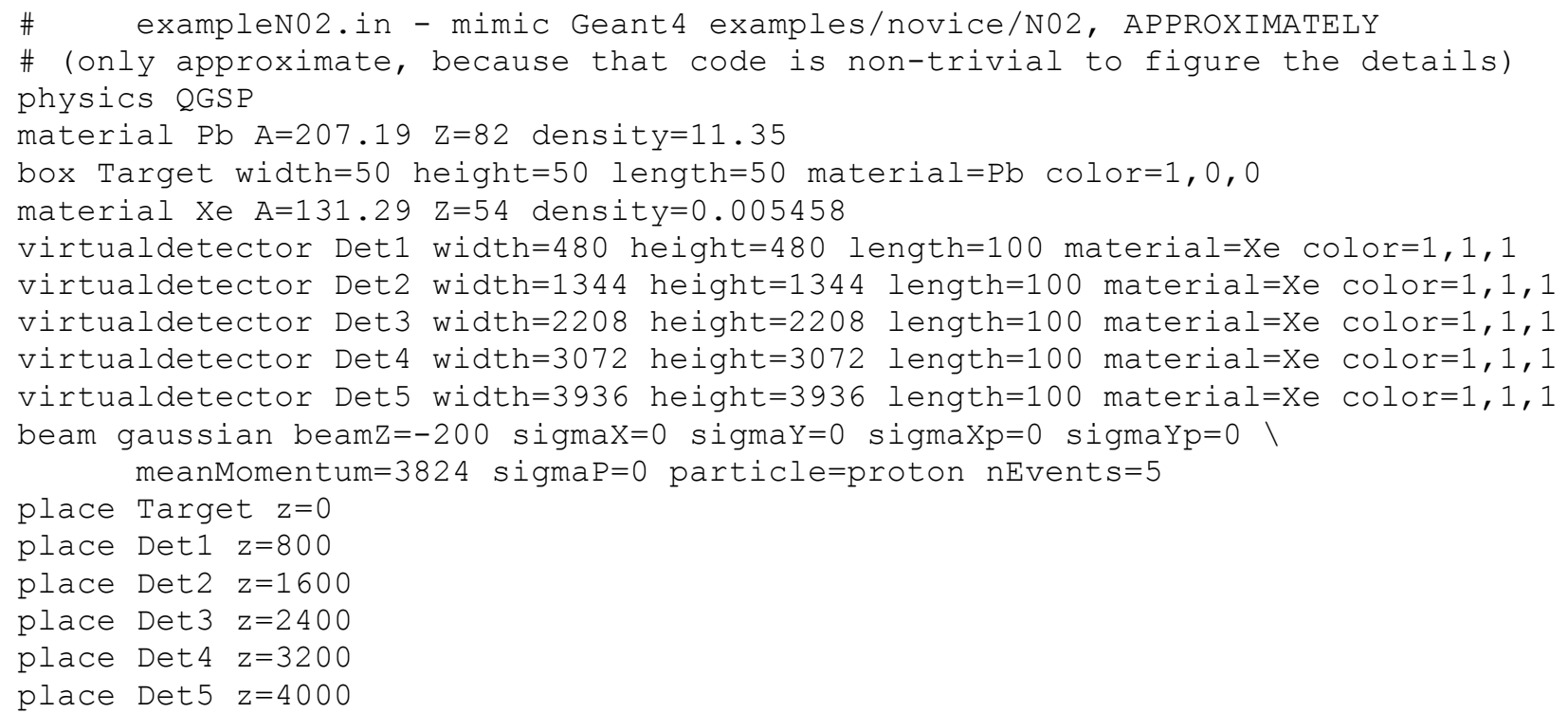

\section{6 triplet.sh - tune a quad triplet for point-to-point focus}

This shell script requires the bash shell and the following programs:

- Gbeamline (http://g4beamline.muonsinc.com )

- gminuit (http://muonsinc.com ComputerPrograms/gminuit)

- gnuplot (available from your Linux distribution or http://sourceforge.net )

- tclsh and wish (available from your Linux distribution)

The gminuit program is configured to have the following parameters: $\mathrm{P}, \mathrm{dP}$, sigmaXp, sigmaYp, QF, QD. The first four determine the initial beam (which emanates from a point), and the last two determine 
the gradients of the three quads. The Chisquared to be minimized is (sigmaX $\left.{ }^{\wedge} 2+\operatorname{sigmaY}^{\wedge} 2\right)$ evaluated at $Z=6000$, so the minimum Chisquared will be a point focus. Because of the front-to-back symmetry, the two horizontal-focusing quads have the same gradient.

Gminuit presents the user with a window containing sliders and edit-boxes for the parameters, plus buttons to execute the script once and to fit the parameters (see the gminuit documentation for details):

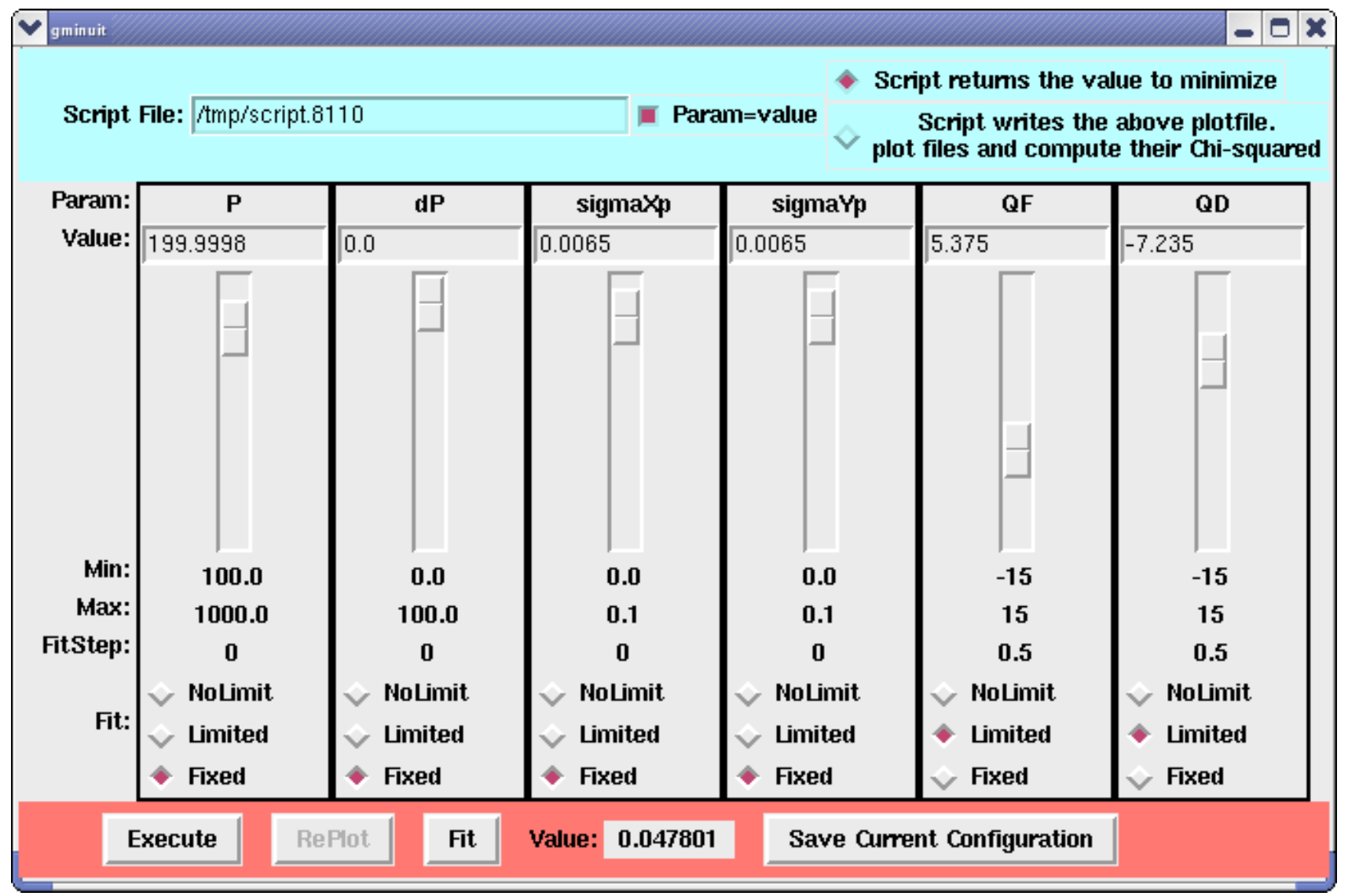

The script run by gminuit computes the Chisquared to be minimized, and also plots the profile of the beam (as traditional, $3 *$ sigmaX is on top and $-3 *$ sigmaY is below the axis, with the apertures of the quads plotted in green): 


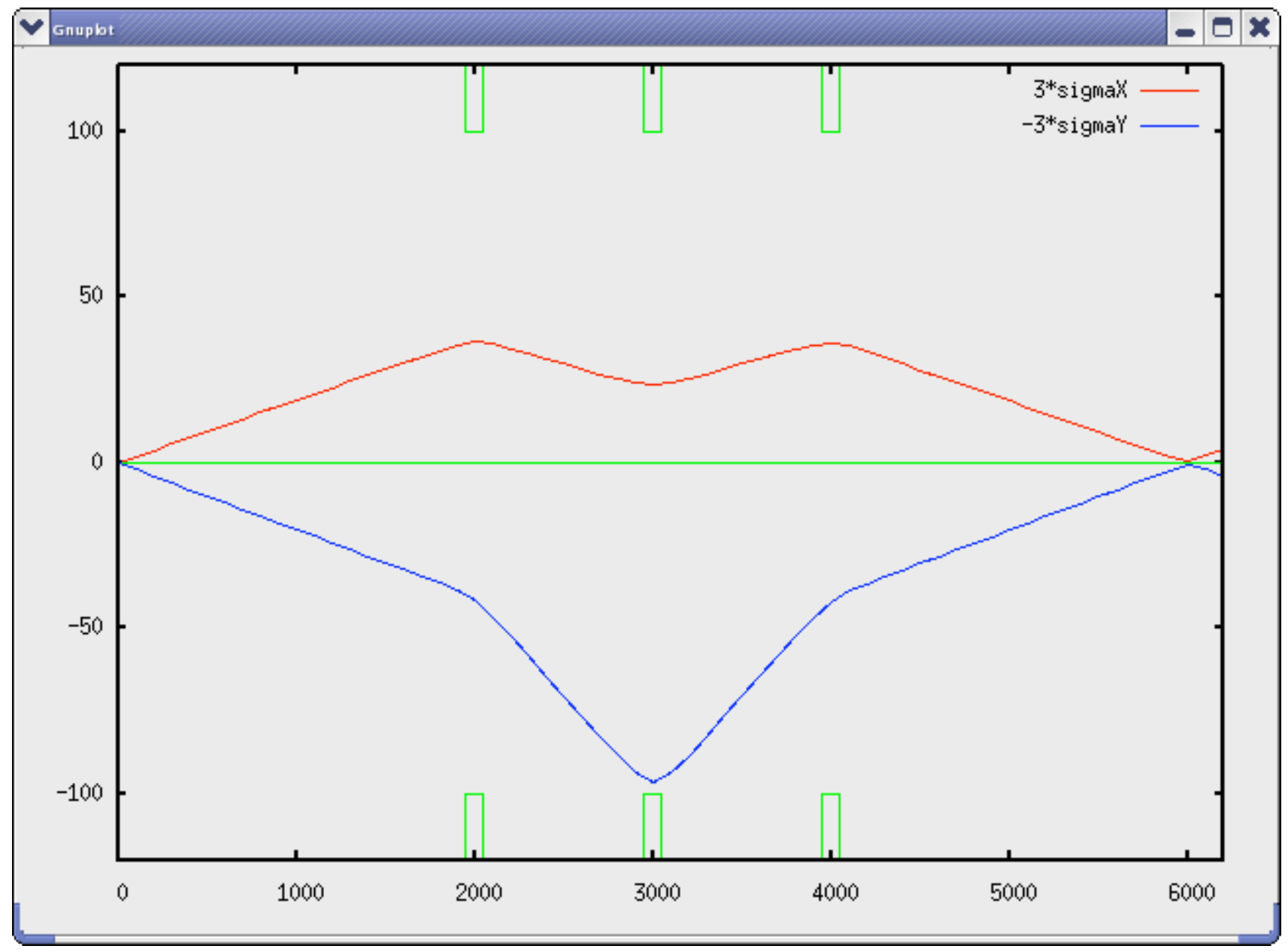

In practice, a human user can find the best tune better than the minuit minimization engine. This is primarily because the human can watch the effect of parameter changes on the entire profile plot, and use experience and knowledge that minuit does not have.

\section{7 emittancematch.sh - attempt to match a quad triplet into a solenoid}

This script is similar to triplet.sh, except it adds a solenoid after the quad triplet. This is incomplete, but demonstrates the gnuplot commands necessary to plot the profile, emittance, and beta of the beam.

\subsection{ExampleAUG05 - the MICE Muon Beam Line}

NOTE: this input file is intended for use on a Linux cluster, and expects two parameters on the command-line to manage events and HistoScope output files: first and last (defaults are 0 and 1000).

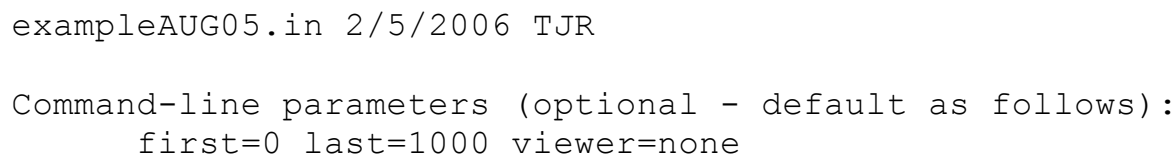




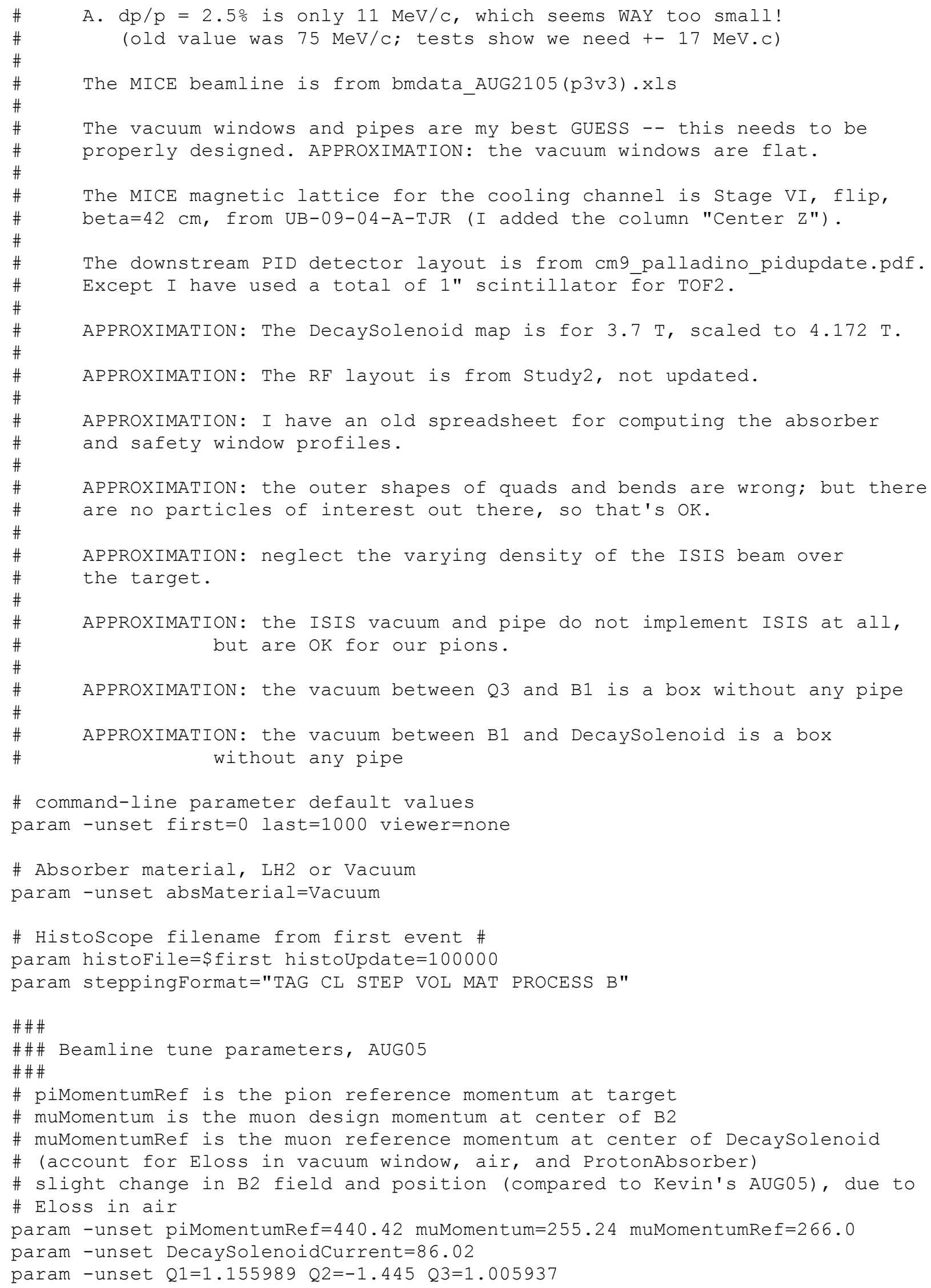




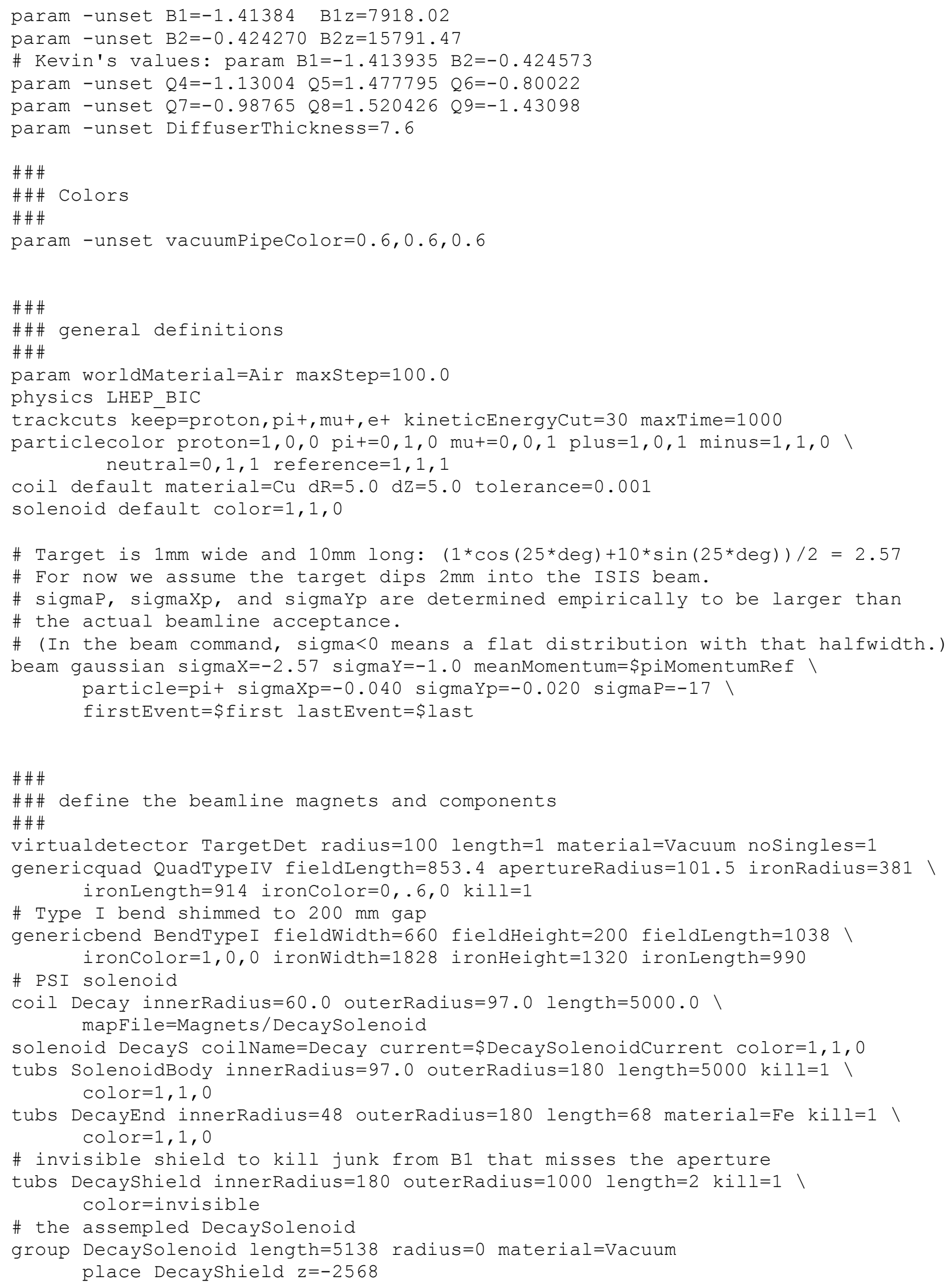




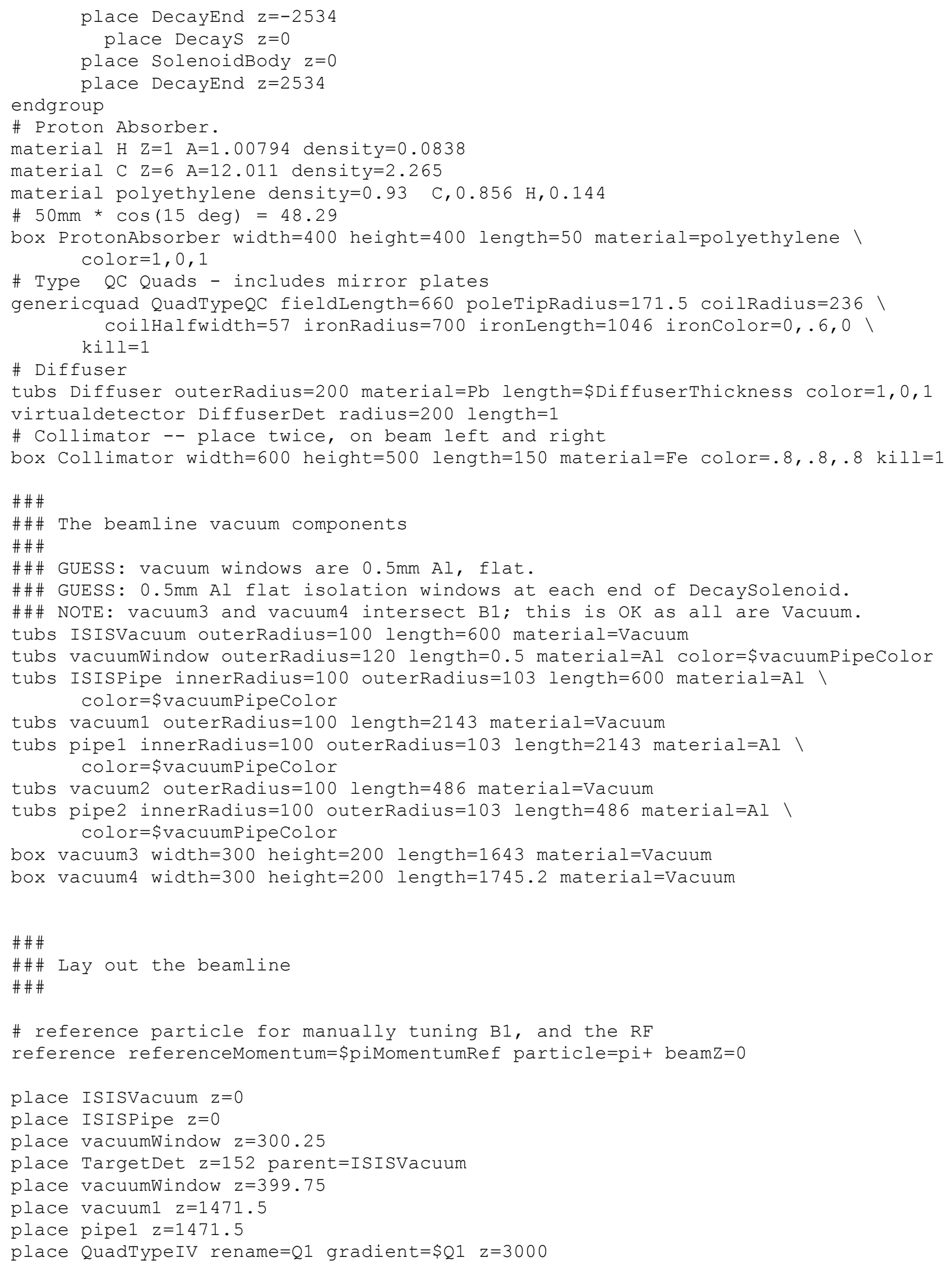




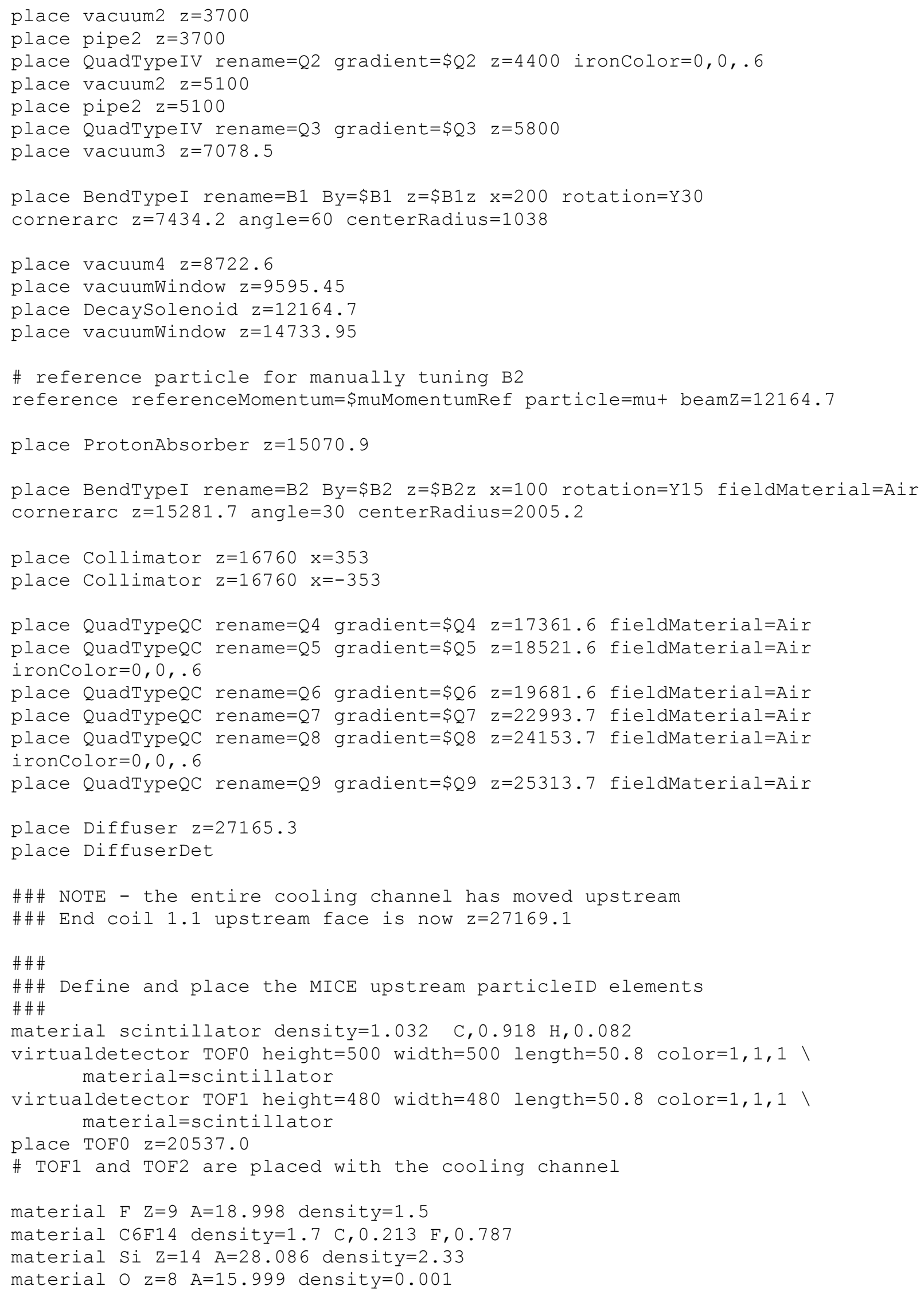


material quartz density=2.20 Si,0.467 0,0.533

box CherenkovlFront width=400 height=400 length=1 color=1,0,0 material=Al

virtualdetector Cherenkov1 width=400 height=400 length=20 color=0,0,1 । material $=\mathrm{C} 6 \mathrm{~F} 14$

box Cherenkov1Window width $=400$ height $=400$ length=2 color=0,1,0 material=quartz box CherenkovlLight width=550 height=550 length=478 material=Air color=1, 1,1

place Cherenkovifront $\mathrm{z}=20762.9$

place Cherenkov1

place CherenkoviWindow

place CherenkovlLight

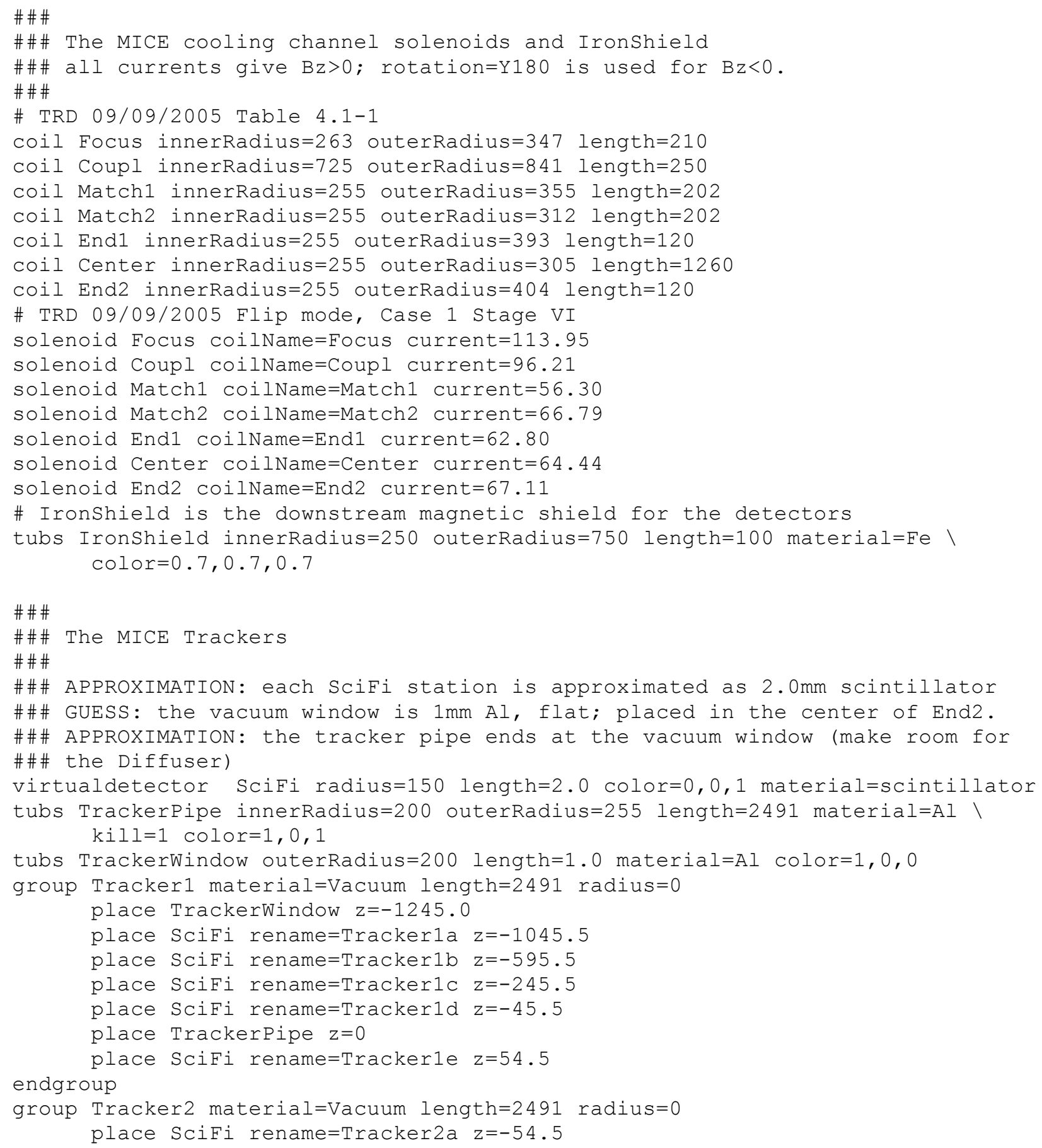




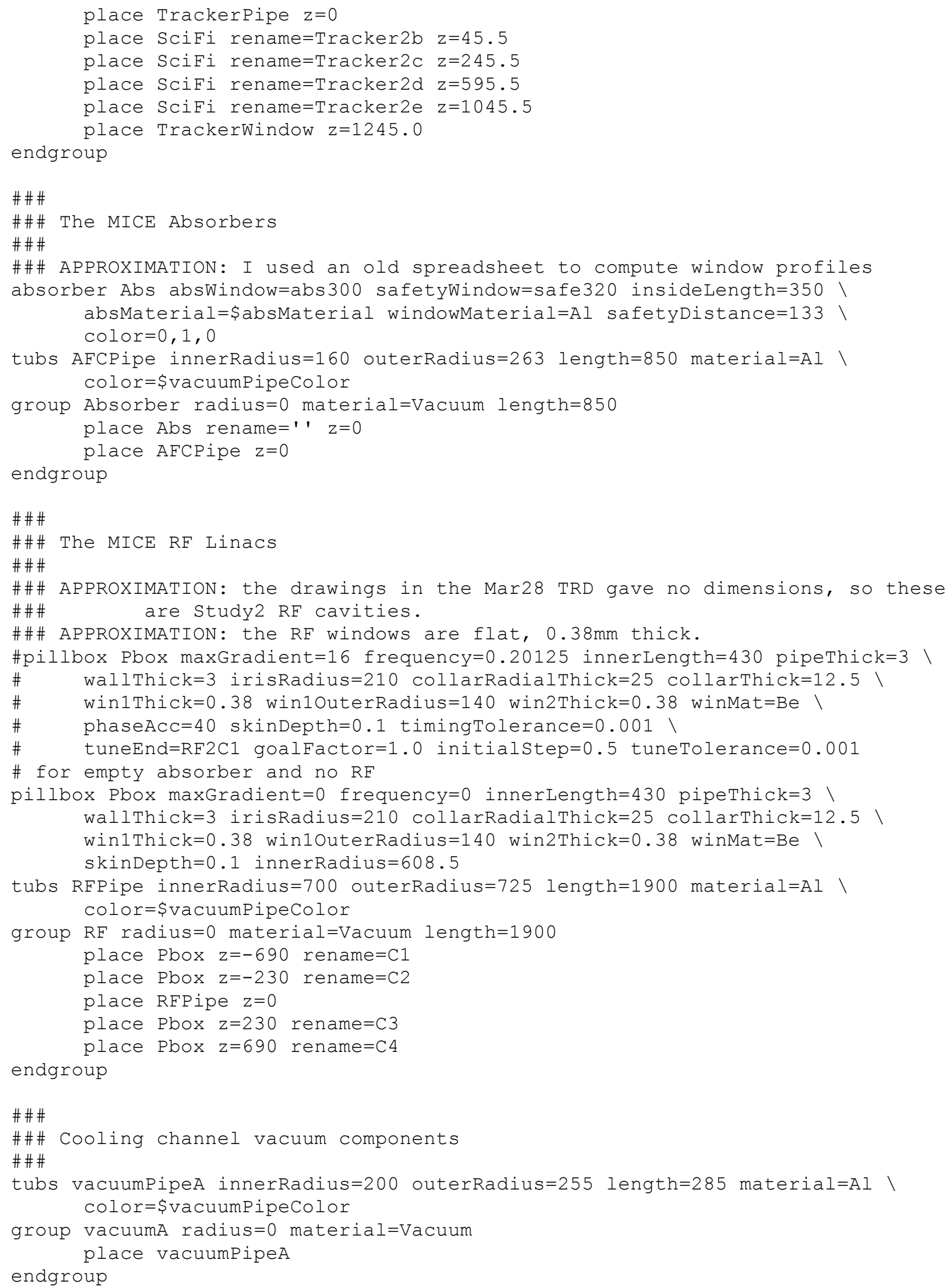




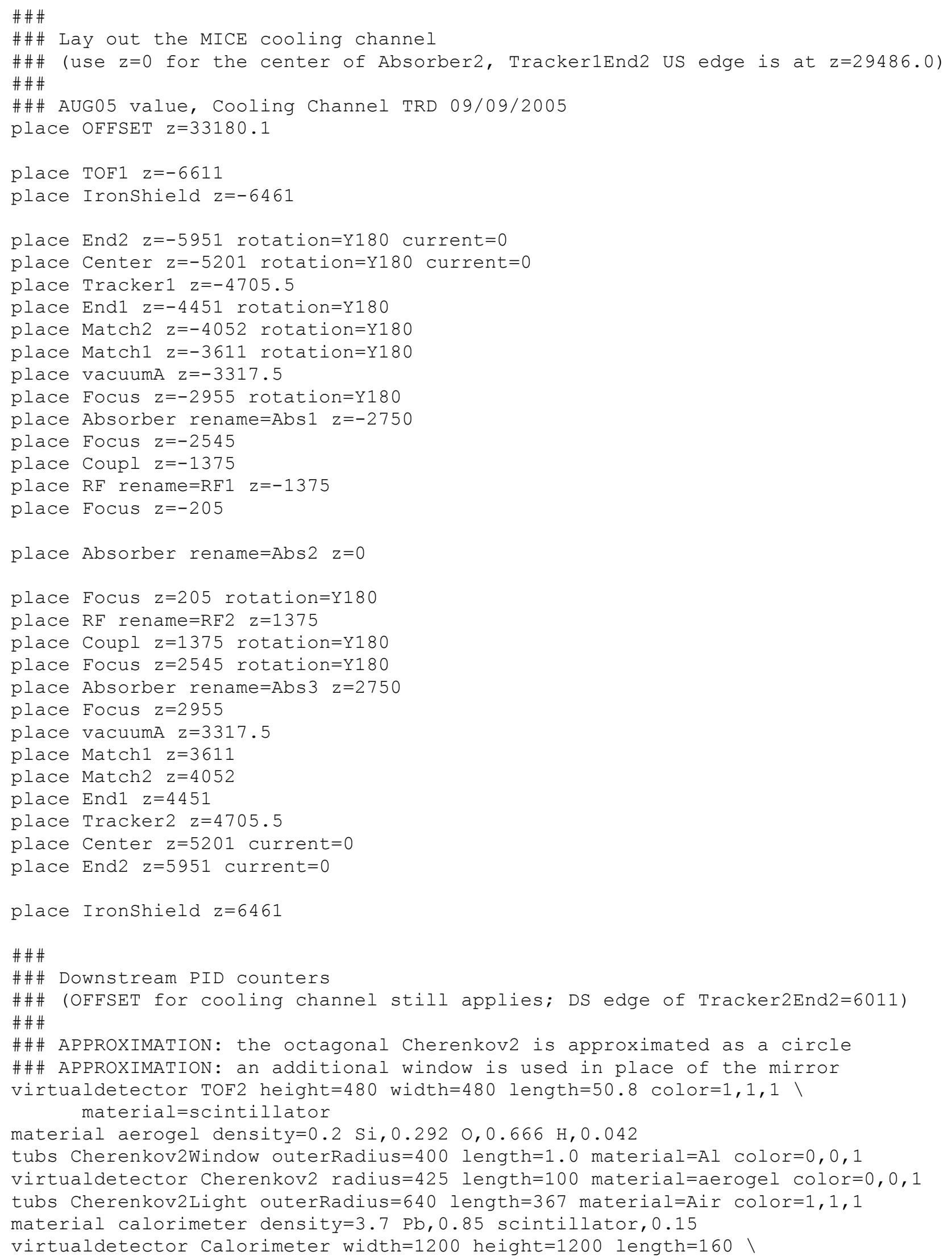




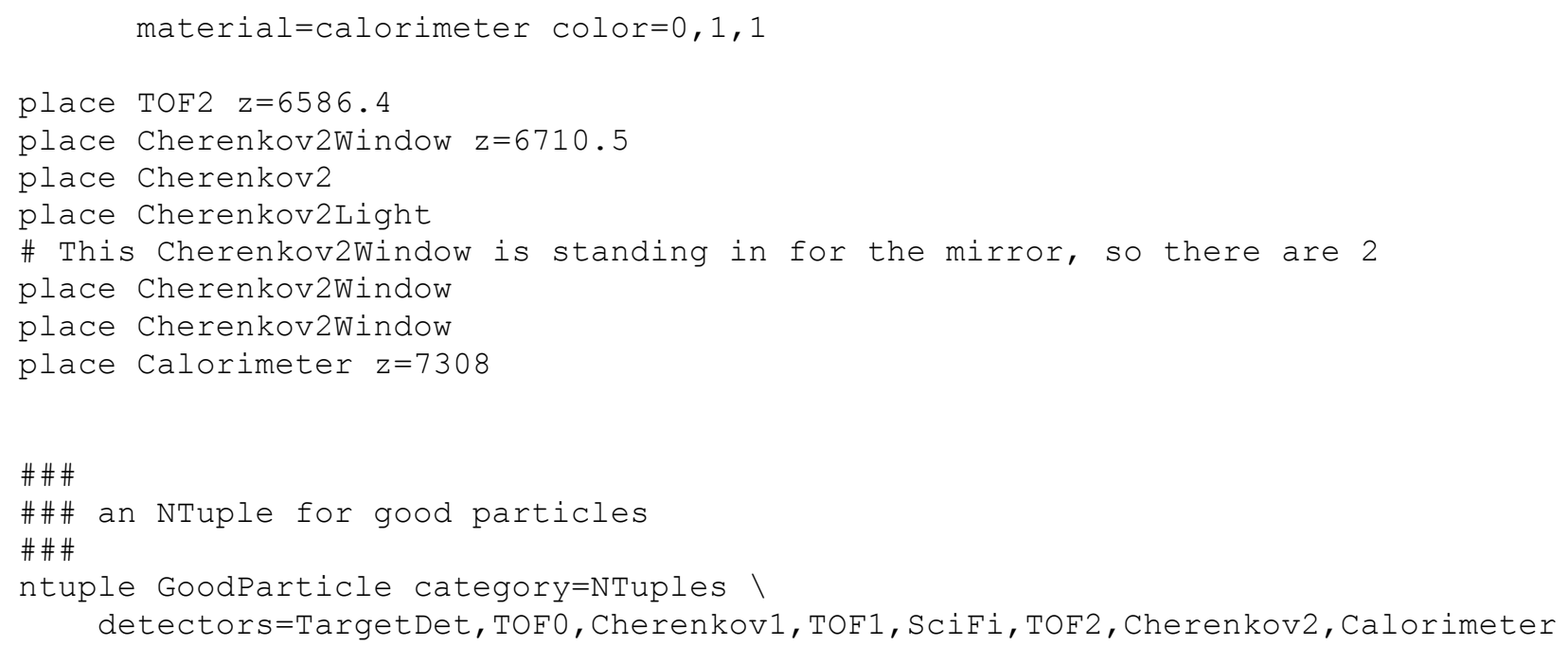




\section{Tips and Techniques}

These generally useful tips and techniques have been found useful while using G4beamline to simulate several dozen different systems, including suggestions from numerous users.

\subsection{Getting Help on Using G4beamline}

The primary means of obtaining assistance in using G4beamline is our user's forum, http://www.muonsinc.com/tiki-view forum.php?forumId=1. You can also send email to mailto:inquiries@muonsinc.com, or to Tom Roberts, the primary author of G4beamline mailto:tjrob@muonsinc.com.

Some questions are more about Geant4 than G4beamline. In particular, detailed questions about physics processes and/or physics lists are in this category. For such questions, you can use the previous paragraph, but we will most likely redirect you to the Geant4 forums:

http:/hypernews.slac.stanford.edu/HyperNews/geant4/cindex. It's quite appropriate for users of G4beamline to join the Geant4 forums as well.

\subsection{Reporting Bugs in G4beamline}

The primary means of reporting bugs in G4beamline is our user's forum, http://www.muonsinc.com/tiki-view forum.php?forumId=1. You can also send email to Tom Roberts, the primary author of G4beamline mailto:tjrob@muonsinc.com.

\subsection{Requesting New Features in G4beamline}

The primary means of requesting new features in G4beamline is our user's forum, http://www.muonsinc.com/tiki-view forum.php?forumId=1. You can also send email to Tom Roberts, the primary author of G4beamline mailto:tjrob@muonsinc.com.

\subsection{Getting Help on Individual G4beamline Commands}

The source code of each G4beamline command includes help text for the command and its individual arguments; it is printed by the help command. This text is the primary source of information about the command, and is copied into this User's Guide and into the help text of the G4beamline GUI. You can obtain help on each command in several ways:

1. From a command line type " $g 4 b l$-" on one line, and then "help command" on a new line in response to the $c m d$ : prompt.

2. Run the G4beamline GUI, and if the help text is not displayed, push the Help button. You can then scroll around and find the desired command.

3. Look in section 5 of this document.

Note that the definitive source is number 1 , so if there is a question about changes (e.g. in different versions of G4beamline), that is the method to use. In particular, the help text in the GUI and in this document can get out of date. 


\subsection{In what Directory should I Work?}

In general, it is a bad idea to put your simulations inside the G4beamline install directory - that makes it difficult to update G4beamline; similarly, the G4beamlineExamples directory is not a good place to work (except to run the examples). It is usually a good idea to work in your \$HOME directory, and to make a separate directory for each simulation; this is especially true if you are using the GUI. I have a single directory in my \$HOME called g4work under which I create a new directory for each new simulation, using a descriptive name.

\subsection{Files Common to Multiple Simulations}

Many G4beamline commands reference additional files. In some cases there will be a collection of different simulations that share common files, such as field maps, window profiles, etc. It may be convenient to put these files into a single directory called "Common" at the same level as the simulation directories. Then in each input file, reference these common files as "../Common/filename.ext".

\subsection{Basic Execution in a Command-Line Environment}

Most modern desktop environments permit the user to have multiple command-line windows open simultaneously. This can significantly improve your productivity while developing a new G4beamline simulation, or optimizing parameters of an existing one. Multiple windows are not necessary, and what I describe in parallel can be performed sequentially in a single command-line window; it is noticeably more efficient with multiple windows, however.

Usually when developing a new simulation, or optimizing the parameters or configuration of a simulation, one wants to see histograms or plots from each run before tweaking the input.file and running another test run. The idea is to run enough events to get statistically meaningful plots, but to not take too long so one can iterate rapidly through choices. For a simple system being simulated, I can often perform the loop of "edit, simulate, histogram, choose new values" in less than a minute per step. I do this with four windows open simultaneously, all in the same directory:

1. A command-line window in which I run G4beamline simulations.

2. A command-line window in which I run " $g 4 b l$ - " and to which I type "help command" whenever I need help on a command or its arguments.

3. A command-line window in which I edit my current input.file. I happen to use vi, but you should use the editor you are most familiar with.

4. A command-line window in which I run historoot. This can be window 1 if historoot is put in the background.

I rarely have a big enough monitor to see all four windows at once, but I can see all of one and parts of the others, so I can rapidly switch windows with a mouse click. The basic sequence is:

1. W3 (editor): If it's not open already, open input.file in the editor and prepare the next version to try. Use W2 (help) for help if necessary. When finished, save the file to disk without exiting the editor.

2. W1 (command prompt): run "g4bl input.file" and wait for it to finish. If there was an error, go back to step 1. 
3. W4 (historoot): do File/OpenFile and open g4beamline.root (or whatever file the simulation wrote). Select the desired NTuple and set the expressions to plot (if necessary - the plot configuration will remain from the previous iteration), and then push CreatePlot.

4. W4 (historoot): do File/CloseAllFiles. This is important to avoid confusion. On Windows this is essential, because if historoot has the Root file open, g4beamline won't be able to write to it and the next step 2 will fail for that reason.

5. Examine the plot and determine what to do next. Go back to step 1.

By using separate command-line windows, the command-line history in each will permit you to avoid re-typing commands, reducing typos and speeding things up considerably. By keeping historoot and the editor open, you avoid the delay of re-configuring them each time.

When constructing a new geometry, plots are usually not needed, and the GUI is better suited to viewing the system easily. So in window 1 I type "g4blgui input.file" and push its Run button instead of running $g 4 b l$ directly (with the best viewer selected). W4 (historoot) is not used. I find that the GUI is easier to use than the command-line when using visualization (though it works fine to type " $g 4 b l$ input.file viewer=best", and then "/run/beamOn 10" to the Geant4 Idle> prompt).

\subsection{Basic Execution in a GUI Environment}

Execution in a GUI environment, such as Windows, is similar to that in a command-line environment discussed above. The basic idea is to keep windows open so you don't constantly have to re-configure them.

Usually when developing a new simulation, or optimizing the parameters or configuration of a simulation, one wants to see histograms or plots from each run before tweaking the input.file and running another test run. The idea is to run enough events to get statistically meaningful plots, but to not take too long so one can iterate rapidly through choices. For a simple system being simulated, I can often perform the loop of "edit, simulate, histogram, choose new values" in less than a minute per step. I do this with four windows open simultaneously, all in the same directory:

1. The G4beamline GUI window.

2. (Optional) The G4beamline User's Guide in a PDF viewer. A PDF viewer makes it easier to scan the text for command names. Can be omitted and the help text of W1 can be used instead.

3. An editor window to edit the current input.file.

4. The historoot window.

I rarely have a big enough monitor to see all four windows at once, but I can see all of one and parts of the others, so I can rapidly switch windows with a mouse click. The basic sequence is:

1. W3 (editor): If it's not open already, open input.file in the editor and prepare the next version to try. Use W2 (help) for help if necessary. When finished, save the file to disk without exiting the editor.

2. W1 (G4beamline GUI): If input.file is not already selected, do so with the Browse button. Push the Run button to run the simulation. If there was an error, go back to step 1 .

3. W4 (historoot): do File/OpenFile and open g4beamline.root (or whatever file the simulation wrote). Select the desired NTuple and push CreatePlot (the plot configuration will remain from the previous iteration). 
4. W4 (historoot): do File/CloseAllFiles. This is important to avoid confusion. On Windows this is essential, because if historoot has the .root file open, g4beamline won't be able to write to it and the next step 2 will fail for that reason.

5. Examine the plot and determine what to do next. Go back to step 1.

\subsection{Putting Shielding into a Simulation}

G4beamline is a realistic simulation program, and just like a real beamline, one must provide appropriate shielding. While obviously there are no radiation issues in a simulation, it is necessary to provide shielding that is sufficient to prevent particles from traveling in unexpected places. This is particularly troublesome for secondary particles from decays and interactions, as they often have sufficient transverse momentum to quickly leave many beamline apertures, and even get outside the outer dimensions of magnets. Particles that go in unexpected places and directions can confuse plots and histograms, and can waste CPU time on irrelevant particles.

Probably the best and simplest approach is to use the radiusCut argument to the start, corner, and cornerarc commands. This puts a virtual cylinder around the centerline of the beamline, killing all particles that exceed the current radiusCut. You may need to use an unusually large radiusCut inside a bending magnet paired with a cornerarc, so be sure to check this visually. If everything within the radius $\mathrm{Cut}$ is either the beam aperture or elements with kill $=1$, then this will ensure that unusual paths outside the nominal beam aperture only extend between elements, which is usually sufficient. Make sure the elements fill the radiusCut, or particles could go around them. Note that a corner without object or rotation can be used to change the radius $\mathrm{Cut}$ at a specified value of $\mathrm{z}$ (centerline coordinates).

Another common approach is to use the kill=1 argument to bending magnets, quadrupole magnets, beam pipes, etc. This makes them kill any particles that hit their solid parts, providing shielding for many situations. You can add specific objects just for shielding, such as a box, cylinder, or tubs. If you enclose the entire beamline with elements and pipes having kill=1, this is probably sufficient.

In many cases, the beam pipes are not of interest in a simulation, and putting them in is more effort than it is worth. Then you must take care that particles do not take unintended paths. For instance, it is rather common for a particle to take a "shortcut" around a bending magnet, so be sure to check for this. Rings are obviously subject to this. Shielding can be any element with kill=1, most often box or tubs (the latter is particularly convenient as it can be given a hole for the intended beam); it is often convenient to set color=invisible, but don't do that until you have looked at it to know it is appropriately sized and placed. A tubs of any radius, a length of $0.01 \mathrm{~mm}$ or more, and with kill $=1$, is a perfect shield (i.e. kills every particle that hits it).

\subsection{How to Debug a Simulation}

An important debugging aid is the geometry test performed by G4beamline. The results appear in the output just before tracking. In the real world, two objects cannot occupy the same space; the simulated world does not have this restriction, and input-file errors can generate invalid overlaps. In addition, the Geant 4 optimization of tracking requires a strict hierarchical arrangement of volumes. Usually any geometry errors listed should be corrected in your input file before believing the simulation results; in 
some cases this is difficult or impossible, and some exceptions are listed in section 8.2 on Advanced Topics.

The most common debugging technique is to add steppingVerbose $=1$ to the command line (or the Parameters field of the GUI). This causes G4beamline to print (to stdout) the track variables at every step. That usually lets you figure out what is going wrong. Note that by setting steppingFormat you can control what track variables are printed (see the help for details) - this can be very useful when debugging $\mathrm{E}$ and $\mathrm{B}$ fields. In most cases it is best to widen the window before running, so lines are not wrapped - 140 columns is usually sufficient.

Sometimes the problems are subtle, and only rare events look crazy. If you can select those "crazy" events with sliders in historoot, then you can set its EventID field to cause it to write the histo_event.txt file. You can then check the HistoRootEvents checkbox in the GUI, or put an eventcuts command into your input.file. Running with a viewer will then permit you to visualize just the "crazy" events - seeing them will often let you figure out what's wrong.

Note that G4beamline simulations are quite realistic. This means that pions and muons will decay, particles will interact in material objects, and tracks will propagate wherever they can go (even if you don't expect it). Decay neutrinos can look "crazy" if you're not expecting them (so cut on PDGid in the plots, or eliminate them at the source with a trackcuts in your input.file). You may be surprised at the delta-rays (low energy electrons) produced in vacuum windows and other materials (eliminate them with a trackcuts command with kineticEnergyCut $=1$ or higher, or perhaps kill=e-). Tracks can occasionally go in the most amazing places, and you need to install shielding just as in a real accelerator (use the radius $C u t$ as described above, or add a box or tubs with kill=1 and place it appropriately; when complete you can use color=invisible to avoid cluttering your visualizations). It is often appropriate to surround the beam line with beam pipes having kill $=1$ - this avoids spending CPU time on unwanted tracks, and prevents them from taking "shortcuts" and re-appearing as crazy events in plots; add kill=1 to quads and bends, etc.

In my experience, the most common errors in G4beamline simulations and interpreting results are:

1. Failure to cut on PDGid in plots and histograms, and thus confusing secondary particles for the desired (primary) ones.

2. Failure to put shielding in appropriate places and thus having particles go in unexpected places, causing "crazy" events in plots and other confusion.

3. Mistakes in the input file that cause invalid overlaps of objects.

4. Mistakes in the input file that unintentionally omit objects or put them in the wrong place.

5. Inappropriate values of tracking parameters (see section 3) that cause Geant4 to optimize tracking in an unsuitable or insufficiently accurate manner.

\subsection{Geant4 Commands}

Geant 4 has an internal command processor with a large number of commands. For the most part, G4beamline users don't need to use these commands, as G4beamline controls all aspects of the simulation. Nevertheless, there are situations for which a user might want to issue a Geant 4 command. There are two rather different ways to do this: 
1. Simply put the Geant 4 command into the input.file, putting its initial "/" in column 1. Such commands are issued when they are read - that happens before the system is constructed, and for many commands that is too early.

2. Use the g4ui command.

The when argument controls when the command is issued, and all values are after the system is constructed.

The Geant 4 command interpreter is quirky, but there is a way to obtain a list of all its commands: /control/manual. This command can use either method above.

The most common reason to issue a Geant 4 command is to set some verbose level, for debugging or investigating some aspect of Geant 4 (most especially its physics processes). G4beamline sets all verbose levels to 0 initially. Here is the list of commands that G4beamline runs to do that:

/control/verbose 0

/run/verbose 0

/event/verbose 0

/tracking/verbose 0

/hits/verbose 0

$/ \mathrm{material} /$ verbose 0

/process/setVerbose 0 all

/process/verbose 0

/process/eLoss/verbose 0

Another reason to use a Geant 4 command is to control a viewer. Use " $g 4 u i$ when $=4$ " for that. The selected viewer's initialization commands (from viewer.def) will be executed before such commands.

\subsection{Obtaining Plots and Histograms}

There are a number of different ways to generate plots and histograms from G4beamline output files. I generally find HistoRoot to be most convenient, but each user should use the method they are most comfortable with. Both HistoRoot and PAW can fit functions to histograms or plots; excel and some other spreadsheets can do so, perhaps with more overhead.

\section{General Remarks}

In most cases, you must cut on PDGid when generating a plot or histogram (PDGid is the Particle Data Group's ID for the particle of a track, so this is selecting the type of particle to plot). G4beamline simulations are realistic, and particles will decay and interact, generating secondary particles. Without such a cut, secondary particles can greatly confuse the interpretation of a plot. HistoRoot has a specific feature to display the particle types: put "PDGid" into the "Particle Type" field, and whenever the NTuple is scanned to generate the plot, a list of the PDGid-s contained within the slider cuts will be printed, with names for common particles.

To display a bundle of tracks, put "trace $n$ Trace $=100$ format $=$ ascii oneNTuple $=1$ " into your input.file, and then use gnuplot to plot the AllTracks.txt file that G4beamline wrote. gnuplot omits the back-trace line between tracks because the trace command puts a blank line between tracks, specifically for this. 
gnuplot is available for Linux as part of the distribution, for Mac OS via Fink, and for Windows via http://www.gnuplot.info/ .

\section{HistoRoot}

First, install Root [5]. HistoRoot is included in the G4beamline distribution; it is installed as an icon on Windows, and is available via the command line on Linux and Mac OS X (just type "historoot [filename.root]"). HistoRoot requires Root to be installed. By default, all NTuples generated by G4beamline are placed into a single Root file. Note, however, that HistoRoot can also read the ASCII NTuple files written by G4beamline. Generating plots and histograms is straightforward, and the help for HistoRoot should permit you to get started right away. You can quickly and easily plot arbitrary expressions of the NTuple's fields, with cuts applied based on expressions and sliders that affect the plot in real time. For more complicated analyses (e.g. computing masses of multi-particle states), Root can be used as an analysis platform; this is advanced usage and is beyond the scope of this tip.

\section{Gnuplot}

Gnuplot can directly read the ASCII NTuples written by G4beamline (add format=ascii to the command). Note that Gnuplot does not generate histograms; you'll have to use another program to do that. Gnuplot is especially good at displaying multiple trajectories from "trace format =ascii oneNtuple $=1$ ". gnuplot is available for Linux as part of the distribution, for Mac OS via Fink, and for Windows via http://www.gnuplot.info/.

\section{Excel and other Spreadsheet Programs}

Excel and most other spreadsheet programs can read the ASCII NTuples written by G4beamline (add format $=$ ascii to the command). Each line is a row of the NTuple with tabs separating the columns; the first 2-3 lines give comments, column names, and column units (optional).

\section{PAW}

PAW can read the ASCII NTuples written by G4beamline (add format=ascii to the command), possibly after a format conversion.

\subsection{Obtaining Pictures of the System and Events}

G4beamline is a complicated and flexible program, and like all such programs it is subject to "garbage in, garbage out". So it is important to check and verify that your input.file actually represents the simulation you want, and not some other (perhaps unphysical) system. An important tool for this is the visualization implemented by G4beamline - many errors can be found simply by looking at the system to be simulated.

In the command-line environment, you need to have an active $\mathrm{X}$-Windows environment (i.e. the DISPLAY variable must be set to a valid display server). On Windows this is automatic; on Linux it is automatic as long as you run X windows (i.e. bring up a GUI); on a Mac you need to run the X11 application and use an xterm window (in Leopard you can also manually set DISPLAY in a normal Terminal window). Then all you need do is append "viewer=best" to your $g 4 b l$ command, and the OpenInventor viewer will be displayed. Other viewers can be used; get their names by using viewer=best and looking near the end of the output for a list of supported viewers. In the command-line 
environment, you need to type the Geant4 command "/run/beamOn 10" to run 10 events and display them and the system.

In the GUI environment, simply select Viewer:best. Each run will be displayed in the viewer as a single image, so setting events/run determines how many events are shown, and selecting \#runs is how many different runs (pictures) will be made. In the OpenInventor viewer, select File/Escape to quit this image, simulate the next run, and display its image. Other viewers can be selected by selecting and setting

Other.

To save the image into a file, some viewers have a File menu item that can save in various formats (e.g. OpenInventor can save as PostScript). In any viewer, you can take a screenshot of the viewer window and save it to a file:

- Windows: Ctrl-PrtScrn followed by running a program to save the Clipboard in a graphics format (e.g. Paint)

- Linux: a program like ksnapshot

- Mac OS X: the Grab utility (menu: Capture), the Preview utility (menu: File/Grab), or type shiftcommand-4 and select the desired region of the screen

Note that screenshots all save a bitmap, but for some viewers the File/Save menu item generates a vector file that can be smaller and scales better.

\subsection{Warning and Error messages - Which Ones can be ignored}

Errors in the input file generate an error message immediately while reading and listing the input file. They begin with "***" and must be fixed before the simulation will run. These are such things as syntax errors, invalid parameter names, invalid arguments to commands, etc.

Errors and warnings generated during execution are handled by the G4Exception function; they are printed as a 6-line message starting and ending with a row of asterisks - that makes them stand out visually in a long printout. Those that are issued by a routine beginning with "G4" came from Geant4 code, while those issued by a routine beginning with "BL" came from G4beamline code; those that are issued by a command are also from G4beamline code. It is important to distinguish these two cases, as how you obtain help on them may differ.

The severity of the message is a strong indicator of whether it can be ignored, or indicates a serious problem. Note that a Warning issued by many events probably means the simulation cannot be trusted. Warnings on a tiny fraction of the events can probably be safely ignored (unless they are related to specific rare events of interest). Even Errors that abort the track or event might well be ignorable if they occur only rarely. Note that G4beamline suppresses many similar exception printouts, so look at the Exception Summary at the end of the output to see how many actually occurred.

Errors issued by G4beamline code can be discussed in our user's forum, http:/www.muonsinc.com/tikiview_forum.php?forumId=1. You can also send email to mailto:inquiries@muonsinc.com, or to Tom Roberts, the primary author of G4beamline mailto:tjrob@muonsinc.com. 
Errors issued by Geant 4 code can be discussed in the Geant 4 forums (linked to the Geant 4 home page, http://geant4.cern.ch). You can also discuss them as in the previous paragraph (choose one method, not both).

See also section 2.8 .4 on troubleshooting visualization problems. A reasonably complete list of G4beamline error messages is given in Appendix 7. Here are some notes on specific exceptions:

\section{Stuck Track}

If you track very low-energy tracks $(<100 \mathrm{eV})$, and have materials in your simulated world, then you will probably see a number of "Stuck Track" exceptions that kill the track. This occurs because very low energy tracks can get stuck on the surface, taking many extremely small steps $(<1$ micron). Such tracks would really get absorbed into the material, so killing the track is a reasonable thing to do. Such error messages can usually be ignored, unless they dominate your simulation.

\section{Large Primary TrackID}

This exception has a specific cause and cure. Remember that tracking in Geant4 (and thus G4beamline) will often generate secondary tracks, and these must each be assigned a TrackID. To minimize the collision of multiple tracks within an event having identical TrackID-s, secondary tracks by default have TrackIDs starting with 1001 and incrementing thereafter. If the input beam file has a TrackID $\geq 1001$, then confusion can result, so this exception is issued. You could edit your beam file to not have such large TrackID-s, or you can add the following to the beam command that reads the file:

secondaryTrackID $=2001$. This avoids the overlap, and thus the exception; larger values can be used if necessary.

\subsection{Secondary Tracks and Particles}

Unlike most accelerator physics programs, G4beamline includes particle decays and interactions of particles with matter. This means that during a simulation new particle tracks can, and usually will, be created. For instance, whenever the beam goes through a vacuum window, delta-rays (low-energy e $\mathrm{e}^{-}$) will be produced. In some simulations such secondaries are essential (e.g. for a muon beam produced by pion decay), but in others these secondary particles are uninteresting and are merely a nuisance (as, perhaps, are the neutrinos associated with that muon beam). G4beamline has several mechanisms to let you deal with secondaries:

- physics - doStochastics $=0$ will turn off all stochastic processes, which has major implications, but this implicitly inhibits the creation of secondaries

- physics - disable=process will turn off specific physics processes

- trackcuts - killSecondaries=1 will kill all secondaries; keep=list will keep only those particles, killing all others; kill=list will kill those particles

- particlecolor - assigns colors to different particles for the viewers

Note that when generating histograms and plots, you usually need to cut on PDGid (the Particle Data Group's assigned ID for the particle of the track). It is all too easy to look at a histogram, see an outlier, and wonder what is happening - in many cases the outlier is merely a secondary track from a decay or interaction, and is unrelated to the phenomenon of interest. So be warned. 
HistoRoot has a specific feature to display the particle types: Put "PDGid" into the "Particle Type" field, and whenever the NTuple is scanned to generate the plot, a list of the PDGid-s contained within the slider cuts will be printed, with names for common particles.

Here is a list of common (stable) particle PDGid-s (negative for anti-particle):

\begin{tabular}{|c|c|c|c|c|c|c|}
\hline PDGid & Particle & & PDGid & Particle & PDGid & Particle \\
\hline 11 & $\mathrm{e}^{-}$ & & 12 & $v_{\mathrm{e}}$ & 13 & $\mu^{-}$ \\
\hline 14 & $v_{\mu}$ & & 16 & $v_{\tau}$ & 22 & $\gamma$ \\
\hline 111 & $\pi^{0}$ & & 211 & $\pi^{+}$ & 311 & $\mathrm{~K}^{0}$ \\
\hline 321 & $\mathrm{~K}^{+}$ & 2112 & $\mathrm{n}$ & 2212 & $\mathrm{p}$ \\
\hline
\end{tabular}

A much more complete list of particle IDs is in Appendix 6.

\subsection{Finding Example Input Files using the XXX command}

There is at least one test for every G4beamline command. Look in the test directory under the G4beamline installation directory. You can also send an inquiry our user's forum, http://www.muonsinc.com/tiki-view forum.php?forumId=1. You can also send email to mailto:inquiries@muonsinc.com, or to Tom Roberts, the primary author of G4beamline mailto:tjrob@muonsinc.com.

\subsection{Parameterizing the Input File}

This is a general technique applicable to many different situations.

Parameters in G4beamline are names that represent strings. Whenever \$name appears in an argument to a command, the current value of the parameter name will be substituted. Note this is not a general macro substitution, and it only occurs in the values of command arguments. In addition, for all real- and intvalued arguments and in the param command, argument values that are numerical expressions are evaluated after \$name substitution; the standard C math functions can be used. See section 4.3.

Parameters can be defined by the param command, on the command line, and in the Parameters: field of the GUI. These last two methods permit different runs using the same input.file to be different. In all cases a parameter is set with the syntax name=value, without spaces; the value can be enclosed in singleor double-quotes to include whitespace. These examples will use the command-line interface, but the Parameters field of the GUI can be used in the same way.

The basic idea is to use a parameter rather than hard-coding a specific value into the input.file. Usually one wants to provide a default value for the parameter, so it is never undefined. As an example, imagine you want to compare the effects of several different target materials, all with the same size. The input.file could look like this:

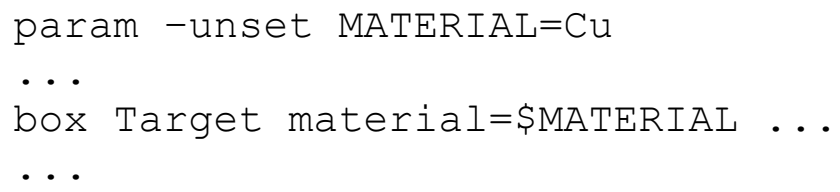


Note the initial "param -unset" command - this provides a default value for the parameter. Commands to run this simulation for several materials would be:

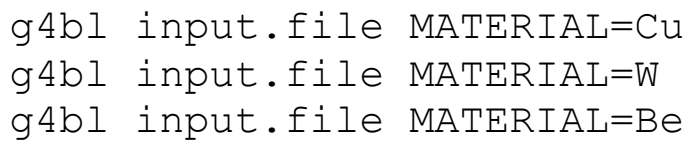

Note that in the previous example the Root output file will be overwritten each time, so one must be careful to obtain all desired output from one run before starting the next. A better input.file will use different output files for each run:

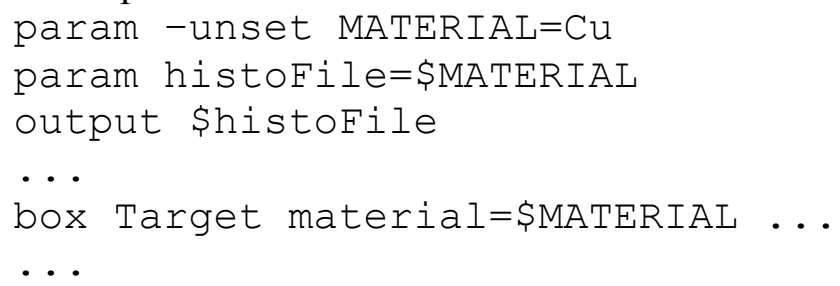

The second param command sets the name of the Root output file to include the material (this command cannot be combined with the first param command and must not have-unset). The output command then re-directs stdout and stderr to files "Cu.out", "W.out", "Be.out", etc.

This can obviously be extended to as many different parameters as desired. It is useful to put them all into the histoFile value, perhaps separated by commas.

\subsection{Setting Fields of Magnets}

If you have a specified field for a solenoid, you need to convert that into a current (density), as that is the argument of the solenoid command that determines the field. You can compute the ampere-turns required, but that is both time consuming and error prone. A better way is to use the probefield command with its input set to a file containing the global coordinates of the solenoid's center. Just guess a value for the current and run the simulation; from the printed value you can take the appropriate ratio and quickly determine the current required to give the desired field. You can also use the printfield command for this.

If, instead, you have a specified current for a given magnet, then you will need the magnet's specifications to determine the field corresponding to that current. Except for a solenoid without iron, there is no simple way to relate field and current. If you have a drawing of the magnet, you could in principle construct a model of the magnet using an EM modeling tool (Tosca, Opera3D, Ansys, ...); that is usually a major effort - the drawing probably gives a value for the integral of $\mathrm{B} \mathrm{dl}$, which should be enough for you to compute the field.

\subsection{Tuning Bending Magnets}

G4beamline is a realistic simulation program, and like a real beamline, the bending magnets in a simulated beamline must be tuned for position, orientation, and field (current). To model a real beamline that has the corners of the beam centerline marked on the floor, a corner or cornerarc command will be used to model each corner. This implies that the corners are fixed and the bending magnet must be positioned and tuned appropriately. The standard criterion is that a reference particle coming into the 
magnet along the centerline of the beam should exit the magnet along the centerline, both measured outside the fringe fields of the magnet. If there are solenoids or other magnets near the bending magnet such that their fringe fields overlap, then the tuning of the bending magnet may depend on their settings. Note that if quadrupoles are used to steer the beam, these remarks apply to them, too.

See also section 2.11 (Tuning the Beamline).

To begin, make sure you have a reference command that puts a reference particle down the centerline into the bending magnet. This implies that if you have multiple bends in your beamline, you should start tuning with the first bend, and proceed downstream.

The usual orientation for a rectangular bending magnet is with its front and rear faces angled at half the total bending angle to the centerlines. It must be placed a short distance behind the corner in order that its fringe fields are accounted for, and must be placed a bit to the outside of the bend so the two centerlines are equidistant from the left and right sides of the aperture.

The usual orientation for a sector bending magnet is with its front and rear faces perpendicular to their centerlines.

Other orientations are possible, and can be used to affect the vertical focusing of the bending magnet. The beamline designer will specify the appropriate angle of the magnet relative to the centerlines.

G4beamline can automatically tune the field of a bending magnet, but cannot tune its position. So the best approach is to put the field tuning into the input file and then tune the position manually - this reduces a 3-parameter problem to two parameters, which goes much faster. Once the position is correct for one reference momentum, other reference momenta can be handled by simply re-tuning the field (or manually adjusting it). Here is an example for a 60-degree left bend:

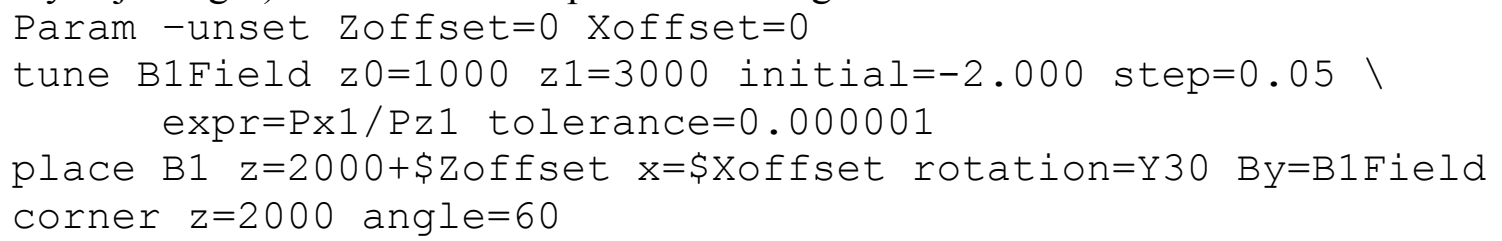

The basic idea is that the tune command will vary the value of B1Field and re-track the tune particle from $\mathrm{z} 0=1000$ until it is parallel to the centerline at $\mathrm{z} 1=3000$, within 1 microradian (tolerance). The user will manually vary Zoffset and Xoffset until the magnet is properly placed so that reference particle is on the centerline at $\mathrm{zl}=3000$ and is equidistant from the horizontal edges of its aperture (these are not independent, due to the rotation of the magnet and the presence of its fringe field; both will be reasonably close to 0 , within $\sim 200 \mathrm{~mm}$ or so). Note the initial value in the tune command must be close enough so the initial tune particle actually reaches $\mathrm{z} 1=3000$ (i.e. does not hit any aperture beforehand).

The idea is to put "Zoffset $=0$ Xoffset $=0$ steppingVerbose $=1$ " onto the command line (or Parameters in the GUI), and re-run the simulation while varying their values until the reference particle is on the centerline at $\mathrm{z} 1=3000$. To speed this up, it is useful to temporarily set the beam command(s) to run one event (nEvents=1). To avoid laborious scanning of a long output file with many steps, it is useful to use the command-line program and pipe it into grep with an appropriate pattern to select a step near or at $\mathrm{z}=3000$ (because step-lengths can vary, it may help to temporarily place an object at $\mathrm{z}=3000$ and grep for it). NOTE: be sure to look at the reference particle, and not any tune or beam particle. 
First keep Xoffset $=0$ and vary Zoffset. You will quickly learn how changes in value relate to distance from the centerline. This usually executes in about 3-5 seconds, and you ought to be able to find the required value of Zoffset in a few minutes. Using the command-line history in your shell greatly speeds this up. To tune Xoffset you must add this command to make the reference particle visible and white: "trackcolor reference=1,1,1" (I put it just after the beam command; you can leave it in). Once you have the value for Zoffset, then add viewer=best to the end of the command and re-run it, typing "/run/beamOn 1" to the Geant4 Idle> prompt (as indicated by the comments preceding it). In OpenInventor, select wireframe mode (right click, DrawStyles/StillDrawStyle/wireframe). Now rotate the image using the mouse until you can see the white reference track inside the bending magnet (ignore the beam track(s) that are other colors); zoom in if necessary, and use a ruler or judge by eye whether or not it is centered left-right. If it is not centered, guess how much change in Xoffset is needed. Then go back to tuning Zoffset with this new value (no viewer).

Once you have values for Zoffset and Xoffset, edit the input.file and put them in.

If you want to handle beams with different momenta, you can parameterize the momentum and B1Field like this (assuming you initially tuned at $200 \mathrm{MeV} / \mathrm{c}$ and B1Field for $200 \mathrm{MeV} / \mathrm{c}$ was $2.10525 \mathrm{Tesla}$ ):

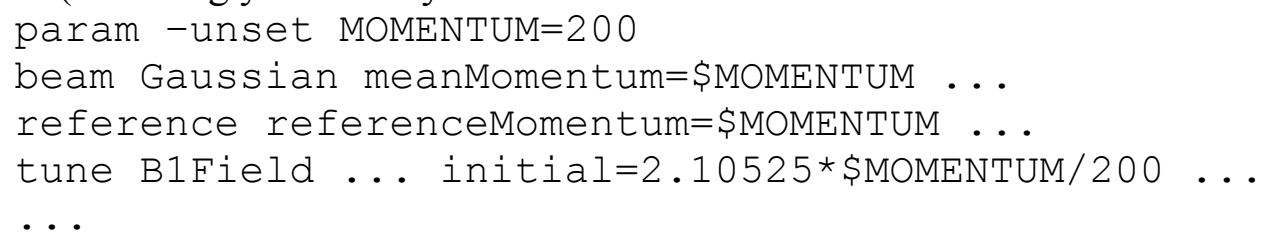

\subsection{Setting the Phase of RF Cavities (pillbox)}

The phase of an RF cavity (pillbox) is determined by its timeOffset and timeIncrement arguments. If timeOffset is not specified it is automatically set to phaseAcc by the Tune particle.

The basic method used to set the phase: when the Tune particle enters the volume of the pillbox its track parameters are saved and an initial value of timeOffset is estimated (based on its current velocity and the distance to the center of the cavity). Then it is tracked to the center of the cavity and its phase is checked (the volume is split in two to ensure the track takes a step ending in the center of the cavity). If the phase is correct within tolerance, nothing further is done and the Tune particle continues being tracked. If the phase is incorrect, the timeOffset value is adjusted via a simple linear solver, the current Tune particle is killed, and a new Tune particle is generated from the saved values. This usually converges in one iteration.

If timeIncrement is nonzero, it is added to timeOffset after tuning is complete. The Tune particle is not re-tracked, so large values will completely confuse the reference particle. timeIncrement is intended for exploring small phase errors of the RF.

See also section 2.11 (Tuning the Beamline). 


\subsection{Tuning the maxGradient of RF Cavities (pillbox)}

When RF cavities are used, it is generally necessary to carefully tune their maxGradient so the output beam has the desired energy or momentum. The operators of the machine arrange for this (either manually or via an automated feedback system). In G4beamline this requires the tune command and is done while tracking the Tune particle, usually in parallel with setting their phases (see previous section).

The tune command defines a "tune variable" and will arrange to save the Tune particle at one Z-position (z0), track the Tune particle to a second Z-position (z1), and then evaluate an expression using track fields. It then varies its tune variable in an attempt to make the expression evaluate to 0.0. This of course requires that some relevant beamline elements between $\mathrm{z} 0$ and $\mathrm{z} 1$ use the tune variable to change their behavior appropriately. In this case we'll vary the maxGradient of all RF cavities between $\mathrm{z} 0$ and $\mathrm{z} 1$, and use an expression at $\mathrm{z} 1$ that makes the Tune particle have the desired momentum, which we'll take as $1 \mathrm{GeV} / \mathrm{c}$ to within $1 \mathrm{MeV} / \mathrm{c}$ :

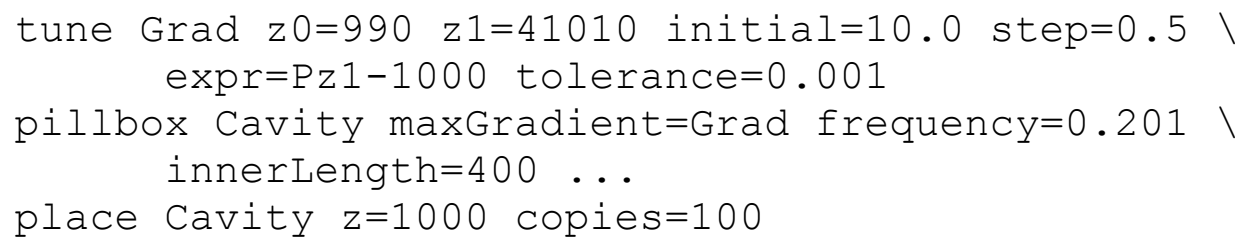

Note that $\mathrm{z} 0$ must come before the first Cavity is placed, and $\mathrm{z} 1$ must be after the last Cavity is placed, at the location where the $1 \mathrm{GeV} / \mathrm{c}$ beam is desired.

See also section 2.11 (Tuning the Beamline).

\subsection{Multiple Jobs in Parallel}

In many cases you will want to run multiple simulations, typically scanning one or more parameters. In this case it is often not necessary to run multiple jobs in parallel (with or without MPI), as you can simply run the different simulations on the available CPUs. In this case nothing special is needed, as long as you keep the different output files from clashing (parameterizing the input file can help, section 7.17).

There certainly are situations in which multiple jobs working on a single simulation are appropriate.

There are two ways to run multiple G4beamline jobs in parallel, all working on a single simulation:

1. Manually or via scripts

2. Via the Message Passing Interface (MPI)

\subsubsection{Manually or via Scripts}

G4beamline is a realistic simulation program, and runs considerably slower than many accelerator simulation programs. While simple simulations can run as fast as $\sim 1000$ events per second, a moderately complicated system might run $\sim 100$ events per second, and complex simulations can run less than 1 event per second. With the advent of Linux clusters and multi-core CPUs, it is now quite common to be able to run multiple jobs in parallel. The key to doing that in G4beamline is that it always seeds the pseudo-random number generator with the event number, just before starting to process the event. The PRNG used comes from CLHEP [2], and has excellent properties for event numbers from 0 to $900,000,000$. 
These basic capabilities are needed to run multiple jobs in parallel:

1. The ability to submit multiple jobs.

This is system dependent. On a multi-core or multi-CPU system, you can simply use multiple terminal windows or multiple GUI windows to run multiple jobs simultaneously. Be sure to keep the jobs separate, including output files. On Linux, Mac OS, and Windows/Cygwin you can use a single terminal window to run several jobs simultaneously "in the background":

g4bl input.file first $=1000000$ last $=1199999>1000000$.out \&

g4bl input.file first $=1200000$ last $=1299999>1200000$.out \&

g4bl input.file first $=1300000$ last $=1399999>1300000$.out \&

On a Linux cluster or other multi-system, you will need to learn how to submit multiple "batch" jobs.

2. The ability to assign a unique range of event numbers to each job.

Do this with parameters in your input.file. I use first and last, and the commands are: param - unset first $=1$ last $=1000$ beam .... firstEvent $=\$$ first lastEvent $=\$$ last

3. The ability to assign a unique Root output file to each job.

Use first in the name of histoFile: param histoFile $=\$$ first

4. The ability to combine all of the Root output files into histograms and plots. Use HistoRoot to read all the root files, and simply select the same NTuple from each file. HistoRoot will combine all selected NTuples into each plot.

Note that this works well up to perhaps 32 parallel jobs; above that it becomes quite cumbersome.

\subsubsection{Via the Message Passing Interface (MPI)}

On systems that implement MPI, it can be used to speed up a simulation by a factor that is almost the number of CPUs available. The advantage is that all outputs from the different instances are combined into a single output (typically one Root file, one stderr, and one stdout). See section 8.5. The program scales essentially linearly up to at least 16 CPUs, and probably more (depends in detail on the system being simulated and on the computer hardware used; more complex systems will scale efficiently to more CPUs). The basic command is:

$$
\text { mpirun -np } 5 \mathrm{~g} 4 \mathrm{bl} \text { input.file [... parameters] }
$$

This will run 5 instances of G4beamline cooperating on a single simulation. As one instance performs only management tasks, it is usually appropriate to start one more instance than the number of available CPUs.

\subsection{Performing a Scan of Values of Some Parameter}

It often happens that one wants to scan over the values of some parameter, looking for maximum transmission, the desired emittance at a given location, or some other aspect of the beamline. The idea is to parameterize the input.file so the parameter can be specified on the command line, and then either manually scan or write a shell script to do the scan. For example:

$$
\text { Param -unset VALUE }=1
$$


... rest of input, using \$VALUE

One can simply do this:

$$
\begin{aligned}
& \text { g4bl input.file VALUE=1.01 } \\
& \text { g4bl input.file VALUE=1.02 } \\
& \text { g4bl input.file VALUE=1.03 } \\
& \text { g4bl input.file VALUE=1.04 }
\end{aligned}
$$

and keep track of the desired property. If the desired property is printed to stdout (e.g. it appears normally, or via a printf command), one can use grep to greatly simplify the search for its value. This method gives a lot of output, and it can be time consuming to scan it all for the desired property of interest. So it is often better to write a short shell script to run G4beamline (with VALUE passed) and then grep for the desired property, or otherwise compute it, and just output that one item.

One can also write a simple shell script to run the jobs in the background (see previous tip). On a 4-core system, it is usually best to run four jobs in the background, do a wait, run four jobs in the background, etc. In the bash shell, wait (no arguments) waits until all background jobs have completed, so this does a reasonably good job of keeping four jobs running, but does not overload the system with too many simultaneous jobs.

\subsection{Visually Scanning Events via the Command Line}

The simplest way to scan events visually is to use the g4blgui program, but it can be done manually via the command line. The OpenInventor driver (OIX) has the ability to exit the viewer and return to the Geant4 command input (File/Escape). This can be used to quickly scan events visually. To do this, first create a text file called beamon.txt containing a large number of identical lines: /run/beamOn 1 $/$ run/beamOn 1 /run/beamOn 1 ... many more identical lines

Then invoke g4beamline like this:

g4bl input.file viewer=best $<$ beamon.txt

Once the viewer appears, it will display the first event. To see the next event, select the File/Escape menu item. This will suspend the OpenInventor viewer, and the Geant4 input routine will read the next line of beamon.txt - that causes it to track one event and refresh the viewer with it. Just keep selecting File/Escape to sequence through events one at a time. This is essentially what the g4blgui program does when a viewer is selected.

\subsection{0ptimizing the Value of Some Parameter(s)}

When designing a new machine or facility, there is frequently the need to optimize some parameters. The program gminuit is available for this, and interfaces well to G4beamline (and to other programs). Download it from http://muonsinc.com in the Computer Programs link at the left.

The basic idea is to write the input.file to have command-line parameters for the parameters to be optimized, and to configure gminuit to vary them. A shell script must be written to run G4beamline and 
then compute a Chi-squared for gminuit to minimize. See the gminuit documentation for details how to do this. Gminuit will optimize any quantity that can be computed in a shell script, and is not limited to G4beamline.

In practice, one finds that program overheads are rather large, so there is a big advantage to requiring fewer iterations. A human can often converge on an optimum faster (fewer iterations) than can gminuit's minimizer. This is especially true if the shell script can present a useful graph to the user rather than just a single value of the Chi-squared. Note also that minimizing an integer-valued expression, such as the \# of particles transported to a given location, is difficult; in gminuit one should set the granularity of MINUIT to 1.0 or more, and run enough particles so that the statistics are reasonably good (at least 1,000 particles, and 10,000 is better).

The examples directory of the G4beamline distribution contains triplet.sh which tunes a quad triplet to achieve a focus at $\mathrm{z}=6000$. Note that it presents the user with a plot of the beam's horizontal and vertical sizes as a function of $\mathrm{z}$ along the beamline - this additional information permits a human to find a solution much faster than the minimizer (the locations of the horizontal and vertical zero-crossings tell you which way to change the quads' values). Even so, using gminuit in manual mode is significantly easier than it would be to do this by manually typing each command (gminuit presents the user with a simple way to change the values of the variables and to execute the program once).

\subsection{Using Two or More Reference Particles}

Unlike most accelerator programs, G4beamline realistically handles particle decays and interactions. This makes it suitable to simulate muon beams from a primary proton beam all the way to a muon experiment or cooling channel. This means that the portion of the beamline just after the production target should be optimized for pions, and the portion at the end should be optimized for muons. The best way to do this is to use two reference particles, one starting at the production target with particle $=$ pi + , and one starting at a suitable downstream point with particle $=\mathrm{mu}+$. The key point is that no tuning should be done in the region where both reference particles are tracked. In exampleAUG05.in, the pion reference hits an aperture and is killed. If this does not happen naturally, you could use a particlefilter to kill the pion reference at an appropriate place. Tuning will probably get confused if multiple reference particles enter the tune region.

\subsection{Fitting to Plots and Histograms in HistoRoot}

A major advantage of using HistoRoot is the fact that it is based on Root [5] and has all of the Root features at its disposal. This includes the ability to fit functions to plots and histograms. The Root user interface is quirky, but quite functional.

To fit a function to a histogram, first generate the plot containing the histogram. Right-click on the histogram itself (one of its bins, not the canvas and not any axis or other object - the cursor changes from cross to arrow when clicking will select the histogram; that's what you want), and then select FitPanel. The FitPanel lets you select from among a number of pre-defined functions and a user-defined function:

\section{Name $\quad$ Description}




\begin{tabular}{|c|l|}
\hline gaus & A Gaussian distribution. \\
\hline gausn & A normalized Gaussian distribution. \\
\hline expo & An exponential distribution. \\
\hline landau & A Landau distribution. \\
\hline landaun & A normalized Landau distribution. \\
\hline pol0- po19 & A polynomial of order 0 through 9. \\
\hline user & $\begin{array}{l}\text { A user-defined function: use "[0]", "[1]", ... for the parameters to be fitted, and } \\
\text { make it a function of the variable "x" (or "x" and "y"). For instance, here is a } \\
1-d \text { Gaussian: }[0]^{*} \exp \left(-0.5 *(x-[1])^{*}(x-[1]) /([2] *[2])\right)(\text { parameters are: constant, } \\
\text { mean, sigma). Note that after typing a user expression you MUST do "Set } \\
\text { parameters", or you will get an error "Function with name ... does not exist". }\end{array}$ \\
\hline
\end{tabular}

There is a button to set the initial values and ranges of the parameters for the selected function. There are a number of options. If you select "Use range", then the double-slider below becomes active and will set the range of the fit (displayed visually in the plot as you move the slider ends). When fitting, it is useful to check the plot's menu item Options/FitParameters so the fit results are displayed in the statistics panel of the plot (you can drag its edges to make it larger, and its font will scale). For further details, see the Root documentation.

Note that if you want to play with backgrounds, you can select Options/CanEditHistograms - then with the mouse you can drag any histogram bin's value up or down as you wish. You can then re-fit to see how your changes affect the results.

\subsection{Interfacing to Other Programs}

Many different file formats are used in physics today, and no program can implement all of them. G4beamline supports ASCII file formats that are suitable for interfacing to other programs, including both track files and field maps. A conversion program in C or Java can easily be written to read an ASCII file and write another in a different format (e.g. new order of items, different units, etc.). Root files can also be converted - Root [5] is quite powerful, and can easily be scripted using C++ macros. It is straightforward to open a root file, select a TNtuple from it, loop over its rows, and write a new Root file and TNtuple (or ASCII file) in a new format. The Root documentation and examples contain numerous macros of this sort. If you have need for an interface that cannot easily be implemented, you can suggest a new feature for G4beamline to implement it directly (see section 7.3).

\subsection{EventID and TrackID, and Encoding Information in them}

In G4beamline, EventID is an integer between -2 and 2,147,483,647 (0x7FFFFFFF), inclusive. Note, however, that EventID-s greater than 16777215 (0xFFFFFF) cannot be precisely represented in a float and their values in any NTuple will be rounded. Values -2 and -1 are reserved for the Tune and Reference particles.

In G4beamline, TrackID is an integer between 1 and 2,147,483,647 (0x7FFFFFFF), inclusive. Similarly, TrackID-s greater than 16777215 (0xFFFFFF) cannot be precisely represented in a float and their values in any NTuple will be rounded. In addition, the beam command will issue a warning for every primary 
TrackID greater or equal to the value of secondaryTrackID (such a primary TrackID could be confused with a secondary's automatically-generated TrackID).

Note there is no requirement that either EventID or TrackID be unique or sequential. When G4beamline generates events, the EventID-s are assigned sequentially from 1, the primary TrackID-s are assigned sequentially from 1 within each event, and secondary TrackID-s are assigned sequentially from the secondaryTrackID argument to the beam command.

This means that when generating a beam file to be read into G4beamline, you can assign both EventID-s and TrackID-s in whatever manner you like, subject to the above constraints. For instance, you could assign bins to the phase space of a generated beam and set either EventID or TrackID to the bin number of each track in the beam file. After running G4beamline to determine which tracks are accepted by the system, a plot of the acceptance can be made by post-processing the output file with knowledge of how many tracks were generated in each bin.

\subsection{Examining Outlier Events}

It often happens that some sort of puzzling event occurs, or an outlier entry appears in some histogram. Here is a method to find such events and then visualize them. As a simple example, consider this bending magnet (red) followed by a virtualdetector (green), with 100 beam tracks shown (blue):

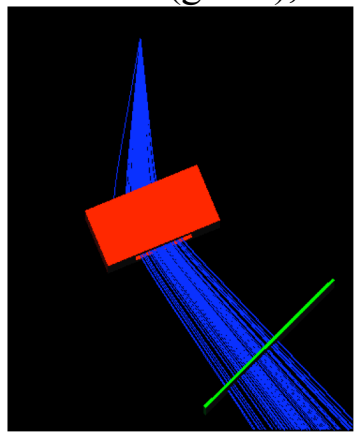

This looks fine, until one runs 1,000 events and histograms $\mathrm{x}$ in the virtualdetector - clearly there is an outlier track at $\mathrm{x} \sim 1500$.

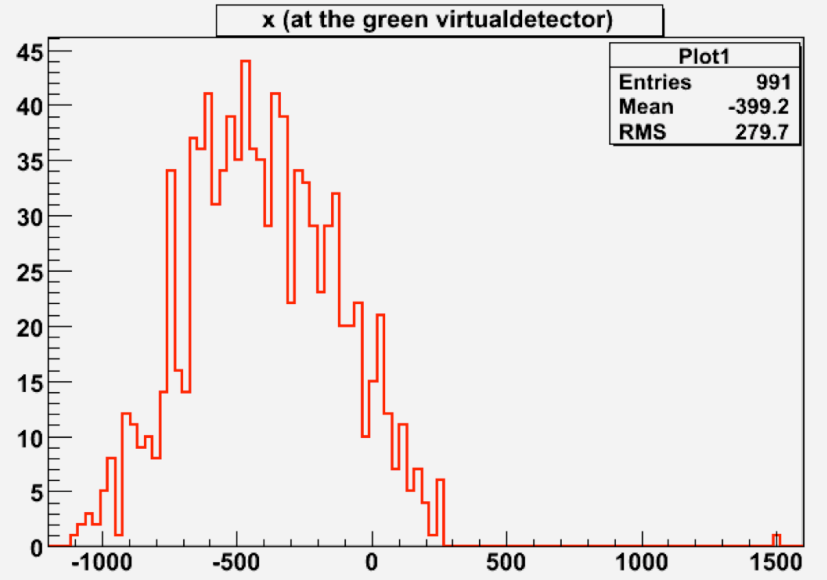

The way to find this event is to use a slider and the EventID features in historoot to select the outlier event and write a histo_events.txt file: 


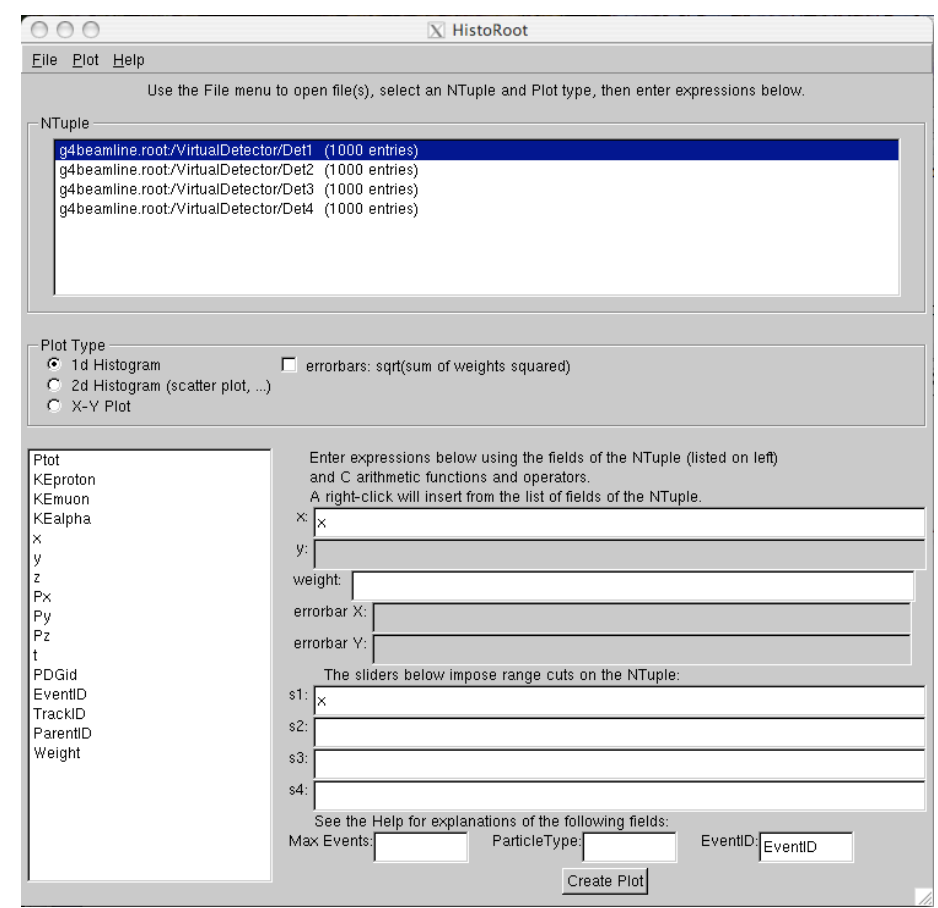

Note that slider $\mathrm{S} 1$ has the same expression as the histogram $\mathrm{x}$ - this permits you to use $\mathrm{S} 1$ to select just the outlier event. As the EventID field contains an expression that evaluates to the event \# of the NTuple row, historoot will write all selected EventID-s to the file histo_events.txt (re-writing it repeatedly as the slider is changed). After selecting just the outlier entry, its EventID will be the only entry in the file.

It is then straightforward to run G4beamline in visualization mode with the eventcuts command to select just those events listed in the histo_events.txt file:

$$
\text { g4bl file.in 'eventcuts file=histo_events.txt' viewer=best }
$$

(The eventcuts command could be edited into file.in instead of being placed on the command-line; in g4blgui the checkbox "HistoRoot Events" puts that eventcuts command into the command-line.) The result is clear and obvious -- this simulation clearly needs shielding around the bending magnet:

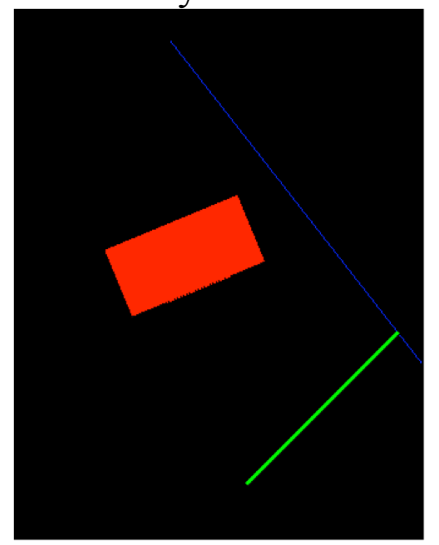

Note: this assumes the default treatment of pseudo-random numbers: the generator is seeded with the event \# before each event. This won't work if you use the randomseed command to change that. 
Note: If your input beam is a file, and that file has multiple tracks with the same event number, G4beamline will treat each track as a separate event with that event number (see the description of beams above). That means that if an event selected via eventcuts has (say) three beam tracks, only one of them need satisfy the conditions used to select the event. The other two tracks with that event \# will be selected by eventcuts but may well look puzzling, as they probably don't satisfy the conditions, and will appear as separate images (events) in the visualization. Just ignore them.

\subsection{Increasing the Number of Events Displayed Visually}

By default, the maximum number of events displayed in a viewer is 100 . To increase this limit to 1000 events, execute this command: g4ui when=4 "/vis/scene/endOfEventAction accumulate 1000"

\subsection{Building G4beamline, Adding Your Own Code}

There are several reasons why you might want to build G4beamline from source:

- You want to run it on an unsupported system or OS.

- You want to optimize it for your system (e.g. a 64-bit build).

- You want to add your own new feature or code.

Before you can add your own code to G4beamline, you must build it from source. Read BUILD.txt and README-*.txt (in the doc directory of any distribution) for details. Note the required tools and the details of the build procedure differ for different OSs.

Once you have built G4beamline from source, test that g4blmake works on your system:

cd test

. $/$ test 72

If this says "test-72: compiling user code omitted" then there is an error in your configuration - you probably need to run setup again (from the top directory of the source distribution you built). If test72 takes 20-30 seconds and prints nothing but its 1-line synopsis, then it succeeded (all tests succeed silently but print an error on failure).

Now you are ready to add your own code. First, create a directory in which to work; for this example I'll call it testing:

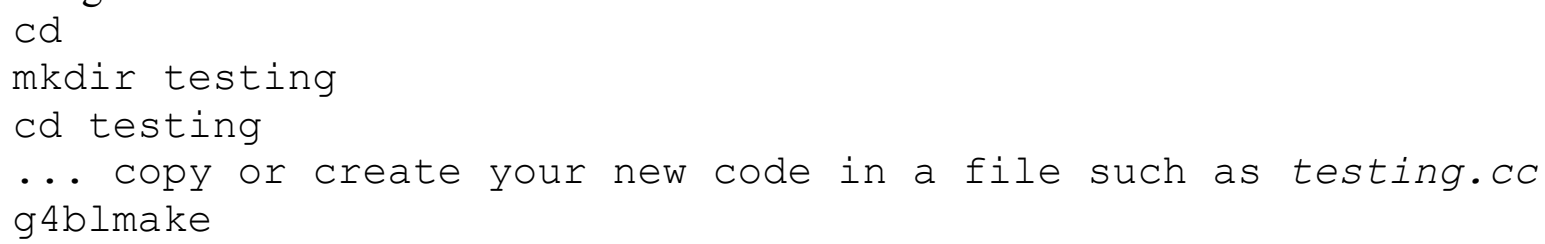

By default this compiles *.cc *.cpp *.C into a new build of G4beamline. Add "-v" to display the details.

If you need an additional library, list all of the files you need on the command line:

G4blmake [-v] *.cc/some/other/library.a

You can put the corresponding "-I/some/other/include/dir" either in the environment variable

CPPFLAGS, or directly on the g4blmake command line (before the .cc files that use it). 
If you have some Fortran source, then you will need a Fortran compiler that is compatible with the $\mathrm{C}++$ compiler on your system. You will also have to write the $\mathrm{C}++$ that calls the Fortran routine(s) correctly (functions are extern "C" in lower-case with an underscore appended, scalar arguments are passed by reference, multiple array indexes are in different order, etc. Google " $\mathrm{C}++$ calling Fortran" for suggestions.). In this case, it is easiest to construct a Makefile to build the pieces (this uses the system .f.o rule to compile the file fortran.f, then link it into G4beamline):

Makefile:

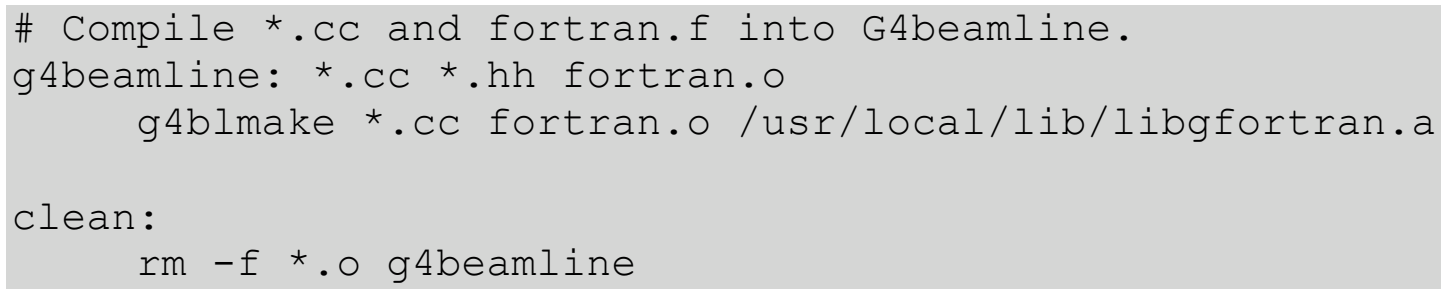

Once your private build of G4beamline succeeds, to run it just follow the directions g4blmake printed: export G4BEAMLINE=`pwd'/g4beamline

Now the $g 4 b l$ script will run your private version. You can verify this by looking at the heading that g4beamline prints out during startup: the version will show both the G4beamline version and your login id. You can also do "help mycommand", or just use mycommand.

Some brief suggestions for writing G4beamline code:

- Point your browser to $<$ install $>/ \mathrm{doc} / \mathrm{html} / \mathrm{index} . \mathrm{html}$ - this will browse the Doxygen documentation for the G4beamline classes.

- Class names beginning "BL" are G4beamline infrastructure classes.

- Class names beginning "BLCMD" are G4beamline commands.

- Filenames are based on the class names by appending .hh or .cc.

- Because commands are modular and independent, your new feature should be packaged in a command. That is, your code should not do anything until and unless the input file uses your command. As a bonus, arguments to that command can pass user-specified values to your code.

- There are many commands in the source. Base your command on an appropriate one.

- Multiple features should be in multiple commands. Note that even such complex features as a space charge computation are contained in a command.

- In general, G4beamline commands are contained in a single file not referenced by any other code (BLCMDphysics and BLCMDcoil are exceptions). In particular, there are no thh files for command classes; the declarations are at the top of the .cc file.

- All classes that implement a command are derived from BLCommand; they register themselves as commands via a static initializer and their default constructor. When properly written, simply compiling your MyCommand.cc via g4blmake will enable its command to be used in any input.file.

- Because commands are so modular, multiple developers can be working on multiple commands in parallel, without conflicts. Only modifications to infrastructure classes require coordination.

- Contributing a command to the G4beamline distribution is very simple, and consists merely of having the development team test it, and then copy its source file into the build directory.

- An exception to using a command is usertrackfilter. This command was specifically designed to use simplified user-supplied code. See section 8.4. 


\subsection{Displaying Magnetic Field Lines}

The fieldlines command will display magnetic field lines. Due to the difficulty of finding fields without user assistance, you must specify a point (in global coordinates) near which to start the field lines; this point is used as the center of a circle of specified radius. The plane of the circle is normal to the $\mathrm{B}$ field at its center. Within the circle, field lines are placed with density that is inversely proportional to $|\mathrm{B}|$. You must also specify the approximate number of field lines to draw; due to the algorithm used this is only approximate.

Within the circle, the initial points of the field lines are allocated as follows: an NxN square grid is placed so it circumscribes the circle; initially all points outside the circle are excluded. An initial value of scale is guessed. As each line is placed, all grid points within scale/|B| of its location in the circle are excluded. The next point is placed at the un-excluded grid point that is closest to the center of the circle, and nearby grid points are excluded. This continues until all grid points have been excluded. If the total number of field lines placed is within a factor of 2 of the desired number, this set is accepted and the field lines are drawn. If the number placed is too small, scale is reduced and the procedure is repeated; if the number placed is too large, scale is increased and the procedure is repeated. At most 10 iterations are permitted. This implies that asking for fewer than 5 lines, or more than 1,000 lines, is likely to be ineffective. This algorithm gives a good distribution of field lines for solenoids and other simple fields.

Once the lines are placed within the circle, for each one the B field is traced in both directions; there are three criteria for stopping drawing a given half-line:

a. The line intersects the plane of the circle (i.e. it has come back to the plane, but outside the magnet).

b. The value of $|\mathrm{B}|$ falls below minField.

c. The field line leaves the simulated world.

There are additional arguments to the fieldlines command that control the drawing. 


\section{Advanced Topics}

\subsection{Writing Scripts Using G4Beamline}

By using parameters in your input.file you can automate running G4beamline programs, without having to script the editing of your input.file.

\subsubsection{Using a Linux Cluster or Multi-CPU System}

Because the pseudo-random number generator is seeded with the Event number before the start of each event, all that is necessary to run jobs in parallel is to ensure that they do not run the same events and that they write to different output files. The arguments firstEvent and lastEvent to the beam command facilitate this. The basic idea is to use parameters for firstEvent and lastEvent and pass them in on the command-line, and write a shell script to submit multiple jobs to the cluster with disjoint ranges for each job. Here the output file corresponds to the first event number. The input.file looks like this:

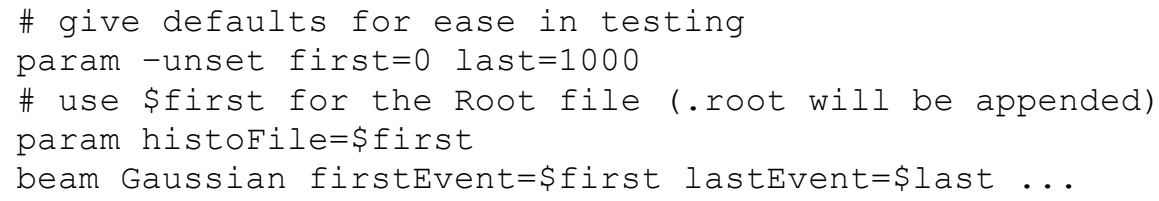

Any other output files (e.g. format=ascii for virtualdetector, zntuple, timentuple, etc.) should be set to $\$$ first as well, so multiple instances of the program won't overwrite output files.

The simulation is run like this on a 4-CPU system (bash shell):

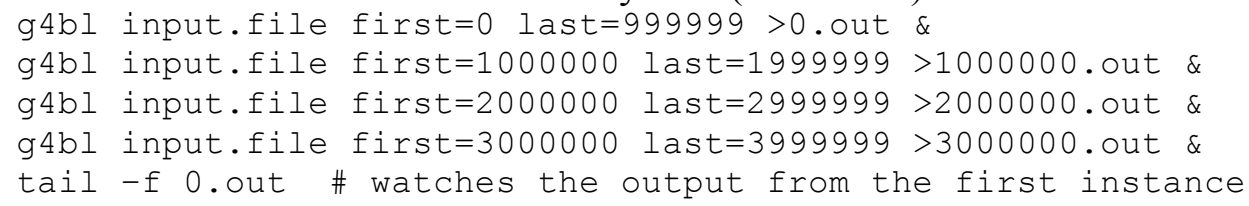

The output files will be 0.root, 1000000.root, 2000000.root, and 3000000.root. The historoot program can be used to generate histograms:

historoot *.root

You can select the 4 instances of a given NTuple and historoot will generate histograms and plots containing results from all 4 million events.

The ExampleAUG05 is a real-world example using this technique.

\subsubsection{Using the gminuit Program to Tune Values}

The gminuit program (http://www.muonsinc.com [ComputerPrograms/Gminuit]) was specifically designed to permit the easy tuning of values in an input.file for G4beamline. Because any argument to any G4beamline command can be set from a parameter, and any parameter can be set on the G4beamline command line, gminuit can be used to tune any value in your input.file based on any criterion you can script. It is possible to determine the gminuit value to minimize by either reading the output from G4beamline with steppingVerbose=1 (tailor steppingFormat to suit), or by reading the file 
generated by virtualdetector format $=$ ascii or trace format $=$ ascii. Remember that most shells only do integer arithmetic, so it is appropriate to use a tcl or perl script to interpret the G4beamline output and return a value for gminuit to minimize. See the gminuit documentation for details. The file examples/triplet.sh is an example that uses gminuit to tune a quad triplet.

\subsection{Violating the Rules on Geometrical Intersections}

Geant 4 tracks particles through the geometry hierarchy by looking for the next geometrical boundary along the track's path, and arranging so there is a step that ends at each boundary crossed by the track. To keep the search for boundaries efficient, Geant4 only looks for the outer boundary of the current volume and for boundaries of all daughter volumes of the current volume. So if sibling volumes A and B overlap, if the track enters A first, then it will be tracked in A through the region of intersection; but if it enters B first then it will be tracked in B through the region of intersection. Similarly, if a daughter A1 of A extends outside volume A, then if the track is in A1 it can be tracked in A1 even outside of A; but if it comes in from the sibling or parent of A it won't enter A1 until it enters A.

While violating the rules is risky and discouraged, if you are careful they can be violated when necessary (e.g. it is not possible or too difficult to describe the geometry correctly). If the invalid intersections don't matter, then they won't affect the result of the simulation. They won't matter if all volumes involved have the same material and none of them is "active" (in G4Beamline that means they aren't a virtualdetector). They also won't matter if the regions of intersection are in places where no particles are tracked (e.g. far from the beamline aperture). And they won't matter if either of the intersecting volumes has kill $=1$.

The automatic geometry test in G4Beamline verifies the geometry hierarchy by testing points on the surface of each element's volume, verifying that each point is inside the parent volume of the element, and is outside every sibling volume. The points are selected to test "corners" first, and then points are randomly distributed over the faces of the surface of the volume. This is not perfect, but it does catch nearly all of the invalid intersections.

Geant 4 also has other methods of verifying the geometry hierarchy. See the Geant4 user's manual [1]. They involve shooting geantinos through the detector and verifying that the boundaries each one crosses are ordered properly. They can be executed using the $g 4 u i$ command.

\subsection{Making a Movie}

G4beamline now supports the ability to make a movie of the beam, with the camera sitting on the reference particle watching the other beam particles "dance" around nearby. The implementation is quite flexible, and permits the user to specify multiple simultaneous panes, each displaying an arbitrary pair of expressions using the track variables. An example movie is viewable at http://g4beamline.muonsinc.com. Here is its first frame: 


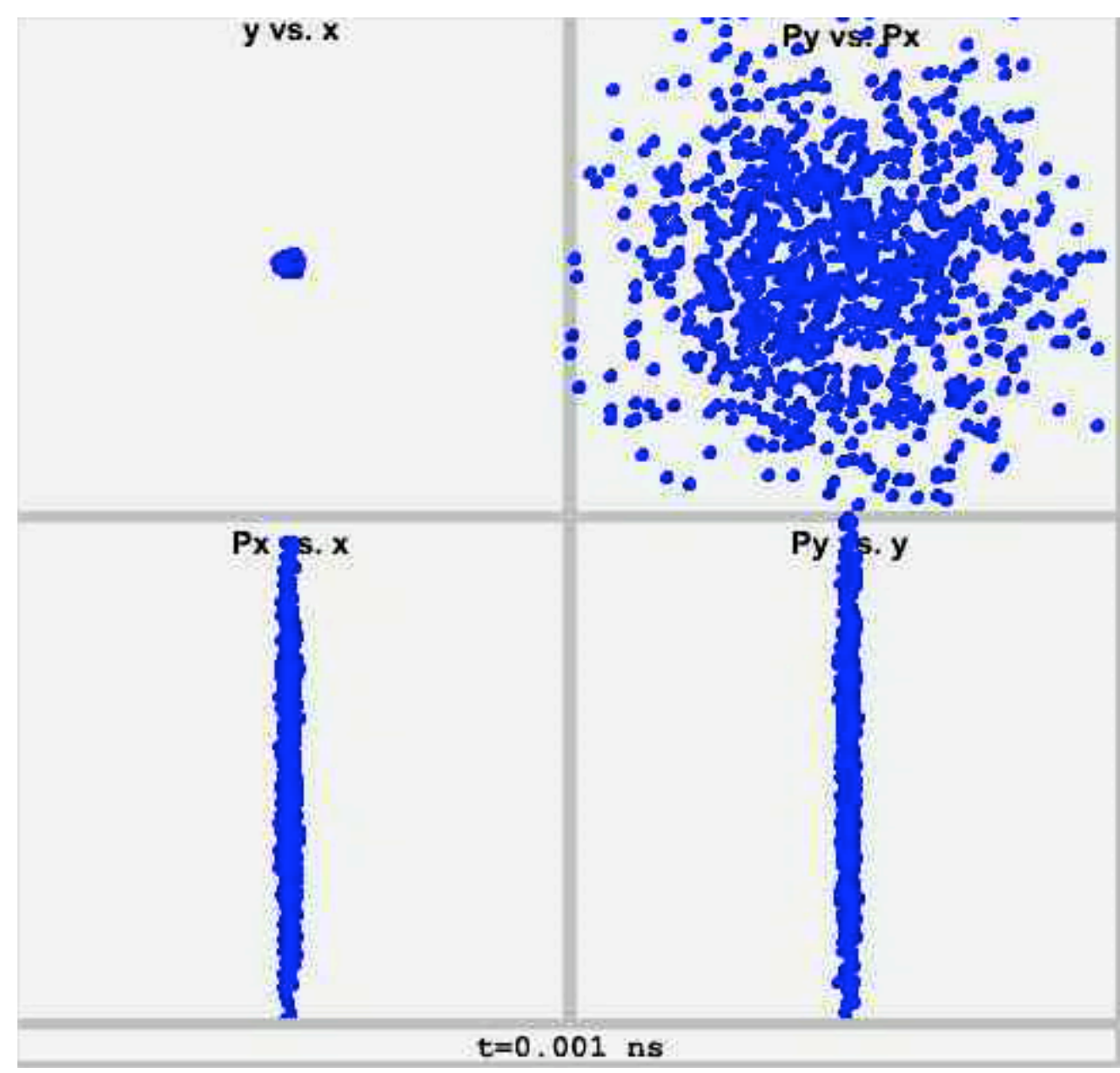

This movie is of example1.in, in which a Gaussian muon beam propagates through free space into four virtualdetector-s (which are invisible in the movie). The movie is 10 seconds long, during which the " $y$ vs. x" pane's particles grow considerably, and the two lower panes' ellipses rotate in the usual manner. A handful of decay electrons and neutrinos become visible.

Making a movie requires the following prerequisites:

1. Installation/configuration requirements:

a. G4beamline version 1.16 (or later).

b. Root version 5.20 (or later).

c. A C++ compiler that Root can use in ACLiC mode.

d. You must use the command-line to run g4blmovie; on Windows this requires Cygwin [7].

e. The ffmpeg program [8] must be installed (it converts a series of frame images into a movie).

2. G4beamline simulation requirements:

a. There must be a reference particle.

b. The movie command must be included. Note that once you have a working simulation, this can be given on the command line: g4bl input.file movie...

3. g4blmovie command-line program

a. It is included in the G4beamline distribution (1.16 and later). 
b. Note that so far this has been used only on Mac OS X; it is expected to work on other platforms but has not yet been tested.

The basic approach is to first run a G4beamline simulation, with the input.file containing the movie command, and with a Root output file. The movie command arranges to generate several NTuples in the Root file that will be plotted to make the movie. This is quite similar to the trace command that traces all tracks into a single NTuple, so the output file can grow quite large. Note that a movie containing more than a few hundred particles tends to be so crowded that details are not visible. Once the simulation is complete, the g4blmovie command is run, which has its own input file to describe the movie. This program uses Root to read the movie NTuples and generate a series of image files; it then runs ffmpeg to convert them into the desired movie, and then deletes the image files. Note that movies can get large, and the production process requires about triple the disk space of the final movie; a 10second movie at 24 frames/second is typically about a megabyte long (these rather un-natural images don't compress very well).

The new g4blmovie command has one argument, an input file to describe the movie. This gives great flexibility in organizing the picture as a number of panes, each displaying a pair of expressions using the track variables. The panes are laid out in rows, with no inherent limit on either the number of panes in a row or on the number of rows, but too many of either will probably make the movie unwatchable. The input file is quite similar to that of G4beamline, except that quoted arguments are not allowed, spaces are not permitted (or needed) in any argument, and there are no parameters.

\begin{tabular}{|c|c|c|}
\hline \multicolumn{3}{|c|}{ g4blmovie input file commands } \\
\hline Command & Argument & Description \\
\hline \multirow[t]{14}{*}{ setup } & & This command must be first. It sets basic parameters for the movie. \\
\hline & outputFile & $\begin{array}{l}\text { The output movie file. Its extension determines the format - any } \\
\text { format supported by ffmpeg can be used. The following extensions } \\
\text { are known to work: .mov, .swf, .avi. Beware: spaces are not allowed. }\end{array}$ \\
\hline & windowWidth & The movie window width, in pixels. All panes scale to this value. \\
\hline & windowHeight & The movie window height, in pixels. All panes scale to this value. \\
\hline & tMin & The simulation's start time of the movie (ns). \\
\hline & tMax & The simulation's end time of the movie (ns). \\
\hline & duration & The real time duration of the movie in seconds (10). \\
\hline & frameRate & The frame rate of the movie, frames/sec (24). \\
\hline & textHeight & $\begin{array}{l}\text { The default height of text, in pixels (15). Changeable in other } \\
\text { commands. }\end{array}$ \\
\hline & background & $\begin{array}{l}\text { The background color (\#F3F3F3 - light gray). Changeable in other } \\
\text { commands. }\end{array}$ \\
\hline & borderColor & The color of the borders between panes ( $\# \mathrm{C} 0 \mathrm{C} 0 \mathrm{C} 0$ ). \\
\hline & borderSize & The size of the borders, in pixels (5). \\
\hline & marker & $\begin{array}{l}\text { The marker to use ( } 21 \text { - the square marker). This marker is used for } \\
\text { all particles (but see the particle command below to color them). Note } \\
\text { that other markers, such as } 20 \text { (filled circle), can greatly slow down } \\
\text { the movie production. }\end{array}$ \\
\hline & markerSize & The relative size of the marker (1.0). \\
\hline
\end{tabular}




\begin{tabular}{|c|c|c|}
\hline & pictureType & $\begin{array}{l}\text { The extension of the pictures used to generate the movie (jpg). Other } \\
\text { types known to both Root and ffmpeg can be used, but jpg has given } \\
\text { the best performance. }\end{array}$ \\
\hline \multirow[t]{2}{*}{ particle } & & Sets the color for each particle type. \\
\hline & * & $\begin{array}{l}\text { Arguments are of the form: } 13=\# 0000 \mathrm{FF} \text {, where } 13 \text { is the PDGid of } \\
\text { the particle, and } \# 0000 \mathrm{FF} \text { is the color ( } 6 \text { hex digits, } 2 \text { each for red, } \\
\text { green, and blue; } \# 000000 \text { is black, } \# \mathrm{FFFFFF} \text { is white, and } \# 0000 \mathrm{FF} \text { is } \\
\text { bright blue). The '\#' is required. PDGid } 0 \text { applies to all unset particles } \\
\text { (defaults to black). }\end{array}$ \\
\hline \multirow[t]{11}{*}{ plot } & & Displays a plot of two expressions. \\
\hline & filename & $\begin{array}{l}\text { The name of the Root file to use. If omitted, uses the most recently } \\
\text { opened Root file (i.e. from the previous command). Note the NTuple } \\
\text { names to use are fixed: Movie/Reference and Movie/Tracks. }\end{array}$ \\
\hline & title & $\begin{array}{l}\text { Title of the plot (defaults to " } y \text { vs. } x \text { ", where } x \text { and } y \text { are the } \\
\text { expressions). }\end{array}$ \\
\hline & $\mathrm{x}$ & $\begin{array}{l}\text { Expression involving the track variables to plot horizontally. } \\
\text { Variables: x, y, z, Px, Py, Pz, t, PDGid, EventID, TrackID, ParentID, } \\
\text { Weight. The standard C functions and operators can be used. }\end{array}$ \\
\hline & $\mathrm{y}$ & Expression involving the track variables to plot vertically. \\
\hline & $\mathrm{xMin}$ & Left limit of the plot, in whatever coordinates are used by $\mathrm{x}$. \\
\hline & $\mathrm{xMax}$ & Right limit of the plot, in whatever coordinates are used by $\mathrm{x}$. \\
\hline & yMin & Bottom limit of the plot, in whatever coordinates are used by $y$. \\
\hline & yMax & Top limit of the plot, in whatever coordinates are used by $y$. \\
\hline & background & Color of the background (setup value) \\
\hline & textHeight & $\begin{array}{l}\text { Height of text in pixels. If omitted, the value from the setup command } \\
\text { is used. }\end{array}$ \\
\hline \multirow[t]{10}{*}{ sideview } & & $\begin{array}{l}\text { Displays a side view of the apparatus using Reference Coordinates, } \\
\text { with a moving marker that indicates where the reference particle is } \\
\text { located at the current time. }\end{array}$ \\
\hline & markerSize & The relative size of the marker (1.0). \\
\hline & filename & $\begin{array}{l}\text { The name of the Root file to use. If omitted, uses the most recently } \\
\text { opened Root file (i.e. from the previous command). Note the NTuple } \\
\text { names to use are fixed: Movie/Reference and Movie/Elements. }\end{array}$ \\
\hline & zMin & Left limit of the plot, in Reference Coordinates (synonym is xMin). \\
\hline & zMax & Right limit of the plot, in Reference Coordinates (synonym is xMax). \\
\hline & yMin & Bottom limit of the plot, in Reference Coordinates. \\
\hline & yMax & Top limit of the plot, in Reference Coordinates. \\
\hline & title & Title of the view ("')). \\
\hline & textHeight & Height of text in pixels (setup value). \\
\hline & background & Color of the background (setup value) \\
\hline \multirow[t]{2}{*}{ row } & & Initiates a new row of plots. \\
\hline & height & $\begin{array}{l}\text { The relative height of this row. The first row has a height of 1.0, } \\
\text { which sets the scale. }\end{array}$ \\
\hline \multirow[t]{2}{*}{ space } & (none) & Displays like a plot, but is empty. \\
\hline & title & Title of the pad (“"). \\
\hline
\end{tabular}




\begin{tabular}{|l|l|l|}
\hline \multirow{4}{*}{ time } & textHeight & Height of text in pixels (setup value). \\
\cline { 2 - 3 } & background & Color of the background (setup value) \\
\cline { 2 - 3 } & (none) & Displays like a plot, but contains the current time (in ns). \\
\cline { 2 - 3 } & title & Title of the pad ('"). \\
\cline { 2 - 3 } & textHeight & Height of text in pixels (setup value). \\
\cline { 2 - 3 } & background & Color of the background (setup value) \\
\hline Position & (none) & $\begin{array}{l}\text { Displays like a plot, but contains the current Z position of the } \\
\text { reference particle (mm). }\end{array}$ \\
\cline { 2 - 3 } & title & Title of the pad ('"'). \\
\cline { 2 - 3 } & textHeight & Height of text in pixels (setup value). \\
\cline { 2 - 3 } & background & Color of the background (setup value) \\
\hline
\end{tabular}

Within a row, all plots share equally the total width of the window. The rows are laid out to use the entire height of the window, but the fraction of each row is determined by its height/totalHeight (totalHeight is the sum of height for all rows). There can be a different number of plots in each row. You can use windowWidth, windowHeight, the row height-s, and the number of panes per row to have both large and small panes in one movie. Usually each pane should be approximately square. To play with the layout, it is helpful to temporarily set tMax and duration to small values to speed up the production until the layout is what you want. Many simulations start at $\mathrm{t}=0$, and for them it is best to set $t$ Min to a small value (e.g. 0.001) so the initial tracks show up in the viewer when the file is opened but not playing.

Here is the examples/movie.in file that generated the example1 movie referenced above:

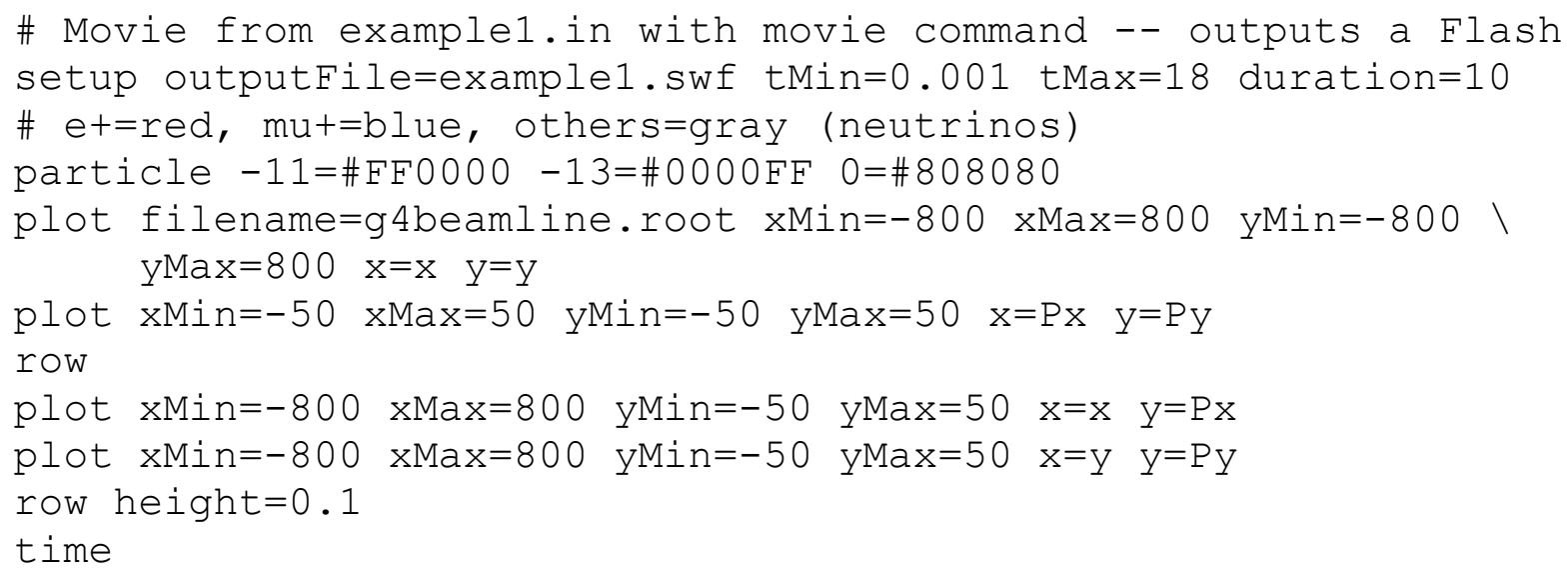

Here are the commands used to generate and display that movie:

cd g4beamline-1.16-Darwin-g++/examples

g4bl examplel.in movie

g4blmovie movie.in

open example1.swf 


\subsection{User Code for the usertrackfilter Command}

The usertrackfilter command requires that the user compile code for use with G4beamline. The usertrackfilter element permits the user to supply code that applies to every track that enters the physical volume of the element; this code can:

- Kill tracks, based on arbitrary criteria.

- Modify the momentum, time, and/or weight of tracks.

- Create secondary tracks.

Doing this requires:

- G4beamline built from source on your system.

- A basic understanding of how to write and compile $\mathrm{C}++$ code.

- On Windows, the Cygwin system must be installed.

- On all OSs, a command-line window is used.

The basic idea is to compile your code directly into G4beamline. An example is provided in the directory UserCode in the G4beamline installation directory (if you don't know where that is, type the command " $\mathrm{g} 4 \mathrm{bl}$-dir"). It contains the following files:

\begin{tabular}{|l|l|}
\hline Filename & Description \\
\hline ExampleFilter.cc & Code for the example filter. \\
\hline test.in & Input file to test the example filter. \\
\hline
\end{tabular}

These instructions are for the bash shell; modify them appropriately for other shells. These are all basic UNIX commands, and if you don't understand them you probably need the assistance of someone more experienced in UNIX and C++ code development. After installing and building G4beamline from source (see the appendices, BUILD.txt, and the README files), you should first try running the example:

cd directory-for-your-simulation

mkdir UserTrackFilter

$\mathrm{cp}$ 'g4bl -dir'/UserCode/*.

g4blmake

export G4BEAMLINE $=\$ P W D / g 4$ beamline

g4bl test.in

This last command should end by printing:

Run complete 10 Events 0 seconds

ExampleFilter::complete killed 6 tracks, created 51 tracks

Examplefilter::complete killed 6 tracks, created 51 tracks

Once the example works, you can modify it to suit your needs (the example is quite silly and unphysical; it is intended only to show what can be done). Things to remember:

- The source files to be compiled are arguments to g4blmake; they default to *.cc *.cpp *.C (i.e. the common extensions for $\mathrm{C}++$ source files).

- Any additional libraries your code requires can be passed in the environment variable EXTRALIBS, or can be simply appended to the g4blmake command line.

- Any additional compiler flags (e.g. - I/some/dir) can be passed in the environment variable CPPFLAGS.

- Your filter code must implement a class derived from UserTrackFilter, and must have a static instance (to register it with G4beamline). 
- Read the comments in UserTrackFilter.hh to understand the interface to G4beamline, and to see what types of things your code can do.

- More than one derived class can be defined in the source files, either in a single .cc file or in separate .cc files. They must have different filterName()-s.

- You can add other libraries if you wish. For example, your class could query an external database indexed by eventID and trackID, and kill unwanted tracks as indicated by the database. (To do that your class would connect to the database in setup(), query it in filter(), and disconnect in complete().)

- It's best to keep your code simple, as it is rather difficult to debug large programs like G4beamline.

\subsection{Multiple Instances of G4beamline using MPI}

The Message Passing Interface (MPI) is a standard interface that permits multiple jobs on multiple computer systems to cooperate in a single computation. One executes a single MPI job that simulates the events specified in the input.file, using multiple jobs running simultaneously, collecting their output into a single set of NTuples (in a single Root file for Root NTuples). Only the tracking is parallelized, but that is "trivially parallelizable" and the overheads are low; it scales quite well to many processors (likely 50 or more, but this depends on the details of both the system being simulated and the computer hardware). On a Mac Pro running Mac OS X with 4 cores, the management and communication overhead is only a few percent.

G4beamline implements MPI when the argument "-enable-mpi" is given to configure when building the program. This is the default on Mac OS X 10.5 and later, which includes all necessary MPI support; on other systems it must be specified at the start of the build process (which requires an installation from source).

G4beamline is written such that if you just run it by itself, via the $g 4 b l$ script, or via the $g 4 b l g u i$ program, it runs without MPI as a single, local process. But if it is run via mpirun (or the equivalent for other MPI implementations), then it runs the specified number of instances and operates in MPI mode.

In MPI terminology, each instance of the program is called a "rank", which is a non-negative integer unique to each instance of the program. For G4beamline, rank number 0 is the master controlling instance, and all other ranks are worker instances (nodes). The usual command is:

\section{mpirun -np 5 g4bl input.file [... parameters]}

This command acts very nearly like a single G4beamline program simulating input.file, except it uses 5 processes to speed up the computation. It does the following:

1. Starts 5 instances of the g4bl script, using mpirun's internal mechanism to start them.

2. Each instance of $\mathrm{g} 4 \mathrm{bl}$ configures its environment and runs g4beamline.

3. Each instance of g4beamline determines that it was run via mpirun, and gets its rank number from the MPI component of the OS.

4. The rank 0 instance does the following:

a. Reads input.file and the parameters, constructs the geometry, the physics list, etc.

b. Creates an output file for each NTuple (one file for all Root NTuples). 
c. It waits for messages from worker nodes, of which there are two basic types:

i. A message indicating the worker is idle; rank 0 responds by sending it a block of beam tracks to process, sending 0 tracks if none are left.

ii. A message containing NTuple rows; rank 0 responds by writing them to the appropriate NTuple.

d. When all events have been processed, and all worker nodes have indicated they are idle, rank 0 terminates any remaining workers (usually none) and exits itself.

5. The instances with rank $>0$ do the following:

a. Reads input.file and the parameters, constructs the geometry, the physics list, etc.

b. Configures all NTuples to send their rows to rank 0 rather than writing to any file.

c. Sends a message to rank 0 indicating it is idle.

d. Waits for a message from rank 0 containing a block of beam tracks to process.

e. If the message contains 0 beam tracks, sends any un-sent NTuple rows to rank 0 (i.e. flushing their buffers), and then exits.

f. Otherwise, processes the received beam tracks.

g. Loops back to $\mathrm{c}$.

The following parameters control this:

MPI_PackTracks Number of beam tracks per message (default $=10$ ).

MPI_PackNTuples Number of NTuple rows per message (default=100).

MPI_ProbeSleep Sleep time (milliseconds) of the sleep in a loop calling MPI_Iprobe();

nonzero permits better termination behavior (default $=1$ ).

Note that the rank 0 instance only does management tasks, and performs no tracking. On a Mac Pro with 4 cores, it is appropriate to run 5 processes, as rank 0 uses only a few percent of a CPU. 


\section{File Formats}

Note that ASCII file formats begin with comments ('\#' in column 1) that give both the field names and their units. The historoot program is able to use these comments to give names to the fields of such files (it ignores the units, which are intended for humans).

\subsection{BLTrackFile (generated by virtualdetector, read by beam)}

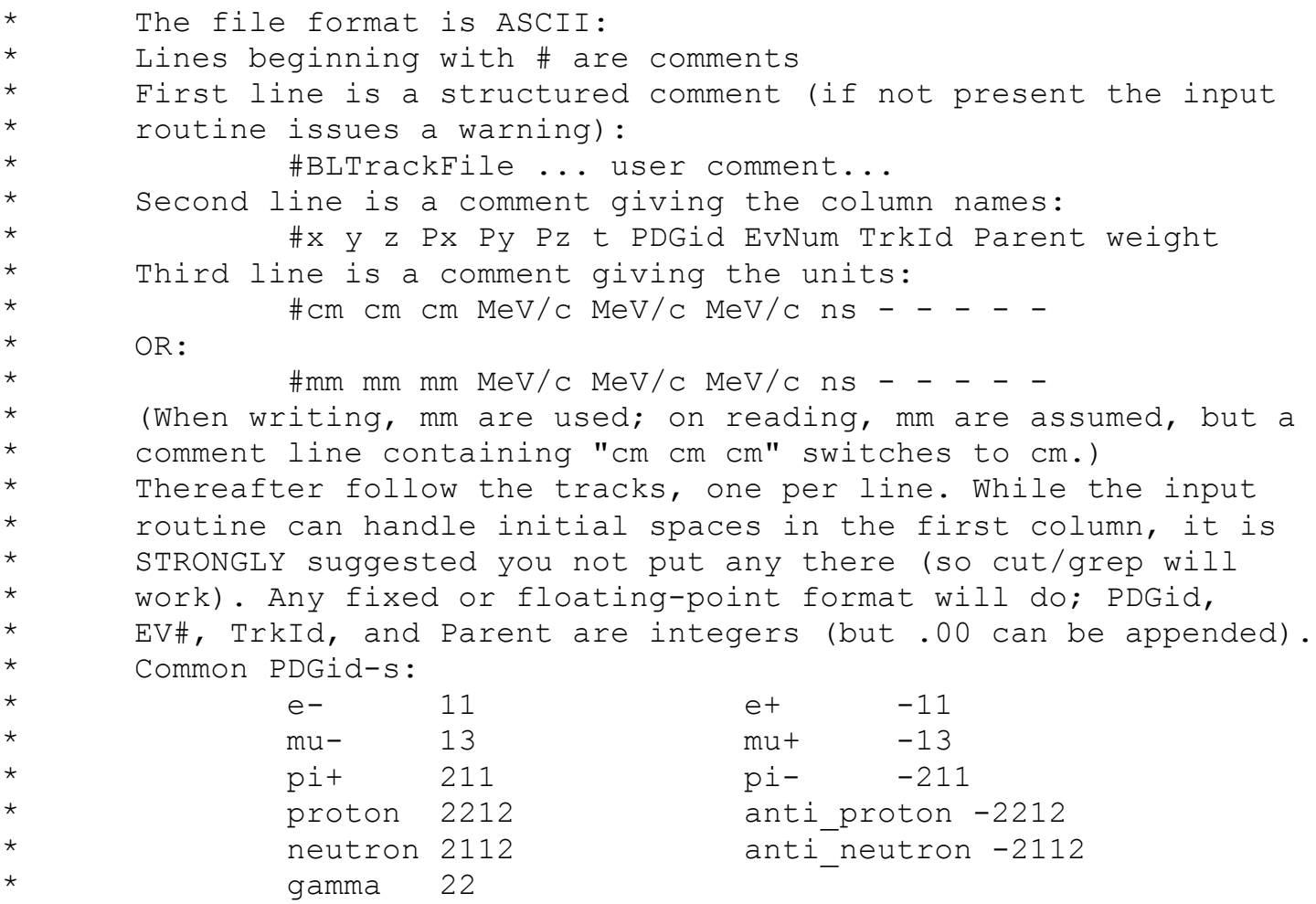

\subsection{Trace File}

When writing track traces with format=ascii in the trace command, the output is as follows:

The first 12 columns are identical to those of BLTrackFile

Columns 13,14,15 give Bx,By,Bz in Tesla

Columns 16,17,18 vive Ex,Ey,Ez in MegaVolts/meter

The first 3 comment lines are similar to those of BLTrackFile, giving a comment, the column names, and their units. All values are in the selected coordinates from the trace command (centerline, global, or reference).

\subsection{HistoScope (generated by NTuples, read by beam)}

NOTE: HistoScope I/O is deprecated, and will disappear from the next major release. The HistoScope program is no longer supported, and the old versions do not run on modern versions of Linux. 
The HistoScope file format is handled by the HistoScope library [3] and the companion histo program. G4Beamline makes no changes to this format.

Each NTuple in HistoScope has a specified set of variables (fields), and in the histo program they are presented by name. The names and units used for G4Beamline virtualdetector NTuples are:

X,Y,Z (mm)
Px,Py,Pz $(\mathrm{MeV} / \mathrm{c})$
T (ns)

PDGid $(11=\mathrm{e}-$, 13=mu-, 22=gamma, 211=pi+, 2212=proton, ... $)$

EventNum (inexact above 16,777,215)

TrackID

ParentID $(0=>$ primary particle $)$

Weight $($ default $=1.0)$

\subsection{FOR009.DAT}

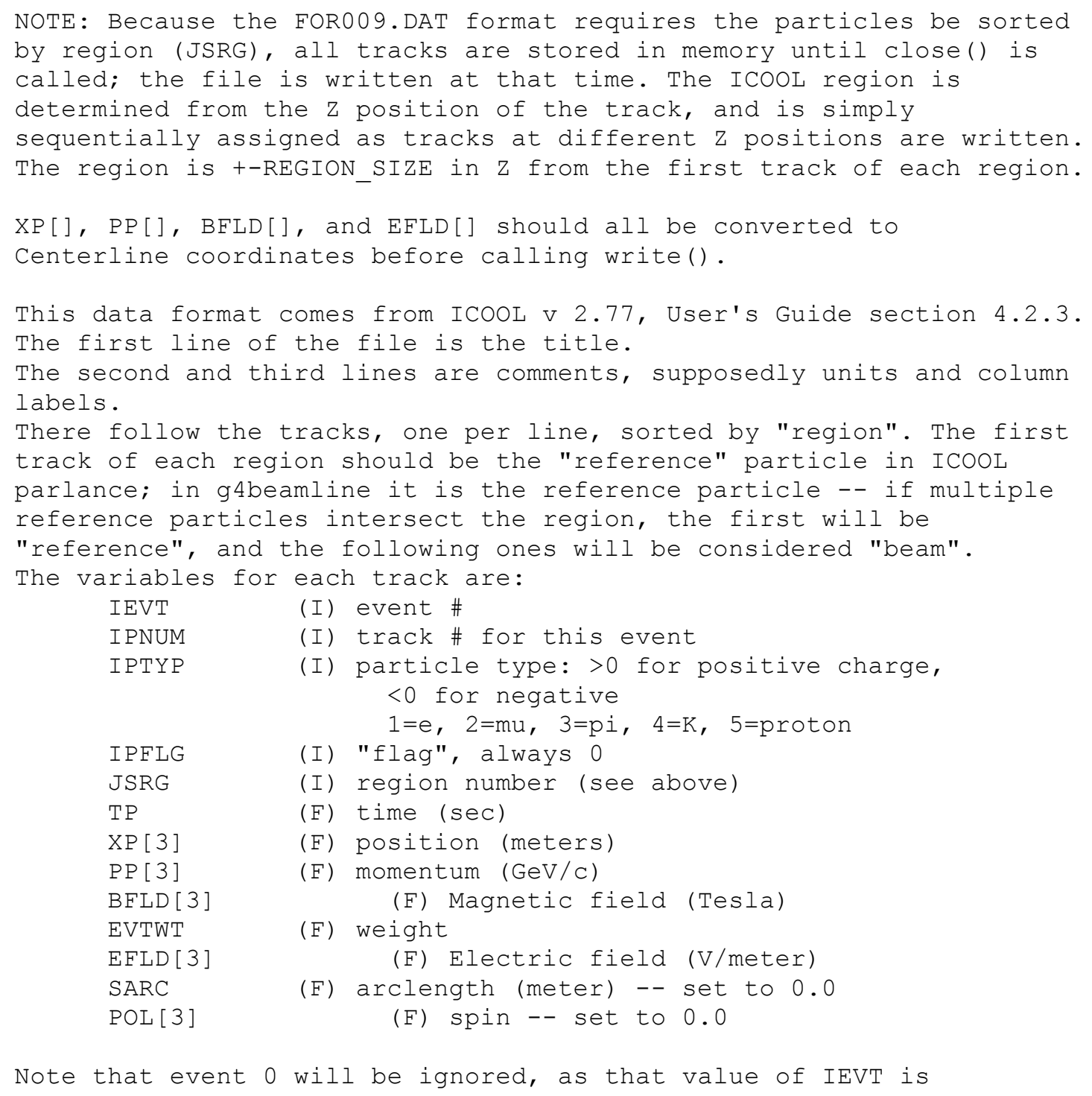


reserved for the reference particle.

\title{
9.5 BLFieldMap
} \\ Blank lines, and lines beginning with \# or * are comments. Lines \\ beginning with * are printed to stdout. Units are mm for coordinates, \\ Tesla for B, and MegaVolts/meter for $E$; use normB and normE if the \\ data points use different units. \\ The input file starts with a set of commands to define the parameters \\ of the map, followed by blocks of lines containing the values of the \\ field components. The field component names depend on the type of map \\ (grid: Bx,By,Bz,Ex,Ey,Ez; Cylinder: Br,Bz,Er,Ez). \\ Each command has a specific list of arguments to define parameters \\ of the map.
}

BEWARE: the parsing is not exhaustive. For instance, invalid arguments are silently ignored (which means you must verify the spelling and capitalization of argument names). Correct inputs will yield correct results, but invalid inputs may not be detected and may yield seemingly-correct but unintended results.

The first command is usually a param command, which has the following arguments:
maxline
The maximum number of characters per line (default=1023)
current
The current corresponding to this map (default=1.0)
gradient The gradient corresponding to the map (default=1.0)
normE A normalization factor for E components (default=1.0)
normB A normalization factor for B components (default=1.0)

Two types of maps are implemented: grid and cylinder.

grid maps are a 3-D grid, with each block of data being a single

$X-Y$ plane; within a block the lines are $Y$ and the columns of each line

are values along $\mathrm{X}$.

The grid command has the following arguments:

Xo The $\mathrm{X}$ value for the first value in each line

Y0 The $Y$ value for the first line in each block

Z0 The $\mathrm{Z}$ value for the first block of each field component

nX The number of columns per line

nY The number of lines per block

nz The number of blocks per field component

$\mathrm{dX}$ The $\mathrm{X}$ increment between values in each line

dY The $Y$ increment between lines

$\mathrm{dz} \quad$ The $\mathrm{Z}$ increment between blocks

tolerance The tolerance for pointwise data (default=0.01 mm)

After the grid command, the following optional commands can be given: extendX flip $=.$. .

extendy flip=...

extendz flip=...

These commands permit a half-map to be extended to the full map

around $X=0, Y=0$, or $Z=0$ respectively. The optional flip argument is a comma-separated list of field components whose signs will be inverted for negative values of the coordinate. For example, "extendz flip=Bx,Ex" means the map from $Z=0$ to $Z=(n Z-1) * d Z$ is extended symmetrically around $\mathrm{Z}=0$ to negative $\mathrm{Z}$ values, flipping the signs of $\mathrm{Bx}$ and Ex when $\mathrm{Z}<0$. 
This could be followed by "extendx flip=Bx,Ex", and the field flips will be the products of both commands.

cylinder maps are a 2-D map with rotational symmetry around the $Z$ axis. Each field component has a single block with lines being $Z$ and the columns being $R$.

The cylinder command has the following arguments:

zo The $z$ value for the first line in each block

$\mathrm{nR}$ The number of columns per line

nz The number of lines per block

$\mathrm{dR} \quad$ The $\mathrm{R}$ increment between colums

$\mathrm{dz} \quad$ The $\mathrm{Z}$ increment between lines

tolerance The tolerance for pointwise data (default=0.01 mm) After the cylinder command, the following optional commands can be given: extendz flip=...

This command behaves the same as for the grid map.

After the commands, each block consists of a line containing the name of the field component, followed by the lines of the block. The values within a line can be separated by whitespace or a ',' followed by optional whitespace.

Field components that are not given are set to 0.0 everywhere. Missing values will be considered to be 0.0 .

For grid maps the first block is for $Z=Z 0$, and successive blocks increment $Z$ by $d Z$; the first line in a block is for $Y=Y O$ and the first column in each line is for $\mathrm{X}=\mathrm{X} 0$.

For cylinder maps, the first line in each block is for $\mathrm{Z}=\mathrm{z} 0$ and the first column in each line is for $\mathrm{R}=0$.

Instead of the blocked input format, a pointwise data format can be used. This is introduced by a line containing the command "data", followed by the individual points of the map, one per line. for a grid field, each line contains values for $\mathrm{X}, \mathrm{Y}, \mathrm{Z}, \mathrm{Bx}, \mathrm{By}, \mathrm{Bz}, \mathrm{Ex}, \mathrm{Ey}, \mathrm{Ez}$ separated by either a comma and optional whitespece or by whitespace. for a cylinder field each line contains values for $\mathrm{R}, \mathrm{Z}, \mathrm{Br}, \mathrm{Bz}, \mathrm{Er}, \mathrm{Ez}$. The order of the points does not matter; omitted grid points will be 0.0 , and for duplicates the last entry wins. If there is no E field, the Ex,Ey,Ez or Er,Ez entries should be omitted on every line. NOTE: every line's $X, Y, Z$ or $R, Z$ must be on a grid point as specified by the arguments to the grid or cylinder commands, to within the tolerance specified; if not, an error message is printed and the input line is ignored.

For time=dependent fields, the "time" command is used: time [period=12]

period, if given, is in nanoseconds, and causes the interval [0, period) to be extended forever (before and after the values given). Because of the interpolation used, at least two points beyond the interval boundaries should be provided; there need not be a point at either boundary (but usually there are).

Following the time command are lines containing 2 or 3 doubles: $t$ B E

where $t$ is the time (nanoseconds), and $B$ and $E$ are factors for the fields. If $E$ is omitted, the value for $B$ is used. These values will be interpolated in time with a cubic spline that can handle either uniform or non-uniform spacing of points along $t$. 


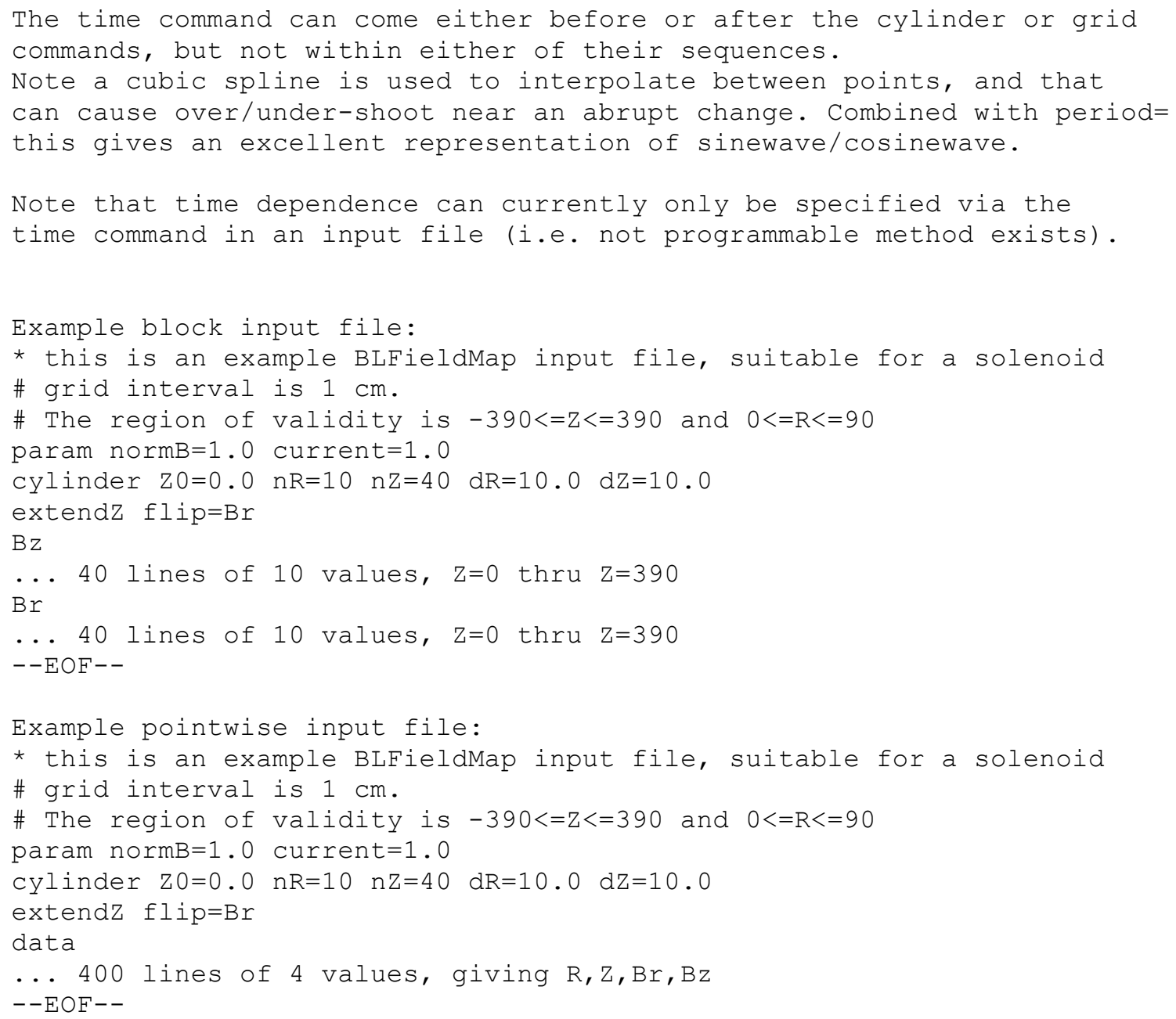

\subsection{Window Files}

Used by the absorber command.

* The file format is a series of lines:

* $\quad$ First character \# means comment, * means printed comment.

* $\quad$ Comment and blank lines are ignored. Units are mm.

* The first line contains the 4 flange variables:

* $\quad$ innerR outerR insideZ outsideZ

* The remaining lines contain 3 values for a given radius:

* $\quad \mathrm{rzt}$

* The first line must have $r=0.0$ and have the largest $\mathrm{z}$ value

* and the largest $\mathrm{z}+\mathrm{t}$ value. $\mathrm{z}$ is the inside of the window,

* $\quad z+t$ is the outside of the window. All values must be positive.

* $\quad$ Successive $r$ values must increase by at least $0.010 \mathrm{~mm}$.

* Successive $\mathrm{z}$ values and $\mathrm{z}+\mathrm{t}$ values must decrease by at least

* $\quad 0.010 \mathrm{~mm}$. 
* $\quad$ flangeInnerRadius should equal the last $r$ value.

* $\quad$ Any $\mathrm{z}$ origin may be used (it will be subtracted away).

* This is intended to be easy to interface to window design

* $\quad$ spreadsheets (export a list of 3 columns delimited by spaces, then

* add the appropriate comments and flange values at the top).

\subsection{Root Files}

The standard Root file format is used. All G4beamline outputs are TNtuple-s combined into a single Root file whose name is given by the parameter histoFile. The TNtuple fields are the same as for BLTrackFile and the Trace file above. 


\section{Acknowledgments}

The genesis of G4beamline was my desire to learn modern physics simulation codes, and Geant 4 seemed to be the best choice. Parts of G4beamline's design were inspired by the Beam Tools developed at Fermilab [6] by Daniel Elvira, Paul Lebrun, Panagiotis Spentzouris, and others, even though almost none of their code remains. The LISAPhysicsList came from the Geant 4 collaboration, and the MICEPhysicsList came from G4MICE developed by the MICE collaboration. The source files from these external sources retain the original authors' comments and disclaimers; they total less than $1 \%$ of the G4beamline source code.

Geant4 [1] is a flexible and versatile toolkit for simulating the passage of particles through matter and electromagnetic fields. This program literally would not have been possible without it. Thanks to the entire Geant 4 collaboration. This product includes software developed by Members of the Geant 4 Collaboration (http://cern.ch/geant4).

CLHEP [2] is a comprehensive class library for High Energy Physics, and G4beamline literally would not have been possible without it. Thanks to all of the CLHEP editors and authors.

The HistoScope program [3] is a marvelous program for generating and manipulating histograms. It has a fine graphical user interface and can do just about everything one might want to do with histograms, $\mathrm{X}-\mathrm{Y}$ plots, scatter plots, 2-d histograms, and NTuples. Much thanks to the authors: Mark Edel, Konstantine Iourha, Joy Kyriakopulos, Paul Lebrun, Jeff Kallenbach, and Baolin Ren; thanks also to Fermilab for making it available. Unfortunately, it is no longer supported. It was the inspiration for HistoRoot.

Root [5] is a programming environment intended for data analysis. It supports data files with a tree structure, 1-D and 2-D histograms, X-Y plots, and NTuples. Accompanying G4beamline is historoot, a root program that provides a graphical user interface to the histogram capabilities of Root - it was inspired by the capabilities of HistoScope, with a completely different user interface. Of course, the native Root tools can be used as well. Thanks to all of the Root developers.

The Coin 3-D graphics library [9] is licensed under the Gnu Public License, maintained by Kongsberg SIM AS. This implements by far the best visualization driver for simulated systems and events.

The $\mathrm{X}$-windows graphics libraries and the basic gcc libraries and compiler are also released under the GPL and/or LGPL. Thanks to the entire open source community for making such a comprehensive software development environment freely available. 


\title{
Appendix 1 - README.txt
}

\author{
G4beamline \\ by Tom Roberts \\ Copyright (C) 2003-2010 by Tom Roberts. \\ All rights reserved.
}

http: //g4 beamline.muonsinc.com

\section{LICENSE}

This program is free software; you can redistribute it and/or modify it under the terms of the GNU General Public License as published by the Free Software Foundation; either version 2 of the License, or (at your option) any later version.

This program is distributed in the hope that it will be useful, but WITHOUT ANY WARRANTY; without even the implied warranty of MERCHANTABILITY Or FITNESS FOR A PARTICULAR PURPOSE. See the GNU General Public License for more details.

http://www.gnu.org/copyleft/gpl.html

GENERAL

The general reference for G4beamline is the user's guide, located in the doc directory named G4beamlineUsersGuide.pdf.

It is also available on the web: http://g4beamline.muonsinc.com

INSTALLATION

There is a "Quick Start" section in the user's guide. For details, see the README-*.txt file for your OS (appendices of the user's guide).

INITIAL TESTING

Be sure you have added the G4beamline programs to your PATH (see README-*.txt).

Execute the following commands (in a Cygwin shell on Windows): cd g4beamline-VERSION/test

./loop

This will run a series of tests, with 1 line of description for each, hopefully ending with "All Tests Passed" and starting over. If this fails, see README-*.txt for a description of what is required to run G4beamline. 
For further assistance, you can join the G4beamline forum at http: //g4beamline.muonsinc.com .

NOTE: On Windows you will need to setup the Cygwin environment, and put g4beamline-VERSION/bin into your PATH (see README-Windows.txt). The Cygwin environment is not needed to run G4beamline itself, it is needed just for building the program and running the tests.

RUNNING THE PROGRAM -- GUI

Simply double-click the G4beamline icon. On Windows it is placed on your desktop

and in the Start/G4beamline menu. On Mac OS $\mathrm{x}$ it is placed where you dragged (copied) it when you installed it. On Linux the setup script puts it onto your desktop. Historoot is the same.

To run the GUI program via the command line, be sure you have added G4beamline's

bin directory to your PATH (see README-*.txt) and execute: g4blgui

This requires Java and a display ( $\mathrm{X}$-Windows on Linux and Mac OS $\mathrm{X}$, nothing special on Windows). Its opening screen decribes how to use it.

To run the examples, simply push the Browse button, navigate to the install directory / examples, and select examplel.in (or other *.in file). Then select the desired viewer (if any), and push the Run button. On Windows, a copy of "G4beamline Examples" is put into "My Documents" (Windows Xp) or "Documents" (Windows Vista).

RUNNING THE PROGRAM -- COMMAND-LINE

For command-line use (Linux, Mac os $\mathrm{x}$, and Windows with Cygwin), be sure you have added G4beamline's bin directory to your PATH (see README-*.txt). Then cd to whatever directory you plan to use for developing your simulation(s), and execute: g4bl -

After a few seconds it should type (with obvious variations): G4BL_DIR=/Users/g4bl/G4beamline G4LEDATA=/Users/g4bl/G4 beamline/data/G4EMLOW6.2 $\mathrm{G} 4 \mathrm{NDL}=/$ Users / 4 bl/G4beamline/data/G4NDL3.13 G4LEVELGAMMADATA=/Users/g $4 \mathrm{bl} / \mathrm{G} 4$ beamline/data/PhotonEvaporation2 . 0 DYLD_LIBRARY_PATH=/Users/g4bl/G4beamline/LibraryBinaries/Darwin-g++:

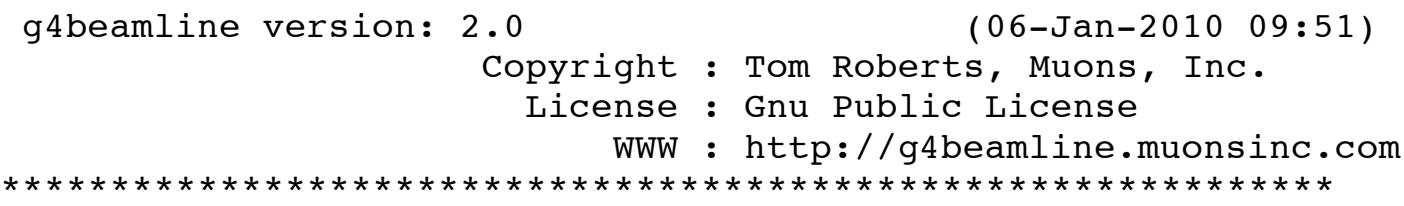

2/11/11 TJR G4Beamline User's Guide 


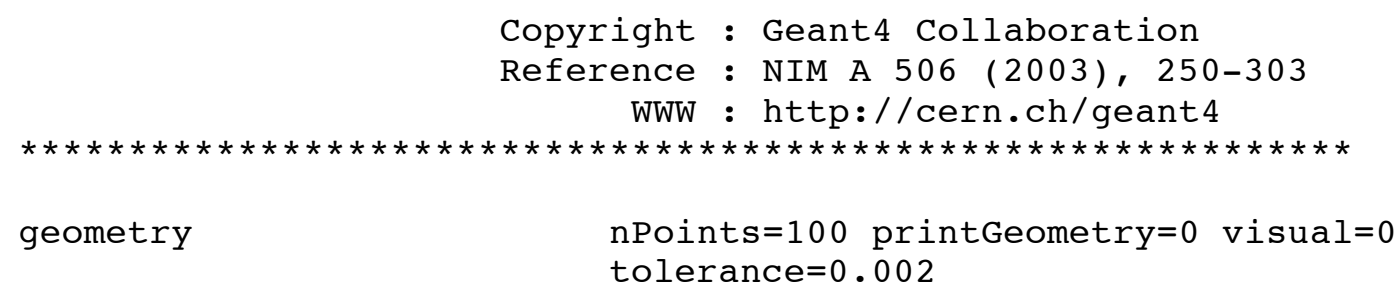

cmd : tolerance $=0.002$

The program is now ready for input. Type "help" to get a short list of the input-file commands, or "help *" for a detailed description of the commands. This is a useful way to get help on commands when editing your input file(s). Type ${ }^{\wedge} \mathrm{C}$ to exit back to a shell prompt.

EXAMPLE 1

The first example input file is examplel.in. It is a simple file to track muons through 1-meter drift spaces into 4 detectors. To visualize its geometry using openInventor, execute:

cd g4beamline-VERSION/examples

$\mathrm{g} 4 \mathrm{bl}$ examplel.in viewer=best

To run the beam through the geometry, execute:

cd g4beamline-VERSION/examples

g4bl examplel.in

This will generate a Root file named g4beamline.root. To view it do:

cd g4beamline-VERSION/examples

historoot $g 4$ beamline.root

EXAMPLE 2

The second example input file is a 4-cell cooling channel based on the beginning of the Study 2 SFoFo channel. Use it just like example1 above.

OTHER EXAMPLES

There are other examples in the examples directory. All are executed as above. 


\title{
Appendix 2 - README-Linux.txt
}

\author{
G4beamline on Linux (Intel) \\ G4beamline 2.02 April 2010 TJR \\ Before installing G4beamline, you should first install: \\ openmotif -- should be available in your Linux distribution. Some \\ distributions install this automatically, some don't. \\ It provides libXm.so.
}

You may need to install these:

x11-deprecated-libs -- should be available in your Linux distribution, but the name may be different. Some distributions install this automatically, some don't. It provides libxp.so.

compat-libstdc++.i386 -- should be available in your Linux distribution, but the name may be different. Provides an older versin of /usr/lib/libstdc++.so.

You probably want to install the following:

Root - http://root.cern.ch

Select "Download Binaries, and download their production version. Any recent version of Root should work; 5.20, 5.22, and 5.24 have been tested.

Be sure the ROOTSYS variable is set in the environment (best done in your .profile or .bash_profile). Root is only necessary to run historoot, G4beamline itself does not need Root installed. Root can be installed after G4beamline, if desired.

You may want to install the following:

tcl - should be available in your Linux distribution. Required by most of the tests, but g4beamline itself will run without it. tcl can be installed after G4beamline, if desired. Many distributions install this automatically.

java - http://java.sun.com Required only for g4blgui. Select "Java SE", and all you need is the Java Runtime Environment (JRE); get the latest. If you have installed the development kit (JDK) that includes the JRE. Java can be installed after G4beamline, if desired. Most distributions install this automatically.

To install G4beamline, download the tarball and un-tar it in your HOME directory:

tar -xzf g4beamline-2.02-Linux-g++.tgz

this will create a directory \$HOME/g4beamline-2.02-Linux-g++ .

You need to put the G4beamline programs into your PATH. The simplest way to do this is: cd g4beamline-2.02-Linux-g++ 
.$/$ setup

The setup script will guide you through the process; it puts 2-line programs into the directory you specify. It also creates .desktop files for the G4beamline and Historoot applications, and copies them to your Desktop (if any) .

After running setup, you can double-click on the G4beamline icon to run it via the GUI, and you can run the command-line version.

Once g4beamline has been put into your PATH, to run G4beamline simply do: g4bl input.file [name=value [...]]

The default format for NTuples is now Root. To create histograms from NTuples you can use Root in the usual way. That is rather complicated and un-obvious, so a new root-based program is included to assist you: historoot. Just double-click the Historoot icon. With g4beamline in your PATH, just type: historoot [file.root] [ ...]

this will permit you to open Root files, select an NTuple, and create plots using expressions involving the NTuple variables and $\mathrm{C}$ functions and operators. Four sliders are available to impose cuts on arbitrary expressions. Plots can be saved in many formats, including PS, PDF, GIF, and JPEG. HistoRoot can also handle ASCII and . CSV files in the proper format (as are all ASCII files written by G4beamline).

For visualization, the OpenInventor viewer works fine, use viewer=best or viewer=OIX. Other viewers are available.

\section{TROUBLESHOOTING}

This is g4beamline-2.02-Linux-g++.

Here is the output of ldd on Scientific Linux Fermi 4.5, showing which shared libraries are used, and where they come from.

\$ pwd

/home/tjrob/g4bl/G4beamline/bin/Linux-g++

$\$$ ldd g4beamline

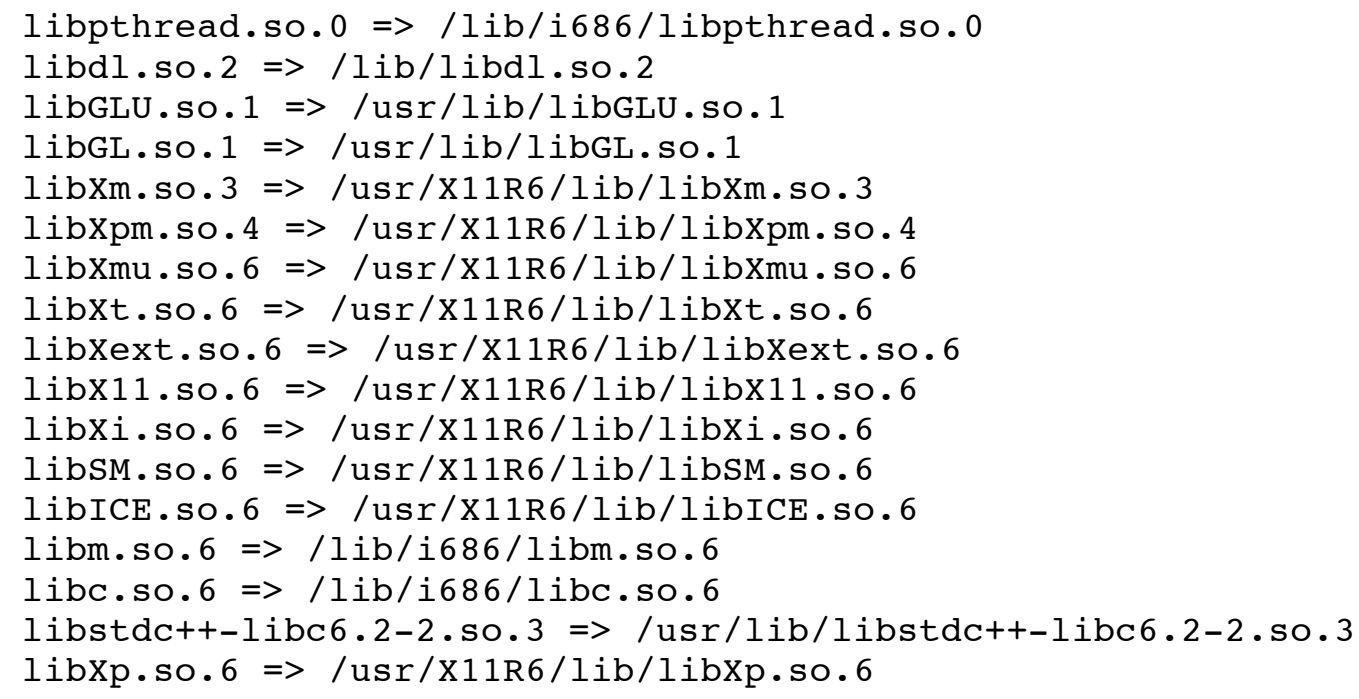




\section{$/$ lib/ld-linux.so.2 => /lib/ld-linux.so.2}

Note that some motif/X11 shared libraries are included in the G4beamline distribution (and remain local to it). This reduces the need for users to install precisely the same version of these libraries as was used to build g4beamline.

Here are the RPMs that provide these libraries in Scientific Linux Fermi 4.5: ++ rpm -qf /lib/tls/libpthread.so.0

glibc-2.3.4-2.36

$++\mathrm{rpm}-\mathrm{qf} / \mathrm{lib} / \mathrm{libdl}$.so.2

glibc-2.3.4-2.36

++ rpm -qf /usr/x11R6/lib/libGLU.so.1

xorg-x11-Mesa-libGLU-6.8.2-1.EL.19

++ rpm-qf /usr/X11R6/lib/libGL.so.1

xorg-x11-Mesa-libGL-6.8.2-1.EL.19

++ rpm -qf /usr/X11R6/lib/libXm.so.3

openmotif-2.2.3-10.1.el4

++ rpm -qf /usr/X11R6/lib/libXpm.so.4

xorg-x11-libs-6.8.2-1.EL.19

++ rpm -qf /usr/X11R6/lib/libXmu.so.6

xorg-x11-libs-6.8.2-1.EL.19

++ rpm-qf /usr/X11R6/lib/libxt.so.6

xorg-x11-libs-6.8.2-1.EL.19

++ rpm -qf /usr/X11R6/lib/libXext.so.6

xorg-x11-libs-6.8.2-1.EL.19

++ rpm -qf /usr/X11R6/lib/libX11.so.6

xorg-x11-libs-6.8.2-1.EL. 19

++ rpm-qf /usr/X11R6/lib/libXi.so.6

xorg-x11-libs-6.8.2-1.EL.19

++ rpm -qf /usr/X11R6/lib/libsM.so.6

xorg-x11-libs-6.8.2-1.EL.19

++ rpm -qf /usr/x11R6/lib/libICE.so.6

xorg-x11-libs-6.8.2-1.EL.19

++ rpm -qf /lib/tls/libm.so.6

glibc-2.3.4-2.36

++ rpm -qf /lib/tls/libc.so.6

glibc-2.3.4-2.36

++ rpm -qf /lib/ld-linux.so.2

glibc-2.3.4-2.36

++ rpm -qf /usr/lib/libstdc++.so. 6

libstdc++-3.4.6-8

++ rpm -qf /lib/libgcc_s.so.1

libgcc $-3.4 .6-8$

++ rpm -qf /usr/X11R6/lib/libXxf86vm.so.1

xorg-x11-libs-6.8.2-1.EL.19

++ rpm -qf /usr/X11R6/lib/libXp.so.6

xorg-x11-deprecated-libs-6.8.2-1.EL. 19 


\title{
Appendix 3 - README-Windows.txt
}

\author{
G4 beamline on Windows XP and Windows Vista \\ G4beamline 2.02 April 2010 TJR \\ Before installing G4beamline, you must first install: \\ Java - http://java.sun.com \\ Select "Java SE", and all you need is the Java Runtime Environment \\ (JRE); get the latest. If you have installed the development kit \\ (JDK), that includes the JRE.
}

You may want to install:

Root - http://root.cern.ch

Select "Download Binaries, and download their production version.

Any recent version of Root should work; 5.20 and 5.24 have been tested.

Install it into the default directory, C: \Root . Really only needed

to run historoot, G4beamline itself does not need Root installed.

Cygwin - http://cygwin.com

This is a UNIX-like command-line environment for Windows.

Select "Install Cygwin now". This will download Cygwin's

setup.exe. Run it, select a mirror site, and you will have the

opportunity to select packages. You should select at least one

editor of your choice (vi, emacs, or ...). You must also select

tcltk, which is in the Libs category. You can add or remove

packages at any time by re-running the Cygwin setup.exe.

Install it into the default directory, c: lcygwin

To install G4beamline, download G4beamline-2.02.msi and run it. Follow the usual

directions for installing packages into Windows. The installer will install shortcuts for G4beamline and Historoot onto your desktop and into the start menu.

If you have installed Cygwin, then if you want to run G4beamline from

its command line, do this in a Cygwin shell:

cd "C:/Program Files/MuonsInc/G4beamline/bin"

- / setup

The setup script will guide you through adding the G4beamline programs into directories in your Cygwin PATH. When prompted for a directory, be sure to respond with a full path that begins with a drive, and use forward-slashes (e.g. C:/cygwin/usr/bin) -- this is Cygwin "mixed" format, and is required so both Cygin tools and Windows tools can find the directories and files.

The default format for NTuples is now Root. To create histograms from NTuples you can use Root in the usual way. That is rather complicated and un-obvious, so a new root-based program is included to assist you: historoot.

It will permit you to open Root files, select an NTuple, and create plots using expressions involving the NTuple variables and $\mathrm{C}$ functions and operators. Four sliders are available to impose cuts on arbitrary expressions. Plots can be saved in many formats, including PS, PDF, GIF, and JPEG. HistoRoot can also handle ASCII and . csv files in the proper format (as are

2/11/11 TJR G4Beamline User's Guide 
all ASCII files written by G4beamline).

For visualization, the OpenInventor viewer works fine, simply select "best" from the viewer panel of the G4beamline window. Other viewers are available. 


\title{
Appendix 4 - README-MacOSX.txt
}

\author{
G4beamline on Mac OS X (Intel) \\ G4beamline 2.02 April 2010 TJR \\ This version of G4beamline was built and tested on Leopard (Mac OS X 10.5.8); \\ it runs on Snow Leopard (Mac OS X 10.6.2). \\ G4beamline has been used in the past on Tiger $(10.4 . x)$, but now must be built \\ from source to run there (see BUILD.txt). \\ Note: PowerPC binaries are not available. It is expected that installing \\ G4beamline from source should work on older Macs running Tiger or later, \\ and at least one user has had success doing this. \\ Before installing G4beamline, you should first install: \\ X11 - part of the OS, available on the installation DVD. G4beamline will \\ not run without it. Installed by default on Leopard and Snow Leopard, \\ but on Tiger you must install it manually.
}

You probably want to install this:

Root - http://root.cern.ch

Select "Download Binaries, and download their production version. Any recent version of Root should work; 5.20, 5.22, and 5.24 have been tested.

Be sure the ROOTSYS variable is set in the environment (best done

in your .profile or .bash_profile). Really only needed to run

historoot, G4beamline itself does not need Root installed.

You may want to install these:

tcl - available as part of Mac os x. Required by most of the tests, but g4beamline itself will run without it.

To install G4beamline, download the installer.dmg from http: //g4beamline.muonsinc.com

Open it, and drag the application icons into /Applications (requires

administrator privileges; if you can't do that, drag them into your Home). You can then drag them into the Dock, if desired.

You can run them in the usual way by double-clicking on their icons.

If you want to run these programs from the command line, you need to put the G4beamline programs into your PATH. The simplest way to do this is:

cd /Applications/G4beamline.app/Contents/Resources - / setup

The setup script will guide you through the process; it places 2-line shell scripts into the directory you specify (e.g./Users/\$USER/bin). You can also add the /Applications/G4beamline.app/Contents/Resources/bin directory into your PATH manually (e.g. in \$HOME/.bash_profile).

Once g4beamline has been put into your PATH, to run G4beamline simply do:
2/11/11 TJR
G4Beamline User's Guide
156 
g4bl input.file [name=value $[\ldots]$ ]

You can also run the Graphical User Interface:

g4blgui [input.file]

NOTE: On Tiger you may need to do this from an X11 xterm window (this distribution does not run on Tiger, but this applies if you build it from source).

The default format for NTuples is now Root. To create histograms from NTuples you can use Root in the usual way. That is rather complicated and un-obvious, so a new root-based program is included to assist you: historoot. Double-click on the Historoot icon, or with g4beamline in your PATH, type:

historoot [file.root] [ ...]

this will permit you to open Root files, select an NTuple, and create plots using expressions involving the NTuple variables and $\mathrm{C}$ functions and operators. Four sliders are available to impose cuts on arbitrary expressions. Plots can be saved in many formats, including PS, PDF, GIF, and JPEG. HistoRoot can also handle ASCII and . CSV files in the proper format (as are all ASCII files written by G4beamline).

For visualization, the openInventor viewer works fine, use viewer=best or viewer=OIX. Other viewers are available and work quite similar to Linux. Note that all viewers use X11, so on Tiger you must run G4beamline from an X11 xterm window, or must have X11 running and supply the DISPLAY manually. This is also true of historoot.

TROUBLESHOOTING

Here is the result of "otool -L" on Mac OS X 10.5 .8 (Intel). This shows what shared libraries are used. Libraries in /usr are from the os; those in /sw are from Fink.

$\$$ otool -L g4beamline

g4 beamline:

/usr//lib/libmpi_cxx.0.dylib (compatibility version 1.0.0, current version $1.0 .0)$

/usr//lib/libmpi.0.dylib (compatibility version 1.0.0, current version $1.0 .0)$

$1.3 .0)$

$1.2 .0)$

/usr/X11/lib/libGLU.1.dylib (compatibility version 1.3.0, current version

/usr/X11/lib/libGL.1.dylib (compatibility version 1.2.0, current version

/sw/lib/libXm.2.dylib (compatibility version 3.0.0, current version 3.1.0) $16.0 .0)$

/usr/X11/lib/libxpm.4.dylib (compatibility version 16.0.0, current version $9.0 .0)$

$7.0 .0)$

/usr/X11/lib/libXmu.6.dylib (compatibility version 9.0.0, current version

/usr/X11/lib/libxt.6.dylib (compatibility version 7.0.0, current version

/usr/x11/lib/libXext.6.dylib (compatibility version 11.0 .0 , current version 11.0 .0 ) 
$9.0 .0)$

/usr/X11/lib/libX11.6.dylib (compatibility version 9.0.0, current version /usr/X11/lib/libXi.6.dylib (compatibility version 7.0 .0 , current version $7.0 .0)$

/usr/X11/lib/libsM.6.dylib (compatibility version 7.0.0, current version $7.0 .0)$

/usr/X11/lib/libICE.6.dylib (compatibility version 10.0.0, current version $10.0 .0)$

/usr/lib/libSystem.B.dylib (compatibility version 1.0.0, current version $111.1 .4)$

/usr/lib/libstdc++.6.dylib (compatibility version 7.0.0, current version $7.4 .0)$ $1.0 .0)$

/usr/lib/libgcc_s.1.dylib (compatibility version 1.0.0, current version

Note that libxm.2.dylib is included in the G4beamline distribution and should not be a problem. If it is a problem, it comes from Fink in lesstif. 


\section{Appendix 5 - Annotated Output from Example1}

Note that Geant4 outputs considerable detail to stdout. Annotations below are headings inserted into the output text.

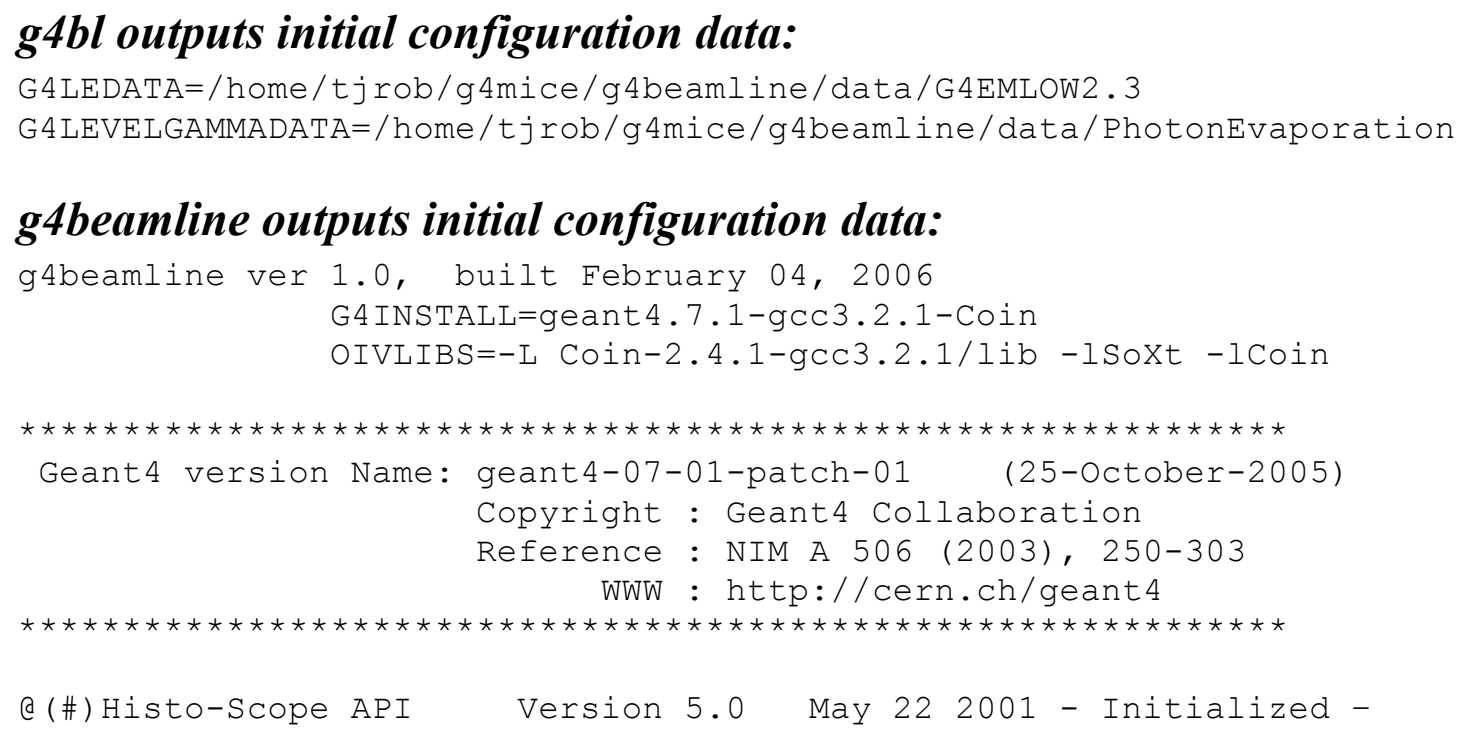

\section{g4beamline outputs data derived from input.file:}

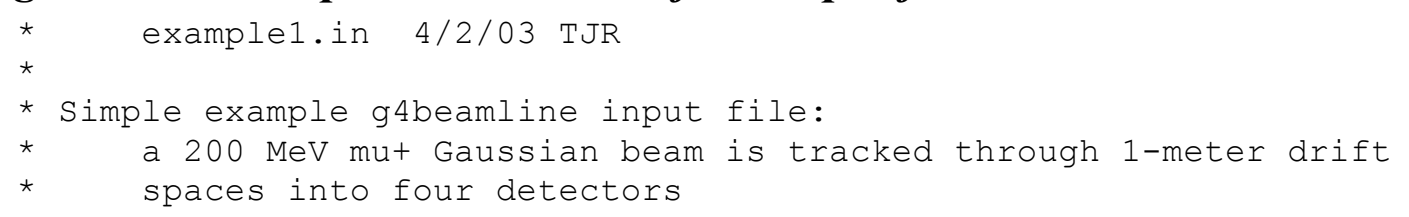

\section{Geant4 physics processes identify themselves:}

You are using the simulation engine reuse library: PACK 2.4

You are using the simulation engine: QGSP 2.8

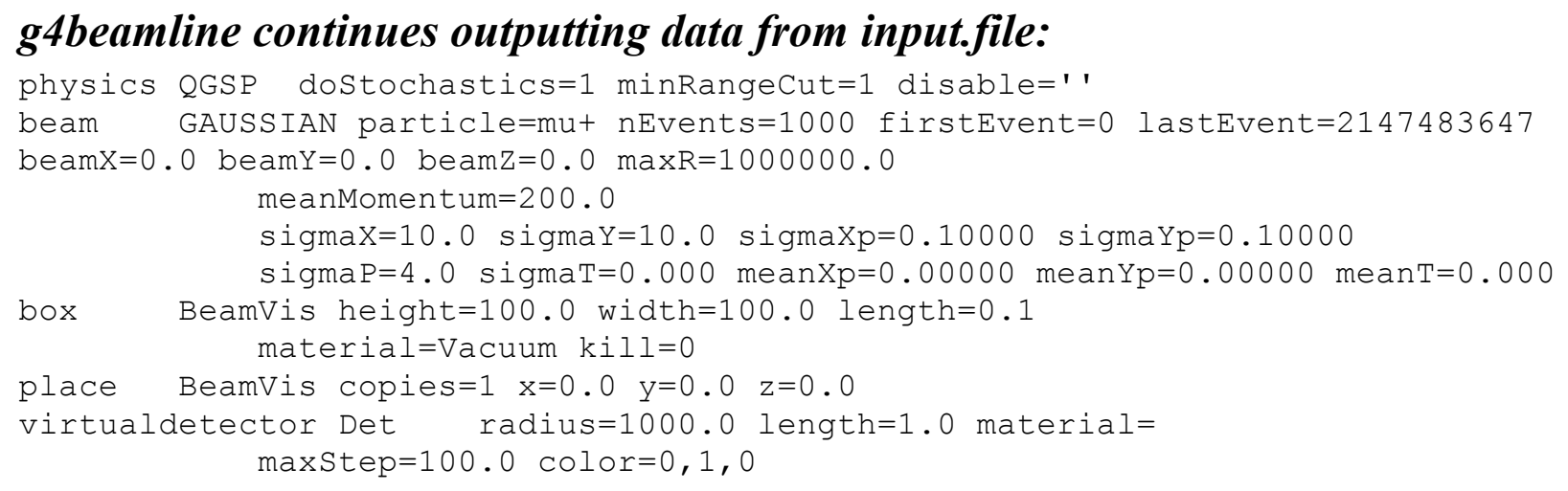




\begin{tabular}{|c|c|c|c|c|c|}
\hline & Det & opies $=1 \quad x=0.0$ & $y=0.0$ & $z=1000.0$ & re \\
\hline & م & pies $=1 \quad x=0.0$ & $y=0.0$ & $z=2000.0$ & $=' D$ \\
\hline & ת & copies $=1 \quad x=0.0$ & $y=0.0$ & $z=3000.0$ & re \\
\hline & $\mathrm{De}$ & copies $=1 \quad x=0.0$ & $y=0.0$ & $z=4000.0$ & rename $=$ \\
\hline
\end{tabular}

\section{At the end of the input file, g4beamline summarizes the values of all defined} parameters :

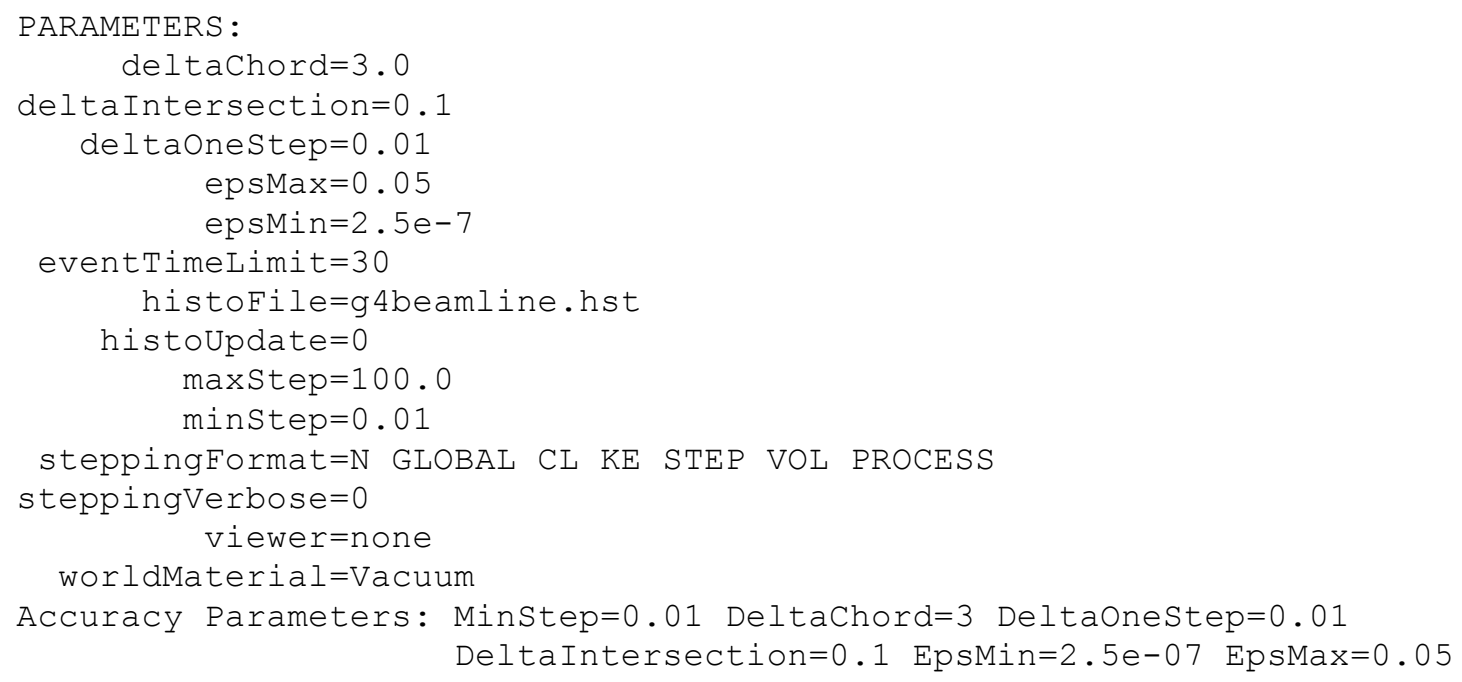

g4beamline outputs data related to element construction:

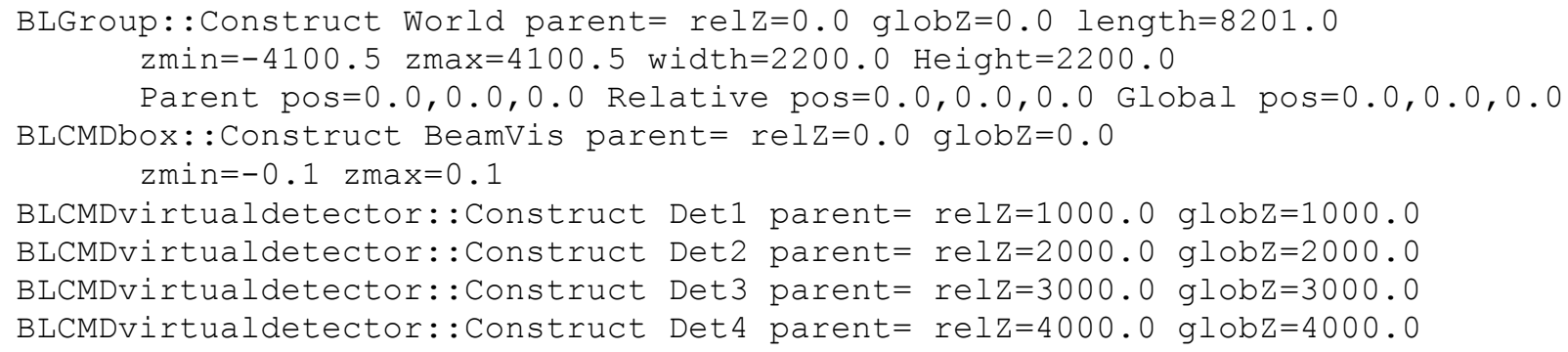

\section{g4beamline performs its geometry test:}

Geometry test nPoints $=100$ tolerance=0.002 mm:

Testing geometry for children of group 'World':

Total geometry errors $=0 \quad 0$ seconds

\section{g4beamline indicates its status:}

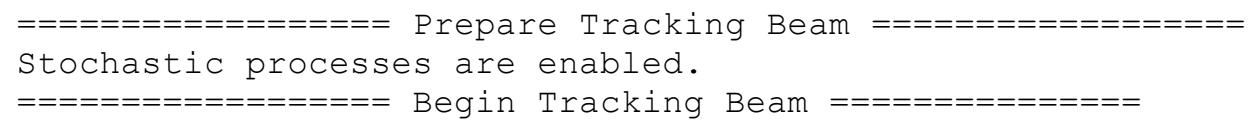

\section{g4beamline outputs a summary of events completed:}

$\begin{array}{lllll}\text { Event } 0 \text { Completed } 1 \text { events realtime=1 } & \mathrm{sec} & 1.0 \mathrm{ev} / \mathrm{sec} \\ \text { Event } 1 \text { Completed } & 2 \text { events } & \text { realTime=1 } \mathrm{sec} & 2.0 \mathrm{ev} / \mathrm{sec}\end{array}$

2/11/11 TJR G4Beamline User's Guide 


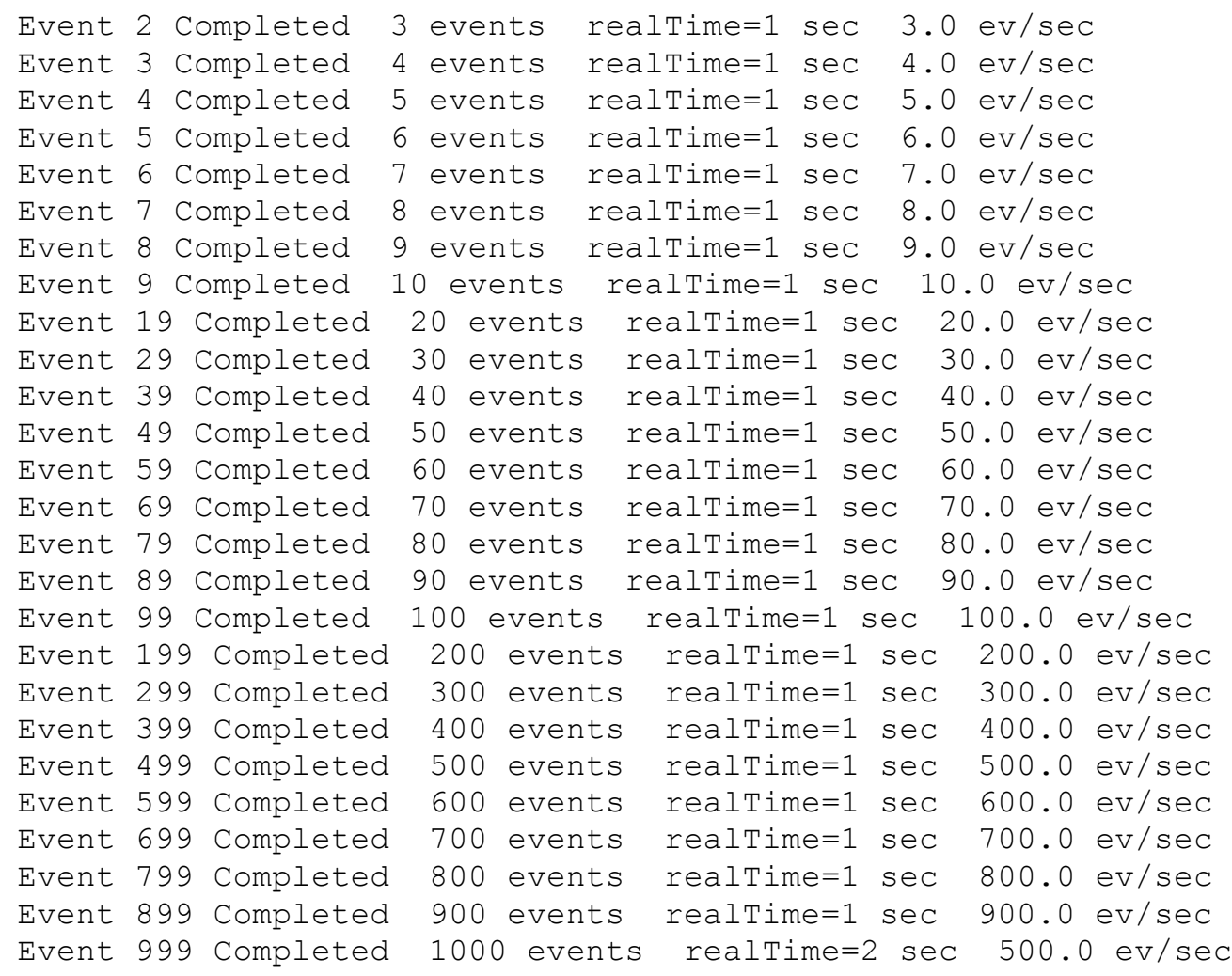

\section{g4beamline outputs a summary of the run:}

$\begin{array}{lr}\text { Run complete } 1000 \text { Events } & 2 \text { seconds } \\ \text { NTuple Det1 } & 1002 \text { entries } \\ \text { NTuple Det2 } & 1003 \text { entries } \\ \text { NTuple Det3 } & 998 \text { entries } \\ \text { NTuple Det4 } & 950 \text { entries } \\ \text { NTuple wrote Root File 'g4beamline.root' } \\ \text { g4beamline: simulation complete -- exiting }\end{array}$




\title{
Appendix 6 - Particle IDs
}

\author{
B+ PDGid $=521$ \\ B- PDGid $=-521$ \\ B0 PDGid=511 \\ Bs0 PDGid=531 \\ D+ PDGid $=411$ \\ D- PDGid $=-411$ \\ D0 PDGid=421 \\ Ds+ PDGid $=431$ \\ Ds- PDGid=-431 \\ GenericIon PDGid=0 \\ He3 PDGid=1000020030 \\ $\mathrm{J} / \mathrm{psi}$ PDGid $=443$ \\ $\mathrm{N}(1440)+$ PDGid $=12212$ \\ $\mathrm{N}(1440) 0$ PDGid=12112 \\ $\mathrm{N}(1520)+\mathrm{PDGid}=2124$ \\ $\mathrm{N}(1520) 0$ PDGid=1214 \\ $\mathrm{N}(1535)+$ PDGid $=22212$ \\ $\mathrm{N}(1535) 0$ PDGid=22112 \\ $\mathrm{N}(1650)+\mathrm{PDGid}=32212$ \\ $\mathrm{N}(1650) 0$ PDGid $=32112$ \\ $\mathrm{N}(1675)+\mathrm{PDGid}=2216$ \\ $\mathrm{N}(1675) 0$ PDGid=2116 \\ $\mathrm{N}(1680)+$ PDGid $=12216$ \\ $\mathrm{N}(1680) 0$ PDGid=12116 \\ $\mathrm{N}(1700)+$ PDGid $=22124$ \\ $\mathrm{N}(1700) 0$ PDGid=21214 \\ $\mathrm{N}(1710)+$ PDGid $=42212$ \\ $\mathrm{N}(1710) 0$ PDGid $=42112$ \\ $\mathrm{N}(1720)+$ PDGid $=32124$ \\ $\mathrm{N}(1720) 0$ PDGid=31214 \\ $\mathrm{N}(1900)+\mathrm{PDGid}=42124$ \\ $\mathrm{N}(1900) 0$ PDGid $=41214$ \\ $\mathrm{N}(1990)+$ PDGid $=12218$ \\ $\mathrm{N}(1990) 0$ PDGid $=12118$ \\ $\mathrm{N}(2090)+$ PDGid $=52214$ \\ $\mathrm{N}(2090) 0$ PDGid $=52114$ \\ $\mathrm{N}(2190)+\mathrm{PDGid}=2128$ \\ $\mathrm{N}(2190) 0$ PDGid $=1218$ \\ $\mathrm{N}(2220)+\mathrm{PDGid}=100002210$ \\ $\mathrm{N}(2220) 0$ PDGid $=100002110$ \\ $\mathrm{N}(2250)+\mathrm{PDGid}=100012210$ \\ $\mathrm{N}(2250) 0$ PDGid $=100012110$ \\ a0 $(1450)+$ PDGid $=10211$ \\ a0 (1450)- PDGid=-10211 \\ a0(1450)0 PDGid=10111 \\ a0 (980)+ PDGid $=9000211$ \\ a0 (980) - PDGid=-9000211 \\ a0 (980)0 PDGid=9000111 \\ a $1(1260)+$ PDGid $=20213$ \\ a1(1260) - PDGid=-20213 \\ a1 (1260)0 PDGid=20113 \\ a2 (1320) + PDGid $=215$
}


a2 (1320)- PDGid $=-215$

a2 (1320)0 PDGid=115

alpha PDGid=1000020040

anti_B0 PDGid=-511

anti_Bs0 PDGid $=-531$

anti_D0 PDGid $=-421$

anti_N $(144 \overline{0})+$ PDGid $=-12212$

anti-N(1440)0 PDGid=-12112

anti-N(1520)+ PDGid $=-2124$

anti-N(1520)0 PDGid=-1214

anti_N(1535)+ PDGid $=-22212$

anti_N(1535) 0 PDGid $=-22112$

anti_N(1650)+ PDGid $=-32212$

anti ${ }^{-} \mathrm{N}(1650) 0$ PDGid $=-32112$

anti_N $(1675)+$ PDGid $=-2216$

anti_N(1675)0 PDGid=-2116

anti-N(1680)+ PDGid $=-12216$

anti_N(1680)0 PDGid=-12116

anti_N(1700)+ PDGid $=-22124$

anti_N(1700)0 PDGid=-21214

anti_N(1710)+ PDGid $=-42212$

anti-N(1710)0 PDGid=-42112

anti-N(1720)+ PDGid $=-32124$

anti_N(1720)0 PDGid=-31214

anti_N(1900)+ PDGid $=-42124$

anti_N(1900)0 PDGid=-41214

anti_N(1990)+ PDGid $=-12218$

anti_N(1990)0 PDGid $=-12118$

anti_N(2090)+ PDGid $=-52214$

anti_N (2090)0 PDGid=-52114

anti-N(2190)+ PDGid $=-2128$

anti_N(2190)0 PDGid=-1218

anti-N(2220)+ PDGid $=-100002210$

anti_N(2220)0 PDGid $=-100002110$

anti_N(2250)+ PDGid $=-100012210$

anti_N (2250)0 PDGid $=-100012110$

antíi_b_quark PDGid $=-5$

anti_c_quark PDGid=-4

anti_d_quark PDGid=-1

anti_dd $\overline{1}$ diquark PDGid=-1103

anti_delta(1600)+ PDGid $=-32214$

anti_delta $(1600)++$ PDGid $=-32224$

anti_delta(1600)- PDGid $=-31114$

anti_delta(1600)0 PDGid=-32114

anti_delta $(1620)+$ PDGid $=-2122$

anti_delta(1620)++ PDGid $=-2222$

anti_delta(1620)- PDGid $=-1112$

anti_delta(1620)0 PDGid $=-1212$

anti_delta $(1700)+$ PDGid $=-12214$

anti_delta(1700)++ PDGid=-12224

anti_delta(1700)- PDGid $=-11114$

anti_delta(1700)0 PDGid $=-12114$

anti_delta(1900)+ PDGid $=-12122$

anti_delta(1900)++ PDGid $=-12222$

anti_delta(1900)- PDGid $=-11112$

2/11/11 TJR G4Beamline User's Guide 


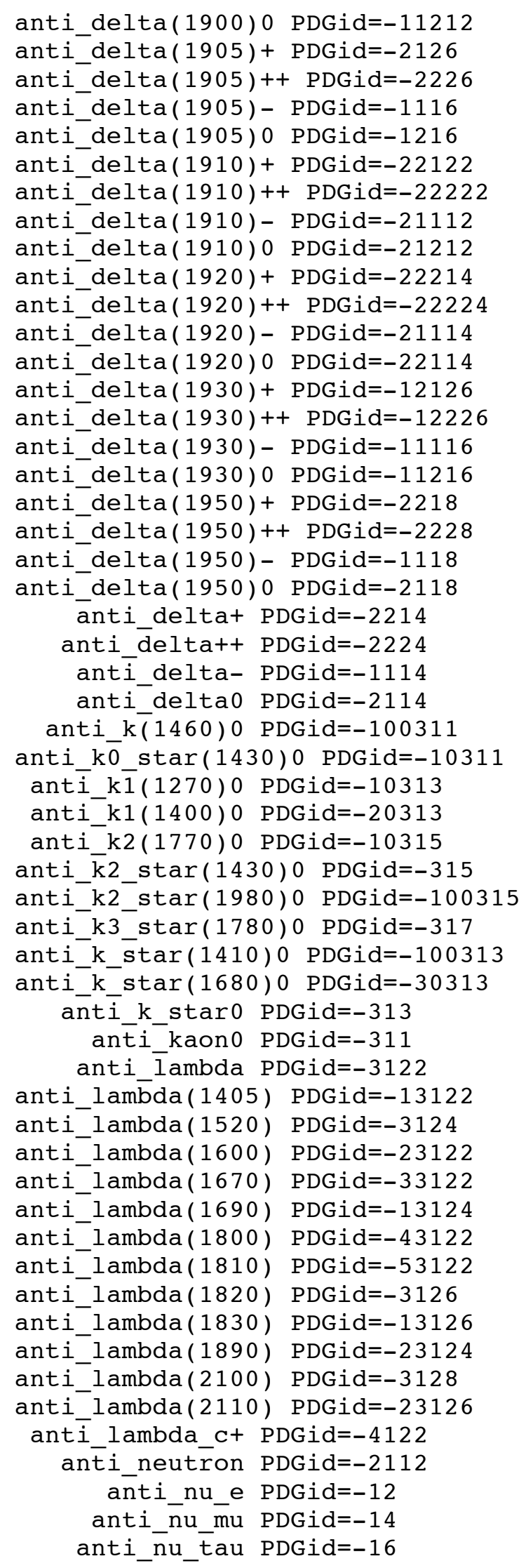




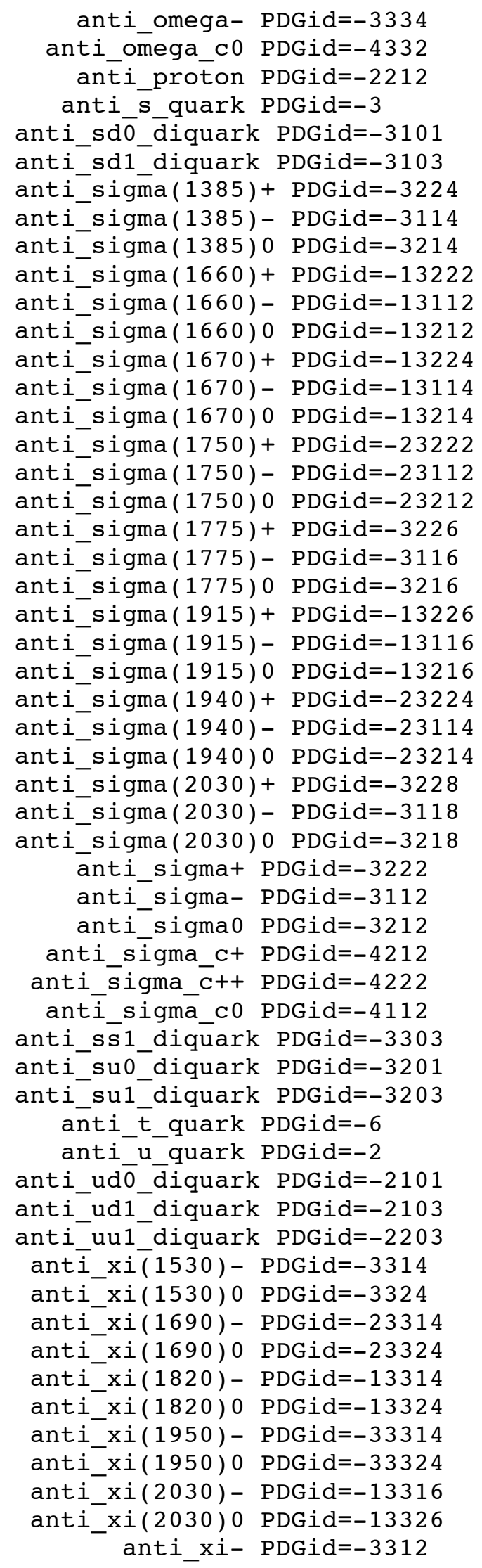

2/11/11 TJR G4Beamline User's Guide 


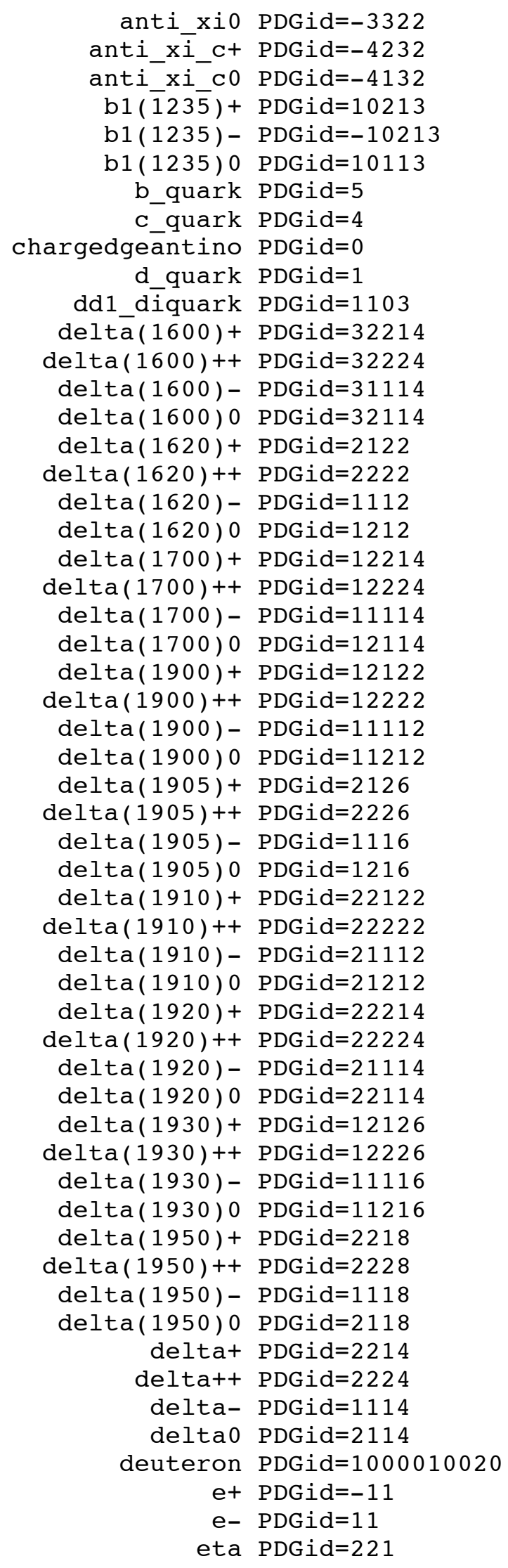




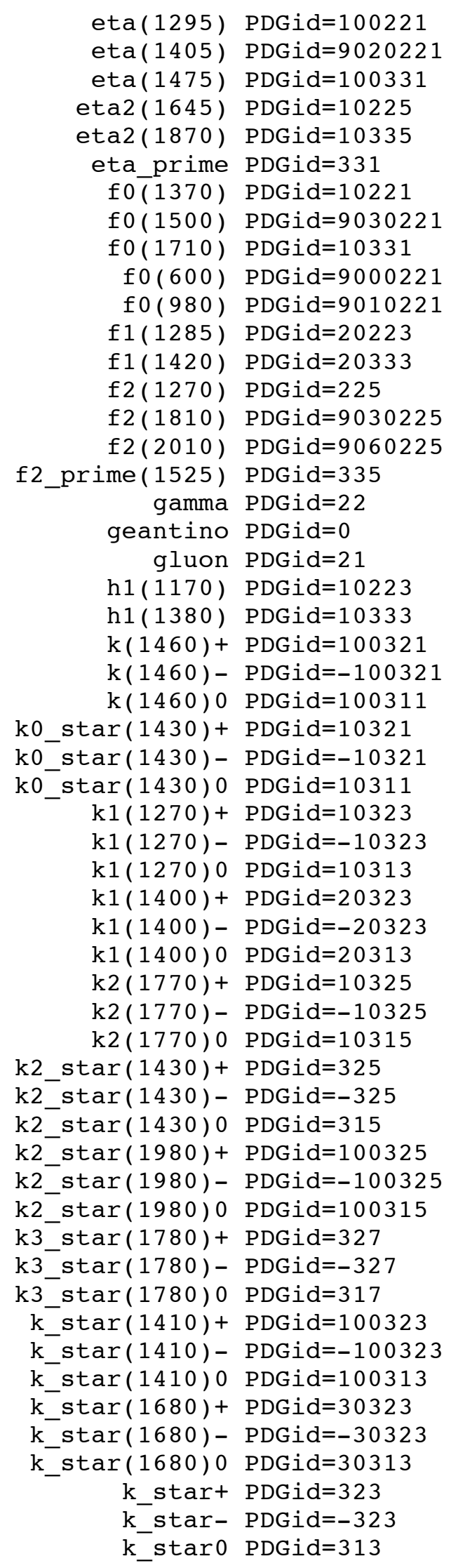




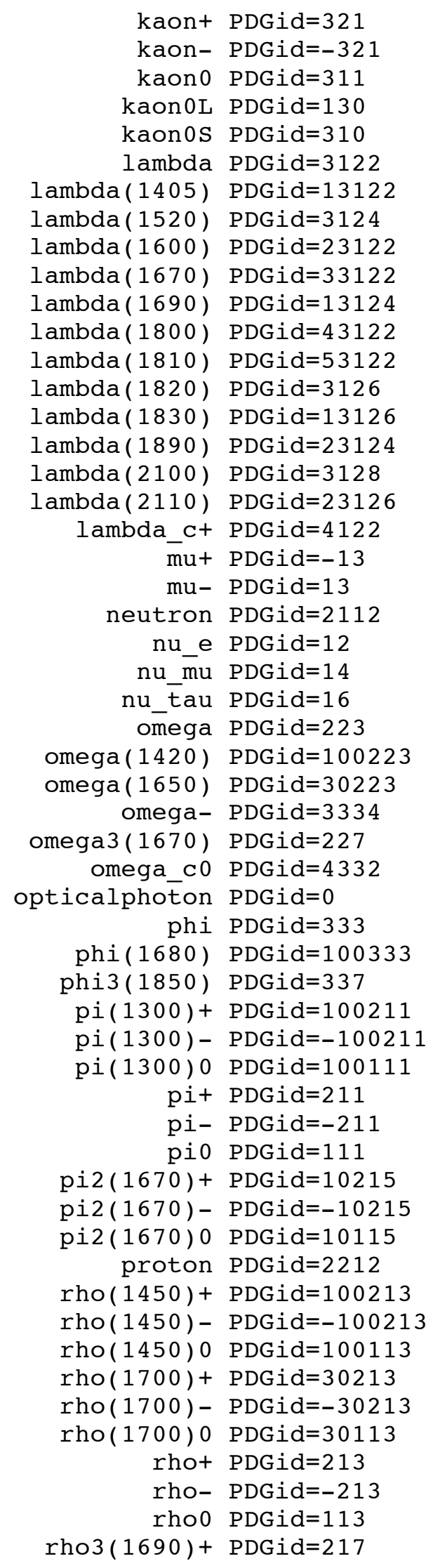




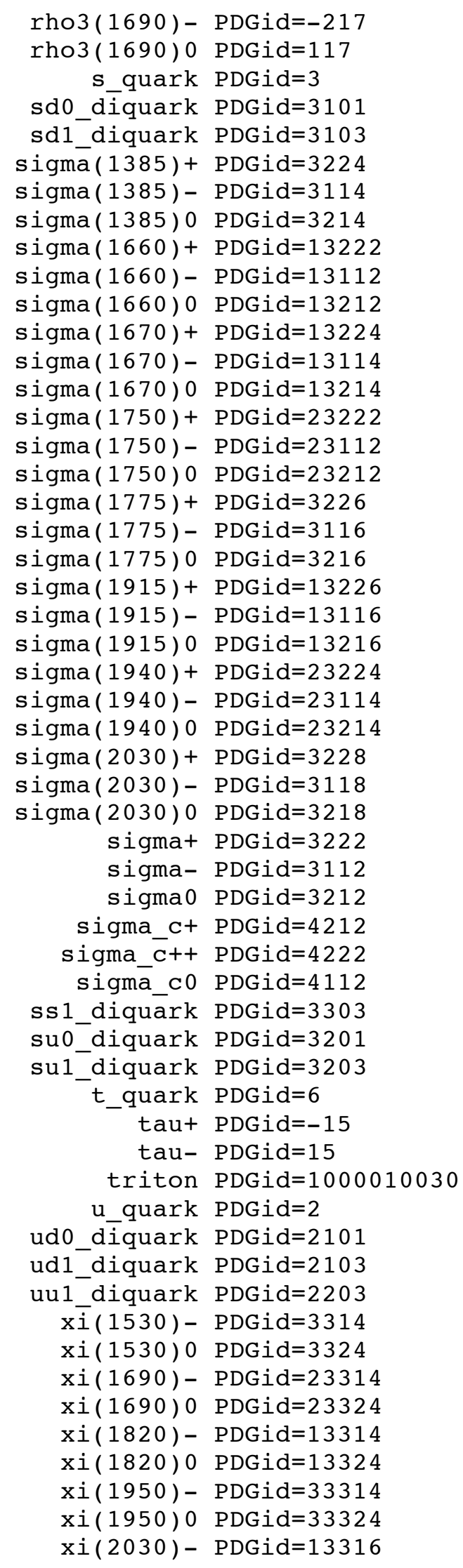




$$
\begin{aligned}
\text { xi(2030)0 } & \text { PDGid }=13326 \\
\text { xi- } & \text { PDGid }=3312 \\
\text { xi0 } & \text { PDGid }=3322 \\
\text { xi_c+ } & \text { PDGid }=4232 \\
\text { xi_c0 } & \text { PDGid }=4132
\end{aligned}
$$




\section{Appendix 7 - Error Messages}

Errors and warnings generated during execution are handled by the G4Exception function; they are printed as a 7-line message starting and ending with a row of asterisks - that makes them stand out visually in a long printout. Those that are issued by a routine beginning with "G4" came from Geant 4 code, while those issued by a routine beginning with "BL" came from G4beamline code; those that are issued by a command name are also from G4beamline code. This list may not be complete; it does not attempt to list all error messages from libraries such as Geant4, CLHEP, GSL, OpenInventor, OpenGL, Xwindows, etc.

\begin{tabular}{|c|c|c|}
\hline Exception & Severity & Discussion \\
\hline 001 & Warning & $\begin{array}{l}\text { G4HadronicProcess - this is a coding error and is being } \\
\text { investigated by the Geant } 4 \text { team. It is rare and can be } \\
\text { ignored unless a large number of tracks are affected }\end{array}$ \\
\hline Alarm Signal & Fatal & $\begin{array}{l}\text { The alarm timer fired. This timer is set } 10 \text { seconds longer } \\
\text { than the eventTimeLimit, and will only fire if no steps were } \\
\text { taken during those extra } 10 \text { seconds. Usually indicates an } \\
\text { infinite loop somewhere in the program. }\end{array}$ \\
\hline Missing material & Fatal & The specified material cannot be found. \\
\hline UnknownParticle & Fatal & The specified particle does not exist. \\
\hline Cannot Tune & Fatal & The pillbox cannot be tuned properly. \\
\hline Tune Iteration Limit & Fatal & The pillbox tuning did not converge. \\
\hline Invalid Step & Fatal & The pillbox tuning made an invalid step. \\
\hline Tuning failed to converge & Fatal & Tuning did not converge. \\
\hline Duplicate trackerplane-s & Fatal & Two or more trackerplane-s have the same name. \\
\hline No Trackers & Fatal & The trackermode command has no trackers to control. \\
\hline Invalid Start Expr & Fatal & The tune command has an invalid argument. \\
\hline Iteration Limit & Fatal & The tune command did not converge within the limit. \\
\hline Invalid Tune Expr & Fatal & The tune command has an invalid argument. \\
\hline Failed to Converge & Fatal & The tune command did not converge. \\
\hline Out of Memory & Fatal & Not enough memory. \\
\hline $\begin{array}{l}\text { Failed to achieve required } \\
\text { accuracy }\end{array}$ & Fatal & $\begin{array}{l}\text { The coil command could not achieve the required accuracy } \\
\text { in constructing the field map, given the limits on its size. }\end{array}$ \\
\hline Material not found & Fatal & The named material does not exist. \\
\hline Invalid Coordinate Type & Fatal & Invalid coordinate argument \\
\hline $\begin{array}{l}\text { Invalid init during } \\
\text { tracking }\end{array}$ & Event & Internal coding error. \\
\hline Invalid start & Fatal & The start command is not valid. \\
\hline $\begin{array}{l}\text { Cannot determine } \\
\text { reference coordinate } \\
\text { segment }\end{array}$ & Event & $\begin{array}{l}\text { The reference coordinates are not unique at the start of this } \\
\text { track. Probably means that radiusCut is omitted or too large } \\
\text { in some corner or cornerarc command. }\end{array}$ \\
\hline $\begin{array}{l}\text { Coordinate Object } \\
\text { Missing }\end{array}$ & Event & Internal coding error. \\
\hline $\begin{array}{l}\text { Reference Coordinates } \\
\text { not available }\end{array}$ & Fatal & $\begin{array}{l}\text { Reference coordinates specified, but no applicable reference } \\
\text { particle was tracked. }\end{array}$ \\
\hline
\end{tabular}




\begin{tabular}{|c|c|c|}
\hline Output File Exists & Fatal & $\begin{array}{l}\text { To prevent confusion BLFieldMap will not overwrite an } \\
\text { existing file. }\end{array}$ \\
\hline Nesting $>64$ & Fatal & Groups can only be nested to 64 levels. \\
\hline Object Already Exists & Fatal & The name of an object is not unique. \\
\hline Already Initialized & Fatal & Internal coding error. \\
\hline No Physics Registered & Fatal & No physics command in the input file. \\
\hline No beam Registered & Fatal & No beam command (or other beam). \\
\hline G4VIS_USE not defined & Fatal & $\begin{array}{l}\text { Attempt to use visualization, but the program has no } \\
\text { visualization compiled into it. }\end{array}$ \\
\hline Coordinates got lost & Event & Internal coding error. \\
\hline Stuck Track & Track & $\begin{array}{l}\text { A track has taken }>100 \text { steps without moving significantly. } \\
\text { This is usually a very low energy track on a boundary. }\end{array}$ \\
\hline Event time limit & Event & $\begin{array}{l}\text { Simulating the event has taken more real time than the } \\
\text { parameter eventTimeLimit. }\end{array}$ \\
\hline Erroneous call to & Fatal & Internal coding error. \\
\hline Cannot find NTuple & Fatal & NTuple name not found in Root file. \\
\hline Cannot run beam & Fatal & Internal coding error. \\
\hline Geometry not closed & Fatal & Internal coding error. \\
\hline Cannot read viewers.def & Fatal & $\begin{array}{l}\text { Visualization requested, but cannot find the file viewers.def } \\
\text { that defines the viewers (it is normally in the G4beamline } \\
\text { install directory). }\end{array}$ \\
\hline Viewer not defined & Fatal & A viewer was requested that does not exist. \\
\hline Large Primary TrackID & Warning & $\begin{array}{l}\text { A beam track was read with TrackID }>1000 \text {, which can be } \\
\text { confused with new secondary tracks having such TrackID-s. } \\
\text { See section } 7.14 \text {. }\end{array}$ \\
\hline
\end{tabular}




\section{References}

[1] Geant4 - http://geant4.cern.ch

[2] CLHEP - http://proj-clhep.web.cern.ch/proj-clhep/

[3] HistoScope - http://fermitools.fnal.gov/abstracts/histoscope/abstract.html

[4] The MICE Collaboration - http://mice.iit.edu/

[5] Root - http://root.cern.ch

[6] "Beam simulation tools for GEANT4 (and neutrino source applications)", hep-ex/0210057

[7] Cygwin - http://www.cygwin.com

[8] ffmpeg - http://www.ffmpeg.org

[9] Coin - The Coin 3D graphics library - http://coin3d.org 


\section{G4beamline Validation}

Tom Roberts Muons, Inc. tjrob@muonsinc.com

January 2011

http://g4beamline.muonsinc.com

Copyright $(\subset)$ 2010, 2011 by Tom Roberts; all rights reserved. 


\section{G4Beamline Validation Contents}

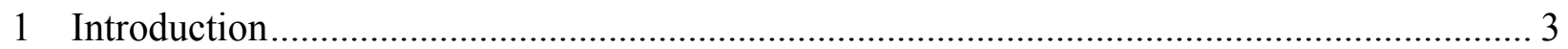

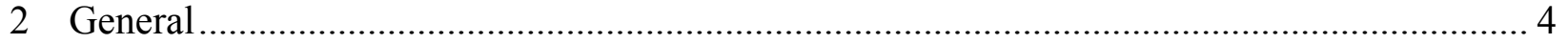

$2.1 \quad$ Element Sizing and Placement............................................................................... 4

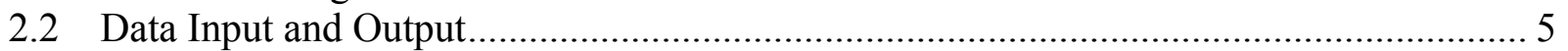

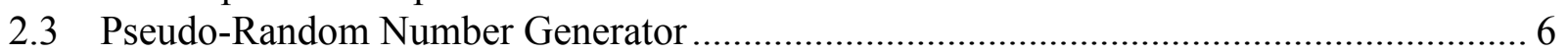

2.4 Regression Tests for G4beamline Releases ............................................................... 6

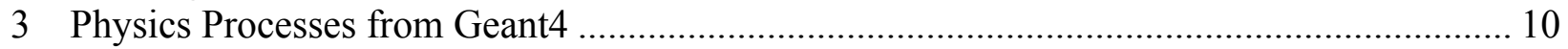

3.1 Particle Transport.......................................................................................... 10

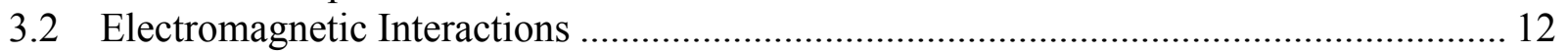

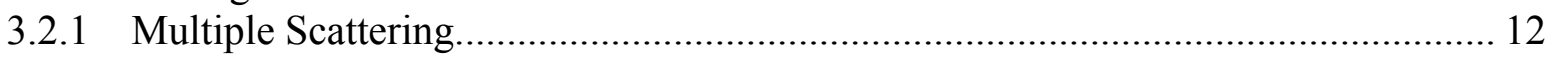

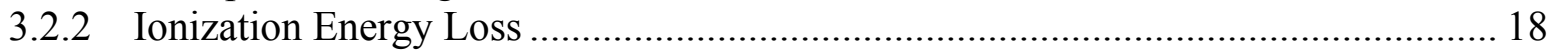

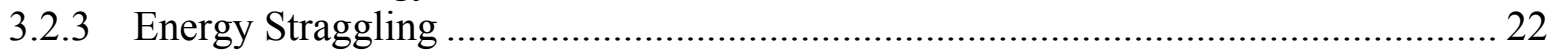

3.2.4 Synchrotron Radiation and Other EM Processes..................................................... 24

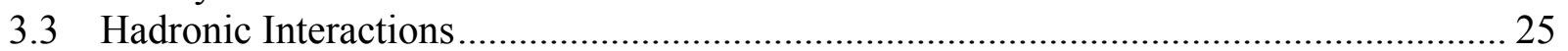

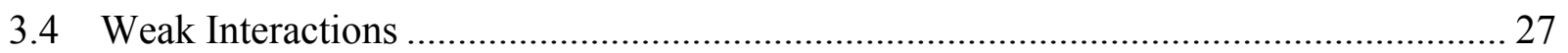

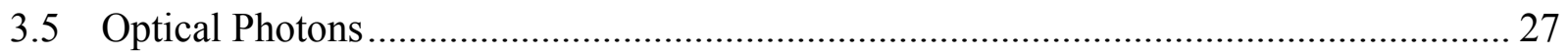

4 Physics Processes Implemented in G4beamline ……......................................................... 28

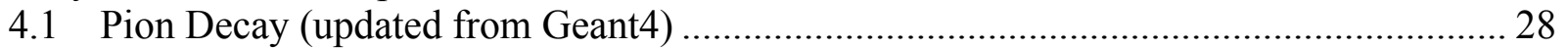

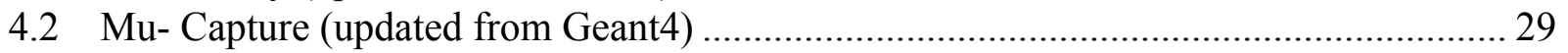

4.3 Bug 1021 (update to Geant4 tracking in E field) ........................................................... 32

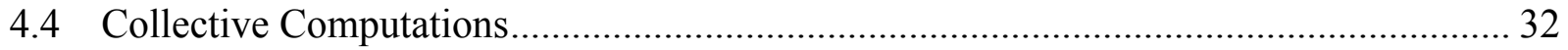

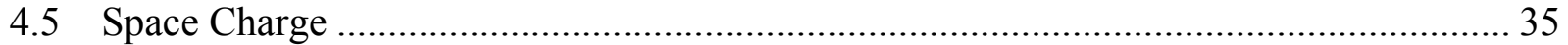

4.5.1 spacechargelw - Lienard-Wiechert computation................................................ 36

4.5.2 spacecharge - Beam-frame Green's Function Convolution via FFTs ....................... 38

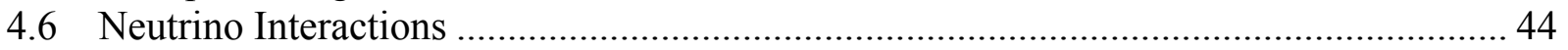

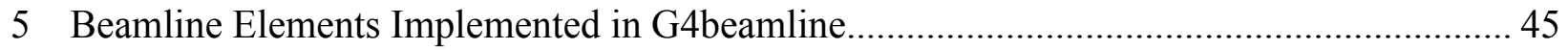

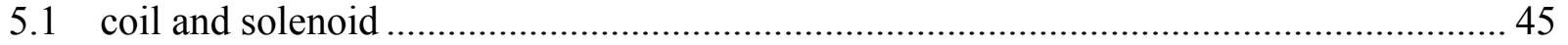

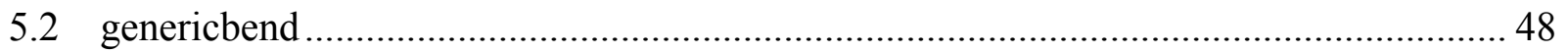

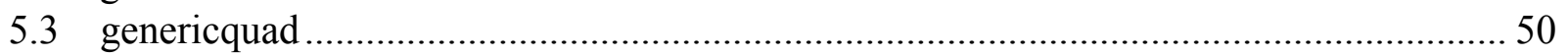

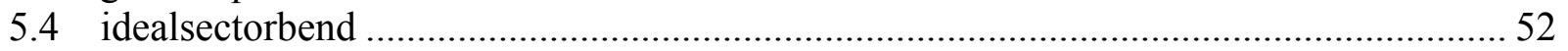

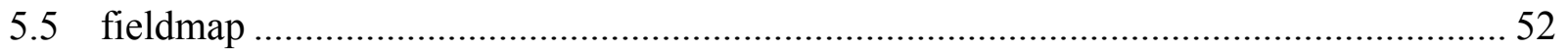

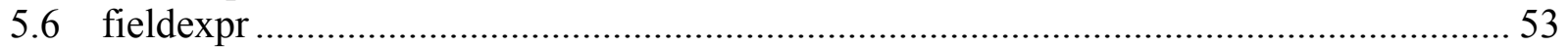

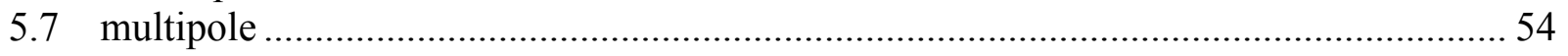

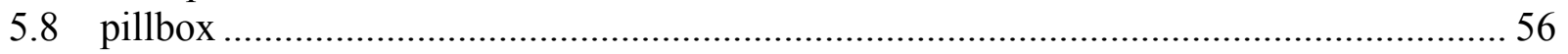

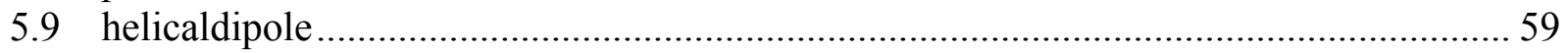

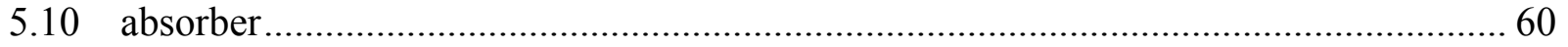

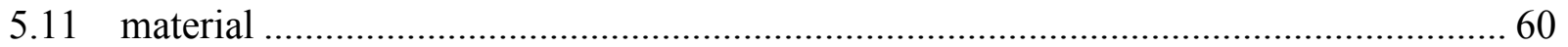

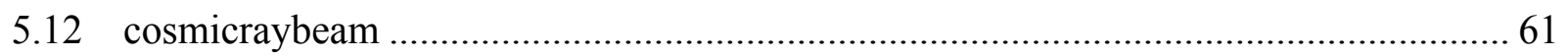

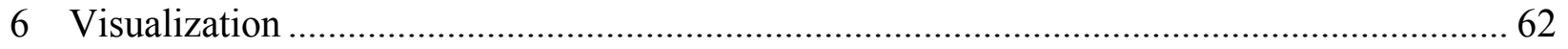

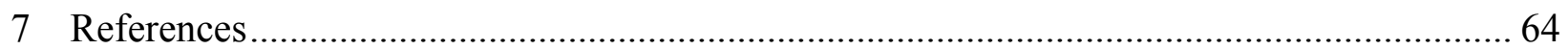




\section{Introduction}

G4Beamline [1] is a single-particle tracking simulation program based on the Geant4 toolkit [2]. Its primary use is evaluating designs of new beamlines and charged-particle beam facilities. So it is important that it simulate real facilities as accurately as possible, and that its accuracy be quantified as well as possible. This document describes the many tests of G4beamline and Geant4 accuracy that have been performed.

As most of the physics processes implemented in G4beamline come directly from Geant4, the validation performed by the Geant 4 collaboration is directly applicable. This document will merely summarize their results, and provide references. Some particularly important processes, such as transport and multiple scattering, have been tested independently, and those results are reported here.

This document does not discuss how to use G4beamline; for that, see the "G4beamline User's Guide" [1]. The input files used for specific tests of G4beamline are included in the Validation directory of the distribution, starting with release 2.8 .

Except as noted, the tests described here were performed using G4beamline 2.06, which uses Geant.9.3p01. Most of the Geant4 Collaboration's tests used earlier versions.

Some authors distinguish between verification and validation, using the first to mean testing that the code performs as intended, and the second to mean its physics accuracy. This document makes no such distinction - if the code is wrong the physics cannot be correct. 


\section{General}

These are general tests, unrelated to specific physics processes or beamline elements.

\subsection{Element Sizing and Placement}

All element sizes and placements are performed with the accuracy of a double; all rotations are specified in degrees, but decimals are permitted, again with the accuracy of a double. Here is a scatter plot of the shadow of a 10-mm square box that kills all tracks that hit it:

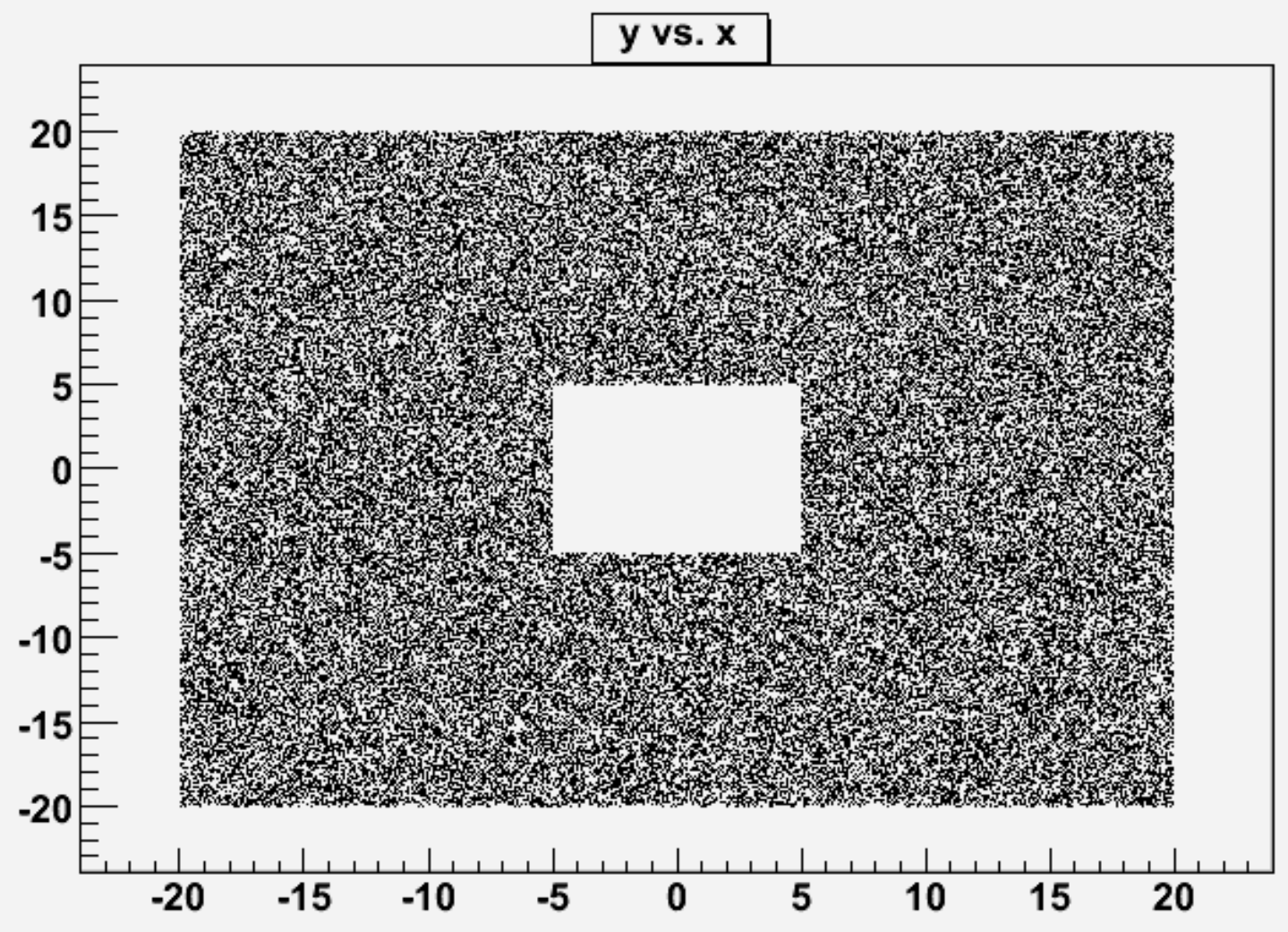

Here is a histogram for events with $-4.9<\mathrm{y}<4.9$ (i.e. within the vertical region of the box), showing the right edge $(\mathrm{x})$ has an accuracy better than 0.2 micron: 


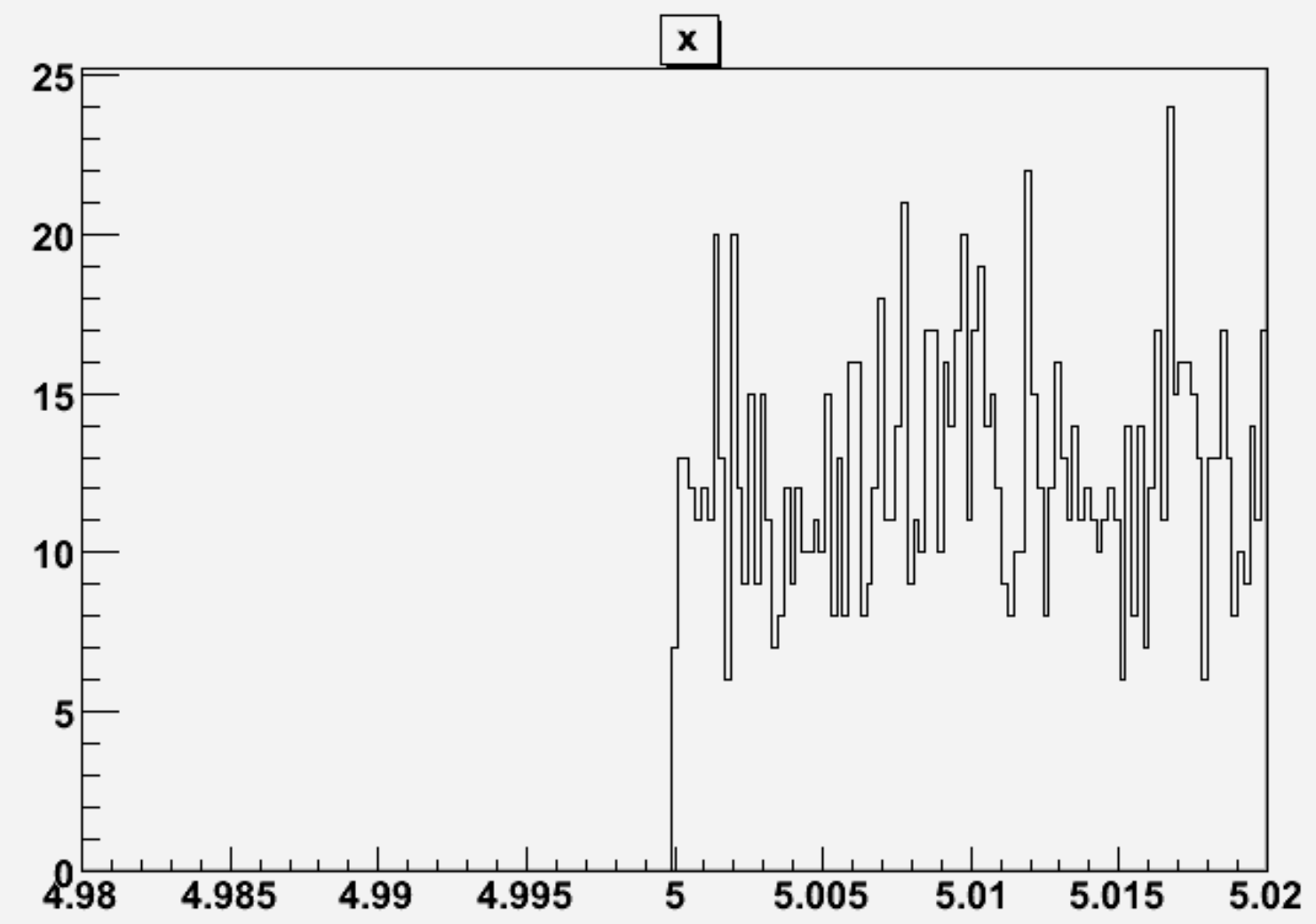

The input file for this test is placement.g4bl:

* placement.g4bl - verify object placement with beam

physics QGSP_BERT

\# this is a uniform beam emanating from a square $20 \mathrm{~mm}$ on a side

beam gaussian sigma $X=-20$ sigma $=-20$ nEvents $=10000000$

box $B$ height $=10$ width $=10$ length $=10$ kill $=1$ color $=0,0,1$

place $B z=100$

virtualdetector Det height $=1000$ width $=1000$ length $=1$ color $=0,1,0$

place Det $z=200$

\subsection{Data Input and Output}

Input tracks from files in Root [3], ASCII, or BLTrackFile format come via NTuples, which have float values. Output is similarly sent via NTuples with float values; for ASCII and BLTrackFile formats the floats are printed in $\% .6 \mathrm{~g}$ format. Here's a test that directly writes $0.001 \mathrm{~mm}$ after reading (spaces added to line up the columns):

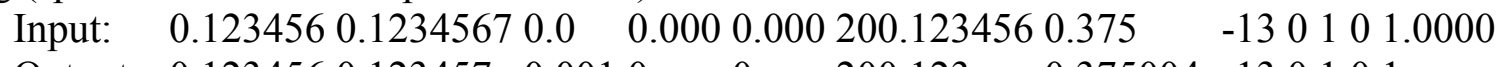

$\begin{array}{llllllllllllllll}\text { Output: } & 0.123456 & 0.123457 & 0.0010 & 0 & 200.123 & 0.375004 & -13 & 0 & 1 & 0 & 1\end{array}$ (The differences are correct.)

The input file for this test is dataio.g4bl:

* dataio.g4bl - test BLTrackFile data accuracy 


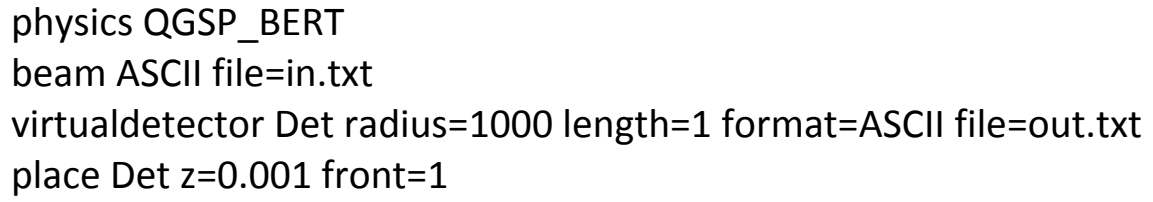

\subsection{Pseudo-Random Number Generator}

G4Beamline uses the default Geant4 random number generator. It is an excellent pseudo-random number generator that essentially guarantees no repeat sequences when seeded by any integer between 0 and 900 million. At the start of each event, G4Beamline seeds the random number generator with the event number. This permits the user to submit multiple jobs in parallel with confidence, as long as no two jobs run the same event numbers. This also permits the user to rerun the same events (as long as the simulation remains unchanged). The randomseed command can be used to change this default behavior.

The random number generator comes from CLHEP [4], and is the default HepJamesRandom generator, described in [5]. It is documented to provide independent random streams for seeds between 0 and 900,000,000 (inclusive), which exceeds the normal range of event numbers in G4beamline. It is not feasible to perform a useful test of this generator here, see the references for details.

\subsection{Regression Tests for G4beamline Releases}

Before every release of G4beamline, a set of tests is run to make sure that the program basically works, and that no previously working features were broken in obvious ways. The goal is to have at least one test for each G4beamline command; that has not quite been reached, as some commands are difficult or impossible to test in an automated manner.

In most cases, the test was written at the same time as the feature being tested. Most tests have simple physical situations and quantitative checks on some value(s) that were generated by the program; in most cases, these internal checks of the test have been verified manually. That is, most of these tests not only verify consistency among releases, but also verify that for at least one instance, the basic operation of the feature is correct.

For example, test78 tests the setdecay command, which permits the user to change the decay parameters of particles (a capability intended for testing and background studies). test78 does the following:

1. Changes pi+ to decay with equal branching ratios into (e+ nu_e) and (mu+ nu_mu), and a lifetime of $0.1 \mathrm{~ns}$.

2. Changes mu+ to decay with a lifetime of $1 \mathrm{E} 20 \mathrm{~ns}$ (i.e. they never decay, to avoid confusing the particle counts).

3. Makes deuterons be unstable (!), decaying into (tau+ gamma) with a lifetime of $0.1 \mathrm{~ns}$.

4. Changes tau + to have a lifetime of 1E20 ns (i.e. they never decay).

5. Runs with a beam of 50 deuterons and 100 pi+, over 10 meters.

6. Checks that at the end of the simulation $(\mathrm{z}=10000 \mathrm{~mm})$ there were $50 \pm 1 \mathrm{tau}+$, $50 \pm 12 \mathrm{mu}+$, and $50 \pm 12 \mathrm{e}+$ (values outside those ranges make the test fail). 
The artificially short lifetimes ensure that all $\mathrm{pi}+$ and all deuterons decay (!), and the \pm 12 values for particle counts accommodate statistical variations. This is a highly unphysical simulation, but the presence of those particle counts at the end does imply that the setdecay command performed as intended. Note that this just tests for basic operation, and details such as energy and momentum conservation are not checked. The setdecay command has several major cases internally, and test 78 was written to check one instance of each - as frequently happens, one of the unit tests of the code became a regression test.

Another example is test 12 testing the multipole command, which creates a beamline element with a multipole magnetic field. It places 5 elements with quadrupole, sextupole, octopole, decapole, and dodecapole fields. It then uses the printfield command to print the $\mathrm{B}_{\mathrm{x}}$ and $\mathrm{B}_{\mathrm{y}}$ fields in the $x-z$ and $y-z$ planes inside all of them, verifying they are correct to within 0.0002 tesla. The multipole command has five internal cases for the different multipoles, and test 12 was written to check one instance of each; this is another unit test turned into a regression test. The values of the fields were checked manually when the test was written.

A third example is test68 testing the polycone command, which generates an element consisting of an absorber shaped as multiple cones in a line. It creates and places a polycone, and then surrounds it with several cylinders that should or should not intersect the polycone, and checks the correctness of those intersections. It also checks that beam propagates through the polycone without error. Unit testing of the polycone command relied heavily on visualization, which cannot easily be translated into automated tests.

These tests are in the directory test of the G4beamline distribution. As of release 2.06 the list of these tests is:

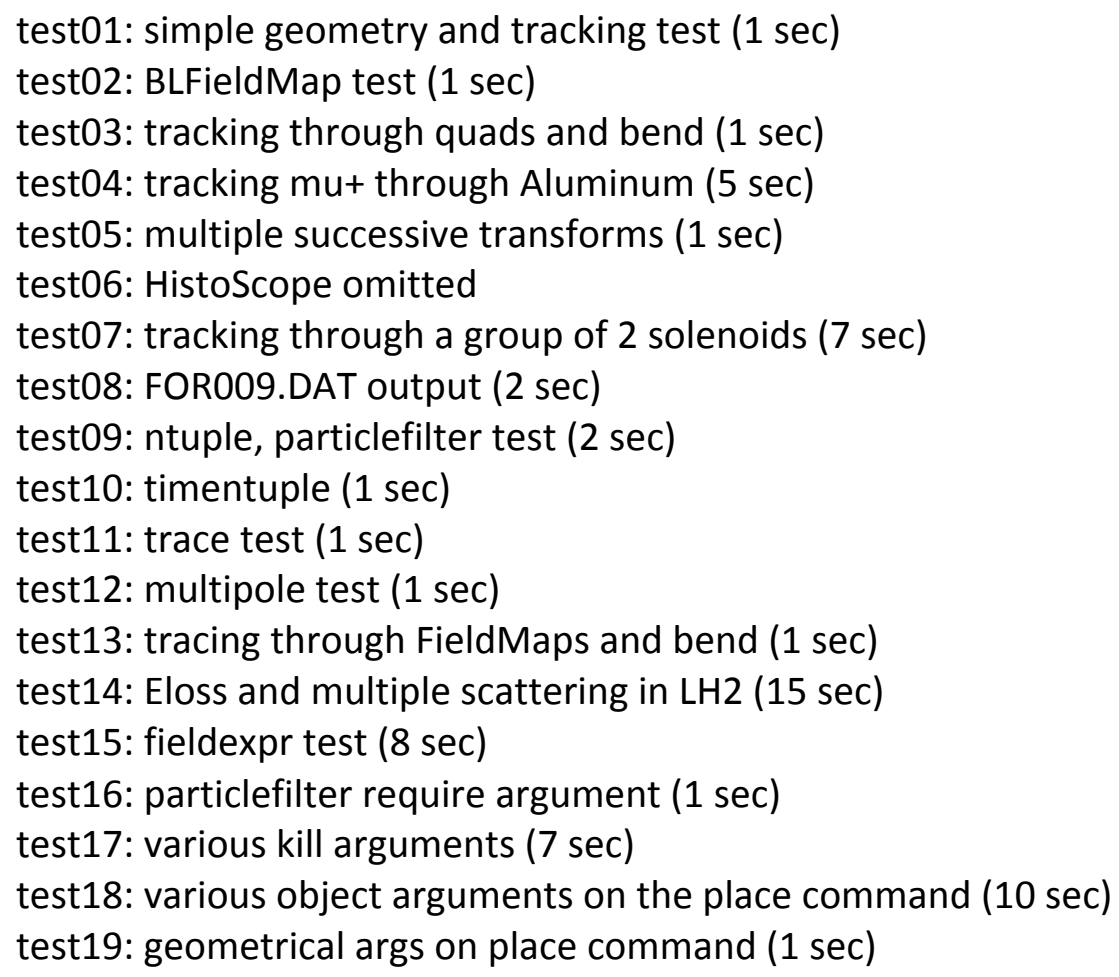


test20: beamlossntuple $(7 \mathrm{sec})$

test21: field of rotated solenoid and solenoid cache file $(7 \mathrm{sec})$

test22: argument expressions (1 sec)

test23: place OFFSET with 2 solenoids $(7 \mathrm{sec})$

test24: virtualdetector NTuple name test $(1 \mathrm{sec})$

test25: particlefilter nWait and referenceWait test $(1 \mathrm{sec})$

test26: ntuple with for009.dat ( $2 \mathrm{sec}$ )

test27: randomseed command (4 sec)

test28: printf $(1 \mathrm{sec})$

test29: 3 nested tune-s $(2 \mathrm{sec})$

test30: tune By of genericbend ( $2 \mathrm{sec}$ )

test31: tune maxGradient of 4 pillbox-es $(2 \mathrm{sec})$

test32: tune reference momentum ( $2 \mathrm{sec}$ )

test33: profile command ( $15 \mathrm{sec})$

test34: element naming $(1 \mathrm{sec})$

test35: tune By of idealsectorbend ( $2 \mathrm{sec}$ )

test36: the if and the define commands $(1 \mathrm{sec})$

test37: zntuple ( $2 \mathrm{sec}$ )

test38: Root input and output ( $6 \mathrm{sec})$

test39: zntuple + beam rotation $(2 \mathrm{sec})$

test40: MICEPhysicsList (1 sec)

test41 -- LISAPhysicsList OMITTED

test42: rotated trace (1 sec)

test43: various coordinates arguments $(2 \mathrm{sec})$

test44: steppingVerbose (1 sec)

test45: psuedo random number generator seeds and sequence $(1 \mathrm{sec})$

test46: do loops and complex (multi-line) if-s (1 sec)

test47: tune By of six idealsectorbends $(2 \mathrm{sec})$

test48: reference coordinates ( $2 \mathrm{sec}$ )

test49: multiple beam commands eventide-s $(1 \mathrm{sec})$

test50: require arguments on NTuple commands (1 sec)

test51: newparticlentuple ( $2 \mathrm{sec})$

test52: tune fieldexpr $(2 \mathrm{sec})$

test53: tune fieldmap ( $2 \mathrm{sec})$

test54: $\mathrm{Zcl}(2 \mathrm{sec})$

test55: compile and run BLMinimize (1 sec)

test56: extrusion $(2 \mathrm{sec})$

test57: eventcuts file $(1 \mathrm{sec})$

test58: NIST material database, output command $(1 \mathrm{sec})$

test59: multiple beam-s and corner-s $(3 \mathrm{sec})$

test60: tracker (4 sec)

test61: linac $(3 \mathrm{sec})$

test62: $120 \mathrm{GeV} / \mathrm{c}$ beam (10 sec)

test63: zntuple tracked both directions (2 sec) 


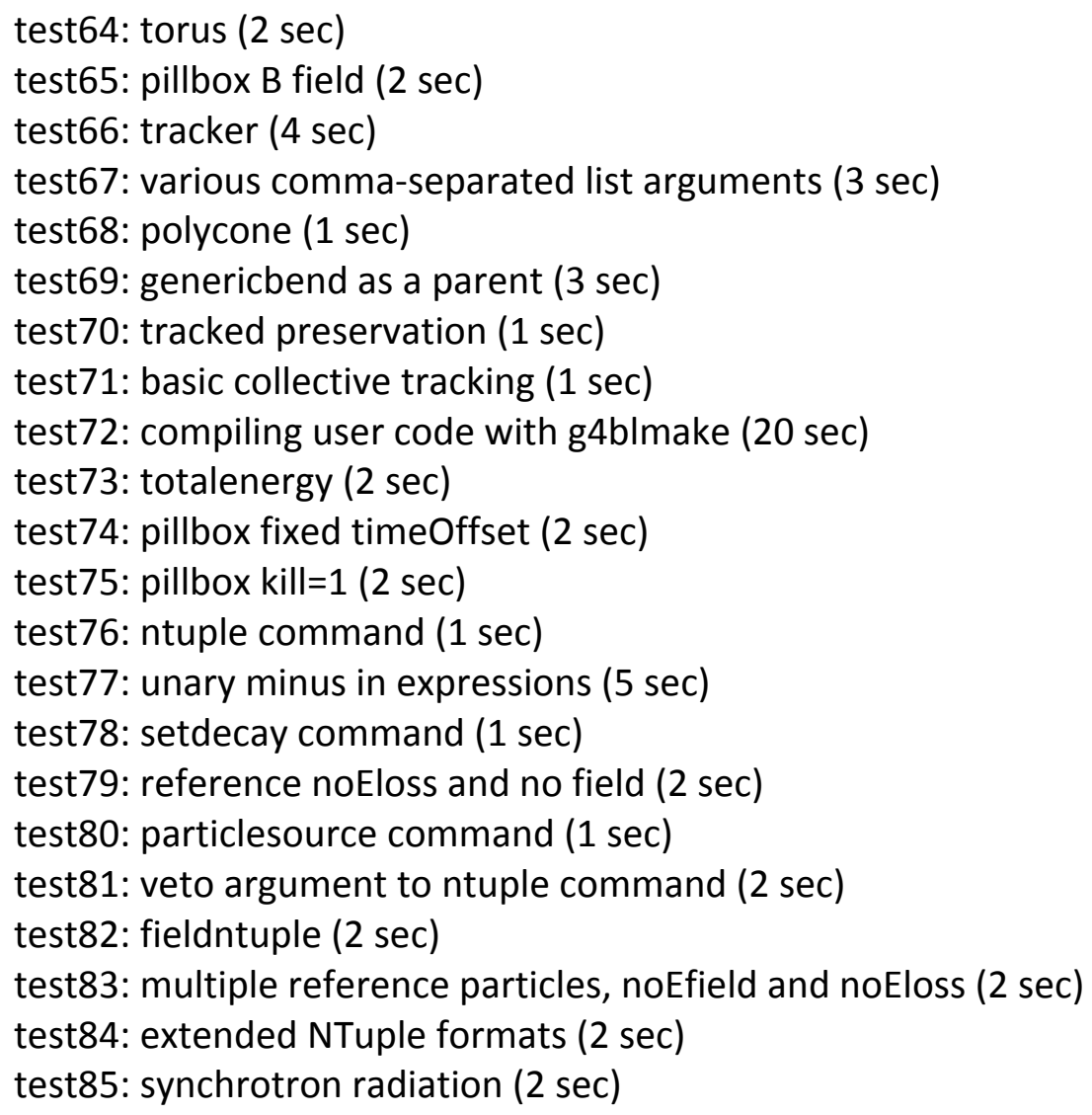




\section{Physics Processes from Geant4}

Most physics processes in G4beamline are taken from the Geant4 toolkit [2] without change. These processes are discussed in this section. See section 4 for physics processes implemented in G4beamline, including modifications of processes from Geant4.

The primary link for "Validation and Testing" of Geant4 from the Geant4 Collaboration is: http://geant4.web.cern.ch/geant4/results/results.shtml.

A more general link to Geant4 "Results \& Publications" is:

http://geant4.web.cern.ch/geant4/results/index.shtml.

The CERN "Simulation Validation Project" is also relevant:

http://lcgapp.cern.ch/project/simu/validation/.

\subsection{Particle Transport}

\section{Test 1}

To check particle transport, one thousand $25 \mathrm{MeV} / \mathrm{c}$ electrons were tracked in the $\mathrm{x}-\mathrm{z}$ plane normal to a uniform 0.01 Tesla magnetic field along $\mathrm{y}$, with initial sigmaXp $=0.1$. These tracks have a circumference of about 52 meters. The maximum step length (maxStep) was varied from $1 \mathrm{~mm}$ to 1 meter, and the position in $\mathrm{x}$ when returning to its starting point is histogrammed for 1000 tracks. Remember that this step is the "physics step", and integrating the equations of motion in the EM field uses smaller steps controlled by the tracking parameters. In this plot, $\mid$ mean $\mid+2 *$ sigma is plotted, providing an upper bound on the error for $>95 \%$ of the tracks (fewer tracks exceed $2 *$ sigma than for a Gaussian); the value for maxStep $=1 \mathrm{~mm}$ cannot be read from the plot; it is 0.015 micron: 


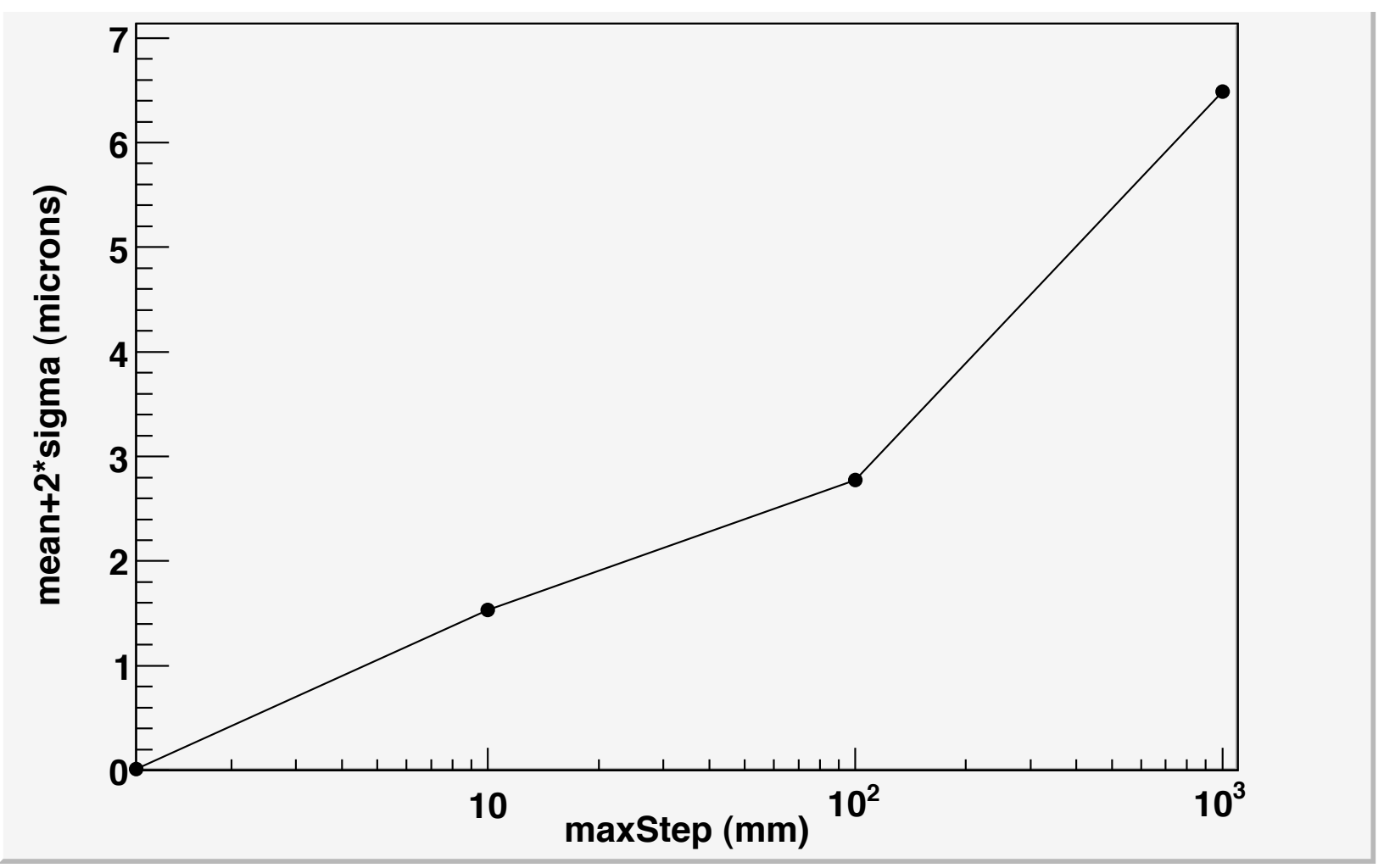

After one circle, the momentum of each track is observed to be within $0.000001 \mathrm{MeV} / \mathrm{c}$ of the initial $25.000000 \mathrm{MeV} / \mathrm{c}$ beam; all tracks have a y value within 0.01 micron of the correct value, 0.0 . The transit time for each track is within $0.0001 \mathrm{~ns}$ of the correct value, $174.8111 \mathrm{~ns}-$ for these parameters the period is (MKS):

$$
\begin{aligned}
\mathrm{T} & =2 \pi \mathrm{m} \gamma /(\mathrm{q} \mathrm{B}) \\
& =6.28318531 * 9.1093821 \times 10^{-31} * 48.934002 /\left(1.6021765 \times 10^{-19} * 0.01\right) \\
& =174.8111 \mathrm{~ns}
\end{aligned}
$$

By inference, the radius of each track is correct within a few microns.

As expected, the real-time speed of tracking depends strongly on the value of maxStep:

\begin{tabular}{|c|c|}
\hline maxStep $(\mathrm{mm})$ & Tracks / sec \\
\hline 1 & 2.1 \\
\hline 10 & 20.8 \\
\hline 100 & 200 \\
\hline 1000 & $>500$ \\
\hline
\end{tabular}

The input file for this test is transport.g4bl:

* $\quad$ transport.g4bl - test G4beamline transport in a uniform B field param -unset maxStep $=100$

physics QGSP_BIC param $\operatorname{maxStep}=\$$ maxStep histoFile $=$ transport $\operatorname{maxStep}=\$$ maxStep output \$histoFile.out \# beam has angular divergence, but all particles come back to $(0,0,0)$. 
beam gaussian particle $=\mathrm{e}-$ meanMomentum $=25$ sigmaXp $=0.1 \mathrm{nEvents}=1000$

fieldexpr Field $B y=0.01$ height $=50000$ width $=50000$ length $=50000$

place Field $\mathrm{z}=0$

virtualdetector Det height $=100$ width $=100$ length $=0.1$ color $=0,1,0$

place Det $\mathrm{z}=0$ front $=1$

particlefilter Filter height $=100$ width $=100$ length $=0.1$ kill=e- $n W a i t=2$

place Filter $\mathrm{z}=0.1$ front $=1$

box $\mathrm{W}$ height $=50000$ width $=50000$ length $=1$ color $=1,0,0$

place $\mathrm{W} z=25000$

\section{Test 2}

A second test is to check that particles move in straight lines through vacuum with no fields. A proton beam with zero emittance and meanXp=0.000001 (i.e. $\mathrm{dx} / \mathrm{dz}=1$ microradian) has every particle at $\mathrm{y}=0.0$ and $\mathrm{x}=10000.0$ at $\mathrm{z}=1 \mathrm{E} 10$, to the accuracy of a float (tracking is performed in double precision, but the NTuple uses float-s). This test has the default maxStep=100 (mm), and took $\sim 800$ seconds to track each particle for $10,000 \mathrm{~km}$ (100 million steps).

\subsection{Electromagnetic Interactions}

The primary link for the Geant4 Collaboration's testing and validation of electromagnetic physics processes is: https://twiki.cern.ch/twiki/bin/view/Geant4/EMValidation. A large body of work is reported there.

\subsubsection{Multiple Scattering}

\section{Test1 - MuScat}

The MuScat experiment [6] ran at TRIUMF to measure $\mu^{+}$multiple scattering in various materials. It was inspired by the need for accurate data to validate simulation programs like G4beamline, specifically for muon cooling studies. It used a $172 \mathrm{MeV} / \mathrm{c} \mu^{+}$beam on several different targets. For liquid hydrogen, the experimenters de-convolved the data using targetempty runs, to remove the effects of the windows. The G4beamline runs use 10 million events each, and the errorbars from G4beamline are negligible compared to those from MuScat. 
MuScat Iata for H2, $109 \mathrm{~mm}$

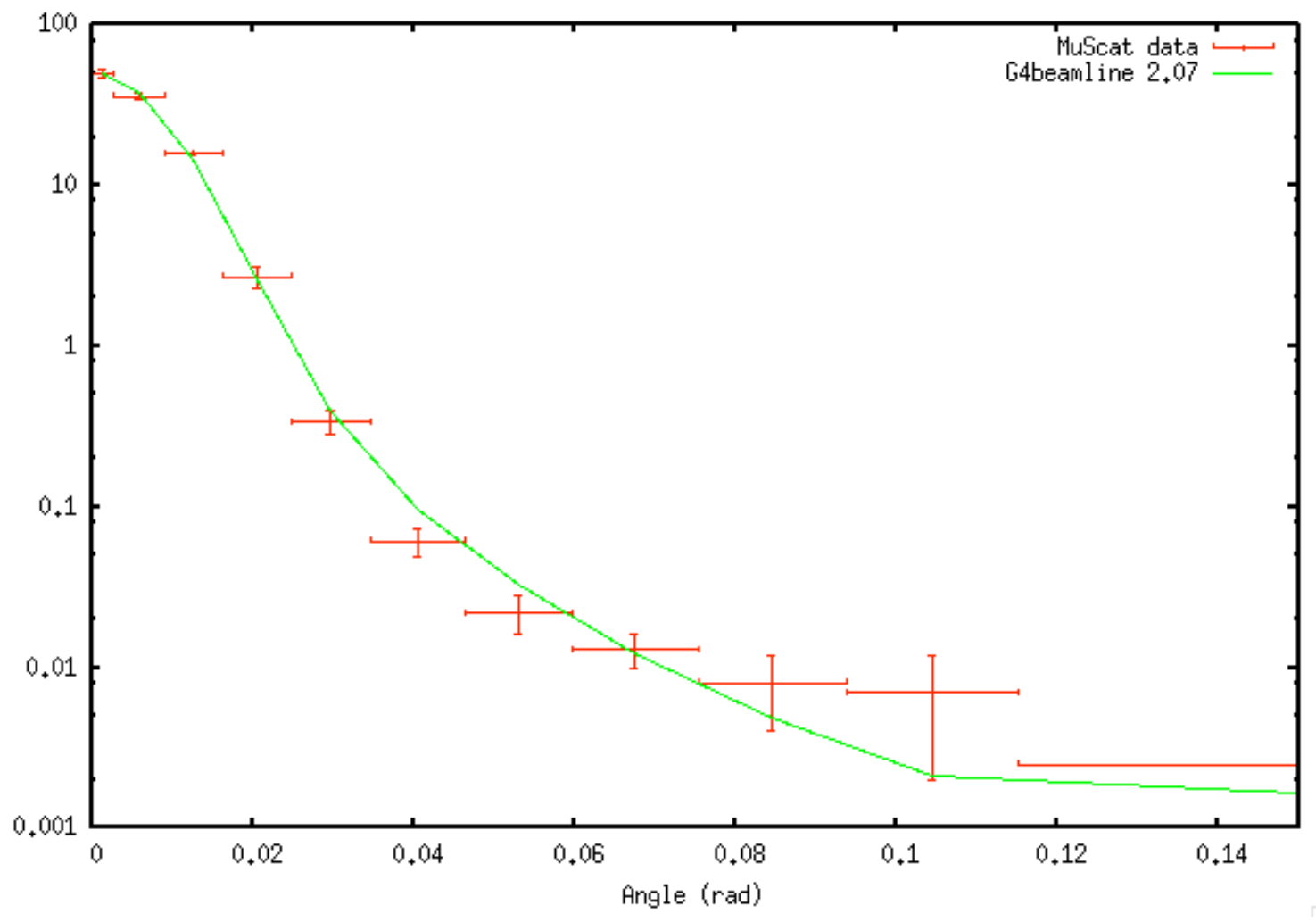

MuScat Data for H2, 159 mm

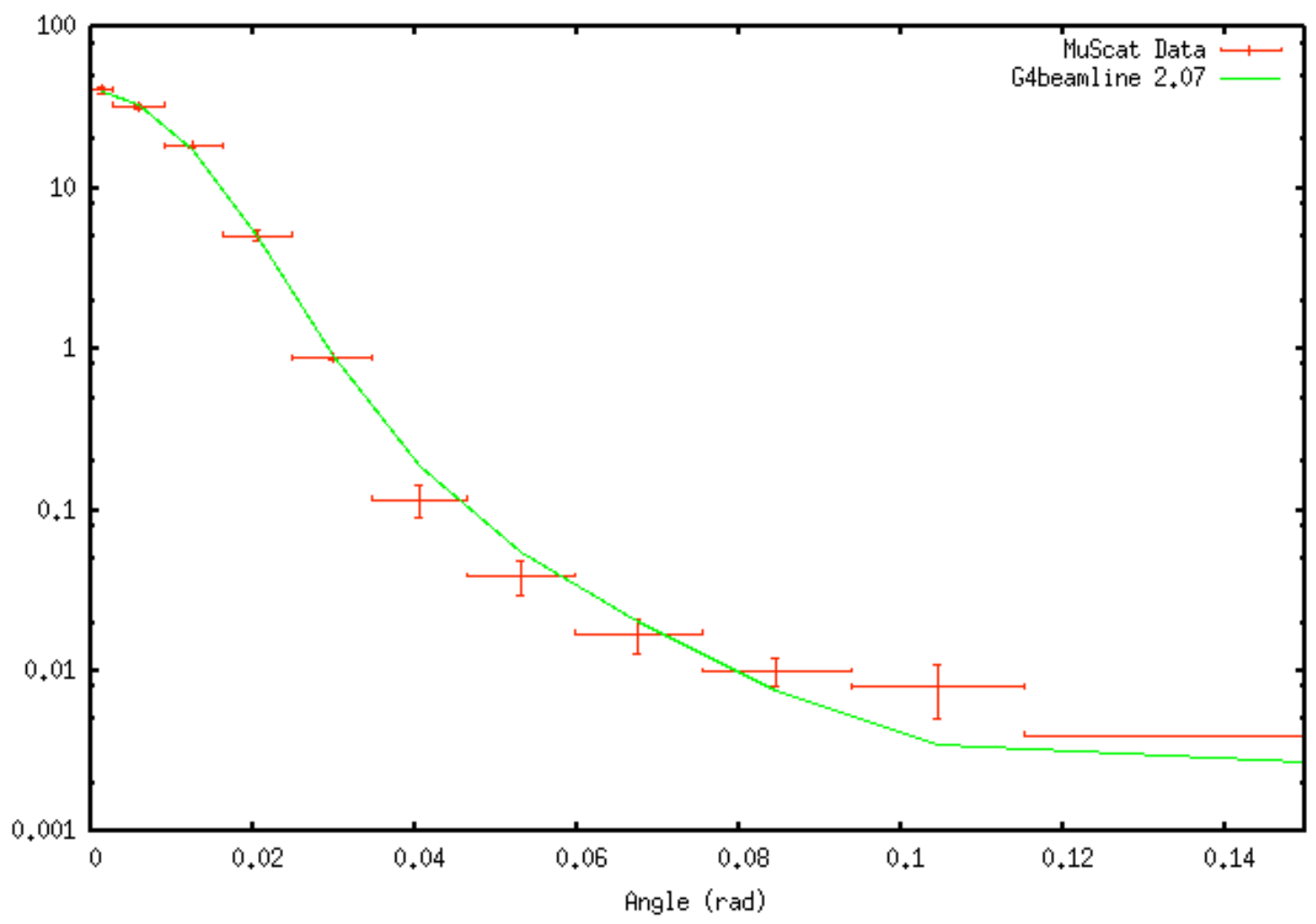


MuScat Data for Be

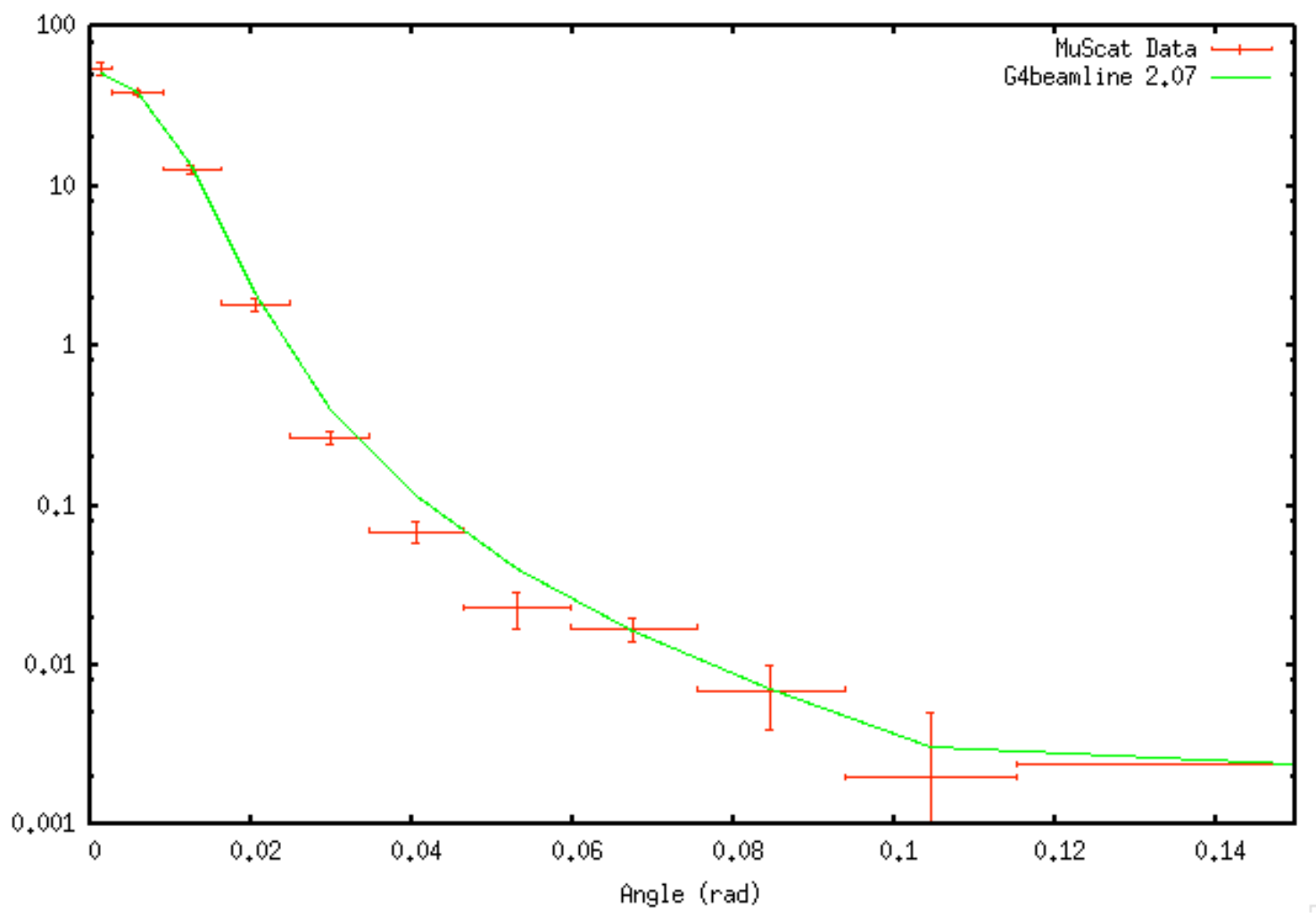

MuScat Data for Li

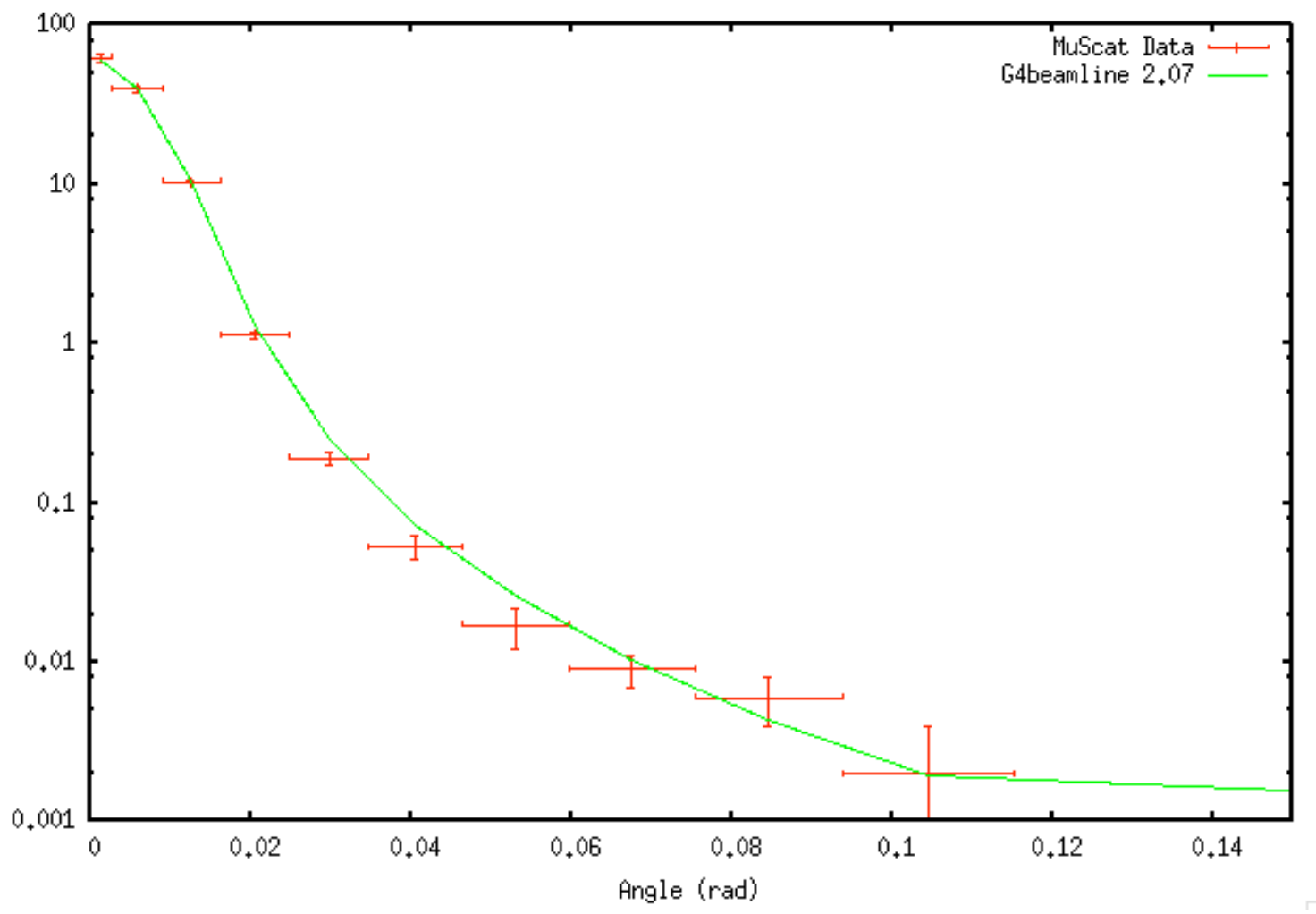


MuScat Data for C

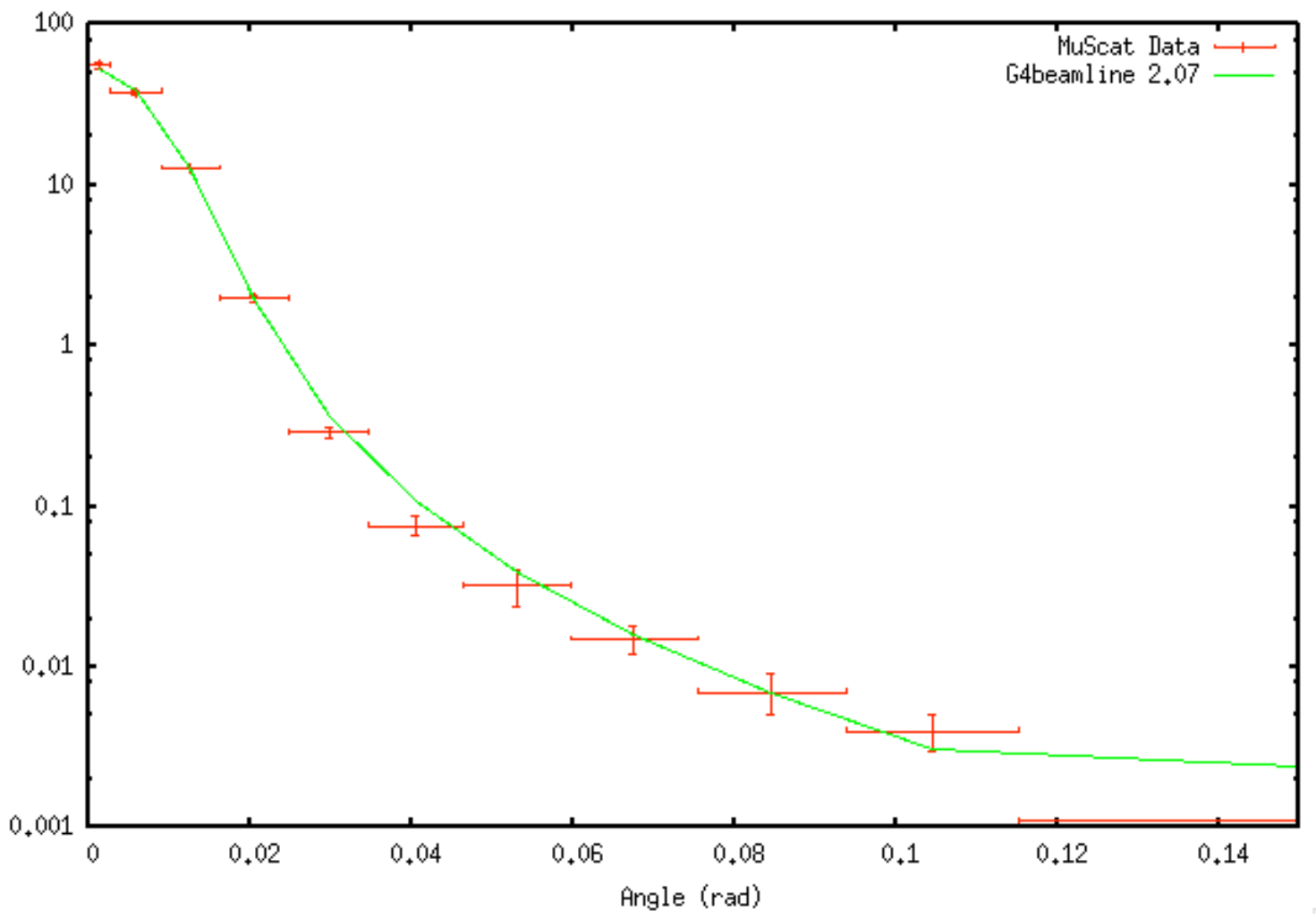

MuScat Data for $\mathrm{Fe}$

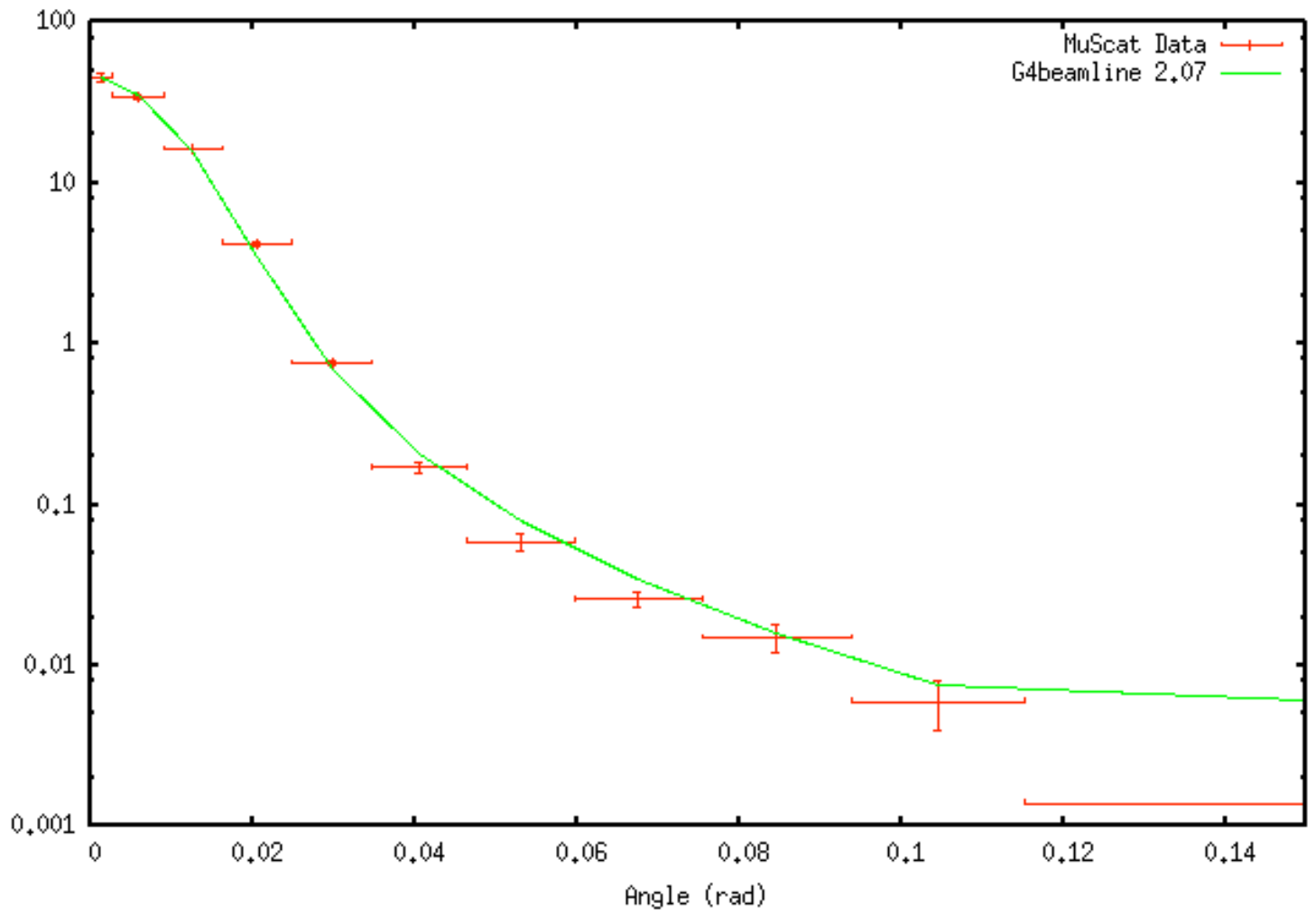


While these plots look reasonable, and certainly reproduce the broad features of the data, when examined quantitatively the correspondence is not so good. The G4beamline data can be considered as a zero-parameter fit to the MuScat data with 11 degrees of freedom, and the corresponding Chisq values are given below. Note the bins for the two datasets are identical, so the errorbars in $\mathrm{x}$ have no effect (the lines for G4beamline data above are drawn point-to-point at the same bin centers as the MuScat data).

\begin{tabular}{|c|c|c|c|}
\hline Target & Chisq & Chisq/DF & Probability \\
\hline $\mathrm{H} 2(109 \mathrm{~mm})$ & 28.8 & 2.62 & $0.3 \%$ \\
\hline $\mathrm{H} 2(159 \mathrm{~mm})$ & 47.1 & 4.28 & $<0.01 \%$ \\
\hline $\mathrm{Be}$ & 63.7 & 5.79 & $<0.01 \%$ \\
\hline $\mathrm{Li}$ & 27.6 & 2.51 & $0.4 \%$ \\
\hline $\mathrm{C}$ & 27.0 & 2.45 & $0.4 \%$ \\
\hline $\mathrm{Fe}$ & 87.5 & 7.96 & $<0.01 \%$ \\
\hline
\end{tabular}

For all six targets, the largest contributions to Chisq come from bins in the range $0.02<$ Angle $<$ 0.05 . These are neither in the center nor in the tail. In all cases, at least $1 / 3$ of the Chisq comes from a single bin; for the three worst targets, more than half of the Chisq comes from a single bin.

The input file for these tests is muscat.g4bl:

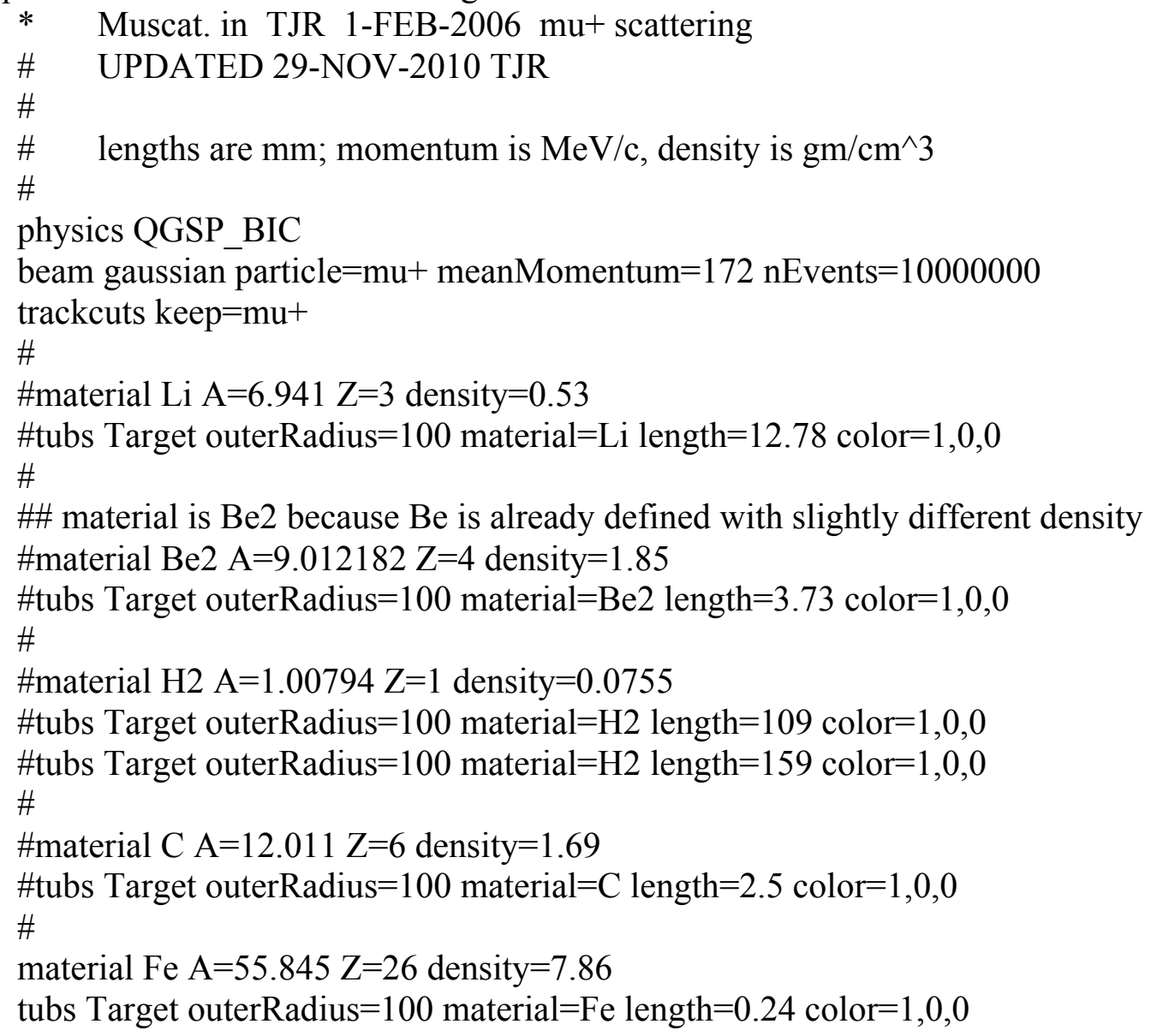


\#

virtualdetector Det radius $=10000$ color $=0,1,0$ format $=$ ascii

place Target $\mathrm{z}=100$

place Det $\mathrm{z}=200$

\section{Test2 - Geant4 Collaboration tests}

The Geant4 electromagnetic physics working group page on testing and validation is located at: http://geant4.web.cern.ch/geant4/collaboration/working_groups/electromagnetic/tests.shtml It contains considerably more tests than just multiple scattering. Two plots from the section on multiple scattering are reproduced below.

Low energy electron scattering in gold. Data are from Phys. Rev. 84 (1951) 634.

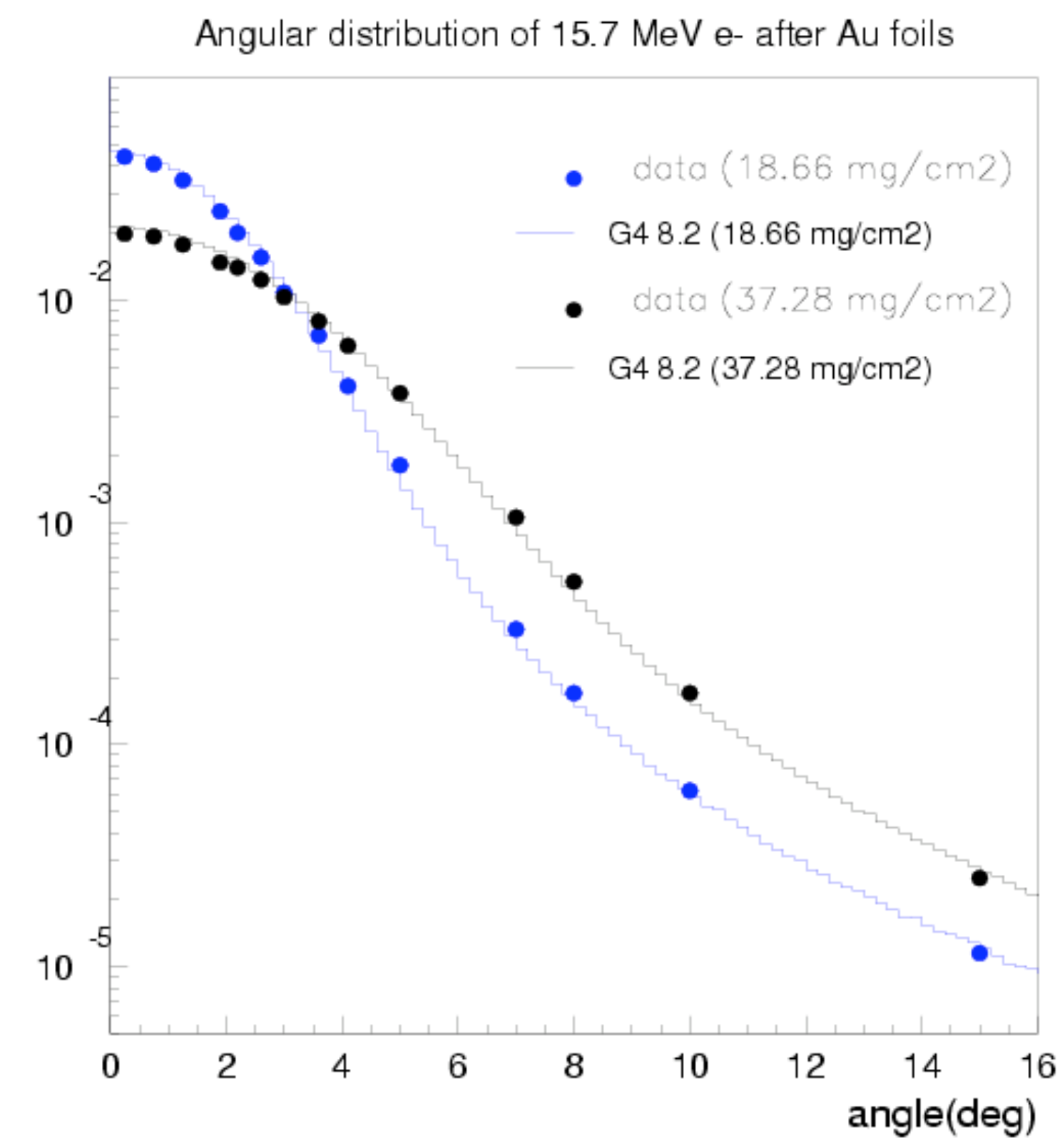


High-energy proton scattering in Be. Data are from Phys. Rev. D 20 (1979) 1584.

theta0 for proton scattering in $32 \mathrm{~mm} \mathrm{Be}$

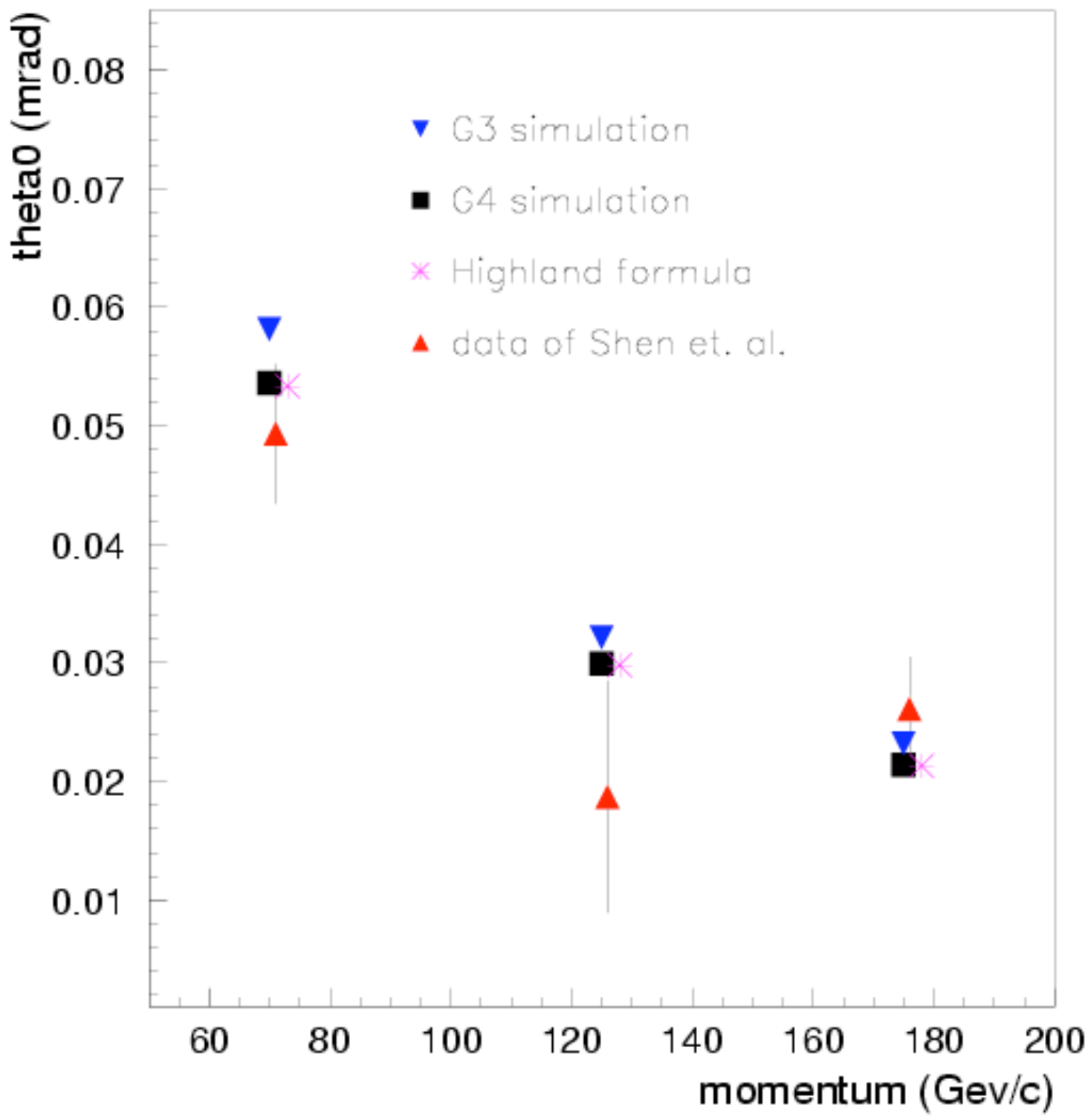

\subsubsection{Ionization Energy Loss}

\section{Test1 Comparison to the PDG model}

The ionization energy loss in Geant4 for $\mu^{+}$tracks well with the PDG model below $\beta \gamma \sim 1000$. Above that, the discrepancy is claimed to be due to nuclear absorption of muons, which still needs to be investigated.

The following plot is from the PDG "Review Tables and Plots" section on "Passage of particles through matter" [7], fig. 27.1. The red dots were computed using G4beamline 1.16; errorbars are 
smaller than the dots. For each point, the thickness of the $\mathrm{Cu}$ absorber was varied so the incident $\mu^{+}$lose about $5 \%$ of their momentum.

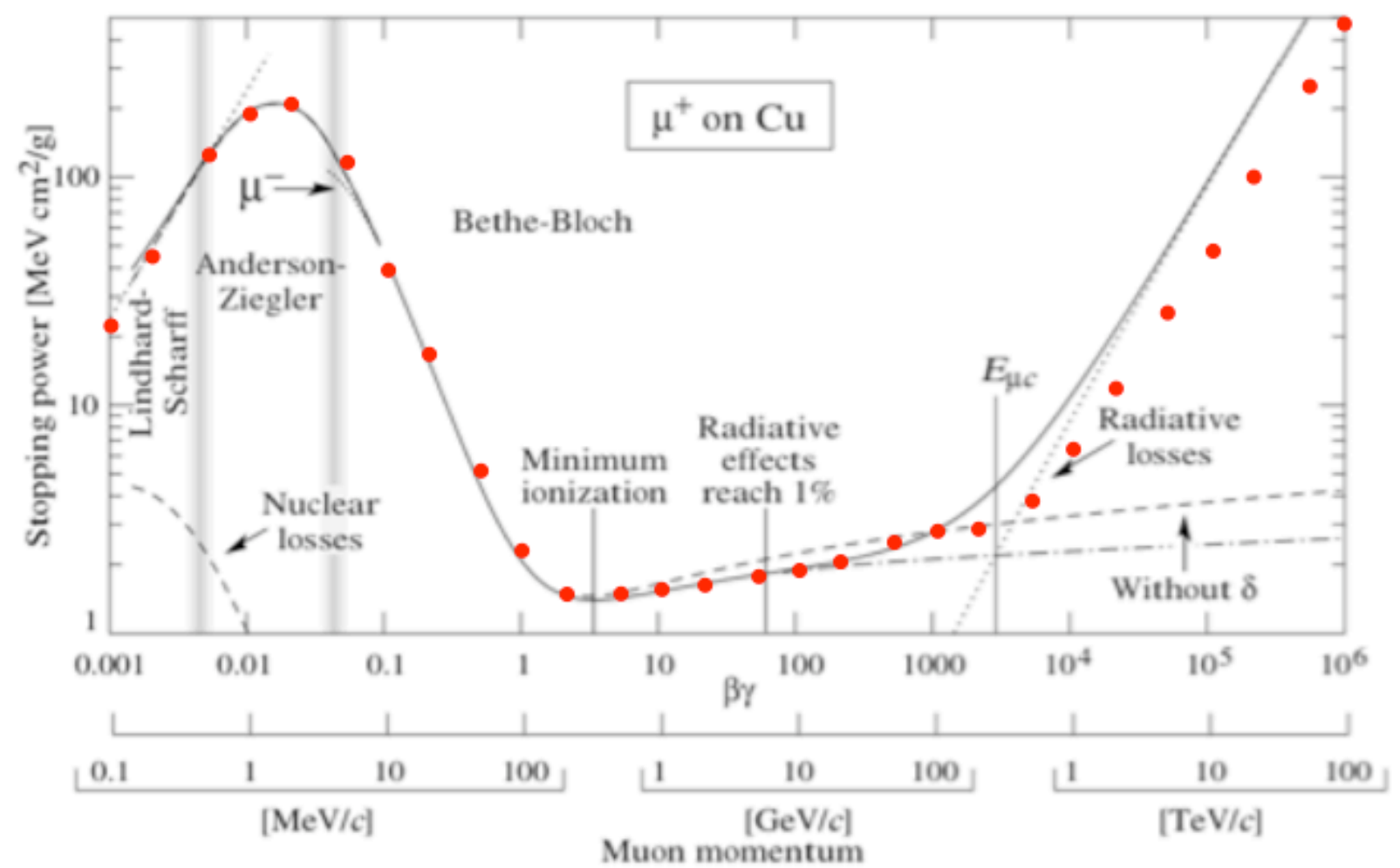

The input file for this test is eloss.g4bl:

\# eloss.g4bl

\# $\quad$ vary Thick so mu+ lose about $5 \%$ of their initial momentum.

param -unset $\mathrm{P}=1.0$ Thick=1.0 Det=1.0.txt

param material=Cu $\mathrm{M}=105.658$

physics QGSP_BERT disable=Decay

trackcuts kill=e+,e-,gamma

beam gaussian particle $=$ mu + meanMomentum $=\$ P$ nEvents $=10000$

tubs Absorber outerRadius $=200$ material $=\$$ material length $=\$$ Thick

place Absorber $\mathrm{z}=1000$

virtualdetector Det radius $=200$ length $=1$ format $=$ ascii file $=\$$ Det

place Det

\section{Test2 Geant4 Collaboration tests}

The Geant4 electromagnetic physics working group page on testing and validation is located at: http://geant4.web.cern.ch/geant4/collaboration/working_groups/electromagnetic/tests.shtml It contains considerably more tests than just ionization energy loss. A few figures are copied below. 
Energy deposition of protons in Xenon gaseous detector; data are from NIM 217 (1983) 277.

\section{$X_{e,} 85 \mathrm{~cm}\left(20^{\circ} \mathrm{C}, 1 \mathrm{~atm}\right)$}

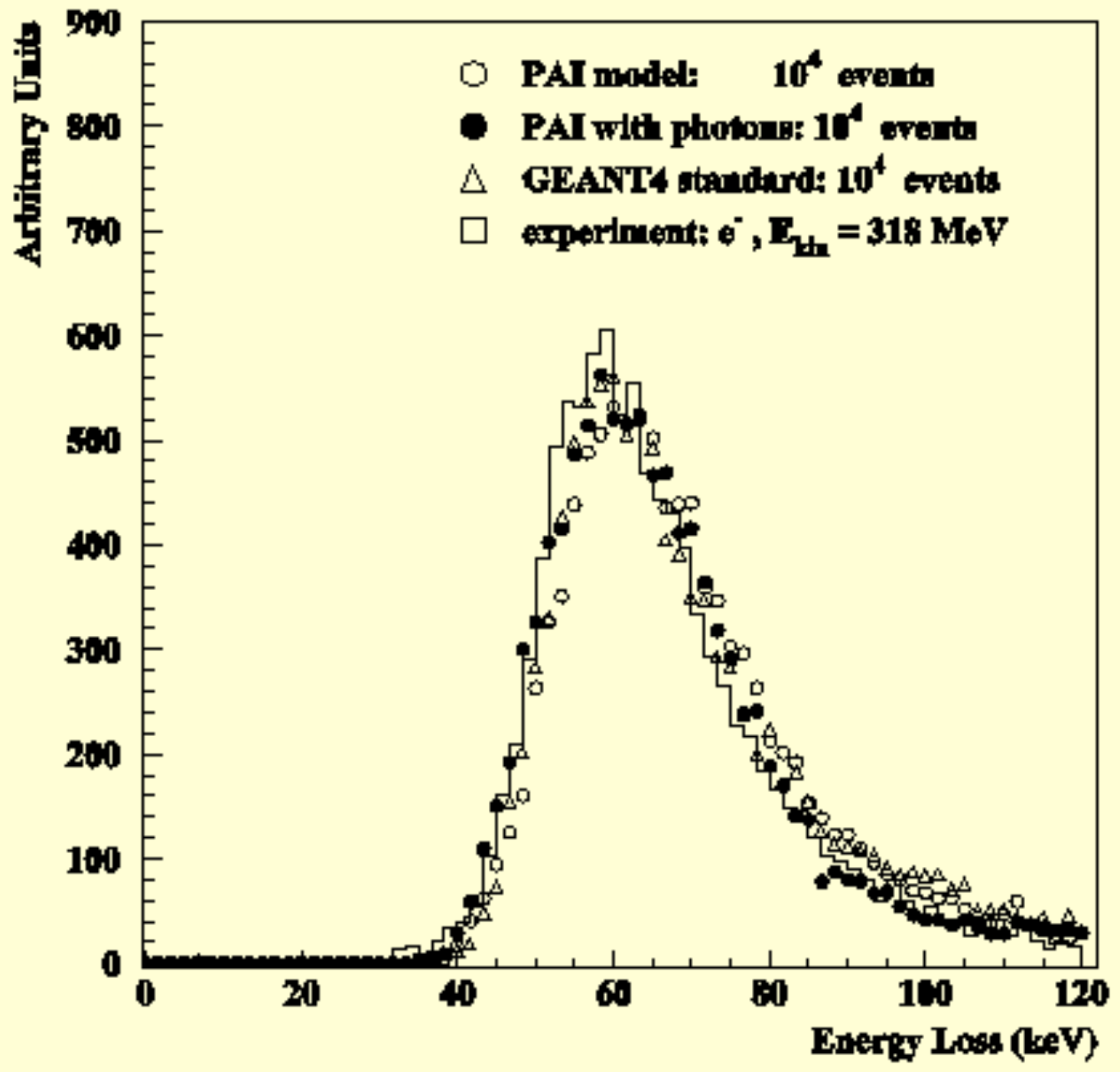


Proton projected range, ratio of the Geant 4 calculation to the NIST data, as a function of energy. Accuracy of the data above $1 \mathrm{MeV}$ is claimed to be about $2 \%$. This plot shows the ratio of the Geant 4 computation to the data; note the suppressed zero.

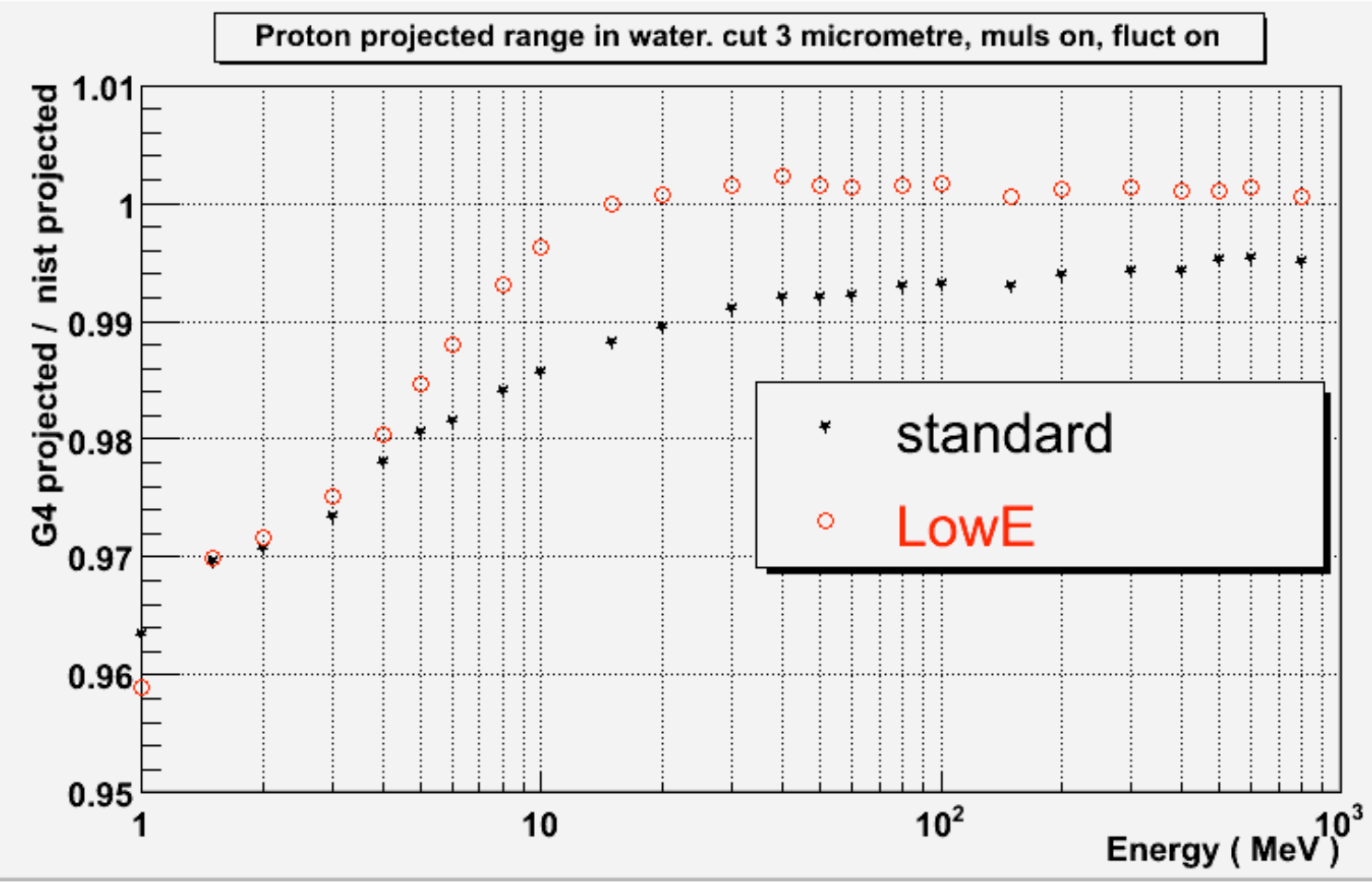


Proton stopping power versus ICRU'49 evaluated data for Geant4 7.1p01. The accuracy of the data above $1 \mathrm{MeV}$ is claimed to be about $2 \%$. These plots show the difference in percent between the data and the Geant4 computation.
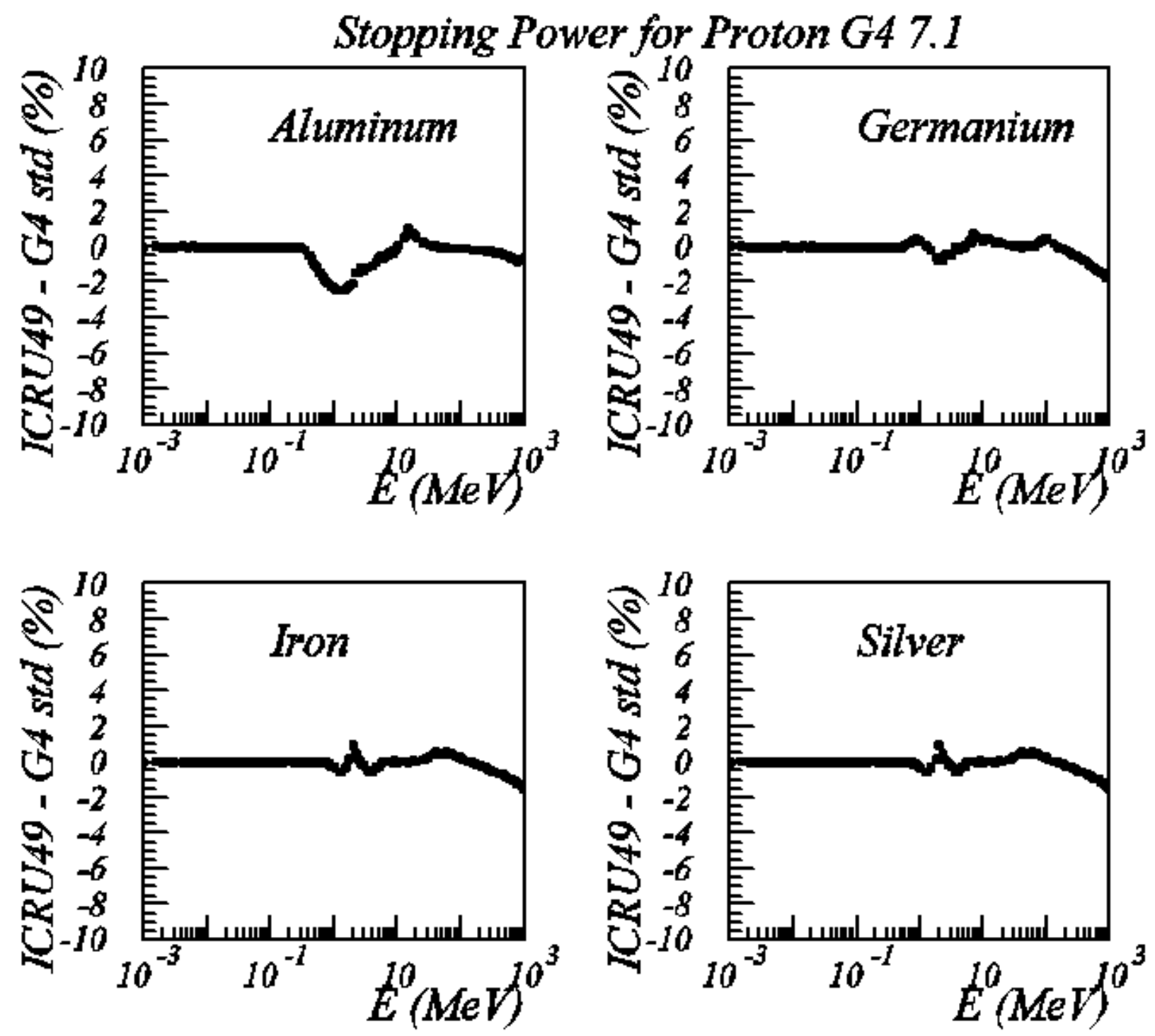

\subsubsection{Energy Straggling}

The following plot is from Tschalär and Maccabee, Phys. Rev. B 1, no. 7, p2863 (1970). It shows the straggling of $19.68 \mathrm{MeV}$ protons after various thicknesses of $\mathrm{Al}$ absorber. The red dots are G4beamline. The G4beamline beam has zero energy width, which is lower than that of their beam ( $\sim 0.03 \mathrm{MeV}$, comparable to their detector resolution), but this is not enough to account for the differences. Clearly, the Geant 4 energy-loss process loses slightly too little energy, and has too little straggling. This simulation has four absorbers in a row so all four plots could be made at once, but the difference between that and a single absorber of $0.497 \mathrm{~g} / \mathrm{cm}^{2}$ is barely visible. 


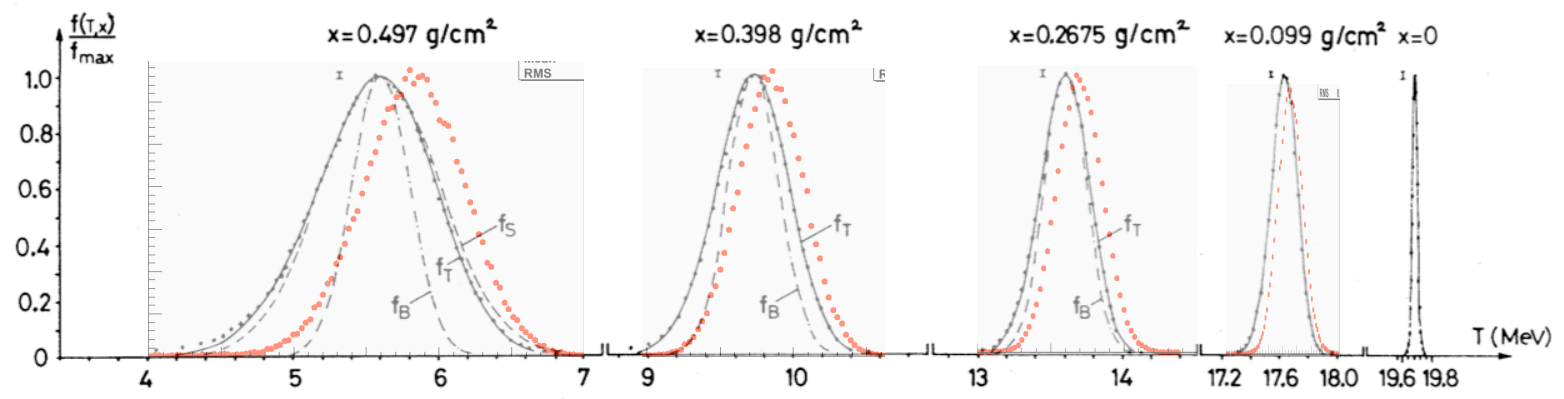

Fig. 3. Energy distributions of $19.68-\mathrm{MeV}$ protons after traversing an aluminum absorber. Curves $f_{B}$ are theoretical predictions of Bohr, $f_{S}$ of Symon, and $f_{T}$ of Payne and Tschalär.

The same difference applies to $49.10 \mathrm{MeV}$ protons after a thicker $\mathrm{Al}$ absorber $\left(2.675 \mathrm{~g} / \mathrm{cm}^{2}\right)$; data are from the same reference as above. The peak energy loss differs by about $1 \mathrm{MeV}$, out of $40 \mathrm{MeV}$ in energy loss, a difference of $2.5 \%$.

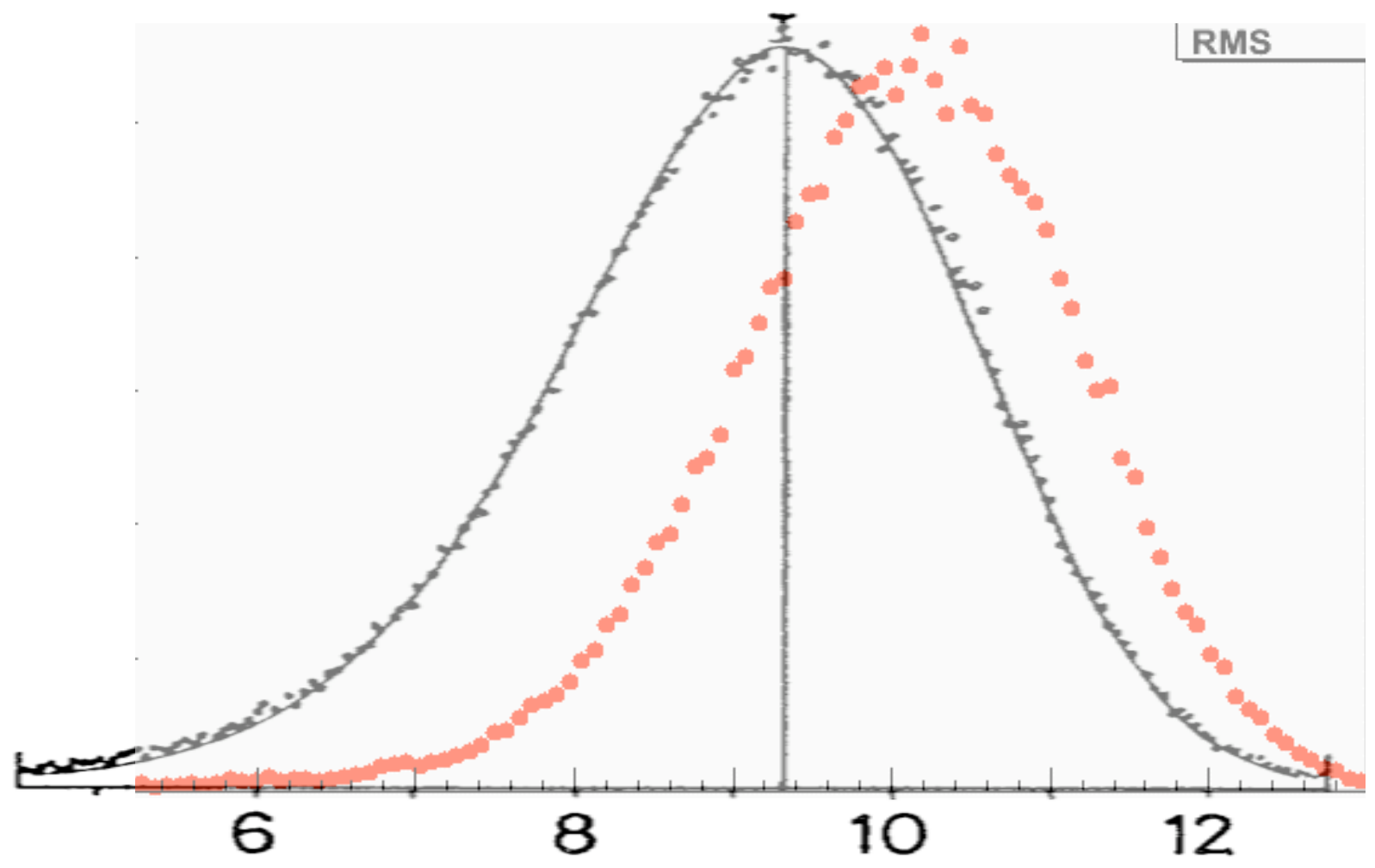

The input file for this test is straggling.g4bl:

\# $\quad$ straggling.g4bl

physics QGSP_BERT

param $\mathrm{DEN}=2.699 \mathrm{M}=938.272 \mathrm{KE}=19.68$

param $\mathrm{P}=\operatorname{sqrt}((\$ \mathrm{KE}+\$ \mathrm{M}) *(\$ \mathrm{KE}+\$ \mathrm{M})-\$ \mathrm{M} * \$ \mathrm{M})$

beam gaussian particle $=$ proton meanMomentum $=\$$ PEvents $=100000$

virtualdetector Det radius $=1000$ length $=0.01$ color $=0,0,1$

place Det $\mathrm{z}=1$ rename $=0$ 
cylinder Abs1 material=Al outerRadius $=1000$ length $=0.099 / \$ D E N * 10$

place Abs1 $\mathrm{z}=\$ \mathrm{Zcl}+10$

place Det rename $=0.099 \mathrm{z}=\$ \mathrm{Zcl}+10$

cylinder Abs2 material $=\mathrm{Al}$ outerRadius $=1000$ length $=(0.2675-0.099) / \$ D E N * 10$

place Abs $2 \mathrm{z}=\$ \mathrm{Zcl}+10$

place Det rename $=0.2675 \mathrm{z}=\$ \mathrm{Zcl}+10$

cylinder Abs3 material $=\mathrm{Al}$ outerRadius $=1000$ length $=(0.398-0.2675) / \$ D E N * 10$

place Abs3 z=\$Zcl+10

place Det rename $=0.398 \mathrm{z}=\$ \mathrm{Zcl}+10$

cylinder Abs4 material=Al outerRadius $=1000$ length $=(0.497-0.398) / \$ D E N * 10$

place Abs4 $\mathrm{z}=\$ \mathrm{Zcl}+10$

place Det rename $=0.497 \mathrm{z}=\$ \mathrm{Zcl}+10$

\subsubsection{Synchrotron Radiation and Other EM Processes}

The Geant4 Collaboration test of synchrotron radiation is in:

http://geant4.web.cern.ch/geant4/collaboration/working_groups/electromagnetic/tests.shtml\#SR.

The plot is copied below.

Synchrotron radiation: comparison of theoretical (smooth curve) and generated (histogram) spectra.
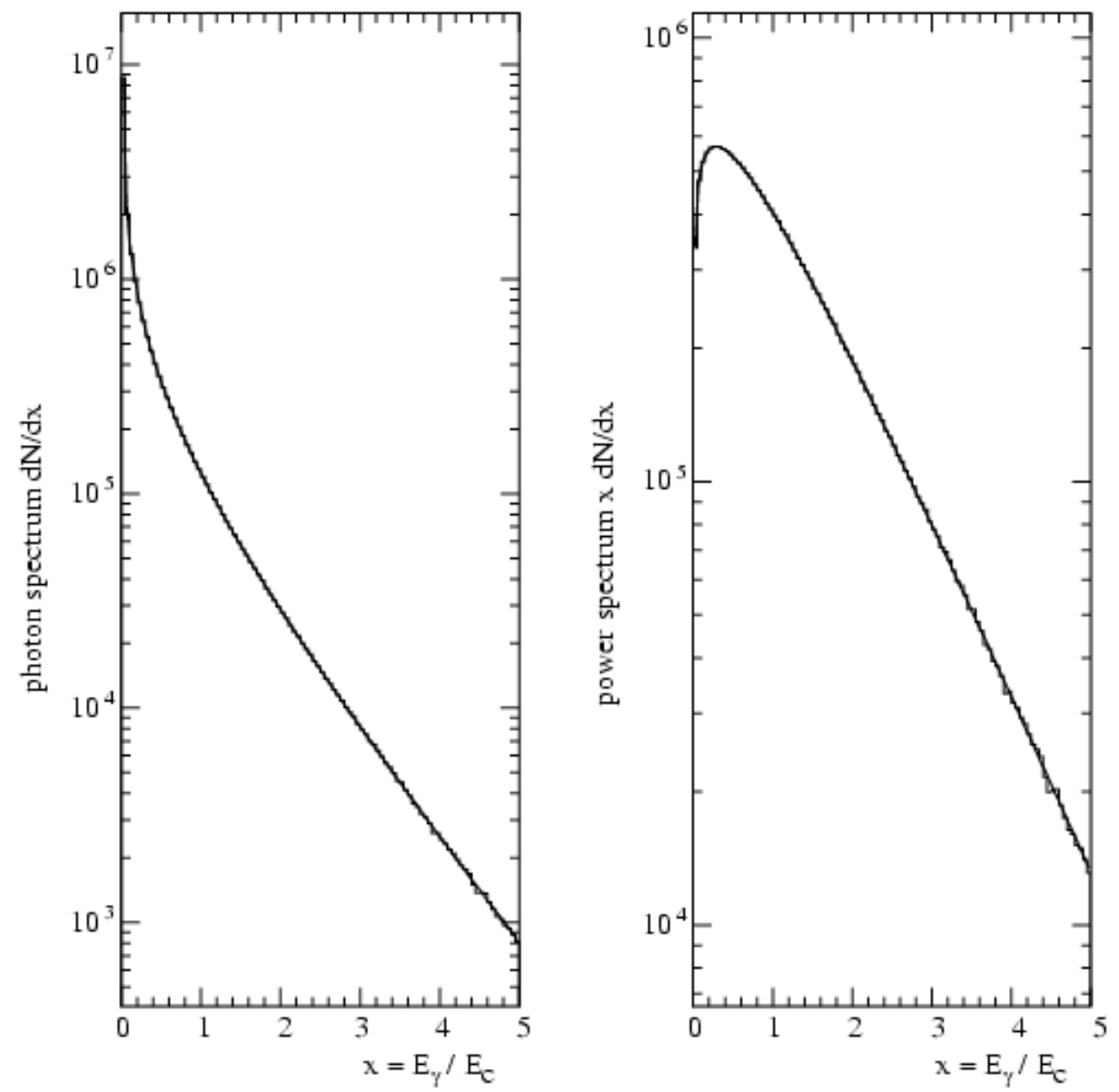


\subsection{Hadronic Interactions}

The primary link for the Geant4 Collaborations webpage on "Physics Validation and Verification" for hadronic physics processes is:

http://geant4.web.cern.ch/geant4/results/validation_plots.htm. A very tiny sample of representative plots is copied here.

Comparison to HARP inclusive proton production data, $\mathrm{p} \mathrm{A} \rightarrow \mathrm{p} \mathrm{X}, 20^{\circ}<$ angle $<50^{\circ}$. The transition region of QGSP_BERT just above $10 \mathrm{GeV}$ is quite evident. Plot is from arXiv:1006.3429.

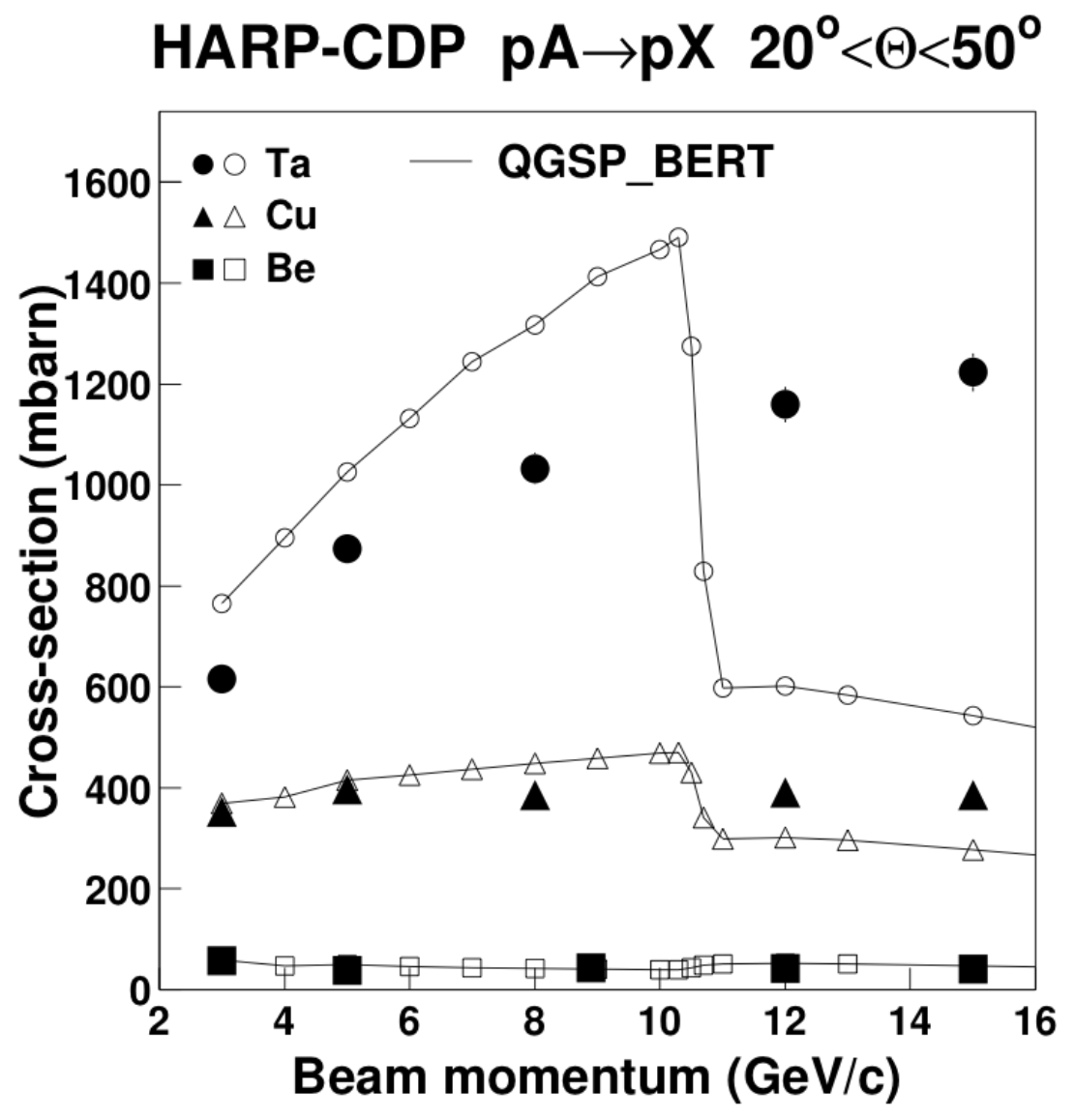


A proton beam of $730 \mathrm{MeV}$ is incident upon a $\mathrm{C}$ target. The double-differential pi+ cross sections are measured vs. angle and kinetic energy. Data are from: D.R.F. Cochran et al., Phys. Rev. D6, 3085 (1972). Error bars are between 8 and 18\%, statistical.

\section{$730 \mathrm{MeV} p$ on C}

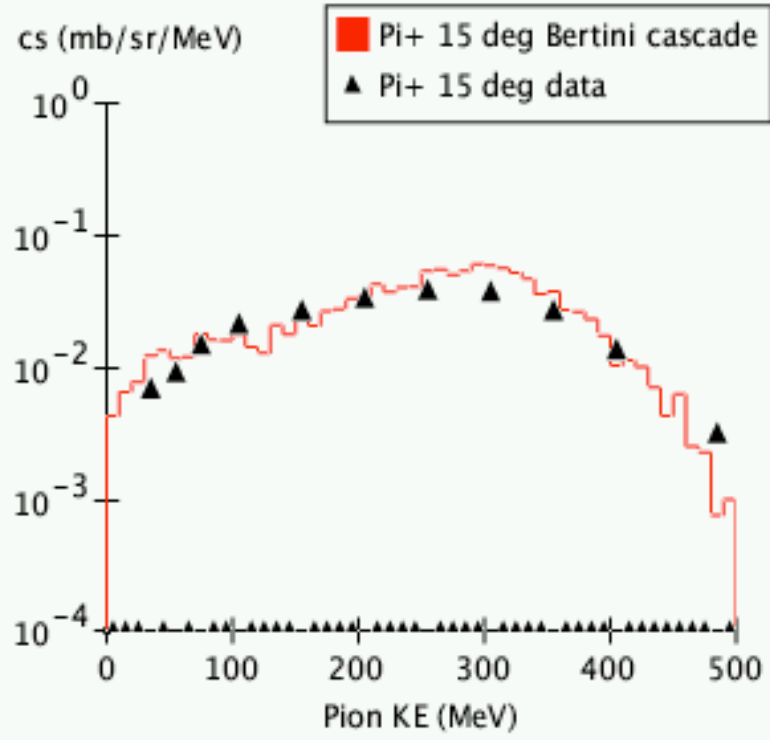

$730 \mathrm{MeV}$ p on C

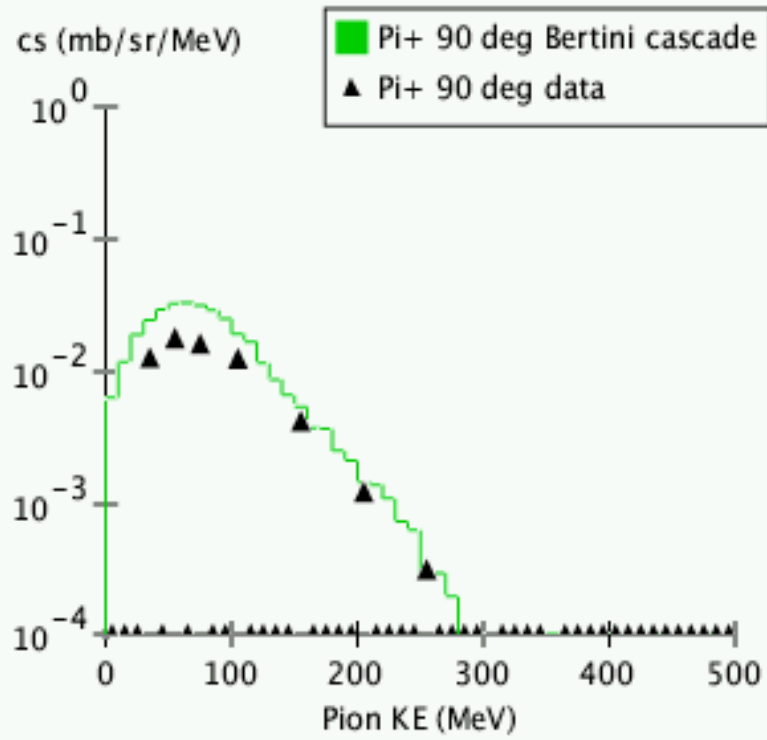

$730 \mathrm{MeV} p$ on $\mathrm{C}$

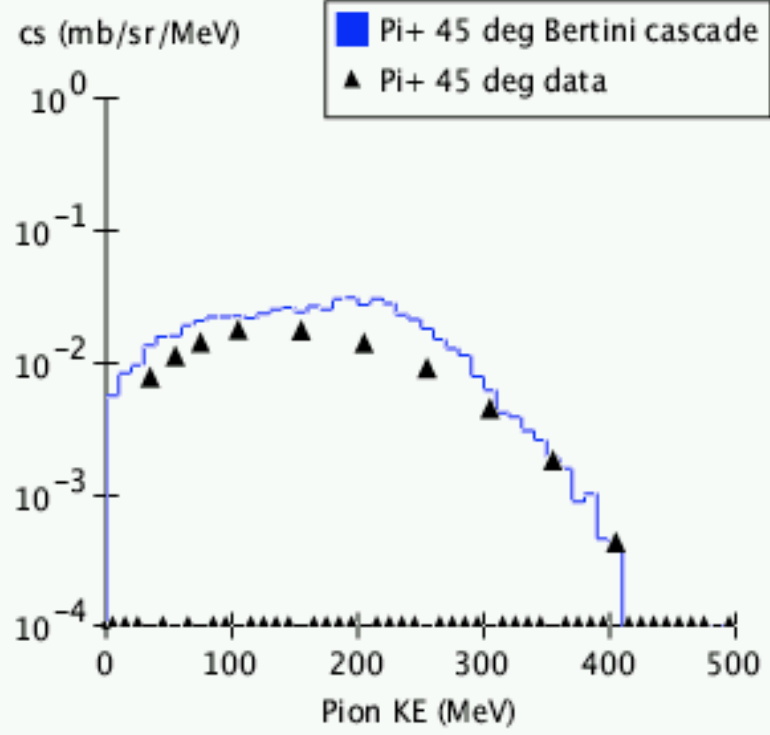

$730 \mathrm{MeV} p$ on C
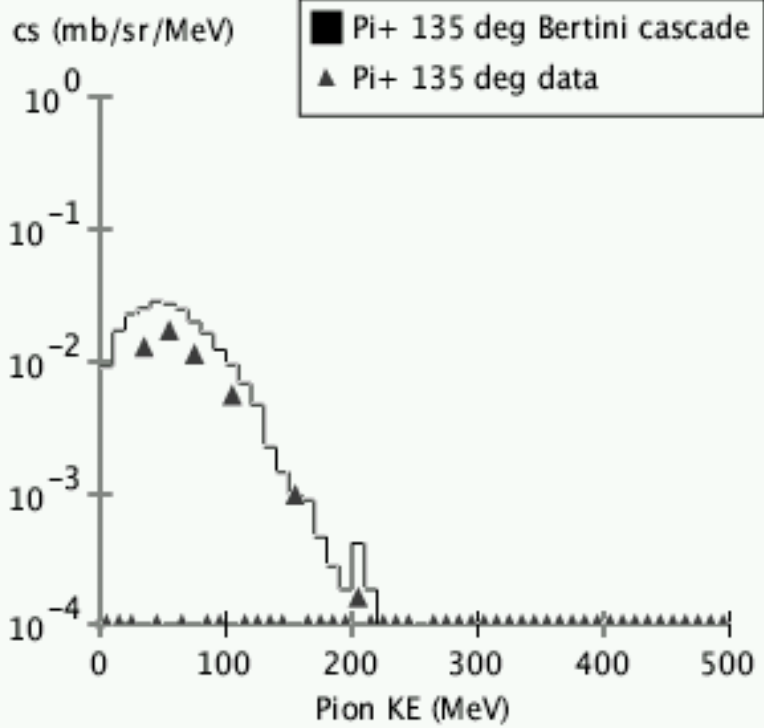
A $100 \mathrm{GeV} / \mathrm{c}$ pi- beam is incident upon an Au target. Rapidity distributions of the final state pi+ are plotted. Data are from: J.J. Whitmore et al., Z. Phys. C62, 199 (1994). Filled points: data, open points: QGS model.

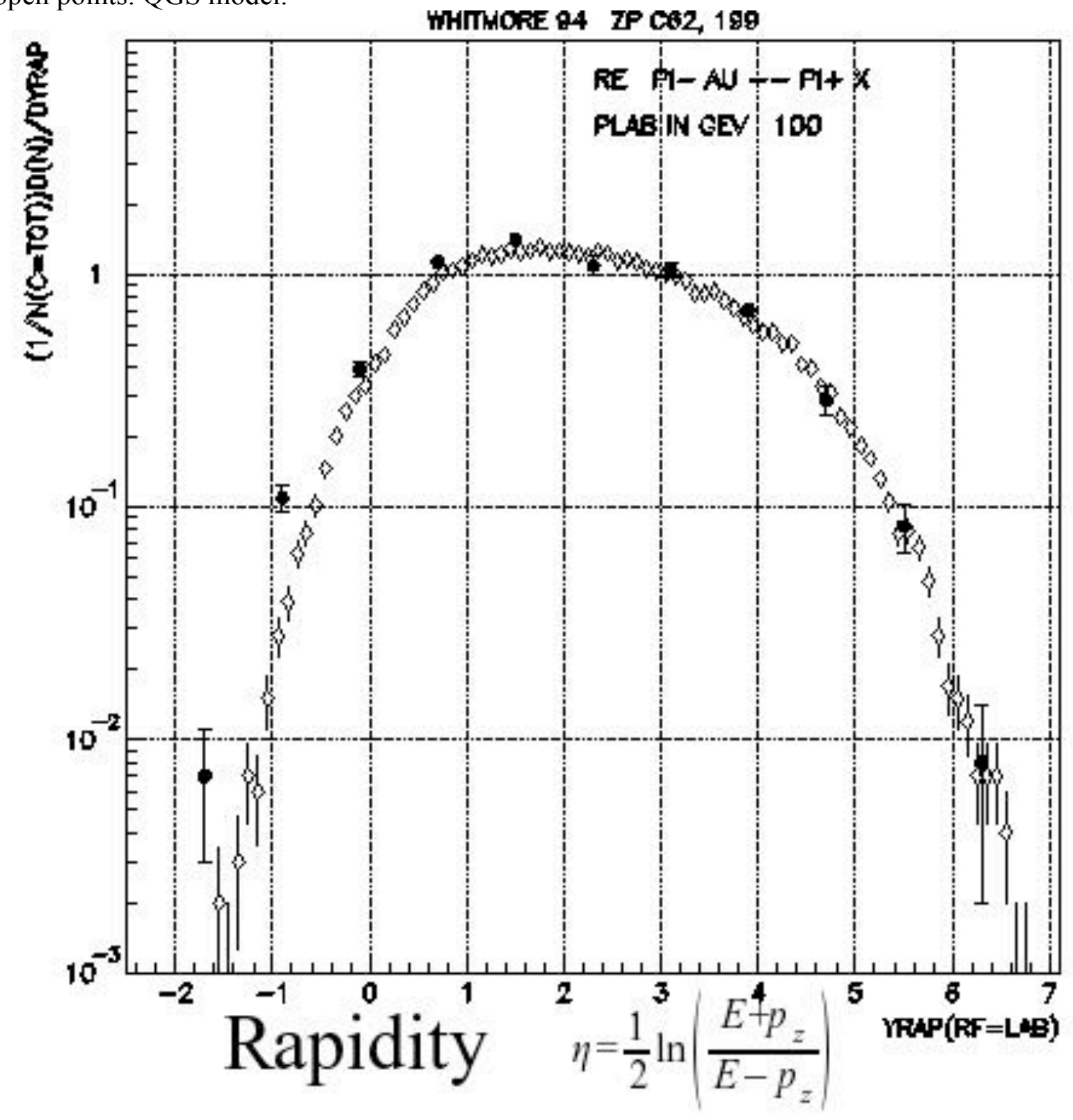

\subsection{Weak Interactions}

The Geant4 Collaboration does not report on validation of weak interaction processes.

\subsection{Optical Photons}

Optical photons are not yet implemented in G4beamline. 


\section{Physics Processes Implemented in G4beamline}

\subsection{Pion Decay (updated from Geant4)}

By default, Geant4 does not include the rare decay modes of charged pions:

$$
\begin{aligned}
& \pi^{+} \rightarrow \mathrm{e}^{+} v_{\mu} \quad(1.230 \mathrm{E}-4) \\
& \pi^{-} \rightarrow \mathrm{e}^{-} \bar{v}_{\mu} \quad(1.230 \mathrm{E}-4)
\end{aligned}
$$

G4beamline adds these modes. The other rare-decay modes have not been implemented, due to the complexity of modeling 3- and 4-body decays; they are a factor of 1000 (or more) below the above. Moreover, unlike these modes, they do not generate additional backgrounds for the Mu2E experiment [8], which is the primary customer for this.

The setdecay command can be used to modify particles' decay modes, including changing their lifetime, adding and removing specific modes, and changing their branching ratios (e.g. for testing background rejection, pions could be forced to always decay into these normally rare modes).

The decay products from $1,000,000 \pi^{+}$decays, with $\mu^{+}$decays inhibited, are:

$$
\begin{array}{rr}
-13 & 999875 \mathrm{mu}+ \\
-11 & 125 \mathrm{e}+ \\
12 & 125 \mathrm{nu} \mathrm{e} \\
14 & 999875 \mathrm{nu} \text { mu }
\end{array}
$$

The decay products from $1,000,000 \pi^{-}$decays, with $\mu^{-}$decays inhibited, are:

$$
\begin{array}{rc}
-14 & 999886 \text { anti_nu_mu } \\
-12 & 114 \text { anti_nu_e } \\
11 & 114 \mathrm{e}- \\
13 & 999886 \mathrm{mu}-
\end{array}
$$


The lifetime of $\pi^{-}$is correct ( $\left.26.033 \mathrm{~ns}\right)$ :

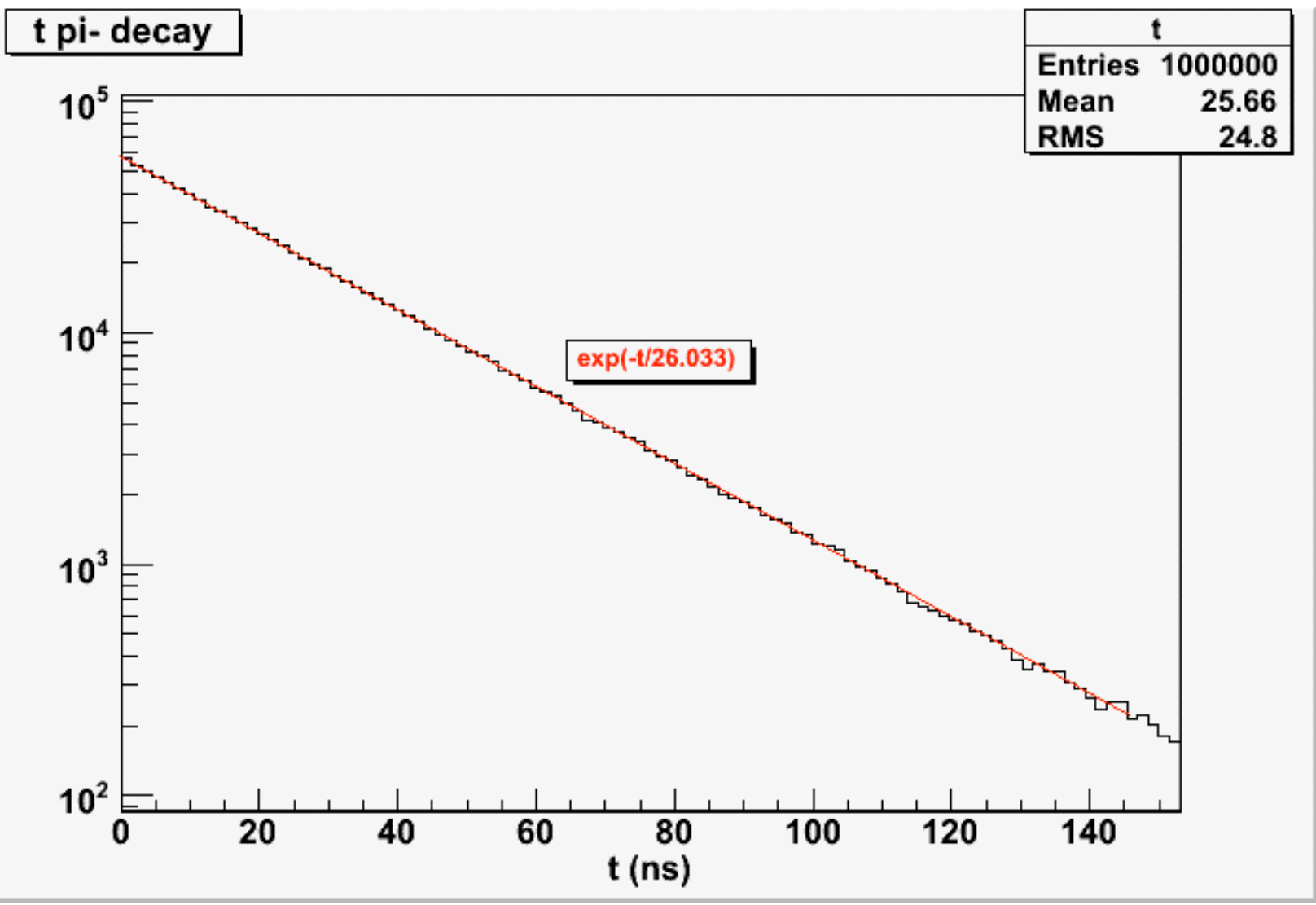

The input file for this test is decay.g4bl:

* decay.g4bl - test pion decay

randomseed time

physics QGSP_BERT

setdecay mu+ lifetime $=1 \mathrm{E} 99$

beam gaussian particle $=$ pi + meanMomentum $=10$ nEvents $=1000000$

newparticlentuple New

box End height=1 width=1 length=1

place End $\mathrm{z}=100000$

\subsection{Mu- Capture (updated from Geant4)}

As mu- capture at rest is very important to the Mu2E experiment [8], considerable testing has been performed. See the report "Analysis of Geant4 Physics Processes for $\mu^{-}$Capture at Rest" [9]. The X-Ray spectrum from neon is reproduced well, as shown in the following figure: 


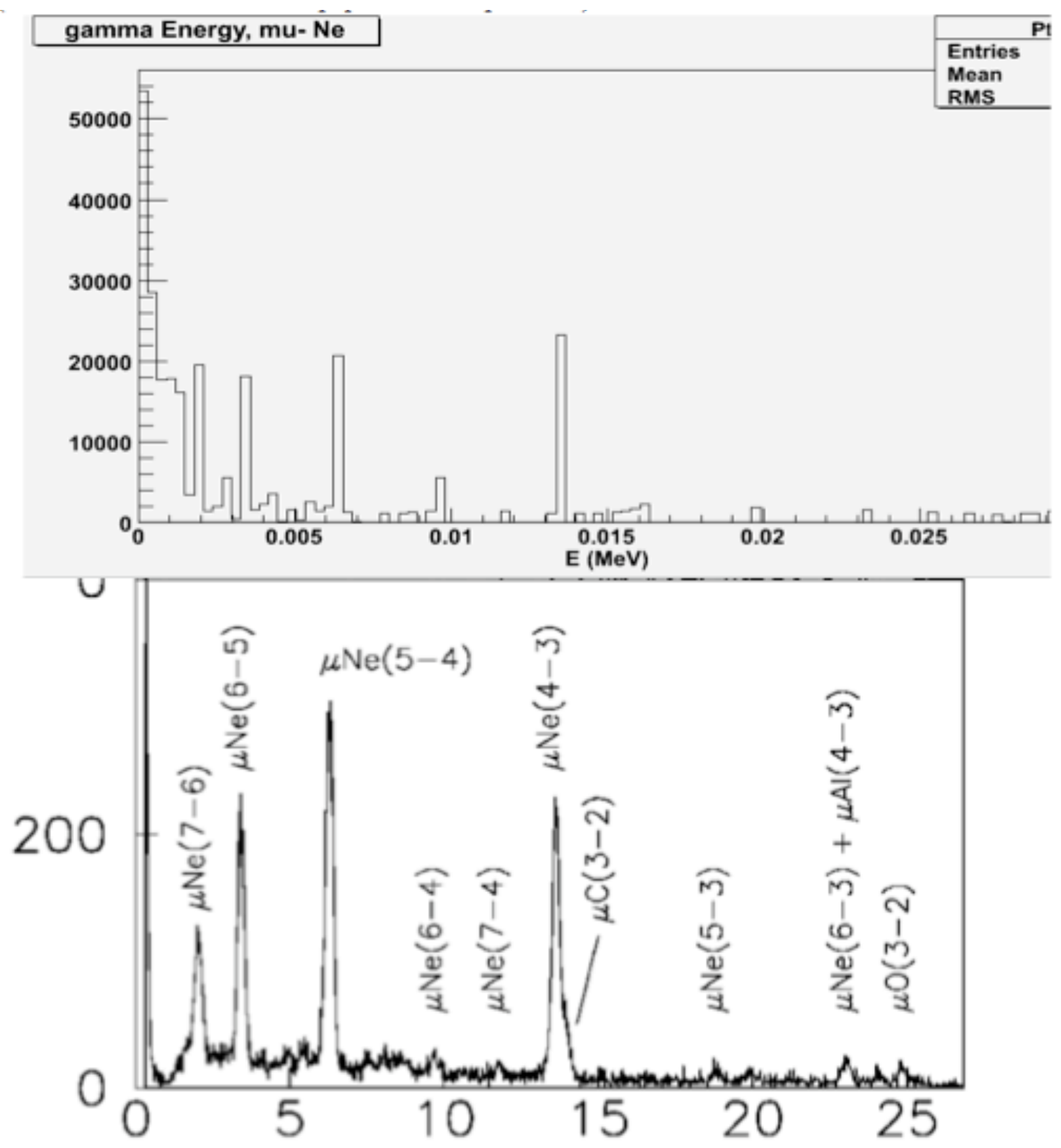

Experimental X-ray Spectrum for $\mu^{-}$Neon, from Kirch et al, Phys. Rev. A59, p3375.

Agreement with the experiment is good, except for an excess below $1 \mathrm{keV}$ where their detector loses efficiency.

The lifetime for decay in orbit is in good agreement with measurements: 


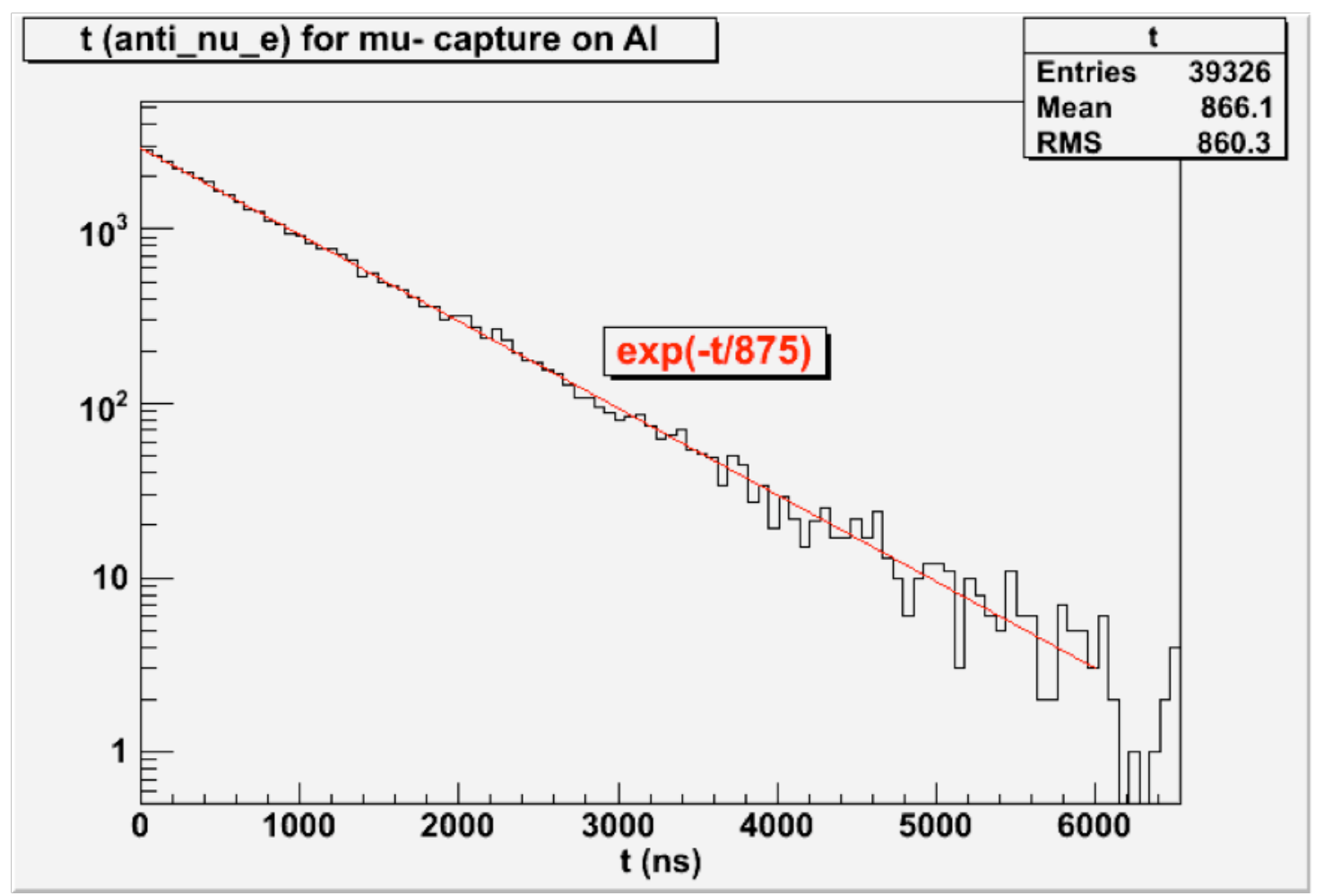

The generation of neutrons from nuclear captures of mu- was found to be incorrect, missing a high-energy tail. A new command muminuscapturefix was implemented to add the missing neutrons with the distribution from MARS [10]. This is a stopgap fix to permit the investigation of neutron backgrounds, and is simply adding isotropic neutrons with an exponential energy distribution fit to the MARS high-energy tail. In the following plot, the lines are for MARS and the MECO simulation; the dots are for G4beamline (available experiments have very poor resolution in this region). 


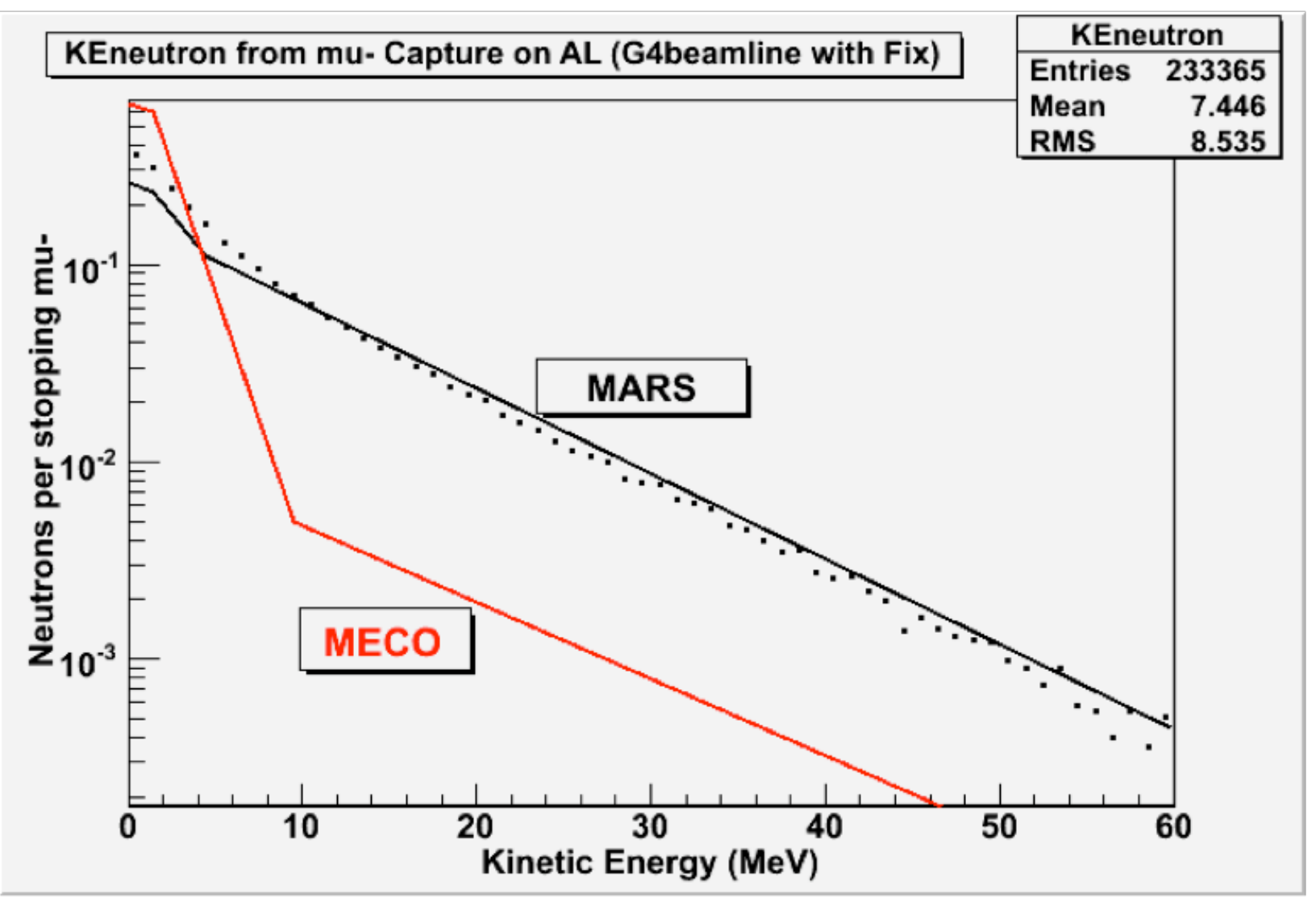

\subsection{Bug 1021 (update to Geant4 tracking in E field)}

Bug 1021 was reported to the Geant4 developers on 20-AUG-2008; at present, it has been assigned but not resolved (it is a rather difficult problem, as it is related to the fundamental design of Geant4 tracking). This bug only affects very low energy charged particles that travel anti-parallel to an E field, stop, and turn around. If the track is just a few degrees from being antiparallel to E, the tracking is OK, but for tracks that do stop, the results can be grotesquely wrong (huge kinetic energies, outrageous time delays). The basic problem is that Geant4 tracks in space, which becomes singular at points where the particle velocity is zero. G4beamline has a workaround for this bug, the bug1021 command, which applies a variable step size as the turnaround is approached, and "jumps" through the turnaround when it is less than 2 microns away. Note that for negative particles in a material with an E field, the appropriate atomic capture-atrest process is not invoked.

\subsection{Collective Computations}

Geant4 is designed and intended for single-particle simulations. But beams inherently have many particles, and in some cases, collective effects are important. G4beamline has an infrastructure that supports collective computations; it is used in the computations of space charge. 
Implementing collective computations requires a completely new RunManager, EventManager, TrackManager, and SteppingManager in the Geant4 tracking code (the original versions are used when collective mode is not invoked, so comparisons can be made easily). The idea is to track all particles essentially in parallel, for steps in time (not space). So every particle is given a new physics process that will suspend it when it reaches the designated step time (it uses the particle's current velocity to limit the step in space). The algorithm used is:

1. Create a vector of the initial (beam) tracks.

2. Find the largest global time in all tracks, and set the step time to it.

3. Loop over the track vector, stepping each active track until it is killed or suspended (at the step time).

4. Call the collectiveComputation() function with the current track vector.

5. Increment the step time by the current value of deltaT.

6. Change all tracks with status $=$ suspended to status $=$ active.

7. Loop back to item 3 until no tracks are active.

The user sets the initial value of deltaT (the time increment between steps); the collectiveComputation() function can modify it, if necessary, depending on the detailed needs of each computation.

This differs from standard Geant4 tracking in the following ways:

- There is a vector of tracks being tracked, not just one track.

- Each track has tracking completely re-started for each time step.

- As far as any Geant4 code is concerned, only a single track is active at any time; as far as the collectiveComputation() is concerned, all tracks are tracked in parallel, with their times equal to the value of the current time step.

- The collectiveComputation() function can be used to perform any sort of computation using the current vector of tracks; each command implements its specific computation, and multiple collective commands can be used in a single simulation. The computation can modify the tracks (e.g. by computing and applying momentum kicks).

- The use of random numbers is quite different from the standard tracking, so comparisons to standard tracking can be statistical only (not on an individual track-by-track basis). All Geant 4 physics processes of interest tolerate this without problems. They of course apply only to each individual track.

Note that when using macroparticles to simulate larger bunches, each macroparticle is tracked as a single particle in the usual way, but their generated fields are multiplied by the macroparticle charge. It is non-trivial to determine what to do when one of the particles decays or interacts; it is easy to greatly increase the number of macroparticles, beyond the capabilities of memory or CPU. At present, the entire macroparticle follows the decay or interaction, which means the statistical accuracy can be poor (many more macroparticles are needed for accuracy).

The following plots compare standard tracking with collective tracking, using a collectiveComputation() that only monitors the tracks. The beam is $100,000 \mu^{+}$with momentum $200 \mathrm{MeV} / \mathrm{c}$ and zero emittance, incident on a $20 \mathrm{~mm}$ slab of iron, followed by a virtualdetector to generate the plots. The collective time step is $0.01 \mathrm{~ns}$. All secondaries and decay products are included in the plots. The standard tracking is plotted as black points with errorbars; the 
collective tracking is plotted in red. The standard run took 103 seconds; the collective run took 181 seconds.
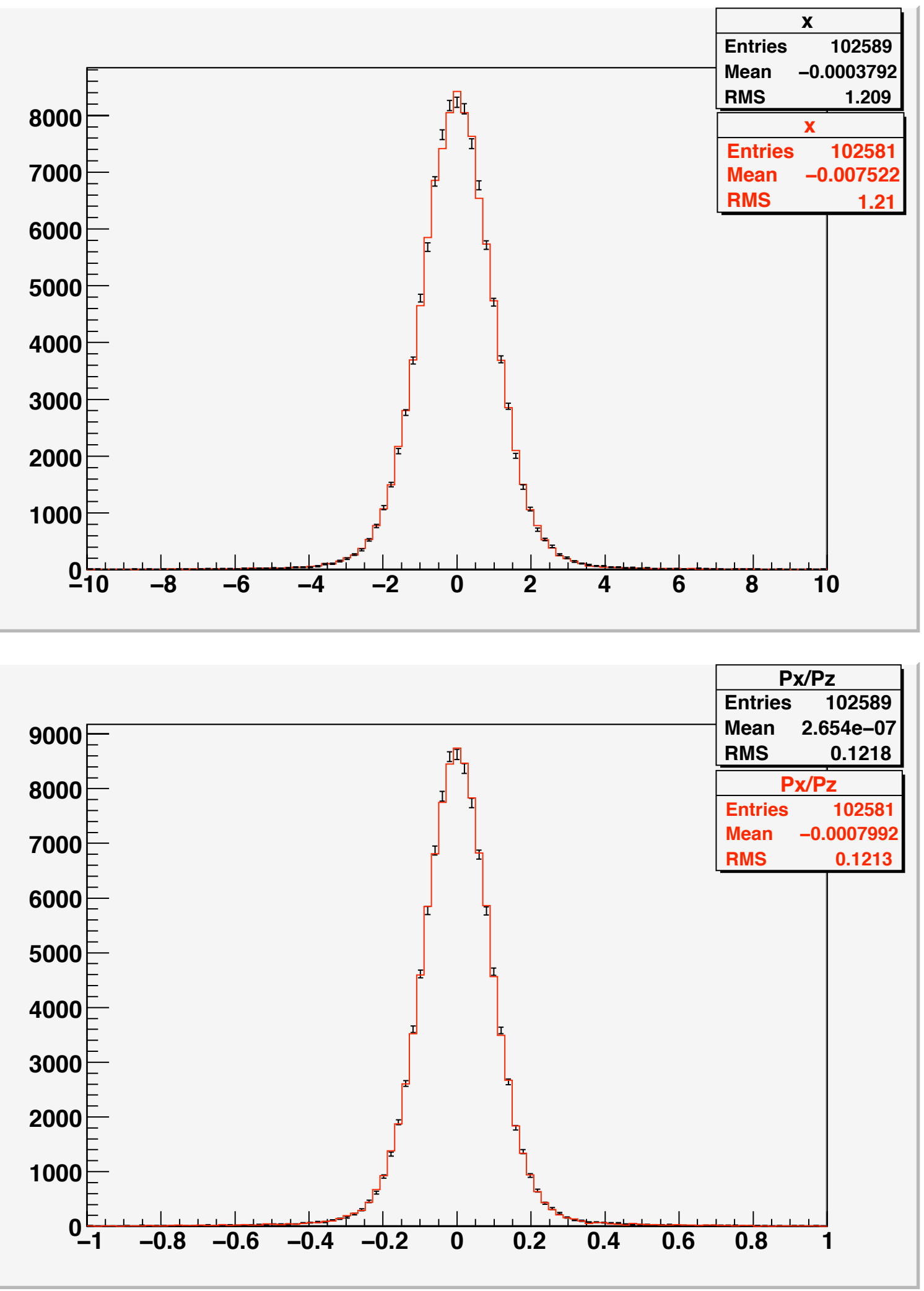


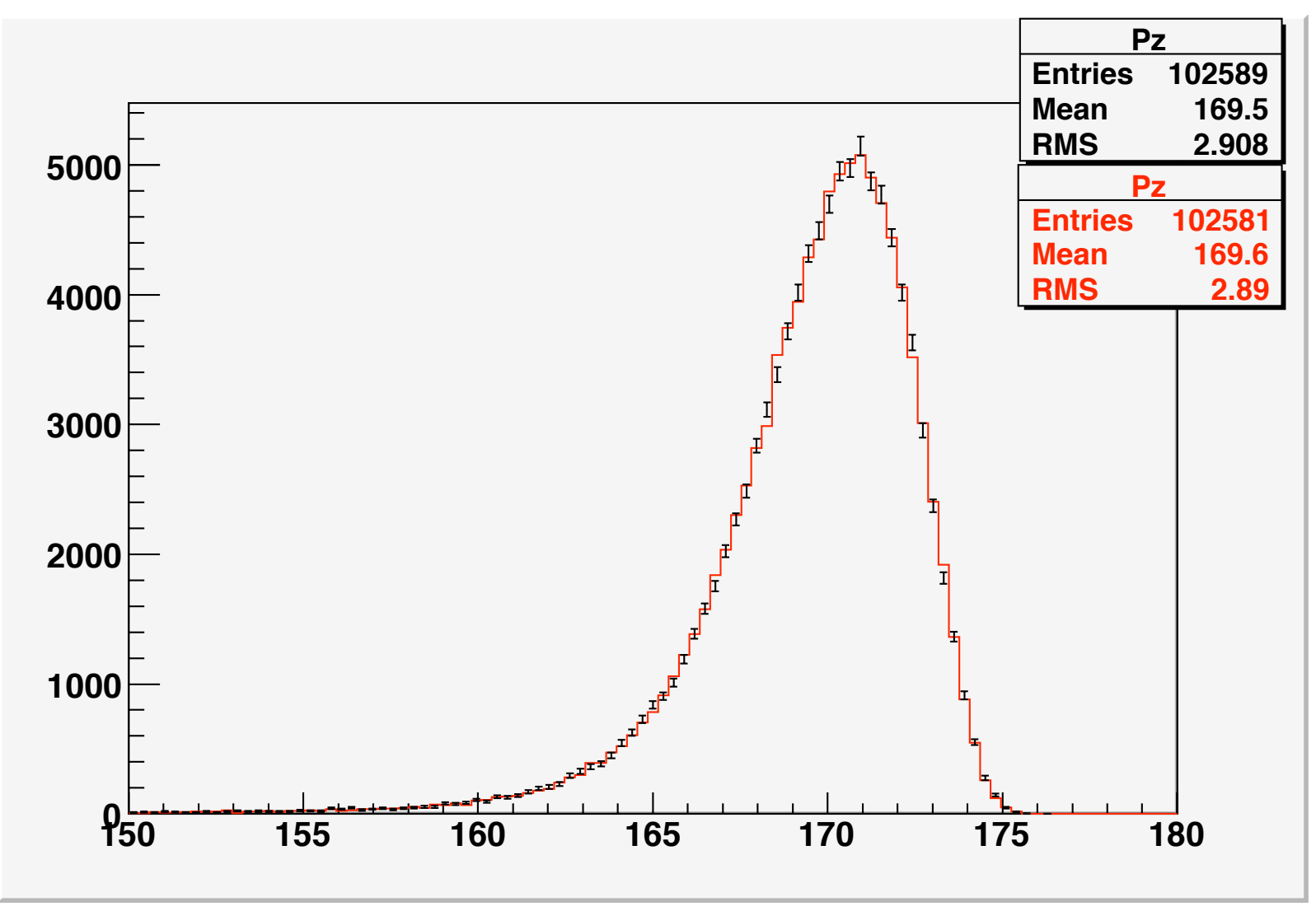

The input file for this test is collective.g4bl:

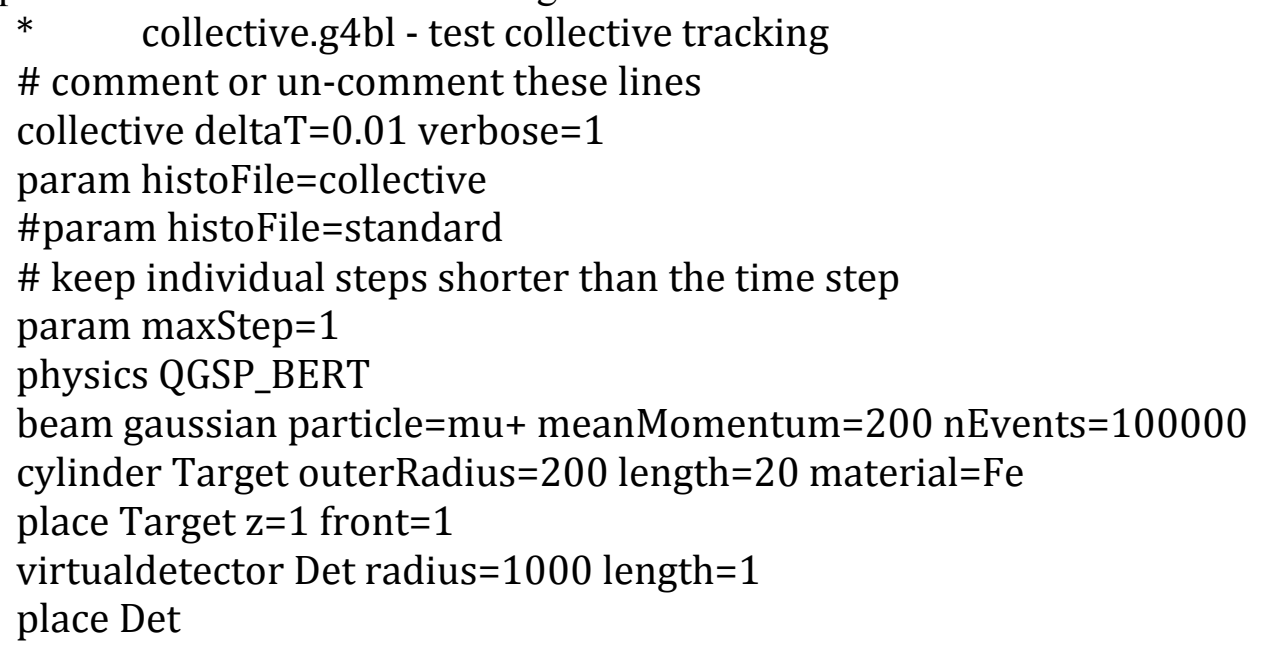

\subsection{Space Charge}

Three different implementations of the space-charge computation have been written and tested:

spacechargelw A computation based on Lienard-Wiechert potentials. This computation is CPU intensive, and in practice is limited to a few hundred macroparticles, 
which is too few for any realistic situation. But for the macroparticles used, it is accurate and correct. Its primary purpose is for testing the other computations.

spacechargeps A computation using a solver for Poisson's equation on a 3-D grid in the beam frame. This computation requires a good approximation to the potential on the grid boundary, which can be difficult in practice. It relies on a Poisson solver written in Fortran, which limits its portability. As it has no benefits over the spacecharge command, and is much more finicky to use, it is not included in any G4beamline release, and is not discussed further.

spacecharge A computation using the Green's function on a 3-D grid in the beam frame, based on convolution via FFTs. This is an excellent, efficient, and stable computation.

For the expansion of a Gaussian bunch of $10^{12} \mu^{+}$expanding in free space, all three computations give the same result (to the width of the lines in the plots), but their CPU time requirements are very different, and they scale much differently with (\# macroparticles), as shown here:
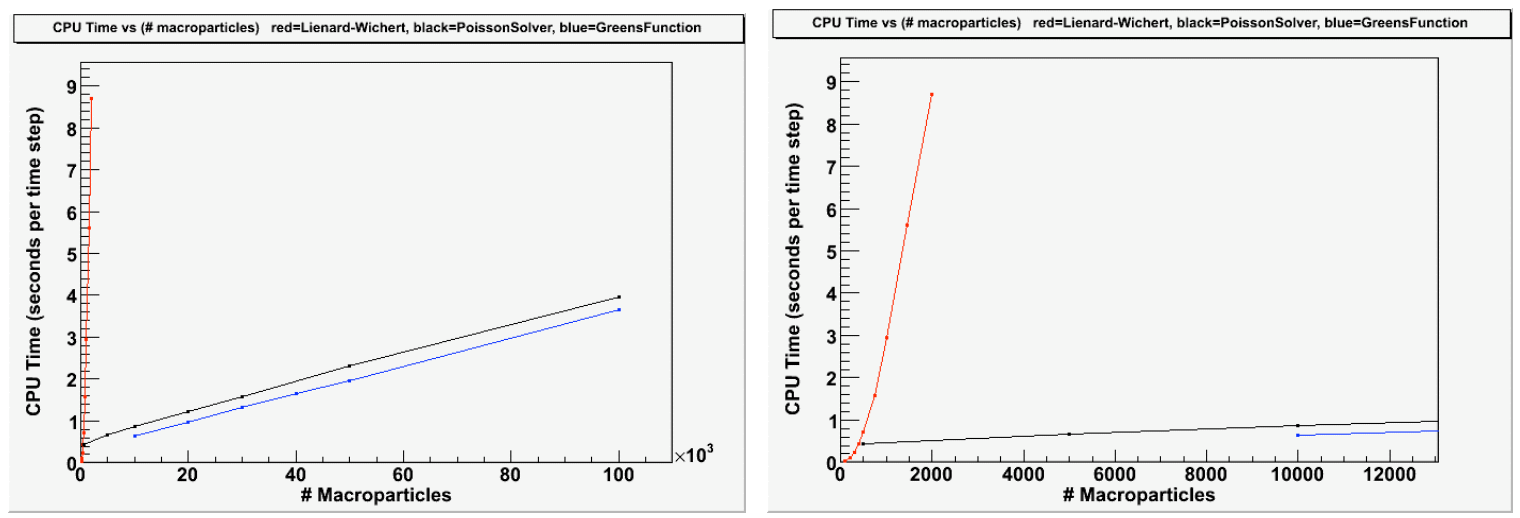

(Same plot, expanded horizontal scale.)

All three computations can handle multiple bunches of arbitrary particles, but spacechargeps and spacecharge require a reference particle for each bunch.

\subsection{1 spacechargelw - Lienard-Wiechert computation}

This computation uses the Lienard-Wichert formulas for E and B fields in the lab. These fields are simply included in the usual G4GlobalField (G4beamline uses a registration technique so many different elements and computations can generate fields, and overlaps are handled correctly and efficiently). Macroparticles are used to simulate much larger bunches than are feasible to track. Note that implementing this required using the collective computation discussed above.

The approximations involved are:

- Macroparticles are used to simulate large bunches.

- Particle trajectories are linearly interpolated between steps, and are occasionally extrapolated linearly for up to one time step from their last step. 
- The radiation term is omitted.

This algorithm scales as (\# macroparticles) $)^{2}$, and is not computationally feasible for more than $\sim 1,000$ macroparticles. It was decided early on that the availability of a rigorously correct computation would permit the testing of other, more efficient approximations and algorithms (it is correct for the macroparticles used; whether that corresponds accurately to a real beam bunch is a different question, usually answered in the negative).

\section{Test 1 - E and B fields from known sources}

A 1 Coulomb charge has an electric field of 8.987551787E9 Volt/meter at a distance of 1 meter. This is tested by generating a single macroparticle with a charge of 1 Coulomb, at rest ${ }^{1}$ at $(\mathrm{x}, \mathrm{y}, \mathrm{z})=(0,0,0)$. At $(\mathrm{x}, \mathrm{y}, \mathrm{z})=(1000,0,0), \mathrm{E}_{\mathrm{x}}=8988 \mathrm{MV} /$ meter; at $(\mathrm{x}, \mathrm{y}, \mathrm{z})=(0,2000,0), \mathrm{Ey}=2247$ $\mathrm{MV} /$ meter; at $(\mathrm{x}, \mathrm{y}, \mathrm{z})=(0,0,-1000), \mathrm{E}_{\mathrm{z}}=-8988 \mathrm{MV} /$ meter. These are the correct values and directions.

A uniform current of $1,000 \mathrm{Amps}$, at radius $0.1 \mathrm{~m}$, has $\mathrm{B}=0.002 \mathrm{~T}$. This is tested by generating 8,001 macroparticles $^{2}$ with charge +0.001 Coulomb, spaced uniformly $1 \mathrm{~cm}$ apart, moving at 10,000 meter/sec in the $+\mathrm{z}$ direction, centered at $\mathrm{z}=0$. At $(\mathrm{x}, \mathrm{y}, \mathrm{z})=(100,0,0), \mathrm{B}_{\mathrm{x}}=0.00199999$ Tesla; at $(\mathrm{x}, \mathrm{y}, \mathrm{z})=(0,200,0), \mathrm{B}_{\mathrm{x}}=-0.000999988$ Tesla. These are the correct values and directions to 5 significant digits.

There is no input file for these tests; they are executed automatically by the code whenever the spacechargelw command is used. There is an assert() to ensure they are correct.

\section{Test 2 - Comparison to a textbook space-charge computation}

The results are compared to a computation in the textbook by M. Reiser, Theory and Design of Charged Particle Beams, section 4.2.1, p200. This is a cylindrical bunch with uniform charge density; the comparison is at the longitudinal center of the bunch, where end effects are seen to be negligible.

The computation scales in many ways, and tests of proper scaling behavior were made for variations in:

- Macroparticle radius

- Macroparticle charge density exponent K

- Time step deltaT

- Total charge in the bunch

- Pz (longitudinal 3-momentum)

- R0 (initial radius of the bunch)

- Particle mass

- Sign of particle charge

The scaling for number of macroparticles (total charge held constant) is violated in minor ways, as described in the next paragraph.

\footnotetext{
${ }^{1}$ This macroparticle is exactly at rest; its trajectory was generated manually, without any tracking.

${ }^{2}$ These macroparticle trajectories were also generated manually, without any tracking.
} 
In general, the algorithm gives more expansion than Reiser's computation. This appears to be due to the finite number of macroparticles - using more macroparticles with less charge in each gives results closer to the computation. The plot below keeps the total charge of the bunch constant, but varies the number of macroparticles from 100 to 10,000. The vertical axis is the scaled radius of the bunch and the horizontal axis is the scaled position along $\mathrm{z}$ of the bunch center; both axes are scaled by factors involving Pz, particle mass, initial radius, and total charge -- see the textbook for details. The three red lines correspond to three initial conditions:

$$
\begin{array}{ll}
\mathrm{dR} / \mathrm{dZ}=0 & \text { momenta parallel to the } Z \text { axis } \\
\mathrm{dR} / \mathrm{dZ}=-1 & \text { focused to a point at } Z=1 \\
\mathrm{dR} / \mathrm{dZ}=-2 & \text { focused to a point at } Z=0.5
\end{array}
$$

The first case is pretty good for just 100 macroparticles. The second case is not too bad for 10,000 macroparticles. The third case clearly needs significantly more than 10,000 macro particles for good accuracy. Unfortunately, this algorithm scales as (\# macroparticles) ${ }^{2}$, and it becomes computationally infeasible to use more macroparticles ${ }^{1}$.

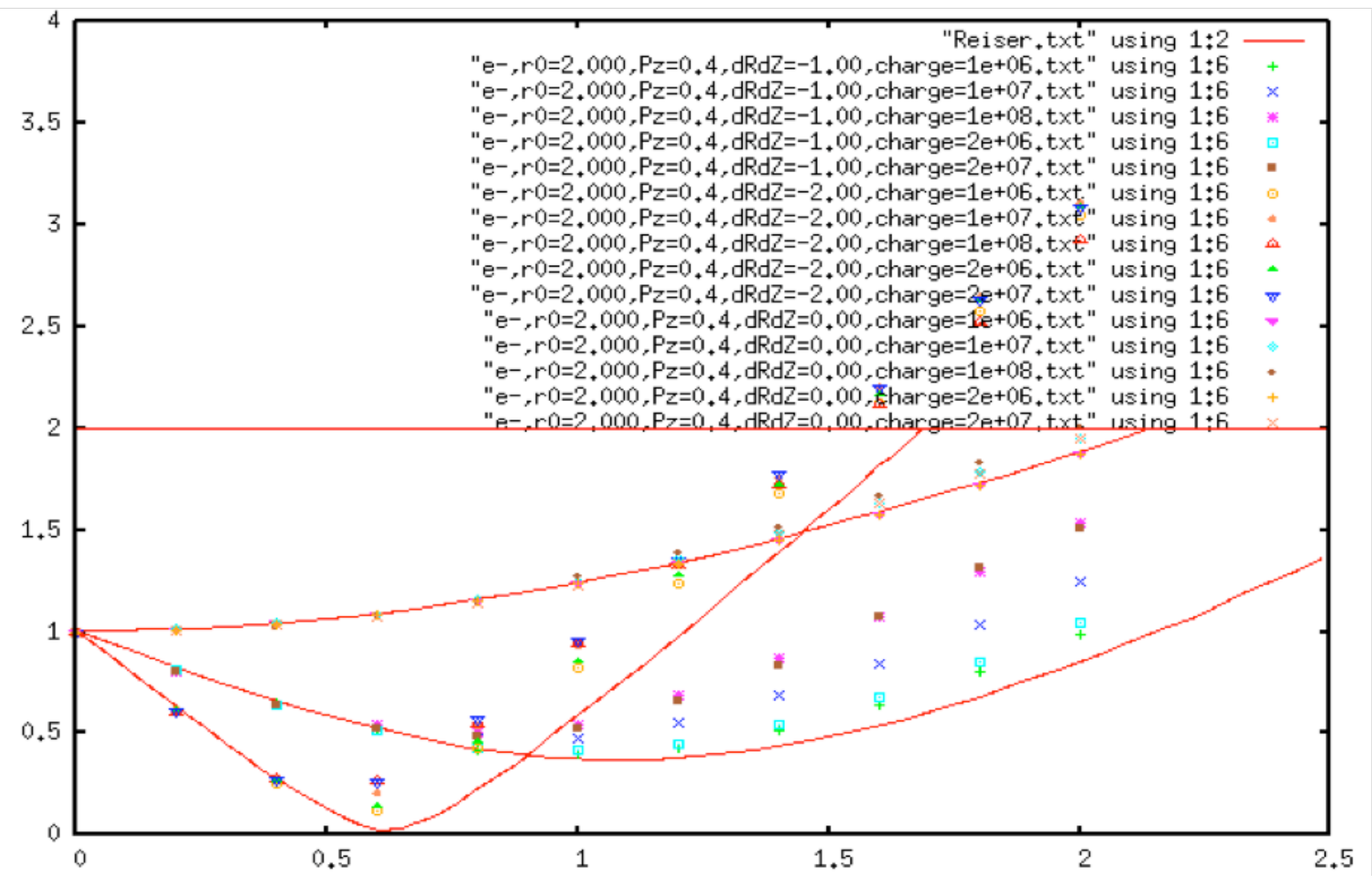

\subsection{2 spacecharge - Beam-frame Green's Function Convolution via FFTs}

This computation inherently requires the beam to be bunched. Macroparticles are used to simulate much larger bunches than are feasible to track. It requires that all particles of a bunch be near to its reference particle, in both position and 3-momentum. The particles of the bunch are boosted to the rest frame of the reference, and a 3-d rectangular grid is defined that contains the

\footnotetext{
${ }^{1}$ The run with 10,000 macroparticles took 14 hours of CPU time, for a mere $400 \mathrm{~mm}$ of travel.
} 
particles. The particles are placed into this grid, and Poisson's equation is solved on the grid for the electrostatic potential in the beam frame. This solution is obtained by convolving the Green's function (infinite boundary conditions) with the source distribution; FFTs are used for efficiency. This is differentiated to obtain the E field, which is then boosted back to the lab to determine the $\mathrm{E}$ and $\mathrm{B}$ fields for tracking. This is performed at each time step to handle the evolution of the particles within the bunch. The grid moves with the reference particle between time steps, and is dynamically sized to keep the $99^{\text {th }}$ percentile of the charge distribution between 0.5 and 0.67 of the grid size (separately in $\mathrm{x}, \mathrm{y}$, and $\mathrm{z}$ ).

FFTW 3.2.2 [11] is used to implement the fast Fourier transforms. The beam-frame grid is doubled in all three dimensions so the cyclical convolution via FFTs yields the correct potential for infinite boundary conditions [12]. As the Green's function includes the boundary conditions, the difficulties of spacechargeps are avoided.

\section{Test 1 - Comparison to known sources}

A 1 Coulomb charge has an electric field of 8.987551787E9 Volt/meter at a distance of 1 meter. This is tested by generating a single macroparticle with a charge of 1 Coulomb, at rest at $(\mathrm{x}, \mathrm{y}, \mathrm{z})=(0,0,0)$. At $(\mathrm{x}, \mathrm{y}, \mathrm{z})=(1000,0,0), \mathrm{E}_{\mathrm{x}}=8987.54 \mathrm{MV} /$ meter.

A uniform current of $1,000 \mathrm{Amps}$, at radius $0.1 \mathrm{~m}$, has $\mathrm{B}=0.002 \mathrm{~T}$. This is tested by generating 501 macroparticles with charge +0.001 Coulomb, spaced uniformly $1 \mathrm{~cm}$ apart, moving at 10,000 meter/sec in the $+\mathrm{z}$ direction, centered at $\mathrm{z}=0$. At $(\mathrm{x}, \mathrm{y}, \mathrm{z})=(100,0,0), \mathrm{B}_{\mathrm{x}}=0.001971$ Tesla.

There is no input file for these tests; they are executed automatically by the code whenever the spacecharge command is used. There is an assert() to ensure they are correct.

\section{Test 2 - Comparison to the Lienard-Wiechert computation}

The ability to compare two different calculations is a powerful capability. This code and its algorithm are so completely different from those in spacechargelw that the agreement shown here gives confidence in the correctness of both. Note, however, that it shares considerable code in common with spacechargeps (everything but the actual solving of Poisson's equation on the grid).

Here are plots of the $\mathrm{E}$ field at a fixed point in space as a function of time, during the transit of a bunch consisting of two Gaussians. The total bunch is $10^{12} \mu^{+}$with $\mathrm{Pz}=200 \mathrm{MeV} / \mathrm{c}$, consisting of two Gaussian distributions in space with $\sigma_{\mathrm{x}}=\sigma_{\mathrm{y}}=2 \mathrm{~mm}, \sigma_{\mathrm{z}}=0.2 \mathrm{~mm}$, separated by $4 \mathrm{~mm}$ along $\mathrm{z}$. The spacechargelw computation is a red line, and the spacecharge computation is black dots; they use exactly the same set of 2,000 macroparticles. It is clear that some spatial resolution is lost, due to the spatial averaging inherent in a grid, but that the computation is correct within the approximation that defines its limitations. $x=2$ is well inside the bunch and the grid; $x=9$ is outside the bunch but inside the grid. 
Ex vs. $t, x=9$, TwoGaussians

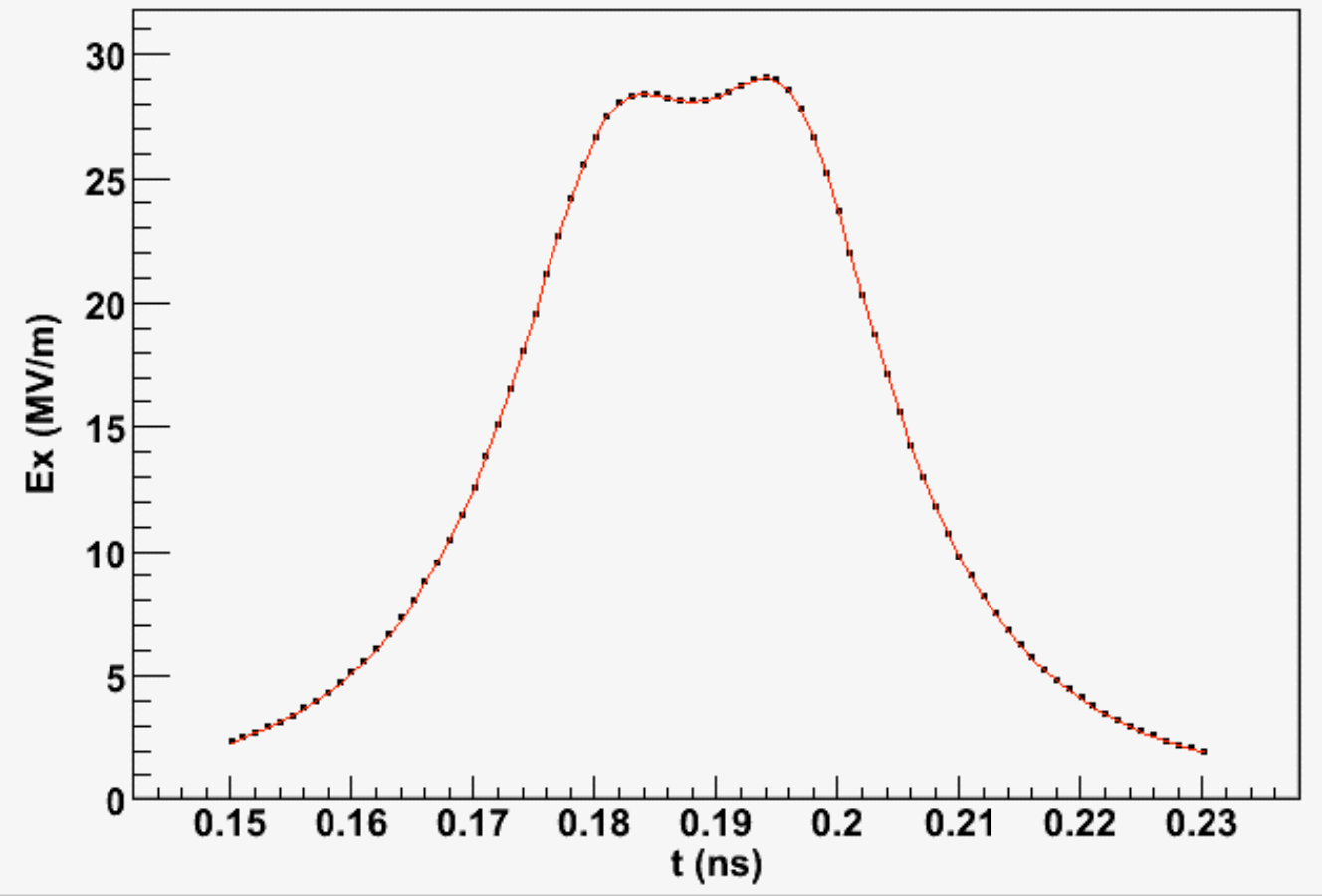

\section{Ex vs. $t, x=2$, TwoGaussians}

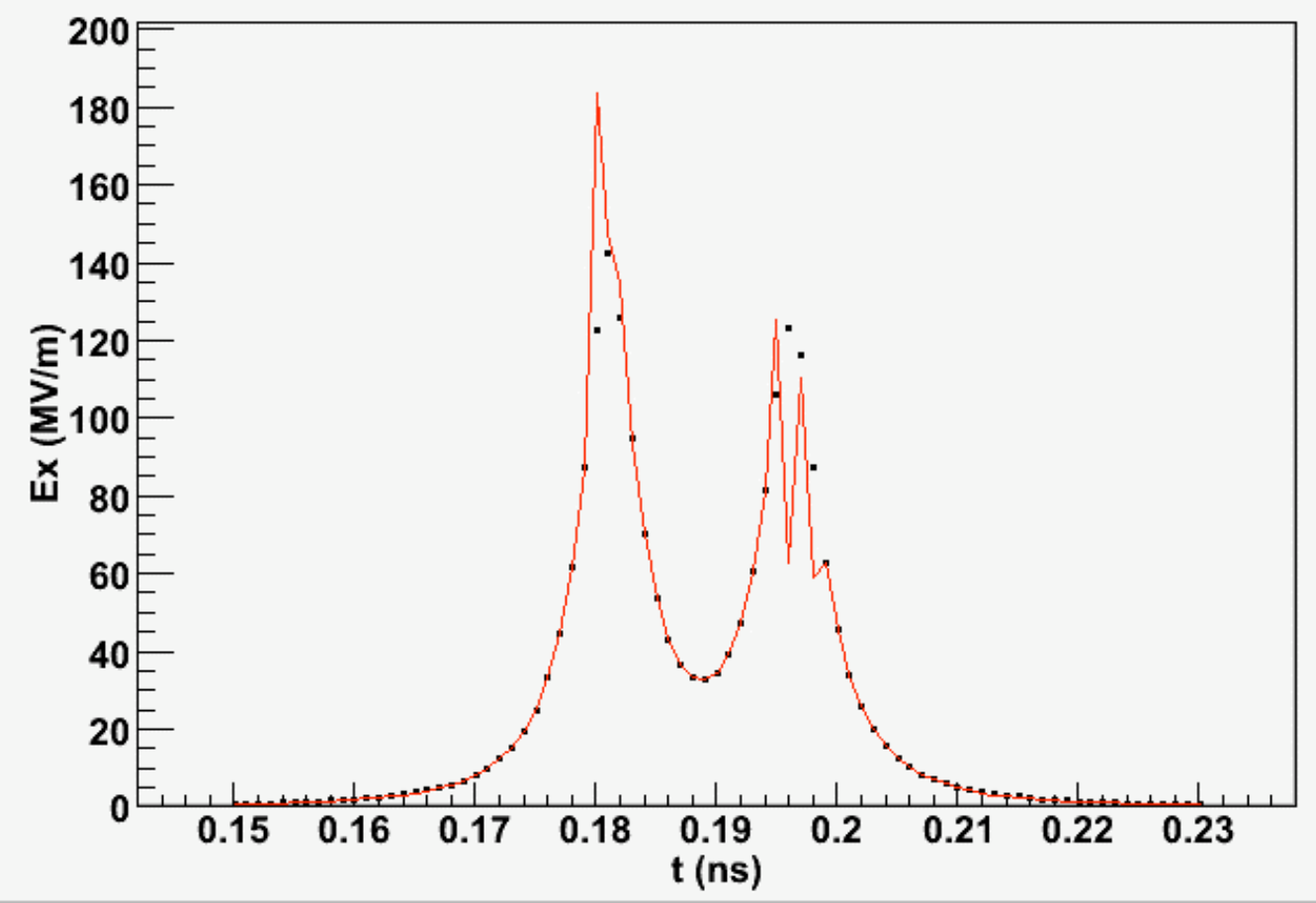




\section{Ez vs. $t, x=2$, TwoGaussians}

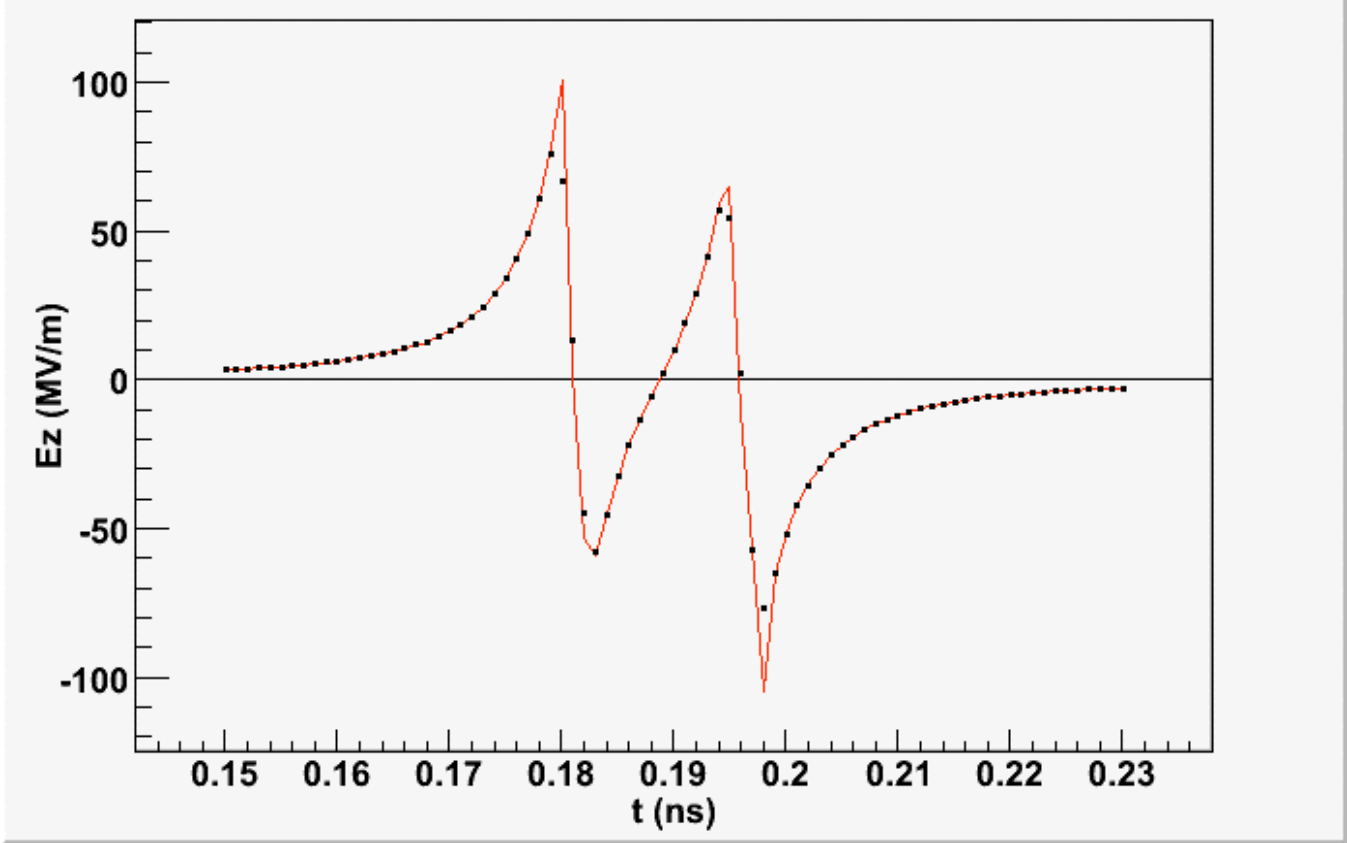

Here is a similar plot, showing $B_{y}$ vs. $t$ at $x=9$, but for a beam momentum of $2,000 \mathrm{MeV} / \mathrm{c}$. The higher momentum increases the value of $\mathrm{B}$, and reduces the time it takes to reach the point at which the fields are sampled; because the particles are now much more relativistic $(\gamma=19)$, the widths of the Gaussians are reduced so they are well resolved (compared to $E_{x}$ at $x=9$ above). Given the G4beamline coordinates with $\mathrm{x}=$ beam left, $\mathrm{y}=\mathrm{up}, \mathrm{z}=$ beam, and positive particles, the sign is correct.

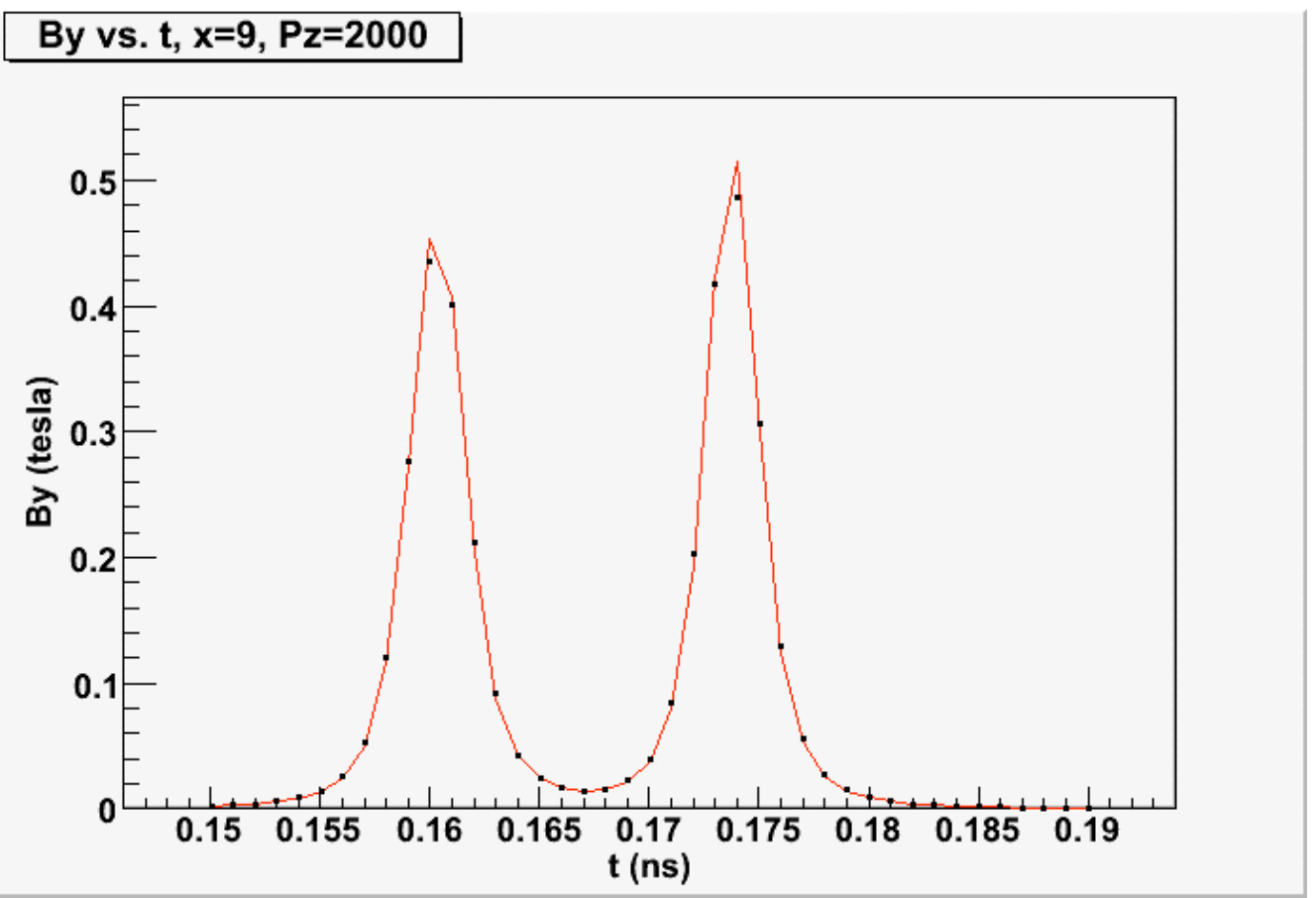


Another comparison is to plot the expansion of a Gaussian bunch with initial parameters $\sigma_{\mathrm{x}}=\sigma_{\mathrm{y}}=\sigma_{\mathrm{z}}=50 \mathrm{~mm}, \sigma_{\mathrm{x}}=\sigma_{\mathrm{y}}=\sigma_{\mathrm{Pz}}=\sigma_{\mathrm{t}}=0$; the bunch consists of $10^{12} \mu^{+}$, each particle has a kinetic energy of $100 \mathrm{keV}$, propagating along $\mathrm{Z}$ in free space. The plot is sigmaX; sigmaY and sigmaZ behave identically. The plot contains 2,000 macroparticles. The KE is so low that this corresponds accurately to a bunch at rest in the lab.

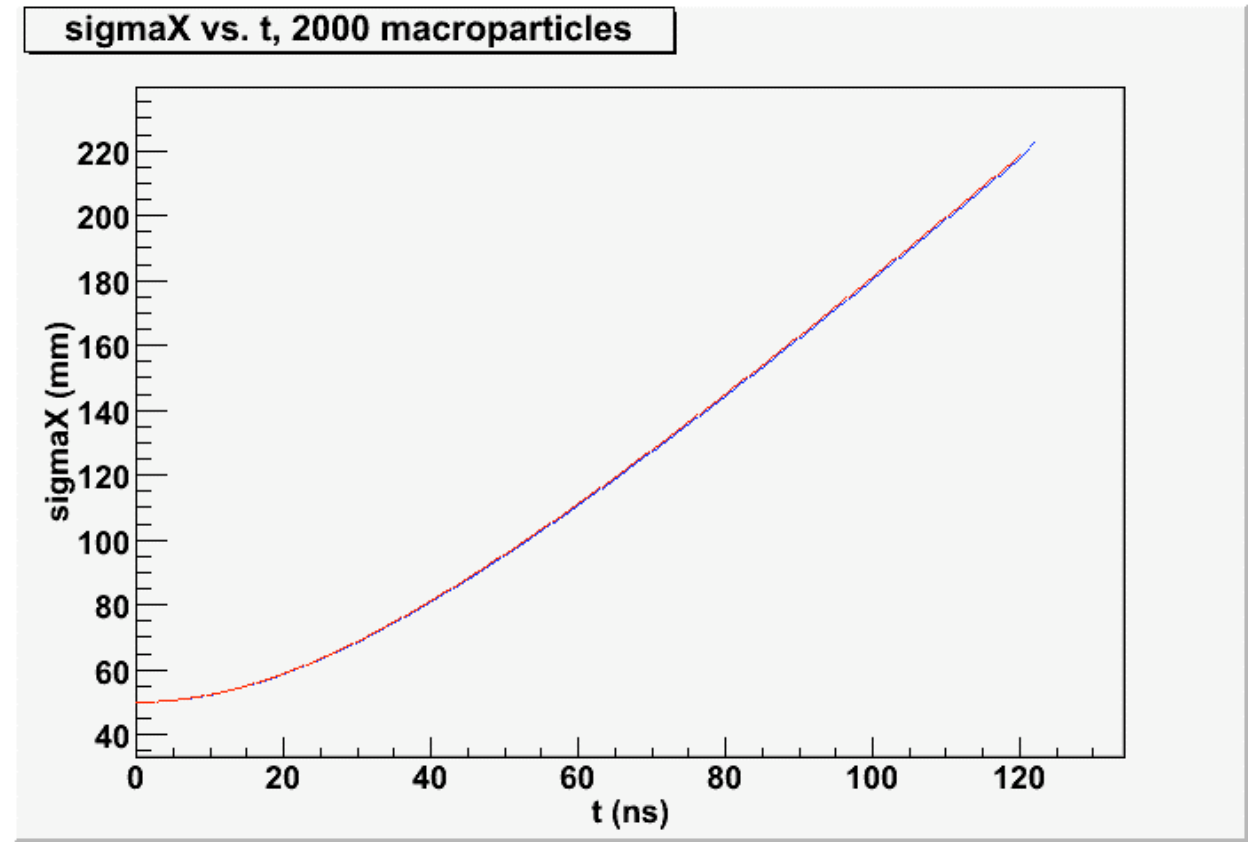

\section{Test 3 - Comparison to a textbook space-charge computation}

The results are compared to a computation in the textbook by M. Reiser, Theory and Design of Charged Particle Beams, section 4.2.1, p200. This is a cylindrical bunch with uniform charge density; the comparison is at the longitudinal center of the bunch, where end effects are seen to be negligible.

The computation scales in many ways, and tests of proper scaling behavior were made for variations in:

- Time step deltaT

- Total charge in the bunch

- Pz (longitudinal 3-momentum)

- R0 (initial radius of the bunch)

- Particle mass

- Sign of particle charge

The plot below keeps the total charge of the bunch constant, but varies the number of macroparticles from 2,000 to 100,000 . The grid is $65 \times 65 \times 513$; its physical size is kept between $3 / 2$ and 2 times the $99^{\text {th }}$ percentile of the bunch dimensions. The vertical axis is the scaled radius of the bunch and the horizontal axis is the scaled position along $\mathrm{z}$ of the bunch center; both axes are scaled by factors involving $\mathrm{Pz}$, particle mass, initial radius, and total charge - see the textbook for details. The three red lines correspond to three initial conditions: 

$\mathrm{dR} / \mathrm{dZ}=0$
momenta parallel to the $\mathrm{Z}$ axis
$\mathrm{dR} / \mathrm{dZ}=-1$
focused to a point at $Z=1$
$\mathrm{dR} / \mathrm{dZ}=-2$
focused to a point at $Z=0.5$

This plot is the best of all, and shows the advantage of being able to efficiently track 100,000 macroparticles. It also shows that using a mere 500 macroparticles gives major inaccuracies.

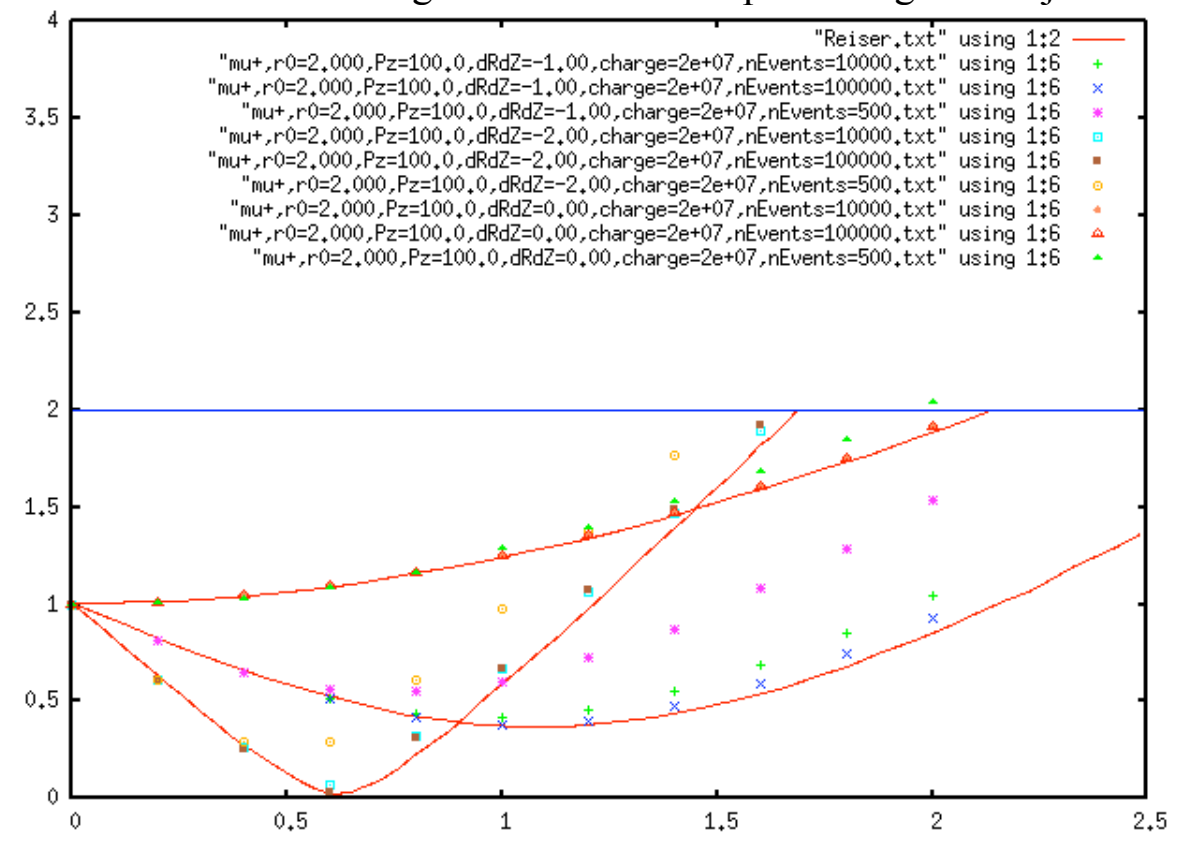

Reducing the grid to $33 \times 33 \times 257$ (factor of 8 fewer grid points) does not affect the agreement very much. This plot has 100,000 macroparticles.

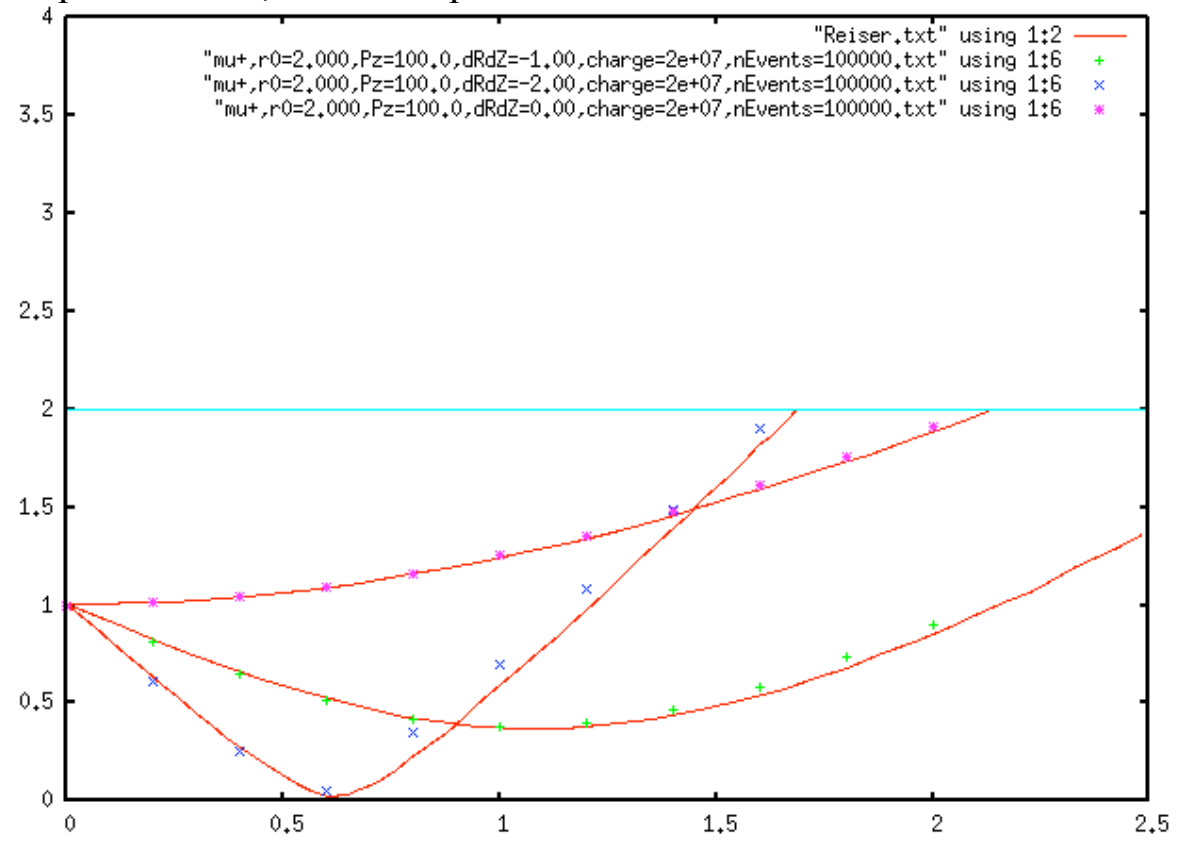




\subsection{Neutrino Interactions}

Neutrino interactions are not yet implemented in G4beamline. It is expected they will be implemented using an external program to model the generation of secondary particles, which will then be tracked as usual by G4beamline. The interaction cross-section will be greatly enhanced. 


\section{Beamline Elements Implemented in G4beamline}

\section{1 coil and solenoid}

The field lines look reasonable (these are in 3-D, and apparent intersections are not real; to really appreciate this you must run it and manipulate the image in real time to look at it from different directions.

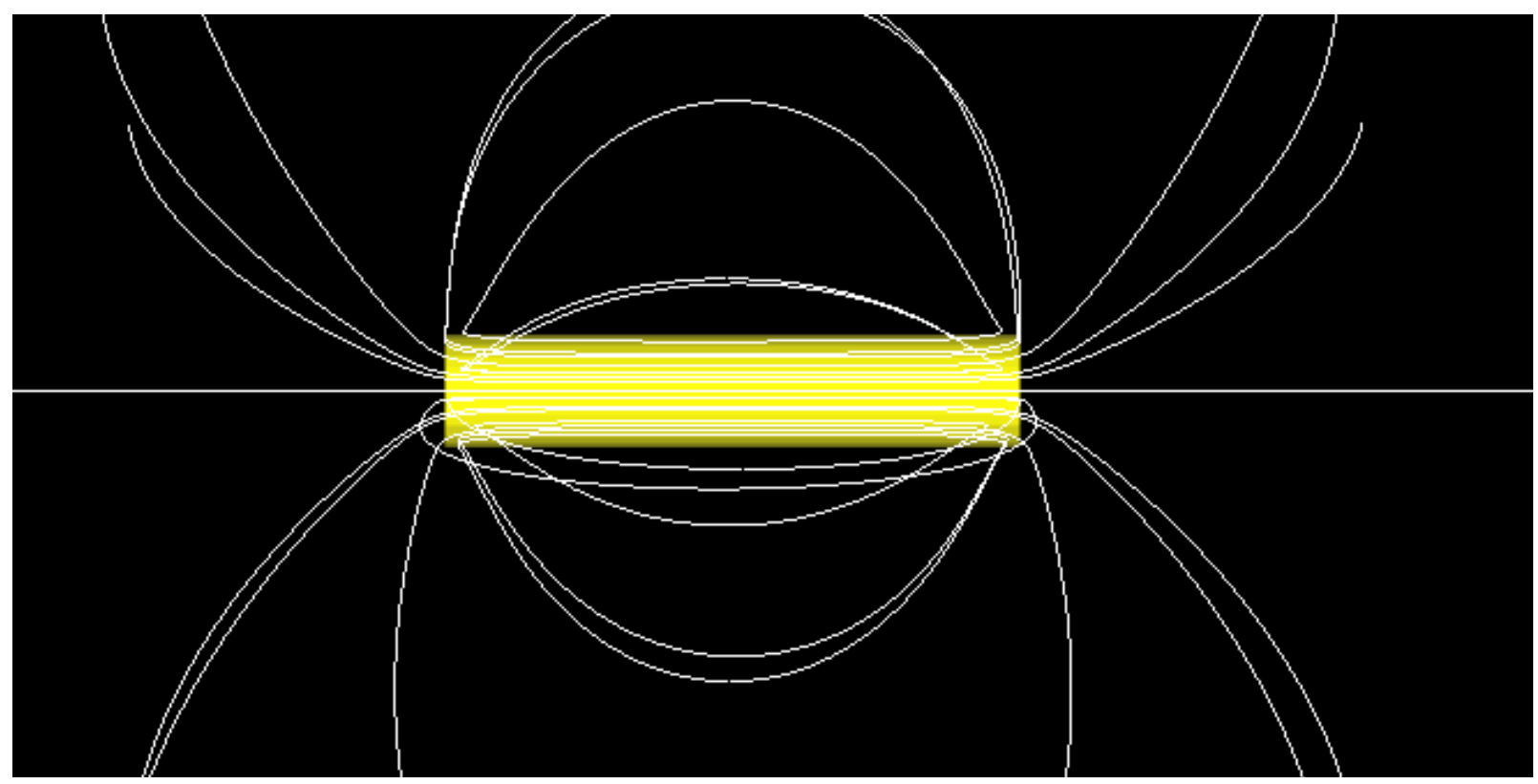

The input file for this plot is solenoid.g4bl:

* $\quad$ solenoid.g4bl - solenoid field lines

physics QGSP_BERT

coil $\mathrm{C}$ innerRadius $=199.5$ outerRadius $=200.5$ length $=2000$

solenoid $S$ coil $=C$ current $=1000$ color $=1,1,0, .5$

place $\mathrm{S} \mathrm{z}=0$

cylinder K outerRadius=20000 length=1 color="

place $\mathrm{Kz}=10000$

printfield field $=\mathrm{Bz}$ layout $=\mathrm{zx}$ drow $=50$ nrow $=100 \mathrm{dcol}=1$ ncol $=1 \mathrm{x}=0 \mathrm{y}=0 \mathrm{z}=0$

fieldlines center $=0,0,0$ radius $=200 \mathrm{dl}=1$ exit $=1$ nLines $=10$ 


\section{Test 2 - Helmholtz Coils field value}

From Reitz and Milford, Foundations of Electromagnetic Theory, p158, the field at the midpoint of a pair of Helmholtz coils is

$$
\mathrm{B}_{\mathrm{z}}=32 \pi \mathrm{N} \mathrm{I} /\left(5^{3 / 2} \text { a } 10\right)
$$

where $I$ is the current in amperes, a is the coil radius in $\mathrm{cm}, \mathrm{N}$ is the number of turns, and $\mathrm{B}_{\mathrm{z}}$ is given in gauss. The coil separation is of course equal to their radius. For $\mathrm{N}=1, \mathrm{I}=10000 \mathrm{~A}, \mathrm{a}=50$ $\mathrm{cm}$, this formula gives 179.84 gauss. G4beamline gives 0.017984 tesla, which is the same.

The input file for this test is helmholtz.g4bl:

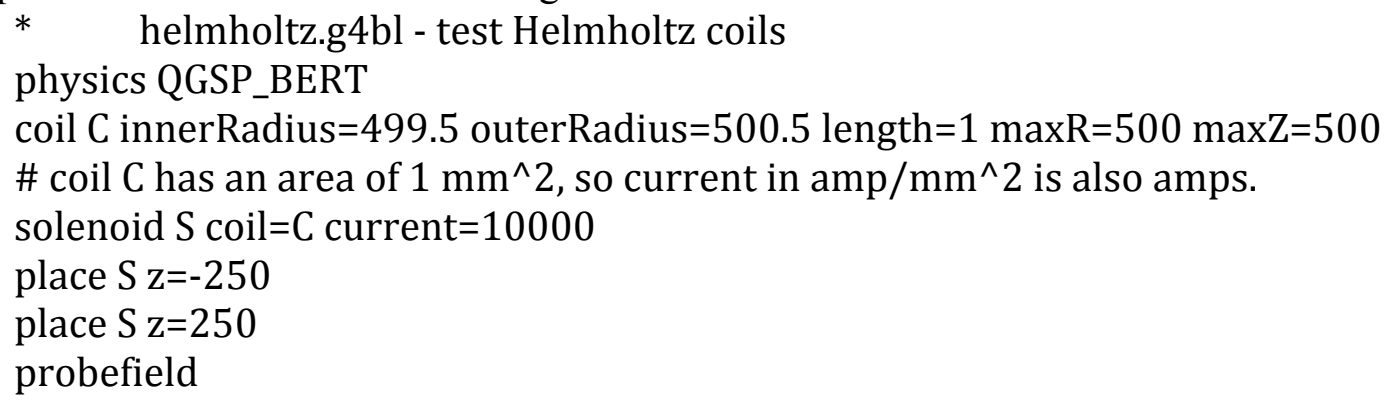

\section{Test 3 - On-Axis Field}

From R. Winch, Electricity and Magnetism, p380, on the axis of a solenoid the B field is:

$$
\mathrm{B}_{\mathrm{z}}=\mu_{0} \mathrm{NI}(\cos \alpha-\cos \beta) / 2 \mathrm{~L}
$$

Where $\mu_{0}$ is $4 \pi \times 10^{-7}, N$ is the number of turns, I is the current in amps, $L$ is the solenoid length in meters, and $\alpha$ and $\beta$ are angles from the point on the axis to the ends of the solenoid; $B_{z}$ is given in tesla. The following plot compares this formula to G4beamline, for a solenoid of radius 200 $\mathrm{mm}$ and a length of $2,000 \mathrm{~mm}$, centered at $\mathrm{z}=0$. The formula is a black line and the G4beamline data are red points: 


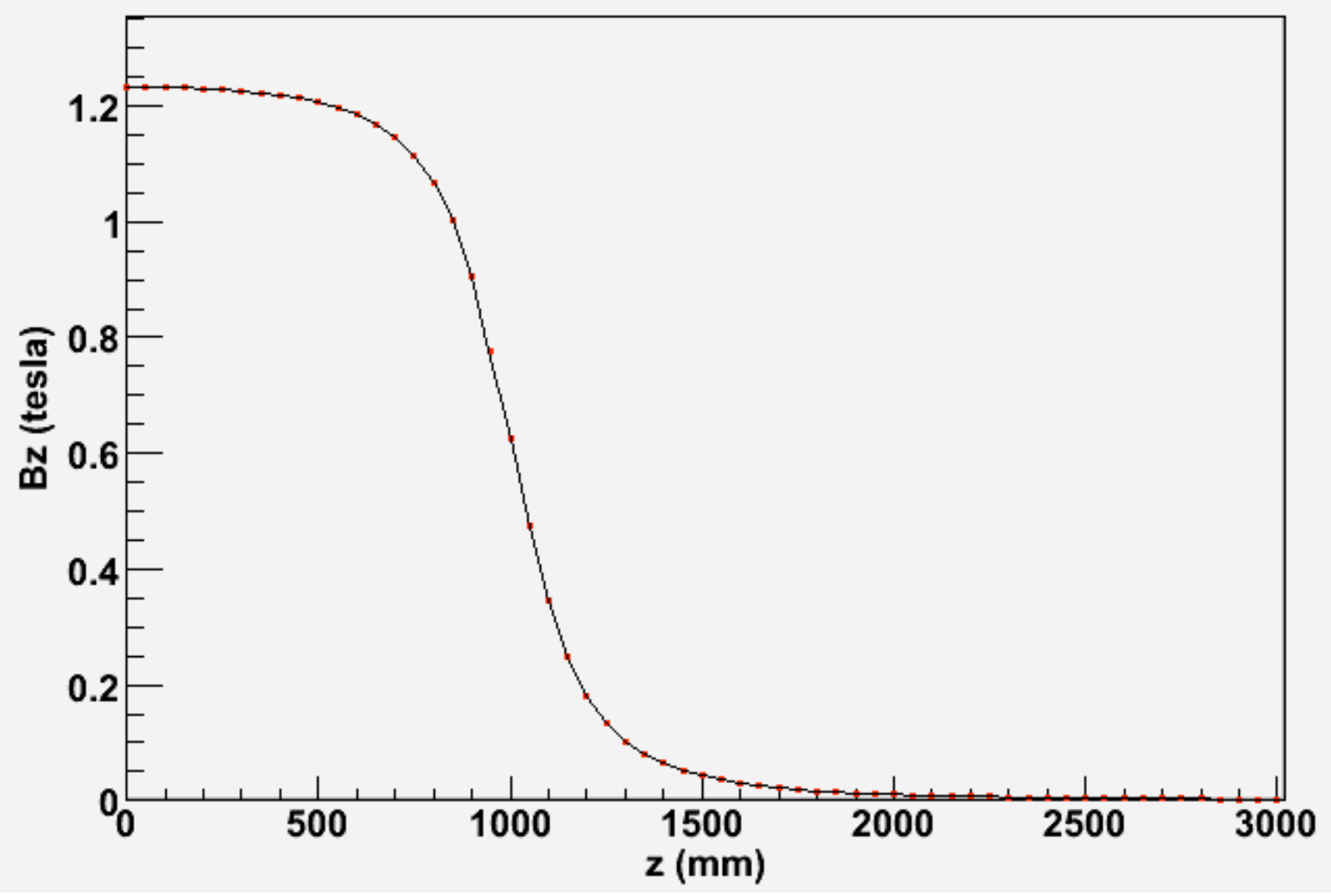

G4beamline creates a field map for the solenoid, in this case using the default tolerance of $0.1 \%$ to determine the grid spacing and extent of the map. Numerically, the two data series above differ by at most 0.0004 tesla $(0.03 \%)$ at every point within the map (which extends beyond the plot to $\mathrm{z}=3755 \mathrm{~mm}$ ), and at most by 0.0007 tesla $(0.06 \%)$ outside the map. The points plotted are not related to the field map's grid spacing.

The input file for this test is solenoid.g4bl:

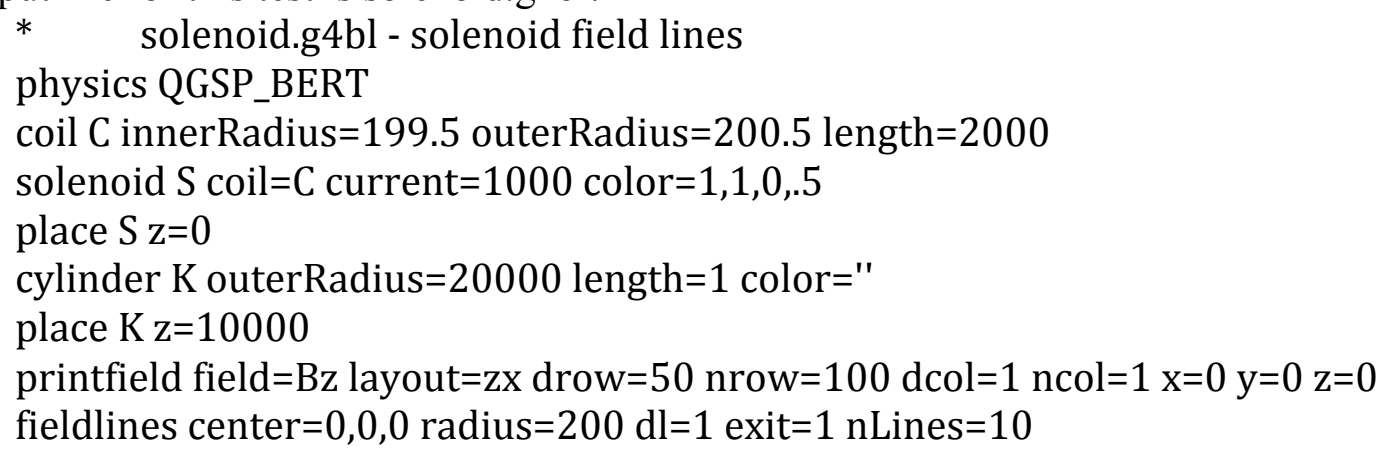

\section{Test 4 - Chaotic B field}

The CERN Courier [13] showed a plot of a chaotic B field from two circular current loops at right angles to each other. The plot of a single field line below is quite similar to the picture they showed. Indeed, moving the initial position of the field line by $0.1 \mathrm{~mm}$ changes its character completely. This is not due to the approximation introduced by the field map of the coil - using 
the exact computation shows similar behavior (but different in detail, as is expected for such chaotic behavior).

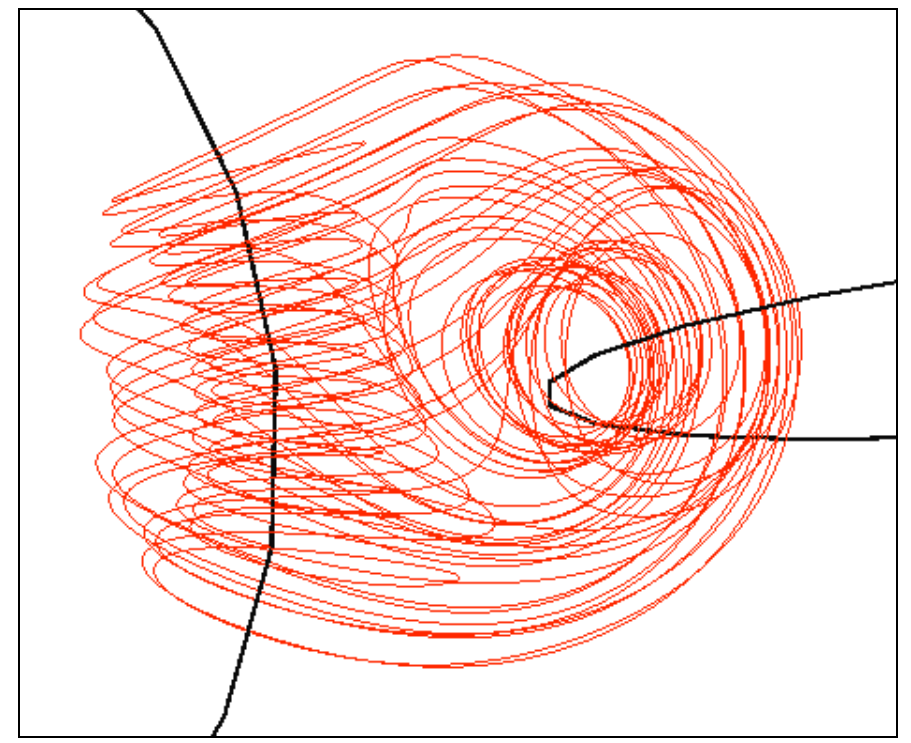

The input file for this test is chaos.g4bl:

* chaos.g4bl

physics QGSP_BERT

coil $\mathrm{C}$ innerRadius $=199.5$ outerRadius $=200.5$ length $=1$ nSheets $=1 \backslash$ exactComputation $=1$

solenoid $S$ coil $=C$ current $=10000$ color $=0,0,0$

box Huge height $=50000$ width $=50000$ length $=50000$ color $=$ invisible

place $S \mathrm{z}=0 \mathrm{x}=250$ parent=Huge

place $S \mathrm{z}=0 \mathrm{x}=-250$ parent=Huge rotation $=\mathrm{Y} 180, \mathrm{X} 90$

place Huge $\mathrm{z}=0$

g4ui when=4 "/vis/viewer/set/background 111 "

fieldlines exit $=1 \mathrm{nLines}=0$ forever $=1 \mathrm{dl}=167.1,0,0$ color $=1,0,0$

\section{2 genericbend}

This plot of field lines shows how artificial the region containing the field is - the field is valid only within the aperture, and within the aperture extended outside. In particular, the field is zero inside the iron. The fringe field does not include effects from the sides, only the top and bottom. The field lines are in 3-D, and this side view does not accurately reflect their density; it does show the behavior of the fringe fields. The field lines look reasonable; to really appreciate this you must run it and manipulate the image in real time to look at it from different directions. 


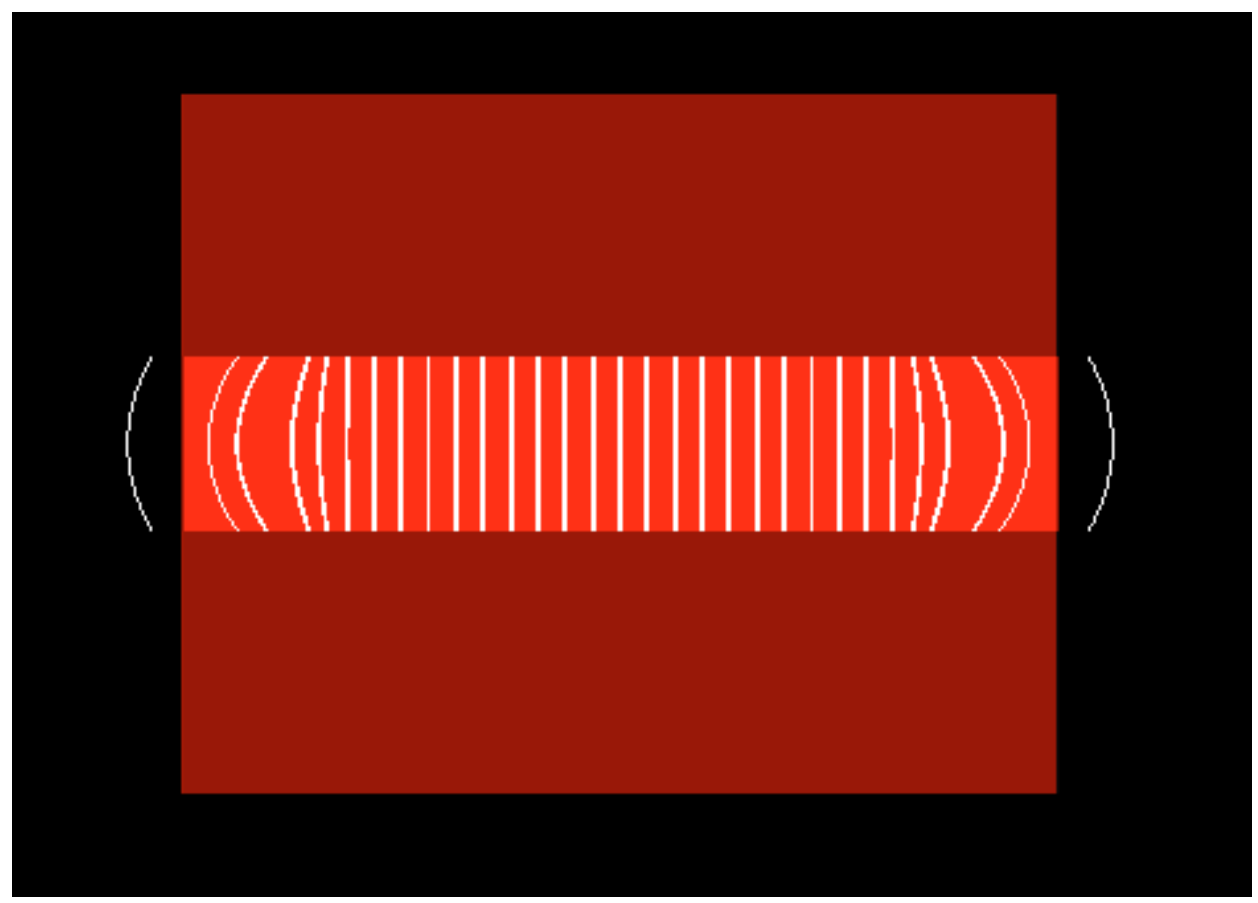

The following plot shows $\mathrm{B}_{\mathrm{y}}$ along the $\mathrm{z}$ axis, as seen by the $v_{\mu}$ beam particle trace.

\section{By vs. $z$}

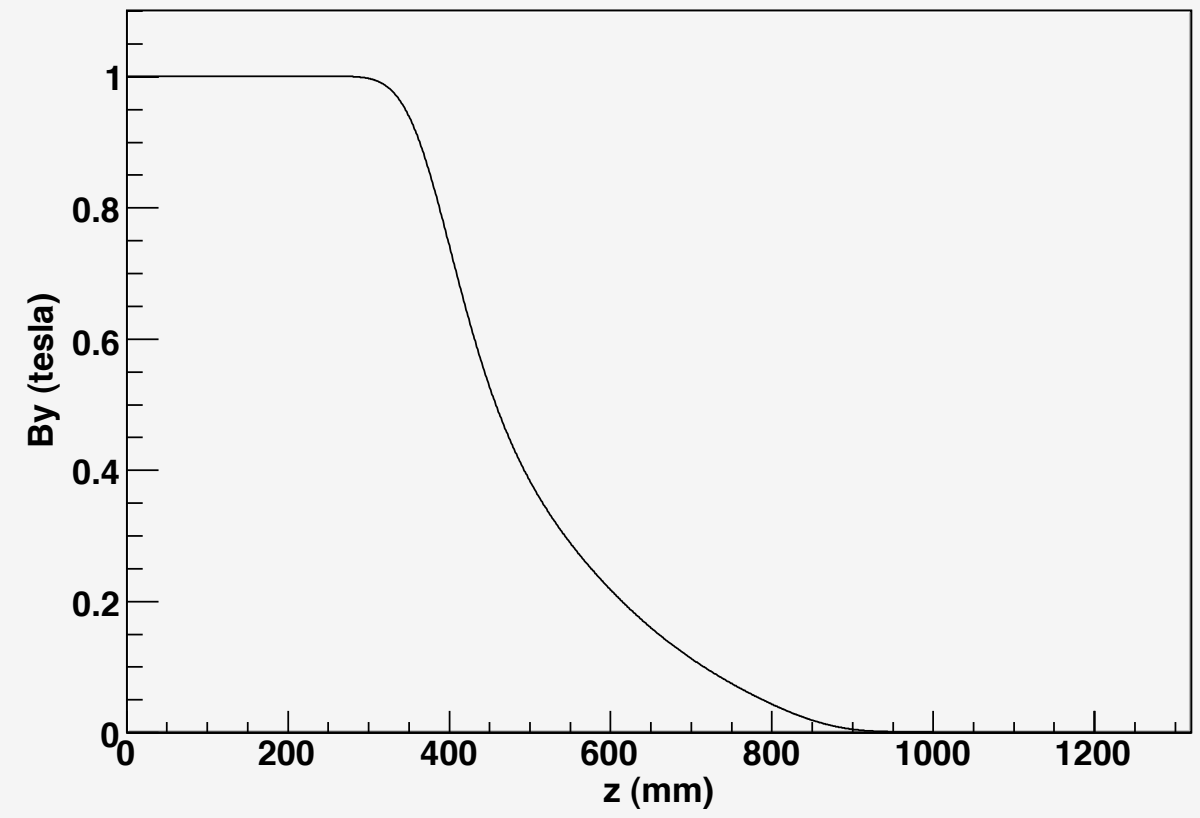

The sum of the $\mathrm{B}_{\mathrm{y}}$ entries in the trace file is 500.501 , corresponding approximately to the field integral $\int \mathrm{B}_{\mathrm{y}} \mathrm{dl}$ in tesla-mm. The correct value is 500.000 , so the fringe-field computation has indeed preserved the field integral, to within the accuracy of this simple integration technique. 
The input file for these two plots is bend.g4bl:

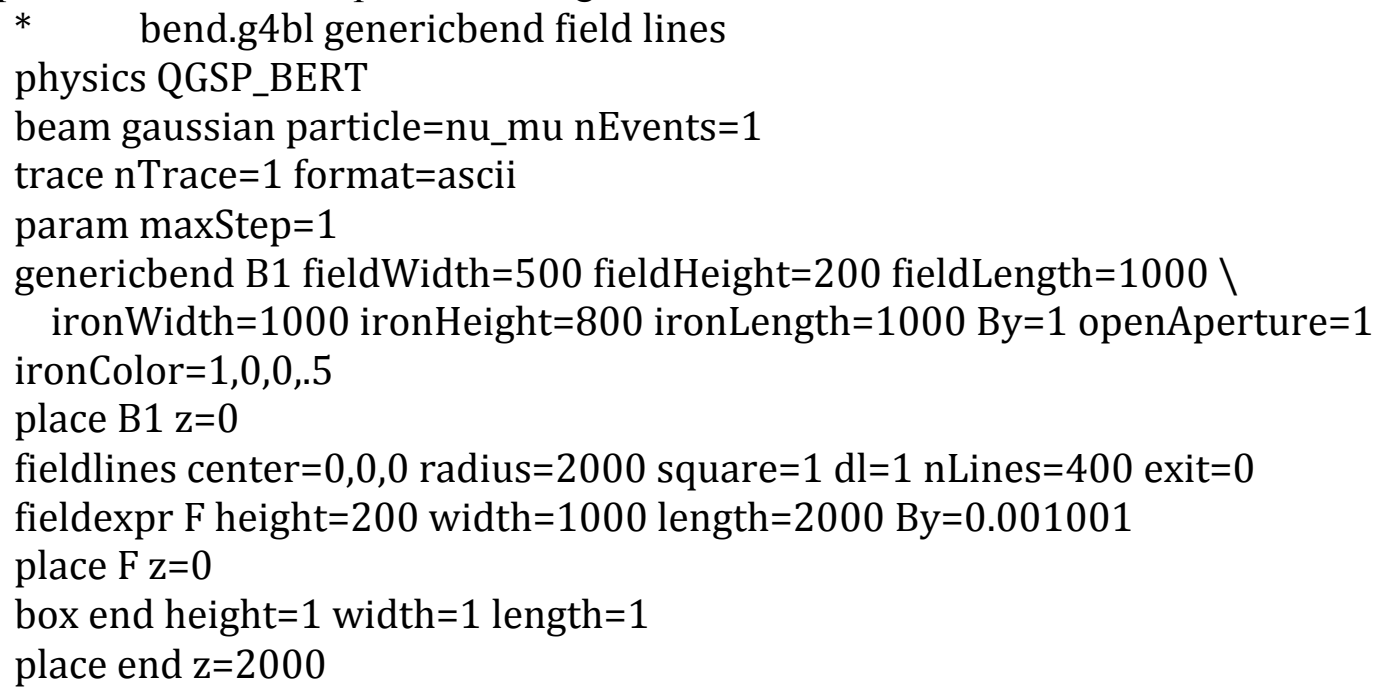

\section{3 genericquad}

This plot of field lines shows how artificial the region containing the field is - the field is valid only within the aperture, and within the aperture extended outside. In particular, the field is zero inside the iron. The field lines are in 3-D, and these views do not accurately reflect their density, but the side view does show the behavior of the fringe fields.
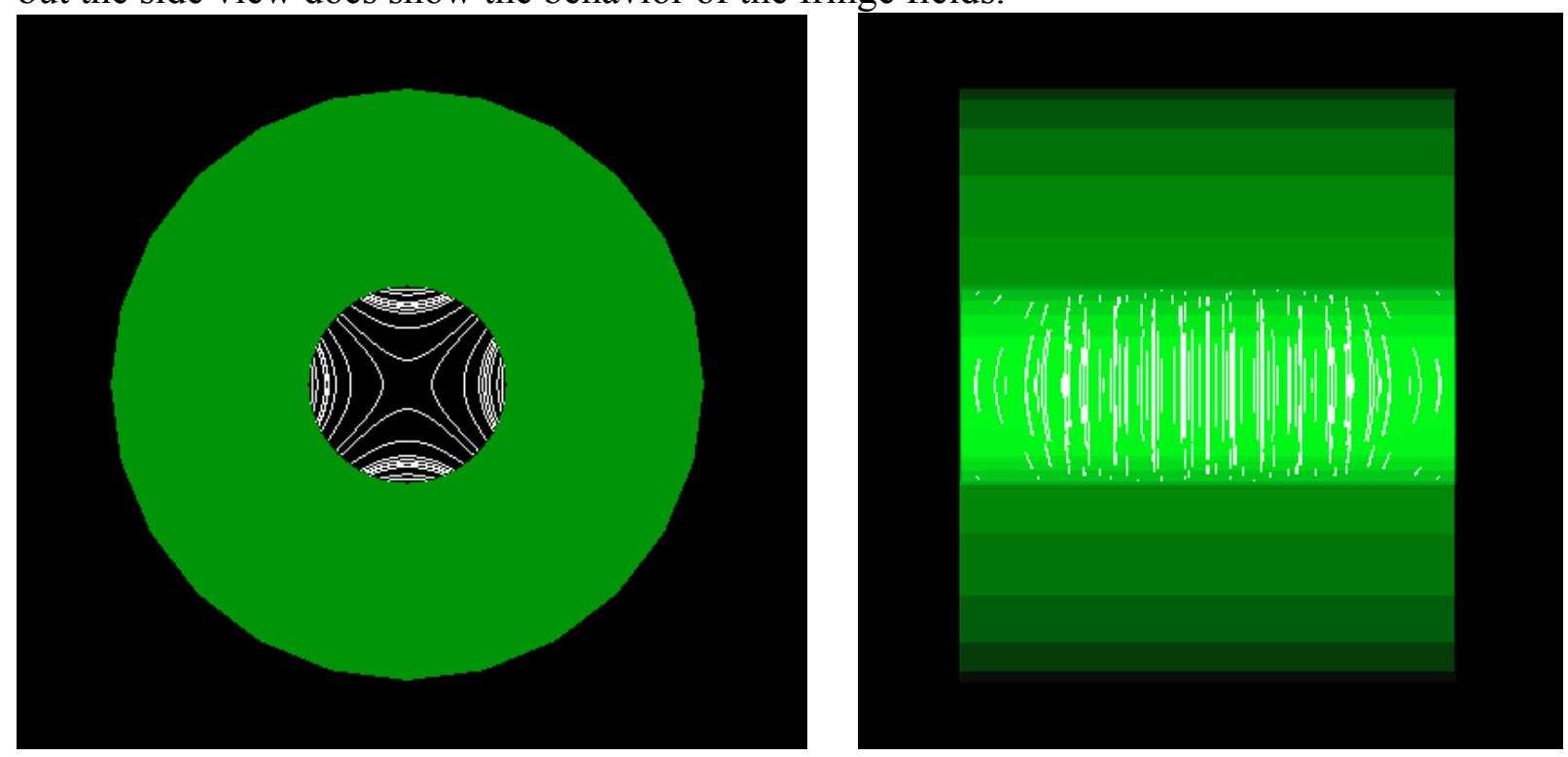

The input file for this test is quad.g4bl:

* $\quad$ quad.g4bl genericbend field lines physics QGSP_BERT genericquad Q1 fieldLength=1000 ironLength=1000 apertureRadius $=200 \backslash$ ironRadius $=600$ gradient $=4$ ironColor $=0,1,0,0.5$ place Q1 z=0

fieldlines center $=50,0,0$ radius $=200$ square $=1 \mathrm{dl}=1$ nLines $=10$ exit $=0$ 
fieldlines center $=-50,0,0$ radius $=200$ square $=1 \mathrm{dl}=1$ nLines $=10$ exit $=0$

fieldlines center $=0,-50,0$ radius $=200$ square $=1 \mathrm{dl}=1$ nLines $=10$ exit $=0$

fieldlines center $=0,50,0$ radius $=200$ square $=1 \mathrm{dl}=1$ nLines $=10$ exit $=1$

\section{Test 2}

Marco Apollonio has compared the G4beamline genericquad field to that of three TypeQC quadrupole magnets in a row. The following plot compares field maps generated by Tosca to the genericquad field. The mirror plates are 1-inch iron plates at the front and back to "reflect" the field and thus reduce the extent of the fringe field along the axis; genericquad has no mirror plates. The Tosca-generated maps were validated against measurements of one magnet, but the details have been lost. This is a large quadrupole magnet with a pole-tip radius of $171 \mathrm{~mm}$ and an overall length of $1,046 \mathrm{~mm}$; its large diameter-to-length ratio implies that fringe fields are important. Drawings of the magnet are on pages 11-12 of http://hep04.phys.iit.edu/cooldemo/micenotes/public/pdf/MICE0065/MICE0065.pdf.

The following plot is of $\mathrm{B}_{\mathrm{y}}$ along a line parallel to the $\mathrm{z}$ axis but off axis in $\mathrm{x}$.

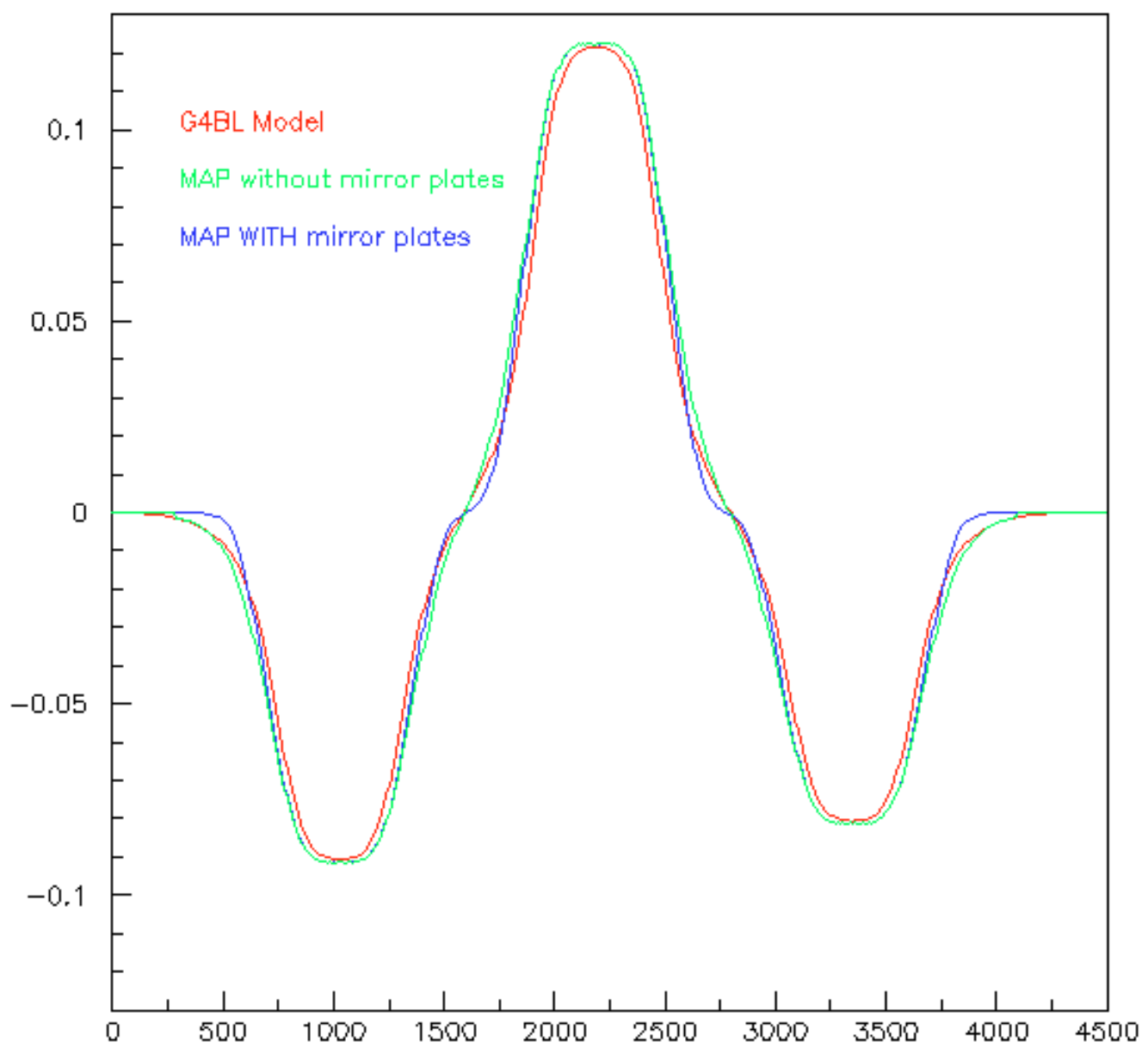




\section{4 idealsectorbend}

This plot of field lines shows how artificial the region containing the field is - the field is valid only within the aperture. In particular, the field is zero inside the iron. This element has no fringe field. The field lines look reasonable; to really appreciate this you must run it and manipulate the image in real time to look at it from different directions.

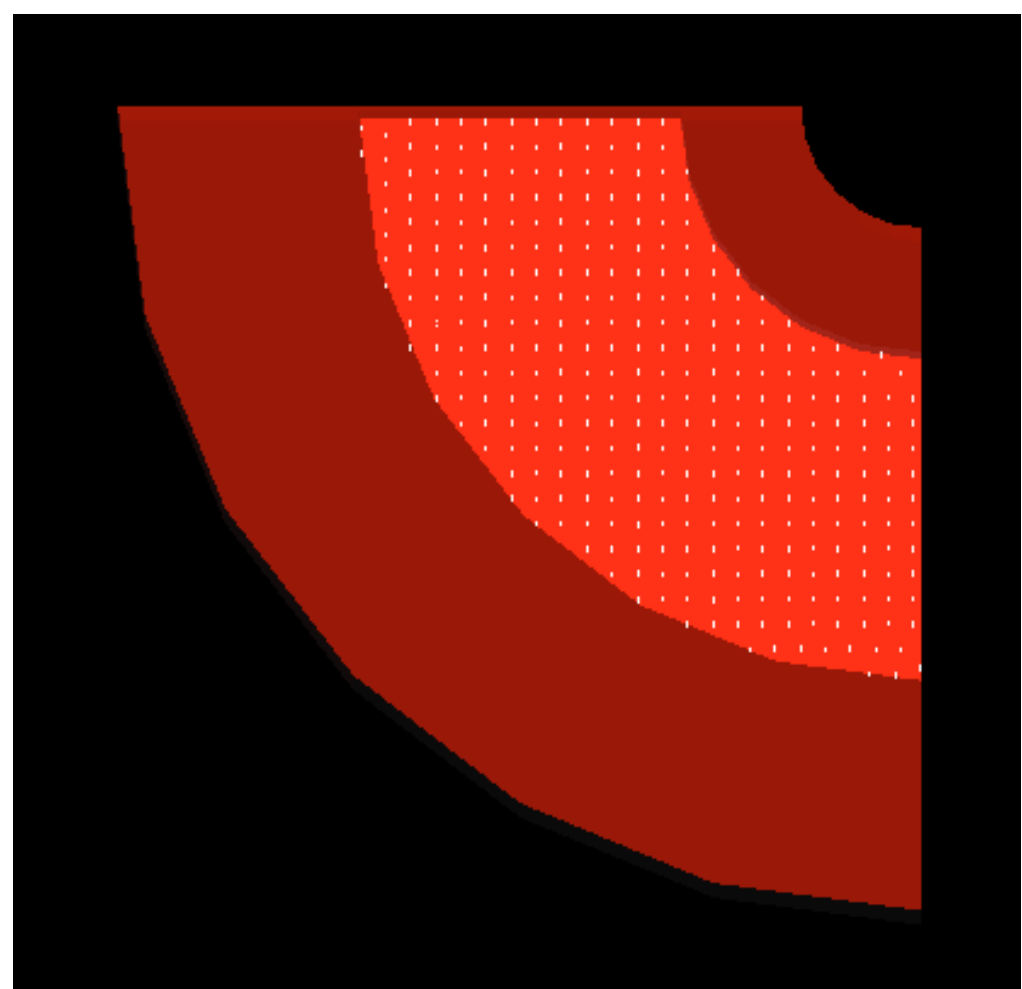

(Some field lines appear to be in the edge of the iron; they are not. This picture is rotated slightly to ensure the field lines are visible.)

The input file for this test is sectorbend.g4bl:

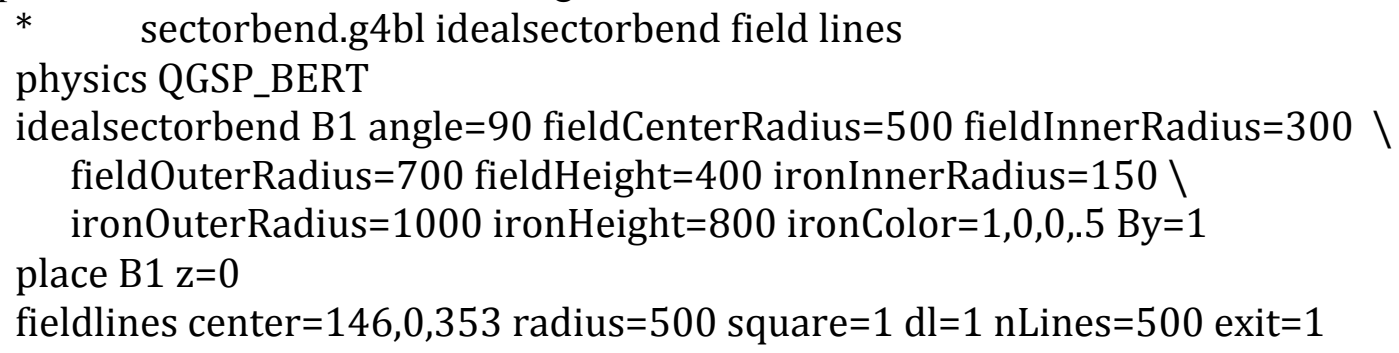

\section{5 fieldmap}

This plot of field lines shows a fieldmap with Bz proportional to $\mathrm{x}^{*} \mathrm{y}$, looking from the $\mathrm{z}$ axis; the field is valid only in a 1-meter cube, shown in dark gray. The field lines look reasonable; to really appreciate this you must run it and manipulate the image in real time to look at it from different directions. 


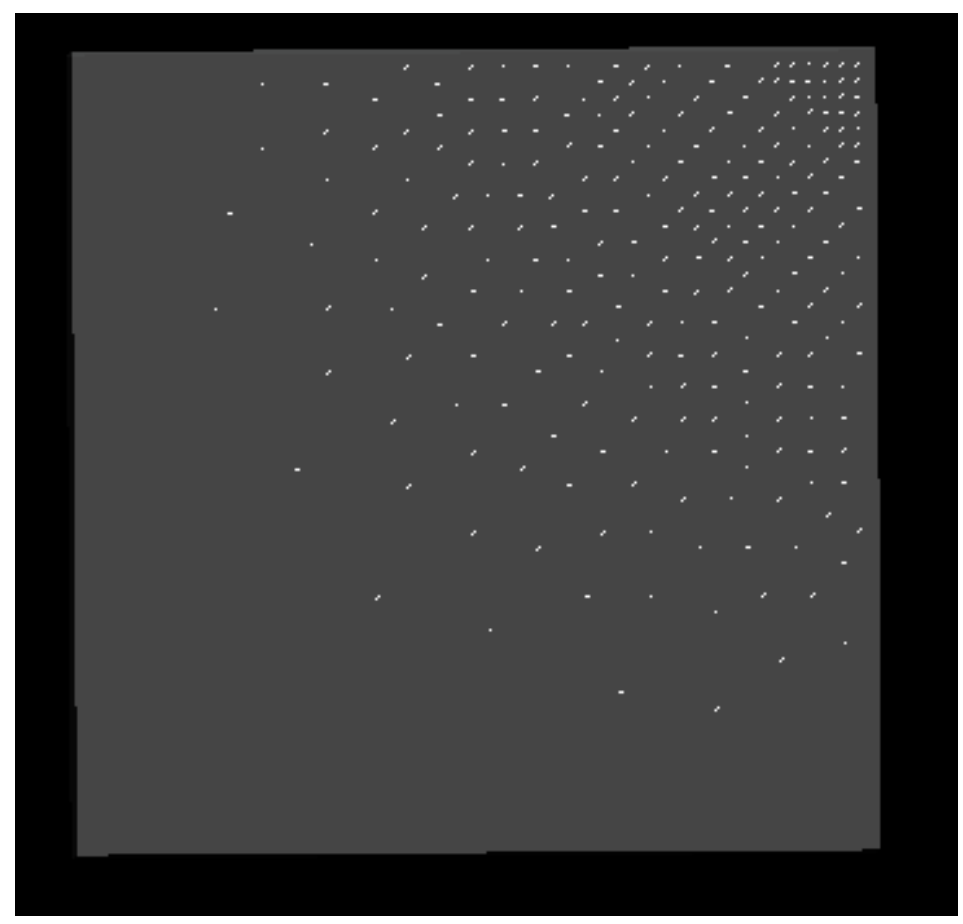

(The image is rotated slightly to ensure the field lines are visible. $\mathrm{x}=0, \mathrm{y}=0$ is in the lower left corner.)

The input file for this test is fieldmap.g4bl:

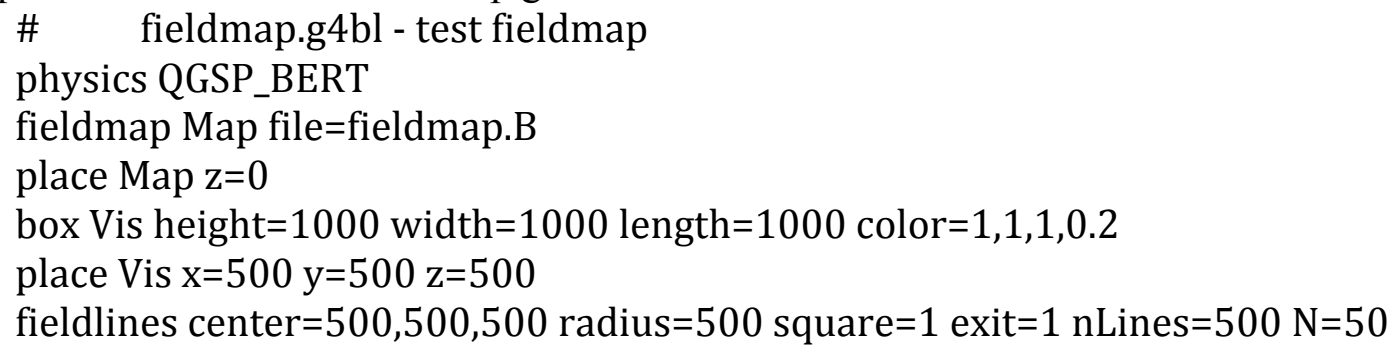

\section{6 fieldexpr}

This plot of field lines shows a fieldmap with Bz proportional to $\mathrm{x}^{*} \mathrm{y}$, looking from the $\mathrm{z}$-axis; the field is valid only in a 1-meter cube, shown in dark green. The field lines look reasonable; to really appreciate this you must run it and manipulate the image in real time to look at it from different directions. 


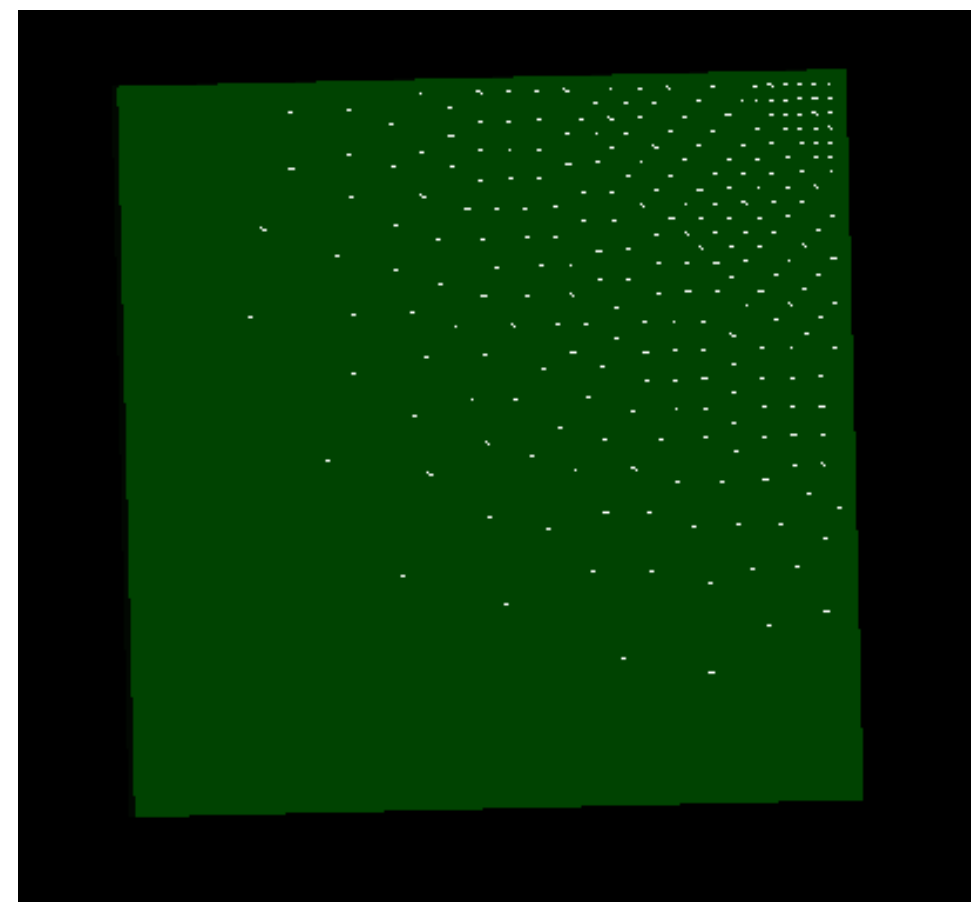

(The image is rotated slightly to ensure the field lines are visible. $\mathrm{x}=0, \mathrm{y}=0$ is in the lower left corner.)

The input file for this test is fieldexpr.g4bl:

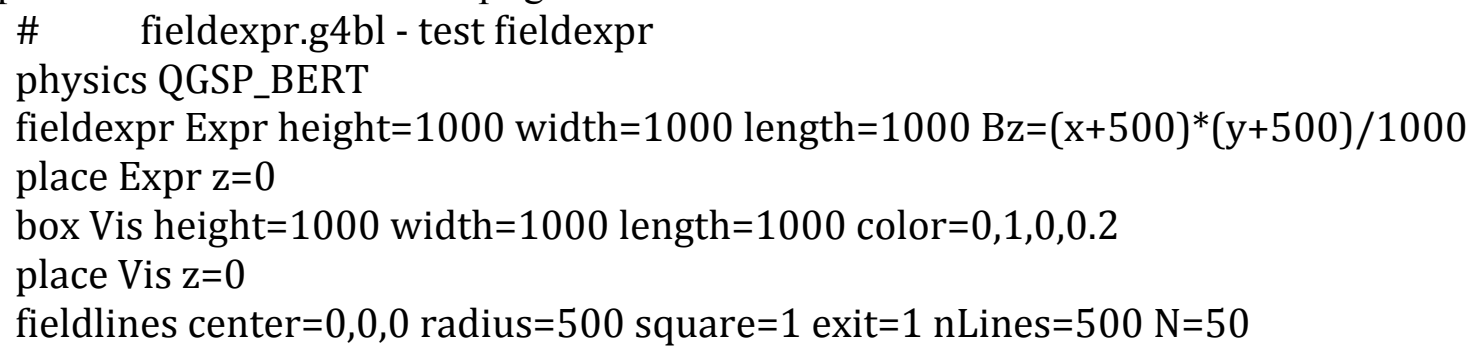

\section{7 multipole}

These plots of field lines show multipoles from dipole thru dodecapole, looking from the z-axis. The field lines look reasonable; to really appreciate this you must run it and manipulate the image in real time to look at it from different directions. The field lines are in 3-D, and these views do not accurately reflect their density. 

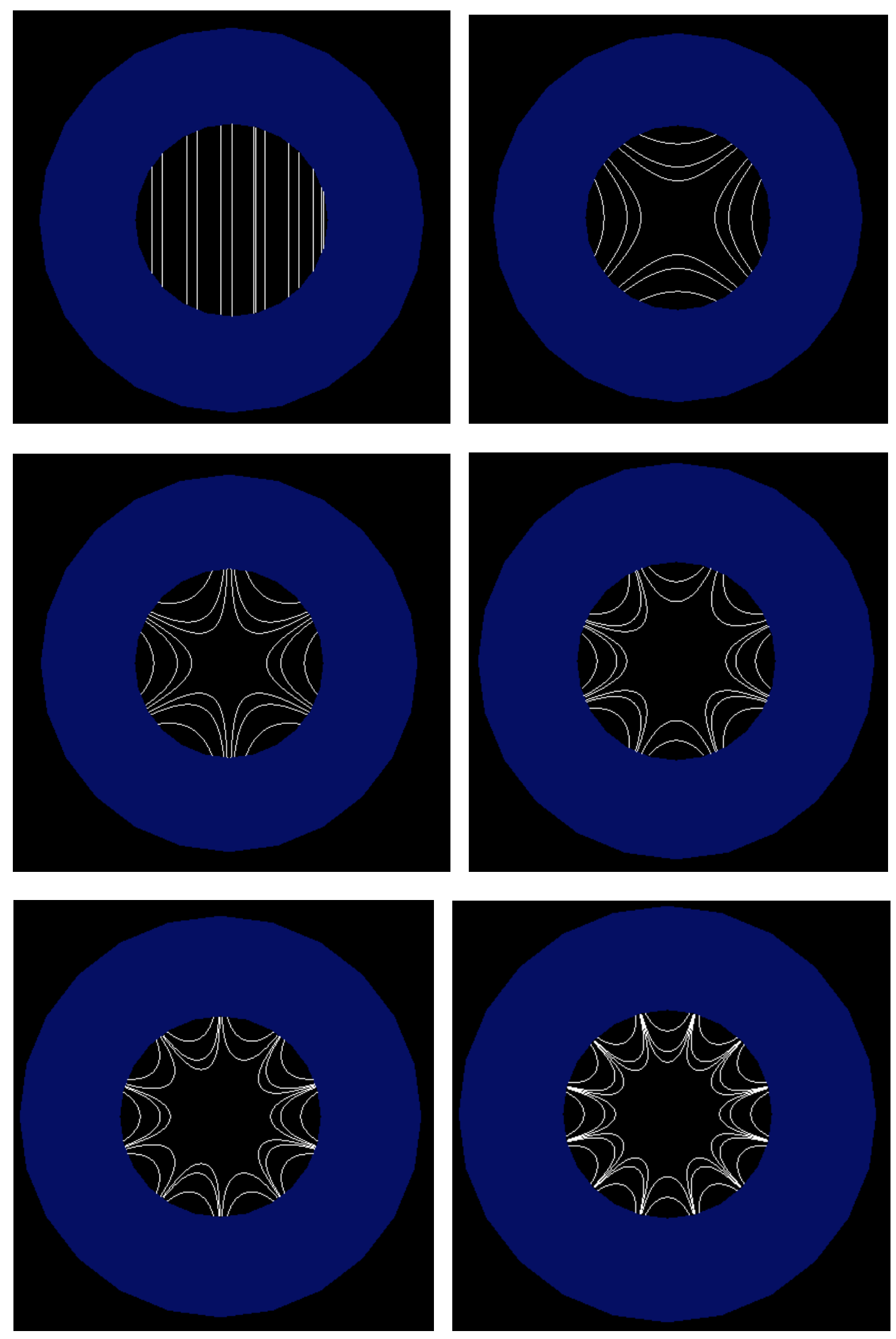
The input file for this test is multipole.g4bl:

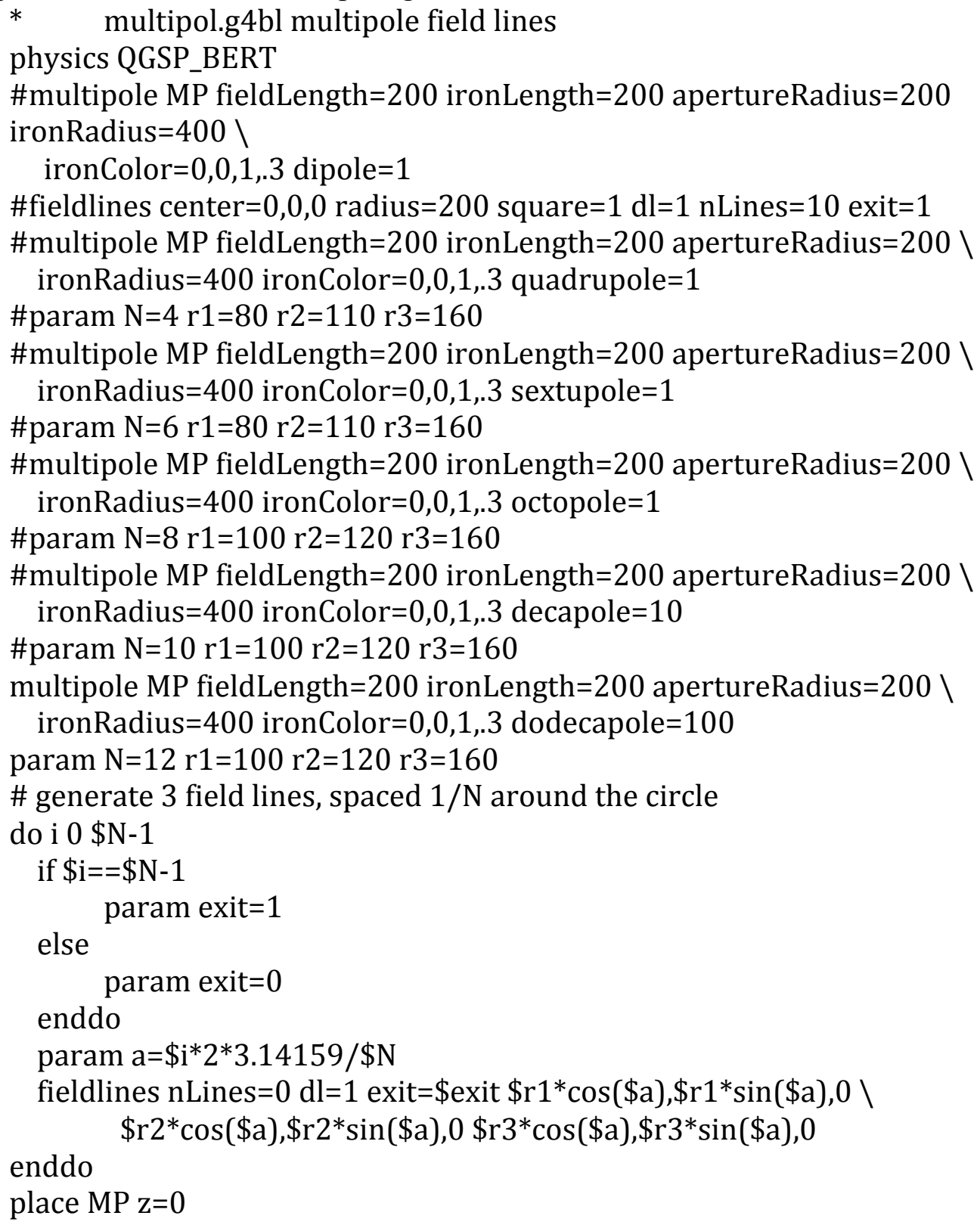

\section{8 pillbox}

These plots of E-field (left) and B-field (right) lines show a pillbox looking from the z-axis, at 90 degrees in the RF cycle (max fields). The field lines look reasonable; to really appreciate this you must run it and manipulate the image in real time to look at it from different directions). The inner red circle is a window for muon accelerators. The field lines are in 3-D, and these views do not accurately reflect their density. 

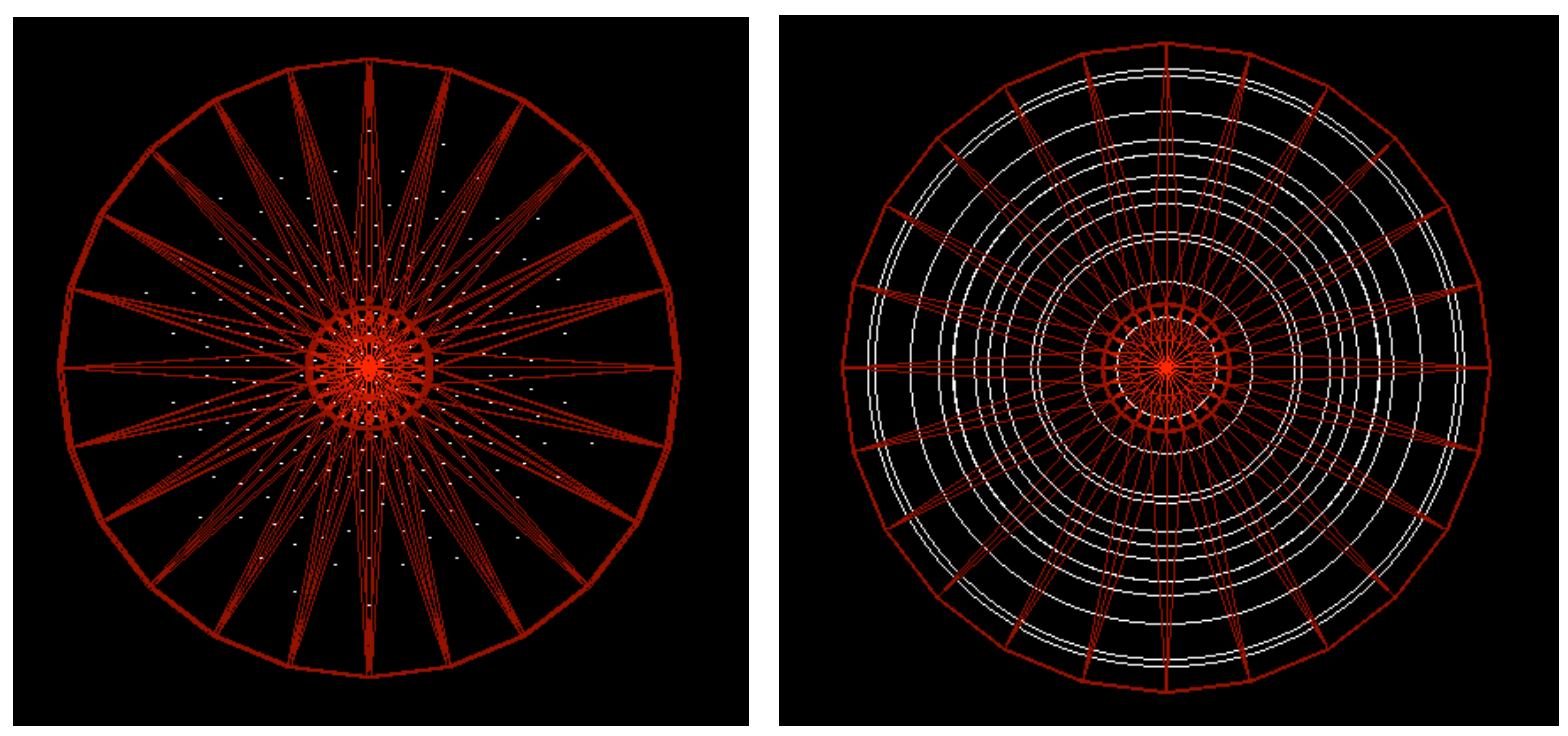

The input file for this test is pillbox.g4bl:

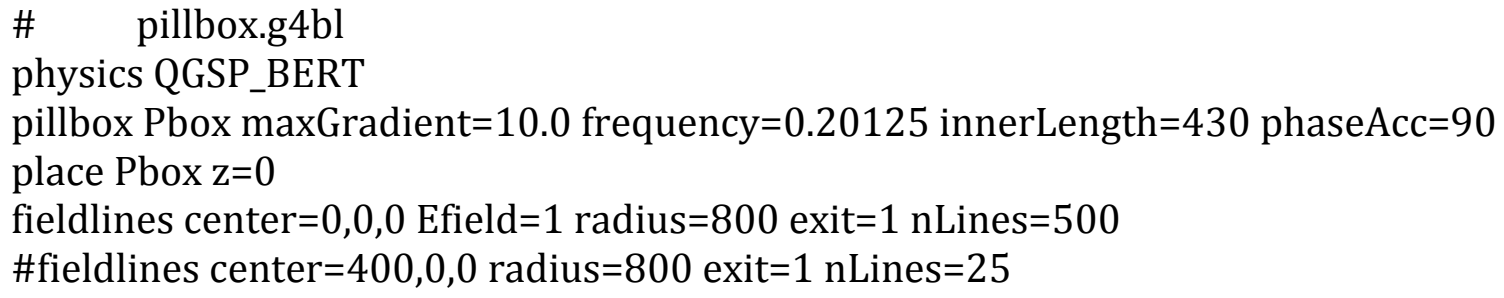

\section{Test 2}

The magnitude of the RF B-field has been compared to a pillbox modeled in Superfish by Milorad Popovic. The value was correct to $0.1 \%$. This was comparing the maximum B-field for a given accelerating voltage in a given pillbox geometry. The details have been lost.

\section{Test 3}

Shahid Ahmed of JLab has tested deflecting/crabbing cavity beam dynamics studies and made a comparison of simulations with different codes. The figures show comparison of deflection and displacement of an $11 \mathrm{GeV}$ electron passing through the axis of the superconducting deflecting cavity.

GPT : General particle tracer code

CST : CST microwave studio particle tracker 

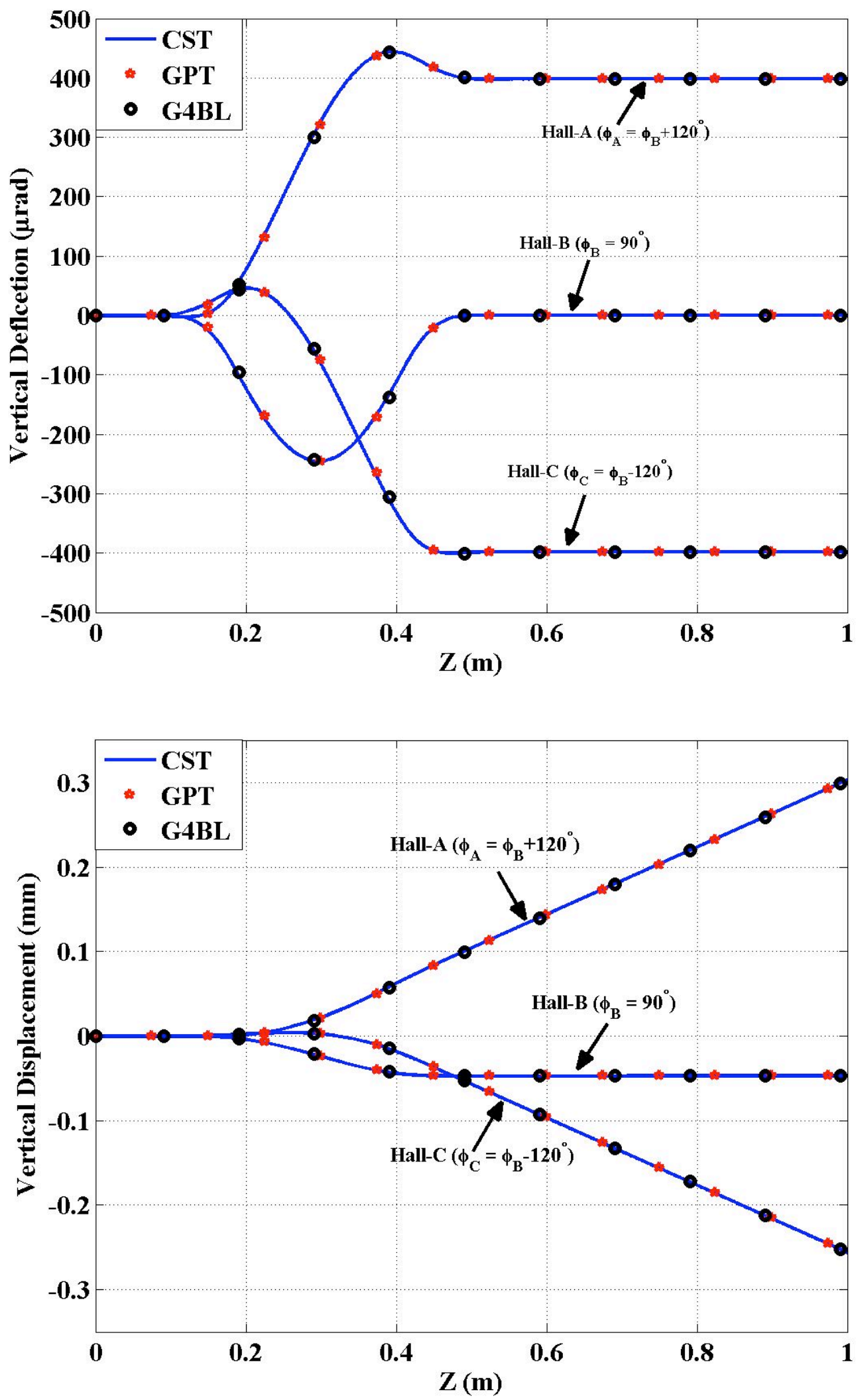


\section{9 helicaldipole}

\section{Test 1 - Field Lines}

Note that the solenoid field is much larger than the helical dipole field, so the field lines do not follow the reference particle's much broader helical trajectory.
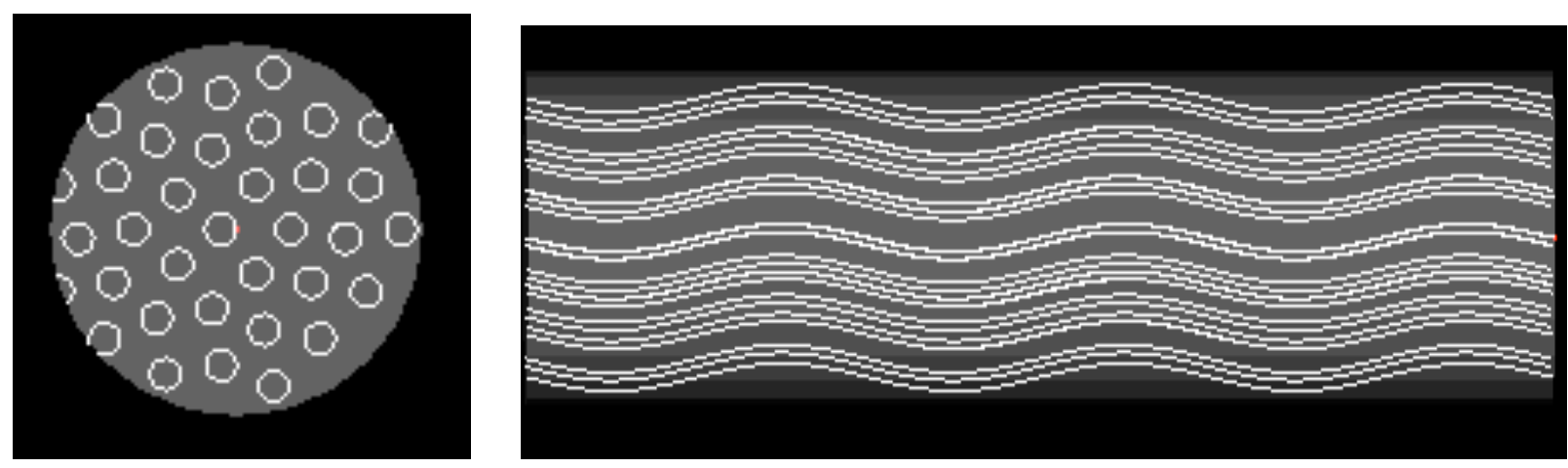

The input file for this test is helicaldipole.g4bl:

* helicaldipole.g4bl

physics QGSP_BERT

helicaldipole HelicalDipoleField radius $=320$ length $=3000.0 \backslash$

model $=1$ Bsolenoid $=5 \mathrm{bQ}=0.0 \mathrm{bD}=1.0$ phi0 $=0.0$ lambda $=1000.0$

place HelicalDipoleField $\mathrm{z}=1500$

tubs Vis outerRadius $=320$ length $=3000$ color $=1,1,1, .3$

place Vis $\mathrm{z}=1500$

fieldlines exit $=1$ center $=0,0,1500$ radius $=320$ nLines $=20 \mathrm{dl}=1$

\section{Test 2 - Comparison to Helical Solenoid}

The helicaldipole command is intended to permit simulation of helical structures involving helical dipole, quadrupole, and sextupole fields. It simply generates these fields according to its parameters. A more realistic way to generate these fields is to use a large number of thin coils with their centers placed along an appropriate helix, which has been named "helical solenoid". Katsuya Yonehara compared these two systems, using the following parameters:

\begin{tabular}{|l|l|l|l|}
\hline Parameter & Unit & Helical Solenoid magnet & helicaldipole module \\
\hline$\kappa$ & & 1.0 & 1.0 \\
\hline$\lambda$ & $\mathrm{mm}$ & 1000 & 1000 \\
\hline Parameters & & HS coil length $=25 \mathrm{~mm}$ & $\mathrm{bd}=1.41412$ \\
& & \# of coils per $\lambda=20$ & $\mathrm{bQ}=-0.210448$ \\
& & HS coil inner radius $=350 \mathrm{~mm}$ & $\mathrm{bsol}=-5.61552$ \\
& & HS coil outer radius $=400 \mathrm{~mm}$ & \\
& & HS coil current $=-256 \mathrm{~A} / \mathrm{mm}^{2}$ & \\
& & Radius of coil center $=225 \mathrm{~mm}$ & \\
\hline
\end{tabular}

The following values of the two systems compare quite well:

\begin{tabular}{|l|l|l|l|}
\hline Value & Unit & Helical Solenoid Magnet & helicaldipole module \\
\hline $\mathrm{b}$ & $\mathrm{T}$ & -1.3084 & -1.3093 \\
\hline
\end{tabular}




\begin{tabular}{|l|l|l|l|}
\hline $\mathrm{b}^{\prime}(=\partial \mathrm{b} / \partial \rho)$ & $\mathrm{T} / \mathrm{m}$ & 0.5349 & 0.5435 \\
\hline $\mathrm{b} / \mathrm{b}^{\prime}$ & $\mathrm{m}$ & -2.4460 & -2.4089 \\
\hline $\mathrm{bz}$ & $\mathrm{T}$ & -4.3069 & -4.3040 \\
\hline $\mathrm{p}$ (from analytical formula) & $\mathrm{GeV} / \mathrm{c}$ & 0.20233 & 0.20208 \\
\hline $\mathrm{p}$ (from particle tracking) & $\mathrm{GeV} / \mathrm{c}$ & 0.20234 & 0.20234 \\
\hline$\hat{D}$ & & 1.4505 & 1.4436 \\
\hline$\eta$ & & 0.4803 & 0.4775 \\
\hline $\mathrm{Q}_{+}$ & & 0.8747 & 0.8747 \\
\hline $\mathrm{Q}_{-}$ & & 0.7847 & 0.7856 \\
\hline
\end{tabular}

The following plots compare the equilibrium orbits for helicaldipole (blue) to the helical solenoid (red). The discrepancies between the two systems are at the $0.01 \mathrm{~mm}$ level.
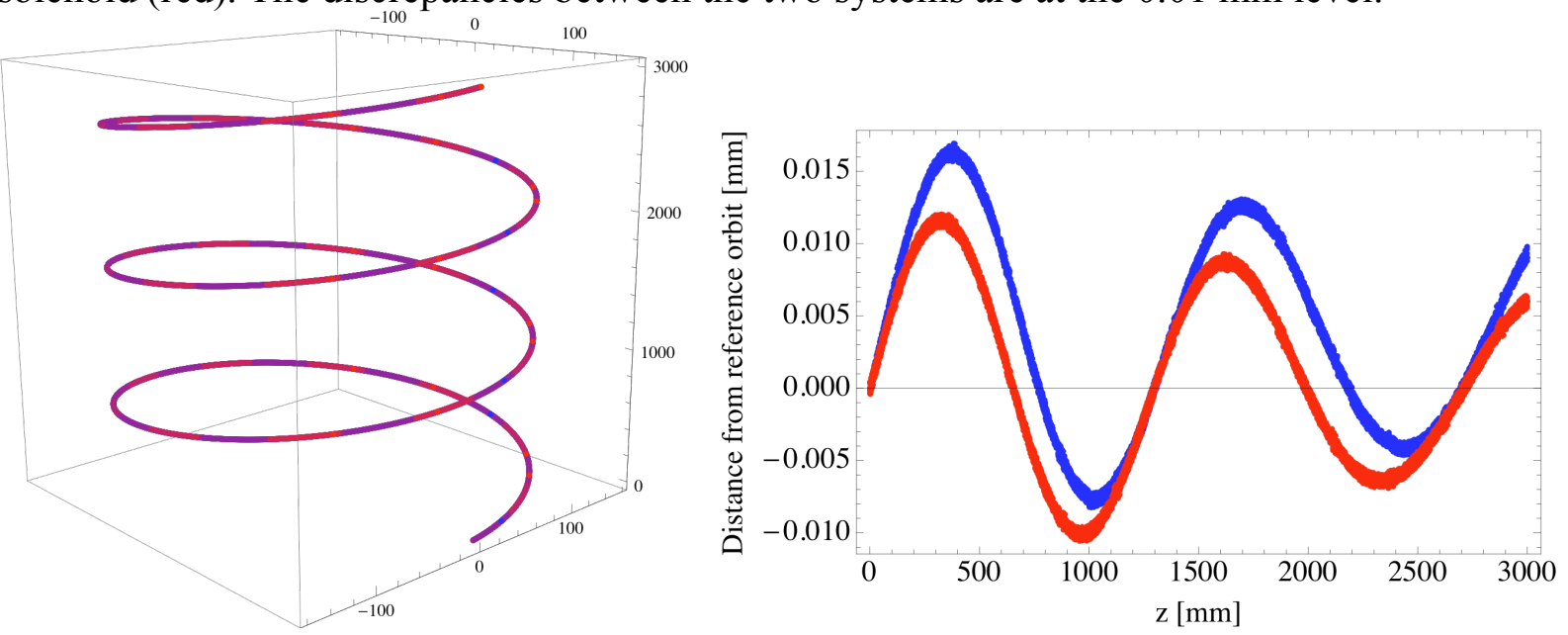

\subsection{0 absorber}

The absorber command uses polycones and tubs to implement a complex absorber geometry.

Visualization and tracking are consistent with its definition. The details have been lost.

\subsection{1 material}

G4beamline materials use the NIST database implemented by the Geant 4 collaboration. Here is a comparison of selected materials between G4beamline and the PDG "Particle Physics Booklet", July 2008:

\begin{tabular}{|c|c|c|c|c|c|c|}
\hline \multirow[t]{2}{*}{ Material } & \multicolumn{2}{|c|}{ Density $\left(\mathrm{g} / \mathrm{cm}^{3}\right)$} & \multicolumn{2}{|c|}{ Radiation Length (m) } & \multicolumn{2}{|c|}{ Nucl. Interact. Length (m) } \\
\hline & G4beamline & PDG & G4beamline & PDG & G4beamline & PDG \\
\hline LH2 & 0.07080 & 0.071 & 8.904 & 8.879 & 4.982 & 7.324 \\
\hline $\mathrm{LiH}$ & 0.820 & 0.820 & 0.971 & 0.971 & 0.732 & 0.830 \\
\hline $\mathrm{He}$ & 0.000166 & 0.000166 & 5,671 & 5,682 & 3,343 & 4,277 \\
\hline $\mathrm{Li}$ & 0.534 & 0.534 & 1.550 & 1.550 & 1.252 & 1.335 \\
\hline $\mathrm{Be}$ & 1.848 & 1.848 & 0.353 & 0.353 & 0.395 & 0.421 \\
\hline $\mathrm{C}$ & 2.000 & 2.210 & 0.213 & 0.193 & 0.401 & 0.193 \\
\hline $\mathrm{Al}$ & 2.699 & 2.699 & 0.0890 & 0.0889 & 0.389 & 0.397 \\
\hline
\end{tabular}




\begin{tabular}{|c|c|c|c|c|c|c|}
\hline $\mathrm{Fe}$ & 7.874 & 7.874 & 0.0176 & 0.0176 & 0.170 & 0.167 \\
\hline $\mathrm{Pb}$ & 11.350 & 11.350 & .00561 & .00561 & 0.182 & 0.175 \\
\hline $\mathrm{U}$ & 18.950 & 18.950 & .003166 & .003166 & 0.114 & 0.110 \\
\hline
\end{tabular}

The densities and radiation lengths are consistent, but the nuclear interactions lengths differ substantially. It's not clear what this means. Note that G4beamline (Geant4) tracking does not use the nuclear interaction length directly, instead it uses a more accurate and much more complicated technique based on cross-sections and densities.

The input file for this test is material.g4bl:

material Ignore density=1 LH2,0.1 He,0.1 Li,0.1 LITHIUM_HYDRIDE,0.1 C,0.1 \ $\mathrm{Be}, 0.1 \mathrm{Al}, 0.1 \mathrm{Fe}, 0.1 \mathrm{U}, 0.1 \mathrm{~Pb}, 0.1$ material

\subsection{2 cosmicraybeam}

This command does not reproduce recent measurements very well (factors of 2-4), and needs to be completely re-done. 


\section{Visualization}

Visualization has been used extensively during the development and application of G4beamline. Literally thousands of images have been examined. Most users use the Open Inventor viewer, but the other supported viewers have all been tested and used at least occasionally. The wireframe mode in Open Inventor sometimes renders an object in reduced wireframe mode (i.e. only real lines are displayed, no surface-tracing triangles are used). In addition, Boolean operations between solids sometimes display incorrectly (a well-known bug in the Geant4 visualization system, which is being fixed). In no cases have the locations or sizes of objects been rendered incorrectly.

\section{Test 1}

As a test of visualization consistency with tracking, a 10-by-10 array of cylinders was constructed, and tracks were sent into it. The cylinders hit by the tracks are identified by the steppingVerbose output, which can be compared to the picture below (1,1 is lower left in red, 10,10 is upper right). Careful examination in the viewer showed which cylinders were hit and which were missed, in agreement with the steppingVerbose output from tracking.

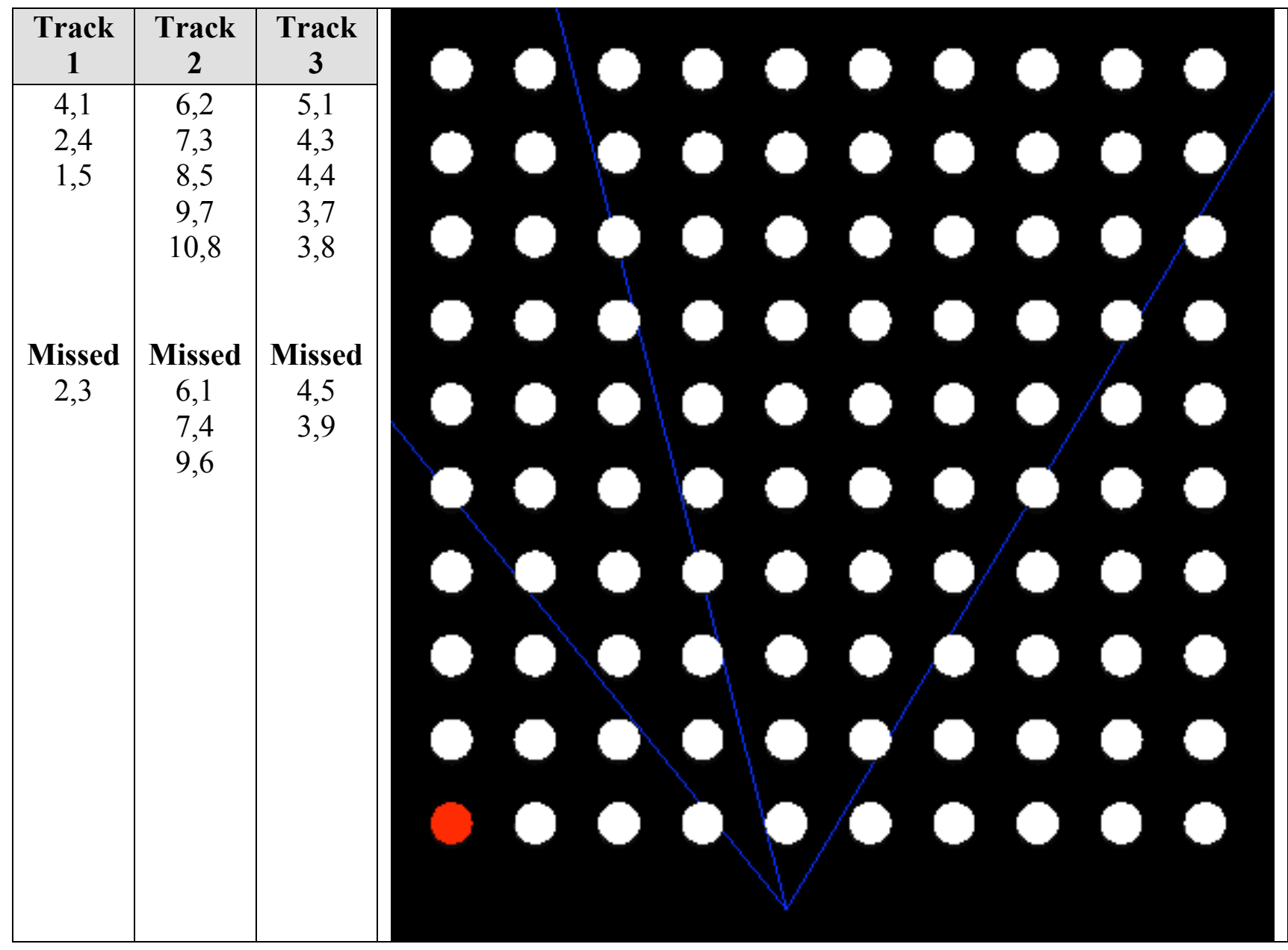


The input file for this test is visualization.g4bl:

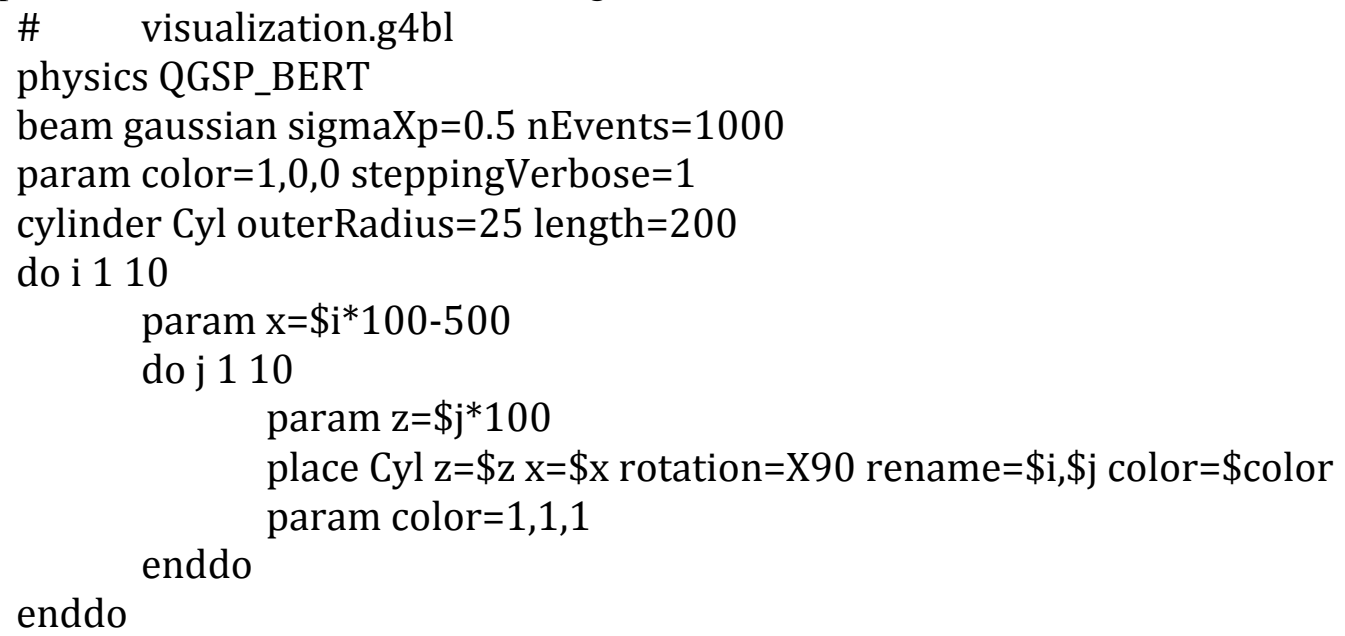




\section{References}

[1] http://g4beamline.muonsinc.com

[2] http://geant4.cern.ch

[3] http://root.cern.ch

[4] http://wwwasd.web.cern.ch/wwwasd/lhc++/clhep/index.html

[5] F. James, Comp. Phys. Comm. 60 (1990) 329.

[6] http://arxiv.org/abs/hep-ex/0512005

[7] http://pdg.lbl.gov/2010/reviews/rpp2010-rev-passage-particles-matter.pdf

[8] http://mu2e.fnal.gov/

[9] T. J. Roberts, "Analysis of Geant4 Physics Processes for $\mu^{-}$Capture at Rest", http://mu2e-docdb.fnal.gov:8080/cgi-bin/ShowDocument?docid=1119

[10] http://www-ap.fnal.gov/MARS/

[11] http://www.fftw.org/

[12] Qiang et al, Phys. Rev. STAB, 9, 044204 (2006).

Hockney and Eastwood, Computer Simulation Using Particles, Hilger, 1988, section 6-5-4, p200.

[13] CERN Courier 50, no. 1, p10 (2010). 